\title{
Imaging Crystal/Spectral Line Search
}

\author{
J. A. Koch
}

February 16, 1998

This is an informal report intended primarily for internal or limited external distribution. The opinions and conclusions stated are those of the author and may or may not be those of the Laboratory.

Work performed under the auspices of the U.S. Department of Energy by the Lawrence Livermore National Laboratory under Contract W-7405-ENG-48. 


\section{DISCLAIMER}

This document was prepared as an account of work sponsored by an agency of the United States Government. Neither the United States Government nor the University of California nor any of their employees, makes any warranty, express or implied, or assumes any legal liability or responsibility for the accuracy, completeness, or usefulness of any information, apparatus, product, or process disclosed, or represents that its use would not infringe privately owned rights. Reference herein to any specific commercial product, process, or service by trade name, trademark, manufacturer, or otherwise, does not necessarily constitute or imply its endorsement, recommendation, or favoring by the United States Government or the University of California. The views and opinions of authors expressed herein do not necessarily state or reflect those of the United States Government or the University of California, and shall not be used for advertising or product endorsement purposes.

This report has been reproduced directly from the best available copy.

Available to DOE and DOE contractors from the

Office of Scientific and Technical Information P.O. Box 62, Oak Ridge. TN 37831

Prices available from (615) 576-8401, FTS 626-8401

Available to the public from the

National Technical Information Service

U.S. Department of Commerce

5285 Port Royal Rd.,

Springfield, VA 22161 
Jeffrey A. Koch

Lawrence Livermore National Laboratory P.O. BOX $808, . L-473$ Livermore, CA 94550

$2 / 16 / 98$

\section{Imaging crystal/spectral Line search}

The following table is a compilation of chance coincidences between $x$-ray line wavelengths and crystal planes which will reflect those wavelengths near normal incidence. The motivation is to explore the possibilities for expanding the range of choices for near normal incidence $x$-ray crystal imaging. The crystal data were taken from five sources:

[1] B. I. Henke et al., Atomic Data and Nuclear Data Tables 54(2) (1993).

[2] H. T. Yamada et al., in Lawrence Berkeley Laboratory Report LBL-22800 (1986).

[3] J. H. Underwood, in "X-ray Data Booklet", LBL/Center for X-ray Optics (1986).

[4] A. Faenov, personal communication (1997). (mica 002 only)

[5] T. Barbee Jr., personal communication (1997). (tungsten 110 only)

$X$-ray spectral data were taken from two sources:

[6] R. L. Kelly, J. Phys. Chem. Ref. Data 16, Supp. 1 (1987) (computer version, $z=1-36$ ).

[7] J. B. Kortright, in "X-ray Data Booklet", LBL/Center for X-ray Optics (1986). (K $\alpha_{1}$ data only, $\mathrm{z}=1-50$ )

A FORTRAN code was written to read in files containing the above data, to systematically search for matches, and to output the results in a useful format. The search constraints were:

- Crystal 2d values were taken from [1] wherever possible except for mica and tungsten; in some cases, $2 d$ values vary from sample to sample (e.g. mica).

- Crystals with unknown Miller indices in [3] were ignored.

- Crystals with 2 d values specified to less than 4 significant figures in [3] were ignored.

- Crystals with 2d values greater than KAP (26.634 $\AA$ ) were ignored.

- The search was limited to bragg angles between 70 and 90 degrees relative to line-center. Angles below $~ 85$ degrees may be good candidates for toroidally-bent crystals.

No attempt was made to verify $2 d$ values, wavelengths, configurations, etc., and any errors in the references listed above will be reproduced here. However, every effort was made to avoid introducing additional errors. No attempt was made to rank the crystals in terms of suitability for highresolution imaging (mechanical properties, integrated reflectivity, mosaic vs. perfect crystal, etc.); the purpose of the compilation is to explore which crystals might be valuable for such further study. 


\begin{tabular}{|c|c|c|c|}
\hline quartz 502 & $2 \mathrm{~d}=1.624 \mathrm{~A}$ & topaz 200 & $2 d=4.638$ \\
\hline LiF 422 & $2 \mathrm{a}=1.652 \mathrm{~A}$ & Al 111 & $2 d=4.676$ \\
\hline corundum 1462 & $2 \mathrm{~d}=1.660 \mathrm{~A}$ & quartz 110 & $2 \mathrm{~d}=4.912$ \\
\hline LiF 420 & $2 \mathrm{~d}=1.801 \AA$ & gypsum 002 & $2 \mathrm{~d}=4.990 \mathrm{t}$ \\
\hline quartz 223 & $2 d=2.024 \AA$ & $\mathrm{NaCl} 200$ & $2 \mathrm{~d}=5.641$ \\
\hline Si 422 & $2 \mathrm{~d}=2.217 \AA$ & calcite 200 & $2 d=6.071$ \\
\hline Ge 422 & $2 \mathrm{~d}=2.310 \AA$ & Si 111 & $2 d=6.271$ \\
\hline quartz 310 & $2 d=2.360 \mathrm{~A}$ & sylvite 200 & $2 \mathrm{~d}=6.292$ \\
\hline quartz 220 & $2 \mathrm{~d}=2.451 \AA$ & fluorite 111 & $2 d=6.308$ \\
\hline topaz 303 & $2 \mathrm{~d}=2.712 \mathrm{~A}$ & Ge 111 & $2 \mathrm{a}=6.532$ \\
\hline corundum 0302 & $2 \mathrm{a}=2.748 \AA$ & $\mathrm{KBr} 200$ & $2 \mathrm{~d}=6.584$ \\
\hline quartz 203 & $2 \mathrm{~d}=2.749 \AA$ & quartz 101 & $2 \alpha=6.687 t$ \\
\hline topaz 006 & $2 \alpha=2.795 \AA$ & graphite 002 & $2 \mathrm{~d}=6.696$ \\
\hline LIF 220 & $2 \mathrm{~d}=2.848 \mathrm{~A}$ & Insb 111 & $2 \mathrm{~d}=7.481$ \\
\hline calcite 422 & $2 \mathrm{~d}=3.034 \mathrm{~A}$ & topaz 002 & $2 \mathrm{~d}=8.374$ \\
\hline quartz 211 & $2 \mathrm{~d}=3.082 \mathrm{~A}$ & quartz 100 & $2 \mathrm{~d}=8.512$ \\
\hline quartz 112 & $2 \mathrm{~d}=3.636 \AA$ & PET 002 & $2 a=8.742$ \\
\hline Si 220 & $2 \mathrm{~d}=3.840 \mathrm{~A}$ & EDT 020 & $2 \mathrm{~d}=8.808$ \\
\hline fluorite 2202 & $2 \mathrm{a}=3.862 \AA$ & $\mathrm{ADP} 101$ & $2 \mathrm{~d}=10.640$ \\
\hline $\mathrm{Ge} 220$ & $2 \mathrm{~d}=4.000 \AA$ & gypsum 020 & $2 \mathrm{~d}=15.185$ \\
\hline LiF 200 & $2 \mathrm{~d}=4.027 \mathrm{~A}$ & beryl 100 & $2 d=15.954$ \\
\hline Al 200 & $2 \mathrm{~d}=4.048 \mathrm{~A}$ & mica 002 & $2 \mathrm{~d}=19.942$ \\
\hline quartz 200 & $2 a=4.246 \mathrm{~A}$ & TAP 100 & $2 d=25.763$ \\
\hline tungsten 1102 & $2 \alpha=4.476 \mathrm{~A}$ & RAP 100 & $2 d=26.116$ \\
\hline quartz 112 & $2 \mathrm{~d}=4.564 \AA$ & $\mathrm{KAP} 100$ & $2 d=26.634$ \\
\hline
\end{tabular}

\begin{tabular}{|c|c|c|c|c|c|c|c|c|c|c|c|}
\hline & charge & configuration & term $j$ & configuration & term $j$ & wave. & crystal & order & $2 d$ & $\theta$ (bragg) \\
\hline ion & & 78 & & & & & & & & & \\
\hline C & 6 & H -like & $10 P$ & $\left(2 P^{*}\right) j=1.5$ & $-1 S$ & (2S) $j=.5$ & 25.559 & TAP 100 & 1 & 25.763 & 82.785 \\
\hline C & 6 & H -like & $10 P$ & $\left(2 \mathrm{P}^{\star}\right) j=1.5$ & $-1 S$ & $(2 S) j=.5$ & 25.559 & RAP 100 & 1 & 26.116 & 78.145 \\
\hline C & 6 & H -like & $10 P$ & $\left(2 P^{\star}\right) j=1.5$ & $-1 S$ & $(2 S) j=.5$ & 25.559 & KAP 100 & 1 & 26.634 & 73.666 \\
\hline $\mathrm{C}$ & 6 & H -like & $9 \mathrm{P}$ & $\left(2 \mathrm{P}^{\star}\right) j=1.5$ & $-1 S$ & $(2 S) j=.5$ & 25.619 & TAP 100 & 1 & 25.763 & 83.939 \\
\hline C & 6 & H -like & $9 P$ & $\left(2 p^{*}\right) j=1.5$ & $-1 S$ & $(2 S) j=.5$ & 25.619 & RAP 100 & 1 & 26.116 & 78.804 \\
\hline C & 6 & H -like & $9 P$ & $\left(2 P^{*}\right) j=1.5$ & $-1 s$ & $(2 S) j=.5$ & 25.619 & KAP 100 & 1 & 26.634 & 74.131 \\
\hline C & 6 & H -like & $8 \mathrm{P}$ & $\left(2 P^{\star}\right) j=1.5$ & $-1 S$ & $(2 S) j=.5$ & 25.705 & TAP 100 & 1 & 25.763 & 86.155 \\
\hline $\mathrm{C}$ & 6 & $\mathrm{H}$-like & $8 \mathrm{P}$ & $\left(2 P^{*}\right) j=1.5$ & $-1 S$ & $(2 S) j=.5$ & 25.705 & RAP 100 & 1 & 26.116 & 79.822 \\
\hline C & 6 & H-like & $8 \mathrm{P}$ & $\left(2 P^{*}\right) j=1.5$ & $-1 S$ & $(2 S) j=.5$ & 25.705 & KAP 100 & 1 & 26.634 & 74.823 \\
\hline c & 6 & H -like & $7 P$ & $\left(2 P^{\star}\right) j=1.5$ & -15 & $(2 S) j=.5$ & 25.830 & RAP 100 & 1 & 26.116 & 81.513 \\
\hline C & 6 & H -like & $7 P$ & $\left(2 \mathrm{P}^{*}\right) j=1.5$ & $-1 S$ & $(2 S) j=.5$ & 25.830 & KAP 100 & 1 & 26.634 & 75.886 \\
\hline $\mathrm{C}$ & 6 & $\mathrm{H}$-like & $6 \mathrm{P}$ & $\left(2 \mathrm{P}^{\star}\right) j=1.5$ & $-1 S$ & $(2 S) j=.5$ & 26.026 & RAP 100 & 1 & 26.116 & 85.242 \\
\hline C & 6 & H -like & $6 \mathrm{P}$ & $\left(2 P^{\star}\right) j=1.5$ & $-1 S$ & $(2 S) j=.5$ & 26.026 & KAP 100 & 1 & 26.634 & 77.734 \\
\hline C & 6 & H -like & $5 P$ & $\left(2 P^{\star}\right) j=1.5$ & $-1 S$ & $(2 S) j=.5$ & 26.357 & KAP 100 & 1 & 26.634 & 81.729 \\
\hline $\mathrm{N}$ & 6 & He-like & $1 S$ & $\left(1 P^{*}\right) j=1.0$ & $-1 S 2$ & $(1 \mathrm{~s}) j=.0$ & 24.898 & TAP 100 & 1 & 25.763 & 75.111 \\
\hline
\end{tabular}




\begin{tabular}{|c|c|c|c|c|}
\hline $\mathrm{N}$ & 6 & He-like & 15 & $3 p$ \\
\hline $\mathrm{N}$ & 6 & He-like & 15 & $3 P$ \\
\hline$N$ & 6 & Fe-like & $1 S$ & $3 P$ \\
\hline $\mathbf{N}$ & 7 & $\mathrm{H}$-like & $11 P$ & \\
\hline $\mathrm{N}$ & 7 & H -like & $10 P$ & \\
\hline $\mathrm{N}$ & 7 & H -Iike & $9 F$ & \\
\hline $\mathrm{N}$ & 7 & H -like & $8 P$ & \\
\hline $\mathrm{N}$ & 7 & H -like & $7 P$ & \\
\hline $\mathrm{N}$ & 7 & H -like & $6 P$ & \\
\hline $\mathrm{N}$ & 7 & H -like & $5 \mathrm{P}$ & \\
\hline $\mathrm{N}$ & 7 & H-like & $4 P$ & \\
\hline $\mathbf{N}$ & 7 & H -like & $2 P$ & \\
\hline $\mathrm{N}$ & 7 & H -like & $2 P$ & \\
\hline $\mathbf{N}$ & 7 & H -like & $2 \mathrm{P}$ & \\
\hline $\mathbf{N}$ & 7 & H -like & $2 P$ & \\
\hline 0 & 7 & He-like & $3 P$ & $4 \mathrm{P}$ \\
\hline 0 & 7 & He-like & 25 & $4 \mathrm{P}$ \\
\hline 0 & 7 & He-1ike & $2 \mathrm{P}$ & $3 D$ \\
\hline 0 & 7 & He-like & $2 P$ & $3 D$ \\
\hline 0 & 7 & He-like & $2 \mathrm{P}$ & $3 P$ \\
\hline 0 & 7 & Fe-like & $2 P$ & $3 P$ \\
\hline 0 & 7 & He-1ike & $2 P$ & 35 \\
\hline 0 & 7 & He-like & $2 P$ & $3 s$ \\
\hline 0 & 7 & He-like & $2 P$ & $3 S$ \\
\hline 0 & 7 & Ke-like & $2 P$ & $3 P$ \\
\hline 0 & 7 & He-like & $2 S$ & $2 \mathrm{P}$ \\
\hline 0 & 7 & He-like & $2 S$ & $2 P$ \\
\hline 0 & 7 & He-like & $2 S$ & $2 P$ \\
\hline 0 & 7 & He-like & $2 P 2$ & \\
\hline 0 & 7 & He-like & $2 \mathrm{P} 2$ & \\
\hline 0 & 8 & H -like & $10 P$ & \\
\hline 0 & 8 & H -like & $9 P$ & \\
\hline 0 & 8 & H -like & 89 & \\
\hline 0 & 8 & H-like & 78 & \\
\hline 0 & 8 & H -like & $6 \mathrm{P}$ & \\
\hline 0 & 8 & H -like & $5 P$ & \\
\hline 0 & 8 & H -like & $4 P$ & \\
\hline 0 & 8 & H -like & $4 P$ & \\
\hline 0 & 8 & H -like & $2 P$ & \\
\hline 0 & 8 & H -like & $2 P$ & \\
\hline$F$ & 8 & He-like & 15 & $9 \mathrm{P}$ \\
\hline $\mathbf{F}$ & 8 & He-like & $1 s$ & $8 \mathrm{P}$ \\
\hline$F$ & 8 & He-like & 15 & $7 P$ \\
\hline$F$ & 8 & $\mathrm{He}-1 \mathrm{ike}$ & 15 & $3 P$ \\
\hline $\mathbf{F}$ & 8 & He-like & 15 & $3 P$ \\
\hline$F$ & 8 & He-like & $2 \mathrm{P}$ & $4 S$ \\
\hline
\end{tabular}

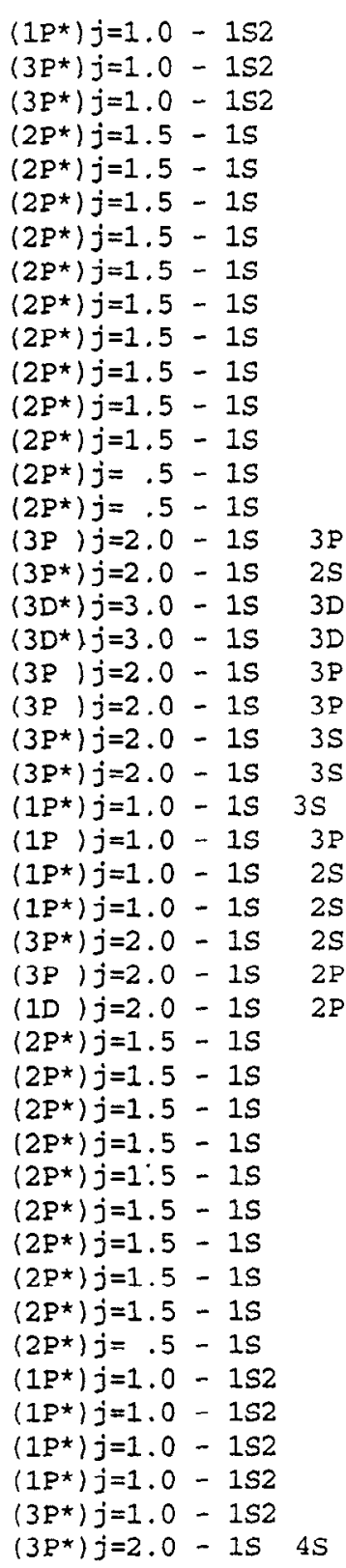

(1S) $j=.0 \quad 24.898 \quad$ RAP 100 (1S) $j=.0 \quad 24.962$ TAP 100 (1S ) $j=.0 \quad 24.962 \quad$ RAP 100 (2S $) j=.5 \quad 18.742$ mica 002 (2S) $j=.5 \quad 18.774$ mica 002 (2S $) j=.5 \quad 18.819$ mica 002 (2S ) $j=.5 \quad 18.882$ mica 002 (2S ) $j=.5 \quad 18.974$ mica 002 (2S $) j=.5 \quad 19.118$ mica 002 (2S) $j=.5 \quad 19.361$ mica 002 (2S $) j=.5 \quad 19.826$ mica 002 (2S $) j=.5 \quad 24.779$ TAP 100 (2S $) j=.5 \quad 24.779 \quad$ RAP 100 (2S) $j=.5 \quad 24.785$ TAP 100 (2S $) j=.5 \quad 24.785 \quad \operatorname{RAP} 100$ $\left(3 P^{\star}\right) j=2.0 \quad 15.439$ beryl 100 (3S) $j=1.0 \quad 15.750$ beryl 100 (3D) $j=3.0 \quad 18.993$ mica 002 (3D) $j=3.0 \quad 18.993$ mica 002 $\left(3 P^{\star}\right) j=2.0 \quad 19.012$ mica 002 $\left(3 P^{*}\right) j=2.0 \quad 19.012$ mica 002 (3s) $j=1.0 \quad 19.045$ mica 002 (3s) $j=1.0 \quad 19.045$ mica 002 (1S $) j=.0 \quad 19.096$ mica 002 (1P*) $j=1.0 \quad 19.164$ mica 002

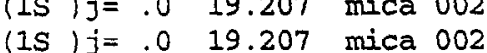
(3s) $j=1.0 \quad 19.301$ mica 002 $\left(3 P^{\star}\right) j=2.0 \quad 19.330$ mica 002 $\left(1 P^{\star}\right) j=1.0 \quad 19.385$ mica 002 (2S $) j=.5 \quad 14.372$ gypsum 020 (2S ) $j=.5 \quad 14.406$ gypsum 020 (2S) $j=.5 \quad 14.454$ gypsum 020 (2S) $j=.5 \quad 14.524$ gypsum 020 (2S ) $j=.5 \quad 14.634$ gypsum 020 (2S ) $j=.5 \quad 14.821$ gYd (2S) $j=.5 \quad 15.176$ gypsum 020 (2S ) $j=.5 \quad 15.176$ beryl 100 (2S ) $j=.5 \quad 18.967$ mica 002 (2S ) $j=.5 \quad 18.972$ mica 002 (1S ) $j=.0 \quad 13.143 \quad \operatorname{KAP} 100$ (1S) $j=.0 \quad 13.185 \quad \operatorname{KAP} 100$ (1S ) $j=.0 \quad 13.244 \quad \mathrm{KAP} 100$ (1s) $j=.0 \quad 14.458$ gypsum 020 (IS ) $j=.0 \quad 14.487$ gypsum 020 (3s) $j=1.0 \quad 15.008$ gypsum 020

\begin{tabular}{|c|c|}
\hline 26.116 & 72.432 \\
\hline 25.763 & 75.675 \\
\hline 26.116 & 72.904 \\
\hline 19.942 & 70.022 \\
\hline 19.942 & 70.293 \\
\hline 19.942 & 70.680 \\
\hline 19.942 & 71.235 \\
\hline 19.942 & 72.075 \\
\hline 19.942 & 73.472 \\
\hline 19.942 & 76.136 \\
\hline 19.942 & 83.817 \\
\hline 25.763 & 74.113 \\
\hline 26.116 & 71.587 \\
\hline 25.763 & 74.162 \\
\hline 26.116 & 71.629 \\
\hline 15.954 & 75.402 \\
\hline 15.954 & 80.828 \\
\hline 19.942 & 72.253 \\
\hline 19.942 & 72.253 \\
\hline 19.942 & 72.433 \\
\hline & \\
\hline 19.942 & 72.750 \\
\hline 19.942 & 73.251 \\
\hline 19.942 & 73.943 \\
\hline 19.942 & 74.396 \\
\hline 19.942 & 74.396 \\
\hline 19.942 & 75.434 \\
\hline 19.942 & 75.769 \\
\hline 19.942 & 76.426 \\
\hline 15.185 & 71.166 \\
\hline 15.185 & 71.568 \\
\hline 15.185 & 72.150 \\
\hline 15.185 & 73.032 \\
\hline 15.185 & 74.518 \\
\hline 15.185 & 77.430 \\
\hline 15.185 & 88.027 \\
\hline 15.954 & 72.033 \\
\hline 19.942 & 72.010 \\
\hline 19.942 & 72.056 \\
\hline 26.634 & 80.728 \\
\hline 26.634 & 81.926 \\
\hline 26.634 & 83.998 \\
\hline 15.185 & 72.199 \\
\hline 15.185 & 72.560 \\
\hline 15.185 & 81.243 \\
\hline
\end{tabular}


F 8 He-like $2 \mathrm{P}$ 4S

F 8 He-like 2P 4S

F 8 He-like 2P $4 \mathrm{~S}$

F 8 He-like 2P $3 S$

F 8 He-like 2P 35

F 8 He-like 2P $3 S$

$F$ He-like 2P $3 S$

$F \quad 8$ He-like 2P $3 P$

F 8 He-like 2P 3P

F 8 He-like 2P2

F 8 He-like 2P2

F 8 He-like $2 S$ 3P

F 8 He-like 2P2

F 8 He-like 2P2

F 8 He-like $2 S$ 2P

F 8 He-like $2 S$ 2P

F 8 He-like $2 S$ 2P

F 8 He-like 2S 2P

F 8 He-like 2S $2 P$

F 9 H-like 3P

F 9 H-like $3 P$

F $9 \mathrm{H}$-like $3 \mathrm{P}$

F $9 \mathrm{H}$-like $3 \mathrm{P}$

F 9 H-like $3 P$

F 9 H -like $3 P$

F 9 H -like 2P

$9 \mathrm{H}$-like 2P

Ne K-alpha(1)

Ne 9 He-like $1 \mathrm{~s}$ 8P

Ne 9 He-like is 7P

Ne 9 He-like 2P2

Ne 9 He-like 2S $2 \mathrm{P}$

Ne 9 He-like $2 S$ 2P

Ne 9 He-like 2S $2 P$

Ne 9 He-like 2S $2 \mathrm{P}$

Ne 9 He-like 2S $2 \mathrm{P}$

Ne 9 He-like 2P2

Ne 9 He-like 2P2

Ne 9 He-like 2P2

Ne 9 He-like 2P2

Ne10 H-like 5P

Ne10 $\mathrm{H}$-like $4 \mathrm{P}$

Ne10 H -like 3P

Ne10 H -like 3P

Ne10 H-like $2 \mathrm{P}$

Ne10 H -like 2P $\left(3 P^{*}\right) j=2.0-1 S \quad 4 S$ $\left(3 P^{\star}\right) j=2.0-1 S \quad 4 S$ $\left(3 P^{*}\right) j=2.0-1 S \quad 4 S$ $\left(3 P^{\star}\right) j=2.0-1 S \quad 3 s$ $\left(3 P^{\star}\right) j=2.0-1 S \quad 3 S$ $\left(3 P^{\star}\right) j=2.0-1 S \quad 3 S$ $\left(3 P^{*}\right) j=2.0-1 S \quad 3 S$ (3D) $j=2.0-1 S \quad 3 P$ (3D) $j=2.0-1 S \quad 3 P$ (1S) $j=.0-1 S \quad 2 P$ (1S) $j=.0-1 S \quad 2 P$ $\left(3 P^{\star}\right) j=2.0-1 S \quad 2 S$ (3P) $j=2.0$ - $1 S$ 2P (1D) $j=2.0-1 S \quad 2 P$ $\left(1 P^{\star}\right) j=1.0-1 S \quad 2 S$ $\left(1 P^{*}\right) j=1.0-1 \mathrm{~s} \quad 2 \mathrm{~s}$ $\left(1 P^{*}\right) j=1.0-1 S \quad 2 S$ $\left(3 P^{*}\right) j=2.0-1 S \quad 2 S$ $\left(3 P^{*}\right) j=2.0-1 S$ $\left(2 P^{*}\right) j=1.5-1 S$ $\left(2 P^{*}\right) j=1.5-1 S$ $\left(2 P^{\star}\right) j=1.5-1 S$ $\left(2 P^{*}\right) j=5-1 S$ $\left(2 P^{\star}\right) j=.5-1 S$ $\left(2 P^{*}\right) j=.5-1 S$ $\left(2 P^{*}\right) j=1.5-1 S$ $\left(2 P^{\star}\right) j=.5-1 S$

$\left(1 P^{*}\right) j=1.0-1 \mathrm{~S} 2$ (1P*) $j=1.0-1 S 2$ (1S ) $j=.0-1 s$ $\left(1 P^{\star}\right) j=1.0-1 S$ $\left(3 P^{*}\right) j=2.0-1 S$ $\left(3 P^{\star}\right) j=2.0-1 S$ $\left(3 P^{*}\right) j=1.0-1 S$ $\left(3 P^{*}\right) j=1.0-1 S$ (3P) $j=2.0-1 S$ (3P) $j=2.0-1 S$ (1D) $j=2.0-1 S$ (1D) $j=2.0-1 s$ $\left(2 P^{\star}\right) j=1.5-1 S$ $\left(2 P^{*}\right) j=1.5-1 S$ $\left(2 P^{*}\right) j=1.5-1 S$ $\left(2 P^{*}\right) j=5-1 S$ $\left(2 P^{\star}\right) j=1.5-1 S$ $(2 P *) j=.5-1 S$
(3S ) $j=1.0 \quad 15.008$ beryl 100 (3S) $j=1.0 \quad 15.008$ (3S) $j=1.0 \quad 15.008$ (3s) $j=1.0 \quad 15.046$ (3S ) $j=1.0 \quad 15.046$ (3S ) $j=1.0 \quad 15.046$ (3S ) $j=1.0 \quad 15.046$ $\left(1 P^{*}\right) j=1.0 \quad 15.076$ $\left(1 P^{\star}\right) j=1.0 \quad 15.076$ $\left(1 P^{\star}\right) j=1.0 \quad 15.155$ $\left(1 P^{*}\right) j=1.0 \quad 15.155$ (3S ) $j=1.0 \quad 15.224$ $\left(1 P^{*}\right) j=1.0 \quad 15.286$ bery 100 (1S) $j=.0 \quad 25.740$ TAP 100

(1S) $j=.0 \quad 25.740$ RAP 100 (1S ) $j=.0 \quad 25.740$ KAP 100 (3S) $j=1.0 \quad 25.790 \quad$ RAP 100 (3S) $j=1.0 \quad 25.790 \quad$ KAP 100 (2S) $j=.5 \quad 12.643$ TAP 100 $(2 S) j=.5 \quad 12.643 \quad$ RAP 100 (2S ) $j=.5 \quad 12.643 \quad \mathrm{KAP} 100$ (2S ) $j=.5 \quad 12.644$ TAP 100 (2S) $j=.5 \quad 12.644 \quad \operatorname{RAP} 100$ (2S ) $j=.5 \quad 12.644 \quad \operatorname{KAP} 100$ (2S ) $j=.5 \quad 14.982$ gypsum 020 (2S $) j=.5 \quad 14.988$ gypsum 020 14.611 gypsum 020

(1S ) $j=.0 \quad 10.513 \quad$ ADP 101 (1S ) $j=.0 \quad 10.565$ ADP 101 $\left(I P^{\star}\right) j=1.0 \quad 12.172 \quad$ TAP 100 (1S) $j=.0 \quad 12.260 \quad \operatorname{TAP} 100$ (3S) $j=1.0 \quad 12.303 \quad$ TAP 100 (3S) $j=1.0 \quad 12.303$ RAP 100 (3S) $j=1.0 \quad 12.309$ TAP 100 (3S) $j=1.0 \quad 12.309 \quad \operatorname{RAP} 100$ $\left(3 P^{*}\right) j=2.0 \quad 12.324 \quad$ TAP 100 $\left(3 P^{*}\right) j=2.0 \quad 12.324 \quad \operatorname{RAP} 100$ $\left(1 P^{*}\right) j=1.0 \quad 12.355 \quad$ TAP 100 (12*) $j=1.0 \quad 12.355 \quad$ RAP 100 (2S) $j=.5 \quad 9.481$ mica 002 (2S ) $j=.5 \quad 9.708$ mica 002 (2S) $j=.5 \quad 10.238$ ADP 101 (2S ) $j=.5 \quad 10.240 \quad$ ADP 101 (2S) $j=.5 \quad 12.132$ TAP 100 (2S $) j=.5 \quad 12.138$ TAP 100
$15.954 \quad 70.170$

$15.185 \quad 81.243$

$15.954 \quad 70.170$

$15.185 \quad 82.242$

$15.954 \quad 70.576$

$15.185 \quad 82.242$

$\begin{array}{ll}15.954 & 70.576\end{array}$

$15.185 \quad 83.131$

$15.954 \quad 70.903$

$15.185 \quad 86.398$

$15.954 \quad 71.790$

$15.954 \quad 72.601$

$15.954 \quad 72.867$

$15.954 \quad 73.361$

$25.763 \quad 87.579$

$26.116 \quad 80.266$

$26.634 \quad 75.113$

$26.116 \quad 80.938$

$26.634 \quad 75.537$

$25.763 \quad 78.957$

$26.116 \quad 75.516$

$26.634 \quad 71.693$

$25.763 \quad 78.981$

$26.116 \quad 75.534$

$26.634 \quad 71.707$

$15.185 \quad 80.621$

$\begin{array}{ll}15.185 & 80.761\end{array}$

$15.185 \quad 74.191$

$10.640 \quad 81.139$

$10.640 \quad 83.193$

$25.763 \quad 70.895$

$25.763 \quad 72.130$

$25.763 \quad 72.764$

$26.116 \quad 70.421$

$25.763 \quad 72.854$

$26.116 \quad 70.500$

$25.763 \quad 73.082$

$26.116 \quad 70.698$

$25.763 \quad 73.562$

$26.116 \quad 71.114$

$19.942 \quad 71.963$

$19.942 \quad 76.811$

$10.640 \quad 74.200$

$10.640 \quad 74.240$

$25.763 \quad 70.359$

$25.763 \quad 70.438$ 


\begin{tabular}{|c|c|c|c|c|c|c|c|c|c|}
\hline Na10 & He-like & $1 \mathrm{~S}$ & $6 \mathrm{P}$ & & & & $\left(1 P^{*}\right) j=1.0$ & $-1 s 2$ & \\
\hline $\mathrm{Na10}$ & $\mathrm{He}-\mathrm{like}$ & IS & $6 P$ & & & & $\left(1 p^{*}\right) j=1.0$ & $-1 S 2$ & \\
\hline Na10 & He-like & $1 \mathrm{~s}$ & $6 P$ & & & & $\left(1 p^{*}\right) j=1.0$ & $-1 S 2$ & \\
\hline $\mathrm{Na} O$ & He-like & $1 S$ & $6 P$ & & & & $\left(1 P^{*}\right) j=1.0$ & $-1 S 2$ & \\
\hline $\mathrm{Na10}$ & He-like & $1 S$ & $5 P$ & & & & $\left(1 P^{\star}\right) j=1.0$ & $-1 S 2$ & \\
\hline $\mathrm{Na10}$ & He-Iike & 1S & $5 P$ & & & & $\left(1 p^{*}\right) j=1.0$ & $-1 S 2$ & \\
\hline $\mathrm{Na10}$ & He-like & IS & $3 P$ & & & & $\left(1 P^{*}\right) j=1.0$ & $-1 S 2$ & \\
\hline Ia10 & He-like & $1 S$ & $3 P$ & & & & $\left(3 P^{*}\right) j=1.0$ & -152 & \\
\hline $\mathrm{Ja10}$ & He-Iike & $2 P$ & $3 P$ & & & & $(3 D) j=3.0$ & $-1 S$ & $3 P$ \\
\hline $\mathrm{Na10}$ & He-like & $2 S$ & $2 F$ & & & & $\left(1 p^{*}\right) j=1.0$ & $-1 S$ & $2 S$ \\
\hline Na10 & He-like & $2 S$ & $2 P$ & & & & $\left(3 P^{*}\right) j=2.0$ & $-1 S$ & $2 S$ \\
\hline 210 & He-like & 292 & & & & & $(3 P) j=2.0$ & $-1 s$ & $2 P$ \\
\hline la10 & He-like & $2 \mathrm{P} 2$ & & & & & (1D) $j=2.0$ & $-1 S$ & $2 P$ \\
\hline $\operatorname{NaII}$ & H-like & $10 \mathrm{P}$ & & & & & $\left(2 \mathrm{P}^{*}\right) j=1.5$ & $-1 S$ & \\
\hline $\operatorname{ta11}$ & H -like & $9 \mathrm{P}$ & & & & & $\left(2 \mathrm{P}^{*}\right) j=1.5$ & $-1 S$ & \\
\hline $\operatorname{va} 11$ & $\mathrm{H}$-like & $8 \mathrm{P}$ & & & & & $\left\langle 2 P^{*}\right\rangle j=1.5$ & -15 & \\
\hline $\mathrm{Na11}$ & $\mathrm{H}$-like & $7 P$ & & & & & $\left(2 \mathrm{P}^{*}\right) j=1.5$ & $-1 S$ & \\
\hline $\mathrm{Na11}$ & H -like & $6 P$ & & & & & $\left(2 \mathrm{P}^{*}\right) j=1.5$ & - IS & \\
\hline $\mathrm{Na} 11$ & H -like & $5 P$ & & & & & $\left(2 P^{*}\right) j=1.5$ & $-1 S$ & \\
\hline $\mathrm{Na} 11$ & H -like & $4 \mathrm{P}$ & & & & & $\left(2 p^{*}\right) j=1.5$ & $-1 S$ & \\
\hline $\operatorname{Na11}$ & H -like & $4 P$ & & & & & $\left(2 P^{*}\right) j=1.5$ & $-1 S$ & \\
\hline $\mathrm{Na11}$ & H-like & $3 P$ & & & & & $\left(2 \mathrm{P}^{\star}\right) j=1.5$ & $-1 S$ & \\
\hline $\operatorname{NaI1}$ & H -like & $3 P$ & & & & & $\left(2 \mathrm{P}^{*}\right) j=1.5$ & $-1 S$ & \\
\hline Na11 & H -like & $3 P$ & & & & & $\left(2 \mathrm{P}^{\star}\right) j=1.5$ & $-1 S$ & \\
\hline Na11 & $H$-like & $3 P$ & & & & & $\left(2 \mathrm{P}^{\star}\right) j=1.5$ & $-1 S$ & \\
\hline $\mathrm{Na} 11$ & H -like & $3 P$ & & & & & $\left(2 P^{\star}\right) j=1.5$ & $-1 S$ & \\
\hline Na11 & H -like & $3 P$ & & & & & $\left(2 P^{\star}\right) j=1.5$ & $-1 S$ & \\
\hline Na11 & H -like & $3 P$ & & & & & $\left(2 P^{*}\right) j=.5$ & $-1 S$ & \\
\hline $\mathrm{Na} 11$ & H-like & $3 P$ & & & & & $\left(2 P^{*}\right) j=.5$ & $-1 S$ & \\
\hline Na11 & H -Iike & $3 P$ & & & & & $\left(2 P^{\star}\right) j=.5$ & $-1 S$ & \\
\hline $\mathrm{Na} 11$ & H -like & $3 P$ & & & & & $\left(2 P^{\star}\right) j=.5$ & -15 & \\
\hline Na11 & H -like & $3 P$ & & & & & $\left(2 P^{\star}\right) j=.5$ & -15 & \\
\hline $\mathrm{Na11}$ & H-like & $3 P$ & & & & & $\left(2 P^{*}\right) j=.5$ & $-1 S$ & \\
\hline $\mathrm{Na} 11$ & H-like & $2 P$ & & & & & $\left(2 \mathrm{P}^{*}\right\rangle j=1.5$ & -15 & \\
\hline Na11 & H -like & $2 P$ & & & & & $\left(2 P^{\star}\right) j=.5$ & $-1 S$ & \\
\hline Mg & & $\mathrm{K}-\mathrm{a}$ & pha & (1) & & & & & \\
\hline Mg10 & Li-like & 15 & $2 S$ & is & $j$ & $3 P$ & $\left(2 P^{*}\right) j=1.5$ & $-1 s 2$ & $2 S$ \\
\hline $\operatorname{Mg} 10$ & Ii-Iike & $1 S$ & $2 P$ & $1 p^{\star}$ & j & $3 P$ & $(2 D) j=2.5$ & $-1 s 2$ & $2 P$ \\
\hline $\operatorname{Mg} 10$ & Li-like & $1 S$ & $2 P$ & $1 p^{\star}$ & ) & $3 P$ & $(2 D) j=2.5$ & $-1 S 2$ & $2 P$ \\
\hline $\operatorname{Mg} 10$ & Li-like & IS & $2 P$ & $3 P^{*}$ & j & $3 P$ & (2S) $j=.5$ & $-1 s 2$ & $2 P$ \\
\hline $\operatorname{Mg} 10$ & Li-like & $1 S$ & $2 P$ & $3 p^{\star}$ & ) & $3 P$ & $(2 S) j=.5$ & -152 & $2 P$ \\
\hline Mg10 & Li-like & $1 S$ & $2 P$ & $39^{*}$ & ) & $3 P$ & $(2 D) j=2.5$ & $-1 S 2$ & $2 \mathrm{P}$ \\
\hline $\operatorname{Mg} 10$ & Li-like & $1 S$ & $2 P$ & $3 P^{*}$ & ) & $3 P$ & $(2 D) j=2.5$ & $-1 s 2$ & $2 P$ \\
\hline $\operatorname{Mg} 10$ & Li-like & $1 \mathrm{~S}$ & $2 \mathrm{P}$ & $(3 P *$ & i & $3 P$ & $(2 \mathrm{P}) j=1.5$ & $-1 s 2$ & $2 \mathrm{P}$ \\
\hline Mg10 & Li-like & IS & $2 P$ & $3 P^{\star}$ & ) & $3 P$ & $(2 P) j=1.5$ & $-1 s 2$ & $2 P$ \\
\hline Mg10 & Li-like & 15 & $2 P$ & $3 F^{*}$ & ) & $3 F$ & $(2 P) j=1.5$ & -152 & $2 P$ \\
\hline
\end{tabular}

\begin{tabular}{|c|c|c|c|c|c|}
\hline$(1 s) j=.0$ & 8.686 & PET 002 & 1 & 8.742 & 83.511 \\
\hline (1s) $j=.0$ & 8.686 & EDT 020 & 1 & 8.808 & 80.453 \\
\hline (s $j j=.0$ & 8.686 & RAP 100 & 3 & 26.116 & 86.181 \\
\hline$(1 s) j=.0$ & 8.686 & KAP 100 & 3 & 26.634 & 78.062 \\
\hline$S) j=.0$ & 8.788 & EDT 020 & 1 & 8.808 & 86.138 \\
\hline is $) j=.0$ & 8.788 & KAP 100 & 3 & 26.634 & 81.835 \\
\hline$S i j=.0$ & 9.433 & mica 002 & 2 & 19.942 & 71.093 \\
\hline$j=.0$ & 9.449 & mica 002 & 2 & 19.942 & 71.378 \\
\hline$j=2.0$ & 10.060 & ADP 101 & 1 & 10.640 & 70.995 \\
\hline$j j=.0$ & 10.119 & $A D P 101$ & 1 & 10.640 & 71.996 \\
\hline$j=1.0$ & 10.157 & ADP 101 & 1 & 10.640 & 72.670 \\
\hline$\left.P^{*}\right) j=2.0$ & 10.170 & $A D P 101$ & 1 & 10.640 & 72.907 \\
\hline$j j=1.0$ & 10.193 & ADP 101 & 1 & 10.640 & 73.333 \\
\hline$j=.5$ & 7.596 & beryl 100 & 2 & 15.954 & 72.220 \\
\hline$j=.5$ & 7.614 & beryl 100 & 2 & 15.954 & 72.649 \\
\hline$j=.5$ & 7.639 & beryl 100 & 2 & 15.954 & 73.261 \\
\hline SS $) j=.5$ & 7.677 & beryl 100 & 2 & 15.954 & 74.237 \\
\hline S $) j=.5$ & 7.735 & beryl 100 & 2 & 15.954 & 75.851 \\
\hline$j j=.5$ & 7.833 & beryl 100 & 2 & 15.954 & 79.097 \\
\hline$j j=.5$ & 8.021 & topaz 002 & 1 & 8.374 & 73.305 \\
\hline$(2 S) j=.5$ & 8.021 & quartz 100 & 1 & 8.512 & 70.444 \\
\hline $2 S) j=.5$ & 8.459 & quartz 100 & 1 & 8.512 & 83.603 \\
\hline$j=.5$ & 8.459 & PET 002 & 1 & 8.742 & 75.381 \\
\hline$j j=.5$ & 8.459 & EDT 020 & 1 & 8.808 & 73.817 \\
\hline $2 S) j=.5$ & 8.459 & TAP 100 & 3 & 25.763 & 80.069 \\
\hline$j j=.5$ & 8.459 & $\operatorname{RAP} 100$ & 3 & 26.116 & 76.337 \\
\hline$j j=.5$ & 8.459 & $\operatorname{KAP} 100$ & 3 & 26.634 & 72.327 \\
\hline$j=.5$ & 8.460 & quartz 100 & 1 & 8.512 & 83.664 \\
\hline$j j=.5$ & 8.460 & PET 002 & 1 & 8.742 & 75.407 \\
\hline$j j=.5$ & 8.460 & EUT 020 & 1 & 8.808 & 73.840 \\
\hline$j j=.5$ & 8.460 & TAP 100 & 3 & 25.763 & 80.108 \\
\hline$j j=.5$ & 8.460 & RAP 100 & 3 & 26.116 & 76.365 \\
\hline .5$) j=.5$ & 8.460 & KAP 100 & 3 & 26.634 & 72.348 \\
\hline 2s $j j=.5$ & 10.023 & ADP 101 & 1 & 10.640 & 70.392 \\
\hline \multirow[t]{2}{*}{$(2 S) j=$} & 10.029 & $A D P 101$ & 1 & 10.640 & 70.489 \\
\hline & 9.890 & mica 002 & 2 & 19.942 & 82.705 \\
\hline$j=.5$ & 7.998 & topaz 002 & 1 & 8.374 & 72.765 \\
\hline$j=1.5$ & 8.035 & topaz 002 & 1 & 8.374 & 73.641 \\
\hline 1.5 & 8.035 & quartz 100 & 1 & 8.512 & 70.728 \\
\hline$j=1.5$ & 8.051 & topaz 002 & 1 & 8.374 & 74.035 \\
\hline$j=1.5$ & 8.051 & quartz 100 & 1 & 8.512 & 71.057 \\
\hline$j=1.5$ & 8.068 & topaz 002 & 1 & 8.374 & 74.463 \\
\hline$j=1.5$ & 8.068 & quartz 100 & 1 & 8.512 & 71.413 \\
\hline$=1.5$ & 8.092 & topaz 002 & 1 & 8.374 & 75.088 \\
\hline$=1.5$ & 8.092 & quartz 100 & 1 & 8.512 & 71.926 \\
\hline$=1.5$ & 8.092 & $\operatorname{TAP} 100$ & 3 & 25.763 & 70.438 \\
\hline
\end{tabular}




\begin{tabular}{|c|c|c|c|c|c|c|c|}
\hline $\operatorname{Mg} 10$ & Li-like & 1S & $2 \mathrm{P} 2$ & & $(4 P) j=1.5$ & $-1 s 2$ & $2 P$ \\
\hline $\mathrm{Mg} 10$ & Li-like & $1 S$ & $2 \mathrm{P} 2$ & & $(4 P) j=2.5$ & $-1 S 2$ & $2 \mathrm{P}$ \\
\hline $\operatorname{Mg} 10$ & Li-like & is & $2 \mathrm{P} 2$ & & $(4 P) j=1.5$ & $-1 S 2$ & $2 \mathrm{P}$ \\
\hline $\operatorname{Mg} 10$ & Li-like & 1S & $2 \mathrm{P} 2$ & & $(4 P) j=.5$ & $-1 s 2$ & $2 \mathrm{P}$ \\
\hline $\operatorname{Mg} 10$ & Li-like & IS & (2S & $2 P<3 P^{*}$ & )) $\left(49^{*}\right) j=.5$ & $-1 s 2$ & $2 S$ \\
\hline $\operatorname{Mg} 11$ & He-like & $1 S$ & $9 P$ & & $\left(1 P^{\star}\right) j=1.0$ & $-1 s 2$ & \\
\hline $\operatorname{Mg} 11$ & He-like & $1 \mathrm{~S}$ & $8 P$ & & $\left(1 P^{\star}\right) j=1.0$ & $-1 S 2$ & \\
\hline $\operatorname{Mg} 11$ & He-Iike & $1 \mathrm{~S}$ & $8 \mathrm{P}$ & & $\left(1 P^{*}\right) j=1.0$ & $-1 s 2$ & \\
\hline $\operatorname{Mg} 11$ & He-like & 1S & $7 P$ & & $\left(1 P^{*}\right) j=1.0$ & $-1 S 2$ & \\
\hline Mg11 & He-like & is & $7 P$ & & $\left(2 P^{*}\right) j=1.0$ & -152 & \\
\hline $\operatorname{Mg11}$ & He-like & $1 S$ & $6 P$ & & $\left(1 P^{*}\right) j=1.0$ & $-1 s 2$ & \\
\hline $\operatorname{Mg} 11$ & He-like & IS & $6 P$ & & $\left(1 P^{*}\right) j=1.0$ & -152 & \\
\hline $\operatorname{Mg} 11$ & He-like & 1S & $5 P$ & & $\left(1 P^{\star}\right) j=1.0$ & $-1 S 2$ & \\
\hline $\operatorname{Mg} 11$ & He-like & Is & $5 P$ & & $\left(1 P^{*}\right) j=1.0$ & $-1 s 2$ & \\
\hline $\operatorname{Mg} 11$ & He-Iike & $1 \mathrm{~S}$ & $4 P$ & & $\left(1 P^{\star}\right) j=1.0$ & $-1 s 2$ & \\
\hline $\operatorname{Mg} 11$ & He-like & is & $4 P$ & & $\left(1 P^{*}\right) j=1.0$ & $-1 S 2$ & \\
\hline $\operatorname{Mg} 11$ & He-like & $1 S$ & $3 P$ & & $\left(18^{\star}\right) j=1.0$ & $-1 s 2$ & \\
\hline $\operatorname{Mg} 11$ & He-like & 1S & $3 P$ & & $\left(3 P^{\star}\right) j=1.0$ & $-1 S 2$ & \\
\hline $\operatorname{Mg} 11$ & He-like & $2 P$ & $3 p$ & & $(3 D) j=3.0$ & $-1 S$ & \\
\hline $\operatorname{Mg} 11$ & He-like & $2 P$ & $3 P$ & & $(3 D) j=3.0$ & $-1 S$ & $P$ \\
\hline $\operatorname{Mg} 11$ & He-like & $2 \mathrm{P}$ & $3 P$ & & $(3 D) j=3.0$ & $-1 S$ & $P$ \\
\hline $\operatorname{Mg} 11$ & He-like & $2 P$ & $3 P$ & & (3D) $j=3.0$ & $-1 S$ & 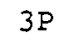 \\
\hline $\operatorname{Mg} 11$ & He-like & $2 P$ & $3 P$ & & $(3 D) j=3.0$ & $-1 S$ & $P$ \\
\hline $\operatorname{Mg} 11$ & He-Iike & $2 P$ & $3 P$ & & $(3 D) j=3.0$ & $-1 S$ & $P$ \\
\hline Mg11 & He-like & $2 \mathrm{P} 2$ & & & $(1 S) j=.0$ & - IS & $2 \mathrm{P}$ \\
\hline $\operatorname{Mg} 11$ & He-like & $2 \mathrm{P} 2$ & & & $(1 S) j=.0$ & $-1 S$ & $2 P$ \\
\hline $\operatorname{Mg} 11$ & He-like & $2 P 2$ & & & $(1 S) j=.0$ & $-1 S$ & $2 \mathrm{P}$ \\
\hline $\operatorname{Mg} 11$ & He-like & $2 \mathrm{P} 2$ & & & $(1 S) j=.0$ & $-1 S$ & $2 P$ \\
\hline $\operatorname{Mg} 11$ & He-like & $2 \mathrm{P} 2$ & & & $(1 \mathrm{~s}) j=.0$ & $-1 S$ & $2 \mathrm{P}$ \\
\hline $\operatorname{Mg} 11$ & He-like & $2 \mathrm{P} 2$ & & & $(1 S) j=.0$ & $-1 S$ & $2 \mathrm{P}$ \\
\hline $\operatorname{Mg} 11$ & He-like & $2 S$ & $2 P$ & & $\left(1 P^{*}\right) j=1.0$ & -15 & $2 s$ \\
\hline $\operatorname{Mg} 11$ & He-like & $2 s$ & $2 P$ & & $\left(1 P^{\star}\right) j=1.0$ & $-1 S$ & 25 \\
\hline $\operatorname{Mg} 11$ & He-like & $2 s$ & $2 \mathrm{P}$ & & $\left(1 p^{*}\right) j=1.0$ & $-1 S$ & $2 S$ \\
\hline $\operatorname{Mg} 11$ & He-Iike & $2 S$ & $2 P$ & & $\left(1 P^{\star}\right) j=1.0$ & $-1 S$ & $2 S$ \\
\hline $\operatorname{Mg} 11$ & He-like & $2 S$ & $2 P$ & & $\left(1 P^{\star}\right) j=1.0$ & $-1 S$ & $2 S$ \\
\hline $\operatorname{Mg} 11$ & He-like & $2 S$ & $2 \mathrm{P}$ & & $\left(1 P^{*}\right) j=1.0$ & $-1 S$ & $2 \mathrm{~S}$ \\
\hline $\operatorname{Mg} 11$ & He-like & $2 S$ & $2 \mathrm{P}$ & & $\left(3 P^{\star}\right) j=1.0$ & $-1 S$ & $2 S$ \\
\hline $\operatorname{Mg} 11$ & He-like & $2 S$ & $2 \mathrm{P}$ & & $\left(3 P^{\star}\right) j=1.0$ & $-1 s$ & $2 S$ \\
\hline $\operatorname{Mg} 11$ & He-like & $2 S$ & $2 \mathrm{P}$ & & $\left(3 P^{\star}\right) j=1.0$ & $-1 S$ & 25 \\
\hline $\operatorname{Mg} 11$ & He-like & $2 S$ & $2 \mathrm{P}$ & & $\left(3 P^{*}\right) j=1.0$ & $-1 S$ & $2 \mathrm{~S}$ \\
\hline Mg11 & He-like & $2 S$ & $2 P$ & & $\left(3 P^{*}\right) j=1.0$ & $-1 S$ & $2 \mathrm{~S}$ \\
\hline $\operatorname{Mg} 11$ & He-like & $2 \mathrm{P} 2$ & & & $(3 P) j=2.0$ & $-1 S$ & $2 \mathrm{P}$ \\
\hline $\operatorname{Mg} 11$ & He-1ike & $2 \mathrm{P} 2$ & & & $(3 P) j=2.0$ & $-1 s$ & $2 P$ \\
\hline $\operatorname{Mg} 11$ & He-like & $2 \mathrm{P} 2$ & & & $(3 P) j=2.0$ & $-1 S$ & $2 \mathrm{P}$ \\
\hline $\mathrm{Mg} 11$ & He-like & $2 \mathrm{P} 2$ & & & $(3 P) j=2.0$ & $-1 S$ & $2 \mathrm{P}$ \\
\hline $\operatorname{Mg} 11$ & He-like & $2 \mathrm{P} 2$ & & & $(3 P) j=2.0$ & $-1 S$ & $2 \mathrm{~F}$ \\
\hline
\end{tabular}

\begin{tabular}{|c|c|c|c|c|c|}
\hline$\left(2 P^{\star}\right) j=.5$ & 9.382 & mica 002 & 2 & 19.942 & 70.208 \\
\hline$\left(2 P^{*}\right) j=1.5$ & 9.383 & mica 002 & 2 & 19.942 & 70.225 \\
\hline$\left(2 \mathrm{P}^{\star}\right) j=1.5$ & 9.384 & mica 002 & 2 & 19.942 & 70.242 \\
\hline$\left(2 P^{*}\right) j=1.5$ & 9.386 & mica 002 & 2 & 19.942 & 70.276 \\
\hline$(2 s) j=.5$ & 9.391 & mica 002 & 2 & 19.942 & 70.361 \\
\hline (1s) j j= .0 & 7.128 & InSb 111 & 1 & 7.481 & 72.329 \\
\hline$(1 s) j=.0$ & 7.156 & Insb 111 & 1 & 7.481 & 73.049 \\
\hline (1s) $j=.0$ & 7.156 & gypsum 020 & 2 & 15.185 & 70.477 \\
\hline$(1 s) j=.0$ & 7.177 & Instb 111 & 1 & 7.481 & 73.610 \\
\hline (1s) $j=.0$ & 7.177 & gypsum 020 & 2 & 15.185 & 70.957 \\
\hline$(1 s) j=.0$ & 7.225 & InSb 111 & 1 & 7.481 & 74.968 \\
\hline$(1 S) j=.0$ & 7.225 & gypsum 020 & 2 & 15.185 & 72.100 \\
\hline$(1 s) j=.0$ & 7.310 & InSb 111 & 1 & 7.481 & 77.726 \\
\hline$(1 s) j=.0$ & 7.310 & gypsum 020 & 2 & 15.185 & 74.321 \\
\hline$(1 S) j=.0$ & 7.473 & InSb 111 & 1 & 7.481 & 87.350 \\
\hline$(1 s) j=.0$ & 7.473 & gypsum 020 & 2 & 15.185 & 79.821 \\
\hline$(1 s) j=.0$ & 7.850 & beryl 100 & 2 & 15.954 & 79.762 \\
\hline (1s) $j=.0$ & 7.862 & beryl 100 & 2 & 15.954 & 80.259 \\
\hline$\left(3 P^{\star}\right) j=2.0$ & 8.445 & quartz 100 & 1 & 8.512 & 82.806 \\
\hline$\left(3 P^{*}\right) j=2.0$ & 8.445 & PET 002 & 1 & 8.742 & 75.022 \\
\hline$\left(3 P^{*}\right) j=2.0$ & 8.445 & EDT 020 & 1 & 8.808 & 73.493 \\
\hline$\left(3 P^{\star}\right) j=2.0$ & 8.445 & TAP 100 & 3 & 25.763 & 79.542 \\
\hline$\left(3 P^{*}\right) j=2.0$ & 8.445 & RAP 100 & 3 & 26.116 & 75.953 \\
\hline$\left(3 P^{*}\right) j=2.0$ & 8.445 & $\operatorname{KAP} 100$ & 3 & 26.634 & 72.032 \\
\hline$\left(1 P^{\star}\right) j=1.0$ & 8.458 & quartz 100 & 1 & 8.512 & 83.543 \\
\hline$\left(1 P^{*}\right) j=1.0$ & 8.458 & PET 002 & 1 & 8.742 & 75.356 \\
\hline$\left(1 P^{*}\right) j=1.0$ & 8.458 & EDT 020 & 1 & 8.808 & 73.794 \\
\hline$\left(1 P^{*}\right) j=1.0$ & 8.458 & TAP 100 & 3 & 25.763 & 80.031 \\
\hline$\left\langle 1 P^{*}\right\rangle j=1.0$ & 8.458 & RAP 100 & 3 & 26.116 & 76.310 \\
\hline$\left(1 P^{*}\right) j=1.0$ & 8.458 & $\operatorname{KAP} 100$ & 3 & 26.634 & 72.306 \\
\hline$(1 s) j=.0$ & 8.494 & quartz 100 & 1 & 8.512 & 86.273 \\
\hline (1s) $j=.0$ & 8.494 & PET 002 & 1 & 8.742 & 76.320 \\
\hline (Is) $j=.0$ & 8.494 & EDT 020 & 1 & 8.808 & 74.655 \\
\hline (1s) $j=.0$ & 8.494 & TAP 100 & 3 & 25.763 & 81.530 \\
\hline$(1 s) j=.0$ & 8.494 & RAP 100 & 3 & 26.116 & 77.349 \\
\hline$(1 \mathrm{~s}) j=.0$ & 8.494 & KAP 100 & 3 & 26.634 & 73.087 \\
\hline$(3 s) j=1.0$ & 8.522 & PET 002 & 1 & 8.742 & 77.119 \\
\hline$(3 s) j=1.0$ & 8.522 & EDT 020 & 1 & 8.808 & 75.359 \\
\hline$(3 s) j=1.0$ & 8.522 & TAP 100 & 3 & 25.763 & 82.910 \\
\hline$(3 S) j=1.0$ & 8.522 & RAP 100 & 3 & 26.116 & 78.220 \\
\hline (3s) $j=1.0$ & 8.522 & KAP 100 & 3 & 26.634 & 73.720 \\
\hline$\left(3 P^{*}\right) j=2.0$ & 8.531 & PET 002 & 1 & 8.742 & 77.386 \\
\hline$\left(3 P^{*}\right) j=2.0$ & 8.531 & EDT 020 & 1 & 8.808 & 75.593 \\
\hline$\left(3 P^{*}\right) j=2.0$ & 8.531 & TAP 100 & 3 & 25.763 & 83.414 \\
\hline$\left(3 P^{*}\right) j=2.0$ & 8.531 & RAP 100 & 3 & 26.116 & 78.514 \\
\hline$\left(3 P^{*}\right) j=2.0$ & 8.531 & KAP 100 & 3 & 26.634 & 73.928 \\
\hline
\end{tabular}


Mg11 He-like 2P2

Mg11 He-like 2P2

Mg11 He-like 2P2

Mg11 He-like 2P2

Mg11 He-like 2P2

Mg11 He-like 2P2

Mg11 He-like 2P2

Mg11 He-like 2P2

Mg11 He-like 2P2

Mg11 He-like 2P2

Mg11 He-like 2S 2P

Mg11 He-like 2S 2P

Mg1I He-like 2S 2P

Mg11 He-like 2S $2 P$

Mg12 H-like 10P

Mg12 H -like 10P

Mg12 H -like $10 P$

Mg12 H -like $10 \mathrm{P}$

Mg12 H -like 10P

Mg12 H -like 10P

Mg12 H -like 10P

Mg12 $\mathrm{H}$-like 10P

Mg12 $\mathrm{H}$-like 9P

Mg12 H -like 9P

Mg12 H-like 9P

Mg12 H-like 9P

Mg12 H-like 9P

Mg12 $\mathrm{H}$-like 9P

Mg12 H -like 9P

Mg12 H-like 9P

$\mathrm{Mg12} \mathrm{H}$-like 8P

Mg12 H -like 8P

Mg12 H -like 8P

Mg12 H -like 8P

Mg12 $\mathrm{H}$-like $8 \mathrm{P}$

Mg12 H -like 8P

Mg12 H -like 8P

Mg12 H -like 8P

Mg12 H -like 7P

$\mathrm{Mg} 12 \mathrm{H}$-like $7 \mathrm{P}$

Mg12 $\mathrm{H}$-like $7 \mathrm{P}$

Mg12 H -like 7P

Mg12 $\mathrm{H}$-like 7P

Mg12 H -like $7 P$

Mg12 H-like 7P

Mg12 H-like $6 \mathrm{P}$
(1D) $j=2.0-1 \mathrm{~s}$

(ID) $j=2.0-1 S$

(1D) $j=2.0-1 s$

(ID) $j=2.0-1 \mathrm{~s}$

(ID) $j=2.0-15$

(3P) $j=2.0-1 S$

(3P) $j=2.0-1 S$

(3P) $j=2.0-1 S$

(3P) $j=2.0-1 S$

(3P) $j=2.0-1 S$

$\left(3 P^{*}\right) j=1.0-1 S$

$\left(3 P^{*}\right) j=1.0-1 S$

$\left(3 P^{*}\right) j=1.0-1 S$

$\left(3 P^{*}\right) j=1.0-1 S$

$\left(2 P^{\star}\right) j=1.5-1 S$

$\left(2 P^{*}\right) j=1.5-1 S$

$\left(2 P^{*}\right) j=1.5-1 S$

$\left(2 P^{*}\right) j=1.5-1 S$

$\left(2 P^{\star}\right) j=1.5-1 S$

$\left(2 P^{\star}\right) j=1.5-1 S$

$\left(2 P^{*}\right) j=1.5-1 S$

$\left(2 P^{\star}\right) j=1.5-1 S$

$\left(2 P^{*}\right) j=1.5-1 \mathrm{~S}$

$\left(2 P^{*}\right) j=1.5-1 S$

$\left(2 P^{*}\right) j=1.5-1 S$

$\left(2 P^{\star}\right) j=1.5-1 S$

$\left(2 P^{\star}\right) j=1.5-1 S$

$\left(2 P^{*}\right) j=1.5-1 S$

$\left(2 P^{\star}\right) j=1.5-1 S$

$\left(2 P^{\star}\right) j=1.5-1 S$

$\left(2 P^{*}\right) j=1.5-1 S$

$\left(2 P^{*}\right) j=1.5-1 \mathrm{~S}$

(2P*) $j=1.5-1 S$

$\left(2 P^{\star}\right) j=1.5-1 S$

$\left(2 P^{\star}\right) j=1.5-1 S$

$\left(2 P^{*}\right) j=1.5-1 S$

$\left(2 P^{\star}\right) j=1.5-15$

$\left(2 \mathrm{P}^{\star}\right) j=1.5-1 \mathrm{~S}$

$\left(2 P^{\star}\right) j=1.5-1 S$

$\left(2 P^{\star}\right) j=1.5-1 S$

$\left(2 P^{\star}\right) j=1.5-1 S$

$\left(2 P^{\star}\right) j=1.5-1 S$

$\left(2 \mathrm{P}^{\star}\right) j=1.5-1 \mathrm{~S}$

$\left(2 P^{*}\right) j=1.5-1 S$

$\left(2 P^{\star}\right) j=1.5-1 S$

$\left(2 P^{\star}\right) j=1.5-1 \mathrm{~S}$

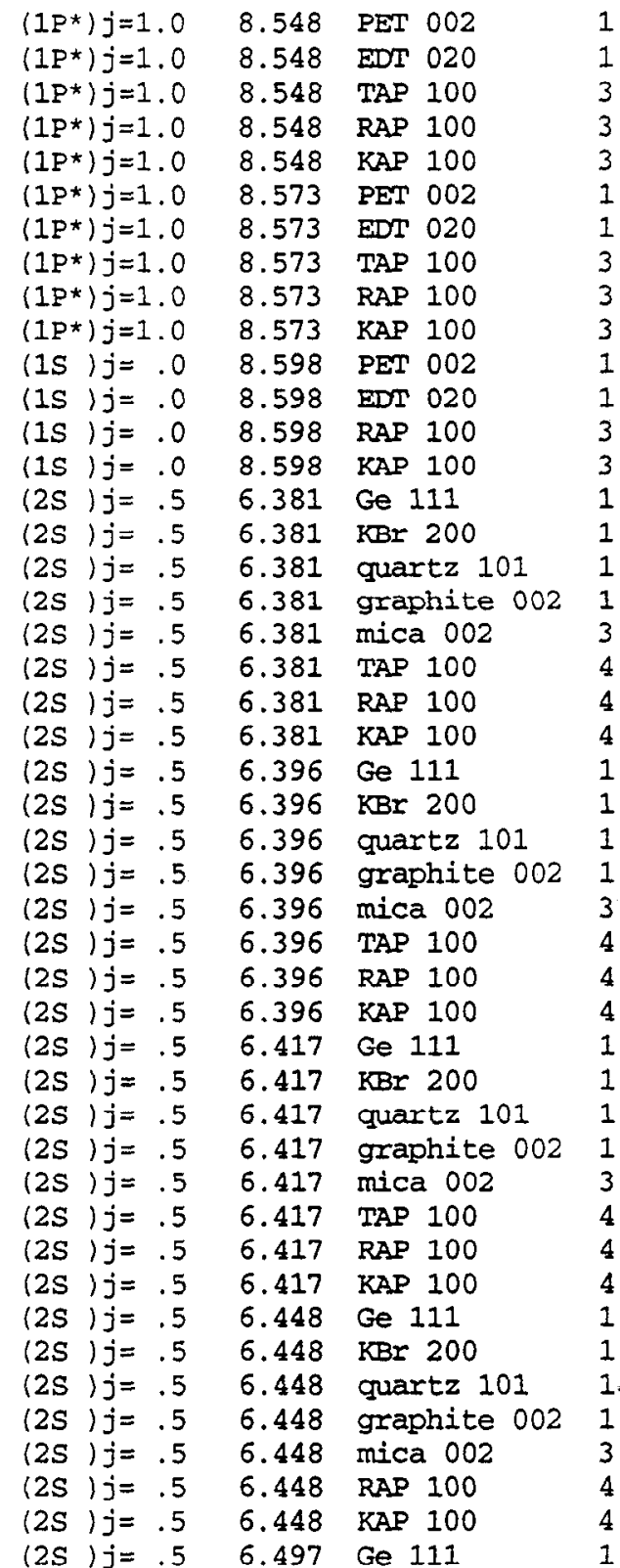

$8.742 \quad 77.907$

$8.808 \quad 76.044$

$25.763 \quad 84.491$

$26.116 \quad 79.090$

26.63474 .329

$8.742 \quad 78.716$

$8.808 \quad 76.735$

$25.763 \quad 86.651$

$26.116 \quad 79.997$

$26.634 \quad 74.938$

$8.742 \quad 79.586$

$8.808 \quad 77.464$

$26.116 \quad 80.993$

$26.634 \quad 75.572$

6.53277 .656

6.58475 .735

$6.687 \quad 72.600$

$6.696 \quad 72.356$

$19.942 \quad 73.726$

$25.763 \quad 82.190$

$26.116 \quad 77.777$

26.63473 .400

6.53278 .288

$6.584 \quad 76.275$

$6.687 \quad 73.035$

$6.696 \quad 72.784$

$19.942 \quad 74.194$

$25.763 \quad 83.242$

$26.116 \quad 78.415$

$26.634 \quad 73.858$

$\begin{array}{ll}6.532 & 79.233\end{array}$

6.58477 .068

$6.687 \quad 73.663$

$6.696 \quad 73.402$

$19.942 \quad 74.873$

$25.763 \quad 85.078$

$26.116 \quad 79.372$

26.63474 .521

6.53280 .801

$6.584 \quad 78.334$

$6.687 \quad 74.635$

$6.696 \quad 74.358$

$19.942 \quad 75.933$

$26.116 \quad 80.965$

$26.634 \quad 75.555$

$6.532 \quad 84.066$ 


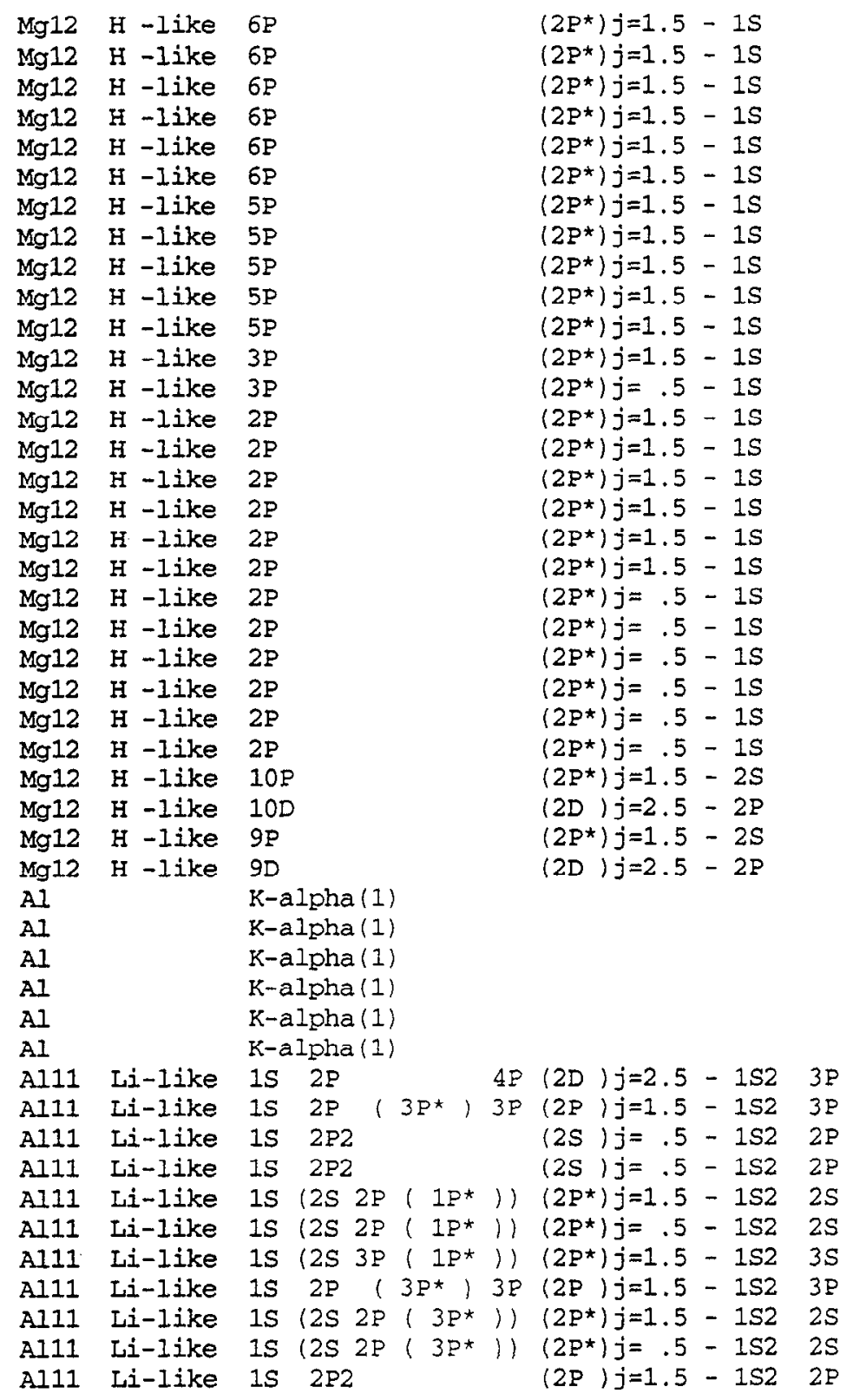

\begin{tabular}{|c|c|c|}
\hline$(2 S) j=.5$ & 6.497 & $\mathrm{KBr} 200$ \\
\hline$(2 S) j=.5$ & 6.497 & quartz 101 \\
\hline$(2 s) j=.5$ & 6.497 & graphite 002 \\
\hline (2s) $j=.5$ & 6.497 & mica 002 \\
\hline $2.5 j=.5$ & 6.497 & $\operatorname{RAP} 100$ \\
\hline s $j j=.5$ & 6.497 & $\operatorname{KAP} 100$ \\
\hline$j=.5$ & 6.580 & $\operatorname{KBr} 200$ \\
\hline S. $j j=.5$ & 6.580 & quartz 101 \\
\hline$(2 s) j=.5$ & 6.580 & graphite 002 \\
\hline$(2 s) j=.5$ & 6.580 & mica 002 \\
\hline$(2 S) j=.5$ & 6.580 & $\operatorname{KAP} 100$ \\
\hline (2s) $j=.5$ & 7.106 & InSb 111 \\
\hline$(2 S) j=.5$ & 7.107 & InSb 111 \\
\hline$S j=.5$ & 8.419 & guartz 100 \\
\hline$S) j=.5$ & 8.419 & PET 002 \\
\hline$s) j=.5$ & 8.419 & EDT 020 \\
\hline$s j j=.5$ & 8.419 & TAP 100 \\
\hline $25 i j=.5$ & 8.419 & $\operatorname{RAP} 100$ \\
\hline $2 S i j=.5$ & 8.419 & $\operatorname{KAP} 100$ \\
\hline$(2 S) j=.5$ & 8.425 & quartz 100 \\
\hline$(2 S) j=.5$ & 8.425 & PET 002 \\
\hline (2s) $j=.5$ & 8.425 & EDT 020 \\
\hline (2s) $j=.5$ & 8.425 & TAP 100 \\
\hline $5 j j=.5$ & 8.425 & $\operatorname{RAP} 100$ \\
\hline$j=.5$ & 8.425 & KAP 100 \\
\hline$j=.5$ & 26.305 & $\operatorname{KAP} 100$ \\
\hline$P * / j=1.5$ & 26.355 & $\operatorname{KAP} 100$ \\
\hline$j=.5$ & 26.564 & $\operatorname{KAP} 100$ \\
\hline *) $j=1.5$ & 26.616 & $\operatorname{KAP} 100$ \\
\hline & 8.340 & topaz 002 \\
\hline & 8.340 & quartz 100 \\
\hline & 8.340 & PET 002 \\
\hline & 8.340 & EDT 020 \\
\hline & 8.340 & $\operatorname{TAP} 100$ \\
\hline & 8.340 & RAP 100 \\
\hline$\left.P^{*}\right) j=1.5$ & 7.765 & bery 100 \\
\hline$j=1.5$ & 7.773 & beryl 100 \\
\hline *) $j=.5$ & 7.796 & beryl 100 \\
\hline$j=j=1.5$ & 7.800 & beryl 100 \\
\hline$j=.5$ & 7.810 & beryl 100 \\
\hline$\langle 2 s) j=.5$ & 7.811 & beryl 100 \\
\hline$(2 s) j=.5$ & 7.815 & beryl 100 \\
\hline $\left.2 P^{*}\right) j=1.5$ & 7.827 & beryl 100 \\
\hline es $j j=.5$ & 7.846 & beryl 100 \\
\hline S $j j=.5$ & 7.849 & beryl 100 \\
\hline$j=1.5$ & 7.856 & beryl 100 \\
\hline
\end{tabular}

$6.584 \quad 80.675$

$6.687 \quad 76.309$

$6.696 \quad 75.996$

$19.942 \quad 77.791$

$26.116 \quad 84.325$

$26.634 \quad 77.355$

$6.584 \quad 88.003$

$6.687 \quad 79.737$

$6.696 \quad 79.320$

$19.942 \quad 81.838$

$26.634 \quad 81.193$

$\begin{array}{ll}7.481 & 71.782\end{array}$

$7.481 \quad 71.806$

$8.512 \quad 81.523$

$8.742 \quad 74.376$

$8.808 \quad 72.908$

$25.763 \quad 78.626$

$26.116 \quad 75.264$

$26.634 \quad 71.496$

8.51281 .801

$8.742 \quad 74.523$

$8.808 \quad 73.042$

$25.763 \quad 78.830$

$26.116 \quad 75.420$

$26.634 \quad 71.618$

$26.634 \quad 80.985$

$26.634 \quad 81.700$

$26.634 \quad 85.845$

$26.634 \quad 87.893$

$8.374 \quad 84.805$

$8.512 \quad 78.449$

$8.742 \quad 72.548$

$8.808 \quad 71.231$

$25.763 \quad 76.195$

$26.116 \quad 73.333$

$15.954 \quad 76.761$

$15.954 \quad 77.014$

$\begin{array}{lll}15.954 & 77.771\end{array}$

$15.954 \quad 77.908$

15.954. 78.255

$15.954 \quad 78.291$

$15.954 \quad 78.433$

$15.954 \quad 78.871$

$\begin{array}{lll}15.954 & 79.602\end{array}$

$15.954 \quad 79.722$

$15.954 \quad 80.008$ 


\begin{tabular}{|c|c|c|c|c|c|c|}
\hline Al11 & Li-like & $1 S$ & $2 P 2$ & $(2 D) j=1.5$ & $-1,52$ & $2 P$ \\
\hline A111 & Ii-like & is & $2 \mathrm{P} 2$ & $(2 D) j=1.5$ & $-1 S 2$ & $2 P$ \\
\hline Al11 & Li-like & 1S & $2 \mathrm{P} 2$ & $(2 D) j=2.5$ & -1.52 & 29 \\
\hline A111 & Li-like & $1 S$ & $2 P 2$ & $(2 D) j=2.5$ & -152 & $2 P$ \\
\hline Al12 & He-Iike & IS & $10 P$ & $\left(1 P^{\star}\right) j=1.0$ & $-1 s 2$ & \\
\hline $\mathrm{A} 112$ & He-like & is & $10 P$ & $\left(1 P^{\star}\right) j=1.0$ & -152 & \\
\hline A112 & He-like & $1 \mathrm{~S}$ & $10 P$ & $\left(1 P^{\star}\right) j=1.0$ & $-1 s 2$ & \\
\hline Al12 & He-like & 15 & $10 P$ & $\left(1 P^{\star}\right) j=1.0$ & $-1 s 2$ & \\
\hline A 112 & He-like & 1S & $9 P$ & $\left(1 Q^{\star}\right) j=1.0$ & -152 & \\
\hline A 112 & He-like & is & $9 P$ & $\left(1 P^{*}\right) j=1.0$ & $-1,52$ & \\
\hline A112 & He-like & 15 & $9 P$ & $\left(1 P^{\star}\right) j=1.0$ & -152 & \\
\hline Al12 & He-like & is & $9 P$ & $\left(1 P^{*}\right) j=1.0$ & $-1 s 2$ & \\
\hline Al12 & He-like & $1 S$ & $7 P$ & $\left(1 P^{\star}\right) j=1.0$ & $-1 S 2$ & \\
\hline A112 & He-like & 15 & $7 P$ & $\left(1 P^{*}\right) j=1.0$ & $-1 s 2$ & \\
\hline A 112 & He-like & is & $7 P$ & $\left(1 P^{\star}\right) j=1.0$ & $-1 s 2$ & \\
\hline A 112 & He-like & $1 S$ & $7 P$ & $\left(1 P^{\star}\right) j=1.0$ & $-1 s 2$ & \\
\hline A112 & He-like & 1s & $7 \mathrm{P}$ & $\left(1 P^{*}\right) j=1.0$ & -152 & \\
\hline A 112 & He-like & 1S & $6 P$ & $\left(1 P^{*}\right) j=1.0$ & $-1 s 2$ & \\
\hline Al12 & He-like & 1S & $6 \mathrm{P}$ & $\left(1 P^{*}\right) j=1.0$ & $-1 s 2$ & \\
\hline A112 & He-like & 15 & 69 & $\left(1 P^{*}\right) j=1.0$ & -152 & \\
\hline A112 & He-like & $1 S$ & $6 P$ & $\left(1 P^{\star}\right) j=1.0$ & $-1 S 2$ & \\
\hline A.112 & He-1ike & 1s & $5 P$ & $\left(1 P^{*}\right) j=1.0$ & $-1 s 2$ & \\
\hline A112 & He-like & $1 S$ & $5 P$ & $\left(1 P^{*}\right) j=1.0$ & $-1 s 2$ & \\
\hline A112 & He-like & 1S & $5 P$ & $\left(1 P^{\star}\right) j=1.0$ & $-1 s 2$ & \\
\hline A112 & He-like & $1 S$ & $5 P$ & $\left(1 P^{\star}\right) j=1.0$ & $-1 s 2$ & \\
\hline Al12 & He-like & $1 s$ & $5 P$ & $\left(1 P^{*}\right) j=1.0$ & $-1 s 2$ & \\
\hline A112 & He-Iike & $1 S$ & $5 P$ & $\left(1 P^{*}\right) j=1.0$ & $-1 s 2$ & \\
\hline A112 & He-like & $1 S$ & $4 P$ & $\left(1 P^{\star}\right) j=1.0$ & $-1 s 2$ & \\
\hline Al12 & He-like & $1 S$ & $4 \mathrm{P}$ & $\left(1 P^{*}\right) j=1.0$ & $-1 S 2$ & \\
\hline A 112 & Fe-like & is & $4 \mathrm{P}$ & $\left(1 P^{*}\right) j=1.0$ & $-1 s 2$ & \\
\hline A.12 & He-like & $1 S$ & $4 P$ & $\left(1 P^{\star}\right) j=1.0$ & $-1 s 2$ & \\
\hline A 112 & He-like & $1 S$ & $4 \mathrm{P}$ & $\left(1 P^{*}\right) j=1.0$ & $-1 s 2$ & \\
\hline A112 & He-like & $1 S$ & $4 \mathrm{~F}$ & $\left(1 P^{*}\right) j=1.0$ & -152 & \\
\hline A112 & He-1ike & $1 \mathrm{~s}$ & $4 P$ & $\left(1 P^{\star}\right) j=1.0$ & $-1 s 2$ & \\
\hline Al12 & He-like & 15 & $4 \mathrm{P}$ & $\left(1 \mathrm{P}^{\star}\right) j=1.0$ & $-1 s 2$ & \\
\hline A112 & He-like & is & $3 P$ & $\left(1 P^{\star}\right) j=1.0$ & -152 & \\
\hline $\mathrm{A} 112$ & He-like & 15 & $3 P$ & $\left(1 P^{\star}\right) j=1.0$ & $-1 s 2$ & \\
\hline Al12 & He-like & $1 \mathrm{~s}$ & $3 P$ & $\left(1 P^{\star}\right) j=1.0$ & $-1 s 2$ & \\
\hline A112 & He-like & $1 S$ & $3 P$ & $\left(1 P^{*}\right) j=1.0$ & -152 & \\
\hline Al12 & He-like & $1 \mathrm{~s}$ & $3 P$ & $\left(3 P^{*}\right) j=1.0$ & $-1 S 2$ & \\
\hline Al12 & He-like & $1 S$ & $3 P$ & $\left(3 P^{\star}\right) j=1.0$ & $-1 s 2$ & \\
\hline A112 & He-like & $1 \mathrm{~s}$ & $3 P$ & $\left(3 P^{\star}\right) j=1.0$ & $-1 s 2$ & \\
\hline A.112 & He-Iike & is & $3 P$ & $\left(3 P^{\star}\right) j=1.0$ & $-1 \mathrm{~s} 2$ & \\
\hline Al12 & He-like & $2 \mathrm{P} 2$ & & $(1 s) j=.0$ & -15 & $2 P$ \\
\hline AI12 & He-like & $2 \mathrm{P} 2$ & & (1s) $j=.0$ & $-1 s$ & $2 P$ \\
\hline Al12 & He-like & $2 s$ & $2 P$ & $\left(1 P^{*}\right) j=1.0$ & $-1 s$ & $2 S$ \\
\hline
\end{tabular}

\begin{tabular}{|c|c|c|}
\hline $\left.2 P^{*}\right) j=.5$ & 7.875 & topaz 002 \\
\hline$\left(2 P^{\star}\right) j=.5$ & 7.875 & beryl 100 \\
\hline $\left.2 p^{*}\right) j=1.5$ & 7.878 & topaz 002 \\
\hline$\left.p^{*}\right) j=1.5$ & 7.878 & beryl 100 \\
\hline$s j j=.0$ & 5.992 & calcite 200 \\
\hline$j=.0$ & 5.992 & Si 111 \\
\hline$j=.0$ & 5.992 & sylvite 200 \\
\hline$j=.0$ & 5.992 & eluorite 111 \\
\hline$j=.0$ & 6.009 & calcite 200 \\
\hline$j=.0$ & 6.009 & Si 111 \\
\hline$S) j=.0$ & 6.009 & sylvite 200 \\
\hline$j=.0$ & 6.009 & Eluorite 111 \\
\hline$j=.0$ & 6.059 & calcite 200 \\
\hline$j=.0$ & 6.059 & Si 111 \\
\hline$j=.0$ & 6.059 & sylvite 200 \\
\hline$j=.0$ & 6.059 & fluorite 111 \\
\hline$j=.0$ & 6.059 & $\operatorname{TAP} 100$ \\
\hline$j=.0$ & 6.100 & si 111 \\
\hline$j=.0$ & 6.100 & sylvite 200 \\
\hline$j=.0$ & 6.100 & fluorite 111 \\
\hline$j=.0$ & 6.100 & TAP 100 \\
\hline$j=.0$ & 6.174 & Si 111 \\
\hline$j=.0$ & 6.174 & sylvite 200 \\
\hline$j=.0$ & 6.174 & Eluorite 111 \\
\hline$j=.0$ & 6.174 & Ge 111 \\
\hline$j j=.0$ & 6.174 & $\operatorname{TAP} 100$ \\
\hline$\eta j=.0$ & 6.174 & RAP 100 \\
\hline$j j=.0$ & 6.313 & Ge 111 \\
\hline$j=.0$ & 6.313 & $\mathrm{KBr} 200$ \\
\hline$j=.0$ & 6.313 & quartz 101 \\
\hline$j=.0$ & 6.313 & graphite 002 \\
\hline$\rangle j=.0$ & 6.313 & mica 002 \\
\hline$j=.0$ & 6.313 & TAP 100 \\
\hline$j j=.0$ & 6.313 & RAP 100 \\
\hline$j j=.0$ & 6.313 & $\operatorname{KAP} 100$ \\
\hline$j=.0$ & 6.635 & quartz 101 \\
\hline$j=.0$ & 6.635 & graphite 002 \\
\hline$j j=.0$ & 6.635 & mica 002 \\
\hline$j j=.0$ & 6.635 & KAP 100 \\
\hline$j j=.0$ & 6.644 & quartz 101 \\
\hline$j=.0$ & 6.644 & graphite 002 \\
\hline .0 & 6.644 & mica 002 \\
\hline .0 & 6.644 & $\operatorname{KAP} 100$ \\
\hline & 7.191 & InSto 111 \\
\hline$=1.0$ & 7.191 & sum 020 \\
\hline & 7.228 & 111 \\
\hline
\end{tabular}

$8.374 \quad 70.121$

$15.954 \quad 80.828$

8.37470 .181

$15.954 \quad 80.964$

6.07180 .747

$6.271 \quad 72.845$

$6.292 \quad 72.236$

6.30871 .788

6.07181 .805

6.27173 .380

$6.292 \quad 72.750$

$6.308 \quad 72.288$

6.07186 .397

$\begin{array}{ll}6.271 & 75.059\end{array}$

6.29274 .359

$6.308 \quad 73.848$

$25.763 \quad 70.174$

$6.271 \quad 76.589$

$6.292 \quad 75.809$

$6.308 \quad 75.246$

$25.763 \quad 71.279$

$6.271 \quad 79.909$

$6.292 \quad 78.886$

$6.308 \quad 78.169$

6.53270 .943

$25.763 \quad 73.453$

$26.116 \quad 71.019$

$6.532 \quad 75.122$

$6.584 \quad 73.504$

$6.687 \quad 70.747$

$6.696 \quad 70.528$

$19.942 \quad 71.751$

$25.763 \quad 78.569$

$26.116 \quad 75.221$

$26.634 \quad 71.462$

$6.687 \quad 82.850$

$6.696 \quad 82.260$

$19.942 \quad 86.509$

$26.634 \quad 85.185$

$6.687 \quad 83.499$

$6.696 \quad 82.855$

$19.942 \quad 88.186$

$26.634 \quad 86.218$

$\begin{array}{lll}7.481 & 73.994\end{array}$

$15.185 \quad 71.284$

$7.481 \quad 75.057$ 


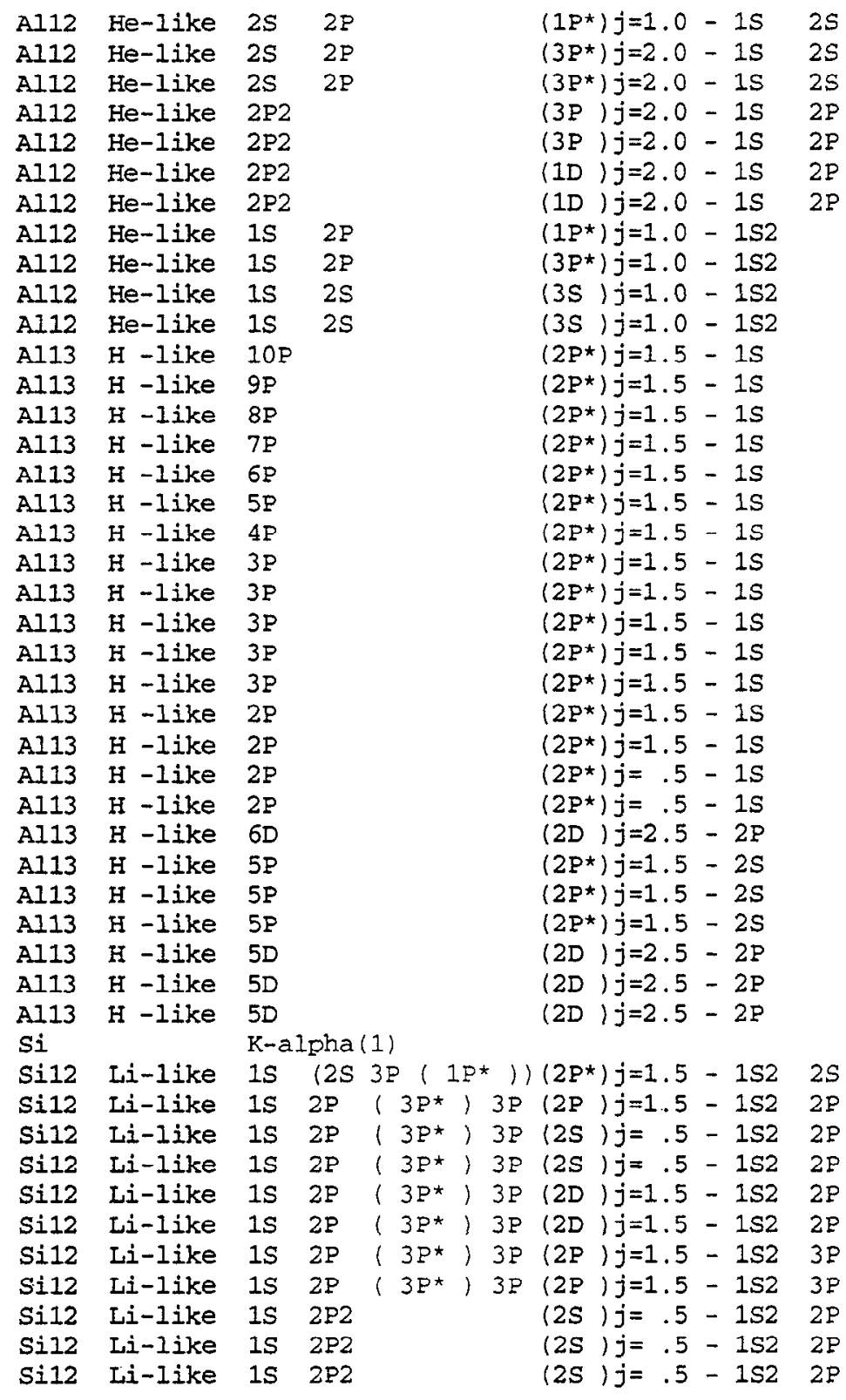

\begin{tabular}{|c|c|c|}
\hline Is $j j=.0$ & 7.228 & gypsum 020 \\
\hline $35 / j=1.0$ & 7.251 & InSb 111 \\
\hline$j=1.0$ & 7.251 & gypsum 020 \\
\hline$j=2.0$ & 7.260 & InSb 111 \\
\hline$j=2.0$ & 7.260 & gypsum 020 \\
\hline$j j=1.0$ & 7.274 & InSb 111 \\
\hline$j=1.0$ & 7.274 & gypsum 020 \\
\hline$j=.0$ & 7.757 & beryl 100 \\
\hline$j=.0$ & 7.806 & beryl 100 \\
\hline$\nu j=.0$ & 7.872 & topaz 002 \\
\hline$j j=.0$ & 7.872 & beryl 100 \\
\hline$j j=.5$ & 5.435 & NaCl 200 \\
\hline$j=.5$ & 5.448 & $\mathrm{NaCl} 200$ \\
\hline$j=.5$ & 5.466 & $\mathrm{NaCl} 200$ \\
\hline$j=.5$ & 5.493 & $\mathrm{NaCl} 200$ \\
\hline$j=.5$ & 5.534 & $\mathrm{NaCl} 200$ \\
\hline$j=.5$ & 5.605 & $\mathrm{NaCl} 200$ \\
\hline$i j=.5$ & 5.739 & calcite 200 \\
\hline$j j=.5$ & 6.053 & calcite 200 \\
\hline$j=.5$ & 6.053 & si 111 \\
\hline$j=.5$ & 6.053 & sylvite 200 \\
\hline$j j=.5$ & 6.053 & fluorite 111 \\
\hline$j=.5$ & 6.053 & TAP 100 \\
\hline$j=.5$ & 7.171 & InSb 111 \\
\hline$j=.5$ & 7.171 & gypsum 020 \\
\hline$j=.5$ & 7.176 & InSt 111 \\
\hline$j=.5$ & 7.176 & gypsum 020 \\
\hline$j=1.5$ & 24.251 & TAP 100 \\
\hline$j j=.5$ & 25.597 & TAP 100 \\
\hline$j=.5$ & 25.597 & RAP 100 \\
\hline$j=.5$ & 25.597 & $\operatorname{KAP} 100$ \\
\hline$j=1.5$ & 25.662 & TAP 100 \\
\hline$j=1.5$ & 25.662 & RAP 100 \\
\hline$j=1.5$ & 25.662 & KAP 100 \\
\hline & 7.126 & Insb 111 \\
\hline$j=.5$ & 5.768 & calcite 200 \\
\hline$j=1.5$ & 5.7 & calcite 200 \\
\hline$=.5$ & 5.803 & calcite 200 \\
\hline$=1.5$ & 5.808 & calcite 200 \\
\hline$=.5$ & 5.812 & calcite 200 \\
\hline$=1.5$ & 5.816 & calcite 200 \\
\hline$=1.5$ & 6.661 & quartz 101 \\
\hline$=1.5$ & 6.661 & graphite 002 \\
\hline & 6.678 & quartz 101 \\
\hline$=.5$ & 6.678 & graphite 002 \\
\hline$=1.5$ & 6.681 & quartz 101 \\
\hline
\end{tabular}

$15.185 \quad 72.174$

$7.481 \quad 75.756$

$15.185 \quad 72.750$

$\begin{array}{ll}7.481 & 76.039\end{array}$

$15.185 \quad 72.981$

7.48176 .490

$15.185 \quad 73.346$

$15.954 \quad 76.512$

$15.954 \quad 78.115$

8.37470 .060

$15.954 \quad 80.693$

5.64174 .468

$5.641 \quad 74.969$

$5.641 \quad 75.691$

$5.641 \quad 76.846$

$5.641 \quad 78.823$

$5.641 \quad 83.523$

$6.071 \quad 70.964$

$6.071 \quad 85.587$

$6.271 \quad 74.848$

$6.292 \quad 74.157$

$6.308 \quad 73.653$

$25.763 \quad 70.018$

$\begin{array}{ll}7.481 & 73.448\end{array}$

$\begin{array}{ll}15.185 & 70.819\end{array}$

7.481
73.583

$15.185 \quad 70.934$

$25.763 \quad 70.273$

$25.763 \quad 83.492$

$26.116 \quad 78.558$

$26.634 \quad 73.959$

$25.763 \quad 84.925$

$26.116 \quad 79.301$

$26.634 \quad 74.473$

$7.481 \quad 72.271$

$6.071 \quad 71.822$

$6.071 \quad 72.594$

6.07172 .912

$6.071 \quad 73.074$

$6.071 \quad 73.204$

$6.071 \quad 73.335$

$6.687 \quad 84.946$

$6.696 \quad 84.139$

$6.687 \quad 87.027$

$6.696 \quad 85.798$

$6.687 \quad 87.573$ 


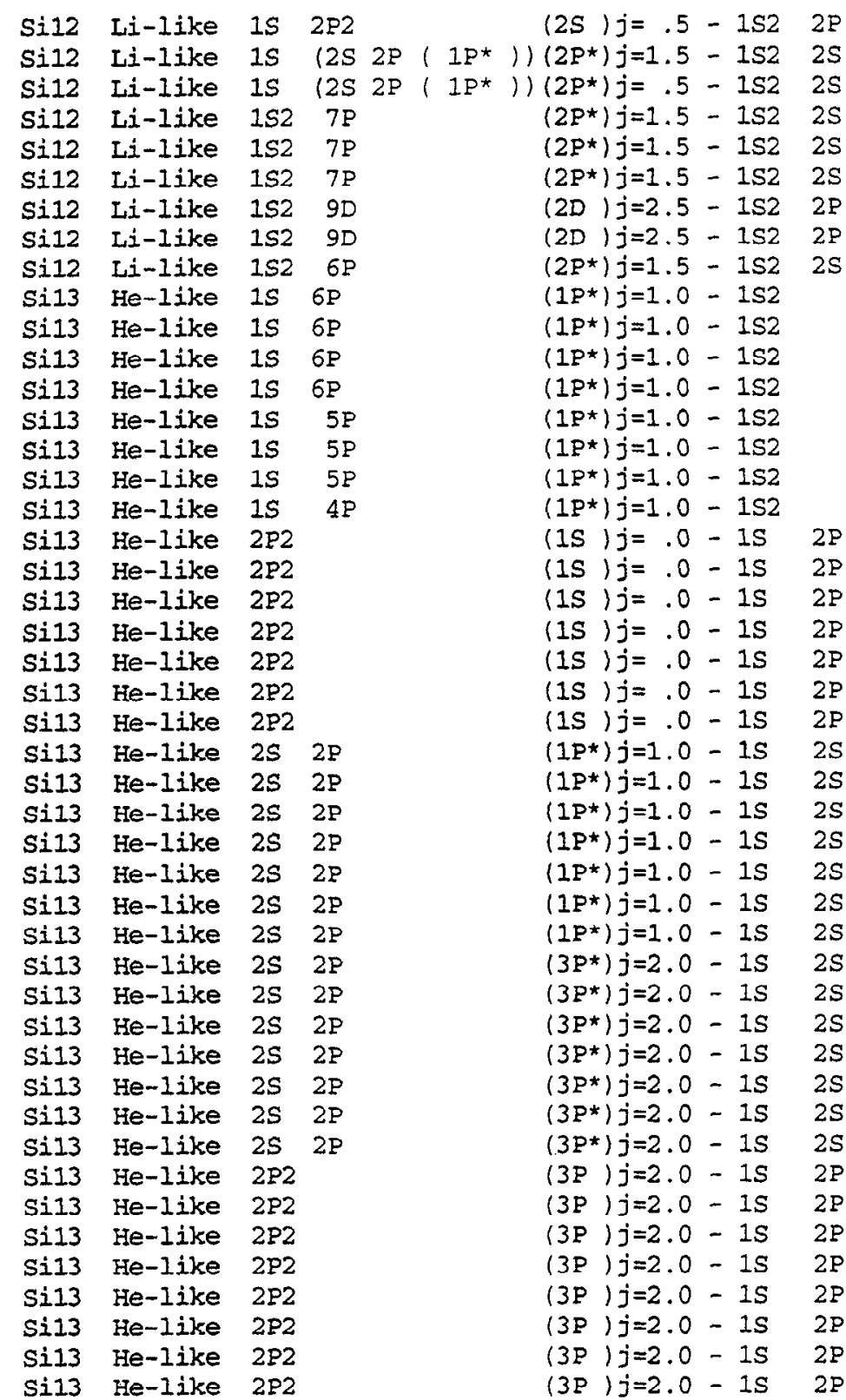

\begin{tabular}{|c|c|c|}
\hline $\left.2 P^{*}\right) j=1.5$ & 6.681 & graphite 002 \\
\hline $2 S j j=.5$ & 6.688 & graphite 002 \\
\hline $2 S j j=.5$ & 6.689 & graphite 002 \\
\hline $2 S\rangle j=.5$ & 25.655 & TAP 100 \\
\hline $2 S / j=.5$ & 25.655 & RAP 100 \\
\hline $2 S / j=.5$ & 25.655 & KAP 100 \\
\hline $\left.2 P^{\star}\right) j=1.5$ & 26.030 & $\operatorname{RAP} 100$ \\
\hline$\left.p^{\star}\right) j=1.5$ & 26.030 & KAP 100 \\
\hline$S i j=.5$ & 26.460 & $\operatorname{KAP} 100$ \\
\hline$s\rangle j=.0$ & 5.223 & ADP 101 \\
\hline$j j=.0$ & 5.223 & beryl 100 \\
\hline $5 j j=.0$ & 5.223 & $\operatorname{RAP} 100$ \\
\hline .5$) j=.0$ & 5.223 & KAP 100 \\
\hline (s) $j=.0$ & 5.285 & ADP 101 \\
\hline (s) $j=.0$ & 5.285 & beryl 100 \\
\hline (s) $j=.0$ & 5.285 & KAP 100 \\
\hline (s) $j=.0$ & 5.404 & $\mathrm{NaCl} 200$ \\
\hline$\left(P^{*}\right) j=1.0$ & 6.197 & Si 111 \\
\hline$j=1.0$ & 6.197 & sylvite 200 \\
\hline$q^{*} j j=1.0$ & 6.197 & fluorite 111 \\
\hline$\left.p^{*}\right) j=1.0$ & 6.197 & Ge 111 \\
\hline$j=1.0$ & 6.197 & $\mathrm{KBI} 200$ \\
\hline *) $j=1.0$ & 6.197 & TAP 100 \\
\hline *) $j=1.0$ & 6.197 & RAP 100 \\
\hline$(s) j=.0$ & 6.224 & Si 111 \\
\hline .S $j j=.0$ & 6.224 & sylvite 200 \\
\hline L $) j=.0$ & 6.224 & Eluorite 111 \\
\hline (s) $j=.0$ & 6.224 & Ge 111 \\
\hline $5 j=.0$ & 6.224 & $\mathrm{KBr} 200$ \\
\hline$j j=.0$ & 6.224 & TAP 100 \\
\hline$j j=.0$ & 6.224 & $\operatorname{RAP} 100$ \\
\hline$j j=1.0$ & 6.244 & Si 111 \\
\hline$j j=1.0$ & 6.244 & sylvite 200 \\
\hline$j j=1.0$ & 6.244 & Eluorite 111 \\
\hline$; j=1.0$ & 6.244 & $\mathrm{Ge} 111$ \\
\hline$j j=1.0$ & 6.244 & $\mathrm{KBI} 200$ \\
\hline$j j=1.0$ & 6.244 & TAP 100 \\
\hline$j j=1.0$ & 6.244 & RAP 100 \\
\hline$\star j j=2.0$ & 6.251 & Si 111 \\
\hline$\star j j=2.0$ & 6.251 & sylvite 200 \\
\hline$j=2.0$ & 6.251 & fluorite 111 \\
\hline *) $j=2.0$ & 6.251 & Ge 111 \\
\hline$j=2.0$ & 6.251 & $\mathrm{KBr} 200$ \\
\hline * $j=2.0$ & 6.251 & mica 002 \\
\hline$\star j j=2.0$ & 6.251 & TAP 100 \\
\hline & & \\
\hline
\end{tabular}

$6.696 \quad 86.164$

$6.696 \quad 87.199$

$6.696 \quad 87.380$

$25.763 \quad 84.752$

$26.116 \quad 79.219$

$26.634 \quad 74.417$

$26.116 \quad 85.349$

26.63477 .775

$26.634 \quad 83.447$

$10.640 \quad 79.042$

$15.954 \quad 79.154$

$26.116 \quad 89.499$

$26.634 \quad 78.670$

$10.640 \quad 83.424$

15.95483 .614

$26.634 \quad 82.817$

$5.641 \quad 73.333$

6.27181 .189

6.29280 .031

$6.308 \quad 79.236$

$6.532 \quad 71.571$

6.58470 .258

$25.763 \quad 74.187$

$26.116 \quad 71.650$

6.27182 .981

6.29281 .569

$\begin{array}{ll}6.308 & 80.639\end{array}$

6.53272 .335

6.58470 .965

$25.763 \quad 75.094$

$26.116 \quad 72.418$

6.27184 .681

$6.292 \quad 82.918$

$6.308 \quad 81.831$

6.53272 .923

6.58471 .507

$25.763 \quad 75.802$

$26.116 \quad 73.009$

6.27185 .423

$6.292 \quad 83.456$

$6.308 \quad 82.292$

$.6 .532 \quad 73.133$

$6.584 \quad 71.700$

$19.942 \quad 70.115$

$25.763 \quad 76.058$

$26.116 \quad 73.220$

Page 11 


\begin{tabular}{|c|c|c|c|}
\hline Si13 & He-like & $2 \mathrm{P} 2$ & \\
\hline Si13 & He-Ijke & $2 \mathrm{P} 2$ & \\
\hline Si13 & He-like & $2 \mathrm{P} 2$ & \\
\hline Si13 & He-like & $2 \mathrm{P} 2$ & \\
\hline Si13 & He-like & $2 \mathrm{P} 2$ & \\
\hline Si13 & He-like & $2 \mathrm{P} 2$ & \\
\hline Si13 & He-like & $2 \mathrm{P2}$ & \\
\hline $\operatorname{si13}$ & He-like & $2 \mathrm{P} 2$ & \\
\hline Si13 & He-like & $2 \mathrm{P} 2$ & \\
\hline $\operatorname{si13}$ & He-like & 15 & $2 P$ \\
\hline $\operatorname{si13}$ & He-like & $1 \mathrm{~S}$ & $2 P$ \\
\hline Si13 & He-Iike & $1 S$ & $2 \mathrm{P}$ \\
\hline Si13 & He-like & $1 s$ & $2 P$ \\
\hline $\operatorname{si13}$ & He-1ike & $1 \mathrm{~s}$ & $2 P$ \\
\hline $\operatorname{Sil} 4$ & $\mathrm{H}-1 \mathrm{ike}$ & $10 P$ & \\
\hline Si14 & H -like & $10 P$ & \\
\hline Si14 & H -like & $9 P$ & \\
\hline $\operatorname{si14}$ & H -like & $9 P$ & \\
\hline Si14 & H -like & $9 P$ & \\
\hline Si14 & H -like & $8 P$ & \\
\hline Si14 & H -like & $8 P$ & \\
\hline $\mathrm{Si14}$ & H -like & $8 P$ & \\
\hline Si14 & $\mathrm{H}$-like & $7 P$ & \\
\hline Sil4 & H-like & $7 P$ & \\
\hline $\operatorname{Si14}$ & $\mathrm{H}$-like & $7 P$ & \\
\hline Si14 & H -like & $6 P$ & \\
\hline Si14 & H -like & $6 \mathrm{P}$ & \\
\hline Si14 & H -like & $6 P$ & \\
\hline Sil4 & $\mathrm{H}$-like & $6 \mathrm{P}$ & \\
\hline Si14 & H -like & $5 P$ & \\
\hline Si14 & $\mathrm{H}$-like & $5 P$ & \\
\hline Sil4 & H -like & $5 P$ & \\
\hline Si14 & H -like & $5 P$ & \\
\hline Sil4 & H-like & $4 \mathrm{P}$ & \\
\hline Sil4 & H -like & $4 \mathrm{P}$ & \\
\hline Si14 & H-like & $4 \mathrm{P}$ & \\
\hline Si14 & H-like & $4 \mathrm{P}$ & \\
\hline Si14 & H -like & $4 \mathrm{P}$ & \\
\hline Si14 & $\mathrm{H}$-like & $3 p$ & \\
\hline Si14 & $\mathrm{H}-1 \mathrm{ike}$ & $3 p$ & \\
\hline Si14 & $\mathrm{H}$-like & $3 P$ & \\
\hline Sil4 & H -like & $3 P$ & \\
\hline Sil4 & H -like & $3 P$ & \\
\hline Si14 & H -like & $3 P$ & \\
\hline Si14 & H -like & $3 p$ & \\
\hline Sil4 & H -like & $3 P$ & \\
\hline
\end{tabular}

$(1 D) j=2.0-1 S$
$(1 D) j=2.0-1 S$
$(1 D) j=2.0-1 S$
$(1 D) j=2.0-1 S$
$(1 D) j=2.0-1 S$
$(1 D) j=2.0-1 S$
$(1 D) j=2.0-1 S$
$(1 D) j=2.0-1 S$
$(1 D) j=2.0-1 S$
$\left(1 P^{\star}\right) j=1.0-1 S 2$
$\left(1 P^{\star}\right) j=1.0-1 S 2$
$\left(1 P^{\star}\right) j=1.0-1 S 2$
$\left(1 P^{\star}\right) j=1.0-1 S 2$
$\left(3 P^{\star}\right) j=1.0-1 S 2$
$\left(2 P^{\star}\right) j=1.5-1 S$
$\left(2 P^{\star}\right) j=1.5-1 S$
$\left(2 P^{\star}\right) j=1.5-1 S$
$\left(2 P^{\star}\right) j=1.5-1 S$
$\left(2 P^{\star}\right) j=1.5-1 S$
$\left(2 P^{\star}\right) j=1.5-1 S$
$\left(2 P^{\star}\right) j=1.5-1 S$
$\left(2 P^{\star}\right) j=1.5-1 S$
$\left(2 P^{\star}\right) j=1.5-1 S$
$\left(2 P^{\star}\right) j=1.5-1 S$
$\left(2 P^{\star}\right) j=1.5-1 S$
$\left(2 P^{\star}\right) j=1.5-1 S$
$\left(2 P^{\star}\right) j=1.5-1 S$
$\left(2 P^{\star}\right) j=1.5-1 S$
$\left(2 P^{\star}\right) j=1.5-1 S$
$\left(2 P^{\star}\right) j=1.5-1 S$
$\left(2 P^{\star}\right) j=1.5-1 S$
$\left(2 P^{\star}\right) j=1.5-1 S$
$\left(2 P^{\star}\right) j=1.5-1 S$
$\left(2 P^{\star}\right) j=1.5-1 S$
$\left(2 P^{\star}\right) j=1.5-1 S$
$\left(2 P^{\star}\right) j=1.5-1 S$
$\left(2 P^{\star}\right) j=1.5-1 S$
$\left(2 P^{\star}\right) j=1.5-1 S$
$\left(2 P^{\star}\right) j=1.5-1 S$
$\left(2 P^{\star}\right) j=1.5-1 S$
$\left(2 P^{\star}\right) j=1.5-1 S$
$\left(2 P^{\star}\right) j=1.5-1 S$
$\left(2 P^{\star}\right) j=.5-1 S$
$\left(2 P^{\star}\right) j=.5-1 S$
$\left(2 P^{\star}\right) j=.5-1 S$
$\left(2 P^{\star}\right) j=.5-1 S$

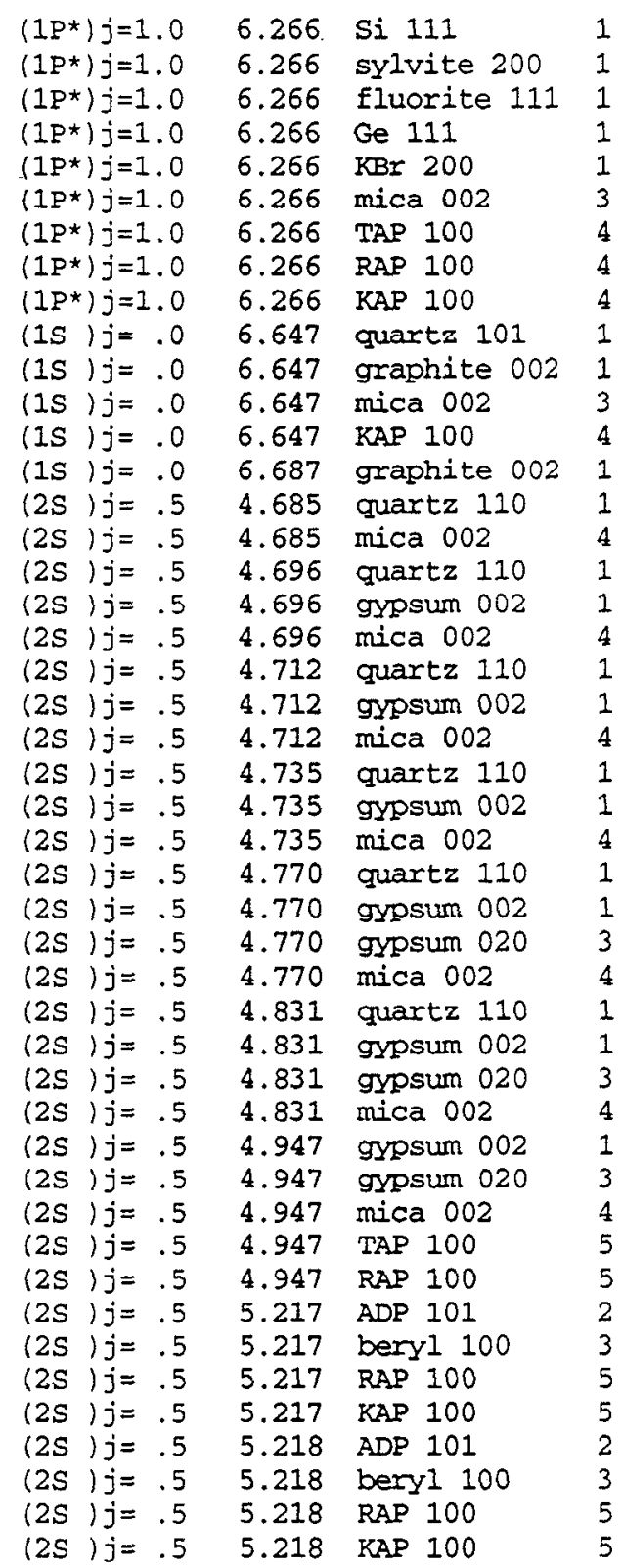

$6.271 \quad 87.712$

$6.292 \quad 84.789$

$6.308 \quad 83.385$

6.53273 .593

6.58472 .120

$19.942 \quad 70.499$

$25.763 \quad 76.623$

$26.116 \quad 73.682$

$26.634 \quad 70.229$

$6.687 \quad 83.730$

$6.696 \quad 83.064$

$19.942 \quad 89.426$

$26.634 \quad 86.632$

$6.696 \quad 87.029$

$4.912 \quad 72.513$

$19.942 \quad 70.005$

$4.912 \quad 72.945$

$4.990 \quad 70.234$

$19.942 \quad 70.379$

$4.912 \quad 73.594$

$4.990 \quad 70.785$

$19.942 \quad 70.934$

$4.912 \quad 74.572$

$4.990 \quad 71.604$

$19.942 \quad 71.760$

4.91276 .190

$4.990 \quad 72.923$

$15.185 \quad 70.455$

$19.942 \quad 73.092$

$4.912 \quad 79.580$

$4.990 \quad 75.497$

$15.185 \quad 72.636$

$19.942 \quad 75.699$

$4.990 \quad 82.473$

$\begin{array}{rr}15.185 & 77.781\end{array}$

$19.942 \quad 82.875$

$25.763 \quad 73.760$

$26.116 \quad 71.284$

$10.640 \quad 78.707$

15.954 .78 .816

$26.116 \quad 87.208$

$26.634 \quad 78.347$

$10.640 \quad 78.762$

$15.954 \quad 78.871$

$26.116 \quad 87.443$

26.63478 .400 


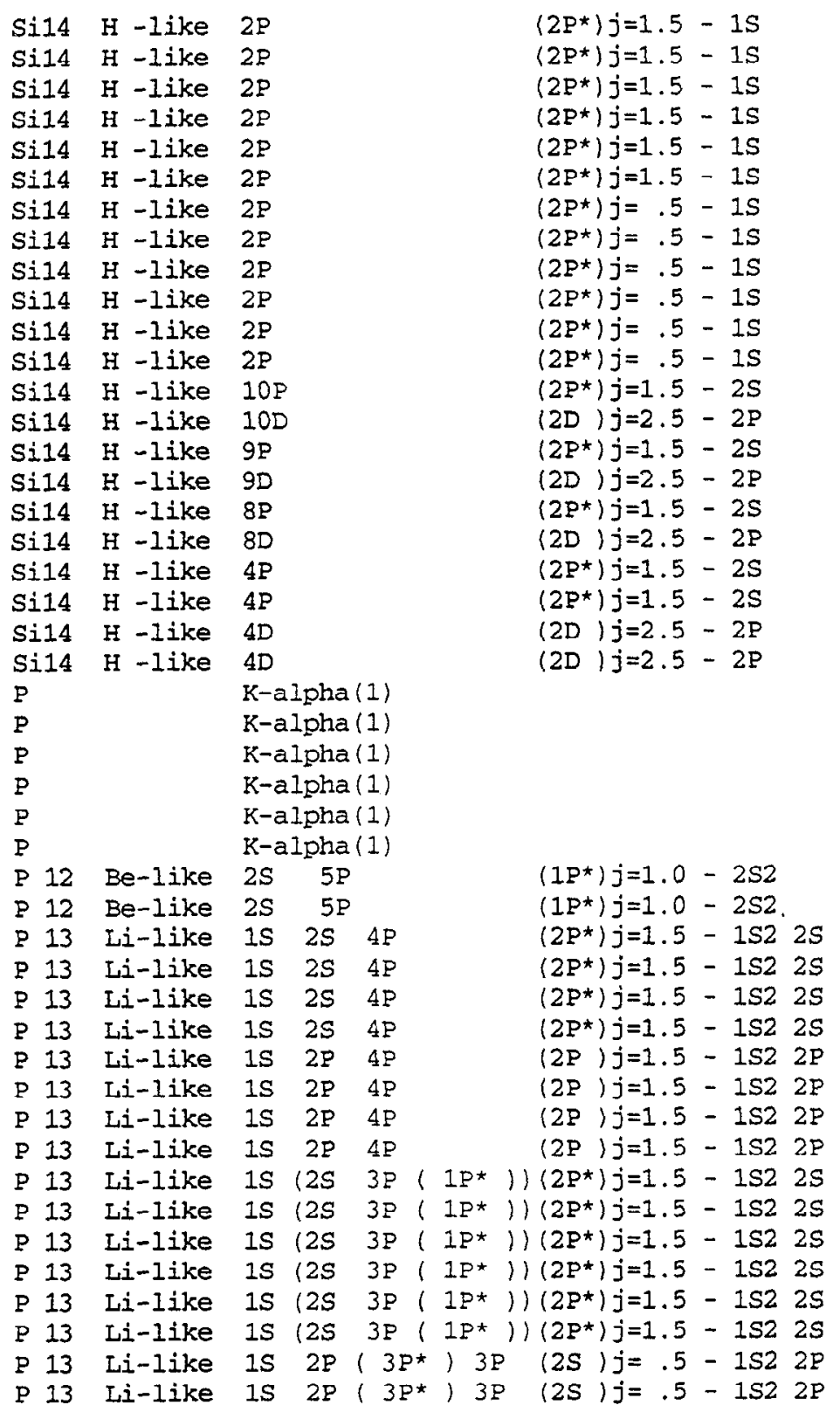

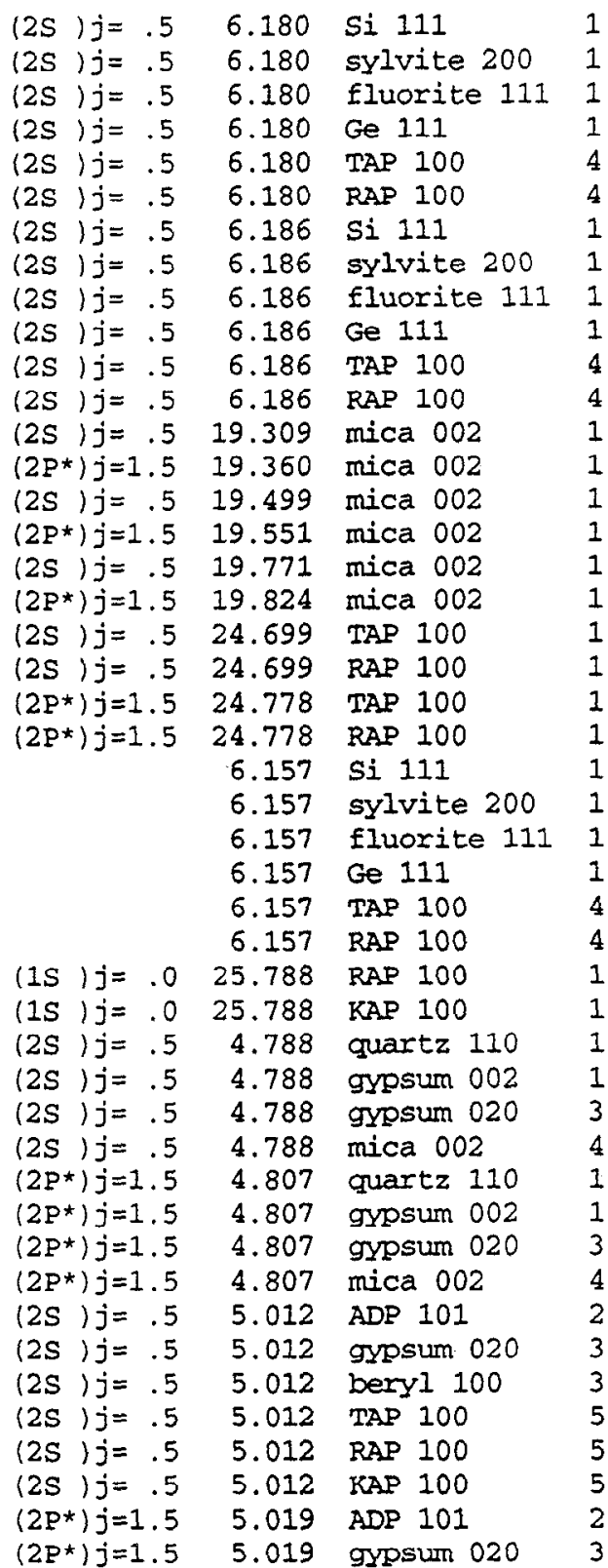

\begin{tabular}{|c|c|}
\hline 71 & \\
\hline & \\
\hline & \\
\hline & \\
\hline 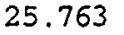 & \\
\hline & \\
\hline & \\
\hline & \\
\hline & \\
\hline 6.5 & 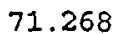 \\
\hline & \\
\hline & \\
\hline . & \\
\hline 9.5 & \\
\hline & \\
\hline & \\
\hline & \\
\hline & \\
\hline & \\
\hline 6.1 & 71 \\
\hline . & , \\
\hline 5. & \\
\hline 6 & \\
\hline 6.2 & \\
\hline & \\
\hline & \\
\hline 5.76 & 72 \\
\hline .1 & \\
\hline 5.1 & \\
\hline 6 & \\
\hline 4.9 & \\
\hline & \\
\hline & \\
\hline 9.94 & 7 \\
\hline 4.91 & 10. \\
\hline 4.9 & 74 \\
\hline 3.1 & 71 \\
\hline 9.9 & 74 \\
\hline & \\
\hline 15.185 & 81. \\
\hline 15.95 & 70.46 \\
\hline .7 & \\
\hline & \\
\hline & \\
\hline & \\
\hline & \\
\hline
\end{tabular}




\begin{tabular}{|c|c|c|c|c|c|c|c|c|c|}
\hline ? & 13 & Li-like & $1 \mathrm{~S}$ & $2 P$ & $\left(3 P^{*}\right) 3 P$ & $(2 S)$ & $j=.5$ & -152 & $2 \mathrm{P}$ \\
\hline $\mathrm{P}$ & 13 & Li-like & 1S & $2 P$ & $\left(3 P^{*}\right) 3 P$ & $(2 S)$ & $j=.5$ & $-1 S 2$ & $2 \mathrm{P}$ \\
\hline $\mathrm{P}$ & 13 & Li-like & $1 S$ & $2 P$ & $\left(3 P^{\star}\right)$ & (2S) & $j=.5$ & $-1 S 2$ & $2 P$ \\
\hline$P$ & 13 & Li-like & 1S & $2 P$ & $\left.3 P^{\star}\right)$ & (2S) & $j=.5$ & $-1 S 2$ & $2 \mathrm{P}$ \\
\hline $\mathrm{P}$ & 13 & Li-like & is & $2 \mathrm{P}$ & $\left(3 P^{*}\right)$ & $(2 \mathrm{P})$ & $j=1.5$ & $-1 s 2$ & $2 P$ \\
\hline$P$ & 1 & Li-like & 1S & $2 P$ & $(3 P *)$ & $(2 \mathrm{P}$ & $j=1.5$ & - IS2 & $2 P$ \\
\hline $\mathbf{P}$ & 1 & Li-like & $1 S$ & $2 \mathrm{P}$ & $3 P^{*} 1$ & (2P & $j j=1.5$ & $-1 S 2$ & $2 \mathrm{P}$ \\
\hline P & 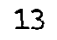 & Li-like & $1 \mathrm{~s}$ & $2 P$ & $3 P * 1$ & $(2 \mathrm{P}$ & $j j=1.5$ & -152 & $2 P$ \\
\hline$P$ & 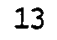 & Li-like & $1 S$ & $2 \mathrm{P}$ & $3 P^{*}, 3 P$ & $(2 \mathrm{P})$ & $j j=1.5$ & $-1 s 2$ & $2 \mathrm{P}$ \\
\hline $\mathbf{P}$ & & Li-like & 1S & $2 \mathrm{P}$ & $\left(3 P^{*}\right) 3 P$ & $(2 \mathrm{P})$ & $j j=1.5$ & -1.52 & $2 P$ \\
\hline P & $I_{0}$ & Li-like & $1 S$ & $2 \mathrm{P}$ & $\left(3 P^{\star}\right) 3 F$ & $(2 \mathrm{D})$ & $j=2.5$ & $-1 s 2$ & $2 P$ \\
\hline$P$ & 1. & Ii-1ike & 15 & $2 \mathrm{P}$ & $(3 P *) 3 E$ & $(2 \mathrm{D})$ & $j j=2.5$ & $-1 s 2$ & $2 \mathrm{P}$ \\
\hline$P$ & 13 & Li-Iike & 15 & $2 \mathrm{P}$ & $\left(3 P^{*}\right) 3 P$ & $(2 D)$ & $j=2.5$ & $-1 s 2$ & $2 \mathrm{P}$ \\
\hline$P$ & 1 & Li-like & $1 \mathrm{~s}$ & $2 P$ & $\left(3 P^{*}\right) 3 P$ & $(2 D)$ & $j=2.5$ & -152 & $2 F$ \\
\hline$P$ & 1 & Li-like & 15 & $2 P$ & $\left(3 P^{*}\right) 3 P$ & $(2 D$ & $j j=2.5$ & $-1 S 2$ & $2 P$ \\
\hline$P$ & 1 & Li-like & 15 & $2 P$ & $\left(3 P^{*}\right) 3 P$ & $(2 D$ & $j j=2.5$ & $-1 s 2$ & $2 \mathrm{P}$ \\
\hline$P$ & 13 & Li-like & 15 & $2 \mathrm{P}$ & $\left(1 P^{*}\right) 3 E$ & $(2 S$ & $j=.5$ & $-1 s 2$ & $P$ \\
\hline $\mathrm{P}$ & 13 & Li-1ike & is & $2 \mathrm{P}$ & $\left(1 P^{*}\right) 3 E$ & $(2 D$ & $j=2.5$ & -152 & $3 P$ \\
\hline$P$ & 13 & Li-like & is & $2 P$ & $19 * / 3 P$ & $(2 \mathrm{P})$ & $j j=.5$ & - 152 & $3 P$ \\
\hline P & 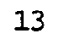 & Li-like & IS & $2 \mathrm{P}$ & $\left(1 P^{*}\right) 3 P$ & $(2 \mathrm{P})$ & $j j=.5$ & $-1 s 2$ & $\mathrm{P}$ \\
\hline $\mathbf{P}$ & 13 & Li-like & $1 S$ & $2 \mathrm{P} 2$ & & $(2 S)$ & $j=.5$ & -152 & 25 \\
\hline P & 13 & Li-like & is & $12 S$ & $2 P$ & )) $\left(2 P^{\star}\right)$ & $j=1.5$ & -152 & $S$ \\
\hline $\mathbf{P}$ & 13 & Li-like & $1 \mathrm{~S}$ & $(2 S$ & $2 P\left(3 P^{*}\right.$ & )) $\left(2 \mathrm{P}^{\star}\right)$ & $\gamma j=1.5$ & $-1 s 2$ & $2 S$ \\
\hline P & 13 & Li-like & 15 & $2 \mathrm{P} 2$ & & $2 \mathrm{P}$ & $j=1.5$ & $-1 s 2$ & $2 \mathrm{P}$ \\
\hline$P$ & 13 & Li-like & 1S & $2 \mathrm{P} 2$ & & $(2 D)$ & $j=1.5$ & -152 & $2 P$ \\
\hline $\mathrm{P}$ & 13 & Li-1ike & 1s & $2 \mathrm{P} 2$ & & $(2 D)$ & $j=2.5$ & $-1 s 2$ & $P$ \\
\hline$P$ & 13 & Li-like & $1 \mathrm{~S}$ & $2 \mathrm{P} 2$ & & $4 \mathrm{P}$ & $j=2.5$ & -152 & $2 \mathrm{P}$ \\
\hline P & 13 & Li-like & $1 \mathrm{~s} 2$ & $5 D$ & & ) & $j=1.5$ & $-1 S 2$ & $P$ \\
\hline $\mathbf{P}$ & 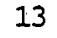 & Li-like & $1 s 2$ & $5 D$ & & $(2 \mathrm{D})$ & $=1.5$ & -152 & $P$ \\
\hline $\mathrm{P}$ & 13 & Li-1ike & $1 \mathrm{~S} 2$ & 50 & & $2 \mathrm{D} !$ & $j=1.5$ & $-1 s 2$ & 5 \\
\hline $\mathbf{P}$ & 13 & Li-like & $1 \mathrm{~S} 2$ & $5 D$ & & $2 \mathrm{DI}$ & $j=2.5$ & $-1 s 2$ & 5 \\
\hline P & 13 & Li-like & $1 \mathrm{~S} 2$ & $5 D$ & & $2 \mathrm{D})$ & $j=2.5$ & $-1 s 2$ & $2 \mathrm{P}$ \\
\hline P & 13 & Li-like & 152 & $5 D$ & & $(2 D)$ & $j=2.5$ & $-1 s 2$ & $2 P$ \\
\hline$P$ & 13 & Li-like & $1 \mathrm{~S} 2$ & $4 \mathrm{P}$ & & $\left(2 \mathrm{P}^{\star}\right)$ & $j=1.5$ & $-1 S 2$ & $S$ \\
\hline P & 14 & He-like & is & $7 \mathrm{P}$ & & 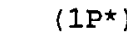 & $j=1.0$ & -152 & \\
\hline$P$ & 14 & He-like & $1 S$ & $7 \mathrm{P}$ & & & $j=1.0$ & $-1 S 2$ & \\
\hline $\mathbf{P}$ & 14 & He-like & is & $7 \mathrm{P}$ & & & $j=1.0$ & $-1 s 2$ & \\
\hline $\mathrm{P}$ & 14 & He-like & 15 & $6 P$ & & & $j=1.0$ & $-1 S 2$ & \\
\hline P & 14 & He-like & $1 \mathrm{~S}$ & $6 \mathrm{P}$ & & * & $j=1.0$ & $-1 S 2$ & \\
\hline$P$ & 14 & He-like & is & $6 \mathrm{P}$ & & & $j=1.0$ & $-1 S 2$ & \\
\hline P & 14 & He-like & is & $5 P$ & & & $j=1.0$ & -152 & \\
\hline$P$ & 14 & He-like & $1 s$ & $5 P$ & & & $j=1.0$ & $-1 S 2$ & \\
\hline $\mathrm{F}$ & & He-like & $1 S$ & $4 \mathrm{P}$ & & & $j=1.0$ & $-1 S 2$ & \\
\hline F & & He-like & is & $3 P$ & & & $j=1.0$ & $-1 S 2$ & \\
\hline & & $\mathrm{He}-1 \mathrm{ike}$ & 1s & $3 P$ & & & $j=1.0$ & -152 & \\
\hline & & He-like & is & $3 P$ & & $(1)^{\star}$ & $j=1.0$ & $-1 S 2$ & \\
\hline
\end{tabular}

\begin{tabular}{|c|c|c|}
\hline$\left(2 P^{*}\right) j=1.5$ & 5.019 & bery 1100 \\
\hline$\left(2 P^{\star}\right) j=1.5$ & 5.019 & TAP 100 \\
\hline$\left(2 P^{*}\right) j=1.5$ & 5.019 & $\operatorname{RAP} 100$ \\
\hline$\left(2 P^{*}\right) j=1.5$ & 5.019 & KAP 100 \\
\hline$\left(2 P^{\star}\right) j=1.5$ & 5.020 & ADP 101 \\
\hline$\left(2 P^{*}\right) j=1.5$ & 5.020 & gypsum 020 \\
\hline$\left(2 P^{*}\right) j=1.5$ & 5.020 & bery 100 \\
\hline$\left(2 P^{*}\right) j=1.5$ & 5.020 & TAP 100 \\
\hline$\left(2 P^{\star}\right) j=1.5$ & 5.020 & RAP 100 \\
\hline$\left(2 P^{*}\right) j=1.5$ & 5.020 & KAP 100 \\
\hline$\left(2 P^{\star}\right) j=1.5$ & 5.039 & ADP 101 \\
\hline$\left(2 P^{*}\right) j=1.5$ & 5.039 & gypsum 020 \\
\hline$\left(2 P^{*}\right) j=1.5$ & 5.039 & beryl 100 \\
\hline$\left(2 P^{\star}\right) j=1.5$ & 5.039 & TAP 100 \\
\hline$\left(2 P^{*}\right) j=1.5$ & 5.039 & $\operatorname{RAP} 100$ \\
\hline$\left(2 P^{*}\right) j=1.5$ & 5.039 & $\operatorname{KAP} 100$ \\
\hline$\left(2 P^{*}\right) j=1.5$ & 5.380 & $\mathrm{NaCl} 200$ \\
\hline$\left(2 P^{*}\right) j=1.5$ & 5.385 & $\mathrm{NaCl} 200$ \\
\hline$\left(2 P^{\star}\right) j=.5$ & 5.764 & calcite 200 \\
\hline$\left(2 P^{\star}\right) j=1.5$ & 5.770 & calcite 200 \\
\hline$\left(2 P^{\star}\right) j=1.5$ & 5.787 & calcite 200 \\
\hline$\langle 2 S\rangle j=.5$ & 5.791 & calcite 200 \\
\hline$(2 S) j=.5$ & 5.816 & calcite 200 \\
\hline$\left(2 P^{\star}\right) j=1.5$ & 5.823 & calcite 200 \\
\hline$\left(2 P^{*}\right) j=.5$ & 5.831 & calcite 200 \\
\hline$\left(2 P^{*}\right) j=1.5$ & 5.836 & calcite 200 \\
\hline$\left(2 P^{\star}\right) j=1.5$ & $\begin{array}{r}5.869 \\
25.103\end{array}$ & calcite 200 \\
\hline$\left(2 \mathrm{P}^{\star}\right) j=.5$ & 25.103 & TAP 100 \\
\hline$\left(2 P^{\star}\right) j=.5$ & 25.103 & RAP 100 \\
\hline$\left(2 P^{*}\right) j=.5$ & 25.103 & KAP 100 \\
\hline$\left(2 P^{\star}\right) j=1.5$ & 25.169 & TAP 100 \\
\hline$\left(2 P^{*}\right) j=1.5$ & 25.169 & RAP 100 \\
\hline$\left(2 P^{\star}\right) j=1.5$ & 25.169 & $\mathrm{KAP} 100$ \\
\hline$(2 S) j=.5$ & 26.608 & KAP 100 \\
\hline$(1 s) j=.0$ & 4.485 & quartz 112 \\
\hline$(1 S) j=.0$ & 4.485 & topaz 200 \\
\hline$(1 s) j=.0$ & 4.485 & $A 1111$ \\
\hline (1s) $j=.0$ & 4.520 & quartz 112 \\
\hline$(1 S) j=.0$ & 4.520 & topaz 200 \\
\hline$(1 s) j=.0$ & 4.520 & Al 111 \\
\hline$(1 S) j=.0$ & 4.574 & topaz 200 \\
\hline$(1 s) j=.0$ & 4.574 & Al 111 \\
\hline$(1 s) j=.0$ & 4.677 & quartz 110 \\
\hline$(1 s) j=.0$ & 4.918 & gypsum 002 \\
\hline$(1 S) j=.0$ & 4.918 & gypsum 020 \\
\hline 1s $j j=.0$ & 4.918 & mica 002 \\
\hline
\end{tabular}

\begin{tabular}{|c|c|}
\hline & \\
\hline 5.763 & 76.92 \\
\hline 116 & \\
\hline & \\
\hline & \\
\hline & \\
\hline & \\
\hline & \\
\hline & \\
\hline & \\
\hline & \\
\hline 1 & \\
\hline 0. & \\
\hline & \\
\hline 0 & \\
\hline .0 & 71 \\
\hline 6 & 72 \\
\hline 5.641 & 72 \\
\hline 6.071 & 71 \\
\hline 6.071 & 71 \\
\hline 6.071 & 72 \\
\hline 6.071 & 72 \\
\hline 6.071 & \\
\hline 6.071 & \\
\hline 6.071 & \\
\hline 6.071 & \\
\hline 6.071 & \\
\hline 25.763 & \\
\hline .116 & 73 \\
\hline .63 & 70 \\
\hline .763 & 77 \\
\hline .11 & 74 \\
\hline 26.634 & 70 \\
\hline 26.634 & 87 \\
\hline 4.564 & 79 \\
\hline 4.638 & 75 \\
\hline 4.676 & 73 \\
\hline 4.564 & 82 \\
\hline 4.638 & 77 \\
\hline 4.67 & \\
\hline 4.638 & 80 \\
\hline 4.676 & 78 \\
\hline & \\
\hline & \\
\hline & \\
\hline & \\
\hline
\end{tabular}




\begin{tabular}{|c|c|c|c|c|}
\hline$P$ & 14 & He-like & $1 S$ & $3 P$ \\
\hline$P$ & 14 & He-like & 15 & $3 P$ \\
\hline $\mathrm{P}$ & 14 & He-like & 15 & $3 P$ \\
\hline $\mathrm{P}$ & 14 & He-like & 15 & $3 P$ \\
\hline $\mathrm{P}$ & 14 & He-like & 15 & $3 P$ \\
\hline $\mathrm{p}$ & 14 & He-like & 15 & $3 P$ \\
\hline$P$ & 14 & He-like & 15 & $3 P$ \\
\hline P & 14 & He-like & $2 \mathrm{P} 2$ & \\
\hline $\mathrm{P}$ & 14 & He-Iike & 15 & $2 P$ \\
\hline $\mathrm{P}$ & 14 & He-like & 15 & $2 P$ \\
\hline$P$ & 14 & He-like & 15 & $2 S$ \\
\hline $\mathrm{P}$ & 15 & H -like & $10 P$ & \\
\hline$P$ & 15 & H -like & $10 P$ & \\
\hline$P$ & 15 & H -like & $10 P$ & \\
\hline$P$ & 15 & H -like & $9 P$ & \\
\hline $\mathrm{P}$ & 15 & H -like & $9 P$ & \\
\hline$P$ & 15 & H -like & $9 P$ & \\
\hline$P$ & 15 & H -like & $8 P$ & \\
\hline$P$ & 15 & H -like & $8 P$ & \\
\hline$P$ & 15 & H -like & $8 P$ & \\
\hline$P$ & 15 & H -like & $7 P$ & \\
\hline$P$ & 15 & H -like & 79 & \\
\hline$P$ & 15 & H -like & $7 P$ & \\
\hline $\mathrm{P}$ & 15 & H -like & $7 P$ & \\
\hline $\mathrm{P}$ & 15 & H-like & $6 P$ & \\
\hline$P$ & 15 & H -like & $6 P$ & \\
\hline 8 & 15 & H -like & $6 P$ & \\
\hline $\mathrm{P}$ & 15 & H-Iike & $6 P$ & \\
\hline$P$ & 15 & $\mathrm{H}-1 \mathrm{ike}$ & $6 P$ & \\
\hline$P$ & 15 & $\mathrm{H}$-like & $5 P$ & \\
\hline$P$ & 15 & H -like & $5 P$ & \\
\hline $\mathrm{P}$ & 15 & H -like & $5 P$ & \\
\hline $\mathrm{P}$ & 15 & H-Iike & $5 P$ & \\
\hline $\mathrm{P}$ & 15 & $\mathrm{H}$-iike & $5 P$ & \\
\hline $\mathrm{P}$ & 15 & H -like & $4 \mathrm{P}$ & \\
\hline$P$ & 15 & H -like & $4 P$ & \\
\hline$P$ & 15 & $\mathrm{H}-1 \mathrm{ike}$ & $4 P$ & \\
\hline$P$ & 15 & H -like & $4 P$ & \\
\hline$P$ & 15 & H -like & $3 P$ & \\
\hline P & 15 & H -like & $3 p$ & \\
\hline$P$ & 15 & H-like & $3 P$ & \\
\hline $\mathrm{P}$ & 15 & $H$-Iike & $3 P$ & \\
\hline$P$ & .15 & H -like & $3 P$ & \\
\hline$P$ & 15 & H -iike & $3 P$ & \\
\hline$P$ & 15 & H -like & $2 P$ & \\
\hline $\mathrm{P}$ & 15 & H -like & $2 P$ & \\
\hline
\end{tabular}

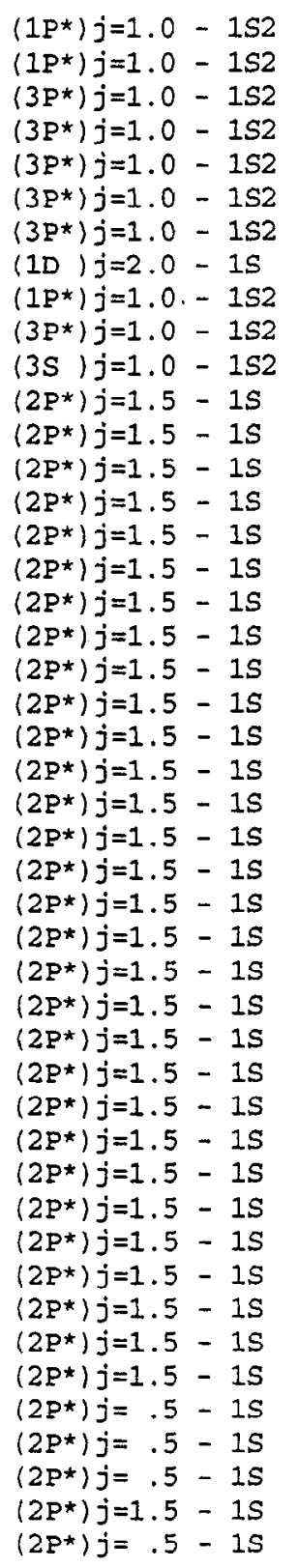

(1P*) j=1.0 - 152

$2 P$

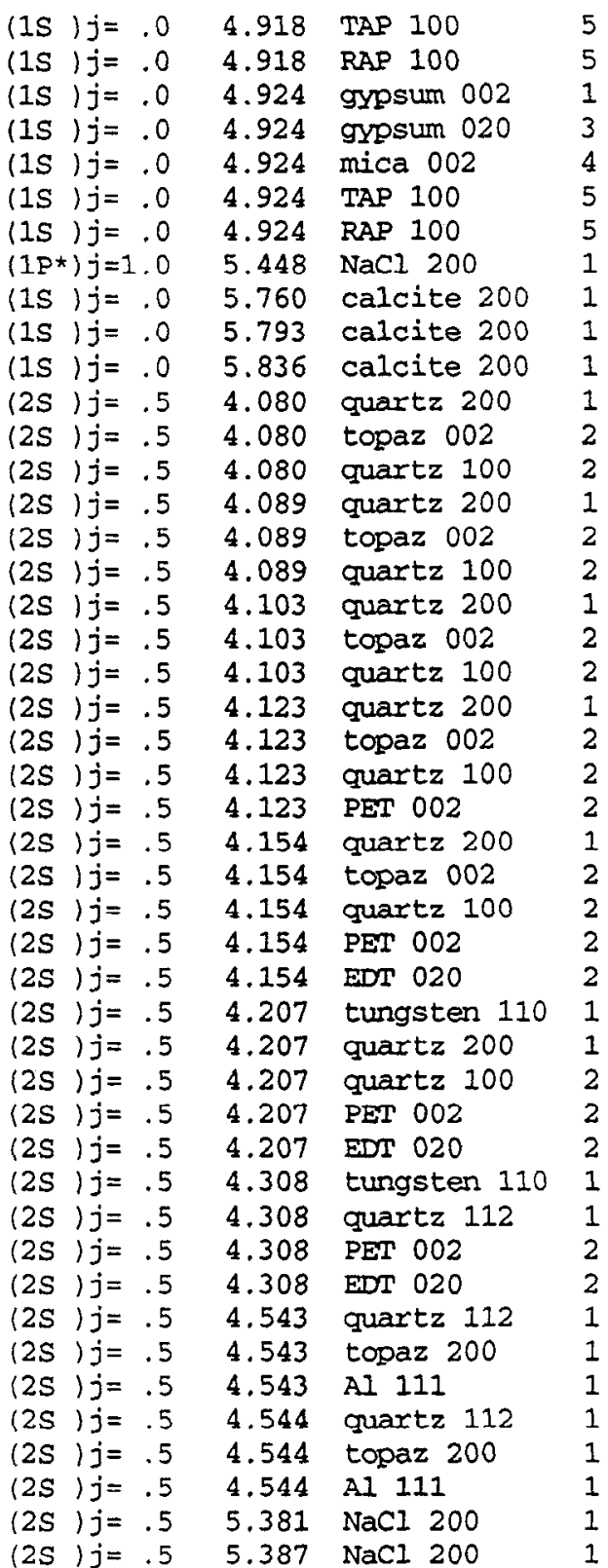




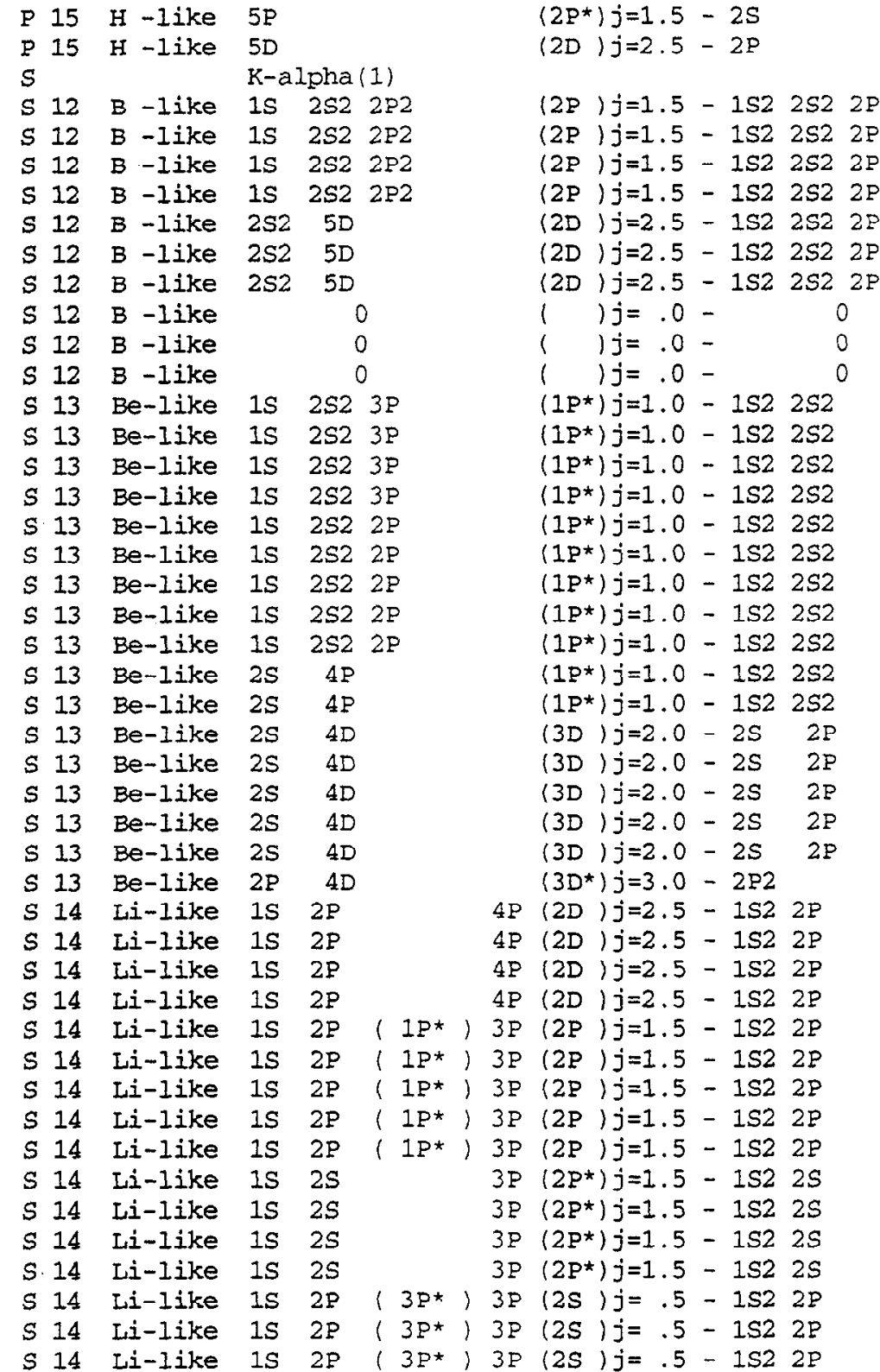

(2S $) j=.5 \quad 19.206$ mica 002 $\left(2 P^{*}\right) j=1.5 \quad 19.271$ mica 002 $5.372 \mathrm{NaCl} 200$

$\left(2 P^{*}\right) j=1.5 \quad 5.180 \quad$ ADP 101

$\left(2 P^{*}\right) j=1.5 \quad 5.180$ beryl 100

$\left(2 P^{\star}\right) j=1.5 \quad 5.180 \quad \operatorname{RAP} 100$

$\left(2 P^{\star}\right) j=1.5 \quad 5.180 \quad \operatorname{KAP} 100$

$\left(2 P^{*}\right) j=1.5 \quad 25.650 \quad$ TAP 100

$\left(2 P^{\star}\right) j=1.5 \quad 25.650 \quad R A P \quad 100$

$\left(2 P^{*}\right) j=1.5 \quad 25.650$ KAP 100

$(\quad j=.0 \quad 4.490$ quartz 112

$(\quad) j=.0 \quad 4.490$ topaz 200

()$j=.0 \quad 4.490$ Al 111

(1s) $j=.0 \quad 4.454$ tungsten 1101

(1S ) $j=.0 \quad 4.454$ quartz 112

(1S ) $j=.0 \quad 4.454$ topaz 200

(1S ) $j=.0 \quad 4.454$ Al 111

(1S) $j=0 \quad 5.137$ ADP 101

(1s) $j=.0 \quad 5.137$ beryl 100

(1S ) $j=.0 \quad 5.137$ TAP 100

(IS) $j=.0 \quad 5.137$ RAP 100

(1S) $j=.0 \quad 5.137$ KAP 100

(1S $) j=.0 \quad 24.590$ TAP 100

(1S) $j=.0 \quad 24.590$ RAP 100

(3P*) $j=1.0 \quad 25.760$ TAP 100

$\left(3 P^{*}\right) j=1.0 \quad 25.760 \quad \mathrm{KAP} \quad 100$

$\left(3 P^{*}\right) j=2.0 \quad 25.830$ RAP 100

(3P*) $j=2.0 \quad 25.830 \quad K A P \quad 100$

(3P) $j=2.0 \quad 26.350$ KAP 100

$\left(2 P^{\star}\right) j=1.5$

$\left(2 P^{*}\right) j=1.5$

$\left(2 P^{*}\right) j=1.5$

$\left(2 P^{*}\right) j=1.5$

$\left(2 P^{\star}\right) j=1.5$

$\left(2 P^{*}\right) j=1.5$

$\left(2 P^{*}\right) j=1.5$

$\left(2 P^{*}\right) j=1.5$

$\left(2 P^{*}\right) j=1.5$

(2S) $j=.5$

(2s) $j=.5$

(2s) $j=.5$

(2S) $j=.5$

$\left(2 P^{*}\right) j=1.5$

$\left(2 P^{*}\right) j=1.5$

$\left(2 P^{*}\right) j=1.5$

\subsection{2 quartz 200}

4.192 quartz 100

4.192 PET 002

4.192 EDT 020

4.370 tungsten 110

4.370 quartz 112

4.370 topaz 200

4.370 EDT 020

4.376 tungsten 110

4.376 quartz 112

4.376 topaz 200

4.376 EDT 020

4.382 tungsten 1101

4.382

4.382
4.370 PET 002
$19.942 \quad 74.385$

$19.942 \quad 75.095$

$5.641 \quad 72.248$

10.640 .76 .826

$15.954 \quad 76.919$

$26.116 \quad 82.626$

$26.634 \quad 76.517$

$25.763 \quad 84.632$

$26.116 \quad 79.160$

$26.634 \quad 74.377$

$4.564 \quad 79.668$

$4.638 \quad 75.487$

$4.676 \quad 73.785$

$4.476 \quad 84.317$

$4.564 \quad 77.395$

$4.638 \quad 73.807$

$4.676 \quad 72.274$

$10.640 \quad 74.928$

$15.954 \quad 75.009$

$25.763 \quad 85.540$

$26.116 \quad 79.576$

$26.634 \quad 74.659$

$25.763 \quad 72.644$

$26.116 \quad 70.317$

$25.763 \quad 89.126$

$26.116 \quad 80.529$

$26.634 \quad 75.281$

$26.116 \quad 81.513$

$26.634 \quad 75.886$

$26.634 \quad 81.625$

$4.246 \quad 80.852$

8.51280 .051

$8.742 \quad 73.546$

$8.808 \quad 72.150$

$4.476 \quad 77.506$

$4.564 \quad 73.235$

$\begin{array}{lll}4.638 & 70.427\end{array}$

$8.742 \quad 88.774$

$8.808 \quad 82.876$

$4.476 \quad 77.866$

$4.564 \quad 73.498$

4.63870 .650

$8.808 \quad 83.536$

$4.476 \quad 78.237$

$4.564 \quad 73.765$

$4.638 \quad 70.875$ 
$S 14$ Li-like $1 S 2 P\left(3 P^{*}\right) 3 P(2 S) j=.5-1 S 22 P$ S 14 Li-like $1 S 2 P\left(3 P^{*}\right) 3 P(2 P) j=.5-1 S 22 P$ $S 14$ Ii-like $1 S 2 P\left(3 P^{*}\right) 3 P(2 P) j=.5-1 S 22 P$ $S 14$ Li-like $1 S 2 P\left(3 P^{*}\right) 3 P(2 P) j=.5-1 S 22 P$ $S 14$ Li-like $1 S 2 P\left(3 P^{*}\right) 3 P(2 P) j=.5-1 S 22 P$ $S 14$ Li-like $1 S 2 P(3 P *) 3 P(2 P) j=.5-1 S 22 P$ $S 14$ Li-1ike $1 S$ $2 P\left(3 P^{*}\right) 3 P(2 P) j=.5-1 S 22 P$ $S 14$ Li-1ike $1 S$ 2P $\left(3 P^{*}\right) 3 P(2 P) j=.5-1 S 22 P$ $S 14$ Li-like $1 S 2 P(3 P *) 3 P(2 P) j=.5-1 S 22 P$ $S 14$ Li-like $1 S 2 P\left(3 P^{*}\right) 3 P(2 D) j=1.5-1 S 22 P$ S 14 Li-like $1 S$ 2P (3P*) $3 P(2 D) j=1.5-1 S 22 P$ $S 14$ Li-1ike $1 S$ 2P (3P*) $3 P(2 D) j=1.5-1 S 22 P$ $S 14 \mathrm{Li}-1 \mathrm{ike}$ is $2 \mathrm{P}\left(3 P^{*}\right) 3 P(2 D) j=1.5-1 S 22 \mathrm{P}$ S 14 Li-1ike 1S. 2P (3P*) $3 P(2 D) j=1.5-1 S 22 P$ $S 14$ Ii-1ike $1 \mathrm{~S} 2 \mathrm{P}\left(3 P^{\star}\right) 3 P(2 D) j=1.5-1 S 22 P$ $S 14$ Li-like $152 P(3 P *) 3 P(2 D) j=1.5-1 S 22 P$ $S 14$ Li-like $1 S 2 P\left(3 P^{*}\right) 3 P(2 D) j=1.5-1 S 22 P$ $S 14$ Li-1ike $1 S 2 P\left(3 P^{*}\right) 3 P(2 D) j=1.5-1 S 22 P$ $S 14$ Li-like $1 S 2 P\left(3 P^{*}\right) 3 P(2 D) j=1.5-1 S 22 P$ $S 14$ Li-like $1 S 2 P\left(1 P^{*}\right) 3 P(2 S) j=.5-1 S 23 P$ $S$ Li-1ike $1 S$ 2P ( $\left.P^{*}\right) 3 P(2 S) j=.5-1 S 23 P$ S 14 Li-like $1 S 2 P\left(1 P^{*}\right) 3 P(2 S) j=.5-1 S 23 P$ S 14 Li-like $1 S 2 P\left(1 P^{*}\right) 3 P(2 S) j=.5-1 S 23 P$ S 14 Li-1ike $1 S$ 2P (1P*) 3P (2S ) j=.5- 1S2 $3 \mathrm{P}$ S 14 Li-like $1 S 2 P\left(1 P^{\star}\right) 3 P(2 S) j=.5-1 S 23 P$ $S 14$ Li-like is $2 P$ ( $\left.1 P^{*}\right) 3 P$ (2D) $j=2.5-1 S 23 P$ $S 14$ Li-like $1 S$ 2P ( $\left.1 P^{\star}\right) 3 P(2 D) j=2.5-1 S 23 P$ $S 14$ Li-like $1 S 2 P$ (1 $\left.P^{\star}\right) 3 P(2 D) j=2.5-1 S 23 P$ $S 14$ Li-like $1 S$ 2P ( IP*) $3 P(2 D) j=2.5-1 S 23 P$ $S 14$ Li-like is $2 P$ ( $\left.1 P^{*}\right) 3 P(2 D) j=2.5-1 S 23 P$ $S 14$ Li-1ike $1 S$ 2P (1P*) $3 P(2 D) j=2.5-1 S 23 P$ 514 Li-1ike is $2 P\left(3 P^{*}\right) 3 P(2 S) j=.5-1 S 23 P$ $S 14$ Li-like is $2 P\left(3 P^{*}\right) 3 P(2 S) j=.5-1 S 23 P$ $S 14$ Li-like $1 S$ $2 P\left(3 P^{*}\right) 3 P(2 S) j=.5-1 S 23 P$ S 14 Li-like $1 S$ 2P (3P*) $3 P(2 S) j=.5-1 S 23 P$ $S 14$ Li-like $1 S$ $2 P$ (3P*) $3 P(2 S) j=.5-1 S 23 P$ $S 14$ Li-like $1 S$ 2P ( $\left.3 P^{*}\right) 3 P(2 S) j=.5-1 S 23 P$ $S 14$ Li-like is 2P2 (2S) $j=.5-1 S 2$ 2P 14 Li-1ike 15 $2 P 2$ S 14 Li-like is 2P2 S 14 Li-like 1S 2P2

S 14 Li-like 1S 2P2

S 14 Li-like $1 \mathrm{~S}$ 2P2

S 14 Li-like $1 S\left(2 S\right.$ 2P( $\left.\left.P^{*}\right)\right)\left(2 P^{*}\right) j=1.5-1 S 22 S$

$S$ I4 Li-like $1 S\left(2 S 2 P\left(1 P^{*}\right)\right)\left(2 P^{*}\right) j=1.5-1 S 22 S$ $\left(2 P^{\star}\right) j=1.5$ $\left(2 P^{*}\right) j=.5$ $\left(2 p^{\star}\right) j=.5$ $\left(2 p^{*}\right) j=.5$ $\left(2 \mathrm{P}^{*}\right) j=.5$ $\left(2 P^{*}\right) j=1.5$ $\left(2 P^{*}\right) j=1.5$ $\left(2 P^{*}\right) j=1.5$

$\left(2 P^{\star}\right) j=1.5$ $\left(2 P^{*}\right) j=.5$ $\left(2 P^{\star}\right) j=.5$ $\left(2 P^{\star}\right) j=.5$ $\left(2 P^{\star}\right) j=.5$ $\left(2 P^{*}\right) j=.5$ $\left(2 P^{*}\right) j=1.5$ $\left(2 P^{\star}\right) j=1.5$ $\left(2 P^{*}\right) j=1.5$ $\left(2 P^{\star}\right) j=1.5$ $\left(2 P^{\star}\right) j=1.5$ $\left(2 P^{*}\right) j=1.5$ $\left(2 P^{*}\right) j=1.5$ $\left(2 P^{\star}\right) j=1.5$ $\left(2 P^{\star}\right) j=1.5$ (2P*) $j=1.5$ $\left(2 P^{\star}\right) j=1.5$ $\left(2 P^{\star}\right) j=1.5$ $\left(2 P^{\star}\right) j=1.5$ $\left(2 P^{*}\right) j=1.5$ $\left(2 P^{\star}\right) j=1.5$ $\left(2 P^{\star}\right) j=1.5$ $\left(2 P^{*}\right) j=1.5$ $\left(2 P^{\star}\right) j=.5$ $\left(2 P^{\star}\right) j=.5$ $\left(2 P^{*}\right) j=.5$ $\left(2 P^{\star}\right) j=.5$ $\left(2 P^{*}\right) j=.5$ $\left(2 P^{\star}\right) j=.5$ $\left(2 P^{\star}\right) j=1.5$ $\left(2 P^{*}\right) j=1.5$ (2P*) $j=1.5$ $\left(2 P^{*}\right) j=1.5$ $\left(2 P^{\star}\right) j=1.5$ $\left(2 P^{*}\right) j=1.5$ (2s) $j=.5$ (2S) $j=.5$ (2S) $j=.5$

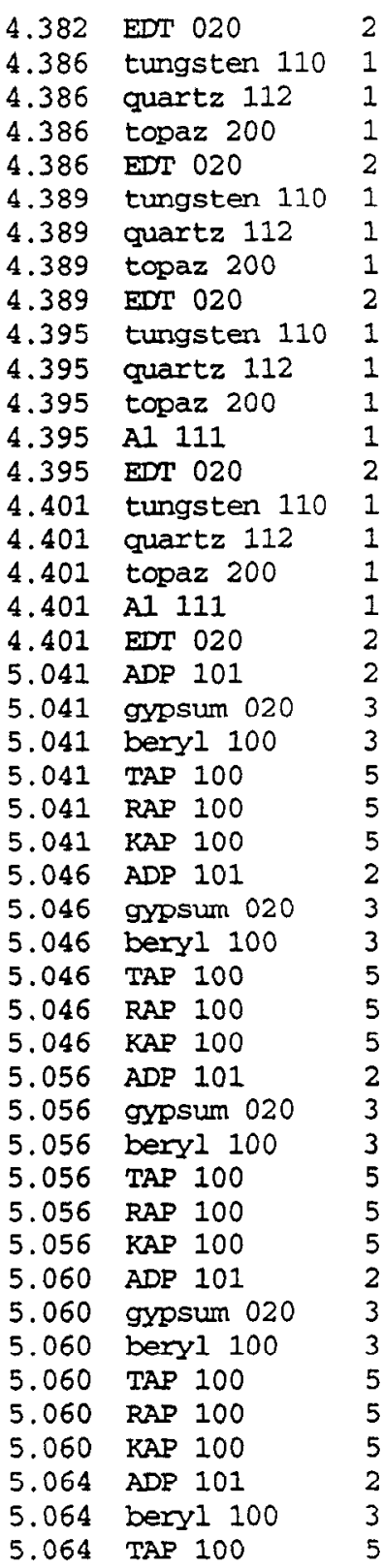

$\begin{array}{rr}8.808 & 84.271 \\ 4.476 & 78.491 \\ 4.564 & 73.946 \\ 4.638 & 71.026 \\ 8.808 & 84.818 \\ 4.476 & 78.685 \\ 4.564 & 74.082 \\ 4.638 & 71.140 \\ 8.808 & 85.270 \\ 4.476 & 79.083 \\ 4.564 & 74.359 \\ 4.638 & 71.371 \\ 4.676 & 70.036 \\ 8.808 & 86.336 \\ 4.476 & 79.497 \\ 4.564 & 74.641 \\ 4.638 & 71.604 \\ 4.676 & 70.252 \\ 8.808 & 87.885 \\ 10.640 & 71.362 \\ 15.185 & 84.821 \\ 15.954 & 71.426 \\ 25.763 & 78.053 \\ 26.116 & 74.822 \\ 26.634 & 71.146 \\ 10.640 & 71.531 \\ 15.185 & 85.491 \\ 15.954 & 71.596 \\ 25.763 & 78.325 \\ 26.116 & 75.033 \\ 26.634 & 71.313 \\ 10.640 & 71.874 \\ 15.185 & 87.289 \\ 15.954 & 71.940 \\ 25.763 & 78.888 \\ 26.116 & 75.464 \\ 26.634 & 71.652 \\ 10.640 & 72.013 \\ 15.185 & 88.529 \\ 15.954 & 72.080 \\ 25.763 & 79.121 \\ 26.116 & 75.640 \\ 26.634 & 71.789 \\ 10.640 & 72.153 \\ 15.954 & 72.220 \\ 25.763 & 79.359\end{array}$ 
S 14 Li-like 1S (2S $\left.2 P\left(1 P^{*}\right)\right)\left(2 P^{*}\right) j=1.5-1 S 22 S$

$S 14$ Li-like $1 S$ (2S $\left.2 P\left(1 P^{*}\right)\right)\left(2 P^{*}\right) j=1.5-1 S 2 S$

$S 14$ Li-like $1 S\left(2 S\right.$ 2P $\left.\left(1 P^{*}\right)\right)\left(2 P^{*}\right) j=1.5-1 S 22 S$

$S 14$ Li-1ike $1 S$ (2S $\left.2 P\left(1 P^{\star}\right)\right)\left(2 P^{\star}\right) j=1.5-1 S 22 S$

$S 14$ Li-like $1 S\left(2 S\right.$ 2P ( $\left.\left.1 P^{*}\right)\right)\left(2 P^{*}\right) j=1.5-1 S 2$ 2S

$S 14$ Li-like $1 S$ (2S $\left.2 P\left(1 P^{*}\right)\right)\left(2 P^{*}\right) j=1.5-1 S 2 S$

$S 14$ Li-like $1 S\left(2 S 2 P\left(1 P^{*}\right)\right)\left(2 P^{\star}\right) j=1.5-1 S 22 S$

$S 14$ Li-like $1 S\left(2 S 2 P\left(1 P^{*}\right)\right)\left(2 P^{*}\right) j=.5-1 S 22 S$

$S$ I4 Li-like $1 S$ (2S $\left.2 \mathrm{P}\left(1 \mathrm{P}^{*}\right)\right)\left(2 \mathrm{P}^{*}\right) j=.5-1 \mathrm{~S} 2 \mathrm{2S}$

$S 14$ Ii-like $1 S\left(2 S 2 P\left(1 P^{*}\right)\right)\left(2 P^{*}\right) j=.5-1 S 22 S$

$S 14$ Li-like $1 S\left(2 S 2 P\left(1 P^{*}\right)\right)\left(2 P^{*}\right) j=.5-1 S 22 S$

$S 14$ Li-like $1 S\left(2 S 2 P\left(1 P^{*}\right)\right)\left\langle 2 P^{*}\right) j=.5-1 S 2$ SS

$S 14$ Li-like $1 S\left(2 S \quad 2 P\left(3 P^{*}\right)\right)\left(2 P^{*}\right) j=1.5-1 S 22 S$

$S 14$ Li-like $1 S\left(2 S\right.$ 2P $\left.\left(3 P^{*}\right)\right)\left(2 P^{*}\right) j=1.5-1 S 22 S$

$S 14$ Li-like $1 S$ (2S $\left.2 P\left(3 P^{*}\right)\right)\left(2 P^{\star}\right) j=1.5-1 S 22 S$

$S 14$ Li-like $1 S\left(2 S \quad 2 P\left(3 P^{*}\right)\right)\left(2 P^{*}\right) j=1.5-1 S 2$ 2S

$S 14$ Li-like $1 S\left(2 S 2 P\left(3 P^{*}\right)\right)\left(2 P^{*}\right) j=1.5-1 S 22 S$

$S 14$ Li-like $1 \mathrm{~S}\left(2 \mathrm{~S} 2 \mathrm{P}\left(3 \mathrm{P}^{*}\right)\right)\left(2 \mathrm{P}^{\star}\right) j=.5-1 S 22 \mathrm{~S}$

$S 14$ Li-like $1 S\left(2 S \quad 2 P\left(3 P^{*}\right)\right)\left(2 P^{\star}\right) j=.5-1 S 22 S$

$S 14$ Li-like $15\left(2 S 2 P\left(3 P^{*}\right)\right)\left(2 P^{*}\right) j=.5-1 S 22 S$

$S 14$ Li-like $1 S\left(2 S \quad 2 P\left(3 P^{*}\right)\right)\left(2 P^{*}\right) j=.5-1 S 22 S$

$S 14$ Li-like $1 S\left(2 S \quad P\left(3 P^{*}\right)\right)\left(2 P^{*}\right) j=.5-1 S 22 S$

$S 14$ Li-like 1S 2P2

$S 14$ Li-like 1S 2P2 (2P) $j=1.5-1 S 2$ 2P

S 14 Li-like 1s 2P2

S 14 Li-like 1S 2P2

S 14 Li-like 15 2P2

S 14 Li-like 15 2P2

S 14 Li-like 1S 2P2

S 14 Li-like 1S 2P2

S 14 Li-like 1S 2P2

S 14 Li-like 15 2P2

S 14 Li-like 15 2P2

S 14 Li-like 15 2P2

S 14 Li-like 1S 2P2

S 14 Li-like 1S 2P2

S 14 Li-like 1S 2P2

$S 14$ Li-like $1 S\left(2 S 2 P\left(3 P^{*}\right)\right)\left(4 P^{*}\right) j=15-1 S 22 S$

$S 14$ Li-like $1 S\left(2 S\right.$ 2P $\left(3 P^{*}\right)\left(4 P^{*}\right) j=1.5-1 S 2$ 2S

$S 14$ Li-like $1 S$ (2S $\left.2 P\left(3 P^{*}\right)\right)\left(4 P^{*}\right) j=1.5-1 S 22 S$

S 14 Li-like $1 S$ (2S $\left.2 P\left(3 P^{*}\right)\right)\left(4 P^{*}\right) j=1.5-1 S 22 S$

$S 14$ Li-1ike is (2S $\left.2 P\left(3 P^{*}\right)\right)\left(4 P^{*}\right) j=1.5-1 S 22 S$

S. 14 Li-like 1S 2P2

S 14 Li-like is $2 \mathrm{P} 2$

$S 14$ Li-like 1S 2P2

S 14 Li-like is 2P2

(4P) $j=1.5-1 S 22 P$

$(4 P) j=1.5-1 S 22 P$ (2s) $j=.5$

(2s) $j=.5$

(2s) $j=.5$

(2s) $j=.5$

(2S) $j=.5$

(2S) $j=.5$

(2S ) $j=.5$

(2s) $j=.5$

(2S) $j=.5$

(2S) $j=.5$

(2s) $j=.5$

(2s) $j=.5$

(2S) $j=.5$

(2s) $j=.5$

(2S) $j=.5$

(2S) $j=.5$

(2S) $j=.5$

(2s) $j=.5$

(2S) $j=.5$

(2s) $j=.5$

(2S) $j=.5$

(2s) $j=.5$

$\left(2 P^{\star}\right) j=1.5$

$(2 P *) j=1.5$

$\left(2 P^{\star}\right) j=1.5$

$(2 P *) j=1.5$

$\left(2 P^{*}\right) j=1.5$

$\left(2 P^{*}\right) j=.5$

$\left(2 P^{*}\right) j=.5$

$\left(2 P^{*}\right) j=.5$

$\left(2 P^{\star}\right) j=.5$

$\left(2 P^{*}\right) j=.5$

$\left(2 P^{\star}\right) j=1.5$

$\left(2 P^{*}\right) j=1.5$

$\left(2 P^{\star}\right) j=1.5$

$\left(2 p^{*}\right) j=1.5$

$\left(2 P^{\star}\right) j=1.5$

(2S) $j=.5$

(2s) $j=.5$

(2s) $j=.5$

(2s) $j=.5$

(2S) $j=.5$

$\left(2 P^{*}\right) j=.5$

$\left(2 P^{\star}\right) j=.5$

$\left(2 P^{*}\right) j=.5$

$\left(2 P^{*}\right) j=.5$
5.064 RAP 100

5.064 KAP 100

5.066 ADP 101

5.066 beryl 100

5.066 TAP 100

5.066 RAP 100

$5.066 \mathrm{KAP} 100$

5.067 ADP 101

5.067 beryl 100

5.067 TAP 100

5.067 RAP 100

5.067 KAP 100

5.086 ADP 101

5.086 beryl 100

5.086 TAP 100

5.086 RAP 100

5.086 KAP 100

5.087 ADP 101

5.087 beryl 100

5.087 TAP 100

5.087 RAP 100

5.087 KAP 100

5.090 ADP 101

5.090 beryl 100

5.090 TAP 100

5.090 RAP 100

5.090 KAP 100

5.096 ADP 101

5.096 beryl 100

5.096 TAP 100

5.096 RAP 100

5.096 KAP 100

5.101 ADP 101

5.101 beryl 100

5.101 TAP 100

5.101 RAP 100

5.101 KAP 100

5.122 ADP 101

5.122 bery 100

5.122 TAP 100

5.122 RAP 100

5.122 KAP 100

5.127 ADP 101

5.127 bery 1100
5.127 TAP 100

5.127 RAP 100
$26.116 \quad 75.818$

$26.634 \quad 71.927$

$10.640 \quad 72.224$

$15.954 \quad 72.291$

$25.763 \quad 79.481$

$26.116 \quad 75.907$

$26.634 \quad 71.997$

$10.640 \quad 72.259$

$15.954 \quad 72.326$

$25.763 \quad 79.542$

$26.116 \quad 75.953$

$26.634 \quad 72.032$

$10.640 \quad 72.943$

$15.954 \quad 73.014$

$25.763 \quad 80.778$

$26.116 \quad 76.839$

$26.634 \quad 72.707$

$10.640 \quad 72.980$

$15.954 \quad 73.051$

$25.763 \quad 80.848$

$26.116 \quad 76.887$

26.63472 .743

$10.640 \quad 73.091$

$15.954 \quad 73.162$

$25.763 \quad 81.060$

$26.116 \quad 77.033$

$26.634 \quad 72.852$

$10.640 \quad 73.314$

$15.954 \quad 73.386$

$25.763 \quad 81.500$

$26.116 \quad 77.329$

$26.634 \quad 73.072$

$10.640 \quad 73.503$

$15.954 \quad 73.576$

$25.763 \quad 81.885$

$26.116 \quad 77.582$

26.63473 .258

$10.640 \quad 74.319$

$15.954 \quad 74.396$

$25.763 \quad 83.753$

$26.116 \quad 78.703$

$26.634 \quad 74.061$

$10.640 \quad 74.520$

$15.954 \quad 74.598$

$25.763 \quad 84.286$

$26.116 \quad 78.986$ 
(4P) $j=1.5-1 S 22 P$ (4P ) $j=2.5-1 S 22 B$ $(4 P) j=2.5-1 S 22 P$ $(4 P) j=2.5-1 . S 22 P$ $(4 P) j=2.5-1 S 22 P$ $(4 P) j=2.5-1 S 22 P$ $(4 P) j=1.5-1 S 22 P$ (4P) $j=1.5-1 S 22 P$ (4P) $j=1.5-1 S 22 P$ (4P) $j=1.5-1 S 22 P$ $(4 P) j=1.5-1 S 22 P$ $(4 P) j=.5-1 S 22 P$ $(4 P) j=.5-1 S 22 P$ $(4 P) j=.5-1 S 22 P$ $(4 P) j=.5-1 S 22 P$ $(4 P) j=.5-1 S 22 P$ (2D) $j=2.5-1 S 22 P$ (2S $) j=.5-1 S 22 P$ (1P*) $j=1.0-1 S 2$ $\left(1 P^{\star}\right) j=1.0-152$ $\left(1 P^{*}\right) j=1.0-1 S 2$ $\left(1 P^{*}\right) j=1.0-1 S 2$ (1P*) $j=1.0-1 \mathrm{~S} 2$ $\left(1 P^{*}\right) j=1.0-1.52$ $\left(1 P^{\star}\right) j=1.0-1 \mathrm{~S} 2$ $\left(1 p^{*}\right) j=1.0-1 \mathrm{~S} 2$ $\left(1 P^{*}\right) j=1.0-1 S 2$ $\left(1 P^{*}\right) j=1.0-1 S 2$ $\left(1 P^{\star}\right) j=1.0-1 \mathrm{~S} 2$ $\left(1 P^{*}\right) j=1.0-1 S 2$ $\left(1 P^{*}\right) j=1.0-1 S 2$ $\left(1 P^{\star}\right) j=1.0-1 S 2$ $\left(1 P^{\star}\right) j=1.0-1 \mathrm{~S} 2$ $\left(1 P^{*}\right) j=1.0-1 \mathrm{~S} 2$ $\left(1 P^{*}\right) j=1.0-1 S 2$ $\left(1 P^{*}\right) j=1.0-1 S 2$ $\left(3 P^{*}\right) j=1.0-152$ $\left(3 P^{*}\right) j=1.0-1 S 2$ $\left(3 P^{*}\right) j=1.0-1 S 2$ $\left(3 R^{\star}\right) j=1.0-1 S 2$ $\left(1 P^{*}\right) j=1.0-1 S 2$ $\left(1 D^{*}\right) j=1.0-1 S 2$ $\left(1 P^{\star}\right) j=1.0-1 S 2$ $\left(1 P^{\star}\right) j=1.0-1 S 2$ $\left(1 P^{\star}\right) j=1.0-1 S 2$ $\left(1 P^{\star}\right) j=1.0-1 S 2$ $\left(2 P^{*}\right) j=$.

$\left(2 P^{\star}\right) j=1.5$

$\left(2 P^{\star}\right) j=1.5$

$\left(2 P^{*}\right) j=1.5$

$\left(2 P^{*}\right) j=1.5$

$\left(2 P^{\star}\right) j=1.5$

$\left(2 P^{*}\right) j=1.5$

$\left(2 P^{*}\right) j=1.5$

$(2 P *) j=1.5$

$\left(2 P^{*}\right) j=1.5$

$\left(2 P^{\star}\right) j=1.5$

$\left(2 P^{*}\right) j=1.5$

$\left(2 P^{\star}\right) j=1.5$

$\left(2 P^{\star}\right) j=1.5$

$\left(2 P^{*}\right) j=1.5$

$\left(2 P^{*}\right) j=1.5$

$\left(2 P^{\star}\right) j=1.5$

$\left(2 P^{\star}\right) j=1.5$

(1S) $j=$.0

(1s) $j=$.

(1s) $j=.0$

(1s) $j=.0$

(1s) $j=.0$

(1s) $j=.0$

(Is) $j=.0$

(Is) $j=.0$

(1s) $j=.0$

(1s) $j=.0$

(1s) $j=$.

(Is ) $j=.0$

(1s) $j=.0$

(1s ) $j=.0$

(1s) $j=.0$

(1s) $j=.0$

(IS ) $j=.0$

(1s) $j=.0$

(Is) $j=.0$

(1S) $j=.0$

(1s) $j=.0$

(1S) $j=.0$

(1s) $j=.0$

(1s) $j=.0$

(1s) $j=.0$

(1s) $j=.0$

(1s) $j=.0$

(1s) $j=.0$
5.127 KAP 100

5.128 ADP 101

5.128 beryl 100

5.128 TAP 100

.128 RAP 100

5.128 KAP 100

5.130 ADP 101

5.130 beryl 100

130 TAP 100

5.130 RAP 100

5.130 KAP 100

132 ADP 101

5.132 beryl 100

5.132 TAP 100

5.132 RAP 100

5.132 KAP 100

24.260 TAP 100

3.949 Ge 220

3.949 LiF 200

3.949 Al 200

3.949 topaz 002

3.949 beryl 100

3.949 mica 002

3.998 Ge 220

3.998 LIF 200

3.998 Al 200

3.998 quartz 200

3.998 topaz 002

4.088 quartz 200

4.088 topaz 002

4.088 quartz 100

4.299

4.299

4.299

4.304

4.304

4.304

4.304

5.038

5.038

5.038

5.038

5.038

5.038 tungsten 110

quartz 112

PET 002

EDT 020

tungster 110

PET 002

EDT 020

gypsum 020

beryl 100

TAP 100

RAP 100
$26.634 \quad 74.258$

$10.640 \quad 74.560$

$15.954 \quad 74.638$

$25.763 \quad 84.399$

$26.116 \quad 79.044$

$26.634 \quad 74.297$

$10.640 \quad 74.641$

$15.954 \quad 74.720$

$25.763 \quad 84.632$

$26.116 \quad 79.160$

$26.634 \quad 74.377$

$10.640 \quad 74.723$

$15.954 \quad 74.802$

$25.763 \quad 84.875$

$26.116 \quad 79.277$

$26.634 \quad 74.457$

$25.763 \quad 70.332$

$25.763 \quad 71.488$

$4.000 \quad 80.841$

$4.027 \quad 78.705$

$.4 .048 \quad 77.302$

$8.374 \quad 70.589$

$15.954 \quad 81.930$

$19.942 \quad 81.940$

$4.000 \quad 88.188$

$4.027 \quad 83.120$

$4.048 \quad 80.985$

$4.246 \quad 70.321$

$8.374 \quad 72.719$

$\begin{array}{ll}4.246 & 74.321\end{array}$

$8.374 \quad 77.516$

$8.512 \quad 73.848$

$4.476 \quad 73.833$

4.56470 .379

$8.742 \quad 79.586$

$8.808 \quad 77.464$

$4.476 \quad 74.065$

$4.564 \quad 70.567$

$8.742 \quad 79.955$

$8.808 \quad 77.767$

$10.640 \quad 71.261$

$15.185 \quad 84.457$

$\begin{array}{lll}15.954 & 71.325\end{array}$

$25.763 \quad 77.893$

$26.116 \quad 74.697$

$26.634 \quad 71.047$

Page 19 


\begin{tabular}{|c|c|c|c|c|c|}
\hline S 15 & He-like & $1 S$ & $2 P$ & $\left(3 P^{*}\right) j=1.0$ & $-1 s 2$ \\
\hline S 15 & He-like & $1 S$ & $2 \mathrm{P}$ & $\left(3 P^{*}\right) j=1.0$ & -152 \\
\hline S 15 & He-like & is & $2 \mathrm{P}$ & $\left(3 P^{*}\right) j=1.0$ & $-1 S 2$ \\
\hline S 15 & He-like & $1 \mathrm{~S}$ & $2 \mathrm{P}$ & $\left(3 P^{*}\right) j=1.0$ & -152 \\
\hline S 15 & He-like & $1 \mathrm{~s}$ & $2 \mathrm{P}$ & $\left(3 p^{*}\right) j=1.0$ & $-1 s 2$ \\
\hline S 15 & He-like & 15 & $2 s$ & $(3 S) j=1.0$ & $-1 s 2$ \\
\hline S 15 & He-like & $1 s$ & $2 S$ & $(3 s) j=1.0$ & $-1 s 2$ \\
\hline S 15 . & He-like & $1 S$ & $2 \mathrm{~S}$ & $(3 s) j=1.0$ & $-1 S 2$ \\
\hline S 15 & He-like & $1 S$ & $2 s$ & $(3 s) j=1.0$ & $-1,52$ \\
\hline S 15 & He-like & is & $2 S$ & $(3 s) j=1.0$ & $-1 s 2$ \\
\hline S 16 & H -like & $10 P$ & & $\left(2 P^{\star}\right) j=1.5$ & $-1 s$ \\
\hline S 16 & H -like & $10 P$ & & $\left(2 P^{\star}\right) j=1.5$ & $-1 S$ \\
\hline S 16 & $\mathrm{H}-1 \mathrm{ike}$ & $10 P$ & & $\left(2 P^{*}\right) j=1.5$ & $-1 S$ \\
\hline S 16 & $\mathrm{H}-\mathrm{like}$ & $9 P$ & & $\left(2 \mathrm{P}^{*}\right) j=1.5$ & $-1 S$ \\
\hline S 16 & H-like & $9 P$ & & $\left(2 P^{*}\right) j=1.5$ & $-1 S$ \\
\hline S 16 & $\mathrm{H}$-like & $9 \mathrm{P}$ & & $\left(2 P^{\star}\right) j=1.5$ & $-1 S$ \\
\hline S 16 & H -like & $8 \mathrm{P}$ & & $\left(2 p^{*}\right) j=1.5$ & $-1 S$ \\
\hline S 16 & $\mathrm{H}$-like & $8 P$ & & $\left(2 P^{\star}\right) j=1.5$ & $-1 s$ \\
\hline S 16 & $\mathrm{H}$-like & $8 \mathrm{P}$ & & $\left(2 P^{\star}\right) j=1.5$ & $-1 s$ \\
\hline S 16 & H -like & $7 P$ & & $\left(2 P^{\star}\right) j=1.5$ & $-1 S$ \\
\hline S 16 & H -like & $7 P$ & & $\left(2 P^{\star}\right) j=1.5$ & $-1 S$ \\
\hline S 16 & $\mathrm{H}-1 \mathrm{ike}$ & $7 P$ & & $\left(2 P^{\star}\right) j=1.5$ & $-1 S$ \\
\hline S 16 & H-like & $7 P$ & & $\left(2 P^{*}\right) j=1.5$ & -15 \\
\hline S 16 & $\mathrm{H}$-like & $6 \mathrm{P}$ & & $\left(2 P^{\star}\right) j=1.5$ & -15 \\
\hline S 16 & H -like & $6 \mathrm{P}$ & & $\left(2 P^{*}\right) j=1.5$ & -15 \\
\hline S 16 & H -like & $6 P$ & & $\left(2 P^{*}\right) j=1.5$ & $-1 s$ \\
\hline S 16 & $\mathrm{H}$-like & $6 P$ & & $\left(2 P^{\star}\right) j=1.5$ & $-1 S$ \\
\hline S 16 & H -like & $5 P$ & & $\left(2 P^{*}\right) j=1.5$ & $-1 S$ \\
\hline S 16 & H -like & $5 P$ & & $\left(2 P^{*}\right) j=1.5$ & $-1 S$ \\
\hline S 16 & H -like & $5 P$ & & $\left(2 E^{\star}\right) j=1.5$ & $-1 s$ \\
\hline S 16 & H -like & $5 P$ & & $\left(2 P^{*}\right) j=1.5$ & $-1 s$ \\
\hline S 16 & H-like & $4 \mathrm{P}$ & & $\left(2 P^{*}\right) j=1.5$ & $-1 s$ \\
\hline S 16 & H -like & $4 \mathrm{P}$ & & $\left(2 P^{\star}\right) j=1.5$ & -15 \\
\hline S 16 & $\mathrm{H}$-like & $4 \mathrm{P}$ & & $\left(2 \mathrm{P}^{*}\right) j=1.5$ & $-1 S$ \\
\hline S 16 & H -1ike & $4 \mathrm{P}$ & & $\left(2 P^{*}\right) j=1.5$ & $-1 S$ \\
\hline S 16 & H -like & $4 \mathrm{P}$ & & $\left(2 Q^{*}\right) j=1.5$ & $-1 S$ \\
\hline S 16 & $\mathrm{H}$-like & $4 P$ & & $\left(2 \mathrm{P}^{\star}\right) j=1.5$ & $-1 s$ \\
\hline S 16 & H -like & $3 P$ & & $\left(2 P^{\star}\right) j=1.5$ & $-1 S$ \\
\hline S 16 & H -like & $3 P$ & & $\left(2 \mathrm{P}^{\star}\right) j=1.5$ & $-1 S$ \\
\hline S 16 & H -like & $3 P$ & & $\left(2 \mathrm{P}^{\star}\right) j=1.5$ & $-1 S$ \\
\hline S 16 & H -like & $3 P$ & & $\left(2 P^{\star}\right) j=1.5$ & $-1 S$ \\
\hline S 16 & H -like & $3 P$ & & $\left(2 P^{*}\right) j=1.5$ & $-1 S$ \\
\hline s 16 & H -like & $3 P$ & & $\left(2 P^{*}\right) j=.5$ & $-1 s$ \\
\hline S 16 & H -like & $3 P$ & & $\left(2 P^{*}\right) j=.5$ & $-1 S$ \\
\hline S 16 & H -like & $3 P$ & & $\left(2 P^{\star}\right) j=.5$ & $-1 s$ \\
\hline $\mathrm{S}$ & $\mathrm{H}$-like & $3 P$ & & $\left(2 P^{*}\right) j=.5$ & \\
\hline
\end{tabular}

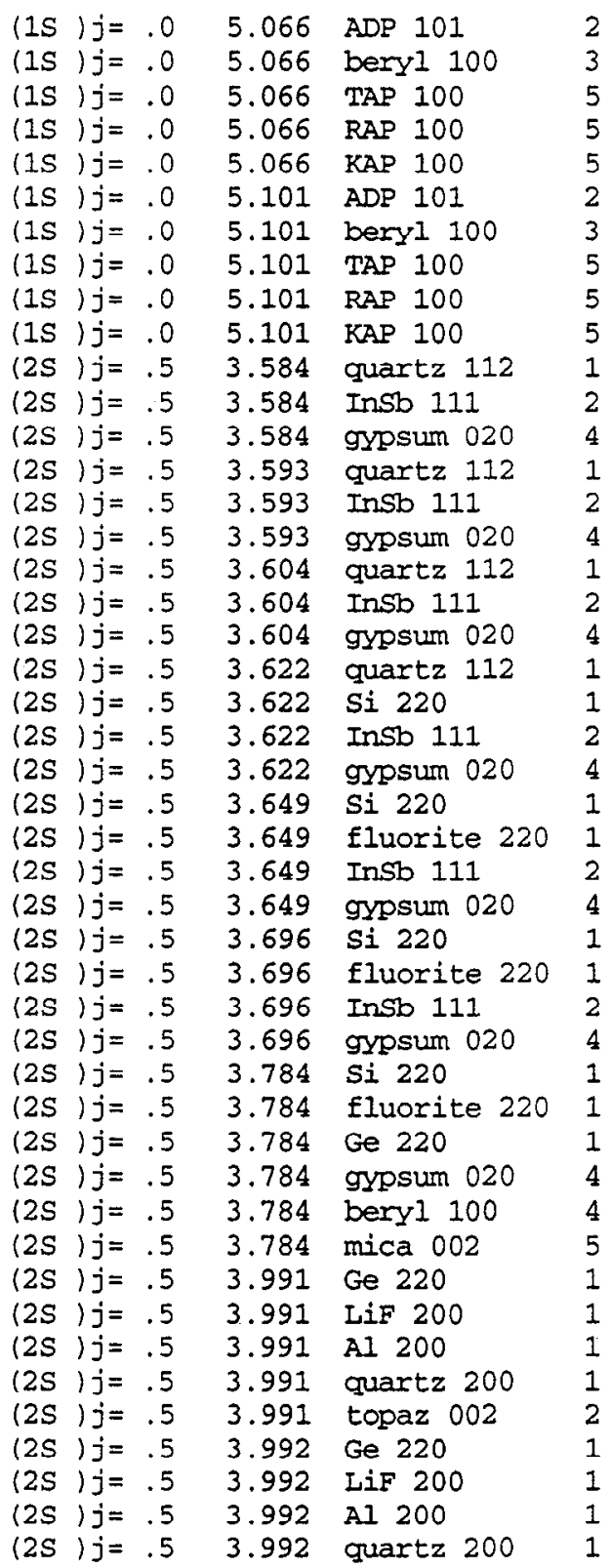

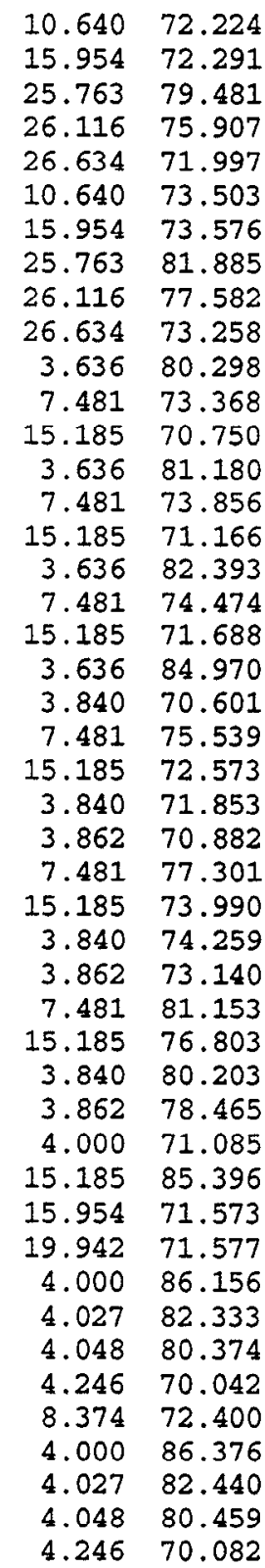




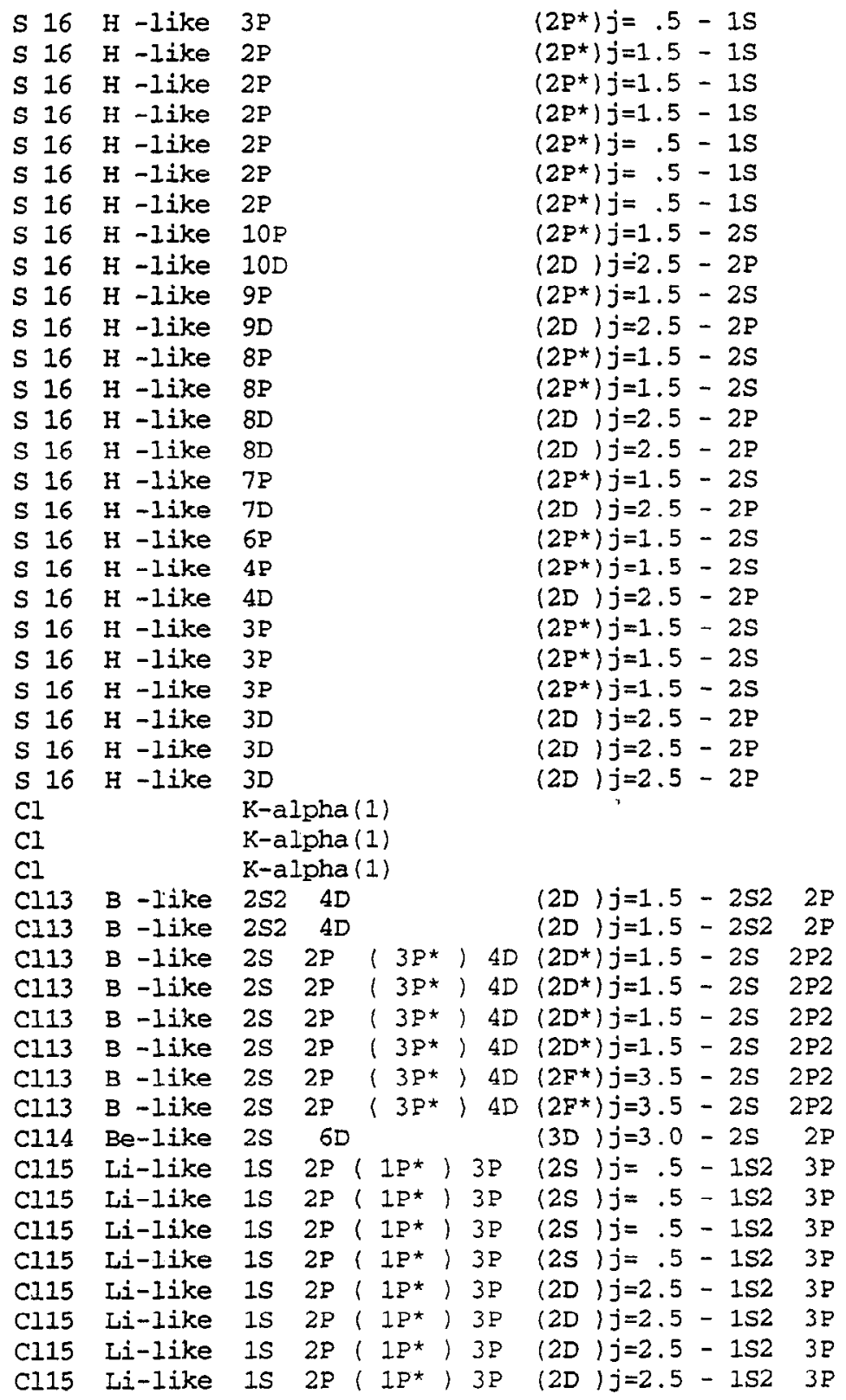

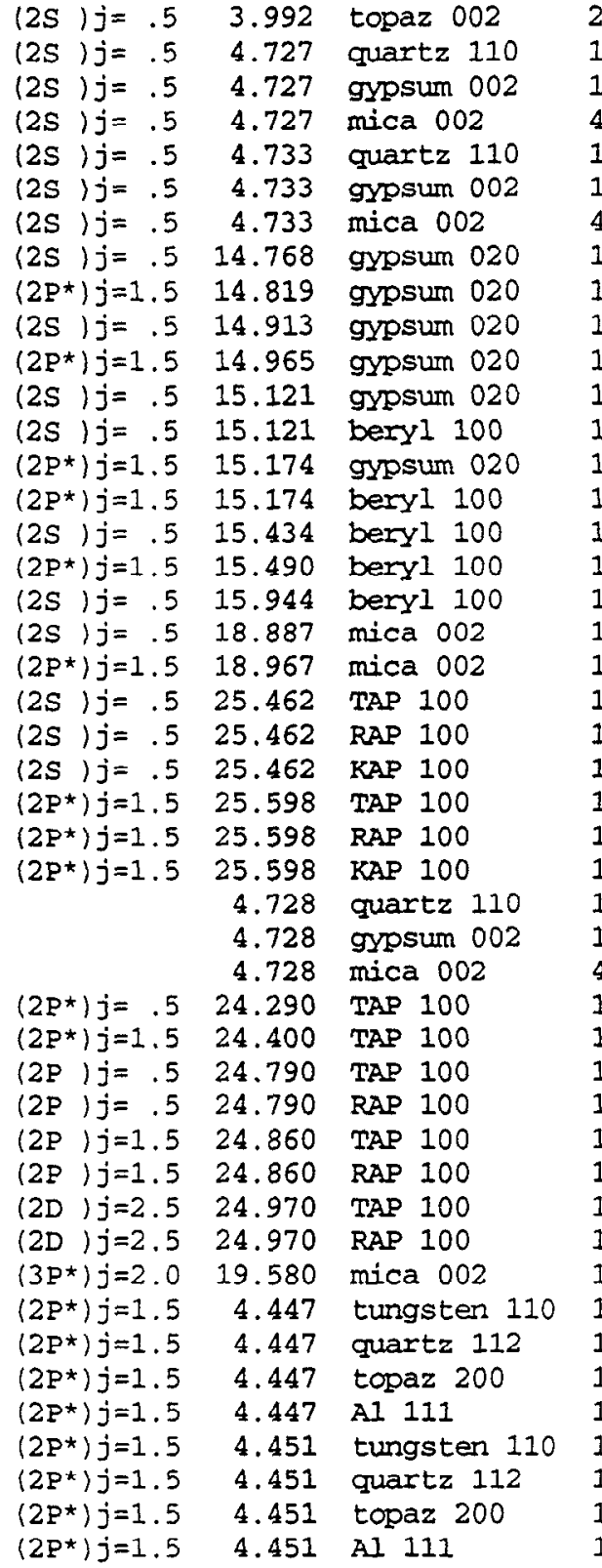

$8.374 \quad 72.445$

$4.912 \quad 74.225$

$4.990 \quad 71.315$

$19.942 \quad 71.469$

$4.912 \quad 74.485$

$4.990 \quad 71.531$

$19.942 \quad 71.687$

$15.185 \quad 76.541$

$15.185 \quad 77.395$

$15.185 \quad 79.139$

$15.185 \quad 80.235$

$15.185 \quad 84.738$

$15.954 \quad 71.403$

$15.185 \quad 87.819$

$15.954 \quad 72.010$

15.95475 .331

$15.954 \quad 76.148$

$15.954 \quad 87.971$

$19.942 \quad 71.280$

$19.942 \quad 72.010$

$25.763 \quad 81.233$

$26.116 \quad 77.151$

$26.634 \quad 72.940$

$25.763 \quad 83.512$

$26.116 \quad 78.569$

$26.634 \quad 73.967$

$4.912 \quad 74.268$

4.99071 .351

$19.942 \quad 71.505$

$25.763 \quad 70.532$

$25.763 \quad 71.279$

$25.763 \quad 74.203$

$26.116 \quad 71.664$

$25.763 \quad 74.785$

$26.116 \quad 72.158$

$25.763 \quad 75.747$

$26.116 \quad 72.964$

$19.942 \quad 79.066$

$4.476 \quad 83.474$

$4.564 \quad 76.999$

$4.638 \quad 73.500$

$4.676 \quad 71.994$

$4.476 \quad 83.942$

$4.564 \quad 77.224$

$4.638 \quad 73.675$

$4.676 \quad 72.154$ 


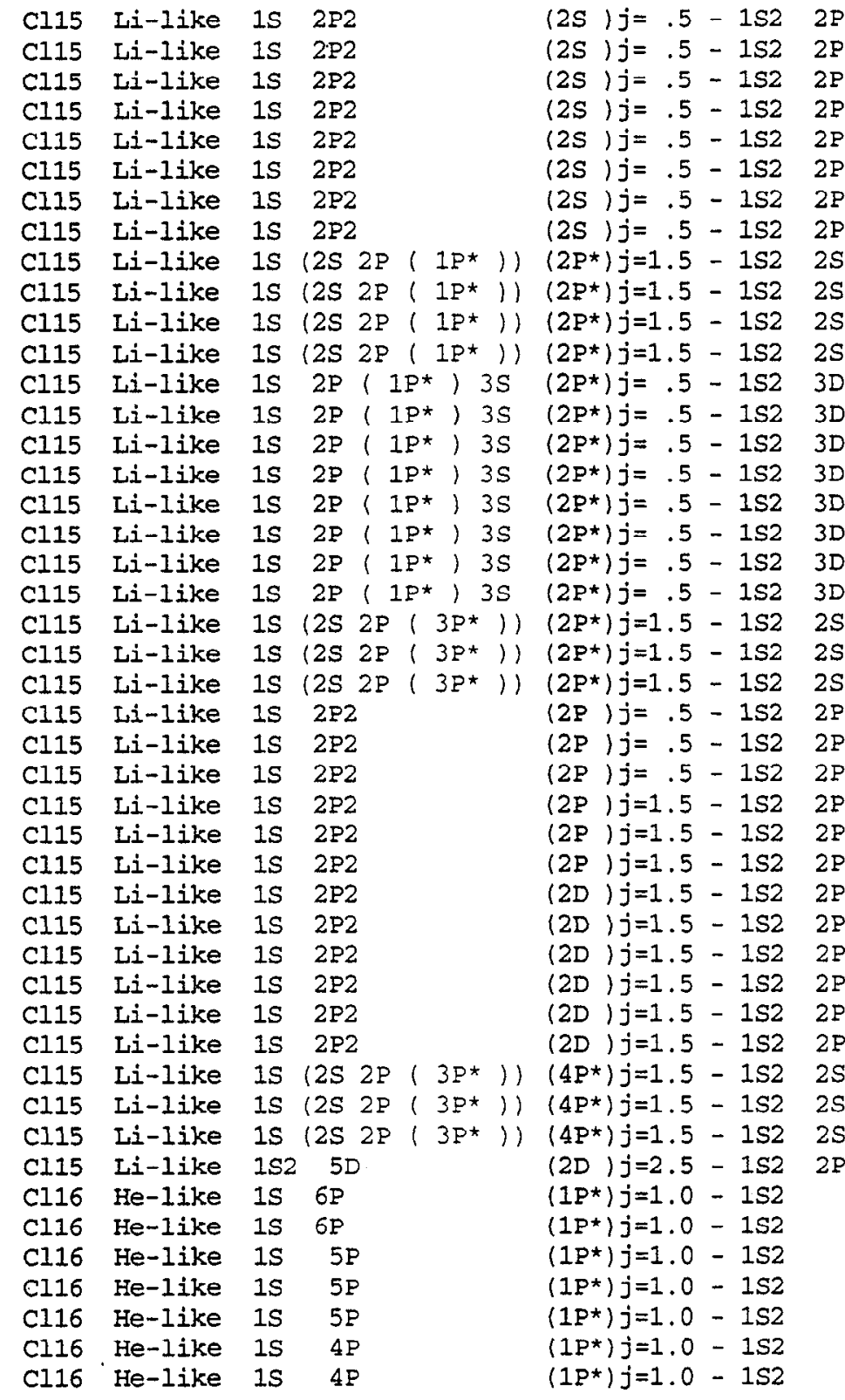

\begin{tabular}{|c|c|c|}
\hline$\left.P^{*}\right) j=.5$ & 4.458 & tungsten 11 \\
\hline$\left(2 P^{*}\right) j=.5$ & 4.458 & quartz 112 \\
\hline $2 P^{*} j j=.5$ & 4.458 & topaz 200 \\
\hline$\left.P^{\star}\right) j=.5$ & 4.458 & $A \perp 111$ \\
\hline$\left.P^{*}\right) j=1.5$ & 4.463 & tungsten 110 \\
\hline$P \star j j=1.5$ & 4.463 & quartz 112 \\
\hline$j=1.5$ & 4.463 & topaz 200 \\
\hline$j=1.5$ & 4.463 & AI 111 \\
\hline$j=.5$ & 4.466 & tungsten 110 \\
\hline$j=.5$ & 4.466 & quartz 112 \\
\hline$j=.5$ & 4.466 & topaz 200 \\
\hline$j=.5$ & 4.466 & A1 111 \\
\hline$D j j=1.5$ & 4.468 & tungsten 110 \\
\hline D $j=1.5$ & 4.468 & quartz 112 \\
\hline$j=1.5$ & 4.468 & topaz 200 \\
\hline$j=1.5$ & 4.468 & Al 111 \\
\hline$j j=2.5$ & 4.469 & tungsten 110 \\
\hline$j=2.5$ & 4.469 & quartz 112 \\
\hline$j j=2.5$ & 4.469 & topaz 200 \\
\hline$j j=2.5$ & 4.469 & A. 111 \\
\hline$j=.5$ & 4.483 & quartz 112 \\
\hline$j=.5$ & 4.483 & topaz 200 \\
\hline$j=.5$ & 4.483 & AI 111 \\
\hline$j=.5$ & 4.485 & quartz 112 \\
\hline$j j=.5$ & 4.485 & topaz 200 \\
\hline$j=.5$ & 4.485 & Al 111 \\
\hline$j=1.5$ & 4.488 & quartz 112 \\
\hline$j=1.5$ & 4.488 & topaz 200 \\
\hline$j=1.5$ & 4.488 & Al 111 \\
\hline$j=.5$ & 4.492 & quartz 112 \\
\hline *) $j=.5$ & 4.492 & topaz 200 \\
\hline$j j=.5$ & 4.492 & AI 111 \\
\hline$j=1.5$ & 4.497 & quartz 112 \\
\hline$j=1.5$ & 4.497 & topaz 200 \\
\hline$j=1.5$ & 4.497 & AI 111 \\
\hline$j=.5$ & 4.521 & quartz 112 \\
\hline$S j j=.5$ & 4.521 & topaz 200 \\
\hline$j=.5$ & 4.521 & Al 111 \\
\hline$j=1.5$ & 18.990 & mica 002 \\
\hline$j=.0$ & 3.481 & quartz 112 \\
\hline$j=.0$ & 3.481 & ADP 101 \\
\hline$j j=.0$ & 3.523 & quartz 112 \\
\hline$j j=.0$ & 3.523 & InSb 111 \\
\hline$j=.0$ & 3.523 & ADP 101 \\
\hline$j=.0$ & 3.603 & quartz 112 \\
\hline$j j=.0$ & 3.603 & InSb 111 \\
\hline
\end{tabular}

$4.476 \quad 84.860$

$4.564 \quad 77.627$

$4.638 \quad 73.985$

$4.676 \quad 72.436$

$4.476 \quad 85.632$

$4.564 \quad 77.924$

$4.638 \quad 74.211$

$4.676 \quad 72.640$

$4.476 \quad 86.169$

4.56478 .105

$4.638 \quad 74.347$

$4.676 \quad 72.764$

$4.476 \quad 86.574$

$4.564 \quad 78.228$

$4.638 \quad 74.439$

$4.676 \quad 72.846$

$4.476 \quad 86.795$

4.56478 .289

$4.638 \quad 74.485$

$4.676 \quad 72.888$

$4.564 \quad 79.189$

$4.638 \quad 75.146$

$4.676 \quad 73.481$

$4.564 \quad 79.324$

$4.638 \quad 75.242$

$4.676 \quad 73.567$

4.56479 .529

4.63875 .388

$4.676 \quad 73.698$

$4.564 \quad 79.809$

$4.638 \quad 75.586$

$4.676 \quad 73.873$

$4.564 \quad 80.170$

$4.638 \quad 75.836$

$4.676 \quad 74.095$

$4.564 \quad 82.129$

$4.638 \quad 77.103$

$4.676 \quad 75.206$

$\begin{array}{lll}19.942 & 72.225\end{array}$

$3.636 \quad 73.210$

$10.640 \quad 78.957$

3.63675 .678

$7.481 \quad 70.365$

$10.640 \quad 83.377$

$3.636 \quad 82.275$

$7.481 \quad 74.417$ 


\begin{tabular}{|c|c|c|c|}
\hline C116 & He-like & $1 S$ & $4 P$ \\
\hline $\mathrm{C} 116$ & He-like & 15 & $3 P$ \\
\hline$C 116$ & He-like & $1 S$ & $3 P$ \\
\hline C116 & He-like & $1 S$ & $3 P$ \\
\hline C116 & He-like & $1 \mathrm{~s}$ & $3 P$ \\
\hline C116 & He-like & $1 s$ & $3 P$ \\
\hline $\mathrm{C} 116$ & He-like & $1 s$ & $3 P$ \\
\hline C116 & He-like & $1 S$ & $3 P$ \\
\hline C116 & He-like & $1 s$ & $3 P$ \\
\hline C116 & He-like & $1 \mathrm{~S}$ & $3 P$ \\
\hline C116 & He-like & 15 & $3 P$ \\
\hline C116 & He-like & $1 S$ & $3 P$ \\
\hline C116 & He-like & $1 S$ & $3 P$ \\
\hline C116 & He-like & $1 S$ & $3 P$ \\
\hline Cl16 & He-like & 15 & $3 P$ \\
\hline C116 & He-like & 15 & $2 P$ \\
\hline C116 & He-like & 1s & $2 P$ \\
\hline C116 & He-like & is & $2 \mathrm{P}$ \\
\hline $\mathrm{C} 116$ & He-like & $1 \mathrm{~s}$ & $2 P$ \\
\hline C)16 & He-like & $1 S$ & $2 \mathrm{P}$ \\
\hline C116 & He-like & 15 & $2 \mathrm{P}$ \\
\hline C116 & He-like & is & $2 \mathrm{P}$ \\
\hline$C 116$ & He-like & $1 S$ & $2 \mathrm{P}$ \\
\hline C116 & He-like & 15 & $2 S$ \\
\hline C116 & He-like & 15 & $2 \mathrm{~S}$ \\
\hline C116 & He-like & 15 & $2 S$ \\
\hline C116 & He-like & Is & $3 P$ \\
\hline $\mathrm{C} 116$ & He-like & $1 S$ & $3 P$ \\
\hline $\mathrm{C} 116$ & He-like & 15 & $3 P$ \\
\hline$C 116$ & He-like & & \\
\hline C116 & He-like & & \\
\hline C116 & He-like & & \\
\hline$C 116$ & He-like & & \\
\hline C116 & He-like & & \\
\hline $\mathrm{C} 117$ & $\mathrm{H}$-like & $10 P$ & \\
\hline $\mathrm{C} 117$ & H -like & $10 P$ & \\
\hline C117 & H -like & $10 P$ & \\
\hline$C 117$ & H -like & $10 P$ & \\
\hline C117 & $\mathrm{H}$-like & $10 \mathrm{P}$ & \\
\hline C117 & H -like & $9 P$ & \\
\hline C117 & H -like & $9 p$ & \\
\hline C117 & H -like & $9 P$ & \\
\hline$C 117$ & H -like & $9 P$ & \\
\hline C117 & H -like & $9 P$ & \\
\hline $\mathrm{C} 117$ & H -like & $8 P$ & \\
\hline $\mathrm{Cl17}$ & H -like & $8 P$ & \\
\hline
\end{tabular}

$\left(1 P^{\star}\right) j=1.0-152$ $\left(1 P^{*}\right) j=1,0-1 \mathrm{~S} 2$ $\left(1 p^{*}\right) j=1.0-1 \mathrm{~S} 2$ $\left.(1)^{*}\right\rangle j=1.0-152$ $\left(1 P^{*}\right) j=1.0-1 S 2$ $\left(1 P^{*}\right) j=1.0-152$ $\left(1 P^{\star}\right) j=1.0-1 S 2$ $\left(1 P^{*}\right) j=1.0-1 S 2$ $\left(3 P^{*}\right) j=1.0-152$ $\left(3 p^{*}\right) j=1.0-152$ $\left(3 P^{*}\right) j=1.0-1 S 2$ $\left(3 E^{*}\right\rangle j=1.0-152$ $(3 P *) j=1.0-1.52$ $\left(3 P^{*}\right) j=1.0-1 S 2$ $(3 P *) j=1.0-152$ $\left(1 p^{*}\right) j=1.0-1 \mathrm{~s} 2$ $\left(1 \mathrm{P}^{*}\right) j=1.0-1 \mathrm{~S} 2$ (1D*) $j=1.0-152$ (1P*) $j=1.0-152$ $\left(3 P^{*}\right) j=1.0-1 \mathrm{~s} 2$ (3P*) $j=1.0-1 S 2$ $\left(3 P^{*}\right) j=1.0-1 S 2$ $\left(3 p^{*}\right) j=1.0-1 S 2$ (3s) $j=1.0-152$ (3s) $j=1.0-152$ (3S) $j=1.0-152$ $\left(3 P^{*}\right) j=2.0-1 S$ $\left(1 P^{*}\right) j=1.0-15$ $\left(1 p^{*}\right) j=1.0-1 \mathrm{~s}$

$(\quad) j=.0-$

( ) $j=.0-$

$j j=.0-$

)$j=.0-$

$(\quad) j=.0-$

$\left(2 \mathrm{P}^{*}\right) j=1.5-1 \mathrm{~S}$

$\left(2 P^{\star}\right) j=1.5-1 S$

(2P*) $j=1.5-1 \mathrm{~s}$

$\left(2 P^{*}\right) j=1.5-1 S$

$\left(2 P^{*}\right) j=1.5-15$

$\left(2 P^{\star}\right) j=1.5-15$

$\left(2 \mathrm{P}^{*}\right) j=1.5-1 \mathrm{~s}$

$\left(2 P^{*}\right) j=1.5-1 S$

$\left(2 p^{*}\right) j=1.5-1 S$

$\left(2 P^{\star}\right) j=1.5-1 \mathrm{~S}$

$\left(2 P^{\star}\right) j=1.5-15$

$\left(2 P^{\star}\right) j=1.5-1 S$

\begin{tabular}{|c|c|c|c|c|}
\hline $1 \mathrm{~s}$ & $j=$ & .0 & 3.603 & gypsum 020 \\
\hline $1 \mathrm{~s}$ & $j j=$ & .0 & 3.789 & $\mathrm{Si} 220$ \\
\hline IS & $j=$ & .0 & 3.789 & fluorite 220 \\
\hline & $j=$ & .0 & 3.789 & Ge 220 \\
\hline & $j=$ & .0 & 3.789 & LiF 200 \\
\hline & $j j=$ & .0 & 3.789 & gypsum 020 \\
\hline & $j=$ & .0 & 3.789 & beryl 100 \\
\hline & $j=$ & .0 & 3.789 & mica 002 \\
\hline & $j=$ & .0 & 3.794 & Si 220 \\
\hline & $j=$ & .0 & 3.794 & fluorite 220 \\
\hline & $j=$ & .0 & 3.794 & Ge 220 \\
\hline & $j=$ & .0 & 3.794 & LiF 200 \\
\hline & $1 j=$ & .0 & 3.794 & gypsum 020 \\
\hline & $i j=$ & .0 & 3.794 & beryl 100 \\
\hline & & .0 & 3.794 & mica 002 \\
\hline & $j=$ & .0 & 4.444 & tungsten 110 \\
\hline 15 & $j=$ & .0 & 4.444 & quartz 112 \\
\hline 10 & $j=$ & .0 & 4.444 & topaz 200 \\
\hline 10 & $j=$ & .0 & 4.444 & Al 111 \\
\hline 15 & $i j=$ & .0 & 4.467 & tungsten 110 \\
\hline & $j j=$ & .0 & 4.467 & quartz 112 \\
\hline & $j j=$ & .0 & 4.467 & topaz 200 \\
\hline & $j=$ & .0 & 4.467 & Al 111 \\
\hline & $j=$ & .0 & 4.497 & quartz 112 \\
\hline (1) & $j=$ & .0 & 4.497 & topaz 200 \\
\hline (15 & $j=$ & .0 & 4.497 & Al 111 \\
\hline & $j=1$ & 1.0 & 24.250 & TAP 100 \\
\hline 11 & $j=$ & .0 & 25.020 & $\operatorname{TAP} 100$ \\
\hline $1 \mathrm{~s}$ & $j=$ & .0 & 25.020 & RAP 100 \\
\hline & $j j=$ & .0 & 3.862 & Ge 220 \\
\hline & $j=$ & .0 & 3.862 & LiF 200 \\
\hline & $j=$ & .0 & 3.862 & Al 200 \\
\hline & $j=$ & .0 & 3.862 & beryl 100 \\
\hline & $j=$ & .0 & 3.862 & mica 002 \\
\hline $2 S$ & $j=$ & .5 & 3.173 & Ge 111 \\
\hline & $j=$ & .5 & 3.173 & $\mathrm{KBr} 200$ \\
\hline & $j=$ & .5 & 3.173 & quartz 101 \\
\hline & $j=$ & .5 & 3.173 & graphite 002 \\
\hline & $j=$ & .5 & 3.173 & beryl 100 \\
\hline & $j=$ & .5 & 3.181 & Ge 111 \\
\hline & $j=$ & .5 & 3.181 & $\mathrm{KBr} 200$ \\
\hline & $j=$ & .5 & 3.181 & quartz 101 \\
\hline & $j=$ & .5 & 3.181 & graphite 002 \\
\hline & & .5 & 3.181 & beryl 100 \\
\hline & $j=$ & .5 & 3.192 & Ge 111 \\
\hline & & & & \\
\hline
\end{tabular}

$15.185 \quad 71.640$

$3.840 \quad 80.652$

$3.862 \quad 78.842$

$4.000 \quad 71.307$

$4.027 \quad 70.203$

$15.185 \quad 86.458$

$15.954 \quad 71.802$

$19.942 \quad 71.806$

$3.840 \quad 81.123$

$3.862 \quad 79.232$

$4.000 \quad 71.532$

$4.027 \quad 70.414$

$15.185 \quad 88.027$

$15.954 \quad 72.033$

$19.942 \quad 72.038$

$4.476 \quad 83.145$

$4.564 \quad 76.832$

$4.638 \quad 73.370$

$4.676 \quad 71.876$

$4.476 \quad 86.366$

$4.564 \quad 78.166$

$4.638 \quad 74.393$

$4.676 \quad 72.805$

$4.564 \quad 80.170$

$4.638 \quad 75.836$

$4.676 \quad 74.095$

$25.763 \quad 70.266$

$25.763 \quad 76.206$

$26.116 \quad 73.342$

$4.000 \quad 74.906$

$4.027 \quad 73.542$

$4.048 \quad 72.564$

$15.954 \quad 75.531$

$19.942 \quad 75.537$

6.53276 .294

6.58474 .548

$6.687 \quad 71.624$

$6.696 \quad 71.393$

$15.954 \quad 83.945$

$6.532 \quad 76.900$

6.58475 .079

$6.687 \quad 72.063$

$\begin{array}{ll}6.696 & 71.827\end{array}$

$15.954 \quad 85.508$

$6.532 \quad 77.780$

6.58475 .842 


\begin{tabular}{|c|c|c|c|c|c|c|c|}
\hline $\mathrm{C} 117$ & $\mathrm{H}$ & -like & $8 \mathrm{P}$ & & $\left(2 \mathrm{P}^{\star}\right) j=1.5$ & $-1 S$ & \\
\hline $\mathrm{Cl17}$ & $\mathrm{H}$ & -like & $8 \mathrm{P}$ & & $\left(2 \mathrm{P}^{\star}\right) j=1.5$ & $-1 S$ & \\
\hline $\mathrm{C} 117$ & $\mathrm{H}$ & -like & $7 P$ & & $\left(2 P^{\star}\right) j=1.5$ & $-1 S$ & \\
\hline $\mathrm{C} 117$ & $\mathrm{H}$ & -like & $7 P$ & & $\left(2 P^{\star}\right) j=1.5$ & $-1 S$ & \\
\hline $\mathrm{Cl17}$ & $\mathrm{H}$ & -like & $7 \mathrm{P}$ & & $\left(2 P^{\star}\right) j=1.5$ & $-1 S$ & \\
\hline Cl17 & $\mathrm{H}$ & -like & $7 P$ & & $\left(2 P^{*}\right) j=1.5$ & -15 & \\
\hline C117 & $\mathrm{H}$ & -like & $6 \mathrm{P}$ & & $\left(2 P^{*}\right) j=1.5$ & $-1 S$ & \\
\hline Cl17 & $\mathrm{H}$ & -like & $6 \mathrm{P}$ & & $\left(2 P^{*}\right) j=1.5$ & $-1 s$ & \\
\hline $\mathrm{Cl} 17$ & $\mathrm{H}$ & -like & $6 \mathrm{P}$ & & $\left(2 P^{*}\right) j=1.5$ & $-1 S$ & \\
\hline C.117 & $\mathrm{H}$ & -like & $6 \mathrm{P}$ & & $\left(2 P^{\star}\right) j=1.5$ & $-1 s$ & \\
\hline $\mathrm{Cl} 17$ & $\mathrm{H}$ & -like & $5 P$ & & $\left(2 P^{*}\right) j=1.5$ & $-1 S$ & \\
\hline C117 & $\mathrm{H}$ & -like & $5 P$ & & $\left(2 P^{\star}\right) j=1.5$ & $-1 S$ & \\
\hline$C \geq 17$ & $\mathrm{H}$ & -like & $5 P$ & & $\left(2 P^{*}\right) j=1.5$ & $-1 S$ & \\
\hline $\mathrm{Cl} 17$ & $\mathrm{H}$ & -like & $4 P$ & & $\left(2 P^{*}\right) j=1.5$ & $-1 S$ & \\
\hline $\mathrm{Cl17}$ & $\mathrm{H}$ & -like & $3 P$ & & $\left(2 P^{*}\right) j=1.5$ & $-1 S$ & \\
\hline C117 & $\mathrm{H}$ & -like & $3 P$ & & $\left(2 P^{*}\right) j=1.5$ & $-1 s$ & \\
\hline Cl17 & $\mathrm{H}$ & -like & $3 P$ & & $\left(2 P^{*}\right) j=1.5$ & $-1 s$ & \\
\hline $\mathrm{Cl17}$ & $\mathrm{H}$ & -like & $3 P$ & & $\left(2 \mathrm{~F}^{*}\right) j=.5$ & $-1 s$ & \\
\hline$C 117$ & $\mathrm{H}$ & -like & $3 P$ & & $\left(2 P^{*}\right) j=.5$ & $-1 S$ & \\
\hline $\mathrm{C} 117$ & $\mathrm{H}$ & -like & $3 P$ & & $\left(2 P^{\star}\right) j=.5$ & $-1 S$ & \\
\hline Cl17 & $\mathrm{H}$ & -like & $2 P$ & & $\left(2 P^{*}\right) j=1.5$ & $-1 S$ & \\
\hline Cl17 & $\mathrm{H}$ & -like & $2 P$ & & $\left(2 P^{*}\right) j=1.5$ & $-1 S$ & \\
\hline $\mathrm{Cl17}$ & $\mathrm{H}$ & -like & $2 P$ & & $\left(2 P^{\star}\right) j=1.5$ & $-I S$ & \\
\hline $\mathrm{C} 117$ & $\mathrm{H}$ & -like & $2 P$ & & $\left(2 P^{\star}\right) j=1.5$ & $-1 s$ & \\
\hline $\mathrm{C} 117$ & $\mathrm{H}$ & -like & 29 & & $\left(2 P^{\star}\right) j=1.5$ & $-1 s$ & \\
\hline $\mathrm{Cl} 17$ & $H$ & -like & $2 P$ & & $\left(2 P^{*}\right) j=.5$ & $-1 S$ & \\
\hline Cl17 & $\mathrm{H}$ & -like & $2 P$ & & $\left(2 P^{\star}\right) j=.5$ & $-1 S$ & \\
\hline $\mathrm{Cl17}$ & $\mathrm{H}$ & -like & $2 \mathrm{P}$ & & $\left(2 P^{\star}\right) j=.5$ & $-1 S$ & \\
\hline Cl17 & $\boldsymbol{H}$ & -1ike & $2 P$ & & $\left(2 P^{*}\right) j=.5$ & $-1 S$ & \\
\hline $\mathrm{C} 117$ & $\mathrm{H}$ & -like & $10 P$ & & $\left(2 P^{\star}\right) j=1.5$ & $-2 s$ & \\
\hline $\mathrm{C} 117$ & $\mathrm{H}$ & -like & $10 D$ & & $(2 D) j=2.5$ & $-2 P$ & \\
\hline $\mathrm{C} 117$ & $\mathrm{H}$ & -1ike & $9 P$ & & $\left(2 P^{*}\right) j=1.5$ & $-2 S$ & \\
\hline C117 & $\mathrm{H}$ & -like & $9 D$ & & $(2 D) j=2.5$ & $-2 P$ & \\
\hline Cl17 & $\mathrm{H}$ & -like & $5 P$ & & $\left(2 P^{\star}\right) j=1.5$ & $-2 s$ & \\
\hline Cl17 & $\mathrm{H}$ & -like & $5 \mathrm{D}$ & & $(2 D) j=2.5$ & $-2 P$ & \\
\hline $\mathrm{Cl17}$ & $\mathrm{H}$ & -like & $5 \mathrm{D}$ & & $(2 D) j=2.5$ & $-2 P$ & \\
\hline Ar & & & K-a.pha (1) & & & & \\
\hline Ar & & & K-alpha (1) & & & & \\
\hline AI & & & $\mathrm{k}$-alpha (1) & & & & \\
\hline Ar & & & K-alpha (1) & & & & \\
\hline $\operatorname{Ar} 12$ & $\mathbf{N}$ & -like & $2 S 22 P 2 \quad(3 P$ & ) $4 D$ & $(4 P) j=2.5$ & $-2 S 2$ & $2 \mathrm{P} 3$ \\
\hline $\operatorname{Ar} 12$ & $\mathrm{~N}$ & -like & $2 S 22 P 2<3 P$ & ) $4 D$ & $(4 P) j=2.5$ & $-2 s 2$ & $2 \mathrm{P} 3$ \\
\hline Ar12 & $\mathrm{N}$ & -like & $2 S 22 P 2(3 P$ & ) $4 D$ & $(4 P) j=2.5$ & $-2 S 2$ & $2 \mathrm{P} 3$ \\
\hline Ar13 & $\mathrm{C}$ & -likse & $2 \mathrm{~S} 22 \mathrm{P}$ & $4 \mathrm{~S}$ & $\left(1 P^{\star}\right) j=1.0$ & $-2 s 2$ & $2 \mathrm{P} 2$ \\
\hline $\operatorname{Ar} 13$ & $\mathrm{C}$ & -like & $2 S 22 P$ & $4 S$ & $\left(1 P^{*}\right) j=1.0$ & $-2 s 2$ & $2 \mathrm{P} 2$ \\
\hline Ar13 & C & -like & $2 S \quad 2 P 2$ & $3 P$ & $\left(3 s^{*}\right) j=1.0$ & $-2 s 2$ & $2 \mathrm{P} 2$ \\
\hline
\end{tabular}

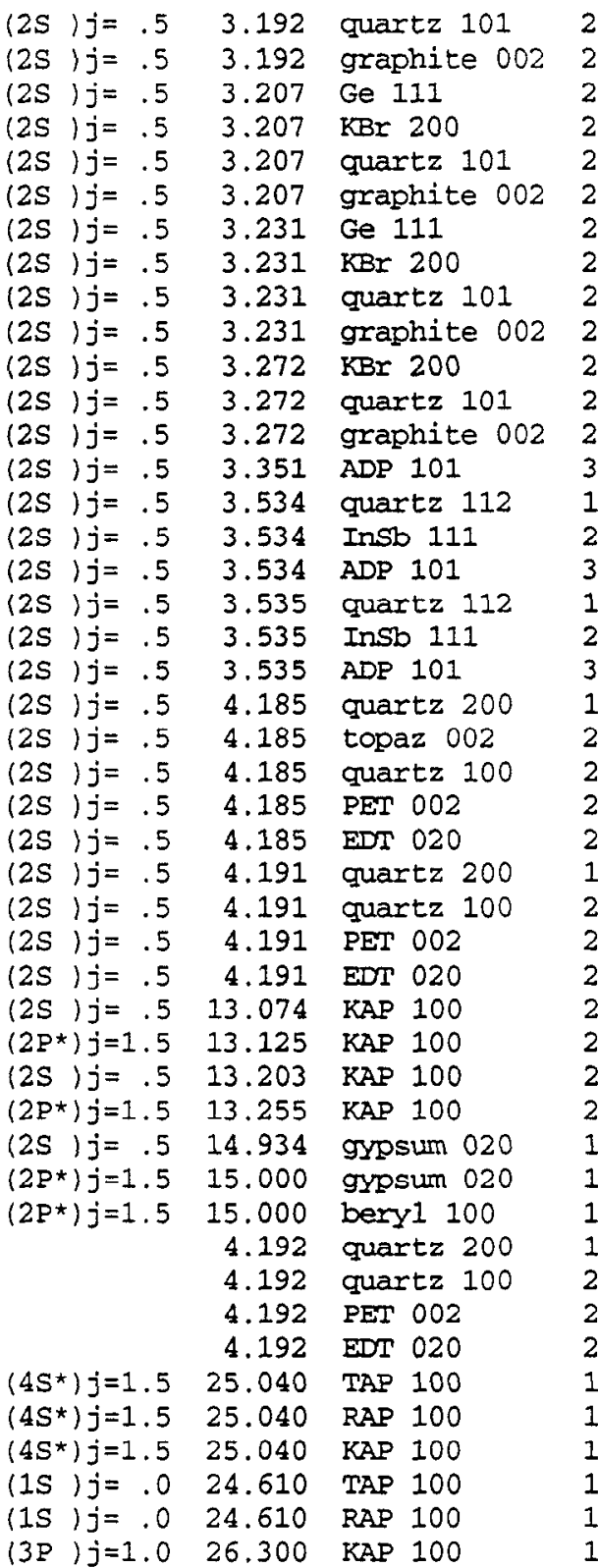

$6.687 \quad 72.686$

$6.695 \quad 72.441$

6.53279 .093

$6.584 \quad 76.952$

$6.687 \quad 73.572$

$6.696 \quad 73.313$

$6.532 \quad 81.604$

6.58478 .953

$6.687 \quad 75.095$

$6.696 \quad 74.808$

$6.584 \quad 83.681$

$6.687 \quad 78.130$

$6.696 \quad 77.769$

$10.640 \quad 70.879$

$3.636 \quad 76.397$

$7.481 \quad 70.873$

$10.640 \quad 85.156$

$3.636 \quad 76.464$

7.48170 .920

$10.640 \quad 85.351$

$4.246 \quad 80.276$

$8.374 \quad 88.229$

$8.512 \quad 79.520$

$8.742 \quad 73.225$

$8.808 \quad 71.855$

$4.246 \quad 80.768$

$8.512 \quad 79.974$

$8.742 \quad 73.500$

$8.808 \quad 72.108$

26.63479 .038

$26.634 \quad 80.259$

$26.634 \quad 82.498$

$26.634 \quad 84.469$

$15.185 \quad 79.568$

$15.185 \quad 81.047$

$15.954 \quad 70.086$

$4.246 \quad 80.852$

$8.512 \quad 80.051$

$8.742 \quad 73.546$

$8.808 \quad 72.150$

$\begin{array}{ll}25.763 & 76.394\end{array}$

$26.116 \quad 73.496$

$26.634 \quad 70.077$

$25.763 \quad 72.794$

$26.116 \quad 70.447$

$26.634 \quad 80.917$ 


\begin{tabular}{|c|c|c|c|c|c|c|c|c|c|}
\hline Ar13 & C -like & $2 S$ & $2 \mathrm{P} 2$ & & $3 P$ & $\left(3 S^{*}\right) j=1.0$ & $-2 s 2$ & $2 \mathrm{P} 2$ & \\
\hline Ar13 & C -1ike & $2 S$ & 2P2 & & $3 P$ & $(3 p *) j=2.0$ & $-2 s 2$ & $2 \mathrm{P} 2$ & \\
\hline Ar14 & B -like & 1s & $2 \$ 2$ & $2 \mathrm{P} 2$ & & $(2 P) j j=1.5$ & -152 & $2 \mathrm{~s} 2$ & $2 \mathrm{P}$ \\
\hline$A \times 14$ & B -like & 1S & $2 \mathrm{~s} 2$ & $2 \mathrm{P} 2$ & & $(2 P) j=1.5$ & $-1 s 2$ & $2 S 2$ & $2 P$ \\
\hline Ar14 & B -like & 15 & $2 s 2$ & $2 \mathrm{P} 2$ & & $(2 P) j=1.5$ & $-1 S 2$ & $2 \mathrm{~S} 2$ & $2 P$ \\
\hline Ar14 & B -like & $2 S$ & $2 P$ & & $3 P$ & $\langle 2 P\rangle j=1.5$ & $-2 s 2$ & $2 P$ & \\
\hline Ar14 & B -like & $2 S$ & $2 \mathrm{P}$ & & $3 P$ & $(2 P) j=1.5$ & $-2 s 2$ & $2 \mathrm{P}$ & \\
\hline Ar14 & B -like & $2 S$ & $2 P$ & & $3 P$ & $(2 P) j=1.5$ & $-2 s 2$ & $2 \mathrm{P}$ & \\
\hline Ar14 & B -like & $2 S$ & $2 P$ & & $3 P$ & $(2 P) j=1.5$ & $-1 s 2$ & $2 S 2$ & $2 P$ \\
\hline Ar14 & B -like & $2 S$ & $2 P$ & & $3 P$ & $(2 P) j=1.5$ & $-1 s 2$ & $2 \mathrm{~S} 2$ & $2 P$ \\
\hline Ar14 & B -like & $2 S$ & $2 \mathrm{P}$ & & $3 P$ & $(2 P) j=1.5$ & $-1 S 2$ & $2 S 2$ & $2 P$ \\
\hline A 15 & Be-like & 15 & $2 s 2$ & $4 \mathrm{P}$ & & $\left(1 P^{\star}\right) j=1.0$ & $-1 S 2$ & $2 \$ 2$ & \\
\hline Ar15 & Be-like & 1S & $2 s 2$ & $4 \mathrm{P}$ & & $\left(19^{\star}\right) j=1.0$ & -152 & $2 \mathrm{~s} 2$ & \\
\hline Ar15 & Be-like & 1S & $2 S 2$ & $3 P$ & & $\left(1 P^{\star}\right) j=1.0$ & $-1 s 2$ & $2 S 2$ & \\
\hline Ar15 & Be-like & 1S & $2 \$ 2$ & $3 P$ & & $\left(1 P^{*}\right) j=1.0$ & -152 & $2 S 2$ & \\
\hline Ar15 & Be-like & 15 & $2 s 2$ & $2 P$ & & $\left(1 P^{\star}\right) j=1.0$ & $-1 s 2$ & $2 s 2$ & \\
\hline Ar15 & Be-like & 15 & $2 S 2$ & $2 P$ & & $\left(1 P^{*}\right) j=1.0$ & $-1 s 2$ & $2 S 2$ & \\
\hline Ar15 & Be-like & 1S & $2 S 2$ & $2 P$ & & $\left(1 P^{\star}\right) j=1.0$ & $-1 s 2$ & $2 \mathrm{~S} 2$ & \\
\hline Ar15 & Be-like & 15 & $2 s 2$ & $2 P$ & & $\left(1 P^{*}\right) j=1.0$ & $-1 s 2$ & $2 \$ 2$ & \\
\hline Ar15 & Be-like & 15 & $2 \mathrm{~S} 2$ & $2 P$ & & $\left(1 P^{\star}\right) j=1.0$ & $-1 s 2$ & $2 s 2$ & \\
\hline Ar15 & Be-like & $1 \mathrm{~S} 2$ & $2 S$ & $3 P$ & & $\left(2 P^{*}\right) j=1.0$ & $-1 s 2$ & $2 S 2$ & \\
\hline Ar15 & Be-like & $1 S 2$ & $2 S$ & $3 P$ & & $\left(1 P^{*}\right) j=1.0$ & $-1 s 2$ & $2 s 2$ & \\
\hline Ar15 & Be-like & 152 & 25 & $3 P$ & & $(1 P *) j=1.0$ & -152 & 252 & \\
\hline Ar15 & Be-like & $1 S 2$ & $2 S$ & $3 D$ & & $(3 D) j=1.0$ & $-1 s 2$ & $2 S$ & $2 \mathrm{P}$ \\
\hline Ar15 & Be-like & 152 & $2 S$ & $3 D$ & & (3D) $j=1.0$ & $-1 s 2$ & $2 S$ & $2 \mathrm{P}$ \\
\hline Ar15 & Be-like & 152 & $2 S$ & $3 D$ & & $(3 D) j=1.0$ & $-1 s 2$ & $2 S$ & $2 \mathrm{P}$ \\
\hline Ar15 & Be-like & $1 \mathrm{~s} 2$ & $2 S$ & 30 & & $(3 D) j=2.0$ & $-1 s 2$ & $2 S$ & $2 P$ \\
\hline Ar15 & Be-like & 152 & $2 S$ & $3 D$ & & $(3 D) j=2.0$ & $-1 s 2$ & $2 S$ & $2 P$ \\
\hline Ar15 & Be-like & 152 & $2 S$ & $3 D$ & & $(3 D) j=3.0$ & -152 & $2 S$ & $2 \mathrm{P}$ \\
\hline Ar15 & Be-like & 152 & $2 S$ & $3 D$ & & $(3 D) j=3.0$ & $-1 s 2$ & $2 S$ & $2 \mathrm{P}$ \\
\hline Ar16 & Li-like & $1 \mathrm{~s}$ & $2 S$ & $4 \mathrm{P}$ & & $\left(2 P^{\star}\right) j=1.5$ & $-1 s 2$ & $2 S$ & \\
\hline Ar16 & Li-like & IS & $2 S$ & $4 \mathrm{P}$ & & $\left(2 P^{*}\right) j=1.5$ & $-1 s 2$ & $2 S$ & \\
\hline Ar16 & Li-like & is & $2 S$ & $4 \mathrm{P}$ & & $(2 P *) j=1.5$ & $-1 s 2$ & $2 S$ & \\
\hline A×16 & Li-like & is & $2 S$ & $4 P$ & & $\left(2 P^{\star}\right) j=1.5$ & $-1 s 2$ & $2 S$ & \\
\hline Ar16 & Li-like & is & $2 \mathrm{P}$ & $4 \mathrm{P}$ & & $(2 D) j=2.5$ & $-1 s 2$ & $2 P$ & \\
\hline Ar16 & Li-like & is & $2 P$ & $4 \mathrm{P}$ & & $(2 D) j=2.5$ & $-1 s 2$ & $2 \mathrm{P}$ & \\
\hline Ar16 & Li-like & $1 S$ & $2 \mathrm{P}$ & $4 P$ & & $(2 D) j=2.5$ & $-1 S 2$ & $2 P$ & \\
\hline Ar16 & Li-like & is & $2 S$ & & $3 P$ & $(2 P *) j=1.5$ & $-1 s 2$ & $2 S$ & \\
\hline Ar16 & Li-like & is & $2 P$ & $\left(3 P^{*}\right.$ & ) $3 P$ & $(2 P) j=1.5$ & $-1 s 2$ & $2 P$ & \\
\hline Ar16 & Li-like & $1 \mathrm{~S}$ & $2 \mathrm{P}$ & $\left(32^{\star}\right.$ & $3 P$ & $(2 P) j=1.5$ & $-1 s 2$ & $2 \mathrm{P}$ & \\
\hline Ar16 & Li-like & is & $2 \mathrm{P}$ & $\left(3 P^{*}\right.$ & ) $3 P$ & $(2 D) j=2.5$ & $-1 \$ 2$ & $2 P$ & \\
\hline Ar16 & Li-like & $1 \mathrm{~s}$ & $2 P$ & $\left(32^{\star}\right.$ & $3 P$ & $(2 D) j=2.5$ & -152 & $2 \mathrm{P}$ & \\
\hline Ar16 & Li-like & $1 \mathrm{~S}$ & $2 \mathrm{P} 2$ & & & $(2 s) j=.5$ & - 152 & $2 P$ & \\
\hline Ar16 & Li-like & 1S & $2 \mathrm{P} 2$ & & & $(2 s) j=.5$ & $-1 s 2$ & $2 \mathrm{P}$ & \\
\hline Ar16 & Li-like & $1 \mathrm{~S}$ & $2 \mathrm{P} 2$ & & & $(2 S) j=.5$ & $-1 s 2$ & $2 \mathrm{P}$ & \\
\hline Ar16 & Li-like & 1S & $2 P 2$ & & & $(2 S) j=.5$ & $-1 s 2$ & $2 P$ & \\
\hline
\end{tabular}

\begin{tabular}{|c|c|c|c|c|c|}
\hline$(3 P) j=2.0$ & 26.360 & KAP 100 & 1 & 26.634 & 81.774 \\
\hline$(3 P) j=2.0$ & 26.530 & KAP 100 & 1 & 26.634 & 84.935 \\
\hline$P^{*} j j=1.5$ & 4.058 & quartz 200 & 1 & 4.246 & 72.886 \\
\hline *) $j=1.5$ & 4.058 & topaz 002 & 2 & 8.374 & 75.741 \\
\hline *) $j=1.5$ & 4.058 & quartz 100 & 2 & 8.512 & 72.454 \\
\hline$e^{*} j=.5$ & 25.420 & $\operatorname{TAP} 100$ & 1 & 25.763 & 80.640 \\
\hline$\left.P^{*}\right) j=.5$ & 25.420 & RAP 100 & 1 & 26.116 & 76.743 \\
\hline$\left.P^{*}\right) j=.5$ & 25.420 & $\operatorname{KAP} 100$ & 1 & 26.634 & 72.634 \\
\hline$\left.P^{*}\right) j=1.5$ & 25.580 & TAP 100 & 1 & 25.763 & 83.167 \\
\hline$P^{\star} / j=1.5$ & 25.580 & RAP 100 & 1 & 26.116 & 78.372 \\
\hline$\left.P^{*}\right) j=1.5$ & 25.580 & KAP 100 & 1 & $26: 634$ & 73.827 \\
\hline$s j j=.0$ & 3.330 & quartz 101 & 2 & 6.687 & 84.850 \\
\hline$S) j=.0$ & 3.330 & graphite 002 & 2 & 6.696 & 84.056 \\
\hline$s) j=.0$ & 3.450 & quartz 112 & 1 & 3.636 & 71.594 \\
\hline (s) $j=.0$ & 3.450 & ADP 101 & 3 & 10.640 & 76.592 \\
\hline$S / j=.0$ & 4.010 & LiF 200 & 1 & 4.027 & 84.734 \\
\hline$S i j=.0$ & 4.010 & A1 200 & 1 & 4.048 & 82.143 \\
\hline$s \mid j=.0$ & 4.010 & quartz 200 & 1 & 4.246 & 70.807 \\
\hline$j=.0$ & 4.010 & topaz 002 & 2 & 8.374 & 73.281 \\
\hline$S j j=.0$ & 4.010 & quartz 100 & 2 & 8.512 & 70.424 \\
\hline$s) j=.0$ & 25.050 & TAP 100 & 1 & 25.763 & 76.48 \\
\hline$s) j=.0$ & 25.050 & $\operatorname{RAP} 100$ & 1 & 26.116 & 73.57 \\
\hline$S / j=.0$ & 25.050 & $\operatorname{KAP} 100$ & 1 & 26.634 & 70.14 \\
\hline$\left.P^{\star}\right) j=.0$ & 25.720 & TAP 100 & 1 & 25.763 & 86.689 \\
\hline$p^{*} \mid j=.0$ & 25.720 & RAP 100 & 1 & 26.116 & 80.010 \\
\hline$\left.P^{*}\right) j=.0$ & 25.720 & $\operatorname{KAP} 100$ & 1 & 26.634 & 74.946 \\
\hline$\left.P^{*}\right) j=1.0$ & 25.840 & RAP 100 & 1 & 26.116 & 81.663 \\
\hline$\left.P^{*}\right) j=1.0$ & 25.840 & $\operatorname{KAP} 100$ & 1 & 26.634 & 75.975 \\
\hline *) $j=2.0$ & 26.000 & RAP 100 & 1 & 26.116 & 84.598 \\
\hline *) $j=2.0$ & 26.000 & KAP 100 & 1 & 26.634 & 77.474 \\
\hline$j=.5$ & 3.250 & Ge 111 & 2 & 6.532 & 84.32 \\
\hline$j j=.5$ & 3.250 & $\operatorname{KBr} 200$ & 2 & 6.584 & 80.83 \\
\hline$j j=.5$ & 3.250 & quartz 101 & 2 & 6.687 & 76.41 \\
\hline$j j=.5$ & 3.250 & graphite 002 & 2 & 6.696 & 76.103 \\
\hline$\left.p^{*}\right) j=1.5$ & 3.268 & $\mathrm{KBI} 200$ & 2 & 6.584 & 83.077 \\
\hline$\left.P^{\star}\right\rangle j=1.5$ & 3.268 & quartz 101 & 2 & 6.687 & 77.80 \\
\hline$\left.P^{*}\right) j=1.5$ & 3.268 & graphite 002 & 2 & 6.696 & 77.45 \\
\hline$j j=.5$ & 3.392 & ADP 101 & 3 & 10.640 & 73.01 \\
\hline *) $j=1.5$ & 3.420 & quartz 112 & 1 & 3.636 & 70.15 \\
\hline *) $j=1.5$ & 3.420 & ADP 101 & 3 & 10.640 & 74.64 \\
\hline *) $j=1.5$ & 3.430 & quartz 112 & 1 & 3.636 & 70.62 \\
\hline *) $j=1.5$ & 3.430 & ADP 101 & 3 & 10.640 & 75.26 \\
\hline$\left.P^{\star}\right) j=.5$ & 3.961 & Ge 220 & 1 & 4.000 & 81.993 \\
\hline$\left.p^{*}\right) j=.5$ & 3.961 & LiF 200 & 1 & 4.027 & 79.612 \\
\hline$\left.P^{*}\right) j=.5$ & 3.961 & A1 200 & 1 & 4.048 & 78.10 \\
\hline$\left(P^{*}\right) j=.5$ & 3.961 & topaz 002 & 2 & 8.374 & 71.08 \\
\hline
\end{tabular}




\begin{tabular}{|c|c|c|c|c|c|c|c|c|}
\hline Ar16 & Li-like & is & $2 \mathrm{P} 2$ & & & $(2 S) j=.5$ & $-1 s 2$ & \\
\hline A×16 & Li-like & 15 & $2 \mathrm{P} 2$ & & & $(2 S) j=.5$ & $-1 s 2$ & \\
\hline Ar16 & Li-like & 1S & $2 \mathrm{P} 2$ & & & $(2 s) j=.5$ & $-1 S 2$ & \\
\hline r16 & Li-like & $1 S$ & $2 \mathrm{P} 2$ & & & $(2 S)$ & $-1 s 2$ & \\
\hline Ar16 & Li-like & 1S & 2P2 & & & $(2 S) j=$ & $-1 s 2$ & \\
\hline$A=16$ & Ii-like & $1 S$ & $2 \mathrm{P} 2$ & & & $(2 s) j=.5$ & $-1 s 2$ & \\
\hline$r 16$ & Li-like & $1 S$ & $2 \mathrm{P} 2$ & & & $(2 S) j=.5$ & - IS2 & \\
\hline 16 & Ii-like & $1 \mathrm{~s}$ & $2 \mathrm{P} 2$ & & & (2S) & $1 \mathrm{~s} 2$ & \\
\hline Ar16 & Ii-like & $1 S$ & i2S & $2 \mathrm{P}$ & $\left(1 P^{*}\right.$ & )) $\left(2 P^{*}\right)$ & $-1 s 2$ & \\
\hline$r 16$ & ike & $1 S$ & (2S & $2 \mathrm{P}$ & $\left(10^{*}\right.$ & $\left\langle 2 P^{\star}\right\rangle$ & $-1 s 2$ & \\
\hline$r 16$ & ike & 1s & (2S & $2 \mathrm{P}$ & $\left(1 P^{*}\right.$ & ) $\left(2 \mathrm{P}^{*}\right)$ & $-1 s 2$ & \\
\hline 216 & Li-like & 15 & 125 & $2 \mathrm{P}$ & $\left(1 P^{\star}\right.$ & ) $\left(2 P^{*}\right) j=1.5$ & $-1 s 2$ & \\
\hline$r 16$ & Li-like & $1 S$ & (2S & $2 \mathrm{P}$ & $\left(1 P^{*}\right.$ & *) $j=1.5$ & -152 & \\
\hline$r$ & Li-like & is & i2s & $2 \mathrm{P}$ & $\left(1 P^{*}\right.$ & $=1.5$ & $-1 s 2$ & \\
\hline$r 1$ & ike & $1 S$ & i2s & $2 \mathrm{P}$ & $\left(1 P^{*}\right.$ & *) $j=.5$ & $-1 s 2$ & \\
\hline$r 16$ & ike & 1s & (2s & $2 \mathrm{P}$ & $\left(12^{*}\right.$ & $\star j j=.5$ & $-1 S 2$ & \\
\hline 21 & ike & is & (2S & $2 \mathrm{P}$ & $(1)$ & *j $j=.5$ & $-1 \$ 2$ & \\
\hline 16 & ike & $1 \mathrm{~s}$ & (2S & $2 \mathrm{P}$ & $(1)$ & )) $\left(2 P^{*}\right) j=.5$ & $-1 s 2$ & \\
\hline-1 & ike & $1 s$ & 125 & $2 P$ & $\left(1 P^{*}\right.$ & $\left.p^{*}\right) j=.5$ & $S 2$ & \\
\hline & ike & is & i2s & $2 \mathrm{P}$ & $\left(1 P^{*}\right.$ & $\left.2 P^{\star}\right) j=.5$ & $-1 s 2$ & \\
\hline & ike & 15 & i2s & $2 P$ & $\left(3 P^{\star}\right.$ & $p^{*} j=1.5$ & $-1 s 2$ & \\
\hline & $\mathrm{Li}$ & is & (2s & $2 P$ & $(3)$ & *) $j=1.5$ & $-1 s 2$ & \\
\hline & $\mathrm{ce}$ & 1s & (2S & $2 \mathrm{P}$ & $(31)$ & *) $j=1.5$ & $-1 s 2$ & \\
\hline$A \simeq 16$ & ike & is & 125 & 29 & $(3 E$ & $=1.5$ & -152 & \\
\hline Ar16 & Li-like & is & (2S & $2 \mathrm{P}$ & $\left(3 P^{*}\right.$ & $=1.5$ & $-1 s 2$ & \\
\hline$A \simeq 16$ & Li-like & 15 & (2S & $2 \mathrm{P}$ & $\left(3 P^{*}\right.$ & $=1.5$ & $-1 s 2$ & \\
\hline$A \simeq 16$ & Li-like & 15 & (2S & $2 \mathrm{P}$ & $\left(3 P^{*}\right.$ & *) $j=.5$ & $-1 s 2$ & \\
\hline$A \simeq 16$ & Li-like & $1 S$ & (2S & $2 P$ & $\left(30^{*}\right.$ & )) $\left(2 P^{\star}\right) j=.5$ & $-1 S 2$ & \\
\hline Ar16 & Li-like & 1S & (2S & $2 \mathrm{P}$ & $\left(3 P^{*}\right.$ & )) $\left(2 \mathrm{P}^{\star}\right) j=.5$ & $-1 s 2$ & \\
\hline Ar16 & ike & is & 125 & $2 p$ & $\left(3 P^{*}\right.$ & $\left(2 P^{*}\right) j=.5$ & $-1 s 2$ & \\
\hline 16 & Li & 15 & (2S & $2 P$ & $\left(3 F^{\star}\right.$ & $\left.e^{*}\right) j=.5$ & -152 & \\
\hline 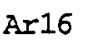 & Li-like & 1s & (2S & $2 \mathrm{P}$ & $(32 *$ & $\left(2 P^{\star}\right) j=.5$ & $-1 s 2$ & \\
\hline & Like & $1 \mathrm{~s}$ & $2 \mathrm{P} 2$ & & & $(2 \mathrm{P})$ & $-1 s 2$ & \\
\hline & ike & 1s & $2 \mathrm{P} 2$ & & & $(2 P$ & $-1 s 2$ & \\
\hline & $L$ & 15 & $2 \mathrm{P} 2$ & & & $2 \mathrm{P}$ & $\$ 2$ & \\
\hline & & $1 \mathrm{~s}$ & $2 \mathrm{P} 2$ & & & 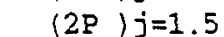 & $\mathrm{s} 2$ & \\
\hline Ar16 & Li-like & is & $2 \mathrm{P} 2$ & & & (2P & -152 & \\
\hline Ar16 & Li- & 15 & $2 \mathrm{P} 2$ & & & $(2 \mathrm{P})$ & $-1 s 2$ & \\
\hline Ar & $\mathrm{Li}-$ & 1S & $2 \mathrm{P} 2$ & & & $=1.5$ & $-1 s 2$ & \\
\hline Ar16 & Li & 1S & $2 \mathrm{P} 2$ & & & $=1.5$ & $-1 \$ 2$ & \\
\hline Ar16 & $\overline{\mathrm{Li}}$ & 15 & $2 \mathrm{P} 2$ & & & $=1.5$ & $-1 s 2$ & \\
\hline Ar 16 & Li-like & is & $2 \mathrm{P} 2$ & & & (2D) & -152 & \\
\hline$A=16$ & {$[i-1 i\}$} & is & $2 \mathrm{P} 2$ & & & $(2 D$ & $-1 s 2$ & \\
\hline & & 15 & $2 \mathrm{P} 2$ & & & (2D & $-1 S 2$ & \\
\hline & & $1 \mathrm{~s}$ & $2 \mathrm{P}^{2}$ & & & (2) & $\$ 2$ & \\
\hline$d \tau]$ & {$[i-1 i$} & is & $2 \mathrm{P} 2$ & & & (2D) $j=2.5$ & 52 & \\
\hline
\end{tabular}

$\left(2 P^{\star}\right) j=.5 \quad 3.961$ beryl 100 $\left(2 P^{\star}\right) j=.5 \quad 3.961$ mica 002 $\left(2 P^{*}\right\rangle j=1.5$ $\left(2 P^{\star}\right) j=1.5$ $\left(2 P^{\star}\right) j=1.5$ $\left(2 P^{\star}\right) j=1.5$ $\left(2 P^{*}\right) j=1.5$ $\left(2 P^{\star}\right) j=1.5$

(2S) $j=.5$ (2s) $j=.5$ (2S) $j=.5$

(2S) $j=.5$

(2S) $j=.5$

(2s) $j=.5$

(2s) $j=.5$

(2S ) $j=.5$

(2S) $j=.5$

(2s) $j=.5$

(2S) $j=.5$

(2s) $j=.5$

(2s) $j=.5$

(2s) $j=.5$

(2S) $j=.5$

(2S) $j=.5$

(2S) $j=.5$

(2s) $j=.5$

(2s) $j=.5$

(2S) $j=.5$

(2S) $j=$.

(2s) $j=.5$

(2s) $j=.5$

(2S) $j=.5$

$\left(2 P^{*}\right) j=1.5$

$\left(2 P^{*}\right) j=1.5$

$\left(2 P^{*}\right) j=1.5$

$\left(2 P^{\star}\right) j=1.5$

$\left(2 P^{*}\right) j=1.5$

$\left(2 P^{*}\right) j=1.5$

$\left(2 P^{*}\right) j=.5$

$\left(2 P^{*}\right) j=.5$

$\left\langle 2 P^{*}\right\rangle j=.5$

$\left(2 P^{\star}\right) j=.5$

$\left(2 P^{\star}\right) j=1.5$

$\left(2 P^{*}\right) j=1.5$

$\left(2 P^{\star}\right) j=1.5$

$\left(2 P^{\star}\right) j=1,5$
3.964 Ge 220

3.964 LiF 200

3.964 AI 200

3.964 topaz 002

3.964 beryl 100

3.967 Ge 220

3.967 Al 200

3.967 topaz 002

3.967 beryl 100

3.967 mica 002

.968 Ge 220

3.968 LiF 200

3.968 AI 200

3.968 topaz 002

3.968 beryl 100

3.968 mica 002

3.981 Ge 220

3.981 LIF 200

3.981 AI 200

3.981 topaz 002

3.981 beryl 100

3.981 mica 002

3.983 Ge 220

3.983 LiF 200

3.983 A] 200

3.983 topaz 002

3.983 beryl 100

3.983 mica 002

3.985 Ge 220

3.985 LiF 200

3.985 Al 200

3.985 topaz 002

3.985 beryl 100

3.985 mica 002

3.989 Ge 220

3.989 LiF 200

3.989 Al 200

3.989 topaz 002

3.992 Ge 220

3.992 LiF 200

3.992 Al 200

3.992 guartz 200
3.964 mica 002

3.967 LiF 200
$15.954 \quad 83.268$

$19.942 \quad 83.280$

$4.000 \quad 82.307$

$4.027 \quad 79.852$

$4.048 \quad 78.307$

$8.374 \quad 71.216$

$15.954 \quad 83.646$

$19.942 \quad 83.659$

$4.000 \quad 82.635$

$4.027 \quad 80.097$

$4.048 \quad 78.519$

$8.374 \quad 71.344$

$15.954 \quad 84.048$

$19.942 \quad 84.062$

$4.000 \quad 82.748$

$4.027 \quad 80.180$

$4.048 \quad 78.590$

$8.374 \quad 71.387$

$15.954 \quad 84.188$

$19.942 \quad 84.203$

$4.000 \quad 84.413$

$4.027 \quad 81.332$

$4.048 \quad 79.561$

$8.374 \quad 71.953$

$15.954 \quad 86.486$

$19.942 \quad 86.509$

$4.000 \quad 84.716$

$4.027 \quad 81.522$

$4.048 \quad 79.719$

$8.374 \quad 72.041$

$15.954 \quad 86.991$

$19.942 \quad 87.018$

$4.000 \quad 85.036$

$4.027 \quad 81.718$

$4.048 \quad 79.878$

$8.374 \quad 72.130$

$15.954 \quad 87.599$

$19.942 \quad 87.634$

$4.000 \quad 85.750$

$4.027 \quad 82.123$

$4.048 \quad 80.206$

$8.374 \quad 72.309$

$4.000 \quad 86.376$

$4.027 \quad 82.440$

$4.048 \quad 80.459$

$4.246 \quad 70.082$ 


\begin{tabular}{|c|c|c|c|c|c|c|c|c|}
\hline Ar16 & Li-like & 15 & $2 \mathrm{P} 2$ & & & (2D ) j=2.5 & -152 & $2 P$ \\
\hline Ar16 & Li-like & $1 \mathrm{~s}$ & $12 \mathrm{~s}$ & $2 P$ & $\left(3 P^{\star}\right.$ & )) $\left(4 P^{\star}\right) j=1.5$ & $-1 s 2$ & $2 S$ \\
\hline$A \times 16$ & Li-like & is & $(2 S$ & $2 P$ & $\left(3 P^{*}\right.$ & )) $\left(4 P^{*}\right) j=1.5$ & -152 & $2 S$ \\
\hline Ar16 & Li-like & $1 S$ & (2S & $2 P$ & $\left(3 P^{*}\right.$ & )) $\left(4 P^{*}\right) j=1.5$ & $-1 s 2$ & $2 \mathrm{~S}$ \\
\hline Ar16 & Li-like & 15 & (2S & $2 \mathrm{P}$ & $\left(3 P^{\star}\right.$ & )$\left(4 P^{*}\right) j=1.5$ & $-1 s 2$ & $2 \mathrm{~S}$ \\
\hline$A=16$ & Ii-like & $1 S$ & (2S & 29 & $\left(3 P^{\star}\right.$ & )) $\left(4 P^{*}\right) j=1.5$ & $-1 s 2$ & $2 S$ \\
\hline Ar16 & Li-like & $1 \mathrm{~s}$ & $2 \mathrm{P} 2$ & & & $(4 P) j=1.5$ & -152 & $2 P$ \\
\hline Ar16 & Li-like & 15 & $2 \mathrm{P} 2$ & & & $(4 P) j=1.5$ & $-1 s 2$ & $2 P$ \\
\hline Ar16 & Ii-like & $1 S$ & $2 \mathrm{P} 2$ & & & $(4 P) j=1.5$ & $-1 s 2$ & $2 \mathrm{P}$ \\
\hline Ar16 & Li-like & 15 & 2P2 & & & $(4 P) j=1.5$ & $-1 s 2$ & $2 \mathrm{P}$ \\
\hline Ar16 & Li-like & $1 S$ & $2 \mathrm{P} 2$ & & & $(4 P) j=1.5$ & $-1 s 2$ & $2 P$ \\
\hline Ar16 & Li-like & is & $2 \mathrm{P} 2$ & & & $\langle 4 P\rangle j=2.5$ & $-1 s 2$ & $2 P$ \\
\hline Ar16 & Li-like & $1 s$ & $2 \mathrm{P} 2$ & & & $(4 P) j=2.5$ & $-1 \$ 2$ & $2 P$ \\
\hline$A=16$ & Ii-like & 15 & $2 \mathrm{P} 2$ & & & $(4 P) j=2.5$ & -152 & $2 \mathrm{P}$ \\
\hline Ar16 & Li-1ike & $1 s$ & $2 \mathrm{P} 2$ & & & $(4 P) j=2.5$ & $-1 s 2$ & $2 \mathrm{P}$ \\
\hline Ar 16 & Li-like & is & 2P2 & & & $(4 P) j=2.5$ & $-1 s 2$ & 29 \\
\hline $\operatorname{Ar} 16$ & Li-like & 1s & $2 \mathrm{P} 2$ & & & $(4 P) j=1.5$ & $-1 s 2$ & $2 \mathrm{P}$ \\
\hline$A=16$ & Li-like & is & $2 \mathrm{P} 2$ & & & $(4 P) j=1.5$ & $-1 s 2$ & $2 P$ \\
\hline$A \times 16$ & Li-like & $1 \mathrm{~s}$ & $2 \mathrm{P} 2$ & & & $(4 P) j=1.5$ & -152 & $2 P$ \\
\hline Axl6 & Li-like & $1 S$ & 2P2 & & & $(4 P) j=1.5$ & $-1 S 2$ & $2 P$ \\
\hline Ar16 & Li-like & 15 & 2P2 & & & $(4 P) j=1.5$ & -152 & $2 \mathrm{P}$ \\
\hline Ar16 & Li-like & 15 & $(2 S$ & $2 P$ & $\left(3 P^{*}\right.$ & )) $\left(4 P^{\star}\right) j=.5$ & $-1 s 2$ & $2 S$ \\
\hline Ar16 & Li-like & 15 & (2S & $2 P$ & $\left(3 P^{*}\right.$ & )) $\left(4 P^{*}\right) j=.5$ & $-1 s 2$ & $2 S$ \\
\hline Ar16 & Li-like & 15 & (2S & $2 P$ & $\left(3 \mathrm{P}^{*}\right.$ & $\int\left(4 P^{*}\right) j=.5$ & $-1 s 2$ & $2 \mathrm{~S}$ \\
\hline Ar16 & Li-like & $1 S$ & (2S & $2 P$ & $\left(3 P^{*}\right.$ & )) $\left(4 P^{*}\right) j=.5$ & $-1 S 2$ & $2 S$ \\
\hline Ar16 & Li-like & $1 \mathrm{~s}$ & $12 \mathrm{~S}$ & $2 \mathrm{P}$ & $\left(3 P^{*}\right.$ & )) $\left(4 P^{*}\right) j=.5$ & $-1 S 2$ & $2 S$ \\
\hline Ar17 & He-like & $1 S$ & $6 \mathrm{P}$ & & & $\left(1 P^{*}\right) j=1.0$ & $-1 s 2$ & \\
\hline $\operatorname{Ar17}$ & He-like & $1 S$ & $6 P$ & & & $\left(1 P^{*}\right) j=1.0$ & $-1 s 2$ & \\
\hline $\operatorname{Ar} 17$ & He-like & $1 s$ & $6 P$ & & & $\left(1 P^{\star}\right) j=1.0$ & $-1 s 2$ & \\
\hline Ar17 & He-like & 15 & $6 P$ & & & $\left(1 p^{*}\right) j=1.0$ & $-1 s 2$ & \\
\hline A 17 & He-like & Is & $6 P$ & & & $\left(1 p^{*}\right) j=1.0$ & $-1 s 2$ & \\
\hline$A \times 17$ & He-like & $1 s$ & $6 P$ & & & $\left(1 P^{\star}\right) j=1.0$ & $-1 s 2$ & \\
\hline $\mathrm{A} \times 17$ & He-like & $1 \mathrm{~s}$ & $5 P$ & & & $\left(1 P^{*}\right) j=1.0$ & $-1 s 2$ & \\
\hline Ar17 & He-like & $1 S$ & $5 P$ & & & $\left(1 P^{*}\right) j=1.0$ & $-1 s 2$ & \\
\hline$A=17$ & He-like & 15 & $5 P$ & & & $(1 \mathrm{D} *) j=1.0$ & $-1 \$ 2$ & \\
\hline $\mathrm{A} \simeq 17$ & He-like & $1 \mathrm{~S}$ & $5 P$ & & & $\left(1 P^{*}\right) j=1.0$ & $-1 s 2$ & \\
\hline Ar17 & He-1ike & 15 & $5 P$ & & & $\left(1 P^{*}\right) j=1.0$ & $-1 s 2$ & \\
\hline A 17 & He-like & $1 \mathrm{~S}$ & $5 P$ & & & $\left(1 P^{*}\right) j=1.0$ & $-1 s 2$ & \\
\hline Ar 17 & He-like & is & $4 \mathrm{P}$ & & & $\left(1 p^{\star}\right) j=1.0$ & $-1 s 2$ & \\
\hline Ar17 & He-like & is & $4 P$ & & & $\left(1 P^{*}\right) j=1.0$ & $-1 s 2$ & \\
\hline Ar17 & He-like & $1 \mathrm{~S}$ & $4 P$ & & & $\left(1 P^{*}\right) j=1.0$ & $-1 s 2$ & \\
\hline Ar17 & He-like & $1 \mathrm{~S}$ & $4 \mathrm{P}$ & & & $\left(1 P^{*}\right) j=1.0$ & $-1 S 2$ & \\
\hline & He-like & 15 & $3 P$ & & & $\left(1 p^{*}\right) j=1.0$ & $-1 s 2$ & \\
\hline Ar 17 & He-like & $1 \mathrm{~s}$ & $3 P$ & & & $\left(3 P^{*}\right) j=1.0$ & $-1 s 2$ & \\
\hline Ar17 & He-like & $1 \mathrm{~s}$ & $2 P$ & & & $\left(1 p^{*}\right) j=1.0$ & $-1 s 2$ & \\
\hline Ar17 & He-like & is & $2 P$ & & & 1.0 & 152 & \\
\hline
\end{tabular}

\begin{tabular}{|c|c|c|}
\hline$\left.P^{*}\right) j=1.5$ & 3.992 & topaz 002 \\
\hline$(2 s) j=.5$ & 4.005 & LiF 200 \\
\hline$(2 S) j=.5$ & 4.005 & Al 200 \\
\hline (S) $j=.5$ & 4.005 & quartz 200 \\
\hline$S j j=.5$ & 4.005 & topaz 002 \\
\hline$j j=.5$ & 4.005 & quartz 100 \\
\hline *) $j=.5$ & 4.010 & LiF 200 \\
\hline$j=.5$ & 4.010 & Al 200 \\
\hline$j=.5$ & 4.010 & quartz 200 \\
\hline$j=.5$ & 4.010 & topaz 002 \\
\hline$j=.5$ & 4.010 & quartz 100 \\
\hline$\left.P^{*}\right) j=1.5$ & 4.012 & LiF 200 \\
\hline$\left.Q^{\star}\right) j=1.5$ & 4.012 & Al 200 \\
\hline$j=1.5$ & 4.012 & quartz 200 \\
\hline$j j=1.5$ & 4.012 & topaz 002 \\
\hline$j=1.5$ & 4.012 & quartz 100 \\
\hline$j j=1.5$ & 4.014 & LiF 200 \\
\hline$j=1.5$ & 4.014 & Al 200 \\
\hline$j=1.5$ & 4.014 & quartz 200 \\
\hline$j=1.5$ & 4.014 & topaz 002 \\
\hline$j=1.5$ & 4.014 & quartz 100 \\
\hline$j=.5$ & 4.015 & LiF 200 \\
\hline$j=.5$ & 4.015 & Al 200 \\
\hline$j=.5$ & 4.015 & quartz 200 \\
\hline$j j=.5$ & 4.015 & topaz 002 \\
\hline$j j=.5$ & 4.015 & quartz 100 \\
\hline$j j=.0$ & 3.095 & Si 111 \\
\hline$j j=.0$ & 3.095 & sylvite 200 \\
\hline$j=.0$ & 3.095 & Eluorite 111 \\
\hline$j=.0$ & 3.095 & Ge 111 \\
\hline$j=.0$ & 3.095 & $\mathrm{KBr} 200$ \\
\hline$j=.0$ & 3.095 & beryl 100 \\
\hline$j=.0$ & 3.128 & Si 111 \\
\hline$j j=.0$ & 3.128 & sylvite 200 \\
\hline$j=.0$ & 3.128 & Eluorite 111 \\
\hline$j=.0$ & 3.128 & Ge 111 \\
\hline$j j=.0$ & 3.128 & $\mathrm{KBr} 200$ \\
\hline$j j=.0$ & 3.128 & beryl 100 \\
\hline$j=.0$ & 3.200 & Ge 111 \\
\hline .0 & 3.200 & $\mathrm{KBr} 200$ \\
\hline$j=.0$ & 3.200 & quartz 101 \\
\hline$j=.0$ & 3.200 & graphite 002 \\
\hline .0 & 3.365 & $\mathrm{ADP} 101$ \\
\hline .0 & 3.370 & ADP 101 \\
\hline .0 & 3.948 & Ge 220 \\
\hline .0 & 3.948 & LiF 200 \\
\hline
\end{tabular}

$8.374 \quad 72.445$

4.02784 .008

$4.048 \quad 81.641$

$4.246 \quad 70.603$

$8.374 \quad 73.045$

8.51270 .224

$4.027 \quad 84.734$

$4.048 \quad 82.143$

$4.246 \quad 70.807$

$8.374 \quad 73.281$

8.51270 .424

$4.027 \quad 85.053$

$4.048 \quad 82.353$

$4.246 \quad 70.890$

$8.374 \quad 73.376$

8.51270 .505

$4.027 \quad 85.395$

$4.048 \quad 82.569$

$4.246 \quad 70.972$

$8.374 \quad 73.472$

$8.512 \quad 70.586$

$4.027 \quad 85.576$

$4.048 \quad 82.679$

$4.246 \quad 71.014$

8.37473 .520

8.51270 .626

6.27180 .781

$6.292 \quad 79.669$

$6.308 \quad 78.900$

$6.532 \quad 71.377$

$\begin{array}{ll}6.584 & 70.078\end{array}$

$15.954 \quad 75.925$

6.27186 .036

$6.292 \quad 83.868$

$6.308 \quad 82.638$

6.53273 .285

$6.584 \quad 71.839$

$15.954 \quad 78.614$

6.53278 .462

6.58476 .423

$6.687 \quad 73.153$

$6.696 \quad 72.900$

$10.640 \quad 71.582$

$10.640 \quad 71.840$

$4.000 \quad 80.751$

$4.027 \quad 78.632$ 


\begin{tabular}{|c|c|c|c|c|c|}
\hline Ar17 & He-like & 1s & $2 \mathrm{P}$ & $\left(1 P^{\star}\right) j=1.0$ & $-1 \$ 2$ \\
\hline Arl7 & He-like & IS & $2 P$ & $\left(1 P^{*}\right) j=1.0$ & $-1 S 2$ \\
\hline $\operatorname{Ar} 17$ & He-like & is & $2 \mathrm{P}$ & $\left(1 P^{\star}\right) j=1.0$ & $-1 s 2$ \\
\hline $\operatorname{Ar} 17$ & He like & 1S & $2 P$ & $\left(1 P^{*}\right) j=1.0$ & $-1 s 2$ \\
\hline Arl7 & He-like & 1s & $2 \mathrm{P}$ & $\left(3 p^{*}\right) j=1.0$ & $-1 s 2$ \\
\hline Ar 17 & He-like & is & $2 P$ & $\left(3 P^{*}\right) j=1.0$ & $-1 s 2$ \\
\hline$A \times 17$ & $\mathrm{He}-1 \mathrm{ike}$ & 15 & $2 P$ & $\left(3 P^{*}\right) j=1.0$ & $-1 S 2$ \\
\hline Ar 17 & He-like & is & $2 \mathrm{P}$ & $\left(3 P^{\star}\right) j=1.0$ & $-1 S 2$ \\
\hline Ar 17 & He-like & is & $2 \mathrm{P}$ & $\left(3 P^{\star}\right) j=1.0$ & $-1 S 2$ \\
\hline Ar17 & He-like & is & $2 P$ & $\left(3 P^{*}\right) j=1.0$ & $-1 s 2$ \\
\hline Ar17 & He-like & 1S & $2 S$ & $(3 s) j=1.0$ & $-1 S 2$ \\
\hline A 17 & He-like & IS & $2 S$ & $(3 s) j=1.0$ & $-1 s 2$ \\
\hline Ar 17 & He-like & is & $2 S$ & $(3 S) j=1.0$ & -152 \\
\hline Ar17 & He-like & Is & $2 S$ & (3s) $j=1.0$ & -152 \\
\hline Ar17 & He-like & is & $2 S$ & $(3 s) j=1.0$ & $-1 s 2$ \\
\hline Ar18 & H -like & $10 \mathrm{P}$ & & $\left(2 P^{\star}\right) j=1.5$ & -15 \\
\hline Ar18 & H-like & $10 P$ & & $\left(2 P^{*}\right) j=1.5$ & $-1 s$ \\
\hline Ar18 & H -like & $10 \mathrm{P}$ & & $\left(2 P^{*}\right) j=1.5$ & $-1 s$ \\
\hline Ar18 & $\mathrm{H}$-like & $10 P$ & & $\left(2 P^{*}\right) j=1.5$ & $-1 s$ \\
\hline Ar18 & H -like & $9 P$ & & $\left(2 P^{*}\right) j=1.5$ & $-1 S$ \\
\hline Ar18 & H -like & 98 & & $\left(2 P^{\star}\right) j=1.5$ & $-1 s$ \\
\hline $\operatorname{Ar} 18$ & $\mathrm{H}-1 \mathrm{ike}$ & $9 P$ & & $\left(2 P^{*}\right) j=1.5$ & $-1 S$ \\
\hline Ar 18 & H -like & $9 P$ & & $\left(2 P^{\star}\right) j=1.5$ & $-1 s$ \\
\hline Ar18 & $\mathrm{H}-1 \mathrm{ike}$ & $8 P$ & & $\left\langle 2 P^{\star}\right\rangle j=I .5$ & -15 \\
\hline $\operatorname{Ar} 18$ & H -like & $8 P$ & & $\left(2 \mathrm{P}^{\star}\right) j=1.5$ & $-1 S$ \\
\hline Ar18 & H-like & $8 \mathrm{P}$ & & $\left(2 P^{*}\right) j=1.5$ & -15 \\
\hline Ar 18 & H -like & $7 P$ & & $\left(2 P^{*}\right) j=1.5$ & $-1 S$ \\
\hline Ar 18 & H -like & $7 P$ & & $\left(2 P^{*}\right) j=1.5$ & $-1 s$ \\
\hline Ar18 & H -like & $7 P$ & & $\left(2 \mathrm{P}^{*}\right) j=1.5$ & $-1 s$ \\
\hline$A=18$ & H -like & $7 \mathrm{P}$ & & $\left(2 P^{*}\right) j=1.5$ & $-1 s$ \\
\hline Aris & H-like & $7 P$ & & $\left(2 P^{*}\right) j=1.5$ & $-1 S$ \\
\hline Ar 18 & H -like & $6 P$ & & $\left(2 P^{*}\right) j=1.5$ & $-1 S$ \\
\hline Ar18 & H-like & $6 \mathrm{P}$ & & $\left(2 P^{\star}\right) j=1.5$ & $-1 S$ \\
\hline Ar18 & H-like & $6 \mathrm{P}$ & & $\left(2 P^{*}\right) j=1.5$ & $-1 S$ \\
\hline Ar18 & $\mathrm{H}$-like & $6 \mathrm{P}$ & & $\left(2 P^{*}\right) j=1.5$ & -15 \\
\hline $\operatorname{Ar} 18$ & H -like & $6 \mathrm{P}$ & & $\left(2 P^{*}\right) j=1.5$ & $-1 S$ \\
\hline $\operatorname{Ar} 18$ & H-like & $5 P$ & & $\left(2 \mathrm{P}^{*}\right) j=1.5$ & -15 \\
\hline Ar 18 & $\mathrm{H}$-like & $5 P$ & & $\left(2 \mathrm{P}^{\star}\right) j=1.5$ & $-1 S$ \\
\hline $\operatorname{Ar} 18$ & $\mathrm{H}$-like & $5 P$ & & $\left(2 P^{\star}\right) j=1.5$ & $-1 s$ \\
\hline $\operatorname{Ar} 18$ & H -like & $5 \mathrm{P}$ & & $\left(2 \mathrm{P}^{\star}\right) j=1.5$ & $-1 s$ \\
\hline Arl8 & H-like & $5 \mathrm{P}$ & & $\left(2 P^{*}\right) j=1.5$ & $-1 s$ \\
\hline Ar 18 & H -like & $5 P$ & & $\left(2 P^{*}\right) j=.5$ & $-1 S$ \\
\hline Ar18 & $\mathrm{H}-1 \mathrm{ike}$ & $5 P$ & & $\left(2 \mathrm{P}^{\star}\right) j=.5$ & -15 \\
\hline Ar 18 & H-like & $5 \mathrm{P}$ & & $\left(2 \mathrm{P}^{*}\right) j=.5$ & $-1 s$ \\
\hline Ar18 & H -like & $5 P$ & & $\left(2 \mathrm{P}^{\star}\right) j=.5$ & - IS \\
\hline Ar18 & $\mathrm{H}$-like & $5 P$ & & $\left(2 P^{\star}\right) j=.5$ & $-1 S$ \\
\hline
\end{tabular}

\begin{tabular}{|c|c|c|c|c|}
\hline (IS & $j=$ & .0 & 3.948 & Al 200 \\
\hline (1S & $j j=$ & .0 & 3.948 & topaz 002 \\
\hline (IS & $j=$ & .0 & 3.948 & beryl 100 \\
\hline (1s & $j=$ & .0 & 3.948 & mica 002 \\
\hline (1S & $j j=$ & .0 & 3.969 & Ge 220 \\
\hline (1S & $j=$ & .0 & 3.969 & LiF 200 \\
\hline (1S & $j j=$ & .0 & 3.969 & Al 200 \\
\hline IIS & $j j=$ & .0 & 3.969 & topaz 002 \\
\hline (1S & $j=$ & .0 & 3.969 & beryl 100 \\
\hline (1s & $j j=$ & .0 & 3.969 & mica 002 \\
\hline IS & $j=$ & .0 & 3.994 & Ge 220 \\
\hline $1 \mathrm{~s}$ & $h j=$ & .0 & 3.994 & LiF 200 \\
\hline (1S & $j=$ & .0 & 3.994 & A. 200 \\
\hline (1s & $j j=$ & .0 & 3.994 & quartz 200 \\
\hline (1S & $j j=$ & .0 & 3.994 & topaz 002 \\
\hline$(2 \mathrm{~S}$ & $y j=$ & .5 & 2.829 & LIF 220 \\
\hline $12 S$ & $j=$ & .5 & 2.829 & quartz 100 \\
\hline$(2 S$ & $j j=$ & .5 & 2.829 & PET 002 \\
\hline $12 \mathrm{~S}$ & $j j=$ & .5 & 2.829 & EDT 020 \\
\hline (2S & $j=$ & .5 & 2.836 & LiF 220 \\
\hline$\langle 2 S$ & $j=$ & .5 & 2.836 & quartz 100 \\
\hline (2S & $j=$ & .5 & 2.836 & PET 002 \\
\hline $12 \mathrm{~s}$ & $j=$ & .5 & 2.836 & EDT 020 \\
\hline $12 S$ & $j=$ & .5 & 2.845 & $\operatorname{LiF} 220$ \\
\hline$(2 S$ & $j=$ & .5 & 2.845 & PET 002 \\
\hline $12 S$ & $j=$ & .5 & 2.845 & EDI 020 \\
\hline (2S & $j=$ & .5 & 2.859 & calcite 422 \\
\hline $12 S$ & $j=$ & .5 & 2.859 & calcite 200 \\
\hline $12 S$ & $j=$ & .5 & 2.859 & PET 002 \\
\hline (2S & $j=$ & .5 & 2.859 & EDT 020 \\
\hline $12 \mathrm{~S}$ & $j=$ & .5 & 2.859 & gypsum 020 \\
\hline (2S & $j=$ & .5 & 2.881 & calcite 422 \\
\hline $12 \mathrm{~S}$ & $j=$ & .5 & 2.881 & calcite 200 \\
\hline (2S & $j j=$ & .5 & 2.881 & PET 002 \\
\hline $12 S$ & $j j=$ & .5 & 2.881 & EDT 020 \\
\hline $12 S$ & $j=$ & .5 & 2.881 & gypsum 020 \\
\hline (2S & $j=$ & .5 & 2.917 & calcite 422 \\
\hline $12 S$ & $j=$ & .5 & 2.917 & quartz 211 \\
\hline $12 S$ & $j=$ & .5 & 2.917 & calcite 200 \\
\hline (2S & $j j=$ & .5 & 2.917 & EDT 020 \\
\hline $12 S$ & $j=$ & .5 & 2.917 & gypsum 020 \\
\hline $12 S$ & $j=$ & .5 & 2.918 & calcite 422 \\
\hline $12 \mathrm{~S}$ & $j=$ & .5 & 2.918 & quartz 211 \\
\hline $12 S$ & $j=$ & .5 & 2.918 & calcite 200 \\
\hline 12 & $j=$ & .5 & 2.918 & EDT 020 \\
\hline (2S & $j=$ & .5 & 2.918 & gypsum 020 \\
\hline
\end{tabular}

$\begin{array}{rr}4.048 & 77.238 \\ 8.374 & 70.548 \\ 15.954 & 81.828 \\ 19.942 & 81.838 \\ 4.000 & 82.862 \\ 4.027 & 80.264 \\ 4.048 & 78.662 \\ 8.374 & 71.430 \\ 15.954 & 84.332 \\ 19.942 & 84.347 \\ 4.000 & 86.861 \\ 4.027 & 82.660 \\ 4.048 & 80.631 \\ 4.246 & 70.161 \\ 8.374 & 72.536 \\ 2.848 & 83.378 \\ 8.512 & 85.608 \\ 8.742 & 76.127 \\ 8.808 & 74.484 \\ 2.848 & 84.738 \\ 8.512 & 88.243 \\ 8.742 & 76.713 \\ 8.808 & 75.003 \\ 2.848 & 87.370 \\ 8.742 & 77.507 \\ 8.808 & 75.698 \\ 3.034 & 70.445 \\ 6.071 & 70.365 \\ 8.742 & 78.850 \\ 8.808 & 76.849 \\ 15.185 & 70.286 \\ 3.034 & 71.727 \\ 6.071 & 71.641 \\ 8.742 & 81.369 \\ 8.808 & 78.892 \\ 15.185 & 71.556 \\ 3.034 & 74.036 \\ 3.082 & 71.167 \\ 6.071 & 73.938 \\ 8.808 & 83.478 \\ 15.185 & 73.840 \\ 3.034 & 74.105 \\ 3.082 & 71.225 \\ 6.071 & 74.006 \\ 15.808 & 83.652 \\ & 73.908\end{array}$ 


\begin{tabular}{|c|c|c|c|c|}
\hline Ar18 & H -like & $4 \mathrm{P}$ & $\left(2 P^{*}\right) j=1.5$ & - Is \\
\hline Ar18 & H -like & $4 P$ & $\left(2 P^{\star}\right) j=1.5$ & $-1 s$ \\
\hline$A \times 18$ & H -like & $4 \mathrm{P}$ & $\left(2 P^{*}\right) j=1.5$ & $-1 s$ \\
\hline Ar18 & H -like & $4 P$ & $\left(2 \mathrm{P}^{\star}\right) j=1.5$ & $-1 S$ \\
\hline Ar18 & H -like & $4 \mathrm{P}$ & $\left(2 P^{\star}\right) j=1.5$ & $-1 S$ \\
\hline A 18 & H -like & $4 P$ & $\left(2 \mathrm{P}^{\star}\right) j=1.5$ & $-1 S$ \\
\hline Ar 18 & H -like & $4 P$ & $\left(2 P^{\star}\right) j=1.5$ & $-1 s$ \\
\hline Ar18 & H -like & $4 \mathrm{P}$ & $\left(2 \mathrm{P}^{\star}\right) j=.5$ & $-1 S$ \\
\hline Ax18 & H -like & $4 P$ & $\left(2 P^{*}\right) j=.5$ & $-1 s$ \\
\hline$A \times 18$ & H -like & $4 P$ & $\left(2 \mathrm{P}^{*}\right) j=.5$ & -15 \\
\hline Ar18 & H -like & $4 \mathrm{P}$ & $\left(2 P^{\star}\right) j=.5$ & -15 \\
\hline Ar18 & H -like & $4 P$ & $\left(2 \mathrm{P}^{\star}\right) j=.5$ & $-1 s$ \\
\hline Ar18 & H -like & $4 \mathrm{P}$ & $\left(2 P^{\star}\right) j=.5$ & -15 \\
\hline Ax 18 & H -like & $4 \mathrm{~F}$ & $\left(2 \mathrm{P}^{\star}\right) j=.5$ & -15 \\
\hline$A \times 18$ & $\mathrm{H}$-like & $3 P$ & $\left(2 \mathrm{P}^{\star}\right) j=1.5$ & $-1 S$ \\
\hline Ar18 & H -like & $3 P$ & $\left(2 P^{\star}\right) j=1.5$ & -15 \\
\hline Ar18 & $\mathrm{H}$-like & $3 P$ & $\left(2 P^{\star}\right) j=1.5$ & -15 \\
\hline Ar18 & H -like & $3 P$ & $\left(2 \mathrm{P}^{*}\right) j=1.5$ & -15 \\
\hline Ar 18 & H -Iike & $3 P$ & $\left(2 P^{*}\right) j=1.5$ & $-1 S$ \\
\hline$A=18$ & H-like & $3 P$ & $\left(2 P^{*}\right) j=1.5$ & $-1 S$ \\
\hline$A \div 18$ & H -like & $3 P$ & $\left(2 P^{\star}\right) j=.5$ & -15 \\
\hline Ar18 & H -like & $3 \mathrm{P}$ & $\left(2 P^{\star}\right) j=.5$ & -15 \\
\hline A 18 & H -like & $3 P$ & $\left(2 P^{*}\right) j=.5$ & $-1 s$ \\
\hline Ar18 & $\mathrm{H}-1$ ike & $3 \mathrm{P}$ & $\left(2 P^{\star}\right) j=.5$ & -15 \\
\hline Ax18 & H-like & $3 P$ & $\left(2 P^{\star}\right) j=.5$ & $-1 S$ \\
\hline A 18 & $H$-like & $3 P$ & $\left(2 P^{*}\right) j=.5$ & $-1 S$ \\
\hline$A \geq 18$ & H-like & $2 P$ & $\left(2 P^{\star}\right) j=1.5$ & $-1 s$ \\
\hline Ar18 & H -like & $2 P$ & $\left(2 P^{\star}\right) j=1.5$ & $-1 s$ \\
\hline Ar18 & H -like & $2 P$ & $\langle 2 P *\rangle j=1.5$ & -15 \\
\hline Ar18 & H -like & $2 \mathrm{P}^{\circ}$ & $\left(2 P^{\star}\right) j=1.5$ & -15 \\
\hline$A=18$ & H -like & $2 \mathrm{P}$ & $\left(2 P^{\star}\right) j=.5$ & -15 \\
\hline Ar 18 & H -like & $2 \mathrm{P}$ & $\left(2 P^{*}\right) j=.5$ & -15 \\
\hline Ar 18 & H -like & $2 P$ & $\left(2 P^{*}\right) j=.5$ & $-1 s$ \\
\hline Ar18 & H -like & $2 \mathrm{P}$ & $\left(2 P^{\star}\right) j=.5$ & $-1 S$ \\
\hline Ar18 & H -like & $7 P$ & $\left(2 P^{\star}\right) j=1.5$ & -25 \\
\hline$A \simeq 18$ & H -like & $7 D$ & $(2 D) j=2.5$ & $-2 P$ \\
\hline Ar18 & it -like & $6 P$ & $\left(2 P^{\star}\right) j=1.5$ & $-2 S$ \\
\hline Ar18 & H -like & $6 \mathrm{P}$ & $(2 P \star) j=1.5$ & $-2 s$ \\
\hline Ar 18 & H -like & $6 \mathrm{P}$ & $\left(2 P^{*}\right) j=1.5$ & $-2 S$ \\
\hline Ar18 & H -like & $6 \mathrm{D}$ & (2D) $j=2.5$ & \\
\hline Ar18 & H -like & $6 D$ & (2D) $j=2.5$ & \\
\hline Ar18 & H -like & $6 D$ & $(2 D) j=2.5$ & $-2 P$ \\
\hline Ar18 & H -like & $5 P$ & $\left(2 P^{\star}\right) j=1.5$ & $-2 S$ \\
\hline $\operatorname{Ar} 18$ & H -like & $4 \mathrm{P}$ & $\left(2 P^{\star}\right) j=1.5$ & $-2 S$ \\
\hline Ar 18 & H -like & $4 \mathrm{D}$ & $(2 D) j=2.5$ & $-2 P$ \\
\hline
\end{tabular}

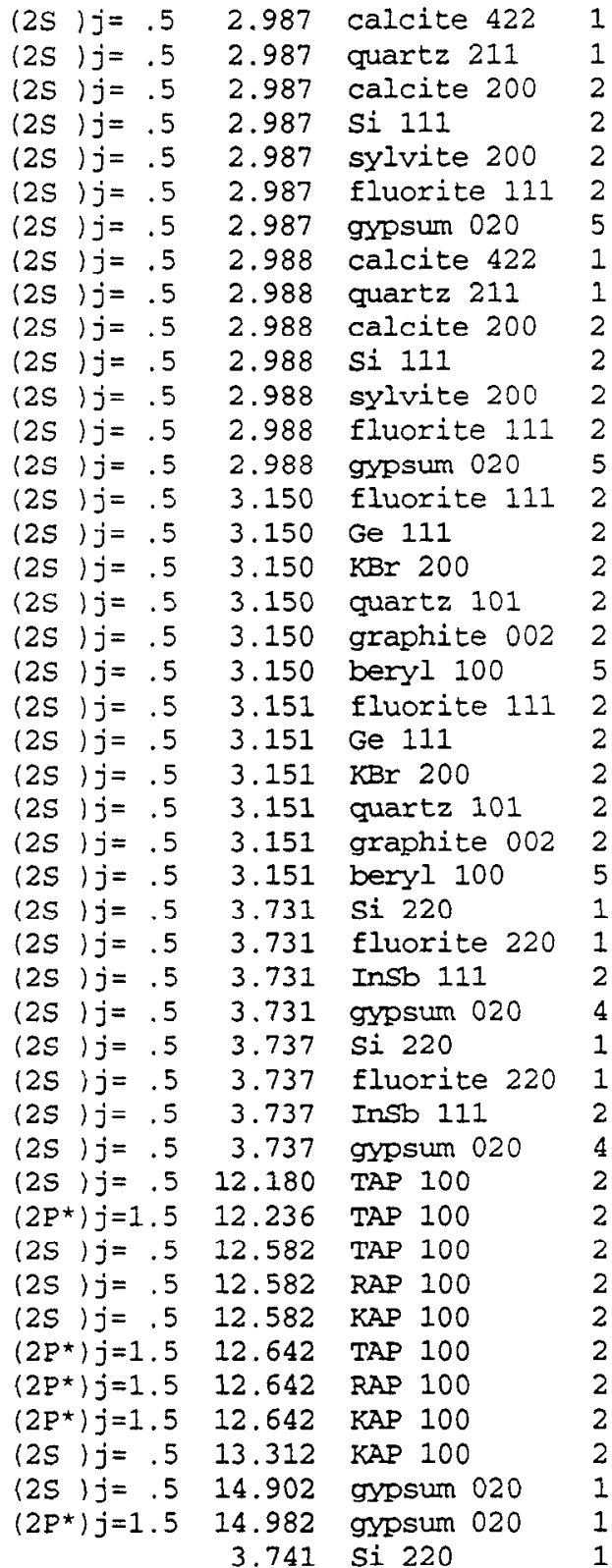

$3.034 \quad 79.902$ $3.082 \quad 75.737$

$6.071 \quad 79.744$

$6.271 \quad 72.296$

$6.292 \quad 71.706$

$6.308 \quad 71.272$

$\begin{array}{lll}15.185 & 79.589\end{array}$

$3.034 \quad 80.010$

$3.082 \quad 75.813$

6.07179 .851

$6.271 \quad 72.356$

$6.292 \quad 71.764$

$6.308 \quad 71.328$

$15.185 \quad 79.694$

$6.308 \quad 87.114$

6.53274 .684

6.58473 .110

$6.687 \quad 70.412$

$6.696 \quad 70.197$

$15.954 \quad 80.828$

$6.308 \quad 87.501$

6.53274 .750

6.58473 .170

$6.587 \quad 70.463$

$6.696 \quad 70.247$

$15.954 \quad 80.941$

$3.840 \quad 76.316$

$3.862 \quad 75.034$

$7.481 \quad 85.916$

$15.185 \quad 79.362$

$3.840 \quad 76.700$

$3.862 \quad 75.383$

$\begin{array}{ll}7.481 & 87.521\end{array}$

$15.185 \quad 79.864$

$25.763 \quad 71.00$

$25.763 \quad 71.785$

$25.763 \quad 77.621$

$26.116 \quad 74.482$

$26.634 \quad 70.875$

$25.763 \quad 78.934$

$26.116 \quad 75.499$

$26.634 \quad 71.679$

$26.634 \quad 88.430$

$15.185 \quad 78.921$

$15.185 \quad 80.621$

$3.840 \quad 76.995$ 
3.741 fluorite $220 \quad 1$ K-alpha (1)

K $14 \mathrm{C}$-1ike $2 S 22 \mathrm{P}\left(2 \mathrm{P}^{\star}\right) 3 \mathrm{D} \quad\left(3 \mathrm{P}^{\star}\right) j=.0-2 S 22 \mathrm{P} 2$ $\mathrm{K} 14 \mathrm{C}$-like 2S2 $2 \mathrm{P}\left(2 \mathrm{P}^{*}\right) 3 \mathrm{D}\left(3 \mathrm{P}^{*}\right) \mathrm{j}=.0-2 \mathrm{~S} 22 \mathrm{P} 2$ K 14 C-like 2S2 $2 P^{2}\left(2 P^{*}\right) 3 D\left(3 P^{*}\right) j=.0-2 S 22 P^{*}$ $K 14 C$-like $2 S 22 P\left(2 P^{\star}\right) 3 D \quad\left(3 P^{\star}\right) j=1.0-2 S 22 P^{*}$ $\mathrm{K} 14 \mathrm{C}-1 \mathrm{ike} 2 \mathrm{~S} 2 \mathrm{PP}\left(2 \mathrm{P}^{*}\right) 3 \mathrm{D}\left(3 \mathrm{P}^{*}\right) \mathrm{j}=1.0-2 \mathrm{~S} 22 \mathrm{P} 2$ K 14 C-like 2S2 2P (2P*) 3D (3P*) j=1.0 - 2S2 2P2 K $14 C$-iike 2S2 2P (2P*) 3D $\left(3 P^{\star}\right) j=2.0-2 S 22 P^{*}$ K $14 \mathrm{C}$-like $2 S 22 \mathrm{P}\left(2 P^{*}\right) 3 \mathrm{D}\left(3 \mathrm{P}^{*}\right) j=2.0-2 \mathrm{~S} 2 \mathrm{PP} 2$ K 14 C-like 2S2 $2 \mathrm{P}\left(2 P^{*}\right) 3 D(3 P *) j=2.0-2 S 22 P 2$ K 14 -like $2 S 22 P$ ( $\left.2 P^{*}\right) 3 D \quad\left(3 D^{*}\right) j=1.0-2 S 22 P^{*}$ K 14 C-like 2S2 $2 P\left(2 P^{*}\right) 3 D\left(3 D^{*}\right) j=1.0-2 S 22 P 2$ K 14 C-like 2S2 2P ( 2P*) 3D $\left(3 D^{*}\right) j=1.0-2 S 22 P 2$ $K 14 C-1 i k e$ 2S2 $2 P\left(2 P^{*}\right) 3 D\left(3 P^{*}\right) j=2.0-2 S 22 P 2$ $\mathrm{K} 14 \mathrm{C}$-like $2 \mathrm{~S} 2 \mathrm{PP}\left(2 \mathrm{P}^{*}\right) 3 \mathrm{D}\left(3 \mathrm{P}^{*}\right) \mathrm{j}=2.0-2 \mathrm{~S} 22 \mathrm{P} 2$ K 14 C-like $2 S 22 P\left(2 P^{*}\right) 3 D\left(3 P^{*}\right) j=2.0-2 S 22 P 2$ $K 14 C$-like $2 S 22 P\left(2 P^{*}\right) 3 D\left(3 D^{*}\right) j=2.0-2 S 22 P 2$ K $14 C$-like $2 S 22 P\left(2 P^{*}\right) 3 D\left(3 D^{\star}\right) j=2.0-2 S 22 P 2$ K $14 C$-like $2 S 22 P\left(2 P^{*}\right) 3 D\left(3 D^{*}\right) j=2.0-2 S 22 P 2$ K 14 C-like $2 S 22 P\left(2 P^{*}\right) 3 D\left(3 D^{*}\right) j=3.0-2 S 22 P 2$ K 14 C-1ike 2S2 2P (2P*) 3D (3D*) j=3.0 - 2S2 2P2 K 14 C-like 2S2 2P (2P*) 3D (3D*) $j=3.0-2 S 22 P 2$ K 14 C-like $2 S 22 P\left(2 P^{*}\right) 3 D\left(3 D^{*}\right) j=1.0-2 S 22 P 2$

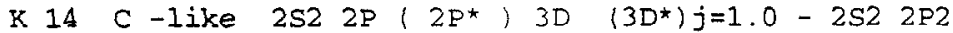
K $14 \mathrm{C}$-like $2 S 22 \mathrm{P}\left(2 \mathrm{P}^{*}\right) 3 \mathrm{D}\left(3 \mathrm{D}^{*}\right) j=2.0-2 S 22 \mathrm{P} 2$ $\mathrm{K} 14 \mathrm{C}$-like $2 S_{2} 2 \mathrm{P}\left(2 \mathrm{P}^{\star}\right) 3 D\left(3 D^{\star}\right) j=2.0-2 S 22 \mathrm{P}^{*}$ K 14 C-like $2 S 22 P\left(2 P^{*}\right) 3 D\left(1 P^{*}\right) j=1.0-2 S 22 P 2$ K $14 \mathrm{C}$-like 2S2 2P (2P*) 3D (1P*) j=1.0 - 2S2 2P2 $K 14$ C -like 2S2 $2 P\left(2 P^{*}\right) 3 D\left(1 F^{*}\right) j=3.0-2 S 22 P 2$ K 14 C-like $2 S 22 P\left(2 P^{*}\right) 3 D\left(1 F^{*}\right) j=3.0-2 S 22 P 2$ K $14 \mathrm{C}$-like 2S2 2P(2P*) $3 D\left(3 F^{*}\right) j=3.0-2 S 22 P 2$

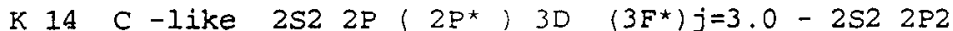
K $14 C$-like $2 S 22 P\left(2 P^{*}\right) 3 D\left(3 F^{*}\right) j=2.0-2 S 22 P 2$ $\mathrm{K} 14 \mathrm{C}$-like $2 \mathrm{S2} 2 \mathrm{P}\left(2 \mathrm{P}^{*}\right) 3 \mathrm{D}\left(3 \mathrm{~F}^{*}\right) j=2.0-2 \mathrm{~S} 22 \mathrm{P2}$ K 14 C-1ike 2S2 2P ( $\left.2 P^{*}\right) 3 D\left(3 D^{*}\right) j=2.0-2 S 22 P 2$ K 14 C-like 2S2 2P (2P*) 3D (1P*)j=1.0 - 2S2 2P2 K 14 C-like 2S2 $2 P\left(2 P^{\star}\right) 3 D\left(3 F^{\star}\right) j=2.0-2 S 22 P 2$

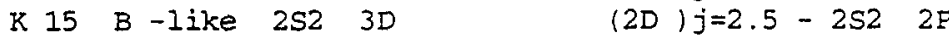
$\mathrm{K} 15$ B-like $2 \mathrm{~S}$ 2P (1P) $3 \mathrm{P}\left(2 \mathrm{D}^{*}\right) j=2.5-2 \mathrm{~S}$ 2P2 $K 15$ B-like $2 S$ 2P ( $1 P$ ) $3 D\left(2 D^{*}\right) j=2.5-2 S$ 2P2 $\mathrm{K} 15 \mathrm{~B}-1 \mathrm{kke} 2 \mathrm{~S} 2 \mathrm{P}$ (3P) $3 \mathrm{D}\left(2 F^{\star}\right) \mathrm{j}=3.5-2 \mathrm{~S} 2 \mathrm{P} 2$ $\mathrm{K} \cdot 15$ B -like $2 \mathrm{~S} 2 \mathrm{P}(3 \mathrm{P}) 3 \mathrm{D}\left(2 \mathrm{~F}^{*}\right) j=3.5-2 \mathrm{~S}$ 2P2 $K 15$ B-like $2 S$ 2P (3P) 3D $\left(2 F^{*}\right) j=2.5-2 S$ 2P2 $\mathrm{K} 15 \mathrm{~B}$-like $2 \mathrm{~S} 2 \mathrm{P}(3 \mathrm{P}), 3 \mathrm{D}\left(2 \mathrm{~F}^{\star}\right) \mathrm{j}=2.5-2 \mathrm{~S} 2 \mathrm{P} 2$ K 15 -like $2 S$ 2P (3P) $3 D\left(2 D^{*}\right) j=2.5-2 S$ 2P2

\subsection{GYPsum 020}

(3P ) $j=1.0 \quad 25.583 \quad$ TAP 100

(3P) $j=1.0 \quad 25.583$ RAP 100

(3P) $j=1.0 \quad 25.583 \quad \mathrm{KAP} 100$

(3P) $j=1.0 \quad 25.592$ TAP 100

(3P) $j=1.0 \quad 25.592 \quad$ RAP 100

(3P) $j=1.0 \quad 25.592 \quad$ KAP 100

(3P) $j=1$

(3P) $j=1.0 \quad 25.607 \quad \operatorname{RAP} 100$

(3P) $j=1.0 \quad 25.607 \quad \mathrm{KAP} 100$

(3P) $j=0$

(3P) $j=.0 \quad 25.696$ RAP 100

$(3 \mathrm{P}) j=.0 \quad 25.696 \mathrm{KAP} 100$

(3P $j=2.0 \quad 25.700$ TAP 100

(3P) $j=2.0 \quad 25.700 \quad$ RAP 100

(3P) $j=2.0 \quad 25.700 \quad$ KAP 100

(3P ) $j=1.0 \quad 25.726 \quad$ TAP 100

(3P) $j=1.0 \quad 25.726$ RAP 100

(3P) $j=2.0 \quad 25.750 \quad$ TAP 100

(3P) $j=2.0 \quad 25.750$ RAP 100

(3P) $j=2.0 \quad 25.750 \quad$ KAP 100

(3P) $j=1.0 \quad 25.784$ RAP 100

(3P) $j=1.0 \quad 25.784$ KAP 100

(3P) $j=2.0 \quad 25.818$ RAP 100

(3P) $j=2.0 \quad 25.818 \quad$ KAP 100

(1D) $j=2.0 \quad 25.887 \quad$ RAP 100

(ID) $j=2.0 \quad 25.887 \quad \mathrm{KAP} 100$

(ID) $j=2.0 \quad 25.909$ RAP 100

(1D) $j=2.0 \quad 25.909$ KAP 100

(3P) $j=2.0 \quad 26.018 \quad$ RAP 100

(3P) $j=2.0 \quad 26.018 \quad \mathrm{KAP} 100$

(3P) $j=2.0 \quad 26.107 \quad$ RAP 100

(3P) $j=2.0 \quad 26.107 \quad$ KAP 100

(ID) $j=2.0 \quad 26.284 \quad \mathrm{KAP} 100$

(IS ) $j=.026 .456$ KAP 100

(ID) $j=2.0 \quad 26.591 \quad K A P \quad 100$

$\left(2 P^{*}\right) j=1.5 \quad 24.328 \quad$ TAP 100

(2P) $j=1.5 \quad 24.770 \quad$ TAP 100

(2P) $j=1.5 \quad 24.770 \quad$ RAP 100

(2D) $j=2.5 \quad 24.889$ TAP 100

(2D) $j=2.5 \quad 24.889 \quad$ RAP 100

(2D) $j=1.5 \quad 24.946 \quad$ TAP 100

(2D) $j=1.5 \quad 24.946 \quad$ RAP 100

(2D) $j=2.5 \quad 25.265$ TAP 100
(3P) $j=1.0 \quad 25.726 \quad \mathrm{KAP} 100$
$3.862 \quad 75.650$

$15.185 \quad 80.257$

$25.763 \quad 83.223$

$26.116 \quad 78.405$

$26.634 \quad 73.850$

$25.763 \quad 83.395$

$26.116 \quad 78.503$

$26.634 \quad 73.920$

$25.763 \quad 83.692$

$26.116 \quad 78.669$

$26.634 \quad 74.037$

$25.763 \quad 85.867$

$26.116 \quad 79.711$

$26.634 \quad 74.749$

$25.763 \quad 85.992$

$26.116 \quad 79.760$

$26.634 \quad 74.782$

$25.763 \quad 86.929$

$26.116 \quad 80.086$

$26.634 \quad 74.996$

$25.763 \quad 88.180$

$26.116 \quad 80.396$

$26.634 \quad 75.197$

$26.116 \quad 80.854$

$26.634 \quad 75.486$

$26.116 \quad 81.336$

$26.634 \quad 75.781$

$26.116 \quad 82.407$

$26.634 \quad 76.398$

$26.116 \quad 82.781$

$26.634 \quad 76.601$

$26.116 \quad 85.035$

$26.634 \quad 77.653$

$26.116 \quad 88.496$

$26.634 \quad 78.583$

$26.634 \quad 80.701$

$26.634 \quad 83.372$

$26.634 \quad 86.744$

$25.763 \quad 70.787$

$25.763 \quad 74.041$

$26.116 \quad 71.525$

$25.763 \quad 75.033$

$26.116 \quad 72.367$

$25.763 \quad 75.532$

$26.116 \quad 72.785$

$25.763 \quad 78.716$ 
$\mathrm{K} 15 \mathrm{~B}-\mathrm{like} 2 \mathrm{~S} 2 \mathrm{P}(3 \mathrm{P}) 3 \mathrm{D}\left(2 \mathrm{D}^{*}\right) \mathrm{j}=2.5-2 \mathrm{~S} 2 \mathrm{P} 2$ $K 15$ B-like $2 S$ 2P ( $3 P$ ) $3 D\left(2 D^{*}\right) j=2.5-2 S$ 2P2

$\mathrm{K} 16$ Be-like

K 16 Be-like

K 16 Be-like

K 17 Ii-like

K 17 Li-like $1 S$ 2P ( $\left.1 P^{*}\right)$ ) $3 S\left(2 P^{*}\right) j=1.5-1 S 23 S$

$\mathrm{K} 17$ Li-like 15 2P2

K 17 Li-like is $2 \mathrm{P} 2$

$\mathrm{K} 17$ Li-like $1 \mathrm{~S}$ 2P2

K 17 Li-like 15 2P2

$\mathrm{K} 17$ Li-like 1S 2P2

K 17 Li-1ike is 2P2

K 17 Li-like $1 S$ ( $2 S)$ (2S $2 \mathrm{P}\left(2 \mathrm{P}^{*}\right) j=1.5-1 \mathrm{~S} 22 \mathrm{~S}$

$K 17$ Li-like $1 S$ ( $2 S$ ) (2S $2 P\left(\left(2 P^{*}\right) j=1.5-1 S 22 S\right.$

K 17 Li-like 15 2P ( $\left.1 P^{*}\right)$ 3S (2P*) $j=1.5-1 S 23 D$

K 17 Li-like is $2 P\left(1 P^{*}\right)$ iS $\left(2 P^{*}\right) j=1.5-1 S 23 D$

K 17 Li-like 15 2P2

K 17 Li-like 1S 2P2

K 17 Li-like 1S 2P2

$\mathrm{K} 17$ Li-like 1S 2P2

K 17 Li-like is 2P2

$\mathrm{K} 17$ Li-like $1 \mathrm{~S}$ 2P2

$\mathrm{K} 17$ Li-like $1 \mathrm{~S} 2 \mathrm{P} 2$

K 17 Li-like 1S $2 \mathrm{P} 2$

K 17 Li-like 15 2P2

K 17 Li-like is $2 \mathrm{P} 2$

K 17 Li-like 1S 2P2

K 17 Li-like 1S 2P2

K 17 Li-like 1S2 $9 P$

K 17 Li-1ike 1S2 $9 P$

K 17 Li-like 1S2 9P

K 17 Li-like 1S2 $8 P$

K 17 Li-like is2 $8 \mathrm{P}$

K 17 Li-like 1S2 8P

K 17 Li-like 1S2 $7 P$

K 17 Li-like 1S2 7P

K 17 Li-like 1S2 9D

K 17 Li-like 1S2 9D

$\mathrm{K} 17$ Li-like 1S2 9D

K 17 Li-like 1S2 8D

K 17 Li-like 1S2 8D

K 17 Li-like 1S2 5D

K 17 Li-like 1S2 5D

K 17 Li-like 1S2 4 P
(2D $) j=2.5 \quad 25.265 \quad R A P 100$ (2D) $j=2.5 \quad 25.265$

$j j=.0 \quad 3.602$

$j=.0 \quad 3.602$

$j=.0$

2S $j j=.5$

(2s) $j=.5$

(2s) $j=.5$

$\left(2 P^{*}\right) j=.5$

$\left(2 P^{\star}\right) j=.5$

$\left(2 P^{*}\right) j=.5$

$\left(2 P^{*}\right) j=1$.

$\left(2 P^{\star}\right) j=1.5$

$\left(2 P^{\star}\right) j=1.5$

(2S) $j=.5$

(2S) $j=.5$

(2D) $j=2.5$

(2D) $j=2.5$

$\left(2 P^{\star}\right) j=1$.

$\left(2 \mathrm{P}^{*}\right) j=1.5$

$\left(2 P^{*}\right) j=$

$(2 P *) j=.5$

$\left(2 P^{*}\right) j=.5$

$\left(2 P^{\star}\right) j=.5$

$\left(2 P^{\star}\right) j=1.5$

$\left(2 P^{*}\right) j=1.5$

$\left(2 P^{*}\right) j=1.5$

$\left(2 P^{*}\right) j=1.5$

$\left(2 P^{*}\right) j=1.5$

(2S) $j=$.

(2s) $j=.5$

(2S) $j=.5$

(2S) $j=$

(2S) $j=$

12.744

(2S) $j=.5 \quad 12$.

(2S) $j=.5 \quad 12.996$

$\left(2 P^{\star}\right) j=.5 \quad 13.025$

$\left(2 P^{*}\right) j=.5 \quad 13.025$

$\left(2 P^{*}\right) j=1.5 \quad 13.080$

$\left(2 P^{\star}\right) j=.5 \quad 13.204$

$\left(2 P^{\star}\right) j=1.5 \quad 13.261$

$\left(2 P^{*}\right) j=.5 \quad 14.715$

$\left(2 P^{*}\right) j=1.5 \quad 14.776$

(2S) $j=.5 \quad 15.755$
KAP 100

quartz 112

Insb 111

sypsum 020

quartz 112

InSb 111

ADP 101

quartz 112

InSb 111

ADP 101

quartz 112

InSb 111

ADP 101

quartz 112

InSb 111

quartz 112

InSb 111

quartz 112

Insb 111

quartz 112

InSb 111

quartz 112

InSb 111

quartz 112

InSto 111

gypsum 020

quartz 112

InSb 111

gypsum 020

TAP 100

RAP 100

KAP 100

TAP 100

RAP 100

KAP 100

RAP 100

KAP 100

RAP 100

KAP 100

KAP 100

KAP 100

KAP 100

gypsum 020

gypsum 020

beryl 100
$26.116 \quad 75.333$

$26.634 \quad 71.550$

$3.636 \quad 82.158$

$\begin{array}{ll}7.481 & 74.360\end{array}$

$15.185 \quad 71.592$

$3.636 \quad 76.464$

$7.481 \quad 70.920$

$10.640 \quad 85.351$

$3.636 \quad 76.874$

$7.481 \quad 71.203$

$10.640 \quad 86.761$

$3.636 \quad 77.154$

$7.481 \quad 71.394$

$10.640 \quad 88.243$

$3.636 \quad 77.369$

$7.481 \quad 71.538$

$3.636 \quad 77.514$

$7.481 \quad 71.635$

$3.636 \quad 78.343$

$\begin{array}{ll}7.481 & 72.178\end{array}$

$3.636 \quad 78.500$

$7.481 \quad 72.278$

$3.636 \quad 78.659$

$7.481 \quad 72.379$

$3.636 \quad 78.984$

$7.481 \quad 72.583$

$15.185 \quad 70.075$

$3.636 \quad 80.680$

$7.481 \quad 73.583$

$15.185 \quad 70.934$

$25.763 \quad 77.497$

$26.116 \quad 74.384$

$26.634 \quad 70.797$

$25.763 \quad 81.621$

$26.116 \quad 77.410$

$26.634 \quad 73.131$

$26.116 \quad 84.414$

$26.634 \quad 77.394$

$26.116 \quad 85.926$

$26.634 \quad 77.979$

$26.634 \quad 79.174$

$26.634 \quad 82.531$

$26.634 \quad 84.744$

$15.185 \quad 75.708$

$15.185 \quad 76.672$

$15.954 \quad 80.941$

Page 31 


\begin{tabular}{|c|c|c|c|}
\hline K 17 & Ii-like & $1 S 2$ & $4 \mathrm{P}$ \\
\hline K 18 & He-like & 15 & $6 P$ \\
\hline K 18 & He-like & $1 S$ & $6 P$ \\
\hline K 18 & He-like & $1 S$ & $6 \mathrm{P}$ \\
\hline K 18 & He-Iike & 1s & $6 P$ \\
\hline K 18 & He-like & 15 & $6 P$ \\
\hline K 18 & He-Iike & 1S & $6 P$ \\
\hline 18 & He-like & IS & $6 P$ \\
\hline K 18 & He-like & $1 S$ & $5 P$ \\
\hline K 18 & He-like & is & $5 \mathrm{P}$ \\
\hline K 18 & He-like & 1S & $5 P$ \\
\hline K 18 & He-iike & 1S & $5 P$ \\
\hline K 18 & He-like & 1S & $5 P$ \\
\hline K 18 & He-like & 15 & $4 \mathrm{P}$ \\
\hline K 18 & He-like & 1s & $4 P$ \\
\hline K 18 & He-like & $1 \mathrm{~S}$ & $4 \mathrm{P}$ \\
\hline K 18 & He-like & 1s & $4 P$ \\
\hline K 18 & He-like & IS & $4 \mathrm{P}$ \\
\hline K 18 & He-like & is & $3 P$ \\
\hline K 18 & He-like & $1 S$ & $3 P$ \\
\hline K 18 & He-like & is & $3 P$ \\
\hline K 18 & He-like & 1s & $3 P$ \\
\hline K 18 & He-like & $1 \mathrm{~S}$ & $3 P$ \\
\hline K 18 & He-like & $1 \mathrm{~S}$ & $3 P$ \\
\hline K 18 & He-like & $1 \mathrm{~s}$ & $3 P$ \\
\hline K 18 & He-like & $1 S$ & $3 P$ \\
\hline K 18 & He-Iike & is & $3 P$ \\
\hline K 18 & He-like & $1 S$ & $3 P$ \\
\hline $\mathrm{K} 18$ & He-like & $1 S$ & $3 P$ \\
\hline K 18 & He-like & 15 & $3 P$ \\
\hline K 18 & He-like & 1s & $3 P$ \\
\hline K 18 & He-like & 1S & $3 P$ \\
\hline K 18 & He-like & 15 & $3 P$ \\
\hline K 18 & He-like & is & $3 P$ \\
\hline K 18 & He-like & 1S & $2 \mathrm{P}$ \\
\hline K 18 & He-like & 1S & $2 P$ \\
\hline K 18 & He-like & IS & $2 \mathrm{P}$ \\
\hline K 18 & He-like & $1 \mathrm{~S}$ & $2 P$ \\
\hline $\mathrm{K} 18$ & He-like & 1s & $2 P$ \\
\hline K 18 & He-like & IS & $2 S$ \\
\hline K 18 & He-like & $1 S$ & $2 S$ \\
\hline K 18 & He-like & $1 \mathrm{~S}$ & $2 S$ \\
\hline K 18 & He-1ike & IS & $3 P$ \\
\hline K 18 & He-like & 15 & $3 P$ \\
\hline K 19 & $\mathrm{H}$-like & $10 \mathrm{P}$ & \\
\hline k 19 & $H-1 j$ & $9 p$ & \\
\hline
\end{tabular}

$\left(2 P^{*}\right) j=.5-1 S 22 S$

$\left(3 P^{*}\right) j=1.0-1 \mathrm{~S} 2$

$\left(3 P^{*}\right) j=1.0-1 S 2$

$\left(3 P^{*}\right) j=1.0-1 S 2$

$\left(3 P^{*}\right) j=1.0-1 S 2$

$\left(3 P^{\star}\right) j=1.0 \quad .1 S 2$

$\left(3 P^{*}\right) j=1.0-1 S 2$

$\left(3 P^{\star}\right) j=1.0-1 S 2$

$\left(3 P^{*}\right) j=1.0-1 S 2$

$(3 P *) j=1.0-1 S 2$

$\left(3 P^{*}\right) j=1.0-1 S 2$

(3P*) $j=1.0-152$

$\left(3 P^{*}\right) j=1.0-1 S 2$

$\left(3 P^{*}\right) j=1.0-1 S 2$

$\left(3 P^{*}\right) j=1.0-1 \mathrm{~S} 2$

$\left(3 P^{*}\right) j=1.0-1 S 2$

$\left(3 P^{*}\right) j=1.0-1 S 2$

$\left(3 P^{*}\right) j=1.0-1 S 2$

$\left(1 P^{*}\right) j=1.0-1 S 2$

$\left(1 P^{*}\right) j=1.0-152$

$\left(1 P^{\star}\right) j=1.0-1 S 2$

$\left(1 P^{*}\right) j=1.0-1 \mathrm{~S} 2$

$\left(1 P^{*}\right) j=1.0-1 S 2$

$\left(1 P^{*}\right) j=1.0-152$

$\left(1 P^{\star}\right) j=1.0-152$

$\left(1 P^{*}\right) j=1.0-1 S 2$

$(3 P *) j=1.0-1 S 2$

$(3 P *) j=1.0-1 S 2$

$\left(3 P^{*}\right) j=1.0-1 S 2$

$\left(3 P^{*}\right) j=1.0-1 S 2$

$\left(3 P^{*}\right) j=1.0-152$

$\left(3 P^{*}\right) j=1.0-1 S 2$

$\left(3 P^{*}\right) j=1.0-152$

$\left(3 P^{*}\right) j=1.0-1 S 2$

$\left(1 P^{*}\right) j=1.0-1 S 2$

$\left(1 P^{*}\right) j=1.0-1 S 2$

$\left(1 P^{*}\right) j=1.0-1 S 2$

$\left(3 P^{*}\right) j=1.0-1 S 2$

$\left(3 P^{*}\right) j=1.0-1 \mathrm{~S} 2$

(3S) $j=1.0-152$

(3s) $j=1.0-1 s 2$

(3S) $j=1.0-1 S 2$

$\left(3 P^{*}\right) j=2.0-1 S 2 S$

$\left(1 P^{*}\right) j=1.0-1 S \quad 2 S$

$\left(2 P^{*}\right) j=1.5-1 S$

$\left(2 p^{*}\right) j=1.5-1 S$

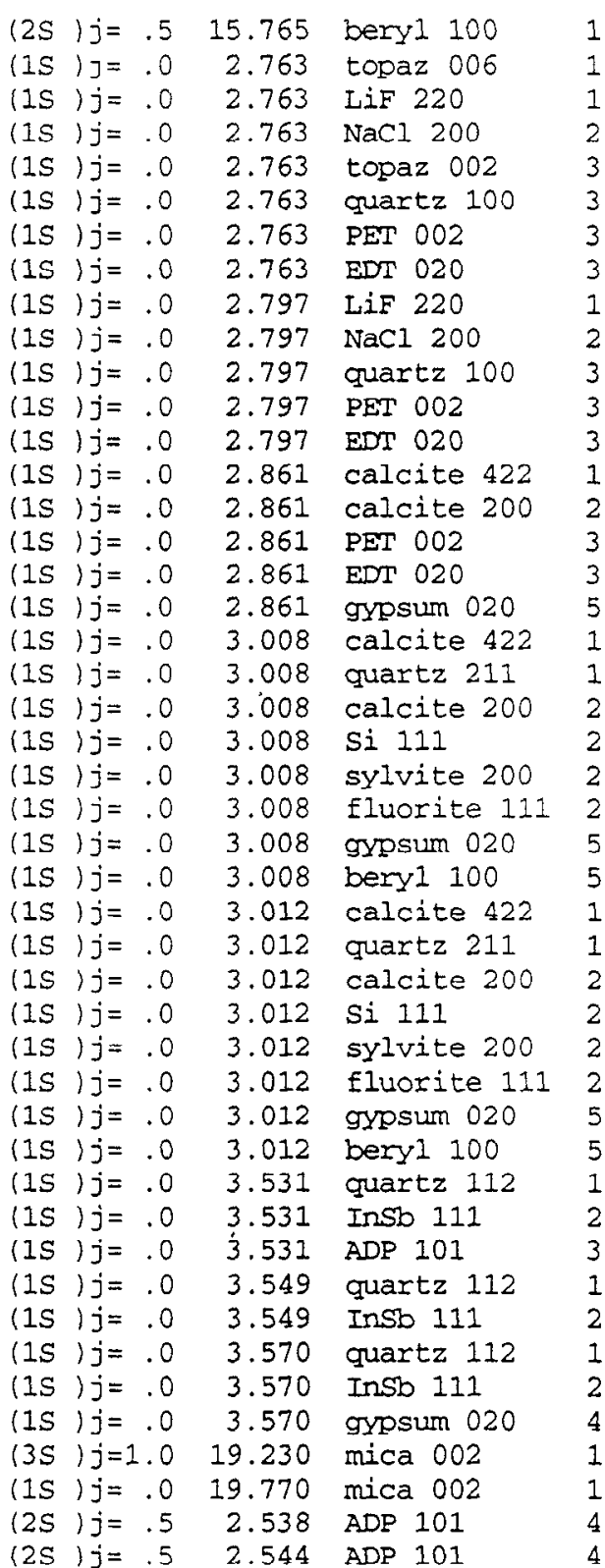

$15.954 \quad 81.172$

$2.795 \quad 81.322$

$2.848 \quad 75.967$

$5.641 \quad 78.411$

$8.374 \quad 81.830$

$8.512 \quad 76.856$

$8.742 \quad 71.474$

$8.808 \quad 70.233$

$2.848 \quad 79.141$

$5.641 \quad 82.599$

$8.512 \quad 80.328$

$8.742 \quad 73.709$

$8.808 \quad 72.299$

$3.034 \quad 70.558$

$\begin{array}{ll}6.071 & 70.478\end{array}$

$8.742 \quad 79.056$

$8.808 \quad 77.022$

$\begin{array}{ll}15.185 & 70.398\end{array}$

$3.034 \quad 82.494$

$3.082 \quad 77.419$

$6.071 \quad 82.282$

$6.271 \quad 73.605$

$6.292 \quad 72.967$

$6.308 \quad 72.499$

$15.185 \quad 82.076$

$15.954 \quad 70.512$

$3.034 \quad 83.096$

3.08277 .765

$.071 \quad 82.866$

$6.271 \quad 73.866$

$6.292 \quad 73.217$

$6.308 \quad 72.742$

$15.185 \quad 82.643$

$15.954 \quad 70.728$

$3.636 \quad 76.197$

$7.481 \quad 70.733$

$10.640 \quad 84.613$

$3.636 \quad 77.441$

$7.481 \quad 71.587$

$3.636 \quad 79.067$

$7.481 \quad 72.634$

$\begin{array}{ll}15.185 & 70.119\end{array}$

$19.942 \quad 74.643$

$19.942 \quad 82.469$

$10.640 \quad 72.580$

$10.640 \quad 73.017$ 


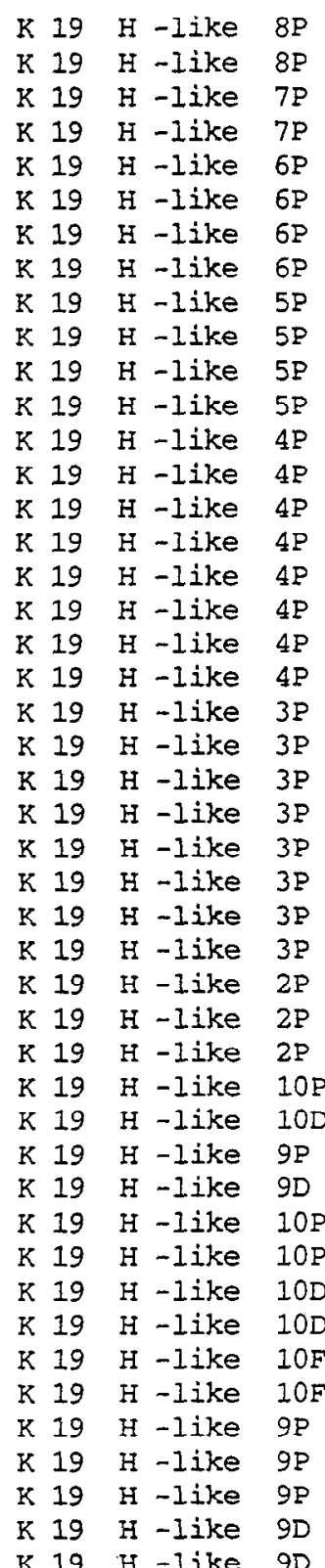

K 19 H -like 9D $\left(2 P^{*}\right) j=1.5-1 S$

$\left(2 P^{*}\right) j=1.5-1 S$

$\left(2 P^{*}\right) j=1.5-1 S$

$\left(2 P^{\star}\right) j=1.5-1 S$

$\left(2 P^{\star}\right) j=1.5-1 S$

$\left(2 P^{*}\right) j=1.5-1 S$

$\left(2 P^{\star}\right) j=1.5-1.5$

$\left(2 P^{*}\right) j=1.5-1$.

$\left(2 P^{\star}\right) j=1.5-1 S$

$\left(2 P^{\star}\right) j=1.5-1 S$

$\left(2 P^{\star}\right) j=1.5-1 S$

$\left(2 P^{\star}\right) j=1.5-1 S$

$\left(2 P^{*}\right) j=1.5-1 S$

$\left(2 P^{\star}\right) j=1.5-15$

$\left(2 P^{*}\right) j=1.5-1 S$

$\left(2 P^{\star}\right) j=1.5-1 S$

$\left(2 P^{*}\right) j=1.5-1 S$

$\left(2 P^{\star}\right) j=1.5-1 S$

$\left(2 P^{*}\right) j=1.5-1 S$

$\left(2 P^{\star}\right) j=1.5-1 S$

$\left(2 P^{\star}\right) j=1.5-1 S$

$\left(2 P^{*}\right) j=1.5-1 S$

$\left(2 P^{\star}\right) j=1.5-1 S$

$\left(2 P^{\star}\right) j=1.5-1 S$

$\left(2 P^{\star}\right) j=.5-1 S$

$\left(2 P^{\star}\right) j=.5-1 S$

$\left(2 P^{\star}\right) j=.5-1 S$

$\left(2 P^{\star}\right) j=.5-1 S$

$\left(2 P^{\star}\right) j=1.5-1 S$

$\left(2 P^{\star}\right) j=1.5-1 S$

$\left(2 P^{*}\right) j=.5-1 S$

$\left(2 P^{\star}\right) j=1.5-2 S$

(2D) $j=2.5-28$

$\left(2 \mathrm{P}^{\star}\right) j=1.5-2 \mathrm{~S}$

(2D) $j=2.5-2$ P

$\left(2 P^{\star}\right) j=1.5-3 S$

$\left(2 P^{*}\right) j=1.5-3 S$

(2D) $j=2.5-3 P$

(2D) $j=2.5-3 P$

$\left(2 F^{\star}\right) j=3.5-3 D$

$\left(2 F^{\star}\right) j=3.5-3 D$

$\left(2 P^{*}\right) j=1.5-3 S$

$\left(2 p^{\star}\right) j=1.5-35$

$\left(2 P^{\star}\right) j=1.5-3 S$

(2D) $j=2.5-3 P$

(2D) $j=2.5-3 P$

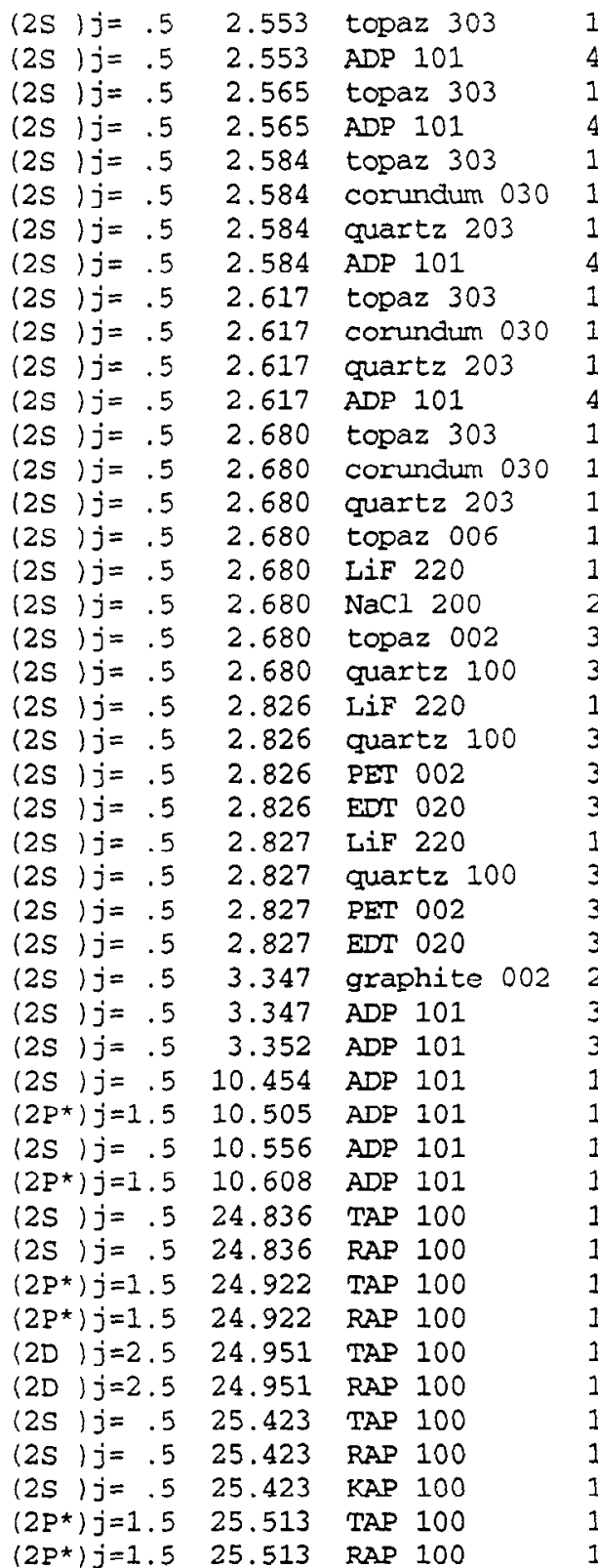

$2.712 \quad 70.283$

$10.640 \quad 73.694$

$2.712 \quad 71.049$

$10.640 \quad 74.641$

$2.712 \quad 72.327$

$2.748 \quad 70.105$

$2.749 \quad 70.048$

$10.640 \quad 76.271$

$2.712 \quad 74.790$

$2.748 \quad 72.237$

$2.749 \quad 72.173$

$10.640 \quad 79.684$

$2.712 \quad 81.190$

$2.748 \quad 77.227$

$2.749 \quad 77.136$

$2.795 \quad 73.507$

$2.848 \quad 70.222$

$5.641 \quad 71.839$

$8.374 \quad 73.763$

$8.512 \quad 70.830$

$2.848 \quad 82.874$

$8.512 \quad 84.877$

$8.742 \quad 75.883$

$8.808 \quad 74.267$

$2.848 \quad 83.038$

$8.512 \quad 85.109$

$8.742 \quad 75.954$

$8.808 \quad 74.339$

$6.696 \quad 88.599$

$10.640 \quad 70.683$

$10.640 \quad 70.929$

$10.640 \quad 79.271$

$10.640 \quad 80.863$

$10.640 \quad 82.796$

$10.640 \quad 85.555$

$25.763 \quad 74.583$

$26.116 \quad 71.987$

$25.763 \quad 75.320$

$26.116 \quad 72.608$

$\begin{array}{lll}25.763 & 75.577\end{array}$

$26.116 \quad 72.822$

$25.763 \quad 80.681$

$26.116 \quad 76.771$

$26.634 \quad 72.656$

$25.763 \quad 82.012$

$26.116 \quad 77.664$ 


\begin{tabular}{|c|c|c|c|c|c|c|c|c|}
\hline K 19 & $\mathrm{H}$-like & \multicolumn{4}{|l|}{$9 D$} & \multicolumn{3}{|c|}{$(2 D) j=2.5-3 P$} \\
\hline K 19 & $\mathrm{H}$-like & $9 F$ & & & & $\left(2 F^{\star}\right) j=3.5$ & & \\
\hline K 19 & $\mathrm{H}$-like & $9 \mathrm{~F}$ & & & & $\left(2 F^{\star}\right) j=3.5$ & $-3 D$ & \\
\hline K 19 & $\mathrm{H}$-like & $9 F$ & & & & $\left(2 F^{\star}\right) j=3.5$ & $-3 D$ & \\
\hline K 19 & $\mathrm{H}$-like & $8 \mathrm{P}$ & & & & $\left(2 P^{*}\right) j=1.5$ & $-3 s$ & \\
\hline K 19 & $\mathrm{H}$-like & $8 \mathrm{D}$ & & & & $(2 D) j=2.5$ & $-3 P$ & \\
\hline K 19 & $\mathrm{H}-1 \mathrm{ike}$ & $8 F$ & & & & $\left(2 F^{\star}\right) j=3.5$ & $-3 D$ & \\
\hline $\mathrm{Ca}$ & & $k-a$ & Lpha & & & & & \\
\hline Ca11 & Ne-Iike & $2 s 2$ & $2 \mathrm{P5}$ & $\left(2 P^{\star} 1\right.$ & 1) $4 D$ & $\left(12^{\star}\right) j=1.0$ & $-2 s 2$ & $2 P 6$ \\
\hline Ca11 & Ne-like & $2 \$ 2$ & $2 \mathrm{P} 5$ & $(2 \mathrm{P} \star 1$ & 1) $4 \mathrm{D}$ & $(12 *) j=1.0$ & $-2 \mathrm{~s} 2$ & $2 P 6$ \\
\hline Ca11 & Ne-like & $2 S 2$ & $2 \mathrm{P5}$ & $(2 P \star 1$ & 1) $4 \mathrm{D}$ & $\left(12^{\star}\right) j=1.0$ & $-2 s 2$ & $2 \mathrm{P} 6$ \\
\hline Ca11 & Ne-like & $2 \mathrm{~s} 2$ & $2 \mathrm{PS}$ & $(2 P \star 2$ & 2) $4 D$ & $(22 *) j=1.0$ & $-2 s 2$ & $2 P 6$ \\
\hline Ca11 & Ne-like & $2 S 2$ & $2 \mathrm{P} 5$ & $(2 P \star 2$ & 2) $4 D$ & $\left(22^{\star}\right) j=1.0$ & -252 & $2 P 6$ \\
\hline Call & $\mathrm{Ne}-1 \mathrm{ike}$ & $2 \mathrm{~S} 2$ & $2 \mathrm{P} 5$ & $(2 P \times 2$ & 2) $4 D$ & $(22 *) j=1.0$ & $-2 s 2$ & $2 P 6$ \\
\hline Cal1 & Ne-like & $2 S 2$ & $2 \mathrm{P} 5$ & $(2 \mathrm{P} * 1$ & 1) $4 S$ & $\left(11^{\star}\right) j=1.0$ & $-2 s 2$ & $2 \mathrm{P} 6$ \\
\hline $\mathrm{Ca} 13$ & o-like & $2 s 2$ & $2 \mathrm{P} 3$ & $\left(2 \mathrm{P}^{*}\right.$ & ) $3 D$ & $\left(3 D^{*}\right) j=3.0$ & $-2 s 2$ & $2 P 4$ \\
\hline $\mathrm{CaI3}$ & o-like & $2 \mathrm{~s} 2$ & $2 \mathrm{P} 3$ & $\left(2 \mathrm{P}^{\star}\right.$ & ) $3 D$ & $\left(3 D^{*}\right) j=3.0$ & $-2 s 2$ & $2 \mathrm{P4}$ \\
\hline Cal3 & o-like & $2 s 2$ & $2 \mathrm{P3}$ & $\left(2 \mathrm{P}^{\star}\right.$ & ) $3 D$ & $\left(3 D^{*}\right) j=3.0$ & $-2 s 2$ & $2 P 4$ \\
\hline Ca13 & o-like & $2 \mathrm{~S} 2$ & $2 \mathrm{P} 3$ & $\left(2 P^{\star}\right.$ & ) $3 D$ & $\left(3 D^{\star}\right) j=2.0$ & -252 & $2 \mathrm{PA}$ \\
\hline Ca13 & o-like & $2 S 2$ & $2 \mathrm{P} 3$ & $\left(2 P^{\star}\right.$ & 30 & $\left(3 D^{*}\right) j=2.0$ & $-2 s 2$ & $2 P 4$ \\
\hline $\mathrm{Cal3}$ & 0 -like & $2 \mathrm{~S} 2$ & $2 \mathrm{P} 3$ & $\left(2 P^{\star}\right.$ & ) $3 D$ & $\left(3 D^{*}\right) j=2.0$ & $-2 s 2$ & $2 \mathrm{PA}$ \\
\hline Ca13 & $0-1 i k e$ & $2 \mathrm{~S} 2$ & $2 \mathrm{P} 3$ & $\left(2 P^{\star}\right.$ & $13 D$ & $\left(3 P^{\star}\right) j=2.0$ & $-2 s 2$ & $2 P 4$ \\
\hline Ca13 & o-like & $2 \mathrm{~S} 2$ & $2 \mathrm{P} 3$ & $\left(2 P^{*}\right.$ & ) $3 D$ & $\left(3 P^{*}\right) j=2.0$ & $-2 s 2$ & $2 P 4$ \\
\hline Ca13 & 0 -like & $2 \mathrm{~S} 2$ & $2 \mathrm{P} 3$ & $\left(2 P^{\star}\right.$ & ) 30 & $\left(3 P^{*}\right) j=2.0$ & $-2 s 2$ & $2 \mathrm{P4}$ \\
\hline Ca13 & o-like & $2 s 2$ & $2 \mathrm{P} 3$ & $\left(2 \mathrm{P}^{\star}\right.$ & ) $3 D$ & $\left(3 D^{*}\right) j=1.0$ & $-2 s 2$ & $2 P 4$ \\
\hline Ca13 & o-like & $2 \mathrm{~S} 2$ & $2 \mathrm{P3}$ & $\left(2 P^{*}\right.$ & ) $3 D$ & $\left(3 D^{\star}\right) j=1.0$ & $-2 s 2$ & $2 \mathrm{P} 4$ \\
\hline $\mathrm{Ca} 13$ & o-like & $2 \mathrm{~s} 2$ & $2 \mathrm{P} 3$ & $\left(2 D^{*}\right.$ & ) $3 D$ & $\left(3 s^{*}\right) j=1.0$ & $-2 s 2$ & $2 P 4$ \\
\hline Cal3 & o-like & $2 s 2$ & $2 \mathrm{P} 3$ & $\left(2 D^{*}\right.$ & ) $3 D$ & $\left(3 s^{*}\right) j=1.0$ & $-2 s 2$ & $2 \mathrm{P} 4$ \\
\hline $\mathrm{Ca} 13$ & $0-1 i k e$ & 252 & $2 \mathrm{P} 3$ & $\left(2 D^{\star}\right.$ & $13 D$ & $\left(3 P^{*}\right) j=1.0$ & $-2 s 2$ & $2 P 4$ \\
\hline Ca13 & o-like & $2 S 2$ & $2 \mathrm{P} 3$ & $\left(2 D^{\star}\right.$ & ) $3 D$ & $\left(3 P^{*}\right) j=1.0$ & $-2 s 2$ & $2 \mathrm{P} 4$ \\
\hline Ca13 & o-like & $2 S 2$ & $2 \mathrm{P} 3$ & $\left(2 D^{*}\right.$ & 30 & $\left(3 R^{*}\right) j=2.0$ & $-2 s 2$ & $2 P 4$ \\
\hline Ca13 & o-1ike & $2 \mathrm{~S} 2$ & $2 \mathrm{P} 3$ & $\left(2 D^{*}\right.$ & $13 D$ & $\left(3 P^{*}\right) j=2.0$ & $-2 s 2$ & $2 P 4$ \\
\hline Cal3 & o-like & $2 \mathrm{~S} 2$ & $2 \mathrm{P} 3$ & $\left(2 D^{*}\right.$ & ) $3 D$ & $\left(3 D^{*}\right) j=3.0$ & $-2 s 2$ & $2 P 4$ \\
\hline $\mathrm{Ca} 13$ & o-like & $2 S 2$ & $2 \mathrm{P} 3$ & $\left(2 D^{\star}\right.$ & ) $3 D$ & $\left(3 D^{*}\right) j=3.0$ & $-2 \$ 2$ & $2 P 4$ \\
\hline Ca13 & o-like & $2 \$ 2$ & $2 \mathrm{P} 3$ & $2 D^{*}$ & ) $3 D$ & $\left(3 D^{*}\right) j=1.0$ & $-2 s 2$ & $2 \mathrm{P} 4$ \\
\hline Ca13 & o-like & $2 \mathrm{~S} 2$ & 283 & $2 D^{\star}$ & 130 & $\left(3 D^{\star}\right) j=2.0$ & $-2 s 2$ & $2 P 4$ \\
\hline Ca13 & o-like & $2 S 2$ & $2 \mathrm{P} 3$ & $\left(2 D^{\star}\right.$ & ) $3 D$ & $\left(3 D^{*}\right) j=1.0$ & $-2 s 2$ & $2 P 4$ \\
\hline $\mathrm{Ca} 13$ & o-like & $2 \mathrm{~S} 2$ & $2 \mathrm{P} 3$ & $\left(2 D^{*}\right.$ & $13 D$ & $\left(1 F^{*}\right) j=3.0$ & $-2 s 2$ & $2 \mathrm{P4}$ \\
\hline Ca13 & o-like & $2 S 2$ & $2 \mathrm{P} 3$ & $\left(2 D^{*}\right.$ & ) $3 D$ & $\left(1 D^{*}\right) j=2.0$ & $-2 s 2$ & $2 P 4$ \\
\hline Cal4 & $\mathrm{N}$-like & $2 S 2$ & $2 \mathrm{P} 2$ & $11 D$ & ) $3 D$ & $(2 F) j=3.5$ & $-2 s 2$ & $2 \mathrm{P} 3$ \\
\hline $\mathrm{Ca} 14$ & $\mathrm{~N}$-like & $2 \mathrm{~S} 2$ & $2 \mathrm{P} 2$ & $13 P$ & ) $3 D$ & $(2 D) j=2.5$ & $-2 s 2$ & $2 P 3$ \\
\hline Ca14 & $\mathrm{N}$-1ike & $2 \mathrm{~S} 2$ & $2 \mathrm{P} 2$ & $(1 \mathrm{D}$ & ) $3 D$ & $(2 D) j=2.5$ & $-2 s 2$ & $2 P 3$ \\
\hline Ca14 & $N$-like & $2 \$ 2$ & 292 & 110 & $13 D$ & (2D) $j=2.5$ & $-2 s 2$ & $2 P 3$ \\
\hline Ca14 & $\mathrm{N}$-like & $2 \mathrm{~S} 2$ & $2 \mathrm{P} 2$ & $13 P$ & 30 & $(2 F) j=3.5$ & $-2 s 2$ & $2 P 3$ \\
\hline Ca14 & $\mathrm{N}$-like & $2 \mathrm{~S} 2$ & $2 \mathrm{P} 2$ & $13 P$ & ) $3 D$ & $(2 F) j=3.5$ & $-2 s 2$ & $2 \mathrm{P} 3$ \\
\hline Cal4 & $N$-like & $2 S 2$ & $2 \mathrm{P} 2$ & $13 P$ & $13 D$ & $(2 D) j=1.5$ & $-2 s 2$ & $2 \mathrm{P} 3$ \\
\hline
\end{tabular}

$\left(2 P^{*}\right) j=1.5 \quad 25.513 \quad \operatorname{KAP} 100$ (2D $j=2.5 \quad 25.544 \quad$ TAP 100 (2D) $j=2.5 \quad 25.544 \quad$ RAP 100 (2D) $j=2.5 \quad 25.544 \quad \mathrm{KAP} 100$ (2S ) $j=.5 \quad 26.292 \quad \mathrm{KAP} 100$ (2P*) $j=1.5 \quad 26.388 \quad$ KAP 100 (2D) $j=2.5 \quad 26.420$ KAP 100 3.359 ADP 101

(1S) $j=.0 \quad 25.327$ TAP 100 (1s) $j=.0 \quad 25.327$ RAP 100 (1S ) $j=.0 \quad 25.327 \quad \operatorname{KAP} 100$ (1S) $j=.0 \quad 25.517$ TAP 100 (1S) $j=.0 \quad 25.517$ RAP 100 (1S) $j=.0 \quad 25.517$ KAP 100 (1S) $j=.0 \quad 26.442$ KAP 100 (3P) $j=2.0 \quad 25.530$ TAP 100 (3P) $j=2.0 \quad 25.530$ RAP 100 (3P) $j=2.0 \quad 25.530 \quad$ KAP 100 (3P) $j=1.0 \quad 25.674$ TAP 100 (3P) $j=1.0 \quad 25.674$ RAP 100 (3P) $j=1.0 \quad 25.674 \quad \mathrm{KAP} 100$ (3P) $j=2.0 \quad 25.710$ TAP 100 (3P) $j=2.0 \quad 25.710$ RAP 100 (3P) $j=2.0 \quad 25.710$ KAP 100 (3P) $j=.0 \quad 25.770$ RAP 100 (3P ) $j=.0 \quad 25.770$ KAP 100 (3P) $j=2.0 \quad 25.880$ RAP (3P) $j=2.0 \quad 25.880 \quad$ KAP 100 (3P) $j=2.0 \quad 25.964 \quad$ RAP 100 (3P) $j=2.0 \quad 25.964 \quad \mathrm{KAP} 100$ (3P) $j=2.0 \quad 26.000 \quad \operatorname{RAP} 100$ (3P) $j=2.0 \quad 26.000$ KAP 100 (3P) $j=2.0 \quad 26.033$ RAP 100 (3P) $j=2.0 \quad 26.033 \quad \mathrm{KAP} 100$ (3P) $j=2.0 \quad 26.124 \quad \mathrm{KAP} 100$ (3P) $j=1.0 \quad 26.219$ KAP 100 (3P) $j=.0 \quad 26.323$ KAP 100 (1D ) $j=2.0 \quad 26.383 \quad \mathrm{KAP} 100$ (1D) $j=2.0 \quad 26.550 \quad \mathrm{KAP} 100$ $\left(2 D^{*}\right) j=2.5 \quad 24.271 \quad$ TAP 100 $\left(2 D^{*}\right) j=2.5 \quad 24.480$ TAP 100 $\left(2 P^{*}\right) j=1.5 \quad 24.623 \quad$ TAP 100 $\left(2 P^{*}\right) j=1.5 \quad 24.623 \quad$ RAP 100 $\left(2 D^{\star}\right) j=2.5 \quad 24.749 \quad$ TAP 100 (2D*) $j=2.5 \quad 24.749$ RAP 100 $\left(2 P^{*}\right) j=.5 \quad 24.895$ TAP 100
$26.634 \quad 73.318$ $25.763 \quad 82.524$ $26.116 \quad 77.986$ $26.634 \quad 73.552$ $26.634 \quad 80.808$ $26.634 \quad 82.207$ $26.634 \quad 82.732$ $10.640 \quad 71.253$ $25.763 \quad 79.444$ $26.116 \quad 75.880$ $26.634 \quad 71.976$ $25.763 \quad 82.076$ $\begin{array}{ll}26.116 & 77.705\end{array}$ $26.634 \quad 73.348$ $26.634 \quad 83.116$ $25.763 \quad 82.288$ $26.116 \quad 77.840$ $26.634 \quad 73.446$ $25.763 \quad 85.236$

$26.116 \quad 79.444$ $26.634 \quad 74.570$ $25.763 \quad 86.324$ $26.116 \quad 79.884$ $26.634 \quad 74.864$ $26.116 \quad 80.663$ $26.634 \quad 75.366$

$26.116 \quad 82.292$

$26.634 \quad 76.334$ $26.116 \quad 83.815$ $26.634 \quad 77.121$ $26.116 \quad 84.598$ $26.634 \quad 77.474$ $26.116 \quad 85.431$ $26.634 \quad 77.805$ $26.634 \quad 78.769$ $26.634 \quad 79.872$ $26.634 \quad 81.236$ $26.634 \quad 82.128$ $26.634 \quad 85.448$ 25.763 .70 .405 $25.763 \quad 71.842$ $25.763 \quad 72.892$ $26.116 \quad 70.533$ $25.763 \quad 73.872$ $26.116 \quad 71.380$ $25.763 \quad 75.085$ 
Ca14 N-like $2 S 22 P 2(3 P) 3 D(2 D) j=1.5-2 S 22 P 3$

Ca14 N-like 2S2 2P2 ( $3 P$ ) $3 D(2 P) j=1.5-2 S 22 P 3$

Ca14 N-Iike 2S2 2P2 ( 3P) 3D (2P) j=1.5 - 2S2 2P3

Ca14 N-like 2S2 2P2 ( $3 \mathrm{P}$ ) $3 \mathrm{D}(2 \mathrm{P}) \mathrm{J}=1.5-2 \mathrm{~S} 22 \mathrm{P} 3$

Ca14 N-like 2S2 $2 \mathrm{P} 2$ ( $1 \mathrm{~S}$ ) $3 \mathrm{~S}(2 \mathrm{~S}) \mathrm{j}=.5-2 \mathrm{~S} 22 \mathrm{P} 3$

Cal4 N-like 2S2 2P2 ( $1 S$ ) 3S (2S) $j=.5-2 S 22 \mathrm{P} 3$

Ca14 $N$-like 2S2 2P2 ( $3 P$ ) $3 S(4 P) j=2.5-2 S 22 P 3$

Ca14 N-like 2S2 2P2 ( $3 P$ ) 3S (4P) j=1.5 - 2S2 2P3

Cal7 Be-like 2S 4P $\quad\left(3 P^{*}\right) j=1.0-2 S 2$

Ca17 Be-like 2P 3P (3P) $j=2.0-2 S \quad 2 P$

Cal7 Be-like 2P $3 P \quad$ (3P) $j=1.0-2 S \quad 2 P$

Cal7 Be-like 2P 3P (3S) $j=1.0-2 S \quad 2 P$

Ca17 Be-like 2P $3 P \quad(3 P) j=2.0-2 S \quad 2 P$

Ca17 Be-like 2P 3P (3S ) $j=1.0-2 S \quad 2 P$

$\begin{array}{lllll}\text { Cal7 Be-like 2P } 3 P & \text { (3P) } j=1.0-2 S & 2 P\end{array}$

Ca17 Be-like 2P 3P (3S $) j=1.0-2 S \quad 2 P$

Cal7 Be-like 2P 3P (3D) $j=3.0-2 S \quad 2 P$

Cal7 Be-like 2S $3 P$

Ca18 Li-like 1S 2P ( $\left.2 P^{*}\right) 3 P(2 D) j=2.5-1 S 2 \quad 2$

Ca18 Li-like $1 \mathrm{~S}$ 2P( $\left.1 \mathrm{P}^{*}\right) 3 \mathrm{P}(2 \mathrm{D}) j=2.5-1 \mathrm{~S} 2$

Ca18 Li-like 1S $2 P\left(1 P^{*}\right) 3 P(2 D) j=2.5-1 S 2 \quad 2 P$

Ca18 Li-like $1 S$ 2P ( IP*) $3 P(2 D) j=2.5-1 S 2$ 2P

Ca18 Li-like $1 S$ 2P( $\left.P^{*}\right) 3 P(2 D) j=2.5-1 S 2 \quad 2 P$

Ca18 Li-like is $2 P\left(1 P^{*}\right) 3 P(2 D) j=2.5-1 S 2 \quad 2 P$

Ca18 Li-like is $2 \mathrm{P}\left(1 \mathrm{P}^{*}\right) 3 \mathrm{P}(2 \mathrm{D}) \mathrm{j}=2.5-1 \mathrm{~S} 2$ 3P

Ca18 Li-like is $2 P\left(1 P^{*}\right) 3 P(2 D) j=2.5-1 S 23 P$

Ca18 Li-like is $2 P\left(1 P^{*}\right) 3 P(2 D) j=2.5-1 S 23 P$

Ca18 Li-like is $2 \mathrm{P}\left(1 \mathrm{P}^{*}\right) 3 \mathrm{P}(2 \mathrm{D}) \mathrm{j}=2.5-1 \mathrm{~S} 23 \mathrm{P}$

Ca18 Li-like $1 S$ 2P ( $1 P^{*}$ ) $3 P$ (2D) $j=2.5-1 S 2$ 3P

Ca18 Li-like 1S 2P2 (2S) $j=.5-1 S 2 \quad 2 P$

Ca18 Li-like 1S 2P2 (2S) $j=.5-1 S 2 \quad 2 P$

Ca18 Li-like $1 S \quad 2 P 2 \quad(2 S) j=.5-1 S 2 \quad 2 P$

Ca18 Li-like 1S 2P2 (2S) $j=.5-1 S 2 \quad 2 P$

Ca18 Li-like 1S 2P2 (2S) j=.5-1S2 2P

Ca18 Li-like 1S $2 \mathrm{P} 2 \quad(2 \mathrm{~S}) \mathrm{j}=.5-1 \mathrm{S2} 2 \mathrm{P}$

Ca18 Li-like 1S 2P2 (2S ) j=.5- 1S2 2P

Ca18 Li-like is $2 \mathrm{P} 2 \quad(2 \mathrm{~S}) j=.5-1 S 2 \quad 2 \mathrm{P}$

Ca18 Li-like is 2P2 (2S ) j=.5- 1S2 29

Ca18 Li-like is 2P2 (2S) $j=.5-1 S 2 \quad 2 P$

Ca18 Li-like 1S (2S 2P ( $\left.\left.1 P^{*}\right)\right)\left(2 P^{*}\right) j=1.5-1 S 2 \quad 2 S$

Ca18 Li-like is (2S 2P(1S $\left.\left.1 P^{\star}\right)\right)\left(2 P^{*}\right) j=1.5-1 S 2 \quad 2 S$

Ca18 Li-like $1 S$ (2S $\left.2 P\left(1 P^{*}\right)\right)\left(2 P^{*}\right) j=1.5-1 S 22 S$

Ca18 Li-like 1S (2S 2P ( 1P*)) (2P*)j=1.5 - 1S2 $2 S$

Ca18 Li-like 1S (2S 2P(3P*) $\left(2 P^{*}\right) j=1.5-1 S 2 \quad 2 S$

Ca18 Li-like is $\left(2 S 2 P\left(3 P^{\star}\right)\right)\left(2 P^{*}\right) j=1.5-1 S 2$ 2S

Ca18 Li-like $1 S$ (2S $\left.2 P\left(3 P^{\star}\right)\right)\left(2 P^{\star}\right) j=1.5-1 S 2$ 2S $\left(2 P^{*}\right) j=.5 \quad 24.895 \quad$ RAP 100

$\left(2 P^{*}\right) j=1.5 \quad 25.441$ TAP 100

$\left(2 P^{*}\right) j=1.5 \quad 25.441 \quad R A P 100$

$\left(2 P^{*}\right) j=1.5 \quad 25.441 \quad \operatorname{KAP} 100$

$\left(2 P^{*}\right) j=.5 \quad 26.180$ KAP 100

$\left(2 P^{*}\right) j=1.5 \quad 26.255 \quad \mathrm{KAP} 100$

$\left\langle 4 S^{*}\right\rangle j=1.5 \quad 26.372 \quad \mathrm{KAP} 100$

$\left(4 S^{*}\right) j=1.5 \quad 26.514 \quad \mathrm{KAP} 100$

(1S ) $j=.0 \quad 14.900$ sypsum 020

$\left(3 P^{*}\right) j=1.0 \quad 19.195$ mica 002

$\left(3 P^{\star}\right) j=1.0 \quad 19.249$ mica 002

$\left(3 P^{\star}\right) j=.0 \quad 19.275$ mica 002

$\left(3 P^{*}\right) j=2.0 \quad 19.296$ mica 002

$\left(3 P^{*}\right) j=1.0 \quad 19.315$ mica 002

$\left(3 P^{\star}\right) j=2.0 \quad 19.345$ mica 002

$\left(3 P^{*}\right) j=2.0 \quad 19.414$ mica 002

$\left(3 P^{*}\right) j=2.0 \quad 19.505$ mica 002

(1s) $j=.0 \quad 19.558$ mica 002

$\left(2 P^{*}\right) j=1.5 \quad 2.750$ topaz 006

$\left(2 P^{*}\right) j=1.5$

2.750 LiF 220

$\left(2 P^{\star}\right) j=1.5 \quad 2.750 \quad \mathrm{NaCl} 200$

$\left(2 P^{*}\right) j=1.5 \quad 2.750$ topaz 002

$\left(2 P^{\star}\right) j=1.5$

2.750 topaz 002
2.750 guartz 100

2.750 PET 002

$\left(2 P^{*}\right) j=1.5$

$\left(2 P^{*}\right) j=1.5$

$\left(2 P^{*}\right) j=1.5$

3.180

Ge 111

$\left(2 P^{\star}\right) j=1.5$

3.180

KBr 200

$\left(2 P^{*}\right) j=1.5$

3.180

quartz 101

3.180 graphite 002

$\left(2 P^{*}\right) j=1.5$

$\left(2 P^{*}\right) j=.5$

$\left(2 P^{*}\right) j=.5$

3.180

beryl 100

3.185

Ge 111

$\left(2 P^{*}\right) j=.5$

3.185

$\left(2 P^{*}\right) j=.5$

3.185

$\left(2 P^{\star}\right) j=.5$

$\left(2 P^{\star}\right) j=1.5$

3.185

3.189

guartz 10

graphite 002

$\left(2 P^{\star}\right) j=1.5$

$\left(2 P^{*}\right) j=1$.

$\left(2 P^{*}\right) j=1.5$

$\left(2 P^{*}\right) j=1.5$

(2S) $j=.5$

(2S) $j=.5$

(2s) $j=.5$

(2s) $j=.5$

(2s) $j=.5$

(2s) $j=.5$

(2S) $j=.5$ quartz 101

3.189 graphite 002

3.189 beryl 100

3.192 Ge 111

$3.192 \mathrm{KBr} 200$

3.192 quartz 101

3.200 Ge 111

$3.200 \mathrm{KBr} 200$

3.200 quartz 101
KBr 200

3.192 graphite 002
$26.116 \quad 72.411$

$25.763 \quad 80.932$

$26.116 \quad 76.945$

$26.634 \quad 72.786$

$26.634 \quad 79.406$

26.63480 .323

$26.634 \quad 81.957$

$26.634 \quad 84.559$

$15.185 \quad 78.882$

$19.942 \quad 74.268$

$19.942 \quad 74.851$

$19.942 \quad 75.139$

$19.942 \quad 75.377$

$19.942 \quad 75.594$

$19.942 \quad 75.945$

$19.942 \quad 76.786$

$19.942 \quad 77.983$

$19.942 \quad 78.738$

$2.795 \quad 79.705$

$2.848 \quad 74.926$

$5.641 \quad 77.163$

$8.374 \quad 80.128$

$8.512 \quad 75.747$

8.74270 .686

6.53276 .822

$6.584 \quad 75.012$

$6.687 \quad 72.008$

$6.696 \quad 71.772$

$15.954 \quad 85.285$

$6.532 \quad 77.213$

$6.584 \quad 75.352$

$6.687 \quad 72.287$

$6.696 \quad 72.048$

$15.954 \quad 86.545$

$6.532 \quad 77.534$

6.58475 .630

$6.687 \quad 72.514$

$6.696 \quad 72.271$

$15.954 \quad 88.075$

$6.532 \quad 77.780$

$6.584 \quad 75.842$

$6.687 \quad 72.686$

$6.696 \quad 72.441$

6.53278 .462

6.58476 .423

$6.687 \quad 73.153$ 
Ca18 Li-like 1S (2S 2P ( $\left.\left.3 P^{*}\right)\right)\left(2 P^{*}\right) j=1.5-1 S 2$ 2S Ca18 Li-like $1 S$ (2S $\left.2 P^{2}\left(3 P^{*}\right)\right)\left(2 P^{*}\right) j=.5-1 S 2 \quad 2 S$

Ca18 Li-like is $\left(2 S 2 P\left(3 P^{*}\right)\right)\left(2 P^{*}\right) j=.5-1 S 2$ 2S

Cal8 Li-like 1S (2S 2P $\left.\left(3 P^{*}\right)\right)\left(2 P^{*}\right) j=.5-1 S 2 \quad 2 S$

Ca18 Ii-like 1S (2S $\left.2 \mathrm{P}\left(3 \mathrm{P}^{*}\right)\right)\left(2 \mathrm{P}^{*}\right) j=5-1 \mathrm{~S} 2 \mathrm{2S}$

Ca18 Li-like $1 S \quad 2 P 2 \quad$ (2D) $j=1.5-1 S 2 \quad 2 P$

Cal8 Li-like is $2 \mathrm{P} 2 \quad$ (2D) $j=1.5-1 S 2 \quad 2 \mathrm{P}$

Cal8 Ii-like 15 2P2

Ca18 Li-like 1S $2 \mathrm{P} 2$

Ca18 Li-like is $2 \mathrm{~F} 2$

Ca18 Li-like 1s 2P2

Ca18 Li-like 1S $2 \mathrm{P2}$

Ca18 Li-like 1s $2 \mathrm{P} 2$

Ca18 Li-like is 2P2

Ca18 Li-like 1S 222

Ca18 Li-like 1S 2P2

Cal8 Li-like is $2 \mathrm{P} 2$

Ca18 Li-like 1S 2P2

Ca18 Li-like 1S 2P2

Ca18 Li-like 1S 2P2

Cal8 Li-like 15 2P2

Ca18 Ii-like 1S 2P2

Ca18 Li-like is 2P2

Ca18 Li-like is $2 \mathrm{P} 2$

Ca18 Li-like is 2P2

Ca18 Li-like 1S2 6D

Cal8 Li-like 1S2 6D

Ca18 Li-like 1S2 6D

Ca18 Li-like 1S2 6D

Ca18 Li-like 152 5P

Ca18 Li-like 1S2 $5 P$

Ca18 Li-like 1S2 5P

Ca18 Li-like 1S2 5D

Ca18 Li-like 1S2 5D

Cal8 Li-like 1S2 4D

Ca18 Li-like 1S2 $4 D$

Ca18 Li-like 1S2 45

Ca18 Li-like 1S2 4S

Ca18 Li-like 1S2 3D

Ca18 Li-like IS2 3D

Ca19 He-like 1S 6P

Ca19 He-like is 6P

Ca19 He-like 1S $5 P$

Ca19 He-like is 4

Cal9 He-like 1S $4 \mathrm{P}$

Ca19 He-like is $3 P$

\begin{tabular}{|c|c|c|}
\hline$S \mid j=$ & 3.200 & graphite 002 \\
\hline$j=.5$ & 3.203 & Ge 111 \\
\hline$j=.5$ & 3.203 & $\mathrm{KBr} 200$ \\
\hline$j=.5$ & 3.203 & guartz 101 \\
\hline .5 & 3.203 & graphite 002 \\
\hline .5 & 3.205 & $\mathrm{Ge} 111$ \\
\hline .5 & 3.205 & $\mathrm{KBr} 200$ \\
\hline .5 & 3.205 & quartz 101 \\
\hline .5 & 3.205 & graphite 002 \\
\hline$=1.5$ & 3.209 & $\mathrm{Ge} 111$ \\
\hline$=1.5$ & 3.209 & $\operatorname{KBr} 200$ \\
\hline 1.5 & 3.209 & quartz 101 \\
\hline 1.5 & 3.209 & graphite 002 \\
\hline$=.5$ & 3.221 & Ge 111 \\
\hline .5 & 3.221 & $\mathrm{KBI} 200$ \\
\hline .5 & 3.221 & quartz 101 \\
\hline .5 & 3.221 & graphite 002 \\
\hline .5 & 3.223 & Ge 111 \\
\hline 1.5 & 3.223 & $\mathrm{KBr} 200$ \\
\hline 1.5 & 3.223 & quartz 101 \\
\hline$j=1.5$ & 3.223 & graphite 002 \\
\hline$j=1.5$ & 3.225 & Ge 111 \\
\hline$j=1.5$ & 3.225 & $\operatorname{KBr} 200$ \\
\hline$j=1.5$ & 3.225 & quartz 101 \\
\hline$j=1.5$ & 3.225 & graphite 002 \\
\hline$j=.5$ & 12.411 & $\operatorname{TAP} 100$ \\
\hline .5 & 12.411 & RAP 100 \\
\hline$j=1.5$ & 12.478 & TAP 100 \\
\hline *) $j=1.5$ & 12.478 & RAP 100 \\
\hline$j j=.5$ & 12.636 & TAP 100 \\
\hline$j j=.5$ & 12.636 & RAP 100 \\
\hline$j . j=.5$ & 12.636 & KAP 100 \\
\hline$*) j=.5$ & 13.118 & $\operatorname{KAP} 100$ \\
\hline$j=1.5$ & 13.191 & $\operatorname{KAP} 100$ \\
\hline .5 & 14.659 & gypsum 020 \\
\hline$j=1.5$ & 14.744 & gypsum 020 \\
\hline .5 & 14.767 & gypsum 020 \\
\hline$=1.5$ & 14.857 & gypsum 020 \\
\hline & 19.646 & mica 002 \\
\hline 1.5 & 19.790 & mica 002 \\
\hline .0 & 2.484 & gypsum 002 \\
\hline$j=.0$ & 2.484 & InSb 111 \\
\hline$j=.0$ & 2.514 & ADP 101 \\
\hline .0 & 2.571 & topaz 303 \\
\hline .0 & 2.571 & ADP 101 \\
\hline .0 & 2.705 & topaz 303 \\
\hline
\end{tabular}

$6.696 \quad 72.900$

6.53278 .728

$6.584 \quad 76.647$

$6.687 \quad 73.331$

6.69673 .076

6.53278 .909

$6.584 \quad 76.798$

$6.687 \quad 73.451$

$6.696 \quad 73.194$

$6.532 \quad 79.280$

$6.584 \quad 77.107$

$6.687 \quad 73.693$

$6.696 \quad 73.432$

6.53280 .478

6.58478 .079

$6.687 \quad 74.442$

6.69674 .168

$6.532 \quad 80.692$

$6.584 \quad 78.248$

$6.687 \quad 74.571$

$6.696 \quad 74.294$

6.53280 .912

$6.584 \quad 78.421$

$6.687 \quad 74.700$

$6.696 \quad 74.421$

$25.763 \quad 74.467$

$26.116 \quad 71.888$

$25.763 \quad 75.621$

$26.116 \quad 72.859$

$25.763 \quad 78.796$

$26.116 \quad 75.394$

$26.634 \quad 71.598$

$26.634 \quad 80.082$

$26.634 \quad 82.112$

$15.185 \quad 74.875$

$15.185 \quad 76.158$

$15.185 \quad 76.525$

$15.185 \quad 78.070$

$19.942 \quad 80.116$

$19.942 \quad 82.921$

$4.990 \quad 84.618$

$7.481 \quad 84.953$

$10.640 \quad 70.929$

2.71271 .443

$10.640 \quad 75.137$

$2.712 \quad 85.882$ 
$\left(1 P^{\star}\right) j=1.0-152$ $\left(1 P^{\star}\right) j=1.0-1 \mathrm{~S} 2$ $\left(1 P^{\star}\right) j=1.0-152$ $\left(1 P^{*}\right) j=1.0-1 S 2$ $\left(1 P^{\star}\right) j=1.0-1 S 2$ $\left(1 P^{*}\right) j=1.0-152$ $\left(1 P^{\star}\right) j=1.0-1 S 2$ $\left(1 P^{\star}\right) j=1.0-152$ $\left(1 P^{*}\right) j=1.0-1 S 2$ $\left(1 P^{\star}\right) j=1.0-1 S 2$ $\left(1 P^{\star}\right) j=1.0-1 S 2$ (1P*) $j=1.0-1 \mathrm{~S} 2$ $\left(3 P^{\star}\right) j=2.0-1 S 2$ $\left(3 P^{*}\right) j=2.0-1 S 2$ $\left(3 P^{*}\right) j=2.0-1 S 2$ $\left\langle 3 P^{*}\right\rangle j=2.0-1 S 2$ $\left(3 P^{*}\right) j=2.0-1 S 2$ $\left(3 P^{*}\right) j=2.0-152$ (3p*) $j=2,0-1 S 2$ $\left(3 P^{*}\right) j=2.0-152$ $\left(3 p^{*}\right) j=2.0-152$ (3S) $j=1.0-1 S 2$ (3s) $j=1.0-152$ (3s) $j=1.0-1 s 2$ (3S) $j=1.0-152$ $\left(2 P^{\star}\right) j=1.5-1 S$ $\left(2 P^{*}\right) j=1.5-1 S$ $\left(2 P^{*}\right) j=1.5-1 S$ $\left(2 P^{\star}\right) j=1.5-15$ $\left(2 P^{*}\right) j=1.5-1 S$ $\left(2 P^{\star}\right) j=1.5-1 S$ $\left(2 P^{*}\right) j=1.5-1 S$ $\left(2 \mathrm{P}^{\star}\right) j=1.5-1 \mathrm{~S}$ $\left(2 P^{*}\right) j=1.5-15$ $\left(2 P^{\star}\right) j=1.5-1 S$ $\left(2 P^{*}\right) j=1.5-1 S$ $\left(2 P^{*}\right) j=1.5-1 S$ $\left(2 P^{*}\right) j=1.5-1 S$ $\left(2 P^{*}\right) j=1.5-1 S$ $\left(2 P^{*}\right) j=1.5-1 S$ $\left(2 P^{\star}\right) j=1.5-1 S$ $\left(2 P^{\star}\right) j=1.5-15$ $\left(2 P^{*}\right) j=1.5-1 S$ $\left(2 P^{\star}\right) j=1.5-1 S$ $\left(2 P^{\star}\right) j=1.5-1 S$ $\left(2 P^{*}\right) j=1.5-1 S$

\begin{tabular}{|c|c|c|c|}
\hline$(1 s) j=$ & .0 & 2.705 & corundum 030 \\
\hline (1s) $j=$ & .0 & 2.705 & quartz 203 \\
\hline (1s) $j=$ & .0 & 2.705 & topaz 006 \\
\hline (1s) $j=$ & .0 & 2.705 & LiF 220 \\
\hline$j j=$ & .0 & 2.705 & $\mathrm{NaCl} 200$ \\
\hline$j=$ & .0 & 2.705 & topaz 002 \\
\hline$j=$ & .0 & 2.705 & quartz 100 \\
\hline$j=$ & .0 & 3.176 & Ge 111 \\
\hline$j=$ & .0 & 3.176 & $\mathrm{KBr} 200$ \\
\hline$i j=$ & .0 & 3.176 & quartz 101 \\
\hline$S \mid j=$ & .0 & 3.176 & graphite 002 \\
\hline (s) $j=$ & .0 & 3.176 & beryl 100 \\
\hline$j j=$ & .0 & 3.188 & $\mathrm{Ge} 111$ \\
\hline$j=$ & .0 & 3.188 & $\mathrm{KBr} 200$ \\
\hline$j=$ & .0 & 3.188 & quartz 101 \\
\hline$j=$ & .0 & 3.188 & graphite 002 \\
\hline$j=$ & .0 & 3.188 & beryl 100 \\
\hline$j j=$ & .0 & 3.192 & Ge 111 \\
\hline$j=$ & .0 & 3.192 & $\mathrm{KBr} 200$ \\
\hline$j j=$ & .0 & 3.192 & quartz 101 \\
\hline$S / j=$ & .0 & 3.192 & graphite 002 \\
\hline$j=$ & .0 & 3.211 & Ge 111 \\
\hline$j=$ & .0 & 3.211 & $\mathrm{KBr} 200$ \\
\hline$j=$ & .0 & 3.211 & quartz 101 \\
\hline$j j=$ & .0 & 3.211 & graphite 002 \\
\hline$j=$ & .5 & 2.314 & quartz 310 \\
\hline$j=$ & .5 & 2.314 & quartz 220 \\
\hline$j j=$ & .5 & 2.314 & topaz 200 \\
\hline$(2 S) j=$ & .5 & 2.314 & Al 111 \\
\hline$j j=$ & .5 & 2.314 & quartz 110 \\
\hline$j j=$ & .5 & 2.331 & quartz 310 \\
\hline $\begin{array}{l}j= \\
j=\end{array}$ & $\begin{array}{l}.5 \\
.5\end{array}$ & $\begin{array}{l}2.331 \\
2.331\end{array}$ & $\begin{array}{l}\text { quartz } 220 \\
\text { Al } 111\end{array}$ \\
\hline$j=$ & .5 & 2.331 & quartz 110 \\
\hline$j=$ & .5 & 2.361 & quartz 220 \\
\hline$j=$ & .5 & 2.361 & quartz 110 \\
\hline$j=$ & .5 & 2.361 & gypsum 002 \\
\hline$j j=$ & .5 & 2.361 & Insb 111 \\
\hline$s) j=$ & .5 & 2.417 & quartz 220 \\
\hline$(2 s) j=$ & .5 & 2.417 & quartz 110 \\
\hline$(2 s) j=$ & .5 & 2.417 & gypsum 002 \\
\hline$j j=$ & .5 & 2.417 & InSb 111 \\
\hline $1 j=$ & .5 & 2.549 & topaz 303 \\
\hline$j j=$ & .5 & 2.549 & $A D P 101$ \\
\hline$j j=$ & .5 & 3.018 & calcite 422 \\
\hline & & & \\
\hline
\end{tabular}

$2.748 \quad 79.851$

$2.749 \quad 79.735$

$2.795 \quad 75.421$

$2.848 \quad 71.767$

5.64173 .546

$8.374 \quad 75.713$

$8.512 \quad 72.432$

6.53276 .518

6.58474 .745

$6.687 \quad 71.787$

$6.696 \quad 71.555$

$15.954 \quad 84.479$

$\begin{array}{lll}6.532 & 77.453\end{array}$

$6.584 \quad 75.560$

$6.687 \quad 72.457$

$6.696 \quad 72.215$

$15.954 \quad 87.599$

6.53277 .780

$6.584 \quad 75.842$

$6.687 \quad 72.686$

$6.696 \quad 72.441$

6.53279 .470

$6.584 \quad 77.264$

$6.687 \quad 73.816$

6.69673 .553

$2.360 \quad 78.669$

2.45170 .753

$4.638 \quad 86.237$

$4.676 \quad 81.783$

$4.912 \quad 70.421$

$2.360 \quad 81.009$

$2.451 \quad 71.997$

$4.676 \quad 85.565$

4.91271 .641

$2.451 \quad 74.425$

$4.912 \quad 74.012$

$4.990 \quad 71.137$

$7.481 \quad 71.227$

$2.451 \quad 80.445$

$4.912 \quad 79.776$

$4.990 \quad 75.636$

$7.481 \quad 75.756$

$2.712 \quad 70.034$

$10.640 \quad 73.390$

$3.034 \quad 84.113$

3.08278 .303 


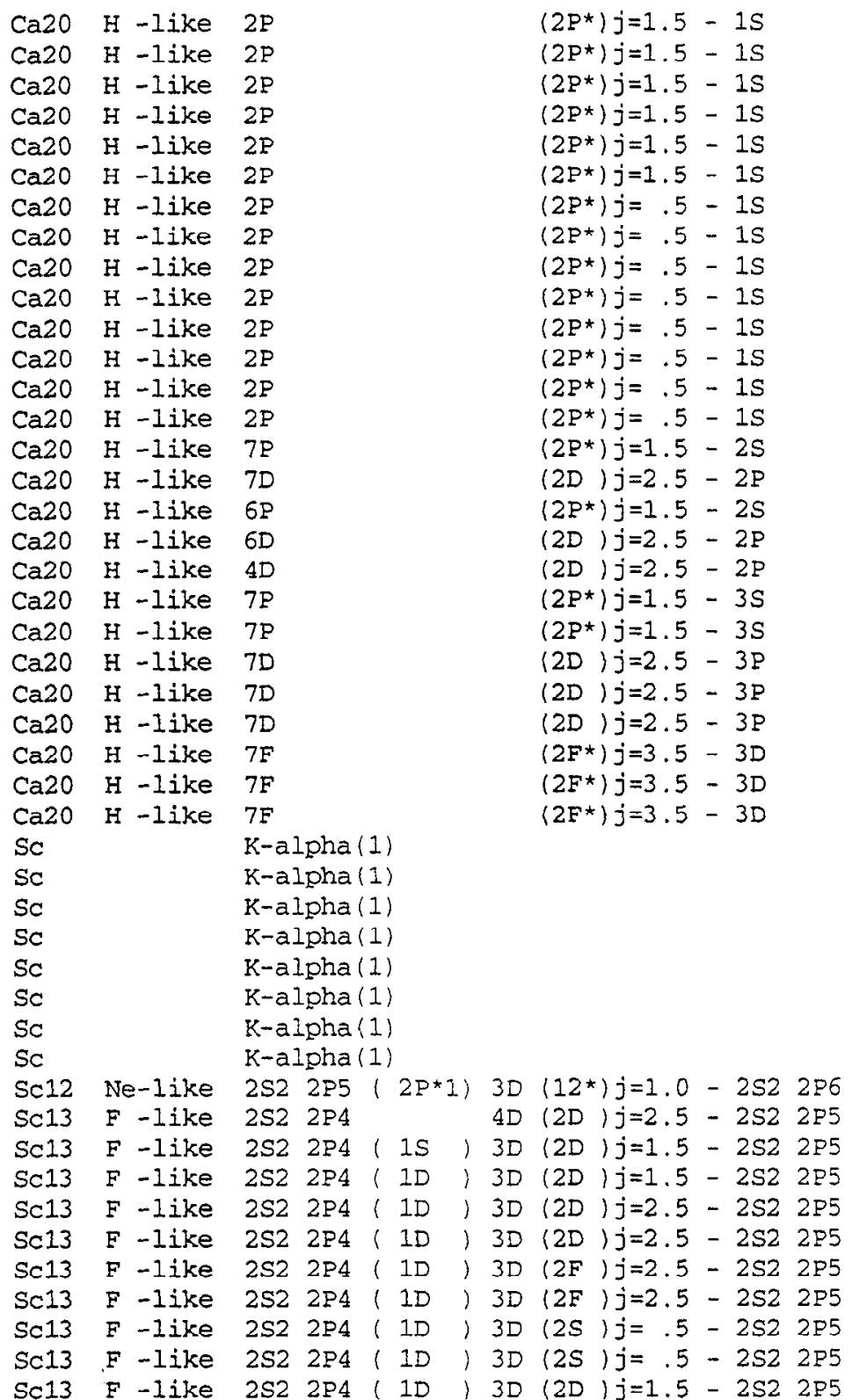

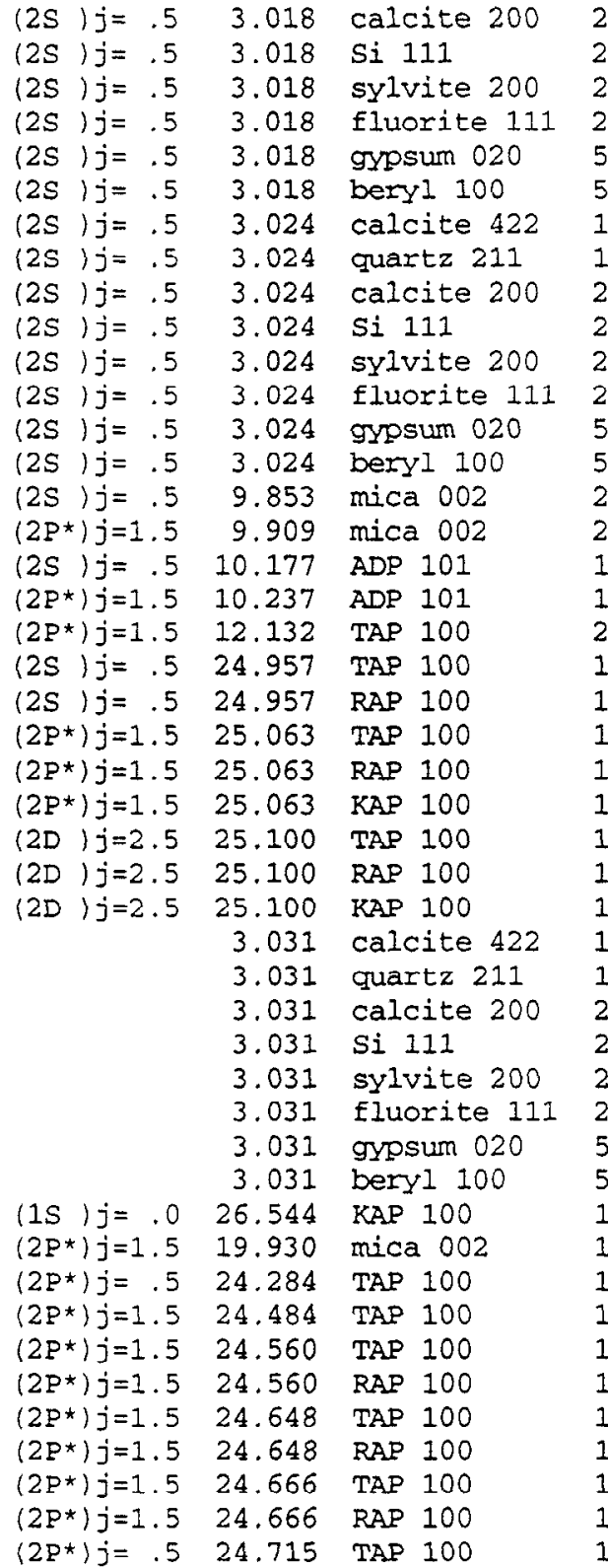

$6.071 \quad 83.845$

$6.271 \quad 74.265$

$6.292 \quad 73.600$

$6.308 \quad 73.113$

$15.185 \quad 83.588$

$15.954 \quad 71.057$

$3.034 \quad 85.347$

3.08278 .867

6.07185 .011

$6.271 \quad 74.674$

$6.292 \quad 73.991$

$6.308 \quad 73.492$

$15.185 \quad 84.697$

$15.954 \quad 71.392$

$19.942 \quad 81.177$

$19.942 \quad 83.607$

$10.640 \quad 73.035$

$10.640 \quad 74.180$

$25.763 \quad 70.359$

$25.763 \quad 75.630$

$26.116 \quad 72.867$

$25.763 \quad 76.613$

$26.116 \quad 73.674$

$26.634 \quad 70.223$

$25.763 \quad 76.973$

$26.116 \quad 73.966$

26.63470 .459

$3.034 \quad 87.452$

$3.082 \quad 79.562$

$6.071 \quad 86.880$

$6.271 \quad 75.166$

6.29274 .460

$6.308 \quad 73.946$

$15.185 \quad 86.398$

$15.954 \quad 71.790$

$26.634 \quad 85.288$

$19.942 \quad 88.012$

$25.763 \quad 70.492$

$25.763 \quad 71.870$

$25.763 \quad 72.422$

$26.116 \quad 70.122$

$25.763 \quad 73.082$

$\begin{array}{ll}26.116 & 70.698\end{array}$

$25.763 \quad 73.220$

$26.116 \quad 70.818$

$25.763 \quad 73.602$ 
Sc13 F-like 2S2 2P4( $1 D$ ) 3D (2D) $j=1.5-2 S 22 P 5$

Sc13 F-like 2S2 2P4 ( $1 D$ ) 3D (2S) $j=.5-2 S 22 P 5$

Sc13 F-like 2S2 2P4 ( $1 D$ ) 3D (2S) $j=.5-2 S 22 \mathrm{P} 5$

Sc13 F-like 2S2 2P4 ( $3 P$ ) 3D (2D) $j=2.5-2 S 22 P 5$

Sc13 F-like 2S2 2P4 ( $3 P$ ) 3D (2D) $j=2.5-2 S 22 P 5$

Sc13 F-like 2S2 2P4 (3P) 3D (2P) j=1.5 - 2S2 2P5

Sc13 $F$-iike 2S2 $2 P 4(3 P) 3 D(2 P) j=1.5-2 S 22 P 5$

Sc13 F-like 2S2 2P4 ( $3 P$ ) $3 D(2 F) j=2.5-2 S 22 P 5$

Sc13 F-like 2S2 2P4 (3P) 3D (2F) $j=2.5-2 S 22 P 5$

Sc13 F-like 2S2 2P4 (3P) 3D (2F) j=2.5-2S2 $2 \mathrm{P} 5$

Sc13 F-like 2S2 2P4 ( $3 P$ ) 3D (2D) $j=1.5-2 S 22 P 5$

Sc13 F-like 2S2 2P4 (3P) 3D (2D) $j=1.5-2 S 22 P 5$

$S c 13 \quad F-1$ ike 2S2 2P4 (3P) 3D (2D) $j=1.5-2 S 22 P 5$

Sc13 F-like 2S2 $2 \mathrm{P} 4$ (3P) 3D (4P) $j=2.5-2 S 22 \mathrm{P} 5$

Sc13 F-like 2S2 2P4 (3P) 3D (4P) $j=2.5-2 S 22 P 5$

SC13 F-1ike 2S2 2P4 (3P) 3D (4P) $j=2.5-2 S 22 P 5$

Sc13 $F$-like 2S2 2P4 ( $3 P$ ) $3 D(2 P) j=.5-2 S 22 P 5$

Sc13 F-like 2S2 2P4 ( $3 P$ ) 3D (2P) $j=.5-2 S 22 P 5$

Sc13 $F$-like 2S2 2P4 (3P) 3D (2P) $j=.5-2 S 22 P 5$

Sc13 F-like 2S2 2P4 (3P) 3D (4P) $j=1.5-2 S 22 P 5$

Sc13 $F$-like 2S2 2P4 ( 3P) 3D (4P) $j=1.5-2 S 22 \mathrm{P} 5$

Sc13 F-like 2S2 2P4 (3P) 3D (4P) $j=1.5-2 S 22 P 5$

ScI3 F-like 2S2 2P4 ( 3P) 3D (4P) $j=.5-2 S 22 P 5$

Sc13 F-like 2S2 2P4 (3P) 3D (4P) $j=.5-2 S 22 P 5$

Sc13 F-like 2S2 2P4 ( $3 P$ ) 3D (4P) $j=.5-2 S 22 P 5$

Sc13 F-like 2S2 2P4 (3P) 3D (2D) $j=1.5-2 S 22 P 5$

$S c 13 F-1 i k e$ 2S2 2PA (3P) 3D (2D) $j=1.5-2 S 22 P 5$

Sc13 F-like 2S2 2P4 ( 3P) 3D (2D) $j=1.5-2 S 22 P 5$

Sc13 $F$-like 2S2 2P4 ( $3 P$ ) 3D (2P) $j=.5-2 S 22 P 5$

SC13 F-like 2S2 2P4 (3P) 3D (2P) $j=.5-2 S 22 P 5$

Sc13 F-like 2S2 2P4 ( 3P) 3D (2P) $j=.5-2 S 22 P 5$

Sc13 F-like $2 S 22 P 4(3 P)$ 3D (4P) $j=1.5-2 S 22 P 5$

Sc13 F-like 2S2 2P4 (3P) 3D (4P) $j=1.5-2 S 22 P 5$

Sc13 F-like 2S2 2P4 ( 3P) 3D (4P) $j=1.5-2 S 2$ 2P5

Sc14 O-like 2S2 2P3 ( 2P*) 3S (1P*) $j=1.0-2 S 22 \mathrm{P} 4$

Sc14 0 -like 2S2 2P3 (2P*) $3 S\left(1 P^{*}\right) j=1.0-2 S 22 P^{*}$

Sc14 0 -like 2S2 2P3 ( $\left.2 P^{*}\right) 3 S\left(3 P^{*}\right) j=2.0-2 S 22 P^{*}$

Sc14 0 -like 2S2 $2 P^{2}\left(2 P^{*}\right) 3 S\left(3 P^{*}\right) j=2.0-2 S 22 P^{*}$

$S c 140$-like $2 S 22 P 3\left(2 D^{*}\right) 3 S\left(3 D^{*}\right) j=3.0-2 S 22 P 4$

Sc14 O-like 2S2 2P3 (2D*) 3S $\left(3 D^{*}\right) j=3.0-2 S 22 P 4$

Sc14 0 -like 2S2 2P3 ( $\left.2 D^{*}\right)$ 3S $\left(3 D^{*}\right) j=3.0-2 S 22 P 4$

Sc14 0 -like 2S2 2P3 ( $\left.2 P^{*}\right) 3 S\left(1 P^{*}\right) j=1.0-2 S 22 P 4$

Sc14 O-like 2S2 2P3 (2P*) 3S (1P*) $j=1.0-2 S 22 P 4$

Sc14 O-like 2S2 2P3 (2P*) 3S (1P*)j=1.0-2S2 $2 P^{*} 4$

$S c 140$-like $2 S 22 P 3\left(2 D^{*}\right) 3 S\left(3 D^{*}\right) j=2.0-2 S 22 P 4$

Sc14 0 -like $2 S 22 P 3\left(2 D^{*}\right) 3 S\left(3 D^{*}\right) j=2.0-2 S 22 P 4$ $\left\langle 2 P^{*}\right\rangle j=.5 \quad 24.899$ TAP 100

$\left\langle 2 P^{*}\right\rangle j=.5 \quad 24.899$ RAP 100

$\left(2 P^{\star}\right) j=1.5 \quad 24.970$ TAP 100

$\left(2 P^{*}\right) j=1.5 \quad 24.970 \quad$ RAP 100

$\left(2 P^{*}\right) j=1.5 \quad 24.998$ TAP 100

$\left(2 P^{*} j j=1.5 \quad 24.998 \quad\right.$ RAP 100

$\left(2 P^{*}\right) j=1.5 \quad 25.079$ TAP 100

$\left(2 P^{*}\right) j=1.5 \quad 25.079 \quad \operatorname{RAP} 100$

$\left(2 P^{*}\right) j=1.5 \quad 25.099 \quad$ TAP 100

$\left(2 P^{*}\right) j=1.5 \quad 25.099 \quad$ RAP 100

$\left(2 P^{*}\right) j=1.5 \quad 25.099$

$\left(2 P^{\star}\right) j=1.5 \quad 25.133 \quad$ TAP 100

$(2 P *) j=1.5 \quad 25.133 \quad$ RAP 100

(2P*) $j=1.5 \quad 25.133$ KAP 100

(2P*)

$\left(2 P^{*}\right) j=1.5 \quad 25.163$ RAP 100

$\left(2 P^{\star}\right) j=1.5 \quad 25.163 \quad \mathrm{KAP} 100$

$\left(2 P^{*}\right) j=1.5 \quad 25.200$ TAP 100

$\left(22^{*}\right) j=1.5 \quad 25.200 \quad$ RAP 100

$\left(2 P^{*}\right) j=1.5 \quad 25.200 \quad \operatorname{KAP} 100$

$\left(2 P^{*}\right) j=1.5 \quad 25.242$ TAP 100

$\left(2 P^{*}\right) j=1.5 \quad 25.242 \quad$ RAP 100

$\left(2 P^{*}\right) j=1.5 \quad 25.242$ KAP 100

$\left(2 P^{*}\right) j=.5 \quad 25.341$ TAP 100

$(2 P *) j=.5 \quad 25.341$ RAP 100

$\left(2 P^{*}\right) j=.5 \quad 25.341$ KAP 100

$\left(2 P^{*}\right) j=.5 \quad 25.400$ TAP 100

$(2 P *) j=.5 \quad 25.400$ RAP 100

$\left(2 P^{\star}\right) j=.5 \quad 25.400$ KAP 100

$\left(2 P^{*}\right) j=.525 .440$ TAP 100

$(2 P *) j=.5 \quad 25.440$ RAP 100

$\left(2 P^{*}\right) j=.5 \quad 25.440$ KAP 100

(3P) $j=2.0 \quad 24.791$ TAP 100

(3P) $j=2.0 \quad 24.791 \quad$ RAP 100

(3P) $j=2.0 \quad 24.971$ TAP 100

(3P) $j=2.0 \quad 24.971$ RAP 100

(3P) $j=2.0 \quad 25.392$ TAP 100

(3P ) $j=2.0 \quad 25.392$ RAP 100

(3P) $j=2.0 \quad 25.392 \quad K A P 100$

(1D) $j=2.0 \quad 25.403$ TAP 100

(ID ) $j=2.0 \quad 25.403 \quad$ RAP 100

(ID) $j=2.0 \quad 25.403$ KAP 100

(3P) $j=2.0 \quad 25.439$ TAP 100

(3P) $j=2.0 \quad 25.439 \quad$ RAP,100
$26.116 \quad 71.148$

$25.763 \quad 75.119$

$26.116 \quad 72.440$

$25.763 \quad 75.747$

$26.116 \quad 72.964$

$25.763 \quad 76.002$

$26.116 \quad 73.175$

$25.763 \quad 76.768$

$26.116 \quad 73.800$

$26.634 \quad 70.325$

$25.763 \quad 76.964$

$26.116 \quad 73.958$

$26.634 \quad 70.453$

$25.763 \quad 77.303$

$26.116 \quad 74.230$

$26.634 \quad 70.673$

$25.763 \quad 77.610$

$26.116 \quad 74.474$

$26.634 \quad 70.869$

$25.763 \quad 78.000$

$26.116 \quad 74.780$

$26.634 \quad 71.113$

$25.763 \quad 78.458$

$26.116 \quad 75.135$

$26.634 \quad 71.394$

$25.763 \quad 79.615$

$26.116 \quad 76.007$

$26.634 \quad 72.074$

$25.763 \quad 80.370$

$26.116 \quad 76.553$

$26.634 \quad 72.491$

$25.763 \quad 80.918$

$26.116 \quad 76.935$

$26.634 \quad 72.779$

$25.763 \quad 74.211$

$26.116 \quad 71.671$

$25.763 \quad 75.756$

$26.116 \quad 72.971$

$25.763 \quad 80.265$

$26.116 \quad 76.477$

$26.634 \quad 72.434$

$25.763 \quad 80.410$

$26.116 \quad 76.581$

$26.634 \quad 72.512$

$25.763 \quad 80.904$

$26.116 \quad 76.926$ 
Sc14 O-like $2 S 22 P^{2}\left(2 D^{*}\right) 3 S\left(3 D^{*}\right) j=2.0-2 S 22 P 4$ Sc14 O-like 2S2 $2 P^{2}\left(2 D^{*}\right) 3 S\left(3 D^{*}\right) j=2.0-2 S 22 P 4$ Sc14 O-like 2S2 $2 P^{3}\left(2 D^{*}\right) 3 S\left(3 D^{*}\right) j=2.0-2 S 22 P 4$ Sc14 O-like 2S2 $2 P^{2}\left(2 D^{*}\right) 3 S\left(3 D^{\star}\right) j=2.0-2 S 22 P 4$ Sc14 0 -like 2S2 $2 \mathrm{P}\left(2 \mathrm{D}^{*}\right) 3 \mathrm{~S}\left(3 \mathrm{D}^{*}\right) j=1.0-2 \mathrm{~S} 22 \mathrm{P} 4$ $S C 140$-like $2 S 22 P 3\left(2 D^{*}\right) 3 S\left(3 D^{*}\right) j=1.0-2 S 22 P 4$ Sc14 O-like 2S2 2P3 (2D*) 3S $\left(3 D^{*}\right) j=1.0-2 S 22 P 4$ Sc14 O-like $2 S 22 P^{2}\left(2 D^{\star}\right) 3 S\left(1 D^{\star}\right) j=2.0-2 S 22 P 4$ Sc14 0 -like $2 S 22 \mathrm{P} 3\left(2 D^{*}\right) 3 S\left(1 D^{*}\right) j=2.0-2 S 22 \mathrm{P} 4$ Sc14 O-like 2S2 2P3 ( $\left.4 S^{*}\right)$ 3S $\left(3 S^{*}\right) j=1.0-2 S 22 P 4$ Sc14 O-like 2S2 $2 P^{2} 3\left(S^{*}\right)$ 3S $\left(3 S^{*}\right) j=1.0-2 S 22 P 4$ Sc14 0 -like $2 S 22 P^{2}\left(2 P^{*}\right) 3 S\left(1 P^{*}\right) j=1.0-2 S 22 P^{*}$ Sc14 O-like 2S2 $2 P^{2}$ ( $\left.2 P^{*}\right)$ 3S (1P*) j=1.0 - 2S2 2P4 Sc14 O-like 2S2 $2 P^{2}$ ( $\left.4 S^{*}\right) 3 S^{*}\left(5 S^{*}\right) j=2.0-2 S 22 P 4$ Sc14 0 -like 2S2 $2 \mathrm{P} 3$ (4S*) $3 S\left(3 S^{*}\right) j=1.0-2 S 22 \mathrm{P} 4$ Sc14 0 -like $2 S 22 P^{2}\left(4 S^{\star}\right) 3 S\left(3 S^{\star}\right) j=1.0-2 S 22 P 4$ Sc14 O-like 2S2 2P3 ( $\left.4 S^{\star}\right) 3 S\left(5 S^{\star}\right) j=2.0-2 S 22 P 4$ Sc17 B-like 2S2 4S (2S ) $j=.5-2 S 2 \quad 2 P$ Sc17 B-like $2 S 2 P\left(3 P^{*}\right) 3 P(2 P) j=.5-2 S 22 P$ Sc17 B -like $2 S 2 P\left(3 P^{*}\right) 3 P(2 P) j=1.5-2 S 2 \quad 2 P$ Sc17 $B$-like $2 S$ 2P (1 $\left.P^{\star}\right) 3 D\left(2 F^{*}\right) j=3.5-2 S \quad 2 P 2$ Sc17 B-like 2S2 3D (2D) $j=1.5-2 S 2 \quad 2 P$ Sc17 B-like $2 S 2 P\left(3 P^{*}\right) 3 D\left(4 D^{*}\right) j=3.5-2 S 2 P 2$ Sc17 $B$-like 2S2 3D (2D) $j=2.5-2 S 2 \quad 2 P$ Sc17 B-like $2 S 2 P$ ( $\left.1 P^{*}\right) 3 D\left(2 D^{*}\right) j=2.5-2 S 2 P 2$ Sc17 B-like 2S 2P (3P*) 3D $\left(2 F^{*}\right) j=3.5-2 S$ 2P2 Sc17 B-like 2S 2P (3P*) 3D $\left(2 F^{*}\right) j=2.5-2 S$ 2P2 Sc18 Be-like $1 S 2$ 2S 3D (1D) j=2.0 - 1S2 $2 S$ 2P Sc19 Li-like $1 S 2 P\left(3 P^{*}\right) \quad 3 P(2 S) j=.5-1 S 23 P$ Sc19 Li-like is $2 P\left(3 P^{*}\right) \quad 3 P(2 S) j=.5-1 S 23 P$ Sc19 Li-like is $2 P\left(3 P^{*}\right) \quad 3 P(2 S) j=.5-1 S 23 P$ Sc19 Li-like $1 S \quad 2 P\left(3 P^{\star}\right) \quad 3 P(2 S) j=.5-1 S 23 P$ Sc19 Li-like is $2 P\left(3 P^{*}\right) \quad 3 P(2 S) j=.5-$ IS2 $3 P$ Sc19 Ii-like 1S $2 P 2 \quad$ (2S) $j=.5-1 S 22 P$ Sc19 Li-like 1S 2P2 (2S) $j=.5-1 S 22 \mathrm{P}$

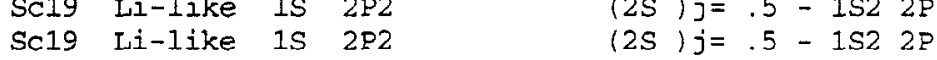
Sc19 Li-1ike 1S 2P2 (2S) $j=.5-1 S 22 \mathrm{P}$ Sc19 Li-like 1S 2P2 (2S) $j=.5-1 S 22 P$ Sc19 Li-like 1S $2 P 2 \quad(2 S) j=.5-1 S 22 P$ Sc19 Ii-like $1 S$ 2P2 (2S ) j=.5 - 1S2 2P Sc19 Ii-like 1S 2P2 (2S) $j=.5-1 S 22 P$ Sc19 Li-like 1S 2P2 (2S ) $j=.5-1 S 22 P$ Sc19 Li-like 1S (2S 2P ( $\left.\left.1 P^{*}\right)\right)\left(2 P^{*}\right) j=1.5-$ IS2 $2 S$ Sc19 Ii-like 1S (2S 2F (1.P*)) $\left(2 P^{*}\right) j=1.5-1 S 22 S$ Sc19 Li-like $1 S\left(2 S 2 P\left(1 P^{*}\right)\right)\left(2 P^{*}\right) j=1.5-1 S 22 S$
(3P) $j=2.0 \quad 25.439 \quad \mathrm{KAP} 100$ (3P) $j=1.0 \quad 25.644$ TAP 100 (3P) $j=1.0 \quad 25.644$ RAP 100 (3P) $j=1.0 \quad 25.644 \quad \mathrm{KAP} 100$ $(3 P) j=.0 \quad 25.680$ TAP 100 (3P) $j=.0 \quad 25.680$ RAP 100 (3P) $j=.025 .680$ KAP 100 (ID) $j=2.0 \quad 25.921$ RAP 100 (1D) $j=2.0 \quad 25.921 \quad K A P \quad 100$ (3P) $j=2.0 \quad 25.985 \quad \operatorname{RAP} 100$ (3P) $j=2.0 \quad 25.985$ KAP 100 (1S ) $j=.0 \quad 26.056 \quad \operatorname{RAP} 100$ (1S) $j=.0 \quad 26.056 \mathrm{KAP} 100$ (3P) $j=2.0 \quad 26.150$ KAP 100 (3P ) $j=1.0 \quad 26.197 \quad \mathrm{KAP} 100$ (3P) $j=.0 \quad 26.224$ KAP 100 (3P) $j=1.0 \quad 26.370$ KAP 100 (2P*) $j=1.5 \quad 14.990$ gypsum 020 $\left(2 P^{*}\right) j=.5 \quad 18.780$ mica 002 $\left(2 P^{\star}\right) j=1.5 \quad 18.830$ mica 002 (2D) $j=2.5 \quad 19.069$ mica 002 $\left(2 P^{*}\right) j=.5 \quad 19.160$ mica 002 (4P) $j=2.5 \quad 19.220$ mica 002 $\left(2 P^{\star}\right) j=1.5 \quad 19.311$ mica 002 (2P) $j=1.5 \quad 19.598$ mica 002 (2D) $j=2.5 \quad 19.651$ mica 002 (2D) $j=1.5 \quad 19.732$ mica 002 $\left(1 P^{*}\right) j=1.0 \quad 18.959$ mica 002 $\left(2 P^{*}\right) j=1.5 \quad 2.875$ calcite 422 $\left(2 P^{\star}\right) j=1.5 \quad 2.875$ calcite 200 $\left(2 P^{\star}\right) j=1.5 \quad 2.875 \quad$ PET 002

(2P*) $j=1.5 \quad 2.875 \quad$ EDT 020

$\left(2 P^{*}\right) j=1.5 \quad 2.875$ gypsum 020

$\left(2 P^{*}\right) j=5 \quad 2.878$ calcite 422

$\left(2 P^{*}\right) j=.5 \quad 2.878$ calcite 200

$\left(2 P^{*}\right) j=.5 \quad 2.878 \quad$ PET 002

$\left(2 P^{*}\right) j=.5 \quad 2.878 \quad$ EDT 020

$\left(2 P^{*}\right) j=.5$

$\left(2 P^{\star}\right) j=1.5$

$\left(2 P^{*}\right) j=1.5$

$\left(2 P^{\star}\right) j=1.5$

2.878 gypsum 020

2.882 calcite 422

2.882 calcite 200

2.882 PET 002

2.882 EDT 020

2.882

gypsum 020

(2S) $j=.5$

2.884 calcite 422

2,884 calcite 200

2.884 PET 002
$26.634 \quad 72.772$

$25.763 \quad 84.491$

$26.116 \quad 79.090$

$26.634 \quad 74.329$

$25.763 \quad 85.400$

$26.116 \quad 79.516$

26.63474 .619

$26.116 \quad 82.994$

26.63476 .713

$26.116 \quad 84.259$

$26.634 \quad 77.326$

$26.116 \quad 86.115$

26.63478 .042

$26.634 \quad 79.060$

$26.634 \quad 79.607$

$26.634 \quad 79.934$

$26.634 \quad 81.926$

$15.185 \quad 80.808$

$19.942 \quad 70.344$

$19.942 \quad 70.776$

$19.942 \quad 72.984$

$19.942 \quad 73.901$

$19.942 \quad 74.535$

$19.942 \quad 75.548$

$19.942 \quad 79.342$

$19.942 \quad 80.200$

$19.942 \quad 81.678$

$19.942 \quad 71.935$

$3.034 \quad 71.369$

6.07171 .285

$8.742 \quad 80.616$

$8.808 \quad 78.300$

$\begin{array}{ll}15.185 & 71.202\end{array}$

3.03471 .547

$6.071 \quad 71.462$

$8.742 \quad 80.984$

$8.808 \quad 78.592$

$15.185 \quad 71.378$

3.03471 .787

6.07171 .701

$8.742 \quad 81.501$

$8.808 \quad 78.994$

$15.185 \quad 71.616$

$3.034 \quad 71.908$

$6.071 \quad 71.822$

$8.742 \quad 81.771$ 
Sc19 Li-like 1S (2S 2P ( 1P* $))\left(2 P^{*}\right) j=1.5-1 S 2$ 2S

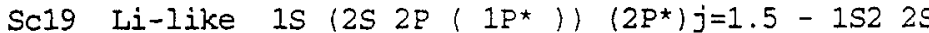
(2P) $j=1.5-1 S 22 P$ Sc19 Li-Iike is 2P2 Sc19 Li-like 1S. 2P2

Sc19 Li-like 1S 2F2 Sc19 Li-like 1S $2 P 2$ Sc19 Li-like is 2P2

Sc19 Li-like 1S $2 P_{2}$

Sc19 Li-like $1 \mathrm{~S} 2 \mathrm{p} 2$

Sc19 Ii-like 1S 2P2

$\operatorname{Sc} 19$ Li-like is $2 \mathrm{P} 2$

Sc19 Li-like 1S 2P2

Sc19 Ii-like 152 9P

Sc19 Li-like 1S2 8P

Sc19 Li-like 1S2 9D

Sc19 Li-like 1S2 7P

Sc19 Li-like 1S2 9D

Sc19 Li-like 1S2 8D

Sc19 Li-like 1S2 8D

Sc19 Li-like 1S2 $4 \mathrm{P}$

Sc19 Li-like 1S2 4P

Sc19 Ii-like 1S2 4P

Sc19 Li-like 1S2 $4 \mathrm{P}$

Sc19 Li-like 1S2 4P

Sc19 Li-like 1S2 $4 P$

Sc19 Li-like 1S2 4D

Sc19 Li-1ike 1S2 4D

Sc19 Li-like 152 4S

Sc20 He-like 15 6P

Sc20 He-like 1S $6 P$

Sc20 He-like 1S $6 \mathrm{P}$

Sc20 He-like 1S 6P

Sc20 He-like 1s 6P

Sc20 He-1ike 1S 5P

Sc20 He-like is 5P

Sc20 He-like 1S $5 P$

Sc20 He-like 1S 5P

Sc20 He-like 15 5P

Sc20 He-like 1S $4 P$

Sc20 He-like 1S $4 \mathrm{P}$

Sc20 He-like 15 4P

Sc20 He-like 1S $4 \mathrm{P}$

Sc20 He-like is $3 P$

$\mathrm{Sc} 20$ He-like $1 \mathrm{~S}$ 3P

Sc20 He-1ike is 3P (2P) $j=1.5-1 S 22 P$ (2P ) $j=1.5-1 S 22 P$ (2P) $j=1.5-1 S 22 \mathrm{P}$ (2P) $j=1.5-1 S 22 \mathrm{P}$ (2D) $j=2.5-1 S 22 P$ (2D) $j=2.5-1 S 22 P$ (2D ) $j=2.5-1 S 22 P$ (2D) $j=2.5-1 S 22 \mathrm{P}$ (2D) $j=2.5-1 S 22$ ? (2D) $j=2.5-1 S 22 P$ $\left(2 \mathrm{P}^{*}\right) j=1.5-1 S 22 S$ $\left(2 P^{*}\right) j=1.5-1 S 22 S$ (2D) j=1.5 - 1S2 $2 P$ $\left(2 P^{*}\right) j=1.5-1 S 22 S$ (2D) $j=2.5-1 S 22 P$ (2D) $j=1.5-1 S 22 P$ (2D) $j=2.5-1 S 22 P$ $\left(2 R^{\star}\right) j=1.5-1522 S$ $\left(2 P^{\star}\right) j=1.5-1 S 22 S$ $\left(2 P^{*}\right) j=1.5-1 S 22 S$ $\left(2 P^{\star}\right) j=.5-1 S 22 S$ $\left(2 P^{*}\right) j=.5-1 S 22 S$ $\left(2 P^{*}\right) j=.5-15225$ (2D) $j=1.5-1 S 22 P$ (2D) $j=2.5-1 S 22 P$ (2S $j=.5-1 S 22 P$ $\left(1 p^{*}\right) j=1.0-152$ $\left(1 P^{\star}\right) j=1.0-152$ $\left(1 P^{\star}\right) j=1.0-1 S 2$ $\left(1 P^{*}\right) j=1.0-1 S 2$ $\left(1 P^{\star}\right) j=1.0-1 S 2$ $\left(1 P^{*}\right) j=1.0-1 \mathrm{~S} 2$ $\left(1 P^{*}\right) j=1.0-1 S 2$ $\left(1 P^{\star}\right) j=1.0-152$ $\left(1 P^{\star}\right) j=1.0-152$ $\left(1 P^{*}\right) j=1.0-1 S 2$ (1P*) $j=1.0-1 S 2$ $\left(1 P^{*}\right) j=1.0-1 S 2$ $\left(1 P^{\star}\right) j=1.0-1 S 2$ $\left(1 P^{*}\right) j=1.0-1 \mathrm{~S} 2$ $\left(1 P^{*}\right) j=1.0-1 S 2$ $\left(1 P^{\star}\right) j=1.0-1 S 2$ (1P*) $j=1.0-1 S 2$

\begin{tabular}{|c|c|c|}
\hline$(2 s) j=.5$ & 2.884 & EDT 020 \\
\hline$(2 S) j=.5$ & 2.884 & gypsum 020 \\
\hline *) $j=1.5$ & 2.896 & calcite 422 \\
\hline *) $j=1.5$ & 2.896 & calcite 200 \\
\hline$j=1.5$ & 2.896 & PET 002 \\
\hline *) $j=1.5$ & 2.896 & EDT 020 \\
\hline$\left.P^{*}\right) j=1.5$ & 2.896 & gypsum 020 \\
\hline$\left.P^{*}\right) j=1.5$ & 2.899 & calcite 422 \\
\hline$\left.P^{*}\right) j=1.5$ & 2.899 & quartz 211 \\
\hline$\left.p^{*}\right) j=1.5$ & 2.899 & calcite 200 \\
\hline$\left.?^{*}\right) j=1.5$ & 2.899 & PET 002 \\
\hline$\left.P^{*}\right) j=1.5$ & 2.899 & EDT 020 \\
\hline$\left.P^{\star}\right) j=1.5$ & 2.899 & gypsum 020 \\
\hline$(2 S) j=.5$ & 10.104 & ADP 101 \\
\hline S $j j=.5$ & 10.240 & ADP 101 \\
\hline$\left.P^{*}\right) j=.5$ & 10.426 & ADP 101 \\
\hline $2 s) j=5$ & 10.443 & ADP 101 \\
\hline$\left.P^{\star}\right) j=1.5$ & 10.481 & ADP 101 \\
\hline$\left.P^{\star}\right) j=.5$ & 10.576 & ADP 101 \\
\hline$\left.p^{*}\right) j=1.5$ & 10.628 & ADP 101 \\
\hline$S / j=.5$ & 12.667 & $\operatorname{TAP} 100$ \\
\hline $2 S) j=.5$ & 12.667 & RAP 100 \\
\hline$(2 S) j=.5$ & 12.667 & KAP 100 \\
\hline $2 S / j=.5$ & 12.678 & $\operatorname{TAP} 100$ \\
\hline S $) j=.5$ & 12.678 & RAP 100 \\
\hline $25 / j=.5$ & 12.678 & KAP 100 \\
\hline $2 P^{*} / j=.5$ & 13.156 & KAP 100 \\
\hline $\left.2 P^{\star}\right) j=1.5$ & 13.241 & KAP 100 \\
\hline$\left.P^{*}\right) j=.5$ & 13.250 & KAP 100 \\
\hline$j j=.0$ & 2.245 & Ge 422 \\
\hline $.5 / j=.0$ & 2.245 & quartz 310 \\
\hline$s) j=.0$ & 2.245 & quartz 112 \\
\hline Ls $/ j=.0$ & 2.245 & topaz 200 \\
\hline$(1 s) j=.0$ & 2.245 & Al 111 \\
\hline$s) j=.0$ & 2.272 & Ge 422 \\
\hline$j j=.0$ & 2.272 & quartz 310 \\
\hline$j j=.0$ & 2.272 & quartz 112 \\
\hline$j=.0$ & 2.272 & topaz 200 \\
\hline$j J=.0$ & 2.272 & Al 111 \\
\hline$j=.0$ & 2.324 & quartz 310 \\
\hline$j j=.0$ & 2.324 & quartz 220 \\
\hline$j j=.0$ & 2.324 & Al 111 \\
\hline$j=.0$ & 2.324 & guartz 110 \\
\hline$j j=.0$ & 2.445 & quartz 220 \\
\hline$j j=.0$ & 2.445 & quartz 110 \\
\hline & & in \\
\hline
\end{tabular}

$8.808 \quad 79.201$ $15.185 \quad 71.736$ $3.034 \quad 72.653$

$6.071 \quad 72.562$

$8.742 \quad 83.628$

$8.808 \quad 80.531$

$\begin{array}{ll}15.185 & 72.473\end{array}$

$3.034 \quad 72.844$

3.08270 .156

$6.071 \quad 72.752$

$8.742 \quad 84.184$

$8.808 \quad 80.894$

$15.185 \quad 72.661$

$10.640 \quad 71.736$

$10.640 \quad 74.240$

$10.640 \quad 78.489$

$10.640 \quad 78.957$

$10.640 \quad 80.082$

$10.640 \quad 83.713$

$10.640 \quad 87.279$

$25.763 \quad 79.529$

$26.116 \quad 75.944$

$6.634 \quad 72.025$

$25.763 \quad 79.802$

$26.116 \quad 76.144$

$26.634 \quad 72.179$

$26.634 \quad 81.082$

$26.634 \quad 83.876$

$26.634 \quad 84.250$

$2.310 \quad 76.376$

$2.360 \quad 72.040$

$4.564 \quad 79.668$

$4.638 \quad 75.487$

$4.676 \quad 73.785$

$2.310 \quad 79.593$

$2.360 \quad 74.304$

$4.564 \quad 84.634$

$4.638 \quad 78.445$

$4.676 \quad 76.354$

$2.360 \quad 79.980$

$2.451 \quad 71.475$

$4.676 \quad 83.727$

4.91271 .130

$2.451 \quad 85.990$

$4.912 \quad 84.575$

$4.990 \quad 78.510$ 


\begin{tabular}{|c|c|c|}
\hline $\mathrm{Sc} 20$ & He-like & 15 \\
\hline $\mathrm{Sc} 20$ & He-like & 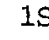 \\
\hline Sc20 & He-like & 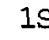 \\
\hline $\mathrm{Sc} 20$ & He-like & 1. \\
\hline Sc20 & He-like & \\
\hline $\operatorname{se} 20$ & He-like & \\
\hline $\mathrm{Sc} 20$ & He-like & \\
\hline $\mathrm{Sc} 20$ & He-Iike & \\
\hline Sc20 & He-like & \\
\hline $\mathrm{Sc} 20$ & He-like & \\
\hline Sc20 & He-Iike & \\
\hline Sc20 & He-like & \\
\hline Sc20 & He-like & \\
\hline Sc20 & He-like & \\
\hline Sc20 & He-like & \\
\hline $\mathrm{Sc} 20$ & He-1ike & \\
\hline Sc20 & He-like & \\
\hline Sc20 & He-Iike & \\
\hline$S c 21$ & $\mathrm{H}$-like & \\
\hline$S c 21$ & H -like & \\
\hline Sc21 & H-like & $5 B$ \\
\hline Sc21 & $\mathrm{H}$-like & 5 \\
\hline $\operatorname{Sc} 21$ & H -like & 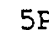 \\
\hline Sc21 & $\mathrm{H}$-like & $5 F$ \\
\hline Sc2I & H-like & \\
\hline $\operatorname{Sc} 21$ & $\mathrm{H}-\mathrm{like}$ & $5 F$ \\
\hline $\operatorname{Sc} 21$ & H -like & \\
\hline$S c 21$ & H -like & \\
\hline Sc21 & H -like & \\
\hline$S c 21$ & H -like & 4 \\
\hline $\operatorname{se} 21$ & H -like & 45 \\
\hline Sc21 & $\mathrm{H}$-like & $4 \mathrm{E}$ \\
\hline $\operatorname{Sc} 21$ & H -like & $4 E$ \\
\hline $\operatorname{Sc} 21$ & $H$-like & 45 \\
\hline $\operatorname{Sc} 21$ & $\mathrm{H}$-like & $4 \mathrm{~F}$ \\
\hline $\mathrm{Sc} 21$ & $\mathrm{H}-1 \mathrm{ike}$ & \\
\hline $\operatorname{Sc} 21$ & H -like & $4 \mathrm{E}$ \\
\hline Sc21 & H -like & \\
\hline $\operatorname{Sc} 21$ & $\mathrm{H}-\mathrm{like}$ & 7 \\
\hline $\operatorname{Sc} 21$ & H -like & \\
\hline Sc21 & H -like & 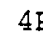 \\
\hline Sc21 & H -like & \\
\hline $\mathrm{Sc} 21$ & $\mathrm{H}-1$ ike & \\
\hline Sc21 & H -like & \\
\hline Sc21 & $\mathrm{H}-\mathrm{like}$ & \\
\hline 21 & H -1ike & \\
\hline
\end{tabular}

$\left(1 P^{*}\right) j=1.0-152$ $\left(1 P^{*}\right) j=1.0-1 S 2$ $\left(1 P^{*}\right) j=1.0-152$ $\left(1 P^{\star}\right) j=1.0-152$ $\left(1 P^{*}\right) j=1.0-152$ $\left(1 P^{*}\right) j=1.0-152$ $\left(3 E^{\star}\right) j=1.0-152$ $\left(3 P^{*}\right) j=1.0-1 S 2$ $(3 P *) j=1.0-152$ $\left(3 F^{*}\right) j=1.0-152$ $\left(3 P^{*}\right) j=1.0-1 S 2$ (3s) $j=1.0-152$ (3s) $j=1.0-1 s 2$ (3s) $j=1.0-1 S 2$ (3s) $j=1.0-1 s 2$ (3S) $j=1.0-1 S 2$ (3S) $j=1.0-1 S 2$ $\left(3 P^{*}\right) j=2.0-1 \mathrm{~s}$ $\left(2 P^{*}\right) j=1.5-1 S$ $\left(2 P^{*}\right) j=1.5-1 S$ $\left(2 P^{*}\right) j=1.5-15$ $\left(2 P^{\star}\right) j=1.5-1 S$ $\left(2 P^{*}\right) j=1.5-1 S$ $\left(2 P^{*}\right) j=1.5-1 S$ $\left(2 P^{*}\right) j=1.5-15$ $\left(2 P^{\star}\right) j=1.5-15$ $\left(2 P^{\star}\right) j=1.5-15$ $\left(2 P^{\star}\right) j=1.5-1 S$ $\left(2 P^{\star}\right) j=1.5-1.5$ $\left(2 P^{*}\right) j=1.5-1 S$ $\left(2 P^{*}\right) j=1.5-1 S$ $\left(2 P^{*}\right) j=1.5-15$ $\left(2 P^{*}\right) j=1.5-15$ $\left(2 P^{\star}\right) j=1.5-15$ $\left(2 P^{\star}\right) j=1.5-15$ $\left(2 P^{*}\right) j=.5-1 S$ $\left(2 P^{*}\right) j=5-1 S$ $\left(2 P^{*}\right) j=.5-1 S$ $\left(2 P^{*}\right) j=.5-1 S$ $\left(2 P^{*}\right) j=.5-15$ $\left(2 P^{*}\right) j=.5-15$ $\left(2 P^{*}\right) j=.5-1 S$ $\left(2 P^{\star}\right) j=.5-15$ $\left(2 P^{*}\right) j=.5-1 S$ $\left(2 P^{\star}\right) j=1.5-1 S$ $\left(2 P^{\star}\right) j=1.5-15$

\begin{tabular}{|c|c|c|c|c|}
\hline (1S & $j j=$ & .0 & 2.445 & InSb 111 \\
\hline (1S & $j j=$ & .0 & 2.872 & calcite 422 \\
\hline (1S & $j j=$ & .0 & 2.872 & calcite 200 \\
\hline (1S & $j j=$ & .0 & 2.872 & PET 002 \\
\hline (1S & $j=$ & .0 & 2.872 & EDT 020 \\
\hline (1s & $j=$ & .0 & 2.872 & gypsum 020 \\
\hline 15 & $j=$ & .0 & 2.886 & calcite 422 \\
\hline $1 \mathrm{~s}$ & $j=$ & .0 & 2.886 & calcite 200 \\
\hline 5 & $j j=$ & .0 & 2.886 & PET 002 \\
\hline$s$ & $j=$ & .0 & 2.886 & EDI 020 \\
\hline (1S & $j=$ & .0 & 2.886 & gypsum 020 \\
\hline (1S & $j=$ & .0 & 2.903 & calcite 422 \\
\hline (1S & $j=$ & .0 & 2.903 & quartz 211 \\
\hline (1) & $j=$ & .0 & 2.903 & calcite 200 \\
\hline is & $j=$ & .0 & 2.903 & PET 002 \\
\hline (1S & $j=$ & .0 & 2.903 & EDT 020 \\
\hline . & $j=$ & .0 & 2.903 & gypsum 020 \\
\hline 35 & $j=1$ & .0 & 15.610 & beryl 100 \\
\hline is & $j j=$ & .5 & 2.140 & Si 422 \\
\hline s & $j=$ & .5 & 2.140 & tungsten 110 \\
\hline is & $j=$ & .5 & 2.140 & Ge 111 \\
\hline S & $j=$ & .5 & 2.140 & $\mathrm{KBr} 200$ \\
\hline $12 S$ & $j=$ & .5 & 2.140 & quartz 101 \\
\hline$\langle 2 S$ & $j=$ & .5 & 2.140 & graphite 002 \\
\hline (2S & $j=$ & .5 & 2.140 & PET 002 \\
\hline $12 S$ & $j=$ & .5 & 2.140 & EDT 020 \\
\hline (2S & $j=$ & .5 & 2.191 & Si 422 \\
\hline $12 S$ & $j=$ & .5 & 2.191 & Ge 422 \\
\hline $12 \mathrm{~s}$ & $j=$ & .5 & 2.191 & tungsten 110 \\
\hline 〈2S & $j=$ & .5 & 2.191 & quartz 112 \\
\hline$(2$ & $j=$ & .5 & 2.191 & topaz 200 \\
\hline & $j=$ & .5 & 2.191 & $\mathrm{KBr} 200$ \\
\hline & $j=$ & .5 & 2.191 & quartz 101 \\
\hline $2 \mathrm{~S}$ & $j=$ & .5 & 2.191 & graphite 002 \\
\hline $12 S$ & $j=$ & .5 & 2.191 & EDT 020 \\
\hline (2S & $j=$ & .5 & 2.192 & Si 422 \\
\hline$(2 S$ & $j=$ & 5 & 2.192 & Ge 422 \\
\hline $12 S$ & $j=$ & .5 & 2.192 & tungsten 110 \\
\hline 0 & $j=$ & .5 & 2.192 & quartz 112 \\
\hline SS & $j=$ & .5 & 2.192 & topaz 200 \\
\hline 12 & $j=$ & .5 & 2.192 & $\mathrm{KBr} 200$ \\
\hline & $j=$ & .5 & 2.192 & guartz 101 \\
\hline & $j=$ & .5 & 2.192 & graphite 002 \\
\hline & $j=$ & .5 & 2.192 & EDT 020 \\
\hline & $i=$ & .5 & 2.311 & quartz 310 \\
\hline & $j=$ & .5 & 2.311 & guartz 220 \\
\hline
\end{tabular}

$\begin{array}{ll}7.481 & 78.662\end{array}$

$3.034 \quad 71.192$

6.07171 .109

$8.742 \quad 80.260$

$8.808 \quad 78.015$

$15.185 \quad 71.027$

$3.034 \quad 72.030$

6.07171 .943

$8.742 \quad 82.051$

$8.808 \quad 79.411$

$15.185 \quad 71.857$

$3.034 \quad 73.102$

$\begin{array}{ll}3.082 & 70.377\end{array}$

6.07173 .009

$8.742 \quad 85.020$

$8.808 \quad 81.402$

$15.185 \quad 72.916$

$15.954 \quad 78.080$

$2.217 \quad 74.855$

$4.476 \quad 72.982$

6.53279 .375

6.58477 .185

$6.687 \quad 73.755$

$6.696 \quad 73.492$

$8.742 \quad 78.288$

$8.808 \quad 76.371$

$2.217 \quad 81.217$

$2.310 \quad 71.529$

$4.476 \quad 78.237$

$\begin{array}{lll}4.564 & 73.765\end{array}$

4.63870 .875

$6.584 \quad 86.688$

$6.687 \quad 79.405$

$6.696 \quad 79.001$

$8.808 \quad 84.271$

$2.217 \quad 81.387$

$2.310 \quad 71.608$

$4.476 \quad 78.363$

$4.564 \quad 73.855$

$4.638 \quad 70.950$

$6.584 \quad 87.175$

$6.687 \quad 79.546$

$6.696 \quad 79.136$

$8.808 \quad 84.537$

$2.360 \quad 78.304$

$2.451 \quad 70.541$ 


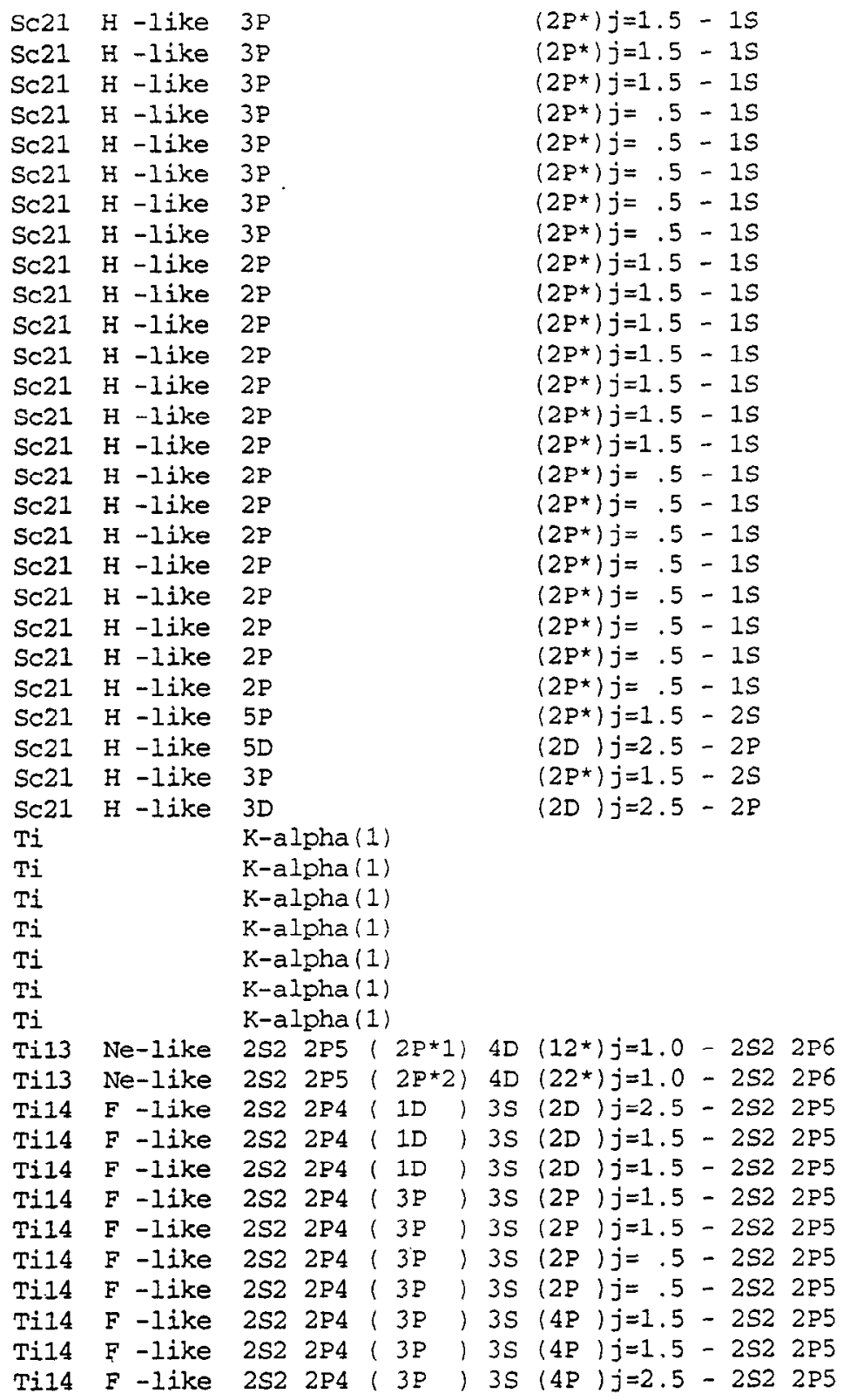

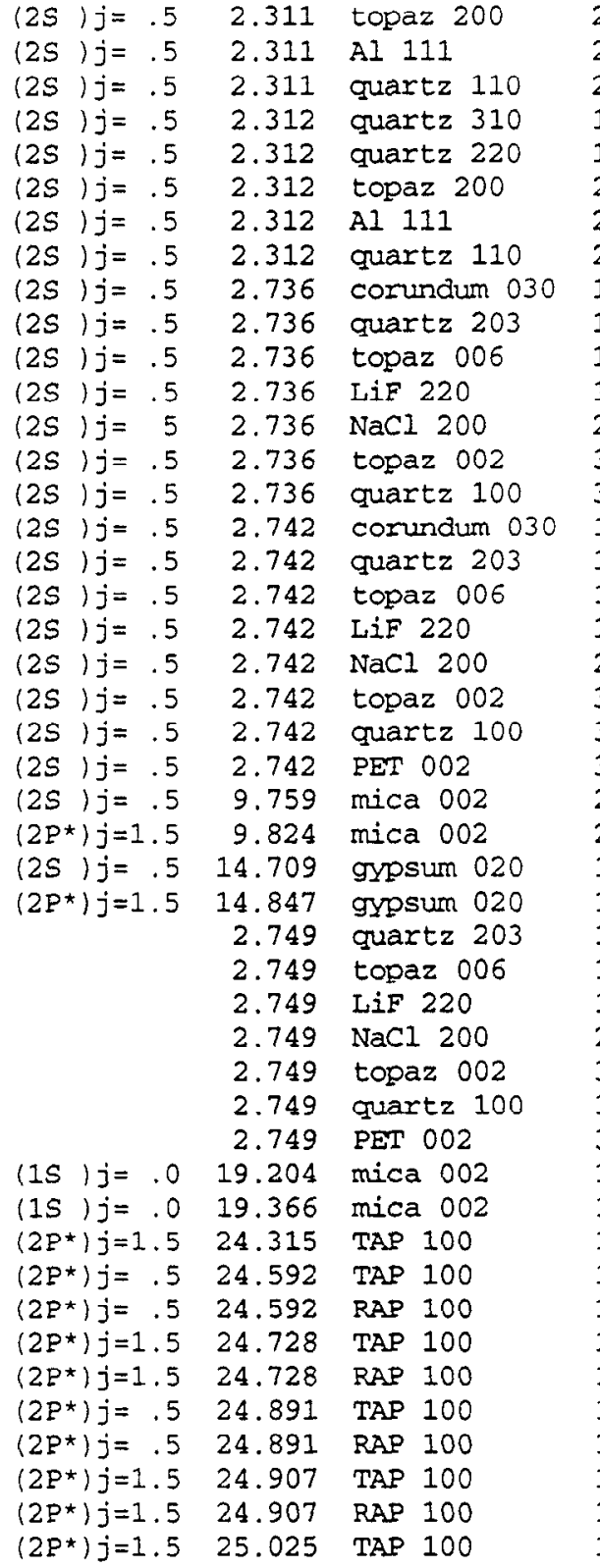

$4.638 \quad 85.239$

$4.676 \quad 81.284$

4.91270 .214

$2.360 \quad 78.424$

2.45170 .611

$4.638 \quad 85.547$

$4.676 \quad 81.447$

$4.912 \quad 70.283$

$2.748 \quad 84.644$

$2.749 \quad 84.426$

$2.795 \quad 78.207$

$2.848 \quad 73.878$

$5.641 \quad 75.940$

8.37478 .573

$8.512 \quad 74.641$

$2.748 \quad 86.213$

$2.749 \quad 85.910$

$2.795 \quad 78.824$

$2.848 \quad 74.319$

5.64176 .451

$8.374 \quad 79.212$

8.51275 .105

8.74270 .216

19.94278 .164

$19.942 \quad 80.149$

$15.185 \quad 75.616$

$15.185 \quad 77.889$

$2.749 \quad 89.022$

$2.795 \quad 79.545$

$2.848 \quad 74.818$

5.64177 .035

$8.374 \quad 79.961$

8.51275 .633

$8.742 \quad 70.603$

$19.942 \quad 74.364$

$19.942 \quad 76.196$

$25.763 \quad 70.699$

$25.763 \quad 72.659$

$26.116 \quad 70.330$

$25.763 \quad 73.704$

$26.116 \quad 71.236$

$25.763 \quad 75.050$

$26.116 \quad 72.382$

$25.763 \quad 75.189$

$26.116 \quad 72.498$

$25.763 \quad 76.253$ 
Ti14 F-like 2S2 2P4 ( $3 P$ ) 3S (4P) j=2.5-2S2 $2 P 5$ Ti14 F-like 2S2 $2 P 4(3 P) 3 S(4 P) j=.5-2 S 22 P 5$ Ti14 F-like 2S2 2P4 (3P) $3 S(4 P) j=.5-2 S 22 P 5$ Ti14 F-like 2S2 2P4 ( $3 P$ ) 3S (4P) $j=.5-2 S 22 P 5$ Ti14 F-like 2S 2P5 $\left(3 P^{*}\right) 3 S\left(2 P^{*}\right) j=.5-2 S 2 P 6$ Ti14 $F$-like $2 S$ 2P5 (3P*) $3 S\left(2 P^{\star}\right) j=.5-2 S \quad 2 P 6$ Ti14 F-like $2 S 2 P 5\left(3 P^{\star}\right) 3 S\left(2 P^{\star}\right) j=.5-2 S 2 P 6$ Ti14 F-like 2S2 2PA ( 3P) 3S (4P) $j=1.5-2 S 22 P 5$ Ti14 $F$-like 2S2 $2 P 4(3 P)$ 3S (4P) $j=1.5-2 S 22 P 5$ Ti14 F-like 2S2 2P4 (3P) 3S (4P) $j=1.5-2 S 2$ 2P5 Ti14 F-like $2 S$ 2P5 ( $\left.3 P^{*}\right)$ 3S $\left(2 P^{*}\right) j=1.5-2 S \quad 2 P 6$ Ti14 F-like 2S 2P5 (3P*) $3 S\left(2 P^{*}\right) j=1.5-2 S 2 P 6$ Ti14 $F$-like $2 S$ 2P5 ( $\left.3 P^{*}\right) 3 S\left(2 P^{*}\right) j=1.5-2 S$ 2P6

Ti15 o-iike

Ti15 o-like

Ti15 0 -like

Ti15 0 -like

Ti15 o-like

Ti15 0 -like

Ti15 0 -like

Ti16 N-like 2S2 2P2 ( $1 S$ ) 3D (2D) $j=1.5-2 S 22 P 3$

Ti16 $N$-like 2S2 2P2 ( $3 P$ ) $3 D(4 P) j=1.5-2 S 22 P 3$

Ti16 $N$-like $2 S 22 P 2$ ( $3 P$ ) $3 D(4 P) j=2.5-2 S 22 P 3$

Ti16 $N$-like $2 S 22 P 2$ ( ID) $3 D(2 F) j=3.5-2 S 22 P 3$

Til6 $N$-like 2S2 2P2 (1D) 3D (2D) j $j=2.5-2 S 22 P 3$

Ti16 $N$-like 2S2 2P2 ( $3 P$ ) 3D (2D) $j=2.5-2 S 22 P 3$

Ti16 N-1ike 2S2 2P2 (1D) 3D (2P) $j=1.5-2 S 2$ 2P3

Ti16 $N$-like $2 S 22 P 2(3 P) 3 D(2 F) j=3.5-2 S 22 P 3$

Ti16 N-like $2 S 22 P 2$ ( $1 D$ ) $3 D(2 D) j=2.5-2 S 22 P 3$

Ti16 $N$-like $2 S 22 \mathrm{P} 2(3 \mathrm{P}) 3 \mathrm{D}(2 \mathrm{D}) \mathrm{j}=2.5-2 S 22 \mathrm{P} 3$

Ti17 $C$-like 2S2 $2 \mathrm{P} \quad 3 \mathrm{D} \quad\left(3 F^{*}\right) j=2.0-2 S 22 \mathrm{P} 2$

Ti17 C-like $2 S$ 2P2 ( $4 P$ P $3 D(3 F) j=4.0-2 S 2 P 3$

Til7 C-like 2S $2 P 2(4 P) 3 D(3 F) j=3.0-2 S$ 2P3

Ti17 C-like 2S2 $2 \mathrm{P}$ 3S (3P*) $j=2.0-2 S 2$ 2P2

Til7 $C$-like $2 S$ 2P2 ( $4 P$ ) $3 S(5 P) j=3.0-2 S$ 2P3

Ti17 C-like $2 S 22 P \quad 3 S \quad\left(3 P^{*}\right) j=2.0-2 S 2 \quad 2 P 2$

Ti17 $C$-like $2 S 2 P 2(2 D) 3 S(3 D) j=2.0-2 S 2 P 3$

Ti17 $C$-like 2S2 $2 \mathrm{P}$ 3S (3P*) $j=1.0-2 S 2 \quad 2 \mathrm{P} 2$

Ti17 $C$-like $2 S 2 \quad 2 P \quad 3 S \quad$ (IP*) $j=1.0-2 S 2 \quad 2 P 2$

Til9 Be-like 1S2 2P 3P

Ti19 Be-like 1S2 2P $3 \mathrm{P}$

Til9 Be-like 152 2P $3 \mathrm{P}$

Ti19 Be-like 152 2P $3 P$

Til9 Be-like 1S2 2P $3 \mathrm{P}$

Ti19 Be-like 1S2 2P 3P

Ti19 Be-like 152 2S $3 P$ $\left(2 P^{*}\right) j=1.5 \quad 25.025 \quad \mathrm{RAP} 100$

$\left(2 P^{*}\right) j=.5 \quad 25.071$

$\left(2 P^{*}\right) j=.5 \quad 25.071$

$\left(2 P^{*}\right) j=.5 \quad 25.071$

(2S) $j=.5 \quad 25.086$

(2S) $j=.5 \quad 25.086$

$\begin{array}{ll}(2 S) j=.5 & 25.086 \\ \left(2 P^{\star}\right) j=.5 & 25.206\end{array}$

$\left(2 P^{\star}\right) j=.5 \quad 25.206$

$\left(2 P^{\star}\right) j=.5 \quad 25.206$

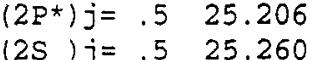

$\begin{array}{ll}(2 S) j=.5 & 25.260 \\ (2 S) j=.5 & 25.260\end{array}$

(2S ) $j=.5 \quad 25.260$

$(\quad j j=.0 \quad 2.721$ cosundum 030

$j j=.0 \quad 2.721$ quartz 203

$(j j=.0 \quad 2.721$ topaz 006

$j=.0 \quad 2.721$ LiF 220

$j=.0 \quad 2.721 \quad \mathrm{NaCl} 200$

$j j=.0 \quad 2.721$ topaz 002

$1 j=.0$

2.721

copaz 002

$\left(2 P^{*}\right) j=5 \quad 19.010$ mica 002

$\left(4 S^{*}\right) j=1.5 \quad 19.089$ mica 002

$\left(4 S^{*}\right) j=1.5 \quad 19.112$ mica 002

$\left(2 D^{*}\right) j=2 \quad 5 \quad 19.210$ mica 002

$\left(2 D^{\star}\right) j=2.5 \quad 19.240$ mica 002

$\left(2 D^{\star}\right) j=2.5 \quad 19.370$ mica 002

$\left(2 Q^{*}\right) j=1.5 \quad 19.450$ mica 002

(2D*) j=2.5 19.551 mica 002

$\left(2 P^{*}\right) j=1.5 \quad 19.570$ mica 002

$\left(2 P^{*}\right) j=1.5 \quad 19.710$ mica 002

(1D) $j=2.0 \quad 18.757$ mica 002

$\left(3 D^{*}\right) j=3.0 \quad 18.799$ mica 002

$\left(3 D^{*}\right) j=2.0 \quad 18.939$ mica 002

(3P) $j=1.0 \quad 19.369$ mica 002

$\left(5 S^{*}\right) j=2.0 \quad 19.415$ mica 002

(3P) $j=2.0 \quad 19.459$ mica 002

$\left(3 D^{*}\right) j=2.0 \quad 19.501$ mica 002

(3P) $j=2.0 \quad 19.651$ mica 002

(1D) $j=2.0 \quad 19.718$ mica 002

$\left(3 P^{*}\right) j=1.0 \quad 15.581$ beryl 100

$\left(3 P^{\star}\right) j=1.0 \quad 15.671$ beryl 100

$\left\langle 3 P^{*}\right\rangle j=2.0 \quad 15.685$ beryl 100

$\left(3 P^{*}\right) j=2.0 \quad 15.738$ beryl 100

$\left(3 P^{\star}\right) j=2.0 \quad 15.742$ bery 100

(3P*) $j=.015 .801$ beryl 100

(IS) $j=.0 \quad 15.831$ bery 100
$26.116 \quad 73.380$

$25.763 \quad 76.690$

$26.116 \quad 73.737$

$26.634 \quad 70.274$

$25.763 \quad 76.836$

$26.116 \quad 73.855$

$26.634 \quad 70.370$

$25.763 \quad 78.064$

$26.116 \quad 74.830$

$26.634 \quad 71.153$

$25.763 \quad 78.659$

$26.116 \quad 75.290$

$26.634 \quad 71.516$

$2.748 \quad 81.962$

$2.749 \quad 81.815$

$2.795 \quad 76.786$

$2.848 \quad 72.825$

$5.641 \quad 74.736$

$8.374 \quad 77.111$

$8.512 \quad 73.536$

$19.942 \quad 72.414$

$19.942 \quad 73.181$

$19.942 \quad 73.411$

$19.942 \quad 74.428$

$19.942 \quad 74.752$

$19.942 \quad 76.244$

$19.942 \quad 77.246$

$19.942 \quad 78.635$

$19.942 \quad 78.916$

$19.942 \quad 81.252$

$\begin{array}{lll}19.942 & 70.149\end{array}$

$19.942 \quad 70.507$

$19.942 \quad 71.751$

$19.942 \quad 76.232$

$19.942 \quad 76.799$

$19.942 \quad 77.364$

$19.942 \quad 77.928$

$19.942 \quad 80.200$

$19.942 \quad 81.404$

$15.954 \quad 77.586$

$15.954 \quad 79.192$

$15.954 \quad 79.464$

$15.954 \quad 80.561$

$15.954 \quad 80.649$

$15.954 \quad 82.059$

$15.954 \quad 82.881$ 


\begin{tabular}{|c|c|c|c|c|c|c|c|c|}
\hline 9 & Be-like & $1 \mathrm{~S} 2$ & 2 & & $3 p$ & $(3 D) j=1.0$ & -152 & \\
\hline Ti19 & Be-like & $1 S 2$ & 2 & $S$ & $3 P$ & $\left(1 P^{*}\right) j=1.0$ & -152 & \\
\hline 19 & Be-like & & & 0 & & $j j=.0$ & - & \\
\hline 19 & Be-like & & & 0 & & $j=.0$ & - & \\
\hline 19 & Be-like & & & 0 & & $j j=.0$ & . & \\
\hline 19 & Be-like & & & 0 & & $j j=.0$ & - & \\
\hline 19 & Be-like & & & 0 & & $j j=.0$ & - & \\
\hline & Be-like & & & 0 & & $j j=.0$ & - & \\
\hline 19 & Be-like & & & 0 & & $j j=.0$ & - & \\
\hline 20 & Li-like & 1s & $2 P$ & $3 P$ & & $(2 P) j=1.5$ & $-1 s 2$ & $3 P$ \\
\hline 20 & Li-like & IS & $2 P$ & $3 P$ & & $(2 \mathrm{P}) j=1.5$ & -152 & $3 P$ \\
\hline 0 & Li-like & is & $2 P$ & $3 P$ & & $(2 P) j=1.5$ & $-1 \$ 2$ & $3 P$ \\
\hline 20 & Li-like & is & $2 \mathrm{P}$ & $3 P$ & & $(2 P) j=1.5$ & -152 & $3 P$ \\
\hline 20 & Li-like & $1 \mathrm{~s}$ & $2 \mathrm{P} 2$ & & & $(2 S) j=.5$ & $-1 S 2$ & $2 \mathrm{P}$ \\
\hline 20 & Li-like & $1 \mathrm{~S}$ & $2 \mathrm{P} 2$ & & & $(2 s) j=.5$ & -152 & $2 E$ \\
\hline 20 & Li-Iike & $1 S$ & $2 P 2$ & & & $(2 s) j=.5$ & $-1 S 2$ & $2 F$ \\
\hline 0 & Li-like & 15 & $2 \mathrm{P} 2$ & & & $(2 S) j=.5$ & $-1 S 2$ & $2 \mathrm{P}$ \\
\hline 20 & Li-like & is & (2S & $2 \mathrm{P}$ & $\left.\left(1 P^{\star}\right)\right)$ & $\left(2 P^{\star}\right) j=.5$ & $-1 s 2$ & $2 S$ \\
\hline 8 & Li-like & $1 \mathrm{~S}$ & (2S & $2 P$ & $\left.\left(1 p^{\star}\right)\right)$ & $\left(2 P^{*}\right) j=.5$ & $-1 S 2$ & $2 S$ \\
\hline 0 & Li-like & 15 & (2s & $2 \mathrm{P}$ & $\left(1 p^{\star}\right)$ & $\left(2 P^{\star}\right) j=.5$ & -152 & $2 S$ \\
\hline 20 & Ii-like & $1 S$ & (2S & $2 \mathrm{P}$ & $\left.\left(1 P^{*}\right)\right)$ & $\left(2 P^{\star}\right) j=.5$ & $-1 \$ 2$ & $2 S$ \\
\hline 20 & Li-like & is & L2S & $2 P$ & $\left.\left(3 p^{*}\right)\right)$ & $\left(2 P^{*}\right) j=.5$ & $-1 S 2$ & $2 S$ \\
\hline 20 & Ii-like & IS & $12 \mathrm{~S}$ & $2 P$ & $\{30 *\})$ & $\left(2 p^{*}\right) j=.5$ & $-1 s 2$ & $2 S$ \\
\hline 20 & Ii-Iike & is & (2s & $2 P$ & $\left.\left(3 P^{\star}\right)\right)$ & $\left(2 \mathrm{P}^{\star}\right) j=.5$ & $-1 s 2$ & $2 \mathrm{~S}$ \\
\hline 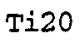 & Ii-Iike & $1 S$ & $12 S$ & $2 P$ & $\left.\left(3 P^{*}\right)\right)$ & $\left(2 P^{\star}\right) j=.5$ & $-1 s 2$ & $2 S$ \\
\hline 20 & Ii-like & 1S & (2S & $2 P$ & $\left.\left(3 P^{\star}\right)\right)$ & $\left(2 p^{*}\right) j=.5$ & -152 & $2 S$ \\
\hline & Li-like & is & (2s & $2 P$ & $\left.\left(3 P^{*}\right)\right)$ & $\left(2 P^{\star}\right) j=.5$ & -152 & $2 S$ \\
\hline 0 & Li-like & $1 S$ & $2 \mathrm{P} 2$ & & & $(2 D) j=1.5$ & -152 & $2 P$ \\
\hline 20 & Li-like & 15 & $2 \mathrm{P} 2$ & & & (2D) $j=1.5$ & -152 & $2 \mathrm{P}$ \\
\hline 20 & Li-like & 1S & $2 \mathrm{P} 2$ & & & (2D) $j=1.5$ & -152 & $2 \mathrm{P}$ \\
\hline 20 & Li-like & IS & $2 \mathrm{P} 2$ & & & $(2 D) j=1.5$ & -152 & $2 \mathrm{P}$ \\
\hline 20 & Li-1ike & $1 \mathrm{~S}$ & $2 \mathrm{P} 2$ & & & $(2 D) j=1.5$ & $-1 s 2$ & $2 P$ \\
\hline 20 & Li-like & $1 \mathrm{~S}$ & $2 \mathrm{P} 2$ & & & (2D) $j=1.5$ & $-1 s 2$ & $2 \mathrm{P}$ \\
\hline 20 & Li-like & $1 S$ & $2 \mathrm{P} 2$ & & & $(2 D) j=2.5$ & $-1 s 2$ & $2 \mathrm{P}$ \\
\hline & Li-like & is & $2 P 2$ & & & $(2 D) j$ & -152 & $2 P$ \\
\hline & Li-like & $1 S$ & $2 \mathrm{P} 2$ & & & $(2 D) j$ & $-1 S 2$ & $2 P$ \\
\hline & Ii-Iike & $1 \mathrm{~S}$ & $2 \mathrm{P} 2$ & & & $(2 D) j=2.5$ & $-1 S 2$ & $2 P$ \\
\hline & Ii-like & IS & $2 \mathrm{P} 2$ & & & $(2 D) j=2.5$ & $-1 S 2$ & $2 \mathrm{P}$ \\
\hline 20 & Li-like & $1 \mathrm{~s}$ & $2 \mathrm{P} 2$ & & & (2D) $j=2.5$ & -152 & $2 \mathrm{P}$ \\
\hline 20 & Li-like & $1 S$ & $(2 S$ & $2 P$ & $\left.\left(3 p^{*}\right)\right)$ & $\left(4 \mathrm{P}^{\star}\right) j=1.5$ & -152 & $2 S$ \\
\hline 20 & Li-like & 15 & $(2 S$ & $2 P$ & $\left.\left(3 P^{*}\right)\right)$ & $\left(4 \mathrm{P}^{*}\right) j=1.5$ & $-1 S 2$ & $2 \mathrm{~S}$ \\
\hline 20 & Li-like & $1 S$ & $(2 S$ & $2 P$ & $\left.\left(3 P^{*}\right)\right)$ & $\left(4 \mathrm{P}^{\star}\right) j=1.5$ & $-1 S 2$ & $2 S$ \\
\hline 20 & Ii-like & $1 S$ & $(2 \mathrm{~S}$ & $2 \mathrm{P}$ & $\left.\left(3 P^{*}\right)\right)$ & $\left(4 \mathrm{P}^{\star}\right) j=1.5$ & -152 & $2 S$ \\
\hline & Li-like & $1 S$ & $12 S$ & $2 F$ & $(3 P *))$ & $\left(4 \mathrm{P}^{*}\right) j=1.5$ & -152 & $2 S$ \\
\hline 20 & Li-like & $1 \mathrm{~S}$ & 125 & $2 z$ & $\left(32^{*} 1\right)$ & $\left(4 \mathrm{P}^{\star}\right) j=1.5$ & $-1,52$ & $2 S$ \\
\hline & Li-like & $1 \mathrm{~S} 2$ & 90 & & & $(2 D) j=1.5$ & -152 & $2 P$ \\
\hline
\end{tabular}

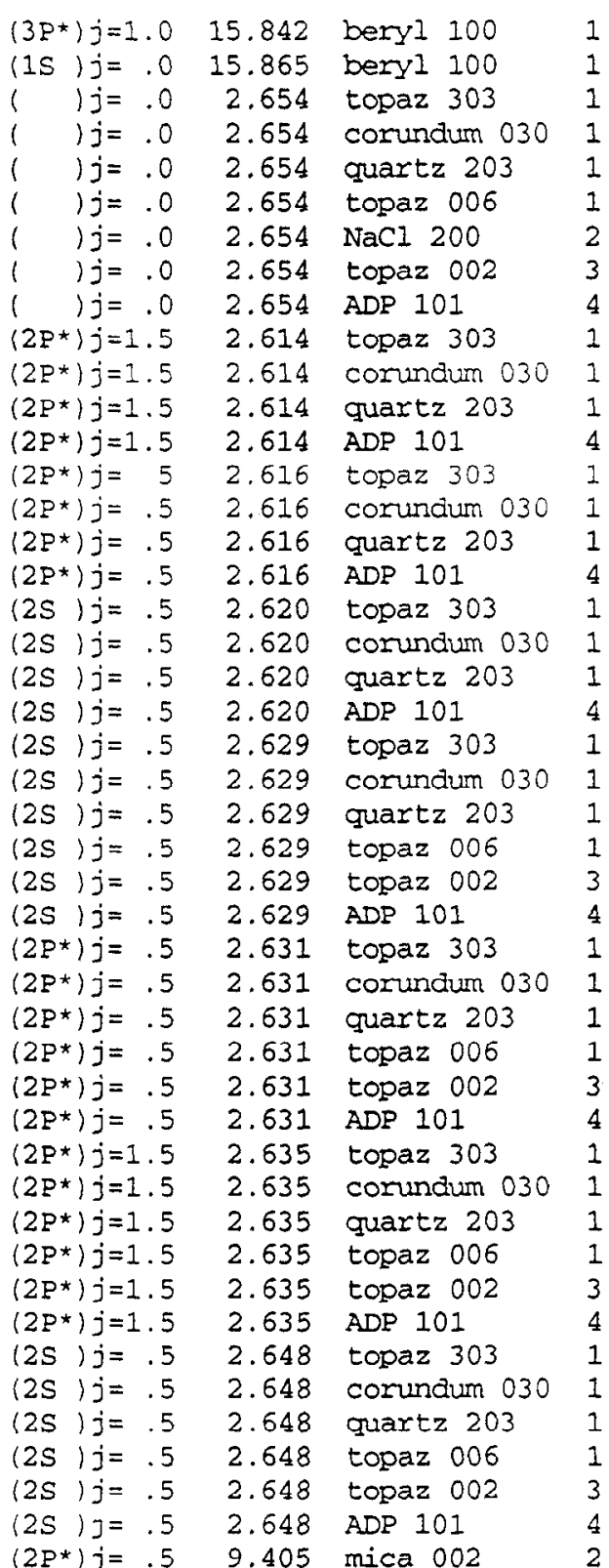

Page 45 
Ti20 Li-like 1S2 $7 \mathrm{P}$

Ti20 Li-like 1S2 9D

Ti20 Li-like IS2 8D

Ti20 Li-like 1S2 8D

Ti20 Li-like 1S2 6P

Ti20 Li-like 1S2 7D

Ti20 Li-like 1S2 6D

Ti20 Li-like 1S2 6D

Ti20 Li-like 1S2 5P

Ti20 Li-like 1S2 5D

Ti20 Li-like 1S2 3P

Ti20 Li-like 1S2 3 F

Ti20 Li-like 1S2 3D

Ti2I He-like IS 5P

Ti21 He-like is 5P

Ti21 He-like is 5P

Ti2I He-like $1 S$ 5P

Ti21 He-like is $5 F$

Ti21 He-like 15 5P

Ti2l He-Iike 1S 5P

Ti21 He-like 1S $5 P$

Ti21 He-like $1 S$ 5P

Ti21 He-like 1S 5P

Ti21 He-like 1S 4P

Ti21 He-like 15 PP

Ti21 He-like 1S 4P

Ti21 He-like is $4 \mathrm{P}$

Ti21 He-like 1S 4P

Ti21 He-like IS $4 \mathrm{P}$

Ti21 He-like 1S 4P

Ti21 He-like 1S 4P

Ti21 He-like 1S $4 \mathrm{P}$

Ti21 He-like 1S $4 \mathrm{P}$

Ti21 He-like is 4P

Ti21 He-like 1S 3P

Ti21 He-like 1S 3P

Ti21 He-like IS $3 \mathrm{P}$

Ti21 He-like 1s 3P

Ti21 He-like IS $3 \mathrm{P}$

Ti21 He-like 1s 3P

Ti21 He-like is 3P

Ti21 He-like 1S 3P

Ti21 He-like 2S $2 \mathrm{P}$

Ti21 He-like $2 S$ 2P

Ti21 He-like 2P2

Ti21 He-like 2S 2P $\left(2 P^{*}\right) j=1.5-1 S 22 S$ (2D) $j=2.5-1 S 22$ P (2D) $j=1.5-1 S 22 P$ (2D) $j=2.5-1 S 22 P$ $\left(2 P^{*}\right) j=1.5-1 S 22 S$ (2D) $j=2.5-1 S 228$ (2D) $j=1.5-1 S 22 P$ (2D) $j=2.5-1 S 22 P$ $\left(2 P^{*}\right) j=1.5-1 S 22 S$ (2D) $j=1.5-1 S 229$ $\left(2 P^{*}\right) j=1.5-1 S 22 S$ $\left(2 P^{\star}\right) j=.5-1 S 22 S$ (2D) $j=1.5-1 S 22 P$ $\left(1 P^{*}\right) j=1.0-1 \mathrm{~S} 2$ $\left(1 P^{\star}\right) j=1.0-1 S 2$ $\left(1 P^{\star}\right) j=1.0-152$ $\left(1 P^{*}\right) j=1.0-1 S 2$ $\left(1 P^{*}\right) j=1.0-1 \mathrm{~S} 2$ $\left(1 P^{\star}\right) j=1.0-1 S 2$ $\left(1 P^{*}\right) j=1.0-1 \mathrm{~S} 2$ $\left(1 P^{*}\right) j=1.0-1 S 2$ $\left(1 P^{\star}\right) j=1.0-1 S 2$ $\left(1 P^{*}\right) j=1.0-1 S 2$ $\left(1 P^{*}\right) j=1.0-1 S 2$ $\left(1 P^{\star}\right) j=1.0-1 S 2$ $\left(1 P^{\star}\right) j=1.0-1 S 2$ $\left(1 P^{*}\right) j=1.0-1 S 2$ $\left(1 P^{\star}\right) j=1.0-1 S 2$ $\left(1 P^{\star}\right) j=1.0-1 S 2$ $\left(1 P^{\star}\right) j=1.0-1 S 2$ $\left(1 P^{\star}\right) j=1.0-1 S 2$ $\left(1 P^{*}\right) j=1.0-1 S 2$ $\left(1 P^{*}\right) j=1.0-1.52$ $\left(1 P^{*}\right) j=1.0-1 S 2$ $\left(1 p^{\star}\right) j=1.0-152$ $\left(1 P^{*}\right) j=1.0-1 S 2$ $\left(1 P^{*}\right) j=1.0-1 S 2$ $\left(1 P^{\star}\right) j=1.0-1 S 2$ $\left(1 P^{\star}\right) j=1.0-1 S 2$ $\left(1 P^{*}\right) j=1.0-1 S 2$ $\left(1 P^{*}\right) j=1.0-1 S 2$ $\left(1 P^{\star}\right) j=1.0-1 S 2$ $\left(3 P^{*}\right) j=2.0-1 S 2 S$ $\left(3 P^{*}\right) j=1.0-1 S \quad 2 S$ (1D) $j=2.0-1 S \quad 2 P$ $\left(3 \mathrm{P}^{\star}\right) j=.0-1 \mathrm{~S} 2 \mathrm{~S}$
(2S) $j=.5 \quad 9.434$ mica 002

$\left(2 P^{*}\right) j=1.5 \quad 9.459$ mica 002

$\left(2 P^{\star}\right) j=.5 \quad 9.534$ mica 002

$(2 P *) j=1.5 \quad 9.591$ mica 002

(2S) $j=.5 \quad 9.733$ mica 002

$\left(2 P^{*}\right) j=1.5 \quad 9.788$ mica 002

$\left(2 P^{*}\right) j=.5 \quad 10.046$ ADP 101

$\left(2 P^{*}\right) j=1.5 \quad 10.109 \quad$ ADP 101

(2S ) $j=.5 \quad 10.278$ ADP 101

$\left(2 P^{*}\right) j=5 \quad 10.620$ ADP 101

(2S) $j=.5 \quad 15.212$ beryl 100

(2S) $j=.5 \quad 15.255$ beryl 100

$\left(2 \mathrm{P}^{*}\right) j=.5 \quad 15.912$ beryl 100

(1S) $j=.0 \quad 2.063$ quartz 200

(IS) $j=.0 \quad 2.063$ Si 111

(1S ) $j=.0$

(1S) $j=$.

(1s) $j=$

(1s) $j=$.0

(1S) $j=.0$

(1S) $j=$

(1s) $j=$.

(1s) $j=.0$

(1S) $j=0$

(IS) $j=.0$

(1S) $j=.0$

(IS) $j=.0$

(1s) $j=.0$

(1S) $j=.0$

(1s) $j=.0$

(1S) $j=.0$

(IS) $j=.0$

(1s) $j=$.

(1S) $j=0$

(IS) $j=0$

(1S) $j=.0$

(1s) $j=.0$

(1s) $j=.0$

(1s) $j=.0$

(1s) $j=.0$

(1s) $j=.0$

(1s) $j=.0$

(3S) $j=1$. 0

(3S) $j=1.0$

$\left.1 P^{*}\right) j=1$. 0

(3S) $j=1.0$
2.063 sylvite 200

063

2.063

2.063

2.063

2.063

2.063

2.063

2. 111

2.111

2.111

2. 111

2.111

2.111

111

2.111

2. 111

2.111

2.221

2. 221

2.221

2.221

2.221

2.221

fluorite 111

Ge 111

topaz 002

quartz 100

PET 002

ADP 101

422

ungsten 110 guartz 200

111

Br 200

quartz 101

graphite 002

quartz 100

PET 002

020

DP 101

422

quartz 310

ungsten 110

quartz 112

Al 111

quartz 101

graphite 002

513 ADP 101

2.517 ADP 101
$19.942 \quad 71.110$

$19.942 \quad 71.559$

$19.942 \quad 72.974$

$19.942 \quad 74.131$

$19.942 \quad 77.456$

$19.942 \quad 79.006$

$10.640 \quad 70.765$

$10.640 \quad 71.822$

$10.640 \quad 75.011$

$10.640 \quad 86.486$

$15.954 \quad 72.457$

$15.954 \quad 72.977$

$15.954 \quad 85.842$

$4.246 \quad 76.346$

$6.271 \quad 80.724$

$6.292 \quad 79.619$

$6.308 \quad 78.853$

$6.532 \quad 71.350$

$6.584 \quad 70.053$

$8.374 \quad 80.208$

$8.512 \quad 75.802$

$8.742 \quad 70.726$

$10.640 \quad 75.802$

$2217 \quad 72.211$

$4.476 \quad 70.605$

$4.246 \quad 83.905$

$6.532 \quad 75.821$

$6.584 \quad 74.128$

$6.687 \quad 71.273$

$6.696 \quad 71.048$

$8.512 \quad 82.753$

$8.742 \quad 74.997$

$8.808 \quad 73.471$

$10.640 \quad 82.753$

$2.310 \quad 74.044$

$2.360 \quad 70.237$

$4.476 \quad 82.933$

$4.564 \quad 76.722$

$4.638 \quad 73.284$

$4.676 \quad 71.797$

$6.687 \quad 85.144$

$6.696 \quad 84.309$

$10.640 \quad 70.473$

$10.640 \quad 70.667$

$10.640 \quad 70.863$

$10.640 \quad 71.127$ 
Ti21 He-like $1 \mathrm{~S}$ 2P

Ti21 He-like 1S 2P

Ti21 He-like is $2 \mathrm{P}$

Ti21 He-like is 2P

Ti21 He-like $1 \mathrm{~S} 2 \mathrm{P}$

Ti21 He-like is 2P

Ti21 He-like is $2 P$

Ti21 He-like 1S $2 \mathrm{P}$

Ti21 He-like 15 2S

Ti21 He-like 1S 2S

Ti21 He-like is 25

Ti21 He-like 1S $2 S$

Ti21 He-like is $2 S$

Ti21 He-like 1S 2S

Ti21 He-like $1 \mathrm{~S} 3 \mathrm{P}$

Ti22 H-like 7P

Ti22 H-like 7P

Ti22 H -like 7P

Ti22 H -like 7P

Ti22 H-like 7P

Ti22 F-like 7P

Ti22 H -like 7P

Ti22 H-like 6P

Ti22 H-like 6P

Ti22 H-like 6P

Ti22 H-like 6P

Ti22 H-like 6P

Ti22 H-like 6P

Ti22 H-like 5P

Ti22 H-like 5P

Ti22 H-like 5P

Ti22 H-like 5P

Ti22 H-like 5P

Ti22 F-like 4P

Ti22 H-like 4P

Ti22 H-like 4P

Ti22 H-like 4P

Ti22 H -like 4P

Ti22 H -like 4P

Ti22 H -like 4P

Ti22 H-like 4P

Ti22 H -like 4P

Ti22 H-like 4P

Ti22 H-like $3 P$

Ti22 H -like 3P

Ti22 H-like 3P $\left(1 P^{*}\right) j=1.0-1 S 2$

$\left(1 P^{\star}\right) j=1.0-1 S 2$

$\left(1 P^{*}\right) j=1.0-1 \mathrm{~s} 2$

$\left(1 P^{*}\right) j=1.0-1 s 2$

(3P*) $j=1.0-152$

$\left(3 P^{*}\right) j=1.0-1 \mathrm{~S} 2$

$\left(3 P^{\star}\right) j=1.0-1 S 2$

$\left(3 P^{*}\right) j=1.0-1.52$

(3S) $j=1.0-1 S 2$

(3s) $j=1.0-1 S 2$

(3S) $j=1.0-1 S 2$

(3s) $j=1.0-152$

(3s) $j=1.0-1 s 2$

(3S) $j=1.0-1 S 2$

(1P*) $j=1.0$ - 15 2S

$\left(2 P^{*}\right) j=1.5-15$

$\left(2 P^{*}\right) j=1.5-1 S$

$\left(2 P^{\star}\right) j=1.5-1 S$

$\left(2 P^{\star}\right) j=1.5-1 S$

$\left(2 P^{*}\right) j=1.5-1 S$

$\left(2 P^{\star}\right) j=1.5-1 S$

$\left(2 P^{\star}\right) j=1.5-1 S$

$\left(2 P^{\star}\right) j=1.5-1 S$

$\left(2 P^{\star}\right) j=1.5-1 S$

$\left(2 P^{\star}\right) j=1.5-1 S$

$\left(2 P^{\star}\right) j=1.5-1 S$

$\left(2 P^{\star}\right) j=1.5-1 S$

$\left(2 P^{*}\right) j=1.5-1 S$

$\left(2 P^{\star}\right) j=1.5-1 S$

$\left(2 P^{*}\right) j=1.5-1 S$

$\left(2 P^{*}\right) j=1.5-1 S$

$\left(2 P^{*}\right) j=1.5-1 S$

$\left(2 P^{\star}\right) j=1.5-1 S$

$\left(2 P^{\star}\right) j=1.5-1 S$

$\left(2 P^{\star}\right) j=1.5-1 S$

$\left(2 P^{*}\right) j=1.5-1 S$

$\left(2 P^{\star}\right) j=1.5-1 S$

$\left(2 P^{\star}\right) j=1.5-1 S$

$\left(2 P^{\star}\right) j=1.5-1 S$

$\left(2 P^{\star}\right) j=1.5-1 S$

$\left(2 P^{*}\right) j=1.5-1 S$

$\left(2 P^{*}\right) j=1.5-1 S$

$\left(2 P^{\star}\right) j=1.5-1 S$

$\left(2 P^{*}\right) j=1.5-1 S$

$\left(2 P^{*}\right) j=1.5-1 S$

$\left(2 P^{*}\right\rangle j=1.5-1 S$

\begin{tabular}{|c|c|c|c|}
\hline $\operatorname{Ls} / j=$ & 0 & 2.610 & topaz 303 \\
\hline$(1 s) j=$ & .0 & 2.610 & corundum 030 \\
\hline$(1 s) j=$ & .0 & 2.610 & quartz 203 \\
\hline $1 s) j=$ & 0 & 2.610 & ADP 101 \\
\hline IS $j j=$ & .0 & 2.622 & topaz 303 \\
\hline$J^{2}$ & .0 & 2.622 & corundum 030 \\
\hline & 0 & 2.622 & quartz 203 \\
\hline$j=$ & .0 & 2.622 & ADP 101 \\
\hline$j=$ & .0 & 2.637 & topaz 303 \\
\hline s $j j=$ & .0 & 2.637 & corundum 030 \\
\hline$j=$ & 0 & 2.637 & quartz 203 \\
\hline$J=$ & .0 & 2.637 & topaz 006 \\
\hline$j=$ & .0 & 2.637 & topaz 002 \\
\hline$y=$ & .0 & 2.637 & $A D P 101$ \\
\hline$j=$ & .0 & 14.520 & gypsum 020 \\
\hline$j=$ & .5 & 1.910 & quartz 223 \\
\hline$j=$ & .5 & 1.910 & Si 220 \\
\hline$j j=$ & .5 & 1.910 & fluorite 220 \\
\hline$j j=$ & .5 & 1.910 & $\mathrm{Ge} 220$ \\
\hline$j=$ & .5 & 1.910 & LiF 200 \\
\hline$j=$ & .5 & 1.910 & Al 200 \\
\hline$j=$ & .5 & 1.910 & calcite 200 \\
\hline$j=$ & .5 & 1.924 & quartz 223 \\
\hline$j=$ & .5 & 1.924 & fluorite 220 \\
\hline$=$ & .5 & 1.924 & $\mathrm{Ge} 220$ \\
\hline$y=$ & .5 & 1.924 & LiF 200 \\
\hline$y=$ & .5 & 1.924 & Al 200 \\
\hline$j=$ & .5 & 1.924 & calcite 200 \\
\hline$j \mathrm{j}$ & 5 & 1.949 & quartz 223 \\
\hline$j=$ & .5 & 1.949 & Ge 220 \\
\hline$j=$ & .5 & 1.949 & LiF 200 \\
\hline$j=$ & .5 & 1.949 & Al 200 \\
\hline$j=$ & .5 & 1.949 & calcite 200 \\
\hline$j=$ & .5 & 1.995 & quartz 223 \\
\hline$j=$ & .5 & 1.995 & Ge 220 \\
\hline 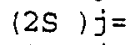 & .5 & 1.995 & LiF 200 \\
\hline$i=$ & .5 & 1.995 & Al 200 \\
\hline$j=$ & .5 & 1.995 & quartz 200 \\
\hline$j=$ & .5 & 1.995 & calcite 200 \\
\hline (2S & .5 & 1.995 & Si 111 \\
\hline$j=$ & .5 & 1.995 & sylvite 200 \\
\hline$\langle 2$ & .5 & 1.995 & Eluorite 111 \\
\hline$(2$ & .5 & 1.995 & topaz 002 \\
\hline & .5 & 2.104 & Si 422 \\
\hline & .5 & 2.104 & tungsten 110 \\
\hline & .5 & 2.104 & guartz 200 \\
\hline
\end{tabular}

$2.712 \quad 74.236$

$2.748 \quad 71.755$

$2.749 \quad 71.702$

$10.640 \quad 78.873$

$2.712 \quad 75.198$

$2.748 \quad 72.582$

$2.749 \quad 72.516$

$10.640 \quad 80.304$

$2.712 \quad 76.494$

$2.748 \quad 73.660$

$2.749 \quad 73.589$

$2.795 \quad 70.643$

8.37470 .858

$10.640 \quad 82.460$

$15.185 \quad 72.981$

2.02470 .678

$3.840 \quad 84.150$

$3.862 \quad 81.542$

$4.000 \quad 72.746$

$4.027 \quad 71.549$

$4.048 \quad 70.678$

$6.071 \quad 70.705$

$2.024 \quad 71.914$

$3.862 \quad 85.120$

$4.000 \quad 74.154$

$4.027 \quad 72.853$

$4.048 \quad 71.914$

6.07171 .943

$2.024 \quad 74.354$

$4.000 \quad 77.033$

$4.027 \quad 75.459$

$4.048 \quad 74.354$

$6.071 \quad 74.387$

2.02480 .289

$4.000 \quad 85.948$

$4.027 \quad 82.227$

$4.048 \quad 80.289$

$4.246 \quad 70.003$

6.07180 .345

$6.271 \quad 72.629$

6.29272 .028

6.30871 .585

$8.374 \quad 72.354$

$2.217 \quad 71.628$

$4.476 \quad 70.073$

$4.246 \quad 82.329$

Page 47 


\begin{tabular}{|c|c|c|}
\hline $\operatorname{Ti22}$ & $\mathrm{H}$ & -like \\
\hline $\operatorname{Ti} 22$ & $\mathrm{H}$ & -like \\
\hline Ti22 & $\mathrm{H}$ & -like \\
\hline $\operatorname{Ti22}$ & $\mathrm{H}$ & -like \\
\hline $\operatorname{Ti22}$ & $\mathrm{H}$ & -like \\
\hline $\operatorname{Ti} 22$ & $\mathrm{H}$ & -like \\
\hline Ti22 & $\mathrm{H}$ & -like \\
\hline Ti22 & $\mathrm{H}$ & -like \\
\hline Ti22 & $\mathrm{H}$ & -like \\
\hline Ti22 & $\mathrm{H}$ & -like \\
\hline Ti22 & $\mathrm{H}$ & -like \\
\hline Ti22 & $\mathrm{H}$ & -like \\
\hline Ti22 & $\mathrm{H}$ & -like \\
\hline $\operatorname{Ti22}$ & $\mathrm{H}$ & -like \\
\hline Ti22 & $\mathrm{H}$ & -like \\
\hline Ti22 & $\mathrm{H}$ & -like \\
\hline $\operatorname{Ti} 22$ & $H$ & $-1 i k e$ \\
\hline $\operatorname{Ti22}$ & H & -like \\
\hline $\mathrm{Ti} 22$ & $\mathrm{H}$ & -like \\
\hline $\mathrm{Ti} 22$ & $\mathrm{H}$ & -like \\
\hline $\operatorname{Ti} 22$ & $\mathrm{H}$ & -like \\
\hline $\operatorname{Ti} 22$ & $\mathrm{H}$ & -like \\
\hline Ti22 & $\mathrm{H}$ & -like \\
\hline $\mathrm{Ti22}$ & $\mathrm{H}$ & -like \\
\hline Ti22 & $\mathrm{H}$ & -like \\
\hline $\mathrm{Ti22}$ & $\mathrm{H}$ & $-1 i k e$ \\
\hline Ti22 & $\mathrm{H}$ & -like \\
\hline $\operatorname{Ti22}$ & $\mathrm{H}$ & -like \\
\hline $\mathrm{Ti} 22$ & $\mathrm{H}$ & -like \\
\hline $\mathrm{Ti} 22$ & $\mathrm{H}$ & -like \\
\hline $\mathrm{Ti} 22$ & $\mathrm{H}$ & -like \\
\hline $\operatorname{Ti} 22$ & H & -like \\
\hline $\operatorname{Ti22}$ & $\mathrm{H}$ & -like \\
\hline Ti22 & $\mathrm{H}$ & -like \\
\hline Ti22 & $\mathrm{H}$ & -like \\
\hline Ti22 & $\mathrm{H}$ & -like \\
\hline $\operatorname{Ti} 22$ & $\mathrm{H}$ & -like \\
\hline $\mathrm{Ti22}$ & $\mathrm{H}$ & -like \\
\hline Ti22 & $\mathrm{H}$ & -like \\
\hline Ti22 & $\mathrm{H}$ & -like \\
\hline Ti22 & $\mathrm{H}$ & -like \\
\hline $\mathrm{Ti} 22$ & $\mathrm{H}$ & -like \\
\hline Ti22 & $\mathrm{H}$ & -like \\
\hline $\mathrm{Ti} 22$ & $\mathrm{H}$ & -like \\
\hline Ti22 & $\mathrm{H}$ & -like \\
\hline
\end{tabular}

$\mathrm{V}$ $\left(2 P^{*}\right) j=1.5-1 S$

$\left(2 P^{*}\right) j=1.5-1 S$

$\left(2 P^{*}\right) j=1.5-1 S$

$\left(2 \mathrm{P}^{*}\right) j=1.5-1 \mathrm{~s}$

$\left(2 P^{*}\right) j=1.5$ - IS

$\left(2 P^{*}\right) j=1.5-1 S$

$\left(2 P^{*}\right) j=1.5-1 S$

$\left(2 P^{*}\right) j=1.5-1 s$

$\left(2 P^{\star}\right) j=.5-1 S$

$\left(2 P^{*}\right) j=.5-15$

$\left(2 P^{\star}\right) j=.5-1 S$

$\left(2 P^{*}\right) j=.5-1 S$

$\left(2 P^{*}\right) j=.5-1 S$

$\left(2 P^{*}\right) j=.5-1 S$

$\left(2 P^{*}\right) j=.5-1 S$

$\left(2 \mathrm{P}^{*}\right) j=.5-1 \mathrm{~S}$

$\left(2 P^{*}\right) j=.5-1 S$

$\left(2 P^{*}\right) j=.5-1 S$

$\left(2 P^{*}\right) j=.5-15$

$\left(2 P^{\star}\right) j=1.5-1 S$

$\left(2 P^{*}\right) j=1.5-1 S$

$\left(2 \mathrm{P}^{*}\right\rangle j=1.5-2 \mathrm{~s}$

$\left(2 P^{*}\right) j=1.5-2 S$

$\left(2 P^{\star}\right) j=1.5-2 S$

(2D) $j=2.5-2 P$

(2D) $j=2.5-2 P$

(2D) $j=2.5-2 P$

(2D) $j=2.5-2 P$

$\left(2 \mathrm{P}^{*}\right) j=1.5-2 S$

$\left(2 P^{*}\right) j=1.5-2 S$

$\left(2 P^{\star}\right) j=1.5-2 S$

$\left(2 P^{\star}\right) j=1.5-2 S$

$\left(2 P^{\star}\right) j=1.5-2 S$

$\left(2 P^{*}\right) j=1.5-2 S$

(2D) $j=2.5-2 P$

(2D) $j=2.5-2 P$

(2D) $j=2.5-25$

(2D) $j=2.5-2 P$

(2D) $j=2.5-2 P$

(2D) $j=2.5-2 P$

$\left(2 P^{*}\right) j=1.5-2 S$

(2D) $j=2.5-2 P$

$\left(2 \mathrm{P}^{*}\right) j=1.5-3 \mathrm{~s}$

(2D) $j=2.5-3 P$

$\left(2 F^{*}\right) j=3.5-3 D$

\begin{tabular}{|c|c|c|}
\hline 2S $) j=$ & 2.104 & Ge 111 \\
\hline$(2 S) j=.5$ & 2.104 & $\mathrm{KBr} 200$ \\
\hline $2 S) j=.5$ & 2.104 & quartz 101 \\
\hline$(2 S) j=5$ & 2.104 & graphite 002 \\
\hline$(2 S) j=.5$ & 2.104 & quartz 100 \\
\hline $2 S / j=.5$ & 2.104 & PET 002 \\
\hline$(2 S) j=.5$ & 2.104 & EDT 020 \\
\hline$(2 s) j=.5$ & 2.104 & $\mathrm{ADP} 101$ \\
\hline $25 / j=.5$ & 2.105 & Si 422 \\
\hline$j j=.5$ & 2.105 & tumgsten 110 \\
\hline$j=.5$ & 2.105 & quartz 200 \\
\hline$j=.5$ & 2.105 & Ge 111 \\
\hline$S) j=.5$ & 2.105 & $\mathrm{KBI} 200$ \\
\hline $2 S / j=.5$ & 2.105 & quartz 101 \\
\hline $2 S / j=.5$ & 2.105 & graphite 002 \\
\hline$(2 S) j=.5$ & 2.105 & quartz 100 \\
\hline$\langle 2 S\rangle j=.5$ & 2.105 & PET 002 \\
\hline$(2 S) j=.5$ & 2.105 & EDT 020 \\
\hline$(2 s) j=.5$ & 2.105 & $A D P 101$ \\
\hline$(2 S) j=.5$ & 2.491 & gypsum 002 \\
\hline$(2 S) j=.5$ & 2.491 & Insto 111 \\
\hline$(2 S) j=.5$ & 8.130 & topaz 002 \\
\hline$(2 S) j=.5$ & 8.130 & quartz 100 \\
\hline $25 / j=.5$ & 8.130 & TAP 100 \\
\hline *) $j=1.5$ & 8.186 & topaz 002 \\
\hline$j=1.5$ & 8.186 & guartz 100 \\
\hline *) $j=1.5$ & 8.186 & TAP 100 \\
\hline *) $j=1$ & 8.186 & $\operatorname{RAP} 100$ \\
\hline$j j=.5$ & 8.398 & quartz 100 \\
\hline$j j=.5$ & 8.398 & PET 002 \\
\hline$\langle 2 s\rangle j=.5$ & 8.398 & EDT 020 \\
\hline$(2 S) j=.5$ & 8.398 & TAP 100 \\
\hline$(2 S) j=.5$ & 8.398 & RAP 100 \\
\hline$(2 S) j=.5$ & 8.398 & KAP 100 \\
\hline$\left(2 P^{*}\right) j=1.5$ & 8.458 & quartz 100 \\
\hline$\left(2 P^{*}\right) j=1.5$ & 8.458 & PET 002 \\
\hline$\left(2 p^{*}\right) j=1.5$ & 8.458 & EDT 020 \\
\hline$\left(2 P^{*}\right) j=1.5$ & 8.458 & TAP 100 \\
\hline *) $j=1.5$ & 8.458 & $\operatorname{RAP} 100$ \\
\hline$j=1.5$ & 8.458 & $\operatorname{KAP} 100$ \\
\hline$j \bar{j}=.5$ & 9.943 & mica 002 \\
\hline$\left(2 P^{\star}\right) j=1.5$ & 10.023 & ADP 101 \\
\hline$(2 S) j=.5$ & 26.242 & KAP 100 \\
\hline$\left(2 P^{*}\right) j=1.5$ & 26.402 & KAP 100 \\
\hline$j=2.5$ & 25.455 & KAP 100 \\
\hline & 2 & ADP 101 \\
\hline
\end{tabular}

\begin{tabular}{|c|c|}
\hline 6.532 & 75.087 \\
\hline 6.584 & 73.473 \\
\hline 6.687 & 70.721 \\
\hline 6.696 & 70.502 \\
\hline 8.512 & 81.387 \\
\hline 8.742 & 74.304 \\
\hline 8.808 & 72.842 \\
\hline 10.640 & 81.387 \\
\hline 2.217 & 71.710 \\
\hline 4.476 & 70.148 \\
\hline 4.246 & 82.534 \\
\hline 6.532 & 75.190 \\
\hline 6.584 & 73.565 \\
\hline 6.687 & 70.799 \\
\hline 6.696 & 70.579 \\
\hline 8.512 & 81.568 \\
\hline 8.742 & 74.401 \\
\hline 8.808 & 72.930 \\
\hline 10.640 & 81.568 \\
\hline 4.990 & 86.755 \\
\hline 7.481 & 87.350 \\
\hline 8.374 & 76.135 \\
\hline 8.512 & 72.770 \\
\hline 25.763 & 71.210 \\
\hline 8.374 & 77.836 \\
\hline 8.512 & 74.092 \\
\hline 25.763 & 72.407 \\
\hline 26.116 & 70.109 \\
\hline 8.512 & 80.612 \\
\hline 8.742 & 73.873 \\
\hline 8.808 & 72.149 \\
\hline 25.763 & 77.936 \\
\hline 26.116 & 74.730 \\
\hline 26.634 & 71.073 \\
\hline 8.512 & 83.543 \\
\hline 8.742 & 75.356 \\
\hline 8.808 & 73.794 \\
\hline 25.763 & 80.031 \\
\hline 26.116 & 76.310 \\
\hline 26.634 & 72.306 \\
\hline 19.942 & 85.705 \\
\hline 10.640 & 70.392 \\
\hline 26.634 & 80.158 \\
\hline 26.634 & 82.432 \\
\hline 26.634 & 83.354 \\
\hline 10.640 & 70.255 \\
\hline
\end{tabular}


$\mathrm{V} 13$ Na-like $2 \mathrm{P} 5 \quad 3 \mathrm{~S} 2 \quad\left(2 \mathrm{P}^{*}\right) j=1.5-2 \mathrm{P} 6 \quad 3 \mathrm{~S}$

$V 14$ Ne-like 2S2 $2 P 5$ (2P*1) 5D (12*)j=1.0 - 2S2 $2 P 6$

$V 14$ Ne-like 2S2 2P5 ( 2P*2) 5D $(22 *) j=1.0-2 S 22 P 6$

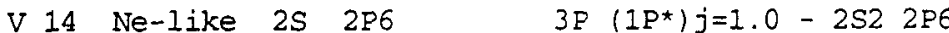

$V 14$ Ne-like $2 S \quad 2 P 6 \quad 3 P\left(3 P^{*}\right) j=1.0-2 S 22 P 6$

$V 15 \quad F$-like $2 S 22 \mathrm{P} 4(1 S) 3 D(2 D) j=2.5-2 S 22 P 5$

$V 15 \quad F$-like $2 S 22 \mathrm{P} 4$ ( $1 S$ ) 3D (2D) j=1.5 - 2S2 2P5

$V 15 \quad F-1 i k e$ 2S2 $2 P 4(1 D) 3 D(2 D) j=1.5-2 S 22 P 5$

$V 15 \quad F$-like 2S2 $2 P 4(1 D) 3 D(2 D) j=2.5-2 S 22 P 5$

$V 15 \quad F-1 i k e$ 2S2 $2 \mathrm{PA}(1 \mathrm{D}), 3 \mathrm{D}(2 \mathrm{~S}) j=.5-2 S 22 \mathrm{P} 5$

$V 15 \quad F-1 i k e$ 2S2 $2 P 4(1 D) 3 D(2 D) j=1.5-2 S 22 P 5$

$V 15 \quad F-l i k e$ 2S2 $2 P 4(1 D) 3 D(2 P) j=1.5-2 S 22 P 5$

$V 15 F$-like 2S2 2P4 ( $3 P$ ) $3 D(2 D) j=2.5-2 S 22 P 5$

$V 15$ F-like $2 S 22 P 4(3 P) 3 D(2 P) j=1.5-2 S 22 P 5$

$V 15 \mathrm{~F}$-like $2 S 22 \mathrm{PA}$ ( $3 \mathrm{P}) 3 \mathrm{D}(2 \mathrm{~F}) j=2.5-2 S 22 \mathrm{P5}$

$V 15 \quad F-1 i k e$ 2S2 $2 \mathrm{P} 4(3 \mathrm{P}) 3 \mathrm{D}(2 \mathrm{D}) j=1.5-2 S 22 \mathrm{P} 5$

$\mathrm{V} 15 \quad \mathrm{~F}$-like $2 \mathrm{~S} 22 \mathrm{P} 4(3 \mathrm{P}) 3 \mathrm{D}(4 \mathrm{P}) \mathrm{j}=2.5-2 \mathrm{~S} 22 \mathrm{PS}$

$V 15$ F-like $2 S 22 P 4(3 P) 3 D(2 P) j=.5-2 S 22 P 5$

$V 15$ F-like $2 S 22 P 4(3 P) 3 D(4 P) j=1.5-2 S 22 P 5$

$V 15 \quad F$-like 2S2 $2 \mathrm{P} 4(3 \mathrm{P}) 3 \mathrm{D}(4 \mathrm{P}) \mathrm{j}=.5-2 \mathrm{~S} 22 \mathrm{PS}$

$V 15$ F-like 2S2 $2 P 4(3 P) 3 D(2 P) j=1.5-2 S 22 P 5$

$V 16$ o-like $2 S 22 \mathrm{P3}\left(4 S^{\star}\right) 3 D\left(3 D^{*}\right) j=2.0-2 S 22 \mathrm{P4}$

$V 160-1 i k e$ 2S2 $2 P^{2}\left(2 P^{*}\right) 3 S\left(3 P^{*}\right) j=2.0-2 S 22 \mathrm{P} 4$

$V 19 \quad B-l i k e$ 2S2 4S $\quad(2 S) j=.5-2 S 22 P$

$V 19$ B-like $2 S 2 P\left(1 P^{*}\right) 3 P$ (2D) $j=2.5-2 S 22 P$

$\mathrm{V} 19 \mathrm{~B}-\mathrm{like} 2 \mathrm{~S} 2 \mathrm{P}\left(3 \mathrm{P}^{\star}\right) 3 \mathrm{P}(2 \mathrm{D}) \mathrm{j}=2.5-2 \mathrm{~S} 2 \mathrm{P}$

$\begin{array}{lll}V & B & B\end{array}-$ like $2 S 2 P\left(3 P^{*}\right) 3 P(2 D) j=2.5-2 S 22 P$

$\mathrm{V} 19 \mathrm{~B}$-1ike $2 \mathrm{~S} 2 \mathrm{P}\left(3 \mathrm{P}^{*}\right) 3 \mathrm{P}(2 \mathrm{P}) j=.5-2 \mathrm{~S} 22 \mathrm{P}$

$V 19 \mathrm{~B}-\mathrm{like} 2 \mathrm{~S} 2 \mathrm{P}\left(3 \mathrm{P}^{*}\right) 3 \mathrm{P}(2 \mathrm{P}) \mathrm{J}=1.5-2 \mathrm{~S} 2 \mathrm{PP}$

$V 19$ B-like $2 S$ 2P (1P*) 3D $\left(2 F^{\star}\right) j=3.5-2 S$ 2P2

$\mathrm{V} 19$ B-like 2S2 30

$V 19$ - 19 lke $2 S 3 P\left(3 D^{*}\right) 3 D(2 D) j=1.5-2 S 22 P$

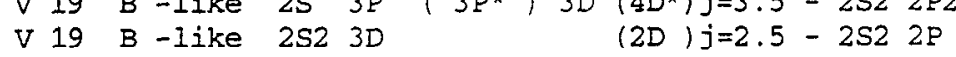

$V 19$ B-like 2S 2P (1P*) 3D (2D*)j=2.5- 2S 2P2

$\mathrm{V} 19 \mathrm{~B}-\mathrm{like} 2 \mathrm{~S} 2 \mathrm{P}\left(3 \mathrm{P}^{*}\right) 3 \mathrm{D}\left(2 F^{*}\right) j=3.5-2 \mathrm{~S}$ 2P2

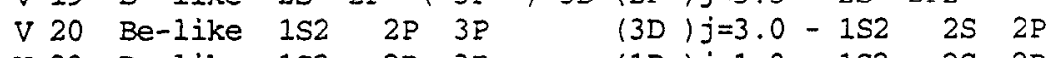

$\checkmark 20$ Be-like 1S2 2P $3 P \quad$ (1P ) $j=1.0-1 S 2$ 2S $2 P$

$V 20 \quad$ Be-like $1 S 2 \quad 2 P \quad 3 P \quad$ (3D) $j=1.0-1 S 2 \quad 2 S \quad 2 P$

20 Be-like $1 S 2$ $2 S$ 3P

$\mathrm{V} 20$ Be-like 1S2 $2 \mathrm{~S} 3 \mathrm{P}$

$\mathrm{V} 20$ Be-like 1s2 2S 3S

$\mathrm{V} 20$ Be-like 1S2 2P $3 \mathrm{P}$

$\mathrm{V} 20$ Be-like 1S2 2S 3D

$\mathrm{V} 20$ Be-like 1S2 2S 3D

$V 20$ Be-like 1S2 2P 3D

$\checkmark 20$ Be-like 1S2 2P 3P $\left(1 P^{*}\right) j=1.0-1 \mathrm{~S} 2$

$\left(3 P^{\star}\right) j=1.0-1 S 2$

(IS ) $j=.0-1 S 2$

(1D) $j=2.0-1 S 2$

(3D) $j=1.0-1 S 2$

(3D) $j=2.0-1 S 2$

(1P*) $j=1.0-1 S 2$
(3D) $j=2.0-1 S 2$
(2S) $j=.5 \quad 24.517$ TAP 100

$(1 S) j=.0 \quad 15.610$ beryl 100

(1s) $j=.0 \quad 15.748$ beryl 100

(1s) $j=.0 \quad 18.782$ mica 002

(1s) $j=.0 \quad 18.870$ mica 002

$\left(2 P^{*}\right) j=1.5 \quad 19.028$ mica 002

$\left(2 P^{*}\right) j=.5 \quad 19.203$ mica 002

$\left(2 P^{*}\right) j=1.5 \quad 19.298$ mica 002

$\left(2 P^{*}\right) j=1.5 \quad 19.366$ mica 002

$\left(2 P^{*}\right) j=1.5 \quad 19.443$ mica 002

$\left(2 P^{*}\right) j=.5 \quad 19.518$ mica 002

$\left(2 P^{*}\right) j=.5 \quad 19.589$ mica 002

$\left(2 P^{*}\right) j=1.5 \quad 19.645$ mica 002

$\left(2 P^{*}\right) j=1.5 \quad 19.671$ mica 002

$\left(2 P^{\star}\right) j=1.5 \quad 19.725$ mica 002

$\left(2 P^{*}\right) j=1.5 \quad 19.757$ mica 002

$\left(2 P^{*}\right) j=1.5 \quad 19.782$ mica 002

$\left(2 P^{\star}\right) j=1.5 \quad 19.800$ mica 002

$\left(2 P^{\star}\right) j=1.5 \quad 19.844$ mica 002

$\left(2 \mathrm{P}^{\star}\right) j=1.5 \quad 19.888$ mica 002

$(2 \mathrm{P} * j=.5 \quad 19.903$ mica 002

(3P) $j=1.0 \quad 18.890$ mica 002

(3P) $j=2.0 \quad 19.730$ mica 002

$\left(2 P^{\star}\right) j=1.5 \quad 12.140 \quad$ TAP 100

$\left(2 P^{*}\right) j=1.5 \quad 14.636$ gypsum 020

$\left(2 P^{*}\right) j=1.5 \quad 15.039$ gypsum 020

$\left(2 P^{*}\right) j=1.5 \quad 15.039$ bery 100

$\left(2 P^{\star}\right) j=.5 \quad 15.296$ beryl 100

$\left(2 P^{*}\right) j=1.5 \quad 15.333$ beryl 100

(2D) $j=2.5 \quad 15.495$ beryl 100

$\left(2 P^{*}\right) j=.515 .560$ beryl 100

(4P) $j=2.5 \quad 15.630$ bery 100

$(2 P *) j=1.5 \quad 15.702$ beryl 100

(2P) $j=1.5 \quad 15.883$ beryl 100

(2D) $j=2.5 \quad 15.924$ beryl 100

$\left(3 P^{*}\right) j=2.0 \quad 14.279$ gypsum 020

$\left(3 P^{*}\right) j=1.0 \quad 14.291$ gypsum 020

(3P*) $j=0 \quad 14.332$ gresum 020

(1s ) $j=.0 \quad 14.360$ gypsum 020

(1s) $j=.0 \quad 14.401$ gypsum 020

(1P*) $j=1.0 \quad 14.503$ gypsum 020

(1P*) $j=1.0 \quad 14.649$ gypsum 020

$\left(3 P^{*}\right) j=.0 \quad 14.829$ gypsum 020

$(3 P *) j=1.0 \quad 14.870$

(3P) $j=1.0 \quad 14.886$

$\left(1 P^{*}\right) j=1.0 \quad 14.929$ gypsum 020

gypsum 020

gypsum 020
$25.763 \quad 72.108$

5.95478 .080

$15.954 \quad 80.783$

$\begin{array}{ll}19.942 & 70.361\end{array}$

$19.942 \quad 71.128$

$19.942 \quad 72.586$

$19.942 \quad 74.353$

$19.942 \quad 75.399$

$19.942 \quad 76.196$

$19.942 \quad 77.156$

$19.942 \quad 78.164$

$19.942 \quad 79.204$

$19.942 \quad 80.099$

$19.942 \quad 80.543$

$19.942 \quad 81.540$

$19.942 \quad 82.190$

$19.942 \quad 82.737$

$19.942 \quad 83.158$

$19.942 \quad 84.317$

$19.942 \quad 85.783$

$19.942 \quad 86.416$

$19.942 \quad 71.307$

$19.942 \quad 81.638$

$25.763 \quad 70.465$

$15.185 \quad 74.546$

$15.185 \quad 82.048$

$15.954 \quad 70.501$

$\begin{array}{ll}15.954 & 73.487\end{array}$

$\begin{array}{lll}15.954 & 73.961\end{array}$

$15.954 \quad 76.223$

$15.954 \quad 77.240$

$15.954 \quad 78.433$

$15.954 \quad 79.803$

$15.954 \quad 84.593$

$15.954 \quad 86.486$

$15.185 \quad 70.108$

$\begin{array}{ll}15.185 & 70.242\end{array}$

$\begin{array}{lll}15.185 & 70.704\end{array}$

$\begin{array}{ll}15.185 & 71.027\end{array}$

$15.185 \quad 71.508$

$\begin{array}{ll}15.185 & 72.763\end{array}$

$15.185 \quad 74.731$

$\begin{array}{ll}15.185 & 77.569\end{array}$

$\begin{array}{ll}15.185 & 78.309\end{array}$

$15.185 \quad 78.611$

$15.185 \quad 79.464$ 


\begin{tabular}{|c|c|c|c|c|c|c|c|c|}
\hline 20 & Be-like & $1 S 2$ & $2 S$ & $3 D$ & & $(3 D) j=3.0$ & -152 & \\
\hline 20 & Be-like & 152 & $2 \mathrm{~S}$ & $3 D$ & & $(3 D) j=2.0$ & $-1 s 2$ & \\
\hline 20 & Be-like & 152 & $2 \mathrm{P}$ & $3 D$ & & $\left(3 p^{*}\right) j=.0$ & $-1 s 2$ & \\
\hline$\checkmark 20$ & Be-1ike & 1S2 & $2 P$ & $3 \mathrm{D}$ & & $\left(3 P^{*}\right) j=.0$ & -152 & \\
\hline 20 & Be-like & 152 & $2 \mathrm{P}$ & $3 D$ & & $\left(3 D^{*}\right) j=2.0$ & $-1 S 2$ & \\
\hline 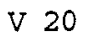 & Be-like & $1 \mathrm{~S} 2$ & $2 \mathrm{P}$ & $3 D$ & & $\left(3 D^{*}\right) j=2.0$ & -152 & \\
\hline$V 20$ & Be-like & $1 \mathrm{~S} 2$ & $2 \mathrm{P}$ & $3 D$ & & $\left(3 D^{\star}\right) j=3.0$ & $-1 s 2$ & \\
\hline 20 & Be-1ike & $1 S 2$ & $2 P$ & $3 D$ & & $\left(3 D^{*}\right) j=3.0$ & -152 & \\
\hline 20 & Be-like & $1 \mathrm{~S} 2$ & $2 P$ & $3 D$ & & $\left(1 F^{\star}\right) j=3.0$ & $-1 s 2$ & \\
\hline 0 & Be-like & $1 S 2$ & $2 P$ & $3 \Gamma$ & & $\left(I D^{*}\right) j=2.0$ & $-1 s 2$ & \\
\hline 20 & Be-like & $1 S 2$ & $2 \mathrm{P}$ & $3 D$ & & $\left.3 F^{\star}\right) j=3.0$ & $-1 S 2$ & \\
\hline 20 & $\mathrm{~B} e-\mathrm{I}$ & $1 s 2$ & $2 s$ & $3 D$ & & 1D $) j=2.0$ &.$\$ 2$ & \\
\hline $\mathrm{V} 20$ & Be-like & $1 S 2$ & $2 S$ & $3 S$ & & $3 S / j=1.0$ & 152 & \\
\hline 0 & Be-like & 152 & $2 P$ & $3 D$ & & $\left.3 E^{*}\right) j=3.0$ & -152 & \\
\hline $\mathrm{V}$ & Be-like & $1 \mathrm{~S} 2$ & $2 S$ & $3 s$ & & $35 \mid j=1.0$ & $-1 s 2$ & \\
\hline $\mathrm{V}$ & Be-like & $1 \mathrm{~S} 2$ & $2 P$ & $3 D$ & & $\left.1 P^{*}\right) j=1.0$ & -152 & \\
\hline $\mathrm{V}$ & $\mathrm{Be}-1$ ike & $1 \mathrm{~S} 2$ & $2 P$ & $3 \mathrm{~S}$ & & $3 p * j j=.0$ & -152 & \\
\hline & $\mathrm{Be}-$ & $1 S 2$ & $2 \mathrm{P}$ & $3 S$ & & $\left.1 P^{*}\right) j=1.0$ & -152 & \\
\hline V & Li-like & $1 S$ & $2 \mathrm{P}$ & $3 \mathrm{P}^{*}$ & ) $3 P$ & $(2 P) j=1.5$ & $-1 s 2$ & \\
\hline V 21 & Li-like & 1s & $2 \mathrm{P}<$ & $3 \mathrm{P}^{*}$ & $3 p$ & $(29) j=1.5$ & -152 & \\
\hline V 21 & $\mathrm{Li}-$ & $1 S$ & $2 P 1$ & $3 P^{*}$ & ) $3 P$ & $(2 P) j=1.5$ & $-1 S 2$ & \\
\hline 21 & ike & 1s & $2 \mathrm{P}$ & $3 P^{*}$ & $3 P$ & $(2 P) j=1.5$ & -152 & \\
\hline$\sqrt{21}$ & ike & is & $2 \mathrm{P} 2$ & & & $\langle 2 s\rangle j=.5$ & $-1 s 2$ & \\
\hline 21 & Li & $1 S$ & $2 \mathrm{P} 2$ & & & $(2 S) j=.5$ & $-1 s 2$ & \\
\hline$\checkmark 21$ & Li & $1 \mathrm{~S}$ & $2 \mathrm{P} 2$ & & & $(2 S) j=.5$ & $-1 S 2$ & \\
\hline 1 & $\mathrm{Li}-$ & $1 \mathrm{~S}$ & $2 \mathrm{P} 2$ & & & $(2 s) j=.5$ & -152 & \\
\hline $\mathrm{V}$ & $\mathrm{Li}$ & $1 \mathrm{~S}$ & (2S & $2 P$ & $1 P^{\star}$ & $\left(2 P^{*}\right) j=1.5$ & $-1 s 2$ & \\
\hline $\mathrm{V}$ & Li- & $1 \mathrm{~s}$ & $(2 S$ & $2 P$ & $1 P^{*}$ & $\left.2 \mathrm{P}^{*}\right)$ & $-1 s 2$ & \\
\hline$\pi$ & Li & Is & $12 \mathrm{~S}$ & $2 \mathrm{P}$ & $1 P^{\star}$ & $\mid\left(2 P^{\star}\right)$ & -152 & \\
\hline V & ike & is & (2S & $2 \mathrm{P}$ & $1 P^{\star}$ & )$\left(2 P^{\star}\right) j=1.5$ & $-1 s 2$ & \\
\hline V 21 & Li-like & $1 \mathrm{~S}$ & $2 \mathrm{P} 2$ & & & $(2 P) j=1.5$ & -152 & \\
\hline V 21 & Li-like & is & $2 \mathrm{P} 2$ & & & $(2 P)$ & $-1 s 2$ & \\
\hline 21 & Li-like & $1 S$ & $2 \mathrm{P} 2$ & & & $(2 P) j$ & $-1 s 2$ & \\
\hline 21 & ike & is & $2 \mathrm{P} 2$ & & & $(2 P)]$ & $-1 S 2$ & \\
\hline 1 & ike & 15 & $2 \mathrm{P} 2$ & & & $(2 D)$ & $-1 S 2$ & \\
\hline V 21 & ke & is & $2 \mathrm{P} 2$ & & & $(2 D)$ & $-1 s 2$ & \\
\hline V 21 & ike & 1s & $2 \mathrm{P} 2$ & & & $(2 D)$ & $-1 s 2$ & \\
\hline $\mathrm{V} 21$ & Li-Iike & 1S & $2 \mathrm{P} 2$ & & & $(2 D)]$ & -152 & \\
\hline V 21 & Li-Iike & is & $2 \mathrm{P} 2$ & & & $(2 D) j$ & -152 & \\
\hline V 21 & Li-like & 1S & $2 \mathrm{P} 2$ & & & $(2 D) j=2.5$ & -152 & \\
\hline$V 2 I$ & Li-1ike & IS & $2 \mathrm{P} 2$ & & & $(2 D) j=2.5$ & $-1 S 2$ & \\
\hline 21 & Li-like & $1 \mathrm{~S}$ & $2 \mathrm{P} 2$ & & & $(2 D) j=2.5$ & $-1 s 2$ & \\
\hline 21 & $\mathrm{Li}$ & is & $2 S<$ & $3 \mathrm{p} *$ & ) & $\left\langle 49^{*}\right\rangle j=1.5$ & -152 & \\
\hline V 21 & Li-like & 1s & $2 S 1$ & $3 P^{*}$ & 2 & $\left(4 P^{*}\right) j=1.5$ & $-1 S 2$ & \\
\hline 21 & Li-like & $1 S$ & $2 S 1$ & $3 P^{\star}$ & $2 P$ & $\left(4 P^{\star}\right) j=1.5$ & $-1 S 2$ & \\
\hline 81 & Li-like & 1s & $2 \mathrm{~s} 1$ & $3 \mathrm{P}^{*}$ & $2 \mathrm{P}$ & $f\left(4 P^{*}\right) j=1.5$ & -152 & \\
\hline
\end{tabular}

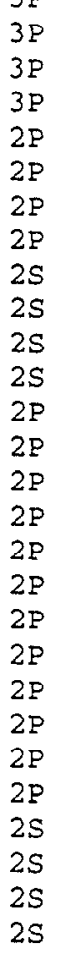
$2 S$ S ( $2 \mathrm{P}$ $P$ P $P$ $2 S$ $2 \mathrm{~S}$ $2 \mathrm{P}$

\begin{tabular}{|c|c|c|}
\hline$\left(3 P^{*}\right) j=2.0$ & 14.976 & gypsum 020 \\
\hline$\left(3 P^{\star}\right) j=2.0$ & 14.986 & gypsum 020 \\
\hline$(3 P) j=1.0$ & 15.051 & gypsum 020 \\
\hline$(3 P) j=1.0$ & 15.051 & beryl 100 \\
\hline $3 P / j=1.0$ & 15.114 & gypsum 020 \\
\hline (p) $j=1.0$ & 15.114 & beryl 100 \\
\hline P $j j=2.0$ & 15.141 & gypsum 020 \\
\hline$p / j=2.0$ & 15.141 & beryl 100 \\
\hline$j j=2.0$ & 15.216 & beryl 100 \\
\hline P $j=1.0$ & 15.229 & beryl 100 \\
\hline P $j j=2.0$ & 15.336 & beryl 100 \\
\hline$\left.q^{\star}\right) j=1.0$ & 15.427 & beryl 100 \\
\hline$\left.p^{*}\right) j=1.0$ & 15.455 & beryl 100 \\
\hline$D \quad j j=2.0$ & 15.551 & beryl 100 \\
\hline$\left.p^{\star}\right) j=2.0$ & 15.580 & beryl 100 \\
\hline is $j j=.0$ & 15.639 & beryl 100 \\
\hline P $j j=1.0$ & 15.884 & beryl 100 \\
\hline D $j j=2.0$ & 15.905 & beryl 100 \\
\hline$\left.p^{*}\right) j=1.5$ & 2.385 & quartz 220 \\
\hline$\left.P^{*}\right) j=1.5$ & 2.385 & quartz 110 \\
\hline$p * j j=1.5$ & 2.385 & gypsum 002 \\
\hline$\left.P^{\star}\right) j=1.5$ & 2.385 & Insb 111 \\
\hline$P^{*} j j=1.5$ & 2.388 & quartz 220 \\
\hline$\left.p^{*}\right) j=1.5$ & 2.388 & quartz 110 \\
\hline$\left.P^{\star}\right) j=1.5$ & 2.388 & gypsum 002 \\
\hline $2 p^{*} j j=1.5$ & 2.388 & InSb 111 \\
\hline $2 s) j=.5$ & 2.390 & quartz 220 \\
\hline $2 S) j=.5$ & 2.390 & quartz 110 \\
\hline $2 s / j=.5$ & 2.390 & gypsum 002 \\
\hline $2 S / j=.5$ & 2.390 & InSb 111 \\
\hline$\left.P^{*}\right) j=1.5$ & 2.399 & quartz 220 \\
\hline$\left.P^{*}\right) j=1.5$ & 2.399 & quartz 110 \\
\hline$p * j=1.5$ & 2.399 & gypsum 002 \\
\hline$p^{*} j j=1.5$ & 2.399 & Inst 111 \\
\hline *) $j=.5$ & 2.401 & cuartz 220 \\
\hline$P^{*} j j=.5$ & 2.401 & quartz 110 \\
\hline $\left.2 P^{\star}\right) j=.5$ & 2.401 & gypsum 002 \\
\hline $2 P^{*} \mid j=.5$ & 2.401 & Insto 111 \\
\hline$\left.p^{*}\right) j=1.5$ & 2.404 & quartz 220 \\
\hline *) $j=1.5$ & 2.404 & cuartz 110 \\
\hline e*) $j=1.5$ & 2.404 & gypsum 002 \\
\hline$\left.p^{*}\right) j=1.5$ & 2.404 & InSb 111 \\
\hline$j=.5$ & 2.414 & guartz 220 \\
\hline$j=.5$ & 2.414 & ouartz 110 \\
\hline$j=.5$ & 2.414 & gypsum 002 \\
\hline s $j j=.5$ & 2.414 & InSb 111 \\
\hline
\end{tabular}

$15.185 \quad 80.483$ $15.185 \quad 80.714$ $15.185 \quad 82.383$ $15.954 \quad 70.631$ $15.185 \quad 84.457$ $15.954 \quad 71.325$ $15.185 \quad 85.637$ $15.954 \quad 71.630$ $15.954 \quad 72.505$ $15.954 \quad 72.661$ $15.954 \quad 74.000$ $15.954 \quad 75.232$ $15.954 \quad 75.632$ $15.954 \quad 77.095$ $15.954 \quad 77.569$ $15.954 \quad 78.596$

$15.954 \quad 84.631$

$15.954 \quad 85.508$

$2.451 \quad 76.673$

4.91276 .190

$4.990 \quad 72.923$

$7.481 \quad 73.023$

$2.451 \quad 76.981$

$4.912 \quad 76.486$

$4.990 \quad 73.159$

$7.481 \quad 73.261$

$2.451 \quad 77.190$

$4.912 \quad 76.687$

$4.990 \quad 73.319$

$7.481 \quad 73.421$

2. $451 \quad 78.177$

$4.912 \quad 77.632$

$4.990 \quad 74.054$

$7.481 \quad 74.162$

$2.451 \quad 78.407$

$4.912 \quad 77.852$

$4.990 \quad 74.222$

7.48174 .331

$2.451 \quad 78.761$

$4.912 \quad 78.189$

$4.990 \quad 74.478$

$7.481 \quad 74.589$

2.45180 .032

$4.912 \quad 79.389$

$4.990 \quad 75.360$

$7.481 \quad 75.478$ 


\begin{tabular}{|c|c|c|c|}
\hline 21 & Li-1ike & $1 s$ & $2 P 2$ \\
\hline 21 & Li-like & 15 & $2 P 2$ \\
\hline 21 & Li-Iike & $1 \mathrm{~s}$ & $2 \mathrm{P} 2$ \\
\hline 21 & Li-like & $1 S$ & $2 P 2$ \\
\hline 21 & Ii-like & 152 & $9 \mathrm{P}$ \\
\hline$\checkmark 21$ & Li-like & $1 S 2$ & $9 P$ \\
\hline$V 2 I$ & Li-1ike & IS2 & $9 P$ \\
\hline V 21 & Li-like & $1 S 2$ & $9 P$ \\
\hline V 21 & Li-like & $1 \mathrm{~S} 2$ & $9 P$ \\
\hline V 21 & Li-like & $1 \mathrm{~S} 2$ & $9 P$ \\
\hline$\checkmark 21$ & Li-like & $1 S 2$ & $8 \mathrm{P}$ \\
\hline $\mathrm{V} 21$ & Li-like & $1 \mathrm{~S} 2$ & $8 P$ \\
\hline V & Li-like & $1 S 2$ & $8 P$ \\
\hline V & Li-like & $1 S 2$ & $8 \mathrm{P}$ \\
\hline V & Li-like & $1 S 2$ & $8 P$ \\
\hline V 21 & Li-like & $1 S 2$ & $8 P$ \\
\hline V 21 & Li-like & $1 S 2$ & 90 \\
\hline$\vee 21$ & Li-like & $1 S 2$ & $9 D$ \\
\hline V 21 & Li-like & $1 \mathrm{~S} 2$ & $9 D$ \\
\hline V & Li-like & $1 S 2$ & $9 \mathrm{D}$ \\
\hline V & Li-like & $1 S 2$ & $9 D$ \\
\hline $\mathrm{V}$ & Li-like & $1 \mathrm{~S} 2$ & $7 P$ \\
\hline V 21 & Li-like & 152 & $7 P$ \\
\hline V 21 & Li-like & IS2 & $7 P$ \\
\hline V 21 & Li-like & $1 S 2$ & $7 P$ \\
\hline V 21 & Li-1ike & $1 S 2$ & $7 P$ \\
\hline V 21 & Li-like & $1 S 2$ & $9 D$ \\
\hline V 21 & Li-like & $1 S 2$ & 90 \\
\hline V 21 & Li-like & $1 S 2$ & $9 D$ \\
\hline V 21 & Li-like & $1 \mathrm{~S} 2$ & $9 D$ \\
\hline V 21 & Li-1ike & IS2 & $9 D$ \\
\hline V 21 & Li-like & $1 S 2$ & 8D \\
\hline V 21 & Li-like & $1 \mathrm{~S} 2$ & $8 D$ \\
\hline V 21 & Li-like & $1 S 2$ & $8 D$ \\
\hline V 21 & Li-like & $1 S 2$ & $8 D$ \\
\hline V 21 & Li-like & $1 S 2$ & $8 \mathrm{D}$ \\
\hline V 21 & Li-like & $1 S 2$ & 8D \\
\hline V 21 & Li-like & $1 S 2$ & $8 \mathrm{D}$ \\
\hline V 21 & Li-like & $1 \mathrm{~S} 2$ & $8 D$ \\
\hline$V 21$ & Li-like & $1 S 2$ & $7 D$ \\
\hline V 21 & Li-like & $1 S 2$ & $6 \mathrm{P}$ \\
\hline V 21 & Li-ijke & $1 S 2$ & $5 \mathrm{D}$ \\
\hline V 21 & Li-like & $1 S 2$ & $5 D$ \\
\hline$V$ & Li-like & 1S2 & $4 \mathrm{P}$ \\
\hline V & Li-like & $1 S 2$ & $4 \mathrm{P}$ \\
\hline 72 & Li-like & $1 S 2$ & $3 D$ \\
\hline
\end{tabular}

(4P) $j=2.5-1 S 2 \quad 2 P$ $(4 P) j=2.5-1522 P$ $(4 P) j=2.5-1 S 2 \quad 2 P$ (4P) $j=2.5-1 S 2 \quad 2 P$ (2Px) $j=1.5-1 S 2 \quad 2 S$ $\left(2 P^{\star}\right) j=1.5-1 S 2 \quad 2 S$ $\left(2 P^{*}\right) j=1.5-1 S 2 \quad 2 S$ $\left\{2 P^{*}\right\} j=1.5-1 S 2 \quad 2 S$ $\left(2 P^{*}\right) j=1.5-1 S 2 \quad 2 S$ $\left(2 \mathrm{P}^{\star}\right) j=1.5-1 \mathrm{~S} 2 \quad 2 \mathrm{~S}$ $\left(2 P^{*}\right) j=1.5-1 S 2 \quad 2 S$ $\left(2 P^{*}\right) j=1.5-1 S 2 \quad 2 S$ $\left(2 P^{*}\right) j=1.5-1 S 2 \quad 2 S$ (2P*) $j=1.5-1 S 2 \quad 2 S$ $\left(2 P^{\star}\right) j=1.5-1 S 2 \quad 2 S$ $\left(2 P^{*}\right) j=1.5-1 S 2 \quad 2 S$ (2D) $j=1.5-1 S 2 \quad 2 P$ (2D) $j=1.5-1 S 2 \quad 2 P$ (2D) $j=1.5-1 S 22 P$ (2D) $j=1.5-1 S 2 \quad 2 P$ (2D) $j=1.5-1 S 2 \quad 2 P$ $\left(2 P^{*}\right) j=1.5-1 S 2 \quad 2 S$ $\left(2 P^{*}\right) j=1.5-1 \mathrm{~S} 2 \quad 2 \mathrm{~S}$ $\left(2 P^{*}\right) j=1.5-1 S 2$ 2S $\left(2 P^{\star}\right) j=1.5-1 S 2 \quad 2 S$ $\left(2 P^{*}\right) j=1.5-1.522 S$ (2D) $j=1.5-1 S 2 \quad 2 P$ (2D) $j=1.5-1 S 2 \quad 2 P$ (2D) $j=1.5-1 \mathrm{~S} 2 \quad 2 \mathrm{P}$ (2D) $j=1.5-1 S 2 \quad 2 P$ (2D) $j=1.5-1 S 2 \quad 2 P$ (2D) $j=1.5$ - IS2 $2 \mathrm{P}$ (2D) $j=1.5-1 S 22 P$ (2D) $j=1.5-1 S 2 \quad 2 P$ (2D) $j=1.5-1 S 22 P$ (2D) $j=15-1 S 2 \quad 2 P$ (2D) $j=1.5-1 S 2 \quad 2 P$ (2D) $j=1.5-1 S 2 \quad 2 \mathrm{P}$ (2D) $j=1.5-1 S 2 \quad 2 P$ (2D) $j=1.5-1 S 2 \quad 2 P$ $\left(2 P^{\star}\right) j=1.5-1 S 2 \quad 2 S$ (2D) $j=1.5-1 S 2 \quad 2 P$ (2D) $j=1.5-1 S 2 \quad 2 P$ $\left(2 P^{\star}\right) j=1.5-1 S 2 \quad 2 S$ $\left(2 P^{*}\right) j=5-1 S 2 \quad 2 S$ (2D) $j=1.5-1 S 2 \quad 2 \mathrm{~g}$

\begin{tabular}{|c|c|c|}
\hline$\left.P^{\star}\right) j=1$ & 2.421 & gua \\
\hline$\left(2 P^{\star}\right) j=1.5$ & 2.421 & quartz 110 \\
\hline$\left(2 P^{\star}\right) j=1.5$ & 2.421 & gypsum 002 \\
\hline$\left(2 P^{\star}\right) j=1.5$ & 2.421 & InSb 111 \\
\hline$(2 S) j=.5$ & 8.288 & topaz 002 \\
\hline$(2 S) j=.5$ & 8.288 & quartz 100 \\
\hline$(2 s) j=.5$ & 8.288 & PET 002 \\
\hline$(2 s) j=.5$ & 8.288 & EDT 020 \\
\hline$(2 s) j=.5$ & 8.288 & TAP 100 \\
\hline$(2 S) j=.5$ & 8.288 & $\operatorname{RAP} 100$ \\
\hline$(2 S) j=.5$ & 8.399 & quartz 100 \\
\hline$(2 S) j=.5$ & 8.399 & PET 002 \\
\hline$(2 s) j=.5$ & 8.399 & EDT 020 \\
\hline$(2 S) j=.5$ & 8.399 & TAP 100 \\
\hline$(2 S) j=.5$ & 8.399 & $\operatorname{RAP} 100$ \\
\hline$(2 S) j=.5$ & 8.399 & KAP 100 \\
\hline$\left(2 P^{*}\right) j=.5$ & 8.527 & PET 002 \\
\hline$\left(2 P^{\star}\right) j=.5$ & 8.527 & EDT 020 \\
\hline$\left(2 P^{\star}\right) j=.5$ & 8.527 & TAP 100 \\
\hline$\left(2 P^{*}\right) j=.5$ & 8.527 & RAP 100 \\
\hline$\left(2 P^{\star}\right) j=.5$ & 8.527 & $\operatorname{KAP} 100$ \\
\hline$(2 s) j=.5$ & 8.576 & PET 002 \\
\hline$(2 s) j=.5$ & 8.576 & EDT 020 \\
\hline$(2 s) j=.5$ & 8.576 & TAP 100 \\
\hline$(2 S) j=.5$ & 8.576 & RAP 100 \\
\hline$(2 s) j=.5$ & 8.576 & KAP 100 \\
\hline$\left(2 P^{\star}\right) j=1.5$ & 8.582 & PET 002 \\
\hline$\left(2 P^{*}\right) j=1.5$ & 8.582 & EDT 020 \\
\hline$\left(2 P^{\star}\right) j=1.5$ & 8.582 & TAP 100 \\
\hline$\left(2 P^{*}\right) j=1.5$ & 8.582 & RAP 100 \\
\hline$\left(2 P^{\star}\right) j=1.5$ & 8.582 & $\operatorname{KAP} 100$ \\
\hline$\left(2 P^{\star}\right) j=.5$ & 8.643 & PET 002 \\
\hline$\left(2 P^{*}\right) j=.5$ & 8.643 & EDT 020 \\
\hline$\left(2 P^{\star}\right) j=.5$ & 8.643 & RAP 100 \\
\hline$\left(2 P^{*}\right) j=.5$ & 8.643 & KAP 100 \\
\hline$\left(2 P^{*}\right) j=1.5$ & 8.703 & PET 002 \\
\hline$\left(2 P^{*}\right) j=1.5$ & 8.703 & EDT 020 \\
\hline$\left(2 P^{\star}\right) j=1.5$ & 8.703 & $\operatorname{RAP} 100$ \\
\hline$\left(2 P^{*}\right) j=1.5$ & 8.703 & KAP 100 \\
\hline$\left(2 P^{*}\right) j=.5$ & 8.826 & KAP 100 \\
\hline$(2 S) j=.5$ & 8.843 & KAP 100 \\
\hline$\left(2 P^{*}\right) j=.5$ & 9.633 & mica 002 \\
\hline$\left(2 P^{*}\right) j=1.5$ & 9.704 & mica 002 \\
\hline $2 s / j=.5$ & 10.401 & $A D P \quad 101$ \\
\hline$j j=.5$ & 10.411 & ADP 101 \\
\hline$\left.P^{\star}\right) J=.5$ & 14.429 & gypsum 02 \\
\hline
\end{tabular}

$2.451 \quad 81.026$

$4.912 \quad 80.316$

$4.990 \quad 76.011$

$\begin{array}{ll}7.481 & 76.134\end{array}$

$8.374 \quad 81.782$

$8.512 \quad 76.826$

$8.742 \quad 71.454$

$8.808 \quad 70.214$

$25.763 \quad 74.819$

$26.116 \quad 72.187$

$8.512 \quad 80.654$

$8.742 \quad 73.897$

$8.808 \quad 72.471$

$25.763 \quad 77.968$

$26.116 \quad 74.755$

$26.634 \quad 71.093$

$8.742 \quad 77.267$

$8.808 \quad 75.488$

$25.763 \quad 83.186$

$26.116 \quad 78.383$

$26.634 \quad 73.835$

$8.742 \quad 78.817$

$8.808 \quad 76.820$

$25.763 \quad 87.013$

$26.116 \quad 80.111$

$26.634 \quad 75.013$

$8.742 \quad 79.021$

$8.808 \quad 76.993$

$25.763 \quad 87.918$

$26.116 \quad 80.344$

26.63475 .163

$8.742 \quad 81.369$

$8.808 \quad 78.892$

$26.116 \quad 83.139$

$26.634 \quad 76.788$

$8.742 \quad 84.586$

$8.808 \quad 81.144$

$26.116 \quad 88.674$

$26.634 \quad 78.605$

$26.634 \quad 83.796$

$26.634 \quad 84.911$

$19.942 \quad 75.039$

$19.942 \quad 76.711$

$10.640 \quad 77.833$

$10.640 \quad 78.091$

$15.185 \quad 71.844$ 


\begin{tabular}{|c|c|c|c|}
\hline V 21 & Li-like & $1 S 2$ & $3 D$ \\
\hline V 21 & Li-like & 152 & $3 D$ \\
\hline V 21 & Li-like & 152 & $3 S$ \\
\hline$V 21$ & Li-like & $1 \mathrm{~S} 2$ & $3 s$ \\
\hline V 21 & Li-like & & 110 \\
\hline V 22 & He-like & $1 S$ & $5 P$ \\
\hline V 22 & He-like & 1S & $5 P$ \\
\hline V 22 & He-like & is & $5 P$ \\
\hline $\mathrm{V} 22$ & He-like & $1 S$ & $4 P$ \\
\hline $\mathrm{V} 22$ & He-like & 15 & $4 P$ \\
\hline$\vee 22$ & He-like & $1 S$ & $4 \mathrm{P}$ \\
\hline$\vee 22$ & $\mathrm{He}$-like & $1 S$ & $4 \mathrm{P}$ \\
\hline V 22 & He-like & is & $4 P$ \\
\hline V 22 & He-like & $1 s$ & $4 \mathrm{P}$ \\
\hline V 22 & He-like & IS & $3 P$ \\
\hline V 22 & He-like & 15 & $3 P$ \\
\hline V 22 & He-like & 15 & $3 P$ \\
\hline V 22 & $\mathrm{He}-1$ ike & 15 & $3 P$ \\
\hline V 22 & He-like & is & $3 P$ \\
\hline V 22 & He-like & $1 \mathrm{~s}$ & $3 P$ \\
\hline V 22 & He-like & IS & $3 P$ \\
\hline V 22 & He-like & $1 S$ & $2 \mathrm{P}$ \\
\hline V 22 & He-like & is & $2 P$ \\
\hline V 22 & He-like & $1 S$ & $2 P$ \\
\hline V 22 & He-like & $1 \mathrm{~S}$ & $2 P$ \\
\hline V 22 & He-like & $1 S$ & 29 \\
\hline V 22 & He-like & $1 S$ & $2 \mathrm{P}$ \\
\hline V 22 & He-like & $1 S$ & $2 P$ \\
\hline V 22 & He-Iike & $1 S$ & $2 P$ \\
\hline V 22 & He-like & $1 \mathrm{~s}$ & $2 S$ \\
\hline V 22 & He-like & $1 \mathrm{~s}$ & $2 S$ \\
\hline V 22 & He-like & $1 S$ & $2 S$ \\
\hline V 22 & He-like & 15 & $2 S$ \\
\hline V 22 & He-like & is & $3 P$ \\
\hline V 22 & He-like & 1s & $3 P$ \\
\hline V 22 & He-like & 1S & $3 P$ \\
\hline V 23 & $\mathrm{H}$-like & 78 & \\
\hline V 23 & H-like & $7 P$ & \\
\hline V 23 & H -like & $7 P$ & \\
\hline V 23 & $\mathrm{H}$-like & $7 P$ & \\
\hline V 23 & $\mathrm{H}$-iike & $6 P$ & \\
\hline V 23 & H-like & $6 P$ & \\
\hline V 23 & H-like & $6 P$ & \\
\hline V 23 & $\mathrm{H}$-like & $6 P$ & \\
\hline V 23 & H -like & $5 P$ & \\
\hline V 23 & H -like & $5 P$ & \\
\hline
\end{tabular}

(2D) $j=2.5-1 S 2 \quad 2 P$ (2D) $j=1.5-1 S 22 P$ (2S) $j=.5-1 S 2 \quad 2 P$ (2S) $j=.5-1 S 2 \quad 2 P$ ( $) j=.0$ 110

$\left(1 P^{*}\right) j=1.0-1 S 2$

$\left(1 P^{\star}\right) j=1.0-152$

$\left(1 P^{*}\right) j=1.0-1 \mathrm{~S} 2$

$\left(1 P^{*}\right) j=1.0-1 S 2$

$\left(1 P^{\star}\right) j=1.0-152$

$\left(1 P^{*}\right) j=1.0-152$

$\left(1 P^{*}\right) j=1.0-1 S 2$

$\left(1 P^{*}\right) j=1.0-1 S 2$

$\left(1 P^{*}\right) j=1.0-152$

$\left(1 P^{*}\right) j=1.0-1 S 2$

$\left(1 P^{*}\right) j=1.0-1 S 2$

$\left(1 P^{*}\right) j=1.0-1 S 2$

$\left(1 P^{\star}\right) j=1.0-152$

$\left(1 P^{*}\right) j=1.0-152$

$\left(1 P^{\star}\right) j=1.0-1 S 2$

$\left(1 P^{*}\right) j=1.0-152$

$\left(1 P^{\star}\right) j=1.0-1 S 2$

$\left(1 P^{\star}\right) j=1.0-152$

$\left(1 P^{*}\right) j=1.0-1 \mathrm{~S} 2$

$\left(1 P^{*}\right) j=1.0-1.52$

$\left(3 P^{*}\right) j=1.0-152$

$\left(3 P^{*}\right) j=1.0-1 S 2$

$\left(3 P^{*}\right) j=1.0-1 S 2$

$\left(3 P^{\star}\right) j=1.0-1 S 2$

(3S) $j=1.0-1 S 2$

(3S) $j=1.0-1 S 2$

(3S) $j=1.0-1 S 2$

(3S) $j=1.0-1 S 2$

$\left(3 P^{*}\right) j=2.0-1 S 2 S$

$\left(3 P^{*}\right) j=20-1 S \quad 2 S$

$\left(1 P^{*}\right) j=1.0-1 S 2 S$

$\left\langle 2 P^{\star}\right\rangle j=1.5-1 S$

(2P*)j=1.5 - $1 \mathrm{~S}$

$\left(2 P^{*}\right) j=1.5-1 S$

$\left(2 P^{*}\right) j=1.5-1 S$

$\left(2 P^{*}\right) j=1.5-1 S$

$\left(2 P^{*}\right) j=1.5-1 S$

$\left(2 P^{*}\right) j=1.5-1 S$

$\left(2 P^{*}\right) j=1.5-1 S$

$\left\{P^{*}\right\} j=1.5-1 S$

$\left(2 P^{\star}\right) j=1.5-1 S$

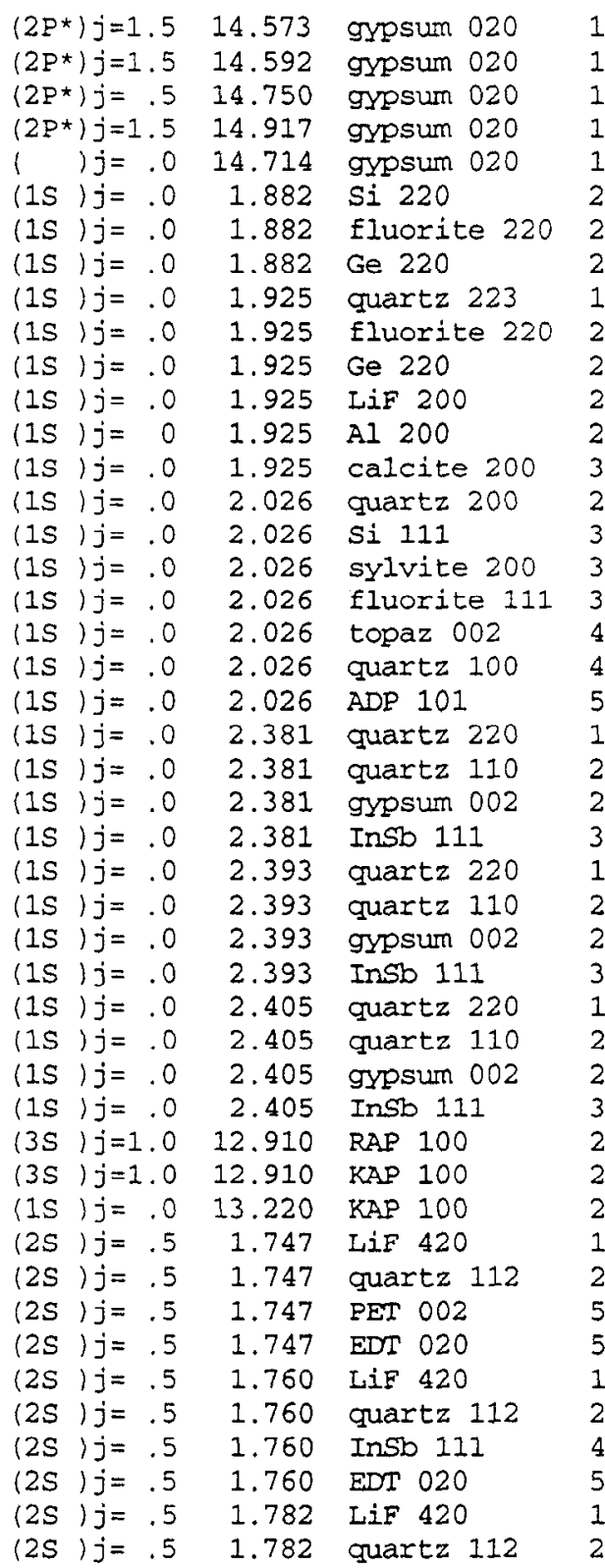

$15.185 \quad 73.678$

$15.185 \quad 73.935$

$15.185 \quad 76.253$

$15.185 \quad 79.220$

$15.185 \quad 75.692$

$3.840 \quad 78.582$

$3.862 \quad 77.065$

$4.000 \quad 70.220$

$2.024 \quad 72.006$

$3.862 \quad 85.482$

$4.000 \quad 74.259$

$4.027 \quad 72.950$

$4.048 \quad 72.006$

$6.071 \quad 72.035$

$4.246 \quad 72.613$

$6.271 \quad 75.748$

$\begin{array}{ll}6.292 & 75.014\end{array}$

$6.308 \quad 74.480$

$8.374 \quad 75.411$

$8.512 \quad 72.188$

$10.640 \quad 72.188$

$2.451 \quad 76.274$

$4.912 \quad 75.804$

$4.990 \quad 72.613$

$7.481 \quad 72.711$

$2.451 \quad 77.511$

$4.912 \quad 76.994$

$4.990 \quad 73.560$

$7.481 \quad 73.664$

$2.451 \quad 78.882$

$4.912 \quad 78.303$

$4.990 \quad 74.564$

$7.481 \quad 74.675$

$26.116 \quad 81.365$

$26.634 \quad 75.798$

$26.634 \quad 83.080$

$1.801 \quad 75.934$

$3.636 \quad 73.935$

$8.742 \quad 87.707$

$8.808 \quad 82.618$

$1.801 \quad 77.751$

$3.636 \quad 75.488$

$7.481 \quad 70.229$

$8.808 \quad 87.558$

$1.801 \quad 81.670$

$\begin{array}{ll}3.636 & 78.579\end{array}$ 


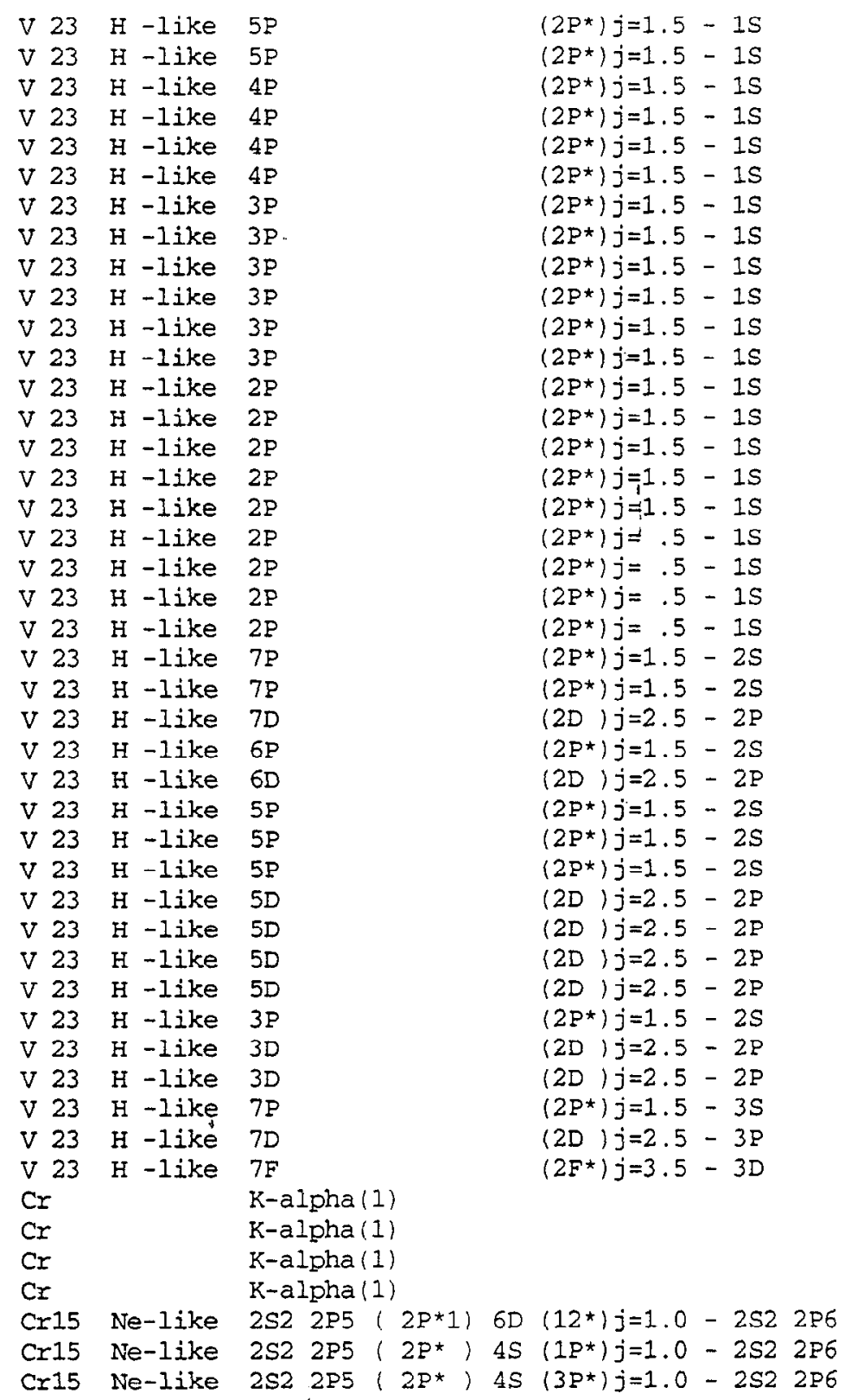

\begin{tabular}{|c|c|c|}
\hline$(2 s) j=.5$ & 1.782 & NaC1 200 \\
\hline $2 s / j=.5$ & 1.782 & InSb 111 \\
\hline$j j=.5$ & 1.825 & Si 220 \\
\hline$j=.5$ & 1.825 & fluorite 220 \\
\hline$j=.5$ & 1.825 & $\mathrm{NaCl} 200$ \\
\hline$j j=.5$ & 1.825 & InSto 111 \\
\hline$j j=.5$ & 1.924 & quartz 223 \\
\hline$j j=.5$ & 1.924 & Eluorite 220 \\
\hline$s j j=.5$ & 1.924 & $\mathrm{Ge} 220$ \\
\hline$j j=.5$ & 1.924 & LiF 200 \\
\hline$j=.5$ & 1.924 & AI 200 \\
\hline$j=.5$ & 1.924 & calcite 200 \\
\hline$j j=.5$ & 2.278 & Ge 422 \\
\hline$j=.5$ & 2.278 & quartz 310 \\
\hline$j j=.5$ & 2.278 & quartz 112 \\
\hline$j=.5$ & 2.278 & topaz 200 \\
\hline$j=.5$ & 2.278 & Al 111 \\
\hline$j=.5$ & 2.283 & Ge 422 \\
\hline$j=.5$ & 2.283 & quartz 310 \\
\hline$j j=.5$ & 2.283 & topaz 200 \\
\hline$j j=.5$ & 2.283 & Al 111 \\
\hline$j j=.5$ & 7.433 & InSb 111 \\
\hline$j=.5$ & 7.433 & gypsum 020 \\
\hline$j=1.5$ & 7.489 & gypsum 020 \\
\hline$j=$ & 7.677 & bery 100 \\
\hline$j=1.5$ & 7.737 & bery 1100 \\
\hline$j=$ & 8.122 & topaz 002 \\
\hline$j j=.5$ & 8.122 & quartz 100 \\
\hline$j j=.5$ & 8.122 & TAP 100 \\
\hline$j j=1.5$ & 8.188 & topaz 002 \\
\hline$j j=1.5$ & 8.188 & quartz 100 \\
\hline$j=1.5$ & 8.188 & TAP 100 \\
\hline$j=1.5$ & 8.188 & RAP 100 \\
\hline$j=.5$ & 12.234 & TAP 100 \\
\hline$j=1.5$ & 12.372 & TAP 100 \\
\hline$j j=1.5$ & 12.372 & RAP 100 \\
\hline$j j=.5$ & 18.831 & mica 002 \\
\hline$j=1.5$ & 18.938 & mica 002 \\
\hline$j j=2.5$ & 18.974 & mica 002 \\
\hline & 2.290 & Ge 422 \\
\hline & 2.290 & quartz 310 \\
\hline & 2.290 & topaz 200 \\
\hline & 2.290 & Al 111 \\
\hline$j=$ & 13.294 & KAP 100 \\
\hline$j=$ & 15.509 & bery 1100 \\
\hline$j=.0$ & 15. & beryl 1 \\
\hline
\end{tabular}

\begin{tabular}{rr}
5.641 & 71.388 \\
7.481 & 72.329 \\
3.840 & 71.901 \\
3.862 & 70.928 \\
5.641 & 76.066 \\
7.481 & 77.371 \\
2.024 & 71.914 \\
3.862 & 85.120 \\
4.000 & 74.154 \\
4.027 & 72.853 \\
4.048 & 71.914 \\
6.071 & 71.943 \\
2.310 & 80.452 \\
2.360 & 74.852 \\
4.564 & 86.607 \\
4.638 & 79.210 \\
4.676 & 76.992 \\
2.310 & 81.231 \\
2.360 & 75.324 \\
4.638 & 79.891 \\
4.676 & 77.548 \\
7.481 & 83.506 \\
15.185 & 78.235 \\
15.185 & 80.529 \\
15.954 & 74.237 \\
15.954 & 75.910 \\
8.374 & 75.908 \\
8.512 & 72.589 \\
25.763 & 71.045 \\
8.374 & 77.901 \\
8.512 & 74.141 \\
25.763 & 72.451 \\
26.116 & 70.148 \\
25.763 & 71.756 \\
25.763 & 73.832 \\
26.116 & 71.346 \\
19.942 & 70.785 \\
19.942 & 71.742 \\
19.942 & 72.075 \\
2.310 & 82.417 \\
2.360 & 75.990 \\
4.638 & 80.898 \\
4.676 & 78.346 \\
15.634 & 86.632 \\
15.954 & 76.436 \\
15.954 & 81.728 \\
\hline
\end{tabular}

Page 53 
Cr15 Ne-like 2S2 $2 \mathrm{P} 5(2 \mathrm{P} * 2) 3 \mathrm{D}\left(22^{*}\right) \mathrm{j}=1.0-2 \mathrm{~S} 2 \quad 2 \mathrm{P} 6$ Cr15 Ne-like 2S2 2P5 ( 2P*2) 3D (21*) $j=1.0-2 S 22 \mathrm{P} 6$ Cr16 F-like 2S2 2P4 ( 3P) $4 D(4 P) j=2.5-2 S 2$ 2P5 Cr16 F-like 2S2 2P4 ( $1 S$ ) $4 S$ (2S) $j=.5-2 S 22 P 5$ Cr16 F-like 2S2 2P4 ( $1 S$ ) 3S (2S) $j=.5-2 S 22 \mathrm{P} 5$ Cr16 F-like 2S2 2P4 (1S) 3S (2S) $j=.5-2 S 22 P 5$ Cr16 F-like 2S2 2P4 (1D) 3S (2D) $j=2.5-2 S 22 P 5$ Cr16 F-like 2S2 $2 P 4(3 P)$ 3S $(2 P) j=.5-2 S 22 P 5$ Cr16 F-like 2S2 2P4 ( $1 D$ ) 3S (2D) $j=1.5-2 S 22 P 5$ Cr16 F-like 2S2 2P4 (3P) 3S (2P) $j=1.5-2 S 22 P 5$ Cr16 F-like 2S2 2P4 ( $3 P$ ) 3S (2P) $j=.5-2 S 22 P 5$ $C r 16 F$-like $2 S 2$ 2P4 ( $3 P$ ) 3S (4P) $j=2.5-2 S 22 P 5$ Cr16 F-like 2S2 2P4 ( 3P) 3S (4P) $j=.5-2 S 22 P 5$ Cr18 N-like 2S2 2P2 ( $1 D)$ ) $3 D(2 F) j=2.5-2 S 22 P 3$ Cr18 N-like 2S2 $2 \mathrm{P} 2(3 \mathrm{P}) 3 \mathrm{D}(4 \mathrm{P}) \mathrm{j}=1.5-2 \mathrm{~S} 22 \mathrm{P} 3$ Cr18 N-like $2 S 22 \mathrm{P} 2(3 \mathrm{P}) 3 \mathrm{D}(4 \mathrm{P}) j=2.5-2 S 22 \mathrm{P} 3$ Cr18 N-like 2S2 2P2 (1D) 3D (2P) j=1.5 - 2S2 2P3 Cr18 N-like 2S2 2P2 ( $1 D$ ) 3D (2F) $j=3.5-2 S 22 P 3$ Cr18 N-like 2S2 2P2 ( $3 P$ ) 3D (2F) j=3.5- 2S2 2 P3 Cr19 C-like 2S2 2P

Cr19 C -like 2S2 2P $3 D$

Cr19 C-like 2S2 2P 3D

Cr19 C-like 2S2 2P

Cr19 C -like 2S2 2P

Cr19 C-like 2S2 2P

Cr19 C-like 2S2 2P

Cr19 C-like 2S2 2P

Cr19 C-like 2S2 $2 P$

Cr19 C-like 2S 2P2

Cr19 C-like 2S2 2P $3 \mathrm{~S}$

Cr20 B - - ike 2S 2P (3P*) $3 P^{*}\left(2 F^{*}\right) j=3.5-2 S-2 P^{*}$

Cr20 B-like 2S 2P ( $\left.3 P^{*}\right) 3 D\left(2 F^{*}\right) j=2.5-2 S \quad 2 P 2$

Cr20 B-like 2S 2P (3P*) 3D $\left(2 D^{*}\right) j=2.5-2 S$ 2P2

Cr20 B-like 2S 2P ( $\left.3 P^{*}\right) \quad 3 D\left(2 D^{*}\right) j=2.5-2 S$ 2P2

Cr20 B-like 2S2 $3 \mathrm{~s}$

Cr20 B-like 2S2 $3 \mathrm{~S}$

Cr21 Be-like 1S2 $2 P \quad 3 P$

Cr21 Be-like 1S2 2P 3P

Cr21 Be-like 1S2 2P $3 F$

Cr21 Be-1ike IS2 2P 3P

Cr21 Be-like 1S2 $2 \mathrm{P}$ 3P

Cr21 Be-like 1S2 2P 3P

Cr21 Be-like 1S2 2P $3 P$

Cr21 Be-like 1S2 2S 3P

Cr21 Be-like IS2 2S 3P

Cr21 Be-like 1S2 $2 S$ 3S

$\left(3 P^{*}\right) j=2,0-2 S 2 \quad 2 P 2$

$\left(3 D^{\star}\right) j=3.0-2 S 22 P 2$

3D $\left(1 P^{\star}\right) j=1.0-2 S 22 P 2$

3D $\left(3 F^{\star}\right) j=3.0-2 S 2 \quad 2 F 2$

3D $\left(3 F^{*}\right) j=3.0-2 S 2 \quad 2 P 2$

3D $\left(1 P^{*}\right) j=1.0-2 S 2 \quad 2 P 2$

$3 D \quad\left(1 P^{*}\right) j=1.0-2 S 22 P 2$

3D $\left(1 D^{\star}\right) j=2.0-2 S 22 P 2$

) $3 D(3 F) j=4.0-2 S 2 P 3$

(2S) $j=.5-2 S 22 P$

(2S) $j=.5-2 S 22 P$

$\left(3 P^{\star}\right) j=1.0-1 S 2 \quad 2 S 2$

$\left(1 P^{*}\right) j=1.0-1 S 22 S 2$ (1s) $j=0 \quad 18.782$ mica 002

(1S) $j=.0 \quad 19.015$ mica 002

$\left(2 P^{*}\right) j=1.5 \quad 14.290$ gypsum 020

$\left(2 P^{*}\right) j=1.5 \quad 14.470$

$\left(2 P^{\star}\right) j=1.5 \quad 18.775$

$\left(2 P^{*}\right) j=.5$

19.038

gypsum 020

$\left(2 D^{*}\right) j=1.5$

$\left(2 P^{\star}\right) j=1.5$

$\left(2 P^{*}\right) j=.5$

$\left(2 P^{*}\right) j=1.5$

19.255

$\left(2 P^{*}\right) j=5$

19.511

19.538

$\left(2 P^{*}\right) j=1$.

$\left(2 P^{\star}\right) j=.5$

$\left(2 D^{\star}\right) j=1.5$

$\left(4 S^{\star}\right) j=1$.

19.714

19.807

19.847

$\left(2 D^{*}\right) j=2$.

$\left(2 D^{\star}\right) j=2$.

15.550

$\left(2 D^{\star}\right) j=2.5$

15.587

(3P) $j=1.0$

15.835

(3P) $j=1.0-1 S 22 S \quad 2 F$

(3P) $j=1.0-1 S 22 S \quad 2 P$

(3S ) $j=1.0-1 S 22 S \quad 2 P$

(3S) $j=1.0-1 S 2$ 2S $2 P$

(3D) $j=3.0-1 S 22 S \quad 2 P$

(3D) $j=3.0-1 S 22 S \quad 2 P$

(3D) $j=1.0-1 S 22 S 2 F$

(IS) $j=.0-1 S 22 S 2 P$
(3P) $j=2.0 \quad 14.809$

(1D) $j=2.0 \quad 14.925$

(3P) $j=2.0 \quad 15.027$

(3P) $j=2.0 \quad 15.027$

(1S) $j=.0 \quad 15.180$

(1S ) $j=.0 \quad 15.180$

(ID) $j=2.0 \quad 15.251$

$\left(3 D^{\star}\right) j=3.0 \quad 15.301$

(3P) $j=2.0 \quad 15.910$

(2D) $j=2.5 \quad 14.447$

(2D) $j=1.5 \quad 14.524$

(2D) $j=1.5 \quad 14.641$

(2D) $j=2.5 \quad 14.669$

$(2 P *) j=1.5 \quad 15.060$

$\left(3 P^{*}\right) j=1.0 \quad 12.909$

$\left(3 P^{*}\right) j=1.0 \quad 12.909$

$\left(3 P^{*}\right) j=2.0 \quad 12.981$

$\left(3 P^{*}\right) j=2.0 \quad 12.981$

$\left(3 P^{*}\right) j=2.0 \quad 13.018$

$\left(3 P^{*}\right) j=2.0 \quad 13.018$

$\left(3 P^{\star}\right) j=$.

(1S $j=013.081$ KAP 100

(1S) $j=$

$\left(1 P^{\star}\right) j=1.0 \quad 13.203$ KAP 100
(3P) $j=2.0 \quad 14.836$

$\left(2 P^{*}\right) j=1.5 \quad 15.060$
$19.942 \quad 70.361$

$19.942 \quad 72.462$

$15.185 \quad 70.230$

$15.185 \quad 72.348$

$19.942 \quad 70.302$

$19.942 \quad 72.682$

$19.942 \quad 74.917$

$19.942 \quad 77.143$

19.94278 .066

19.94278 .447

$19.942 \quad 81.328$

$19.942 \quad 83.329$

$19.942 \quad 84.405$

$15.954 \quad 76.314$

$15.954 \quad 76.482$

15.95476 .605

15.95477 .078

$15.954 \quad 77.687$

$15.954 \quad 82.998$

$15.185 \quad 77.104$

$15.185 \quad 77.223$

$\begin{array}{ll}15.185 & 77.692\end{array}$

$15.185 \quad 79.382$

$15.185 \quad 81.728$

$15.954 \quad 70.372$

15.18588 .530

$15.954 \quad 72.080$

$15.954 \quad 72.928$

15.95473 .551

$15.954 \quad 85.744$

$15.185 \quad 72.064$

$15.185 \quad 73.032$

$15.185 \quad 74.617$

$15.185 \quad 75.021$

$15.185 \quad 82.643$

$15.954 \quad 70.728$

$26.116 \quad 81.336$

$26.634 \quad 75.781$

$26.116 \quad 83.775$

$26.634 \quad 77.102$

$26.116 \quad 85.514$

$26.634 \quad 77.836$

26.63478 .725

$26.634 \quad 79.197$

$26.634 \quad 80.208$

$26.634 \quad 82.498$ 


\begin{tabular}{|c|c|c|c|c|}
\hline $\operatorname{Cr} 21$ & Be-like & $1 s 2$ & & \\
\hline $\operatorname{Cr} 21$ & Be-like & $1 S 2$ & & \\
\hline $\operatorname{Cr} 21$ & Be-like & 152 & & \\
\hline Cr22 & Li-like & 15 & $2 S$ & $2 P$ \\
\hline $\operatorname{Cr} 22$ & Li-like & $1 \mathrm{~S}$ & $2 S$ & $2 P$ \\
\hline Cr22 & Li-like & is & 25 & $2 P$ \\
\hline $\mathrm{C} \times 22$ & Li-like & is & 25 & $2 P$ \\
\hline $\operatorname{Cr} 22$ & Li-like & 1S & $2 S$ & $2 P$ \\
\hline $\mathrm{Cr} 22$ & Li-like & 1S & $2 S$ & $2 P$ \\
\hline $\mathrm{Cr} 22$ & Li-like & IS & $2 S$ & $2 P$ \\
\hline $\mathrm{Cr} 22$ & Li-like & is & $2 S$ & $2 P$ \\
\hline $\mathrm{Cr} 22$ & Li-like & 1S & $2 S$ & $2 P$ \\
\hline $\mathrm{Cr} 22$ & Li-like & 152 & 98 & \\
\hline $\mathrm{Cr} 22$ & Li-like & 152 & $9 P$ & \\
\hline $\operatorname{Cr} 22$ & Li-like & 152 & $8 \mathrm{P}$ & \\
\hline $\mathrm{Cr} 22$ & Li-like & 152 & $9 D$ & \\
\hline $\mathrm{Cr} 22$ & Li-like & 152 & 79 & \\
\hline $\operatorname{Cr} 22$ & Li-like & 152 & $9 D$ & \\
\hline $\operatorname{Cr} 22$ & Li-like & $1 S 2$ & $8 D$ & \\
\hline $\operatorname{cr} 22$ & Li-like & 152 & $8 D$ & \\
\hline $\mathrm{Cr} 22$ & Li-like & 152 & $8 D$ & \\
\hline $\mathrm{Cr} 22$ & Li-like & $1 \mathrm{~S} 2$ & $8 \mathrm{D}$ & \\
\hline $\mathrm{Cr} 22$ & Li-like & 152 & $7 D$ & \\
\hline $\operatorname{Cr} 22$ & Li-like & 152 & $7 D$ & \\
\hline $\operatorname{Cr} 22$ & Ii-like & $1 \mathrm{~S} 2$ & $6 P$ & \\
\hline $\operatorname{Cr} 22$ & Li-like & 152 & $6 P$ & \\
\hline $\operatorname{Cr} 22$ & Li-like & $1 S 2$ & $7 D$ & \\
\hline $\operatorname{Cr} 22$ & Li-like & $1 \mathrm{~s} 2$ & $7 D$ & \\
\hline $\mathrm{Cr} 22$ & Li-like & 152 & $7 D$ & \\
\hline $\mathrm{Cr} 22$ & Li-like & 1S2 & $6 D$ & \\
\hline Cr22 & Li-like & $1 S 2$ & $6 D$ & \\
\hline Cr22 & Li-Iike & $1 S 2$ & $6 D$ & \\
\hline $\mathrm{Cr} 22$ & Li-like & 152 & $6 D$ & \\
\hline $\mathrm{Cr} 22$ & Li-like & $1 S 2$ & $6 \mathrm{D}$ & \\
\hline $\mathrm{Cr} 22$ & Li-like & 152 & $6 D$ & \\
\hline $\mathrm{Cr} 22$ & Li-like & 152 & $6 D$ & \\
\hline $\operatorname{Cr} 22$ & Li-like & $1 \mathrm{~s} 2$ & $6 D$ & \\
\hline $\operatorname{Cr} 22$ & Li-like & 152 & $6 D$ & \\
\hline $\mathrm{Cr} 22$ & Li-like & $1 \mathrm{~s} 2$ & $6 D$ & \\
\hline $\mathrm{Cr} 22$ & Li-like & $1 \mathrm{~S} 2$ & $6 D$ & \\
\hline $\mathrm{Cr} 22$ & Li-like & $1 S 2$ & $6 \mathrm{D}$ & \\
\hline $\mathrm{Cr} 22$ & li-like & 152 & $6 D$ & \\
\hline $\mathrm{Cr} 22$ & Li-like & $1 S 2$ & $5 P$ & \\
\hline $\operatorname{Cr} 22$ & Li-like & $1 S 2$ & $5 P$ & \\
\hline $\mathrm{Cr} 22$ & Li-like & 152 & $5 P$ & \\
\hline Cr22 & Li-like & IS2 & $5 P$ & \\
\hline
\end{tabular}

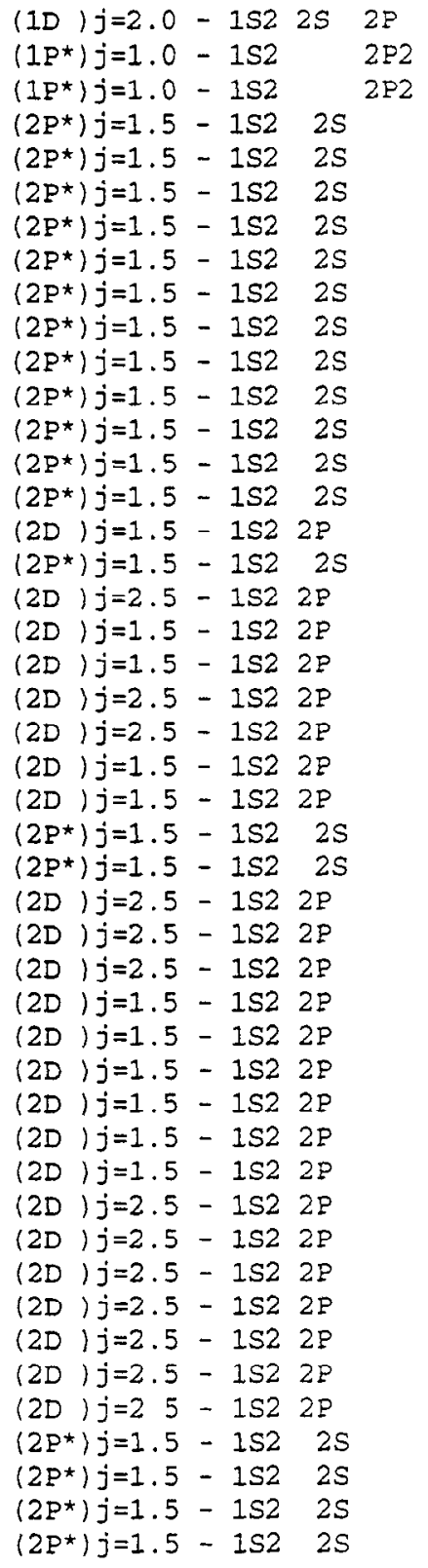

$2 F$

(1D) $j=2.0 \quad 14.457$ gypsum 020

(1s) $j=.0 \quad 14.896$ gypsum 020

(2S) $j=.5 \quad 2.190$ Si 422

(2S) $j=.5 \quad 2.190$ Ge 422

(2S) $j=.5$

(2s) $j=.5$

(2s) $j=.5$

(2S) $j=.5$

(2s) $j=.5$

(2S) $j=.5$

(2S) $j=.5$

(2S) $j=.5$

(2S) $j=.5$

(2S) $j=.5$

$\left(2 P^{*}\right) j=.5$

$(2 S) j=.5$

$\left(2 P^{*}\right) j=1.5$

$\left(2 P^{*}\right) j=.5$

$\left(2 P^{*}\right) j=.5$

$\left(2 P^{*}\right) j=1.5$

$\left(2 P^{*}\right) j=1.5$

$\left(2 P^{*}\right) j=.5$

$\left(2 P^{*}\right) j=.5$

(2S) $j=.5$

(2S) $j=.5$

$\left(2 P^{*}\right) j=1.5$

$\left(2 P^{*}\right) j=1.5$

$\left(2 P^{\star}\right) j=1.5$

$\left(2 P^{\star}\right) j=.5$

$\left(2 P^{\star}\right) j=.5$

$\left(2 P^{\star}\right) j=.5$

$\left(2 P^{*}\right) j=.5$

$\left(2 P^{*}\right) j=.5$

$\left(2 P^{\star}\right) j=.5$

$\left(2 P^{*}\right) j=1.5$

$\left(2 P^{\star}\right) j=1.5$

$\left(2 P^{\star}\right) j=1.5$

$\left(2 P^{*}\right) j=1.5$

$\left(2 P^{*}\right) j=1.5$

$\left(2 P^{*}\right) j=1.5$

$\left(2 P^{\star}\right) j=1.5$

(2S) $j=.5$

(2s) $j=.5$

(2s) $j=.5$

(2s) $j=.5$

8.304 EDT 020
2.190 tungsten 1102

2.190 quartz 112

2.190 topaz 200

$2.190 \mathrm{KBr} 200$

2.190 quartz 101

2.190 graphite 002

2.190 EDT 020

7.562 gypsum 020

7.562 beryl 100

7.664 beryl 100

7.774 beryl 100

7.817 beryl 100

7.828 beryl 100

7.881 topaz 002

7.881 beryl 100

7.936 topaz 002

7.936 beryl 100

8.042 topaz 002

8.042 quartz 100

8.066 topaz 002

8.066 quartz 100

8.100 topaz 002

8.100 quartz 100

8.100 TAP 100

8.304 topaz 002

8.304 quartz 100

8.304 PET 002

8.304 TAP 100

8.304 RAP 100

8.365 topaz 002

8.365 quartz 100

8.365 PET 002

8.365 EDT 020

8.365 TAP 100

8.365 RAP 100

8.365 KAP 100

8.516 PET 002

8.516 EDT 020

8.516 TAP 100

8.516 RAP 100
$26.634 \quad 89.298$

$15.185 \quad 72.187$

$15.185 \quad 78.804$

$2.217 \quad 81.049$

$2.310 \quad 71.451$

$4.476 \quad 78.112$

$4.564 \quad 73.675$

$4.638 \quad 70.799$

$6.584 \quad 86.263$

$6.687 \quad 79.266$

$6.696 \quad 78.867$

$8.808 \quad 84.016$

$15.185 \quad 84.863$

$15.954 \quad 71.437$

$15.954 \quad 73.896$

$15.954 \quad 77.046$

15.95478 .505

$15.954 \quad 78.909$

8.37470 .242

$15.954 \quad 81.102$

$8.374 \quad 71.387$

$15.954 \quad 84.188$

$8.374 \quad 73.812$

$8.512 \quad 70.871$

$8.374 \quad 74.412$

8.51271 .370

$8.374 \quad 75.303$

8.51272 .101

$25.763 \quad 70.598$

$8.374 \quad 82.587$

$8.512 \quad 77.308$

$8.742 \quad 71.786$

$8.808 \quad 70.524$

$25.763 \quad 75.233$

$26.116 \quad 72.535$

$8.374 \quad 87.343$

$8.512 \quad 79.336$

$8.742 \quad 73.112$

$8.808 \quad 71.751$

$25.763 \quad 76.924$

$26.116 \quad 73.926$

$26.634 \quad 70.427$

$8.742 \quad 76.944$

$8.808 \quad 75.206$

$25.763 \quad 82.593$

$26.116 \quad 78.029$ 


\begin{tabular}{|c|c|c|c|}
\hline $\mathrm{Cr} 22$ & Li-1ike & $1 s 2$ & $5 P$ \\
\hline $\mathrm{Cr} 22$ & Li-like & $1 S 2$ & $5 D$ \\
\hline Cr22 & Ii-like & $1 S 2$ & $5 D$ \\
\hline $\mathrm{Cr} 22$ & Li-1ike & 152 & $5 \mathrm{D}$ \\
\hline $\operatorname{Cr} 22$ & Li-like & $1 S 2$ & $4 P$ \\
\hline $\mathrm{Cr} 22$ & Li-like & $1 \mathrm{~S} 2$ & $4 \mathrm{P}$ \\
\hline Cr22 & Li-like & 152 & $4 \mathrm{D}$ \\
\hline $\mathrm{Cr} 22$ & Li-like & 152 & $4 S$ \\
\hline $\operatorname{Cr} 22$ & Li-like & $1 S 2$ & $4 D$ \\
\hline $\operatorname{Cr} 22$ & Li-like & $1 S 2$ & $4 S$ \\
\hline $\operatorname{Cr} 22$ & Ii-like & 1S2 & $3 P$ \\
\hline $\mathrm{Cr} 22$ & Li-Iike & $1 S 2$ & $3 P$ \\
\hline $\mathrm{Cr} 22$ & Li-like & $1 S 2$ & $3 P$ \\
\hline $\mathrm{Cr} 22$ & Li-like & $1 S 2$ & $3 P$ \\
\hline Cr22 & Li-like & IS2 & $3 P$ \\
\hline Cr22 & Li-like & 1S2 & $3 P$ \\
\hline $\mathrm{Cr} 22$ & Li-like & $1 S 2$ & $3 D$ \\
\hline $\operatorname{Cr} 22$ & Li-like & 152 & $3 D$ \\
\hline $\operatorname{Cr} 23$ & He-like & $1 \mathrm{~S}$ & $5 P$ \\
\hline $\mathrm{C} \times 23$ & He-like & 15 & $5 P$ \\
\hline $\operatorname{Cr} 23$ & $\mathrm{He}-1 \mathrm{ike}$ & 1s & $5 P$ \\
\hline $\operatorname{Cr} 23$ & He-like & 1S & $5 P$ \\
\hline Cr23 & He-like & 15 & $4 P$ \\
\hline $\operatorname{Cr} 23$ & He-like & $1 S$ & $4 \mathrm{P}$ \\
\hline $\operatorname{Cr} 23$ & Ho-1ike & $1 \mathrm{~s}$ & $4 \mathrm{P}$ \\
\hline $\operatorname{Cr} 23$ & He-like & $1 S$ & $3 p$ \\
\hline $\mathrm{Cr} 23$ & He-Iike & 15 & $3 \mathrm{P}$ \\
\hline $\operatorname{Cr} 23$ & He-like & $1 S$ & $3 p$ \\
\hline $\mathrm{Cr} 23$ & He-like & $1 \mathrm{~S}$ & $3 P$ \\
\hline $\operatorname{Cr} 23$ & He-like & $1 S$ & $2 P$ \\
\hline $\operatorname{Cr} 23$ & He-like & $1 \mathrm{~s}$ & $2 \mathrm{P}$ \\
\hline $\operatorname{Cr} 23$ & He-like & $1 \mathrm{~S}$ & $2 p$ \\
\hline $\mathrm{Cr} 23$ & He-like & 15 & $2 \mathrm{P}$ \\
\hline $\operatorname{Cr} 23$ & He-like & $1 S$ & $2 P$ \\
\hline $\operatorname{Cr} 23$ & He-like & $1 \mathrm{~S}$ & $2 \mathrm{P}$ \\
\hline $\mathrm{Cr} 23$ & He-like & $1 S$ & $2 P$ \\
\hline $\operatorname{Cr} 23$ & He-1ike & $1 S$ & $2 p$ \\
\hline $\mathrm{Cr} 23$ & He-like & $1 \mathrm{~S}$ & $2 P$ \\
\hline $\mathrm{Cr} 23$ & He-Iike & is & $2 P$ \\
\hline $\mathrm{C} 223$ & He-like & $1 S$ & $2 \mathrm{P}$ \\
\hline $\mathrm{Cr} 23$ & He-like & $1 \mathrm{~s}$ & $2 \mathrm{P}$ \\
\hline $\operatorname{Cr} 23$ & He-like & $1 \mathrm{~s}$ & $2 P$ \\
\hline Cr23 & He-like & 15 & $2 P$ \\
\hline $\mathrm{Cr} 23$ & He-like & 15 & $2 P$ \\
\hline $\mathrm{Cr} 23$ & He-like & 15 & $2 P$ \\
\hline & He-like & 15 & $2 \mathrm{P}$ \\
\hline
\end{tabular}

$\left(2 P^{*}\right) j=1.5-1 S 2 \quad 2 S$ (2D) $j=1.5-1 S 22 P$ (2D) $j=1.5-1 \mathrm{~S} 22 \mathrm{P}$ (2D) $j=2.5-1 S 22 P$ $\left(2 P^{*}\right) j=1.5-1 S 2 \quad 2 S$ $\left(2 P^{*}\right) j=.5-1 S 22 S$ (2D) $j=1.5-1 S 22 P$ (2S) $j=.5-1 S 22 P$ (2D) $j=2.5-1 S 22 P$ $(2 S) j=.5-1 S 22 P$ $\left(2 P^{\star}\right) j=1.5-1 S 2 \quad 2 S$ $\left(2 P^{*}\right) j=1.5-1 S 2 \quad 2 S$ $\left(2 P^{*}\right) j=1.5-1 S 2 \quad 2 S$ $\left(2 P^{\star}\right) j=.5-1 S 2 \quad 2 S$ $\left(2 P^{*}\right) j=.5-1 S 2 \quad 2 S$ $\left(2 P^{*}\right) j=.5-1 S 22 S$ (2D) $j=1.5-1 S 22 \mathrm{P}$ (2D) $j=2.5-1 S 22 P$ $\left(I P^{*}\right) j=1.0-1 S 2$ $\left(1 P^{*}\right) j=1.0-1 \mathrm{~S} 2$ $\left(1 P^{*}\right) j=1.0-1 S 2$ $\left(1 P^{*}\right) j=1.0-1 S 2$ $\left(1 P^{*}\right) j=1.0-1 S 2$ $\left(1 P^{\star}\right) j=1.0-1 S 2$ $\left(1 P^{*}\right) j=1.0-1 S 2$ $\left(1 P^{\star}\right) j=1.0-1 S 2$ $\left(I P^{\star}\right) j=1.0-I S 2$ $\left(1 P^{\star}\right) j=1.0-1 S 2$ $\left(1 P^{*}\right) j=1.0-152$ $\left(1 P^{*}\right) j=1.0-1 S 2$ $\left(1 P^{\star}\right) j=1.0-1 \mathrm{~S} 2$ $\left(1 P^{\star}\right) j=1.0-152$ $\left(1 P^{*}\right) j=1.0-1 \mathrm{~S} 2$ $\left(1 p^{\star}\right) j=1.0-1 S 2$ $\left(19^{*}\right) j=1.0-1 S 2$ $\left(1 P^{*}\right) j=1.0-1 S 2$ $\left(1 P^{*}\right) j=1.0-1 S 2$ $\left(1 P^{\star}\right) j=1.0-1 S 2$ $\left(1 P^{*}\right) j=1.0-1 S 2$ $\left(3 P^{*}\right) j=1.0-1 . S 2$ $\left(3 P^{*}\right) j=1.0-152$ $\left(3 P^{*}\right) j=1.0-152$ $\left(3 P^{*}\right) j=1.0-152$ $\left(3 P^{*}\right) j=1.0-1 S 2$ $\left(3 P^{\star}\right) j=1.0-152$ $\left(3 P^{*}\right) j=1.0-152$
(2S) $j=$. $\left(2 P^{*}\right) j=.5$ $\left(2 P^{\star}\right) j=.5$ $\left(2 P^{\star}\right) j=1.5$

(2S) $j=.5$ (2S) $j=$. $\left(2 P^{*}\right) j=.5$

$\left(2 P^{*}\right) j=$

$\left(2 P^{*}\right) j=1$.

$\left(2 P^{\star}\right) j=1.5$

(2S $) j=$

(2S) $j=$.

(2S $\} j=.5$

(2S) $j=.5$

(2s) $j=.5$

(2S) $j=.5$

$\left\langle 2 P^{\star}\right\rangle j=.5$

$\left(2 P^{*}\right) j=1.5$

(1S) $j=$.

(IS) $j=$.

(1s) $j=.0$

(1S) $J=.0$

(1s) $j=.0$

(1s) $j=.0$

(1s) $j=.0$

(IS ) $j=.0$

(1s) $j=$.

(IS ) $j=.0$

(1s) $j=.0$

(1s) $j=$.

(1s) $j=.0$

(1S) $j=.0$

(1s) $j=.0$

(1s) $j=.0$

(1s) $j=.0$

(Is) $j=.0$

(1S) $j=.0$

(1s) $j=.0$

(1s) $j=$

(1s) $j=.0$

(15) $j=.0$

(1s) $j=.0$

(1S) $j=.0$

(1S) $j=.0$

(1s) $j=.0$

(1S) $j=.0$
$8.516 \mathrm{KAP} 100$

8.778 EDT 020

8.778 KAP 100

8.847 KAP 100

9.488 mica 002

9.498 mica 002

9.806 mica 002

9.891 mica 002

9.960 mica 002

12.613

12.613

12.613

12.656

TAP 100

RAP 100

KAP 100

$12.656 \mathrm{KAP} 100$

13.142 KAP 100

$13.286 \mathrm{KAP} 100$

1.724 LiF 420

1.724 quartz 11

1.724 EDT 020

1.763 LiF 420

1.763 quartz 112

1.763 InSto 111

1.856 Si 220

1.856 fluorite 220

$1.856 \mathrm{NaCl} 200$

.856 InSb 111

2.182

2.182

182 tungsten 110

2.182

2.182

2.182

2.182

2. 182

2.182

2.182

2.192
2.192

2.192

2.192

2.192

2.192

2.192 guartz 112

topaz 200

KBr 200

quartz 101

graphite 002

PET 002

020

Si 422

tungsten 110

guartz 112

topaz 200

quartz 10
$26.634 \quad 73.582$

$8.808 \quad 85.270$

$26.634 \quad 81.392$

$26.634 \quad 85.211$

$19.942 \quad 72.094$

$19.942 \quad 72.281$

$19.942 \quad 79.562$

$19.942 \quad 81.838$

$\begin{array}{lll}19.942 & 82.737\end{array}$

$19.942 \quad 87.308$

$25.763 \quad 78.281$

$26.116 \quad 74.999$

$26.634 \quad 71.287$

$25.763 \quad 79.263$

$26.116 \quad 75.746$

$26.634 \quad 71.872$

$26.634 \quad 80.701$

$26.634 \quad 86.090$

$1.801 \quad 73.185$

$3.636 \quad 71.495$

$8.742 \quad 80.417$

$8.808 \quad 78.141$

$1.801 \quad 78.209$

$3.636 \quad 75.871$

$\begin{array}{lll}7.481 & 70.502\end{array}$

$3.840 \quad 75.165$

3.86273 .979

$5.641 \quad 80.772$

$7.481 \quad 82.923$

$2.217 \quad 79.806$

$2.310 \quad 70.837$

$4.476 \quad 77.156$

$4.564 \quad 72.975$

$\begin{array}{ll}4.638 & 70.207\end{array}$

$6.584 \quad 83.841$

$6.687 \quad 78.213$

$6.696 \quad 77.850$

$8.742 \quad 86.757$

$8.808 \quad 82.272$

$2.217 \quad 81.387$

$2.310 \quad 71.608$

$4.476 \quad 78.363$

$4.564 \quad 73.855$

$4.638 \quad 70.950$

$6.584 \quad 87.175$

$6.687 \quad 79.546$ 


\begin{tabular}{|c|c|c|}
\hline $\operatorname{Cr} 23$ & He-like & IS \\
\hline $\mathrm{Cr} 23$ & He-like & $1 \mathrm{~s}$ \\
\hline $\mathrm{Cr} 23$ & He-like & IS \\
\hline $\mathrm{Cr} 23$ & $\mathrm{He}-1 \mathrm{ike}$ & IS \\
\hline $\operatorname{Cr} 23$ & He-like & is \\
\hline Cr23 & He-like & 15 \\
\hline$C r 23$ & He-like & IS \\
\hline $\mathrm{Cr} 23$ & He-like & $1 S$ \\
\hline $\mathrm{Cr} 23$ & He-like & 15 \\
\hline $\mathrm{Cr} 23$ & He-like & $1 S$ \\
\hline $\operatorname{Cr} 24$ & H -like & $7 P$ \\
\hline $\operatorname{Cr} 24$ & H -like & $7 P$ \\
\hline $\mathrm{Cr} 24$ & $\mathrm{H}$-like & $7 P$ \\
\hline $\mathrm{Cr} 24$ & $\mathrm{H}-1 \mathrm{ike}$ & $7 P$ \\
\hline $\operatorname{Cr} 24$ & H -like & $7 P$ \\
\hline $\operatorname{Cr} 24$ & H -like & $7 P$ \\
\hline $\operatorname{Cr} 24$ & H -like & $7 P$ \\
\hline $\operatorname{Cr} 24$ & $\mathrm{H}$-like & $7 P$ \\
\hline $\operatorname{Cr} 24$ & H -like & $7 P$ \\
\hline Cr24 & H -like & $7 P$ \\
\hline $\mathrm{Cr} 24$ & $\mathrm{H}-$ like & 72 \\
\hline $\operatorname{cr} 24$ & H -like & $6 P$ \\
\hline $\operatorname{Cr} 24$ & H -like & $6 P$ \\
\hline $\mathrm{Cr} 24$ & H -like & $6 P$ \\
\hline $\operatorname{cr} 24$ & H-like & $6 P$ \\
\hline $\operatorname{Cr} 24$ & $\mathrm{H}$-like & $6 P$ \\
\hline$C \times 24$ & H -like & $6 P$ \\
\hline $\operatorname{Cr} 24$ & H -like & $6 P$ \\
\hline$C+24$ & H-like & $6 P$ \\
\hline $\operatorname{Cr} 24$ & $H$-like & $6 P$ \\
\hline $\mathrm{Cr} 24$ & H -like & $6 P$ \\
\hline $\operatorname{Cr} 24$ & H -like & $6 P$ \\
\hline $\operatorname{Cr} 24$ & H -like & $5 P$ \\
\hline $\mathrm{Cr} 24$ & H -like & $5 P$ \\
\hline Cr24 & H -like & $5 P$ \\
\hline $\operatorname{Cr} 24$ & $\mathrm{H}$-like & $5 P$ \\
\hline $\operatorname{Cr} 24$ & $\mathrm{H}$-like & $5 P$ \\
\hline $\mathrm{Cr} 24$ & H -like & $5 P$ \\
\hline Cr24 & H -like & $5 P$ \\
\hline $\operatorname{Cr} 24$ & H -like & $5 P$ \\
\hline $\mathrm{Cr} 24$ & H -like & $5 P$ \\
\hline $\operatorname{Cr} 24$ & H -like & $4 P$ \\
\hline $\operatorname{Cr} 24$ & H -like & $4 P$ \\
\hline $\operatorname{cr} 21$ & H -like & $4 P$ \\
\hline $\operatorname{Cr} 24$ & $\mathrm{H}-$ like & $3 P$ \\
\hline $\operatorname{Cr} 24$ & H -like & $3 p$ \\
\hline
\end{tabular}

$\left(3 P^{*}\right) j=1.0-1 S 2$

$(3 P *) j=1.0-1 S 2$

(3S) $j=1.0-1 S 2$

(3S) $j=1.0-1 S 2$

(3S) $j=1.0-1 S 2$

(3s) $j=1.0-1 s 2$

(3s) $j=1.0-1 s 2$

(3S) $j=1.0-1 S 2$

(3s) $j=1.0-1 S 2$

(3S) $j=1.0-152$

$\left(2 P^{*}\right) j=1.5-1 S$

$\left(2 P^{*}\right) j=1.5-1 S$

$\left(2 P^{*}\right) j=1.5-1 S$

$\left(2 P^{*}\right) j=1.5-1 S$

$\left(2 P^{*}\right) j=1.5-1 S$

$\left(2 P^{\star}\right) j=1.5-1 S$

$\left(2 P^{*}\right) j=1.5-1 S$

$\left(2 P^{\star}\right) j=1.5-1 S$

$\left(2 P^{*}\right) j=1.5-1 S$

$\left(2 P^{\star}\right) j=1.5-1 S$

$\left(2 P^{\star}\right) j=1.5-1 S$

$\left(2 \mathrm{P}^{*}\right) j=1.5-1 \mathrm{~S}$

$\left(2 P^{*}\right) j=1.5-1 S$

$\left(2 P^{\star}\right) j=1.5-1 S$

$\left(2 P^{*}\right) j=1.5-1 S$

$\left(2 P^{\star}\right) j=1.5-1 S$

$\left(2 P^{\star}\right) j=1.5-1 S$

$\left(2 P^{*}\right) j=1.5-1 S$

$\left(2 P^{*}\right) j=1.5-1.5$

$\left(2 P^{\star}\right) j=1.5-15$

$\left(2 P^{*}\right) j=1.5-1 S$

$\left(2 P^{*}\right) j=1.5-1 S$

$\left(2 P^{\star}\right) j=1.5-1 \mathrm{~S}$

$\left(2 P^{*}\right) j=1.5-1 S$

$\left(2 P^{*}\right) j=1.5-1 S$

$\left(2 \mathrm{P}^{\star}\right) j=1.5-1 \mathrm{~S}$

$\left(2 P^{*}\right) j=1.5-1 S$

$\left(2 P^{*}\right) j=1.5-1 S$

$\left(2 P^{*}\right) j=1.5-15$

$\left(2 P^{*}\right) j=1.5-15$

$\left(2 P^{\star}\right) j=1.5-1 S$

$\left(2 P^{*}\right) j=1.5-1 S$

$\left(2 P^{\star}\right) j=1.5-15$

$\left(2 P^{\star}\right) j=1.5-1 S$

- $\quad\left(2 P^{\star}\right) j=1.5-1 S$

$\left(2 P^{*}\right) j=1.5-15$

\begin{tabular}{|c|c|c|c|}
\hline$j=$ & .0 & 2.192 & graphite 002 \\
\hline$j=$ & .0 & 2.192 & EDI 020 \\
\hline$i$ & .0 & 2.203 & Si 422 \\
\hline 1 & .0 & 2.203 & Ge 422 \\
\hline & .0 & 2.203 & tungsten 110 \\
\hline & .0 & 2.203 & quartz 112 \\
\hline & .0 & 2.203 & topaz 200 \\
\hline & .0 & 2.203 & AI 111 \\
\hline & .0 & 2.203 & quartz 101 \\
\hline & .0 & 2.203 & graphite 002 \\
\hline & .5 & 1.603 & quartz 502 \\
\hline & .5 & 1.603 & LiF 422 \\
\hline & .5 & 1.603 & corundum 146 \\
\hline & .5 & 1.603 & quartz 110 \\
\hline & .5 & 1.603 & gypsum 002 \\
\hline & .5 & 1.603 & $\mathrm{Ge} 111$ \\
\hline & .5 & 1.603 & $K B \times 200$ \\
\hline & .5 & 1.603 & quartz 101 \\
\hline & .5 & 1.603 & graphite 002 \\
\hline & .5 & 1.603 & topaz 002 \\
\hline & .5 & 1.603 & quartz 100 \\
\hline & .5 & 1.615 & quartz 502 \\
\hline & .5 & 1.615 & LiF 422 \\
\hline & .5 & 1.615 & corundum 146 \\
\hline & .5 & 1.615 & guartz 110 \\
\hline & .5 & 1.615 & gypsum 002 \\
\hline & .5 & 1.615 & Ge 111 \\
\hline $7 \mathrm{~J}$ & .5 & 1.615 & $\mathrm{KBr} 200$ \\
\hline & .5 & 1.615 & quartz 101 \\
\hline & .5 & 1.615 & graphite 002 \\
\hline & .5 & 1.615 & topaz 002 \\
\hline & .5 & 1.615 & quartz 100 \\
\hline$=$ & .5 & 1.636 & LIF 422 \\
\hline$i=$ & .5 & 1.636 & corundum 146 \\
\hline & .5 & 1.636 & quartz 110 \\
\hline 3 & .5 & 1.636 & gypsum 002 \\
\hline & 5 & 1.636 & $\mathrm{KBr} 200$ \\
\hline 1 & .5 & 1.636 & quartz 101 \\
\hline & .5 & 1.636 & graphite 002 \\
\hline$=$ & .5 & 1.636 & topaz 002 \\
\hline & .5 & 1.636 & quartz 100 \\
\hline & .5 & 1.675 & quartz 100 \\
\hline & .5 & 1.675 & PET 002 \\
\hline & .5 & 1.675 & EDT 020 \\
\hline & .5 & 1.766 & LiF 420 \\
\hline & & & \\
\hline
\end{tabular}

$6.696 \quad 79.136$

$8.808 \quad 84.537$

$2.217 \quad 83.558$

$2.310 \quad 72.493$

$4.476 \quad 79.854$

$4.564 \quad 74.880$

$4.638 \quad 71.801$

$4.676 \quad 70.434$

$6.687 \quad 81.240$

$6.696 \quad 80.754$

1.62480 .776

1.65276 .010

$1.660 \quad 74.942$

$4.912 \quad 78.246$

$4.990 \quad 74.521$

$6.532 \quad 79.001$

6.58476 .875

$6.687 \quad 73.511$

$6.696 \quad 73.253$

$8.374 \quad 73.162$

$8.512 \quad 70.324$

$1.624 \quad 83.965$

$1.652 \quad 77.851$

$1.660 \quad 76.629$

$4.912 \quad 80.526$

$4.990 \quad 76.154$

$6.532 \quad 81.485$

6.58478 .863

$6.687 \quad 75.028$

$6.696 \quad 74.743$

$8.374 \quad 74.643$

8.51271 .561

$1.652 \quad 82.019$

$1.660 \quad 80.245$

$4.912 \quad 87.688$

$4.990 \quad 79.599$

$6.584 \quad 83.681$

$6.687 \quad 78.130$

$6.696 \quad 77.769$

$8.374 \quad 77.643$

$8.512 \quad 73.945$

$8.512 \quad 79.706$

$8.742 \quad 73.339$

$8.808 \quad 71.960$

1.80178 .686

3.63676 .263 
Cr24 H-like 3P

Cr24 H -like 3P

Cr24 H-like 3P

Cr24 H -like 3P

Cr24 H -like 3P

Cr24 H -like 2P

Cr24 H-like 2P

Cr24 F-like 2P

Cr24 H -like 2P

Cr24 H -like 2P

Cr24 H - like 2P

Cr24 H-like 2P

Cr24 H -like 2P

Cr24 H-like 2P

Cr24 H -like 2P

Cr24 H -like 2P

Cr24 H-like 2P

Cr24 H-like 2P

Cr24 H-like 2P

Cr24 H-like 2P

Cr24 H -like 2P

Cr24 H -like 2P

Cr24 H -like $2 \mathrm{P}$

Cr24 H-like 2P

Cr24 H-like 2P

Cr24 H -like 2P

Cr24 H-like 2P

Cr24 H-like 2P

Cr24 H-like 6P

Cr24 H -like 6D

Cr24 H-like 5P

Cr24 H -like 5P

Cr24 H-like 5D

Cr24 H-like 5D

Cr24 H -like 4P

Cr24 H -like 4P

Cr24 H -like 4P

Cr24 H -like 4P

Cr24 H-like 4P

Cr24 H-like 4P

Cr24 H-like 4D

Cr24 H-like 4D

Cr24 H -like 4D

Cr24 H -like 4D

Cr24 H-like 4D

Cr24 H-like 4D $\left(2 \mathrm{P}^{\star}\right) j=1.5-1 \mathrm{~S}$

$\left(2 P^{*}\right) j=.5-1 S$

$\left(2 P^{*}\right) j=.5-1 S$

$\left(2 P^{\star}\right) j=.5-1 S$

$\left(2 P^{*}\right) j=.5-1 S$

$\left(2 P^{\star}\right) j=1.5-1 S$

$\left(2 P^{\star}\right) j=1.5-1 S$

$\left(2 P^{*}\right) j=1.5-1 . S$

$\left(2 P^{\star}\right) j=1.5-1 S$

$\left(2 P^{\star}\right) j=1.5-1 S$

$\left(2 P^{\star}\right) j=1.5-1 S$

$\left(2 P^{*}\right) j=1.5-1 S$

$\left(2 P^{\star}\right) j=1.5-1 S$

$\left(2 P^{*}\right) j=1.5-1 S$

$\left(2 P^{*}\right) j=1.5-1 S$

$\left(2 P^{\star}\right) j=1.5-1 S$

$\left(2 P^{\star}\right) j=1.5-1 S$

$\left(2 P^{\star}\right) j=.5-1 S$

$\left(2 P^{\star}\right) j=.5-1 S$

$\left(2 P^{*}\right) j=.5-1 S$

$\left(2 P^{*}\right) j=.5-1 S$

$\left(2 P^{\star}\right) j=.5-1 S$

$\left(2 P^{*}\right) j=.5-1 S$

$\left(2 \mathrm{P}^{\star}\right) j=.5-1 \mathrm{~S}$

$\left(2 P^{*}\right) j=.5-1 S$

$\left(2 \mathrm{P}^{\star}\right) j=.5-1 \mathrm{~s}$

$\left(2 P^{*}\right) j=.5-1 S$

$\left(2 P^{*}\right) j=.5-1 S$

$\left(2 P^{\star}\right) j=1.5-2 S$

(2D) $j=2.5-2 P$

$\left(2 P^{\star}\right) j=1.5-2 S$

$\left(2 P^{\star}\right) j=1.5-2 S$

(2D) $j=2.5-2 P$

(2D) $j=2.5-2 P$

$\left(2 P^{*}\right) j=1.5-2 S$

$\left(2 P^{\star}\right) j=1.5-2 S$

$\left(2 P^{*}\right) j=1.5-2 S$

$\left(2 P^{*}\right) j=1.5-2 S$

$\left(2 P^{\star}\right) j=1.5-2 S$

$\left(2 P^{\star}\right) j=1.5-2 S$

(2D) $j=2.5-2 P$

(2D) $j=2.5-2 P$

(2D) $j=2.5-2 P$

(2D) $j=2.5-2 P$

(2D) $j=2.5-2 P$

(2D) $j=2.5-2 P$
(2S) $j=$.

(2S) $j=.5$

(2S) $j=.5$

(2S) $j=.5$

(2S) $j=.5$

(2S) $j=.5$

(2S) $j=.5$

(2S) $j=.5$

(2S) $j=.5$

(2S) $j=.5$

(2S) $j=.5$

(2S) $j=.5$

(2S) $j=.5$

(2S) $j=.5$

(2S) $j=.5$

(2S) $j=.5$

(2s) $j=.5$

(2S) $j=.5$

(2S) $j=.5$

(2S) $j=.5$

(2S) $j=.5$

(2S) $j=.5$

(2S) $j=.5$

(2S) $j=.5$

(2S) $j=.5$

(2S) $j=.5$

(2S) $j=.5$

(2S) $j=.5$

(2S) $j=.5$

$\left(2 P^{\star}\right) j=1.5$

(2S) $j=.5$

(2S) $j=.5$

$\left(2 P^{\star}\right) j=1.5$

$\left(2 P^{*}\right) j=1.5$

(2s) $j=.5$

(2S) $j=$.

(2S) $j=.5$

(2S) $j=.5$

(2S) $j=.5$

(2S) $j=.5$

$(2 P *) j=1.5$

$\left(2 P^{*}\right) j=1.5$

$\left(2 P^{\star}\right) j=1.5$

$\left(2 P^{\star}\right) j=1.5$

$\left(2 P^{\star}\right) j=1.5$

$\left(2 P^{\star}\right) j=1.5$
1.766 InSo 111

1.767 LiF 420

1.767 guartz 112

$1.767 \mathrm{NaCl} 200$

1.767 InSb 111

Si 422

2.090 quartz 200

2.090 Si 111

.090 sylvite 200

090 fluorite 111

2.090 Ge 111

$.090 \mathrm{KBr} 200$

.090 topaz 002

2.090 quartz 100

2.090 PET 002

.090 EDT 020

.090 ADP 101

2.096 Si 422

.096 quartz 200

.096 sylvite 200

2.096 fluorite 111

2.096 Ge 111

$.096 \mathrm{KBr} 200$

2.096 quartz 101

2.096 quartz 100

.096 PET 002

2.096 EDT 020

2.096 ADP 101

7.044 InSb 111

.104 InSb 111

7.452 InSb 111

7.452 gypsum 020

.518 gypsum 020

7.518 beryl 100

8.339 topaz 002

8.339 quartz 100

8.339 PET 002

8.339 EDT 020

8.339 TAP 100

8.339

8.419 quartz 100

8.419 PET 002

8.419 EDT O20

8.419 TAP 100

8.419 RAP 100

8.419 KAP 100 $\begin{array}{ll}7.481 & 70.780\end{array}$

$1.801 \quad 78.849$

$3.636 \quad 76.397$

5.64170 .006

$7.481 \quad 70.873$

$2.217 \quad 70.513$

$\begin{array}{lll}4.246 & 79.885\end{array}$

$6.271 \quad 88.977$

$6.292 \quad 85.207$

$6.308 \quad 83.708$

$6.532 \quad 73.717$

$6.584 \quad 72.234$

$8.374 \quad 86.686$

$8.512 \quad 79.156$

$8.742 \quad 73.000$

$8.808 \quad 71.647$

$10.640 \quad 79.156$

$2.217 \quad 70.983$

$4.246 \quad 80.852$

$6.292 \quad 87.957$

$6.308 \quad 85.436$

6.53274 .290

$6.584 \quad 72.754$

$6.687 \quad 70.107$

$8.512 \quad 80.051$

$8.742 \quad 73.546$

$8.808 \quad 72.150$

$10.640 \quad 80.051$

$\begin{array}{ll}7.481 & 70.320\end{array}$

$7.481 \quad 71.733$

$7.481 \quad 84.953$

$15.185 \quad 78.960$

$15.185 \quad 81.967$

$15.954 \quad 70.469$

$8.374 \quad 84.760$

8.51278 .429

$8.742 \quad 72.535$

$8.808 \quad 71.218$

$25.763 \quad 76.178$

$26.116 \quad 73.319$

$8.512 \quad 81.523$

$8.742 \quad 74.376$

$8.808 \quad 72.908$

$25.763 \quad 78.626$

$26.116 \quad 75.264$

$26.634 \quad 71.496$ 


\begin{tabular}{|c|c|c|c|c|c|c|c|}
\hline $\operatorname{Cr} 24$ & $\mathrm{H}$-like & $6 P$ & & & $\left(2 P^{\star}\right) j=1.5$ & $-3 s$ & \\
\hline $\operatorname{Cr} 24$ & $\mathrm{H}-\mathrm{like}$ & $6 \mathrm{D}$ & & & $(2 D) j=2.5$ & $-3 P$ & \\
\hline $\operatorname{Cr} 24$ & $\mathrm{H}$-like & $6 \mathrm{~F}$ & & & $\left(2 F^{\star}\right) j=3.5$ & $-3 D$ & \\
\hline Mn & & K-alpha & & & & & \\
\hline $\mathrm{Mn}$ & & K-alpha & & & & & \\
\hline $\mathrm{Mn}$ & & K-alpha & & & & & \\
\hline Mn & & K-alpha & & & & & \\
\hline Mn & & k-alpha & & & & & \\
\hline $\mathrm{Mn}$ & & K-alpha & & & & & \\
\hline Mn & & K-alpha & & & & & \\
\hline Mn & & K-alpha & & & & & \\
\hline $\mathrm{Mn}$ & & K-alpha & 1) & & & & \\
\hline Mn & & $\mathrm{K}-\mathrm{alph}$ & & & & & \\
\hline $\mathrm{Mn}$ & & K-alpha & & & & & \\
\hline $\operatorname{Mn} 15$ & $\mathrm{Na-like}$ & $2 P 5 \quad 352$ & & & $\left(2 \mathrm{P}^{\star}\right) j=.5$ & $-2 \mathrm{P} 6$ & $3 S$ \\
\hline $\operatorname{Mn} 15$ & Na-1ike & $2 \mathrm{P} 5 \quad 3 \mathrm{~S} 2$ & & & $\left(2 P^{*}\right) j=1.5$ & $-2 p 6$ & $3 s$ \\
\hline $\operatorname{Mn} 16$ & Ne-like & $2 S 2 \quad 2 P 5$ & $(2 \mathrm{P} * 1)$ & $5 D$ & $\left(12^{*}\right) j=1.0$ & $-2 s 2$ & $2 \mathrm{P} 6$ \\
\hline Mn16 & Ne-like & $2 S 2 \quad 2 P 5$ & $\left(2 \mathrm{P}^{\star} 1\right)$ & $5 D$ & $\left(12^{\star}\right) j=1.0$ & $-2 s 2$ & $2 \mathrm{P} 6$ \\
\hline $\operatorname{Mn} 16$ & Ne-like & $2 \mathrm{~S} 2 \quad 2 \mathrm{P} 5$ & $(2 P * 2)$ & $5 D$ & $\left(22^{\star}\right) j=1.0$ & $-2 s 2$ & $2 \mathrm{P} 6$ \\
\hline $\operatorname{Mn} 16$ & Ne-like & $2 S 2 \quad 2 P 5$ & $(2 p * 2)$ & $5 \mathrm{D}$ & $\left(22^{\star}\right) j=1.0$ & $-2 s 2$ & $2 P 6$ \\
\hline $\operatorname{Mn} 16$ & Ne-like & $2 S \quad 2 P 6$ & $3 P$ & & $\left(1 P^{*}\right) j=1.0$ & $-2 s 2$ & $2 \mathrm{P} 6$ \\
\hline $\operatorname{Mn} 16$ & $\mathrm{Ne}-1 \mathrm{ike}$ & $2 \mathrm{P} 6$ & $3 P$ & & $\left(3 P^{*}\right) j=1.0$ & $-2 \$ 2$ & $2 \mathrm{P} 6$ \\
\hline $\operatorname{Mn} 16$ & Ne-like & $2 \mathrm{~S} 2 \quad 2 \mathrm{P} 5$ & $(2 P * 2)$ & $3 S$ & $\left(21^{*}\right) j=1.0$ & $-2 s 2$ & $2 \mathrm{P} 6$ \\
\hline $\operatorname{Mn} 17$ & F-like & $2 \mathrm{~S} 2 \quad 2 \mathrm{P} 4$ & $(15)$ & $4 \mathrm{D}$ & $(2 D) j=2.5$ & $-2 s 2$ & $2 \mathrm{P} 5$ \\
\hline $\operatorname{Mn} 17$ & F-like & $2 S 2 \quad 2 P 4$ & $13 P$ & $4 D$ & $(2 D) j=2.5$ & $-2 s 2$ & $2 P 5$ \\
\hline $\operatorname{Mn} 17$ & F-like & $2 \mathrm{~S} 2 \quad 2 \mathrm{P} 4$ & ( $3 P$ & $4 \mathrm{D}$ & $(2 D) j=2.5$ & $-2 s 2$ & $2 \mathrm{P} 5$ \\
\hline $\operatorname{Mn} 17$ & $F$-like & $2 S 2 \quad 2 P 4$ & $13 P$ & $4 \mathrm{D}$ & $(2 D) j=2.5$ & $-2 s 2$ & $2 \mathrm{P} 5$ \\
\hline $\operatorname{Mn} 17$ & F-like & $2 \mathrm{~S} 2 \quad 2 \mathrm{P} 4$ & 115 & $3 D$ & $(2 D) j=2.5$ & $-2 s 2$ & $2 \mathrm{P} 5$ \\
\hline $\operatorname{Mn} 17$ & F-like & $2 \mathrm{~S} 2 \quad 2 \mathrm{P} 4$ & ( 15 & $3 \mathrm{D}$ & $(2 D)$ & $-2 s 2$ & $2 \mathrm{P} 5$ \\
\hline $\operatorname{Mn} 17$ & F-like & $2 \mathrm{~S} 2 \quad 2 \mathrm{P} 4$ & $11 D$ & $3 \mathrm{D}$ & $(2 \mathrm{D})$ & $-2 s 2$ & $2 \mathrm{P} 5$ \\
\hline $\operatorname{Mn} 17$ & F-like & $2 S 2 \quad 2 P 4$ & $\angle I D$ & $3 D$ & $(2 D):$ & $-2 s 2$ & $2 \mathrm{P} 5$ \\
\hline $\operatorname{Mn} 17$ & F-like & $2 S 2 \quad 2 P 4$ & 110 & $3 D$ & $(2 s) j=.5$ & $-2 s 2$ & $2 \mathrm{P5}$ \\
\hline $\operatorname{Mn} 17$ & $F-1 i k e$ & $2 \mathrm{~S} 2 \quad 2 \mathrm{P} 4$ & ( $1 D$ & $3 D$ & $(2 D) j=1.5$ & $-2 s 2$ & $2 P 5$ \\
\hline $\operatorname{Mn} 17$ & F-like & $2 \mathrm{~S} 2 \quad 2 \mathrm{P} 4$ & $13 P$ & $3 D$ & $(2 D) j=2.5$ & $-2 s 2$ & $2 \mathrm{P} 5$ \\
\hline $\operatorname{Mn} 17$ & F-like & $2 S 2 \quad 2 P 4$ & $13 P$ & $3 D$ & $(2 P) j=1.5$ & $-2 s 2$ & $2 \mathrm{P5}$ \\
\hline $\operatorname{Mn} 17$ & $F$-like & $2 \mathrm{~S} 2 \quad 2 \mathrm{P} 4$ & $(3 P$ & $3 D$ & $(2 F) j=2.5$ & $-2 s 2$ & $2 \mathrm{P} 5$ \\
\hline $\operatorname{Mn} 17$ & $F$-like & $2 \mathrm{~S} 2 \quad 2 \mathrm{P} 4$ & 110 & $3 D$ & $(2 s) j=.5$ & $-2 s 2$ & $2 \mathrm{P5}$ \\
\hline $\operatorname{Mn} 18$ & o-like & $2 \mathrm{~S} 2 \quad 2 \mathrm{P} 3$ & $\left(2 P^{*}\right)$ & $3 D$ & $\left(1 D^{*}\right) j=2.0$ & $-2 s 2$ & $2 \mathrm{P} 4$ \\
\hline Mn18 & o-like & $2 \mathrm{~S} 2 \quad 2 \mathrm{P} 3$ & $\left(2 P^{*}\right)$ & $3 D$ & $\left(3 D^{*}\right)$ & $-2 s 2$ & $2 \mathrm{P} 4$ \\
\hline $\operatorname{Mn} 18$ & o-like & $2 \mathrm{~S} 2 \quad 2 \mathrm{P} 3$ & $\left(2 P^{*}\right)$ & $3 D$ & $\left(3 P^{*}\right) j=2.0$ & $-2 S 2$ & $2 \mathrm{P} 4$ \\
\hline $\operatorname{Mn} 18$ & o-like & $2 \mathrm{~S} 2 \quad 2 \mathrm{P} 3$ & $\left(2 \mathrm{P}^{\star}\right)$ & $3 D$ & $\left(1 D^{*}\right) j=2.0$ & $-2 \$ 2$ & $2 \mathrm{P} 4$ \\
\hline Mn18 & o-like & $2 \mathrm{~S} 2 \quad 2 \mathrm{P} 3$ & $\left(2 D^{\star}\right)$ & $3 D$ & $\left(3 D^{*}\right) j=3.0$ & $-2 s 2$ & $2 \mathrm{P} 4$ \\
\hline $\operatorname{Mn} 18$ & $0-1 i k e$ & $2 \mathrm{~S} 2 \quad 2 \mathrm{P} 3$ & $\left(2 \mathrm{P}^{\star}\right)$ & $3 D$ & $\left(3 P^{*}\right) j=1.0$ & $-2 s 2$ & $2 \mathrm{P} 4$ \\
\hline $\operatorname{Mn} 18$ & o-like & $2 \mathrm{~S} 2 \quad 2 \mathrm{P} 3$ & $\left(2 P^{\star}\right)$ & $3 D$ & $\left(3 P^{\star}\right) j=1.0$ & $-2 \$ 2$ & $2 \mathrm{P} 4$ \\
\hline Mn18 & o-like & $2 \mathrm{~S} 2 \quad 2 \mathrm{P} 3$ & $\left(2 \mathrm{P}^{\star}\right)$ & $3 D$ & $\left(1 P^{*}\right) j=1.0$ & $-2 s 2$ & $2 \mathrm{P} 4$ \\
\hline $\operatorname{Min} 18$ & o -like & $2 \mathrm{~S} 2 \quad 2 \mathrm{P} 3$ & $\left(2 P^{*}\right)$ & $3 D$ & $\left(1 P^{*}\right) j=1.0$ & $-2 s 2$ & $2 \mathrm{P} 4$ \\
\hline
\end{tabular}

\begin{tabular}{|c|c|c|c|c|c|}
\hline$(2 s) j=$ & 18.795 & mica 002 & 1 & 19.942 & 70.473 \\
\hline$\left(2 P^{\star}\right) j=1.5$ & 18.922 & mica 002 & 1 & 19.942 & 71.596 \\
\hline $2 D) j=2.5$ & 18.965 & mica 002 & 1 & 19.942 & 71.991 \\
\hline & 2.102 & Si 422 & 1 & 2.217 & 71.457 \\
\hline & 2.102 & guartz 200 & 2 & 4.246 & 81.915 \\
\hline & 2.102 & fluorite 111 & 3 & 6.308 & 88.453 \\
\hline & 2.102 & $\mathrm{Ge} 111$ & 3 & 6.532 & 74.874 \\
\hline & 2.102 & $\mathrm{KBr} 200$ & 3 & 6.584 & 73.282 \\
\hline & 2.102 & quartz 101 & 3 & 6.687 & 70.558 \\
\hline & 2.102 & graphite 002 & 3 & 6.696 & 70.341 \\
\hline & 2.102 & quartz 100 & 4 & 8.512 & 81.017 \\
\hline & 2.102 & PET 002 & 4 & 8.742 & 74.101 \\
\hline & 2.102 & EDT 020 & 4 & 8.808 & 72.658 \\
\hline & 2.102 & $A D P 101$ & 3 & 10.640 & 81.017 \\
\hline$(2 S) j=.5$ & 19.155 & mica 002 & 1 & 19.942 & 73.850 \\
\hline$(2 S) j=.5$ & 19.450 & mica 002 & 1 & 19.942 & 77.246 \\
\hline$(1 s) j=.0$ & 12.373 & TAP 100 & 2 & 25.763 & 73.848 \\
\hline (Is) $j=.0$ & 12.373 & $\operatorname{RAP} 100$ & 2 & 26.116 & 71.359 \\
\hline$(1 s) j=.0$ & 12.510 & TAP 100 & 2 & 25.763 & 76.206 \\
\hline$(1 s) j=.0$ & 12.510 & RAP 100 & 2 & 26.116 & 73.342 \\
\hline$(1 S) j=.0$ & 15.238 & beryl 100 & 1 & 15.954 & 72.770 \\
\hline (IS) $j=.0$ & 15.314 & beryl 100 & 1 & 15.954 & 73.716 \\
\hline$(1 s) j=.0$ & 18.935 & mica 002 & 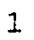 & 19.942 & 71.714 \\
\hline$\left(2 P^{\star}\right) j=1.5$ & 12.181 & TAP 100 & 2 & 25.763 & 71.018 \\
\hline$\left(2 P^{\star}\right) j=1.5$ & 12.643 & TAP 100 & 2 & 25.763 & 78.957 \\
\hline$\left(2 P^{\star}\right) j=1.5$ & 12.643 & RAP 100 & 2 & 26.116 & 75.516 \\
\hline$\left(2 P^{\star}\right) j=1.5$ & 12.643 & KAP 100 & 2 & 26.634 & 71.693 \\
\hline$\left(2 P^{*}\right) j=1.5$ & 15.404 & beryl 100 & 1 & 15.954 & 74.912 \\
\hline$\left(2 P^{\star}\right) j=.5$ & 15.570 & beryl 100 & 1 & 15.954 & 77.404 \\
\hline$\left(2 P^{\star}\right) j=1.5$ & 15.615 & beryl 100 & 1 & 15.954 & 78.168 \\
\hline$\left(2 P^{*}\right) j=1.5$ & 15.670 & beryl 100 & 1 & 15.954 & 79.173 \\
\hline$\left(2 P^{*}\right) j=1.5$ & 15.732 & beryl 100 & 1 & 15.954 & 80.431 \\
\hline$\left(2 P^{*}\right) j=.5$ & 15.826 & beryl 100 & 1 & 15.954 & 82.737 \\
\hline$\left(2 P^{*}\right) j=1.5$ & 15.871 & beryl 100 & 1 & 15.954 & 84.153 \\
\hline *) $j=1.5$ & 15.889 & bery 1100 & 1 & 15.954 & 84.826 \\
\hline$\left(2 P^{\star}\right) j=1.5$ & 15.926 & beryl 100 & 1 & 15.954 & 86.605 \\
\hline$\left(2 P^{*}\right) j=.5$ & 15.946 & bery 100 & 1 & 15.954 & 88.185 \\
\hline$(3 P) j=2.0$ & 14.650 & gypsum 020 & 1 & 15.185 & 74.746 \\
\hline$(3 P) j=2.0$ & 14.698 & gypsum 020 & 1 & 15.185 & 75.450 \\
\hline$(3 P) j=2.0$ & 14.752 & gypsum 020 & 1 & 15.185 & 76.284 \\
\hline$(3 P) j=1.0$ & 14.816 & gypsum 020 & 1 & 15.185 & 77.343 \\
\hline$(3 P) j=2.0$ & 14.877 & gypsum 020 & 1 & 15.185 & 78.440 \\
\hline (1D) $j=2.0$ & 15.024 & gypsum 020 & 1 & 15.185 & 81.649 \\
\hline (ID) $j=2.0$ & 15.024 & beryl 100 & 1 & 15.954 & 70.340 \\
\hline$(1 S) j=.0$ & 15.096 & gypsum 020 & 1 & 15.185 & 83.794 \\
\hline$(1 S) j=.0$ & 15.096 & beryl 100 & 1 & 15.954 & 71.124 \\
\hline
\end{tabular}


Mn18 O-like 2S2 2P3 (2D*) 3D (1D*) j=2.0-2S2 2P4 Mn18 O-like 2S2 2P3 ( 4S*) 3D $\left(3 D^{*}\right) j=3.0-2 S 22 P 4$ Mn18 O-1ike 2S2 2P3 (4S*) 3D (3D*) $j=2.0-2 S 22 P 4$ Mn19 N-like 2S2 2P2 ( 3P ) 3D $(2 F) j=3.5-2 S 22 P 3$ Mn19 N-like 2S2 2P2 ( 3P) 3D (2P) $j=1.5-2 S 22 \mathrm{P} 3$ $\mathrm{Mn} 20$ C-like 2S2 $2 \mathrm{P}$ 3S $\quad\left(3 \mathrm{P}^{*}\right) \mathrm{J}=2.0-2 \mathrm{~S} 2 \quad 2 \mathrm{P} 2$ Mn21 B -like 2S2 4S (2S) $j=.5-2 S 22 P$ $\operatorname{Mn} 21$ B-like $2 S 2 P(3 P) 3 P(2 D) j=1.5-2 S 22 P$ $M$ 21 $B-l i k e$ 2S $2 P$ ( $3 P$ ) $3 P(2 D) j=1.5-2 S 2$ 2P Mn21 $B-1 i k e$ 2S $2 P$ ( $3 P$ ) $3 P(2 D) j=2.5-2 S 22 P$ $M n 21$ B-like $2 S$ 2P (3P) $3 P(2 D) j=2.5-2 S 22 P$ $M \pi 21$ B-like $2 S$ 2P ( $3 P$ ) $3 P(2 P) j=.5-2 S 22 P$ $\operatorname{Mn} 21 \mathrm{~B}-1 \mathrm{ike} 2 \mathrm{~S} 2 \mathrm{P}(3 \mathrm{P}), 3 \mathrm{P}(2 \mathrm{P}) \mathrm{j}=.5-2 S 22 \mathrm{P}$ $\operatorname{Mn} 21$ B -like $2 S 2 P$ ( $3 P$ ) 3P (2P) $j=.5-2 S 22 P$

$M 21$ B-like $2 S$ 2P ( $1 P$ ) 3D $\left(2 D^{*}\right) j=2.5-2 S$ 2P2 $M n 21$ B-like $2 S$ 2P ( $1 P$ ) $3 D\left(2 D^{*}\right) j=2.5-2 S \quad 2 P 2$ Mn21 $B$-like 2S 2P ( $1 P$ ) 3D $\left(2 D^{*}\right) j=2.5-2 S$ 2P2 $\mathrm{Mn21} B$-like $2 S$ 2P ( $1 \mathrm{P}$ ) $3 \mathrm{D}\left(2 \mathrm{~F}^{*}\right) j=3.5-2 \mathrm{~S}$ 2P2 $M 21$ B -like $2 S 2 P$ ( $1 P$ ) $3 D\left(2 F^{*}\right) j=3.5-2 S$ 2P2 $\operatorname{Mn} 21$-like $2 S$ 2P ( $P$, $3 D\left(2 F^{*}\right) j=3.5-2 S$ 2P2 Mn21 B -like 2S2 3D (2D) $j=1.5-2 S 22 P$ Mn21 B -like 2S2 3D (2D) $j=1.5-2 S 2$ 2P Mn21 B-1ike 2S 2P ( $3 P$, 3D $\left(4 D^{*}\right) j=3.5-2 S$ 2P2 $\operatorname{Mn} 21 \mathrm{~B}$-like $2 \mathrm{~S} 2 \mathrm{P}$ ( $3 \mathrm{P}) 3 \mathrm{D}\left(4 \mathrm{D}^{*}\right) \mathrm{j}=3.5-2 \mathrm{~S} 2 \mathrm{P} 2$ Mn21 B -1ike 2S2 3D (2D) $j=2.5-2 S 22 P$ Mn21 B -like 2S2 3D (2D) j=2.5 - 2S2 2P

$M n 21$ - $B$-like $2 S$ 2P ( IP ) 3D $\left(2 D^{*}\right) j=2.5-2 S$ 2P2 $\operatorname{Mn} 21$ B-like $2 S$ 2P (3P) $3 D\left(2 F^{*}\right) j=3.5-2 S$ 2P2 $\mathrm{Mn} 21 \mathrm{~B}-1 \mathrm{kke} 2 \mathrm{~S} 2 \mathrm{P}(3 \mathrm{P}) 3 \mathrm{D}\left(2 \mathrm{~F}^{*}\right) \mathrm{j}=2.5-2 \mathrm{~S}$ 2P2

Mn22 Be-1ike 1S2 2P 3P (ID) $j=2.0-1 S 22 S \quad 2 P$

$\mathrm{Mn} 22$ Be-like 1S2 2S 3D

$\operatorname{Mn} 22$ Be-like 1S2 2S 3D

Mn22 Be-like 1S2 2S 3D

Mn22 Be-like 1S2 2S 3D

$\mathrm{Mn} 22$ Be-1ike 1S2 2P 3D

$\operatorname{Min} 22$ Be-like 1s2 2P $3 \mathrm{D}$

$\mathrm{Mn} 22$ Be-like 1S2 2P $3 \mathrm{P}$

$\operatorname{Mn} 22$ Be-like 1S2 2P 3P

$\mathrm{Mn} 22$ Be-1ike 1S2 2P 3D

Mn22 Be-like 1S2 2P 3D

$\mathrm{Mn} 22$ Be-like 1S2 2P 3D

$\mathrm{Mn} 22$ Be-like 1S2 2P 3D

$\mathrm{Mn} 22$ Be-like 1S2 $2 \mathrm{P}$ 3D

$\mathrm{Mn} 22$ Be-like 1S2 2P 3D

$\mathrm{Mn} 22$ Be-like 1S2 2P 3D

$\mathrm{Mn} 22$ Be-like 1S2 2P 3D
(1D) $j=2.0 \quad 15.188$ beryl 100 (3P ) $j=2.0 \quad 15.238$ beryl 100

(3P ) $j=1.0 \quad 15.403$ beryl 100

(2D*) $j=2.5 \quad 14.364$ gypsum 020

$\left(2 P^{\star}\right) j=1.5 \quad 14.782$ gypsum 020

(3P ) $j=2.0 \quad 14.470 \quad$ gypsum 020

$\left(2 P^{*}\right) j=1.5 \quad 10.040 \quad$ ADP 101

$(2 P *) j=.512 .446$ TAP 100

$\left(2 P^{*}\right) j=.5 \quad 12.446 \quad R A P \quad 100$

$\left(2 P^{\star}\right) j=1.5 \quad 12.496 \quad$ TAP 100

$\left(2 P^{*}\right) j=1.5 \quad 12.496 \quad R A P \quad 100$

$\left(2 P^{*}\right) j=.5 \quad 12.705$ TAP 100

$\left(2 P^{*}\right) j=.5 \quad 12.705$ RAP 100

$\left(2 P^{\star}\right) j=.5 \quad 12.705$ KAP 100

(2D) $j=1.5 \quad 12.742 \quad$ TAP 100

(2D) $j=1.5 \quad 12.742 \quad \operatorname{RAP} 100$

(2D) $j=1.5 \quad 12.742$ KAP 100

(2D) $j=2.5 \quad 12.860$ TAP 100

(2D $j=2.5 \quad 12.860 \quad$ RAP 100

(2D) $j=2.5 \quad 12.860 \quad \operatorname{KAP} 100$

$\left(2 P^{*}\right) j=.5 \quad 12.886 \quad$ RAP 100

$\left.2 P^{*}\right) j=.5 \quad 12.886 \quad \operatorname{KAP} 100$

(4P) $j=2.5 \quad 12.973 \quad$ RAP 100

(4P) $j=2.5 \quad 12.973 \quad \mathrm{KAP} 100$

$\left(2 P^{*}\right) j=1.5 \quad 13.016 \quad$ RAP 100

$\left(2 P^{*}\right) j=1.5 \quad 13.016 \quad \mathrm{KAP} 100$

(2P) $j=1.5 \quad 13.134 \quad \mathrm{KAP} 100$

(2D) $j=2.5 \quad 13.167 \quad \mathrm{KAP} 100$

(2D) $j=1.5 \quad 13.241 \quad \mathrm{KAP} 100$

$\left(1 P^{*}\right) j=1.0 \quad 12.172$ TAP 100

$\left(3 P^{*}\right) j=.0 \quad 12.336$ TAP 100

$(3 P *) j=.0 \quad 12.336 \quad$ RAP 100

$\left(3 P^{*}\right) j=1.0 \quad 12.368 \quad$ TAP 100

$\left(3 P^{*}\right) j=1.0 \quad 12.368$ RAP 100

(3P) $j=1.0 \quad 12.385$ TAP 100

(3P) $j=1.0 \quad 12.385$ RAP 100

$\left(1 P^{*}\right) j=1.0 \quad 12.427$ TAP 100

$\left(1 P^{*}\right) j=1.0 \quad 12.427$ RAP 100

(3P) $j=2.0 \quad 12.447 \quad$ TAP 100

(3P) $j=2.0 \quad 12.447 \quad \operatorname{RAP} 100$

(3P) $j=1.0 \quad 12.488$ TAP 100

(3P) $j=1.0 \quad 12.488$ RAP 100

(3P) $j=2.0 \quad 12.553 \quad$ TAP 100

(3P) $j=2.0 \quad 12.553 \quad \operatorname{RAP} 100$

(3P) $j=2.0 \quad 12.553 \quad \mathrm{KAP} 100$

(3P) $j=2.0 \quad 12.580$ TAP 100
$15.954 \quad 72.173$ $15.954 \quad 72.770$ $15.954 \quad 74.898$

$15.185 \quad 71.073$

$15.185 \quad 76.770$

$15.185 \quad 72.348$

$10.640 \quad 70.667$

$25.763 \quad 75.059$

$26.116 \quad 72.389$

$25.763 \quad 75.947$

$26.116 \quad 73.129$

$25.763 \quad 80.504$

$26.116 \quad 76.647$

$26.634 \quad 72.562$

$25.763 \quad 81.560$

$26.116 \quad 77.369$

$26.634 \quad 73.102$

$25.763 \quad 86.689$

$26.116 \quad 80.010$

$26.634 \quad 74.946$

$26.116 \quad 80.690$

26.63475 .383

$26.116 \quad 83.459$

$26.634 \quad 76.949$

$26.116 \quad 85.403$

$26.634 \quad 77.795$

$26.634 \quad 80.490$

$26.634 \quad 81.392$

$26.634 \quad 83.876$

$25.763 \quad 70.895$

$25.763 \quad 73.266$

$26.116 \quad 70.858$

$25.763 \quad 73.768$

$26.116 \quad 71.291$

$25.763 \quad 74.041$

$26.116 \quad 71.525$

$25.763 \quad 74.735$

$26.116 \quad 72.115$

$25.763 \quad 75.076$

$26.116 \quad 72.403$

$25.763 \quad 75.802$

$26.116 \quad 73.009$

$25.763 \quad 77.033$

$26.116 \quad 74.013$

26.63470 .498

$25.763 \quad 77.579$ 


\begin{tabular}{|c|c|c|c|c|c|c|c|c|c|}
\hline $\operatorname{Mn} 22$ & Be-like & 152 & & $2 \mathrm{P}$ & 30 & $\left(3 D^{\star}\right) j=3.0$ & $-1 S 2$ & & $2 \mathrm{P} 2$ \\
\hline $\operatorname{Mn} 22$ & Be-like & $1 \mathrm{~S} 2$ & & $2 \mathrm{P}$ & $3 D$ & $\left(3 D^{\star}\right) j=3.0$ & $-1 S 2$ & & $2 \mathrm{P} 2$ \\
\hline $\operatorname{Mn} 22$ & Be-like & $1 S 2$ & & $2 \mathrm{P}$ & $3 D$ & $\left(1 F^{*}\right) j=3.0$ & $-1 s 2$ & & $2 \mathrm{P} 2$ \\
\hline $\operatorname{Mrn} 22$ & Be-Iike & $1 \mathrm{~S} 2$ & & $2 F$ & $3 D$ & $\left(1 F^{*}\right) j=3.0$ & $-1 s 2$ & & $2 \mathrm{P} 2$ \\
\hline $\operatorname{Mn} 22$ & Be-like & $1 S 2$ & & $2 \mathrm{P}$ & $3 D$ & $\left(1 F^{*}\right) j=3.0$ & $-1 s 2$ & & $2 \mathrm{P} 2$ \\
\hline $\operatorname{Mn} 22$ & Be-like & $1 S 2$ & & $2 \mathrm{P}$ & $3 D$ & $\left(3 D^{\star}\right) j=1.0$ & $-1 s 2$ & & $2 \mathrm{P} 2$ \\
\hline $\operatorname{Mr} 22$ & Be-like & $1 S 2$ & & $2 \mathrm{P}$ & $3 D$ & $\left(3 D^{\star}\right) j=1.0$ & $-1 s 2$ & & $2 \mathrm{P} 2$ \\
\hline $\operatorname{Mn} 22$ & Be-like & $1 S 2$ & & $2 \mathrm{P}$ & $3 D$ & $\left(3 D^{*}\right) j=1.0$ & $-1 s 2$ & & 242 \\
\hline $\operatorname{Mn} 22$ & Be-like & $1 S 2$ & & $\ddot{2 P}$ & $3 D$ & $\left(3 D^{*}\right) j=2.0$ & $-1 s 2$ & & $2 叉 2$ \\
\hline $\operatorname{Min} 22$ & Be-1ike & $1 \mathrm{~S} 2$ & & $2 F$ & 30 & $\left(3 D^{\star}\right) j=2.0$ & $-1 s 2$ & & $2 \mathrm{P} 2$ \\
\hline $\operatorname{Mn} 22$ & Be-like & 152 & & $2 \mathrm{P}$ & 30 & $\left(3 D^{\star}\right) j=2.0$ & $-1 s 2$ & & $2 P 2$ \\
\hline $\operatorname{Mn} 22$ & Be-like & $1 S 2$ & & $2 \mathrm{P}$ & $3 D$ & $\left(3 D^{*}\right) j=2.0$ & -152 & & $2 \mathrm{P} 2$ \\
\hline $\operatorname{Mn} 22$ & Be-like & $1 S 2$ & & $2 \mathrm{P}$ & 30 & $\left(3 D^{*}\right) j=2.0$ & $-1 s 2$ & & $2 P_{2}$ \\
\hline $\operatorname{Mn} 22$ & Be-like & $1 S 2$ & & $2 P$ & 30 & $\left(3 D^{*}\right) j=2.0$ & $-1 s 2$ & & $2 \mathrm{P} 2$ \\
\hline $\operatorname{Mn} 22$ & Be-like & $1 \mathrm{~S} 2$ & & $2 S$ & 30 & $(1 D) j=2.0$ & $-1 s 2$ & $2 S$ & $2 P$ \\
\hline $\operatorname{Mn} 22$ & Be-like & $1 S 2$ & & $2 S$ & $3 D$ & $(1 D) j=2.0$ & $-1 s 2$ & $2 S$ & $2 P$ \\
\hline $\operatorname{Mn} 22$ & Be-like & 152 & & $2 S$ & $3 D$ & (1D) $j=2.0$ & $-1 s 2$ & $2 S$ & $2 \mathrm{P}$ \\
\hline $\operatorname{Mn} 22$ & Be-1ike & 152 & & $2 \mathrm{P}$ & 30 & $\left(1 D^{*}\right) j=2.0$ & $-1 s 2$ & & $2 \mathrm{P} 2$ \\
\hline $\operatorname{Mn} 22$ & Be-1ike & $1 S 2$ & & $2 \mathrm{P}$ & 30 & $\left(1 D^{*}\right) j=2.0$ & $-1 s 2$ & & $2 \mathrm{P} 2$ \\
\hline $\operatorname{Mn} 22$ & Be-like & $1 \mathrm{~S} 2$ & & $2 \mathrm{P}$ & 3D & $\left(1 D^{\star}\right) j=2.0$ & $-1 s 2$ & & $2 P 2$ \\
\hline $\operatorname{Mn} 22$ & Be-like & $1 S 2$ & & $2 S$ & $3 S$ & $(3 S) j=1.0$ & $-1 s 2$ & $2 S$ & $2 P$ \\
\hline $\operatorname{Mn} 22$ & Be-like & $1 S 2$ & & $2 S$ & $3 S$ & $(3 s) j=1.0$ & $-1 s 2$ & $2 S$ & $2 \mathrm{P}$ \\
\hline $\operatorname{Mn} 22$ & Be-like & $1 \mathrm{~s} 2$ & & $2 P$ & $3 s$ & $\left(1 E^{\star}\right) j=1.0$ & -152 & & 282 \\
\hline $\operatorname{Mn} 22$ & Be-like & & & 0 & & $j=.0$ & - & & 0 \\
\hline $\operatorname{Mn} 22$ & Be-1ike & & & 0 & & $j=.0$ & - & & 0 \\
\hline $\operatorname{Mn} 22$ & Be-like & & & 0 & & $j=.0$ & - & & 0 \\
\hline $\operatorname{Mn} 22$ & Be-like & & & 0 & & $j=.0$ & - & & 0 \\
\hline $\operatorname{Mn} 22$ & Be-like & & & 0 & & $j j=.0$ & - & & 0 \\
\hline $\operatorname{Mn} 23$ & Li-1ike & is & $12 S$ & $2 \mathrm{P}$ & $\left.\left(1 P^{\star}\right)\right)$ & $\left(2 P^{*}\right) j=1.5$ & $-1 s 2$ & $2 S$ & \\
\hline $\operatorname{Mn} 23$ & Li-like & IS & $12 S$ & $2 \mathrm{P}$ & $\left.\left(1 P^{*}\right)\right)$ & $\left(2 P^{*}\right) j=1.5$ & $-1 s 2$ & $2 S$ & \\
\hline $\operatorname{Mn} 23$ & Li-like & is & $12 \mathrm{~S}$ & $2 \mathrm{P}$ & $\left.\left(1 P^{*}\right)\right)$ & $\left(2 P^{*}\right) j=1.5$ & $-1 s 2$ & $2 S$ & \\
\hline $\operatorname{Mn} 23$ & Li-1ike & 1s & (2S & $2 \mathrm{P}$ & $\left.\left(1 p^{*}\right)\right)$ & $\left(2 P^{*}\right) j=1.5$ & $-1 s 2$ & $2 S$ & \\
\hline $\operatorname{Min} 23$ & Li-1ike & $1 s$ & $12 S$ & $2 \mathrm{P}$ & $\left.\left(12^{*}\right)\right)$ & $\left(2 P^{\star}\right) j=1.5$ & $-1 s 2$ & $2 S$ & \\
\hline $\operatorname{Mn} 23$ & Li-like & $1 S$ & $12 S$ & $2 P$ & $\left.\left(1 P^{*}\right)\right)$ & $\left(2 \mathrm{P}^{*}\right) j=1.5$ & - 152 & $2 S$ & \\
\hline $\operatorname{Mn} 23$ & Li-like & is & $12 S$ & $2 \mathrm{P}$ & $\left.\left(1 P^{\star}\right)\right)$ & $\left(2 P^{\star}\right) j=1.5$ & $-1 s 2$ & $2 S$ & \\
\hline $\operatorname{Mn} 23$ & Li-1ike & 1S & (2S & $2 P$ & $(19 *))$ & $\left(2 P^{\star}\right) j=1.5$ & $-1 s 2$ & $2 \mathrm{~S}$ & \\
\hline $\operatorname{Mn} 23$ & Li-like & 1S & $12 S$ & $2 \mathrm{P}$ & $\left.\left(1 P^{*}\right)\right)$ & $j=1.5$ & $-1 s 2$ & $2 S$ & \\
\hline $\operatorname{Mn} 23$ & Li-like & is & $12 S$ & $2 P$ & $\left.\left(1 P^{*}\right)\right)$ & $\left(2 P^{\star}\right) j=1.5$ & $-1 s 2$ & $2 S$ & \\
\hline $\operatorname{Mn} 23$ & Li-like & $1 S$ & (2S & $2 \mathrm{P}$ & $\left(1 P^{*}\right)$ & $\left(2 P^{*}\right) j=1.5$ & $-1 s 2$ & $2 \mathrm{~S}$ & \\
\hline $\operatorname{Mn} 23$ & Li-like & 152 & $9 D$ & & & $(2 D) j=1.5$ & $-1 s 2$ & $2 P$ & \\
\hline $\operatorname{Mn} 23$ & Li-like & $1 \mathrm{~s} 2$ & $7 P$ & & & $\left(2 P^{\star}\right) j=1.5$ & $-1 s 2$ & $2 S$ & \\
\hline $\operatorname{Mn} 23$ & Li-like & $1 S 2$ & $7 P$ & & & $\left(2 P^{*}\right) j=1.5$ & $-1 s 2$ & $2 S$ & \\
\hline $\operatorname{Mn} 23$ & Li-1ike & $1 s 2$ & $9 D$ & & & $(2 D) j=2.5$ & $-1 s 2$ & $2 P$ & \\
\hline $\operatorname{Mn} 23$ & Li-like & $1 \mathrm{~S} 2$ & $9 D$ & & & $(2 D) j=2.5$ & $-1 \leqslant 2$ & $2 \mathrm{P}$ & \\
\hline $\operatorname{Mn} 23$ & Ii-like & $1 S 2$ & $8 \mathrm{D}$ & & & $(2 D) j=1.5$ & -1.52 & $2 \mathrm{P}$ & \\
\hline $\operatorname{Mn} 23$ & Li-1ike & $1 S 2$ & $8 D$ & & & $(2 D) j=1.5$ & $-1 S 2$ & $2 P$ & \\
\hline
\end{tabular}

\begin{tabular}{|c|c|c|}
\hline$(3 P) j=2.0$ & 12.580 & RAP 100 \\
\hline$P / j=2.0$ & 12.580 & KAP 100 \\
\hline$j j=2.0$ & 12.643 & TAP 100 \\
\hline$j j=2.0$ & 12.643 & $\operatorname{RAP} 100$ \\
\hline$D j j=2.0$ & 12.643 & KAP 100 \\
\hline$P / j=1.0$ & 12.656 & TAP 100 \\
\hline$p / j=1.0$ & 12.656 & $\operatorname{RAP} 100$ \\
\hline$P / j=1.0$ & 12.656 & KAP 100 \\
\hline P $/ j=1.0$ & 12.670 & TAP 100 \\
\hline P $/ j=1.0$ & 12.670 & RAP 100 \\
\hline$P / j=1.0$ & 12.670 & KAP 100 \\
\hline P $) j=2.0$ & 12.738 & TAP 100 \\
\hline$P, j=2.0$ & 12.738 & RAP 100 \\
\hline$P \backslash j=2.0$ & 12.738 & KAP 100 \\
\hline$\left.P^{\star}\right) j=1.0$ & 12.800 & $\operatorname{TAP} 100$ \\
\hline$\left.P^{*}\right) j=1.0$ & 12.800 & RAP 100 \\
\hline$\left.P^{\star}\right) j=1.0$ & 12.800 & KAP 100 \\
\hline D $) j=2.0$ & 12.816 & $\operatorname{TAP} 100$ \\
\hline$j j=2.0$ & 12.816 & RAP 100 \\
\hline D $/ j=2.0$ & 12.816 & KAP 100 \\
\hline$\left.P^{*}\right) j=2.0$ & 12.935 & RAP 100 \\
\hline$\left(P^{*}\right) j=2.0$ & 12.935 & $\operatorname{KAP} 100$ \\
\hline LD $j \mathrm{j}=2.0$ & 13.199 & $\operatorname{KAP} 100$ \\
\hline$j=.0$ & 12.507 & TAP 100 \\
\hline$\gamma j=.0$ & 12.507 & $\operatorname{RAP} 100$ \\
\hline$j=.0$ & 12.706 & $\operatorname{TAP} 100$ \\
\hline$j=.0$ & 12.706 & RAP 100 \\
\hline$j=.0$ & 12.706 & KAP 100 \\
\hline$(2 S) j=.5$ & 2.013 & quartz 223 \\
\hline $2 S) j=.5$ & 2.013 & LiF 200 \\
\hline$j j=.5$ & 2.013 & Al 200 \\
\hline$j=.5$ & 2.013 & quartz 200 \\
\hline $2 S j j=.5$ & 2.013 & calcite 200 \\
\hline$(2 S) j=.5$ & 2.013 & Si 111 \\
\hline$(2 S) j=.5$ & 2.013 & sylvite 200 \\
\hline$(2 s) j=.5$ & 2.013 & fluorite 111 \\
\hline$(2 s) j=.5$ & 2.013 & topaz 002 \\
\hline$(2 s) j=.5$ & 2.013 & quartz 100 \\
\hline$(2 s) j=.5$ & 2.013 & ADP 101 \\
\hline$\left(2 P^{*}\right) j=.5$ & 7.110 & InSb 111 \\
\hline$(2 S) j=.5$ & 7.158 & InSb 111 \\
\hline$(2 s) j=.5$ & 7.158 & gypsum 020 \\
\hline$\left(2 P^{\star}\right) j=1.5$ & 7.164 & InSb 111 \\
\hline$\left.P^{*}\right) j=1.5$ & 7.164 & gypsum 020 \\
\hline$\left.p^{*}\right) j=.5$ & 7.208 & InSb 111 \\
\hline$i=5$ & 7.208 & gypsum 020 \\
\hline
\end{tabular}

$\begin{array}{rr}26.116 & 74.449 \\ 26.634 & 70.849 \\ 25.763 & 78.957 \\ 26.116 & 75.516 \\ 26.634 & 71.693 \\ 25.763 & 79.263 \\ 26.116 & 75.746 \\ 26.634 & 71.872 \\ 25.763 & 79.603 \\ 26.116 & 75.998 \\ 26.634 & 72.067 \\ 25.763 & 81.440 \\ 26.116 & 77.289 \\ 26.634 & 73.043 \\ 25.763 & 83.551 \\ 26.116 & 78.592 \\ 26.634 & 73.982 \\ 25.763 & 84.220 \\ 26.116 & 78.952 \\ 26.634 & 74.234 \\ 26.116 & 82.130 \\ 26.634 & 76.243 \\ 26.634 & 82.367 \\ 25.763 & 76.150 \\ 26.116 & 73.296 \\ 25.763 & 80.531 \\ 26.116 & 76.666 \\ 26.634 & 72.577 \\ 2.024 & 84.024 \\ 4.027 & 88.723 \\ 4.048 & 84.024 \\ 4.246 & 71.475 \\ 6.071 & 84.115 \\ 6.271 & 74.366 \\ 6.292 & 73.697 \\ 6.308 & 73.207 \\ 8.374 & 74.060 \\ 8.512 & 71.078 \\ 10.640 & 71.078 \\ 7.481 & 71.880 \\ 7.481 & 73.102 \\ 15.185 & 70.522 \\ 7.481 & 73.261 \\ 15.185 & 70.659 \\ 7.481 & 74.474 \\ 15.185 & 71.688 \\ & \end{array}$ 


\begin{tabular}{|c|c|c|c|}
\hline $\operatorname{Mn} 23$ & Li-like & $1 S 2$ & $8 D$ \\
\hline $\mathrm{Mn} 23$ & Li-1ike & 152 & $8 \mathrm{D}$ \\
\hline $\operatorname{Mn} 23$ & Li-like & 1,52 & $7 D$ \\
\hline $\operatorname{Mn} 23$ & Li-like & $1 S 2$ & $7 D$ \\
\hline $\operatorname{Mn} 23$ & Li-1ike & $1 S 2$ & $6 \mathrm{P}$ \\
\hline $\operatorname{Mn} 23$ & Li-Iike & 152 & $6 P$ \\
\hline $\operatorname{Mn} 23$ & Li-like. & $1 S 2$ & $7 D$ \\
\hline $\operatorname{Mn} 23$ & Li-Iike & $1 S 2$ & $7 D$ \\
\hline $\operatorname{Mn} 23$ & Ii-like & $1 S 2$ & $6 D$ \\
\hline $\operatorname{Mn} 23$ & Li-Iike & 152 & $6 D$ \\
\hline $\operatorname{Mn} 23$ & Li-like & $1 \mathrm{~S} 2$ & $5 p$ \\
\hline $\min 23$ & Ii-like & $1 S 2$ & $5 D$ \\
\hline $\operatorname{Mn} 23$ & Li-like & 152 & $5 D$ \\
\hline $\mathrm{Mn} 23$ & Li-like & $1 \mathrm{S2}$ & $5 \mathrm{D}$ \\
\hline $\operatorname{Mn} 23$ & Li-like & 152 & $5 D$ \\
\hline $\operatorname{Mn} 23$ & Li-like & $1 S 2$ & $5 D$ \\
\hline $\operatorname{Mn} 23$ & Li-like & $1 S 2$ & $4 \mathrm{P}$ \\
\hline $\operatorname{Mn} 23$ & Li-like & 152 & $4 \mathrm{P}$ \\
\hline $\sin 23$ & Li-like & $1 S 2$ & $4 \mathrm{P}$ \\
\hline $\operatorname{Mn} 23$ & Li-like & $1 S 2$ & $4 \mathrm{P}$ \\
\hline $\operatorname{Mn} 23$ & Li-like & IS2 & $4 \mathrm{P}$ \\
\hline $\operatorname{Mn} 23$ & Li-like & $1 S 2$ & $4 \mathrm{P}$ \\
\hline $\mathrm{Mn} 23$ & Li-like & $1 S 2$ & $4 \mathrm{P}$ \\
\hline $\operatorname{Mn} 23$ & Li-like & 152 & $4 \mathrm{P}$ \\
\hline $\operatorname{Mn} 23$ & Li-like & $1 S 2$ & $3 D$ \\
\hline $\operatorname{Mn} 23$ & Li-like & $1 S 2$ & $3 s$ \\
\hline $\operatorname{Mn} 23$ & Li-like & 152 & $3 s$ \\
\hline $\operatorname{Mn} 23$ & Li-Iike & $1 S 2$ & $3 s$ \\
\hline $\operatorname{Mn} 23$ & Li-like & $1 S 2$ & $3 s$ \\
\hline $\operatorname{Mn} 24$ & He-like & 1S & $5 P$ \\
\hline $\operatorname{Mn} 24$ & He-like & is & $5 P$ \\
\hline $\operatorname{Mn} 24$ & He-like & 1S & $5 P$ \\
\hline $\operatorname{Mn} 24$ & He-like & IS & $5 \mathrm{P}$ \\
\hline $\operatorname{Mr} 24$ & He-like & 1s & $5 P$ \\
\hline $\operatorname{Mn} 24$ & He-like & is & $5 P$ \\
\hline $\operatorname{Mn} 24$ & He-like & is & $5 \mathrm{P}$ \\
\hline $\operatorname{Mn} 24$ & He-Iike & $1 S$ & $5 \mathrm{P}$ \\
\hline $\operatorname{Mn} 24$ & He-1ike & is & $5 P$ \\
\hline $\operatorname{Mn} 24$ & He-like & IS & $5 P$ \\
\hline $\operatorname{Mn} 24$ & He-like & $1 \mathrm{~S}$ & $4 \mathrm{P}$ \\
\hline $\operatorname{Mn} 24$ & He-like & is & $4 P$ \\
\hline $\operatorname{Mn} 24$ & He-like & 1S & $4 P$ \\
\hline $\operatorname{Mr} 24$ & He-like & is & $4 P$ \\
\hline $\operatorname{Mn} 24$ & He-like & 1S & $4 \mathrm{P}$ \\
\hline $\operatorname{Mn} 24$ & He-like & IS & $4 P$ \\
\hline Mn & He-like & $1 \mathrm{~s}$ & $4 P$ \\
\hline
\end{tabular}

(2D) $j=2.5-1 S 22 \mathrm{P}$ (2D) $j=2.5-1 S 22 P$ (2D) $j=1.5-1 S 22 P$ (2D) $j=1.5-1 S 22 P$ $\left(2 P^{*}\right) j=1.5-1 S 22 S$ $\left(2 P^{*}\right) j=1.5-1 S 22 S$ (2D) $j=2.5-1 S 22$ P (2D) $j=2.5-1 S 22 P$ (2D) $j=1.5-1 S 22 P$ (2D $) j=2.5-1 S 22 P$ $\left(2 P^{\star}\right) j=1.5-1 S 22 S$ (2D) $j=1.5-1 S 22 P$ (2D) $j=1.5-1$ S2 $2 \mathrm{P}$ (2D) $j=2.5-1 S 22 P$ (2D) $j=2.5-1 S 22 \mathrm{P}$ (2D) $j=2.5-1 S 22 P$ $\left(2 \mathrm{P}^{\star}\right) j=1.5-1 \mathrm{~S} 22 \mathrm{~S}$ $\left(2 P^{\star}\right) j=1.5-1 S 22 S$ $\left(2 P^{*}\right) j=1.5-1 S 22 S$ $\left(2 \mathrm{P}^{\star}\right) j=1.5-1 \mathrm{~S} 22 \mathrm{~S}$ $\left(2 P^{\star}\right) j=.5-1 S 22 S$ $\left(2 P^{\star}\right) j=.5-1 S 22 S$ $\left(2 P^{\star}\right) j=.5-1522 S$ $\left(2 P^{\star}\right) j=.5-1 S 22 S$ (2D) $j=2.5-1 S 22 P$ (2S ) $j=.5-1 S 22 P$ (2S) $j=5-1 S 22 P$ $(2 S) j=.5-1 S 22 P$ (2S) $j=.5-1 S 22$. $\left(1 P^{*}\right) j=1.0-1 \mathrm{~S} 2$ $\left(1 P^{*}\right) j=1.0-152$ $\left(1 P^{*}\right) j=1.0-1 \mathrm{~S} 2$ $\left(1 P^{*}\right) j=1.0-152$ $\left(1 P^{*}\right) j=1.0-1 S 2$ $\left(1 P^{\star}\right) j=1.0-1 S 2$ $\left(1 P^{*}\right) j=1.0-1 \mathrm{~S} 2$ $\left(1 P^{*}\right) j=1.0-152$ $\left(1 P^{*}\right) j=1.0-1 S 2$ $\left(1 P^{*}\right) j=1.0-152$ $\left(1 P^{*}\right) j=1.0-1 S 2$ $\left(1 P^{*}\right) j=1.0-152$ $\left(1 P^{\star}\right) j=1.0-152$ $\left(1 P^{*}\right) j=1.0-152$ (1P*) $j=1.0-152$ $\left(1 P^{\star}\right) j=1.0-152$ $\left(1 P^{*}\right) j=1.0-152$

\begin{tabular}{|c|c|c|}
\hline$\left(2 P^{\star}\right) j=1.5$ & 7.263 & Instb 111 \\
\hline$\left(2 P^{\star}\right) j=1.5$ & 7.263 & grpsum 020 \\
\hline$\left(2 P^{\star}\right) j=.5$ & 7.355 & InSb 111 \\
\hline$\left(2 P^{*}\right) j=.5$ & 7.355 & gxpsum 020 \\
\hline$(2 s) j=.5$ & 7.387 & InSb 111 \\
\hline$(2 s) j=.5$ & 7.387 & gypsum 020 \\
\hline$\left(2 P^{*}\right) j=1.5$ & 7.413 & Insb 111 \\
\hline$\left(2 P^{\star}\right) j=1.5$ & 7.413 & gypsum 020 \\
\hline$\left(2 P^{*}\right) j=.5$ & 7.595 & beryl 100 \\
\hline $2 P *) j=1.5$ & 7.656 & beryl 100 \\
\hline$S i j=.5$ & 7.797 & beryl 100 \\
\hline $\left.2 P^{*}\right) j=.5$ & 8.029 & topaz 002 \\
\hline$\left(2 P^{*}\right) j=.5$ & 8.029 & quartz 100 \\
\hline$\left(2 P^{*}\right) j=1.5$ & 8.090 & topaz 002 \\
\hline$\left(2 P^{*}\right) j=1.5$ & 8.090 & quartz 100 \\
\hline$\left(2 P^{*}\right) j=1.5$ & 8.090 & TAP 100 \\
\hline$(2 S) j=.5$ & 8.689 & PET 002 \\
\hline$(2 s) j=.5$ & 8.689 & EDT 020 \\
\hline$(2 S) j=.5$ & 8.689 & RAP 100 \\
\hline$(2 s) j=.5$ & 8.689 & KAP 100 \\
\hline$(2 s) j=.5$ & 8.699 & PET 002 \\
\hline$(2 s) j=.5$ & 8.699 & EDT 020 \\
\hline$(2 S) j=.5$ & 8.699 & RAP 100 \\
\hline$(25) j=.5$ & 8.699 & KAP 100 \\
\hline $\left.2 P^{*}\right) j=1.5$ & 12.162 & TAP 100 \\
\hline$\left.p^{*}\right) j=.5$ & 12.274 & $\operatorname{TAP} 100$ \\
\hline $\left.2 P^{*}\right) j=.5$ & 12.274 & RAP 100 \\
\hline$\left(2 P^{*}\right) j=1.5$ & 12.439 & TAP 100 \\
\hline$\left(2 P^{\star}\right) j=1.5$ & 12.439 & RAP 100 \\
\hline$(1 s) j=.0$ & 1.584 & quartz 502 \\
\hline$(1 s) j=.0$ & 1.584 & LiF 422 \\
\hline$(1 S) j=.0$ & 1.584 & corundum 146 \\
\hline (S $) j=.0$ & 1.584 & quartz 110 \\
\hline$s j j=.0$ & 1.584 & gypsum 002 \\
\hline$j=.0$ & 1.584 & Ge 111 \\
\hline$j=.0$ & 1.584 & $\mathrm{KBr} 200$ \\
\hline$(1 s) j=.0$ & 1.584 & quartz 101 \\
\hline$(1 s) j=.0$ & 1.584 & graphite 002 \\
\hline (1s) $j=.0$ & 1.584 & topaz 002 \\
\hline$(1 s) j=.0$ & 1.621 & quartz 502 \\
\hline$S / j=.0$ & 1.621 & LiF 422 \\
\hline$S j j=.0$ & 1.621 & corundum 146 \\
\hline$j=.0$. & 1.621 & quartz 110 \\
\hline$j=.0$ & 1.621 & gypsum 002 \\
\hline$j=.0$ & 1.621 & Ge 111 \\
\hline$S i j=.0$ & 1.621 & $\mathrm{KBr} 200$ \\
\hline
\end{tabular}

$7.481 \quad 76.134$ $15.185 \quad 73.058$

$7.481 \quad 79.469$ $15.185 \quad 75.631$ $\begin{array}{ll}7.481 & 80.908\end{array}$ $15.185 \quad 76.639$ $7.481 \quad 82.269$ $15.185 \quad 77.516$ $15.954 \quad 72.197$ $15.954 \quad 73.691$ $15.954 \quad 77.805$ $8.374 \quad 73.496$

$8.512 \quad 70.606$

$8.374 \quad 75.035$

$8.512 \quad 71.883$

$\begin{array}{ll}25.763 & 70.399\end{array}$

$8.742 \quad 83.688$

$8.808 \quad 80.571$

$26.116 \quad 86.490$

26.53478 .156

$8.742 \quad 84.315$

$8.808 \quad 80.977$

$26.116 \quad 87.814$

$26.634 \quad 78.475$

$25.763 \quad 70.760$

$25.763 \quad 72.334$

$26.116 \quad 70.045$

$25.763 \quad 74.939$

$26.116 \quad 72.288$

$1.624 \quad 77.257$

$1.652 \quad 73.504$

$1.660 \quad 72.596$

$4.912 \quad 75.336$

$4.990 \quad 72.233$

6.53275 .929

$6.584 \quad 74.224$

$6.687 \quad 71.354$

$6.696 \quad 71.127$

$8.374 \quad 71.047$

$1.624 \quad 86.517$

$1.652 \quad 78.883$

$1.660 \quad 77.556$

$4.912 \quad 81.900$

$4.990 \quad 77.046$

$6.532 \quad 83.050$

$6.584 \quad 80.001$ 


\begin{tabular}{|c|c|c|c|}
\hline $\operatorname{Mn} 24$ & He-like & $1 S$ & $4 \mathrm{P}$ \\
\hline $\operatorname{Mn} 24$ & He-like & $1 S$ & $4 \mathrm{P}$ \\
\hline $\operatorname{Mr} 24$ & He-like & 1S & $4 \mathrm{P}$ \\
\hline $\operatorname{Mn} 24$ & He-like & $1 S$ & $4 \mathrm{P}$ \\
\hline $\operatorname{Mr} 24$ & He-like & $1 S$ & $3 P$ \\
\hline $\operatorname{Mn} 24$ & He-like & 1s & $3 P$ \\
\hline $\operatorname{Mn} 24$ & He-Iike & $1 \mathrm{~s}$ & $3 P$ \\
\hline $\operatorname{Mn} 24$ & He-like & $1 S$ & $2 P$ \\
\hline $\operatorname{Mn} 24$ & He-like & $1 S$ & $2 P$ \\
\hline $\operatorname{Mn} 24$ & He-like & $1 \mathrm{~s}$ & $2 P$ \\
\hline $\operatorname{Mn} 24$ & He-like & 1S & $2 P$ \\
\hline $\operatorname{Mn} 24$ & He-like & $1 \mathrm{~S}$ & $2 P$ \\
\hline $\operatorname{Mn} 24$ & He-like & 15 & $2 \mathrm{P}$ \\
\hline $\operatorname{Mn} 24$ & He-Iike & $1 \mathrm{~s}$ & $2 P$ \\
\hline $\operatorname{Mn} 24$ & He-like & 1S & $2 \mathrm{P}$ \\
\hline $\operatorname{Mn} 24$ & He-like & $1 S$ & $2 \mathrm{P}$ \\
\hline $\min 24$ & He-like & $1 S$ & $2 \mathrm{P}$ \\
\hline $\operatorname{Mr} 24$ & He-like & 1S & $2 P$ \\
\hline $\operatorname{Mn} 24$ & He-like & $1 \mathrm{~S}$ & $2 P$ \\
\hline $\operatorname{Mn} 24$ & He-like & is & $2 \mathrm{P}$ \\
\hline $\operatorname{Mr} 24$ & He-like & 1S & $2 \mathrm{P}$ \\
\hline $\operatorname{Mn} 24$ & He-like & 1S & $2 \mathrm{P}$ \\
\hline $\operatorname{Mn} 24$ & He-like & $1 \mathrm{~S}$ & $2 \mathrm{P}$ \\
\hline $\min 24$ & He-like & IS & $2 P$ \\
\hline $\sin 24$ & He-like & $1 s$ & $2 P$ \\
\hline $\min 24$ & He-like & 15 & $2 P$ \\
\hline $\operatorname{Mn} 24$ & He-Iike & 15 & $2 \mathrm{P}$ \\
\hline $\operatorname{Mn} 24$ & He-like & 15 & $2 P$ \\
\hline $\operatorname{Mn} 24$ & He-1ike & 15 & $2 S$ \\
\hline $\operatorname{Mn} 24$ & He-like & is & $2 S$ \\
\hline $\min 24$ & He-Iike & 15 & $2 S$ \\
\hline $\sin 24$ & He-like & $1 S$ & $2 S$ \\
\hline $\operatorname{Mr} 24$ & He-1ike & $1 s$ & $2 \mathrm{~S}$ \\
\hline $\operatorname{Mn} 24$ & He-like & 15 & $2 S$ \\
\hline $\operatorname{Mn} 24$ & He-like & 15 & $2 S$ \\
\hline $\operatorname{Mn} 25$ & H -like & $5 P$ & \\
\hline $\operatorname{Mn} 25$ & $\mathrm{H}$-like & $5 P$ & \\
\hline $\operatorname{Mn} 25$ & H -Iike & $5 P$ & \\
\hline $\operatorname{Mn} 25$ & H-like & $5 P$ & \\
\hline $\operatorname{Mn} 25$ & H -like & $5 P$ & \\
\hline $\operatorname{Mn} 25$ & $\mathrm{H}$-like & $5 P$ & \\
\hline $\operatorname{Mn} 25$ & H -like & $5 P$ & \\
\hline $\operatorname{Mn} 25$ & H -like & $5 P$ & \\
\hline $\operatorname{Mn} 25$ & H -like & $5 P$ & \\
\hline $\operatorname{Mn} 25$ & $\mathrm{H}$-like & $4 \mathrm{P}$ & \\
\hline $\sin 25$ & H -like & $4 \mathrm{P}$ & \\
\hline
\end{tabular}

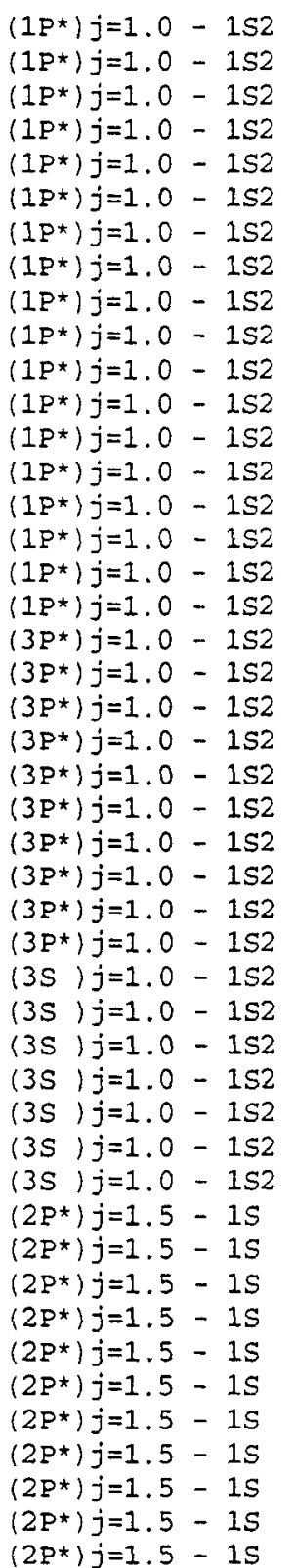

\begin{tabular}{|c|c|c|c|}
\hline (1s) $j=$ & .0 & 1.621 & quartz 101 \\
\hline (1S) $j=$ & .0 & 1.621 & graphite 002 \\
\hline (1S) $j=$ & .0 & 1.621 & topaz 002 \\
\hline (1s) $j=$ & .0 & 1.621 & quartz 100 \\
\hline 15$) j=$ & .0 & 1.706 & LiF 420 \\
\hline LS $j=$ & .0 & 1.706 & PET 002 \\
\hline$j=$ & 0 & 1.706 & EDT 020 \\
\hline$s) j=$ & .0 & 2.006 & quartz 223 \\
\hline$s) j=$ & .0 & 2.006 & LiF 200 \\
\hline$(S) j=$ & .0 & 2.006 & Al 200 \\
\hline (s) $j=$ & .0 & 2.006 & quartz 200 \\
\hline$j=$ & .0 & 2.006 & calcite 200 \\
\hline$j j=$ & .0 & 2.006 & Si 111 \\
\hline$j=$ & .0 & 2.006 & sylvite 200 \\
\hline$j=$ & .0 & 2.006 & Eluorite 111 \\
\hline$j=$ & .0 & 2.006 & topaz 002 \\
\hline$i j=$ & .0 & 2.006 & quartz 100 \\
\hline$j=$ & .0 & 2.006 & ADP 101 \\
\hline$j j=$ & .0 & 2.016 & quartz 223 \\
\hline$j=$ & .0 & 2.016 & Al 200 \\
\hline$j=$ & .0 & 2.016 & quartz 200 \\
\hline$j=$ & .0 & 2.016 & calcite 200 \\
\hline$j=$ & .0 & 2.016 & Si 111 \\
\hline$j=$ & .0 & 2.016 & sylvite 200 \\
\hline$j=$ & .0 & 2.016 & fluorite 111 \\
\hline$j=$ & .0 & 2.016 & topaz 002 \\
\hline $1 \mathrm{~J}=$ & .0 & 2.016 & quartz 100 \\
\hline$j=$ & .0 & 2.016 & ADP 101 \\
\hline$j j=$ & .0 & 2.025 & quartz 200 \\
\hline$j=$ & .0 & 2.025 & Si 111 \\
\hline$j=$ & .0 & 2.025 & sylvite 200 \\
\hline$j=$ & .0 & 2.025 & Eluorite 111 \\
\hline$j=$ & .0 & 2.025 & topaz 002 \\
\hline$j=$ & .0 & 2.025 & quartz 100 \\
\hline$j=$ & .0 & 2.025 & ADP 101 \\
\hline$j j=$ & .5 & 1.506 & calcite 422 \\
\hline$j=$ & .5 & 1.506 & quartz 211 \\
\hline$j=$ & .5 & 1.506 & quartz 112 \\
\hline$j=$ & .5 & 1.506 & topaz 200 \\
\hline$j=$ & .5 & 1.506 & A. 111 \\
\hline$j=$ & .5 & 1.506 & calcite 200 \\
\hline$j=$ & .5 & 1.506 & Si 111 \\
\hline$j=$ & .5 & 1.506 & sylvite 200 \\
\hline 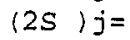 & .5 & 1.506 & fluorite 111 \\
\hline$j=$ & .5 & 1.542 & quartz 502 \\
\hline & 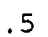 & - & topaz \\
\hline
\end{tabular}

$6.687 \quad 75.846$

$6.696 \quad 75.544$

$8.374 \quad 75.438$

$8.512 \quad 72.210$

$1.801 \quad 71.307$

$8.742 \quad 77.356$

$8.808 \quad 75.567$

$2.024 \quad 82.353$

$4.027 \quad 85.053$

$4.048 \quad 82.353$

$4.246 \quad 70.890$

$6.071 \quad 82.424$

$6.271 \quad 73.669$

$\begin{array}{lll}6.292 & 73.029\end{array}$

$6.308 \quad 72.559$

$8.374 \quad 73.376$

8.51270 .505

$10.640 \quad 70.505$

$2.024 \quad 84.904$

$4.048 \quad 84.904$

$4.246 \quad 71.732$

$6.071 \quad 85.011$

$6.271 \quad 74.674$

$6.292 \quad 73.991$

$6.308 \quad 73.493$

$8.374 \quad 74.361$

$8.512 \quad 71.328$

$10.640 \quad 71.328$

$4.246 \quad 72.523$

$6.271 \quad 75.637$

$6.292 \quad 74.909$

$6.308 \quad 74.379$

$8.374 \quad 75.303$

8.51272 .101

$10.540 \quad 72.101$

$3.034 \quad 83.096$

$3.082 \quad 77.765$

$4.564 \quad 81.858$

$4.638 \quad 76.938$

$4.676 \quad 75.063$

6.07182 .866

$6.271 \quad 73.866$

$6.292 \quad 73.217$

$6.308 \quad 72.742$

1.62471 .715

$4.638 \quad 85.878$ 


\begin{tabular}{|c|c|c|c|}
\hline $\mathrm{Mr} 25$ & $\mathrm{H}$ & -like & $4 F$ \\
\hline $\operatorname{Mn} 25$ & $\mathrm{H}$ & -like & $4 E$ \\
\hline $\operatorname{Mn} 25$ & $\mathrm{H}$ & -like & $4 F$ \\
\hline $\mathrm{Mn} 25$ & $\mathrm{H}$ & -like & 4 \\
\hline $\operatorname{Mn} 25$ & $\mathrm{H}$ & -like & 45 \\
\hline $\operatorname{Mn} 25$ & $\mathrm{H}$ & -like & $4 E$ \\
\hline $\operatorname{Mn} 25$ & $\mathrm{H}$ & -like & $3 E$ \\
\hline $\operatorname{Mn} 25$ & $\mathrm{H}$ & -Iike & 35 \\
\hline $\operatorname{Mn} 25$ & $\mathrm{H}$ & -like & 3 \\
\hline $\operatorname{Mn} 25$ & $\mathrm{H}$ & -1 ike & $3 E$ \\
\hline $\mathrm{Mn} 25$ & $\mathrm{H}$ & -like & $3 E$ \\
\hline $\operatorname{Mn} 25$ & $\mathrm{H}$ & -like & 35 \\
\hline $\operatorname{Mn} 25$ & $\mathrm{H}$ & -like & $3 E$ \\
\hline $\operatorname{Mn} 25$ & $\mathrm{H}$ & -like & 35 \\
\hline $\operatorname{Mn} 25$ & $\mathrm{H}$ & -like & 3 \\
\hline $\operatorname{Mn} 25$ & $\mathrm{H}$ & - like & 35 \\
\hline Mn25 & $\mathrm{H}$ & -like & $2 F$ \\
\hline $\operatorname{Mr} 25$ & $\mathrm{H}$ & -like & $2 E$ \\
\hline $\operatorname{Mn} 25$ & $\mathrm{H}$ & -like & $2 F$ \\
\hline $\operatorname{Mn} 25$ & $\mathrm{H}$ & -like & $2 E$ \\
\hline $\operatorname{Mn} 25$ & $\mathrm{H}$ & -like & $2 F$ \\
\hline $\operatorname{Mn} 25$ & $\mathrm{H}$ & $-1 i k e$ & $2 F$ \\
\hline $\operatorname{Mn} 25$ & $\mathrm{H}$ & -like & 2 \\
\hline $\operatorname{Mn} 25$ & $\mathrm{H}$ & -like & $2 \mathrm{~F}$ \\
\hline $\operatorname{Mn} 25$ & $\mathrm{H}$ & -like & $2 F$ \\
\hline $\operatorname{Mn} 25$ & $\mathrm{H}$ & -like & 2 \\
\hline $\operatorname{Mn} 25$ & $\mathrm{H}$ & -like & 25 \\
\hline $\operatorname{Mn} 25$ & $\mathrm{H}$ & -like & 21 \\
\hline Mn25 & $\mathrm{H}$ & -like & $4 \mathrm{~F}$ \\
\hline $\operatorname{Mn} 25$ & $\mathrm{H}$ & -Iike & 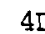 \\
\hline $\operatorname{Mn} 25$ & $H$ & -like & \\
\hline $\operatorname{Mn} 25$ & $\mathrm{H}$ & -like & $3 I$ \\
\hline
\end{tabular}

$\begin{array}{ll}\text { Fe } & \text { K-alpha(1) } \\ \text { Fe } & \text { K-alpha(1) } \\ \text { Fe } & \text { K-alpha(1) } \\ \text { Fe } & \text { K-alpha(1) } \\ \text { Fe } & \text { K-alpha(1) }\end{array}$

Fe15 Mg-like 2P5 3S2 3D $\quad\left(1 P^{*}\right) j=1.0-3 S 2$

Fe16 Na-like 2P5 3S ( $\left.3 P^{*}\right)$ 3D $\left(2 P^{*}\right) j=1.5-2 P 63 S$

Fe16 Na-like 2P5 3S ( $\left.3 P^{\star}\right) 3 D\left(2 P^{*}\right) j=1.5-2 P 63 S$

Fel6 Na-1ike 2P5 ( $2 P * 1) 3 S 3 D\left(\left(2 P^{*}\right) j=1.5-2 P 6 \quad 3 S\right.$

Fe16 Na-like $2 P 5$ 3S (3P*) $3 P \quad(2 S) j=.5-2 P 6 \quad 3 P$

Fe16 Na-like 2P5 3S ( $1 P * 1)$ 3D $\left(2 P^{\star}\right) j=1.5-2 P 6 \quad 3 S$

Fe16 Na-like $2 P 5$ 3S $\left(3 P^{*}\right) 3 P$ (2S $) j=.5-2 P 6$ 3P

Fe16 Na-like 2P5 $3 S\left(1 P^{* 1}\right) 3 D \quad\left(2 P^{*}\right) j=.5-2 P 6 \quad 3 S$

Fe17 Ne-like 2S 2P6 $5 P\left(3 P^{*}\right) j=1.0-2 S 22 P 6$

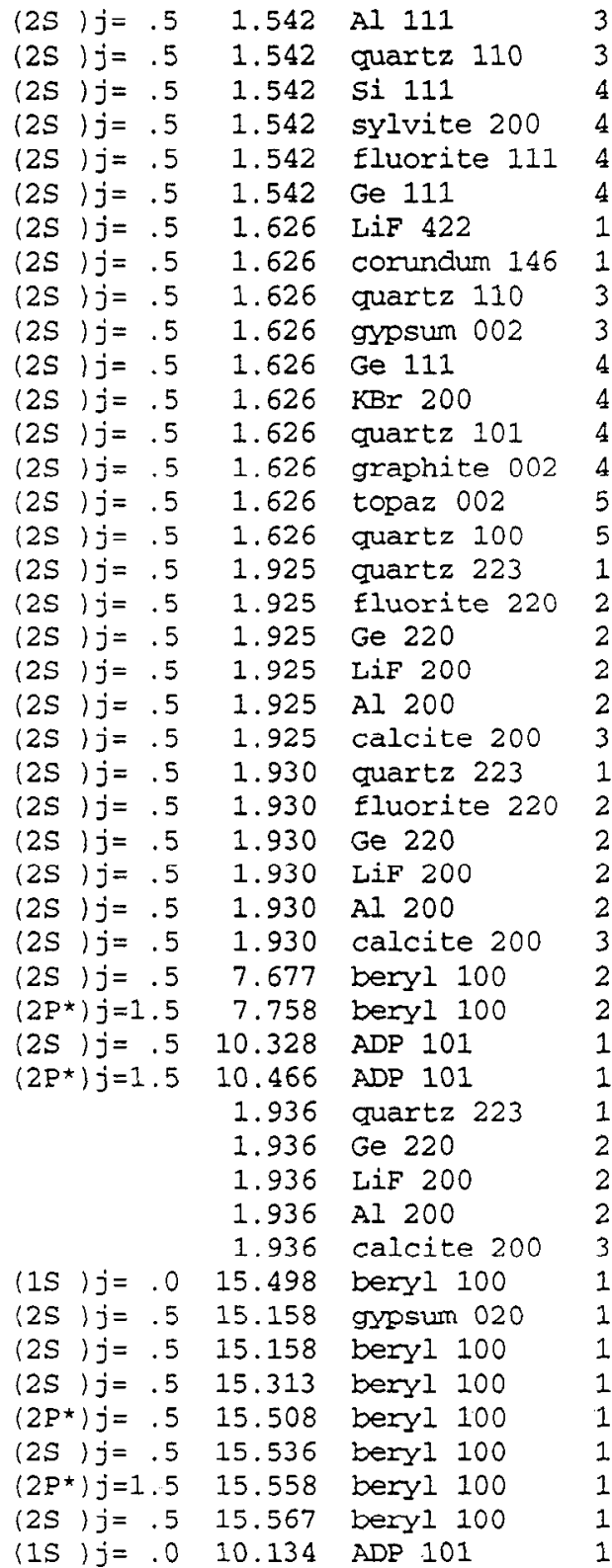

$4.676 \quad 81.61$

4.91270 .352

6.27179 .601

29278.606

$6.308 \quad 77.906$

$6.532 \quad 70.782$

$1.652 \quad 79.821$

$1.660 \quad 78.384$

4.91283 .255

$4.990 \quad 77.838$

$6.532 \quad 84.693$

6.58481 .059

$6.687 \quad 76.565$

6.69676 .246

$8.374 \quad 76.135$

$8.512 \quad 72.770$

$2.024 \quad 72.006$

$3.862 \quad 85.482$

$4.000 \quad 74.259$

$4.027 \quad 72.950$

$4.048 \quad 72.006$

$6.071 \quad 72.035$

$2.024 \quad 72.470$

$3.862 \quad 88.156$

$4.000 \quad 74.796$

$\begin{array}{ll}4.027 & 73.442\end{array}$

$4.048 \quad 72.470$

6.07172 .500

$15.954 \quad 74.237$

$15.954 \quad 76.543$

$10.640 \quad 76.091$

$10.640 \quad 79.624$

$2.024 \quad 73.052$

$4.000 \quad 75.478$

$4.027 \quad 74.062$

$4.048 \quad 73.052$

6.07173 .083

$15.954 \quad 76.268$

$15.185 \quad 86.583$

$15.954 \quad 71.825$

$\begin{array}{ll}15.954 & 73.703\end{array}$

$15.954 \quad 76.420$

$15.954 \quad 76.856$

$15.954 \quad 77.208$

$15.954 \quad 77.354$

$10.640 \quad 72.259$ 
Fe17 Ne-like 2S2 2P5 ( 2P*) 7D (1P*) j=1.0 - 2S2 2P6 Fe17 Ne-like 2S2 2P5 ( 2P*) $7 D\left(3 D^{*}\right) j=1.0-2 S 22 P 6$ Fe17 Ne-like 2S2 2P5 (2P*1) $4 D(12 *) j=1.0-2 S 2$ 2P6 Fe17 Ne-like 2S2 2P5 ( $\left.2 P^{*} 2\right) 4 D(21 *) j=1.0-2 S 22 P 6$ Fel7 Ne-like 2S2 2P5 ( 2P*2) 4D $(22 *) j=1.0-2 S 22 P 6$ Fe17 Ne-like 2S2 2P5 ( 2P*2) $4 D\left(22^{\star}\right) j=1.0-2 S 2$ 2P6 Fe17 Ne-1ike 2S2 2P5 ( $\left.2 P^{*} 1\right) 4 S(11 *) j=1.0-2 S 22 P 6$ Fe17 Ne-like 2S2 2P5 ( $2 P * 1) 4 S(11 *) j=1.0-2 S 22 P 6$ Fe17 Ne-1ike 2S2 2P5 (2P*1) 4S (11*)j=1.0 - 2S2 2P6 Fe17 Ne-like 2S2 2P5 ( $2 P \star 2)$ 4S $(21 *) j=1.0-2 S 22 P 6$ Fe17 Ne-like 2S2 $2 P 5(2 P * 2) 4 S(21 *) j=1.0-2 S 22 P 6$ Fe17 Ne-like 2S2 2P5 ( 2P*2) 4S (21*) j=1.0 - 2S2 $2 P 6$ Fe17 Ne-like 2S2 2P5 ( $2 P * 1) \quad 3 D\left(12^{*}\right) j=1.0-2 S 22 P 6$ Fe17 Ne-1ike 2S2 $2 P 5$ ( $\left.2 P^{*} 1\right) 3 D\left(12^{*}\right) j=1.0-2 S 22 P 6$ Fe17 Ne-like 2S2 2P5 $(2 P * 2)$ 3D $(22 *) j=1.0-2 S 22$ P6 Fe17 Ne-like 2S2 2P5 ( 2F*2) 3D (21*) j=1.0 - 2S2 2P6 Fe18 F-like 2S2 2P4 ( $1 S$ ) 5D (2D) $j=1.5-2 S 22 P 5$ Fel8 F-like 2S2 $2 P 4$ ( $1 S$ ) $5 D(2 D) j=1.5-2 S 22 P 5$ Fe18 F-like 2S2 2P4 ( $1 D$ ) 5D (2S) j=.5- 2S2 2P5 Fe18 $F$-like 2S2 2P4 ( $3 P$ ) 5D (2D) j=2.5 - 2S2 2P5 Fe18 F-like 2S2 2P4 ( 1D) 5D (2D) j=1.5 - 2S2 2P5 Fe18 F-like 2S2 2P4 ( 3P) 5D (4F) j=2.5 - 2S2 2P5 Fe18 $F$-like $2 S 22 P 4$ ( $3 P$ ) 5D (2D) j=1.5 - 2S2 2P5 Fel8 F-like 2S 2P5 ( $\left.1 P^{*}\right) 3 P(2 D) j=2.5-2 S 22 P 5$ Fe18 F-like 2S 2P5 ( $\left.1 P^{*}\right) 3 P(2 D) j=2.5-2 S 22 P 5$ Fe18 $F$-like 2S 2P5 ( 1P*) 3P (2D) $j=2.5-2 S 2$ 2P5 Fe18 F-like 2S 2P5 ( $\left.P^{*}\right)$ 3P (2P) $j=1.5-2 S 22 P 5$ Fe18 $F$-like $2 S$ 2P5 ( $\left.1 P^{\star}\right) 3 P(2 P) j=1.5-2 S 22 P 5$ Fe18 F-like $2 S$ 2P5 (1P*) 3P $(2 P) j=.5-2 S 22 P 5$ Fe18 F-like 2S 2P5 (1P*) 3P (2P) j=.5-2S2 2P5 Fe18 F-like $2 S$ 2P5 ( $P *), 3 P(2 D) j=1.5-2 S 22 P 5$ Fe18 F-like 2S 2P5 ( $\left.1 P^{*}\right)$ ) $P P(2 D) j=1.5-2 S 22 P 5$ Fe18 F-like 2S 2P5 (1P*) 3P (2S $) j=.5-2 S 22 P 5$ Fe18 $F$-like 2S2 2P4 ( 1D) 3D (2P $) j=.5-2 S 22 P 5$ Fe18 F-like 2S2 $2 P 4$ ( $1 D$ ) 3D (2D) $j=1.5-2 S 22 P 5$ Fe18 F-like 2S2 2P4 ( 3P) 3D (2D) $j=2.5-2 S 22 P 5$ Fel8 F-like 2S2 2P4 (3P) 3D ( $4 \mathrm{P}) j=2.5-2 S 22 \mathrm{P} 5$ Fe18 F-like 2S2 2P4 ( $3 P)$ 3D (4D) $j=1.5-2 S 22 P 5$ Fe18 F-like 2S2 2PA ( 1D) 3D (2S $) j=.5-2 S 22 P 5$ Fe18 F-like 2S2 2P4 ( 3P) 3D (4F) j=2.5 - 2S2 2P5 Fe18 $F$-like 2S2 $2 P 4(3 P) 3 D(2 F) j=2.5-2 S 22 P 5$ Fe18 $F$-like 2S2 2P4 ( $3 P$ ) 3D ( $4 P$ ) $j=1.5-2 S 22 P 5$ Fe18 F-like 2S2 2P4 ( 3P) 3D (4P) $j=.5-2 S 22 P 5$ Fe18 F-like 2S2 2P4 ( 3P) 3D (2P) $j=1.5-2 S 2$ 2P5 Fel8 F-Iike 2S2 2P4 ( 3P) 3D (4P) $j=1.5-2 S 22 P 5$ Fe18 $F$-like 2S2 $2 P 4(3 P)$ 3D $(2 P) j=.5-2 S 22 P 5$
(IS $) j=.0 \quad 10.386 \quad$ ADP 101 (IS) $j=.0 \quad 10.506 \quad$ ADP 101 (1S) $j=.0 \quad 12.125$ TAP 100 (IS) $j=.0 \quad 12.264$ TAP 100 (1S) $j=.0 \quad 12.322$ TAP 100 (1S) $j=.0 \quad 12.322 \quad \operatorname{RAP} 100$ (1S $) j=.0 \quad 12.526$ TAP 100 (1S) $j=.0 \quad 12.526$ RAP 100 (1S ) $j=.0 \quad 12.526 \quad \mathrm{KAP} 100$ (1S) $j=.0 \quad 12.678$ TAP 100 (1S) $j=.0 \quad 12.678$ RAP 100 (1S $) j=.0 \quad 12.678$ KAP 100 (1s) $j=.0 \quad 15.013$ gypsum 020 (1s) $j=.0 \quad 15.013$ beryl 100 (1s) $j=.0 \quad 15.260$ beryl 100 (1S) $j=.0 \quad 15.449$ beryl 100 $\left(2 P^{*}\right) j=1.5 \quad 10.192 \quad$ ADP 101 $\left(2 P^{*}\right) j=.5 \quad 10.298 \quad$ ADP 101 $\left(2 P^{*}\right) j=1.5 \quad 10.352 \quad$ ADP 101 $\left(2 P^{*}\right) j=1.5 \quad 10.437 \quad$ ADP 101 $\left(2 P^{*}\right) j=.5 \quad 10.460 \quad$ ADP 101 $\left(2 P^{*}\right) j=1.5 \quad 10.529 \quad$ ADP 101 $\left(2 P^{*}\right) j=.5 \quad 10.543 \quad$ ADP 101 $\left(2 P^{*}\right) j=1.5 \quad 12.847 \quad$ TAP 100 $\left(2 P^{*}\right) j=1.5 \quad 12.847 \quad \operatorname{RAP} 100$ $\left(2 P^{*}\right) j=1.5 \quad 12.847 \quad \mathrm{KAP} 100$ $\left(2 P^{*}\right) j=.5 \quad 13.001$ RAP 100 $\left(2 P^{*}\right) j=.5 \quad 13.001 \quad \operatorname{KAP} 100$ $\left(2 P^{*}\right) j=.5 \quad 13.015 \quad$ RAP 100 $\left(2 P^{*}\right) j=.5 \quad 13.015$ KAP 100 $\left(2 P^{*}\right) j=.5 \quad 13.049$ RAP 100 $\left(2 P^{\star}\right) j=.5 \quad 13.049 \quad \operatorname{KAP} 100$ $\left(2 P^{*}\right) j=1.5 \quad 13.159 \quad \operatorname{KAP} 100$ $\left(2 P^{*}\right) j=.5 \quad 14.344$ gypsum 020 $\left(2 P^{*}\right) j=.5 \quad 14.361$ gypsum 020 $\left(2 P^{\star}\right) j=1.5 \quad 14.373$ gypsum 020 $\left(2 P^{\star}\right) j=1.5 \quad 34.373 \quad 97 \mathrm{~g}$ $\left(2 P^{*}\right) j=1.5 \quad 14.453$ gypsum 020 $\left(2 P^{*}\right) j=.5 \quad 14.467$ gypsum 020 $\left(2 P^{*}\right) j=1.5 \quad 14.485$ gypsum 020 $\left(2 P^{*}\right) j=1.5 \quad 14.536 \quad$ gypsum 020 $\left(2 P^{\star}\right) j=1.5 \quad 14.551$ gypsum 020 $\left(2 P^{\star}\right) j=1.5 \quad 14.581$ gypsum 020 $\left(2 P^{*}\right) j=.5 \quad 14.610$ gypsum 020 $\left(2 P^{*}\right) j=.5 \quad 14.772$ gypsum 020 $\left(2 P^{*}\right) j=.5 \quad 14.868$ gypsum 020
$10.640 \quad 77.456$ $\begin{array}{ll}10.640 & 80.897\end{array}$ $\begin{array}{ll}25.763 & 70.266\end{array}$ $25.763 \quad 72.188$ $25.763 \quad 73.051$ $\begin{array}{ll}26.116 & 70.671\end{array}$ $\begin{array}{ll}25.763 & 76.508\end{array}$ $26.116 \quad 73.589$ $26.634 \quad 70.153$ $\begin{array}{ll}25.763 & 79.802\end{array}$ $26.116 \quad 76.144$ $\begin{array}{ll}26.634 & 72.179\end{array}$ $\begin{array}{ll}15.185 & 81.368\end{array}$ $\begin{array}{ll}15.954 & 70.223\end{array}$ $15.954 \quad 73.038$ $15.954 \quad 75.546$ $10.640 \quad 73.314$ $10.640 \quad 75.434$ $10.640 \quad 76.639$ $10.640 \quad 78.790$ $10.640 \quad 79.446$ $10.640 \quad 81.717$ $10.640 \quad 82.257$ $25.763 \quad 85.806$ $26.116 \quad 79.686$ $26.634 \quad 74.732$ $26.116 \quad 84.645$ $26.634 \quad 77.493$ $26.116 \quad 85.349$ $26.634 \quad 77.775$ $26.116 \quad 87.873$ $26.634 \quad 78.486$ $26.634 \quad 81.165$ $\begin{array}{lll}15.185 & 70.842\end{array}$ $15.185 \quad 71.038$ $\begin{array}{ll}15.185 & 71.178\end{array}$ $\begin{array}{ll}15.185 & 71.724\end{array}$ $\begin{array}{ll}15.185 & 72.137\end{array}$ $\begin{array}{ll}15.185 & 72.310\end{array}$ $\begin{array}{ll}15.185 & 72.535\end{array}$ $15.185 \quad 73.188$ $\begin{array}{ll}15.185 & 73.385\end{array}$ $15.185 \quad 73.786$ $15.185 \quad 74.182$ $15.185 \quad 76.606$ $15.185 \quad 78.272$ 
Fe18 $F$-like 2S2 2P4 ( $1 S$ ) 3S (2S) $j=.5-2 S 22 P 5$

Fe18 F-like 2S2 2P4 ( $1 D) 3 S(2 D) j=1.5-2 S 22 P 5$

Fe18 F-like 2S2 2P4 ( $1 D$ ) 3S (2D) $j=2.5-2 S 2$ 2P5

Fe18 $F$-like 2S2 2P4 ( $3 P$ ) $3 S(2 P) j=.5-2 S 22 P 5$

Fe18 $F$-like 2S2 2P4 ( $3 P$ ) $3 S(4 P) j=1.5-2 S 22 P 5$

Fe18 F-like 2S2 2P4 (1D) 3S (2D) $j=1.5-2 S 2$ 2P5

Fe19 0 -like 1S 2S2 2P5 (3P*) $j=2.0-1 S 2$ 2S2 2P4

Fe19 0 -iike 1s 2S2 2P5

Fe19 O-like 1S 2S2 2P5

Fe19 O-like 1s 2s2 2P5

Fe19 0 -like 1S 2S2 2P5

Fe19 $O$-iike 1S 2S2 2P5

Fe19 0 -like 1S 2S2 2P5

Fe19 O-like 1S 2\$2 2 P5

Fe19 0 -like 1S 2S2 2P5

Fe19 O -like 1S 2S2 2P5

Fe19 O-like 1S 2S2 2P5

Fe19 O-like is 2S2 2P5

Fe19 0 -like 2S2 $2 P_{3}\left(2 P^{*}\right) 5 D\left(1 F^{*}\right) j=3.0-1 S 2$ 2S2 2P4

Fe19 O-like 2S2 2P3 (2P*) SD (3F*) j=3.0 - 1S2 2S2 2P4

Fe19 O-like 2S2 2P3 ( $\left.2 D^{*}\right) 5 D\left(3 D^{*}\right) j=3.0-1 S 22 S 22 P 4$

Fe19 O-like 2S2 2P3 ( $\left.2 P^{*}\right) 5 D\left(1 P^{*}\right) j=1.0-2 S 22 P 4$

Fe19 0 -like 2S2 2P3 ( 2P*) 5D (1F*) j=3.0 - 2S2 2P4

Fe19 0 -like 2S2 2P3 ( 2D*) 5D $\left(3 D^{\star}\right) j=2.0-1 S 22 S 22 P 4$

Fe19 O-like 2S2 2P3 ( 2D*) 5D $\left(3 F^{*}\right) j=3.0-1 S 22 S 22 P 4$

Fe19 0 -like 2S2 2P3 ( $\left.2 P^{*}\right) 5 D\left(3 F^{*}\right) j=3.0-2 S 22 P 4$

Fe19 0 -like 2S2 2P3 (2D*) 5D $\left(3 S^{*}\right) j=1.0-2 S 22 P 4$

Fe19 O-like 2S2 2P3 ( 2D*) $5 D\left(3 D^{*}\right) j=2.0-2 S 22 P 4$

Fe19 O-like 2S2 2P3 ( $\left.4 S^{*}\right) 5 D\left(3 D^{*}\right) j=3.0-1 S 22 S 22 P 4$

Fe19 0 -like 2S2 $2 P^{2}\left(4 S^{*}\right) 5 D\left(3 D^{*}\right) j=3.0-1 S 22 S 22 P 4$

Fe19 O-like 2S2 2P3 ( $\left.4 S^{\star}\right) 5 D\left(3 D^{\star}\right) j=1.0-2 S 22 P 4$

Fe19 O-like 2S2 2P3 ( $\left.4 S^{*}\right)$ 5D $\left(3 D^{*}\right) j=2.0-2 S 22 P 4$

Fe19 O-like 2S2 2P3 (2P*) $4 D\left(3 D^{*}\right) j=2.0-2 S 22 P 4$

Fe19 O-1ike 2S2 2P3 (2P*) $4 D\left(3 P^{*}\right) j=1.0-2 S 22 P 4$

Fe19 O-like 2S2 2P3 ( $\left.2 P^{*}\right) 4 D\left(3 D^{*}\right) j=1.0-2 S 22 P^{*}$

Fe19 O-like 2S2 2P3 ( $\left.2 D^{*}\right) 4 D\left(3 S^{*}\right) j=1.0-1 S 2$ 2S2 2P4

Fe19 0 -like $2 S 22 P 3\left(2 D^{*}\right) 4 D\left(3 S^{*}\right) j=1.0-1 S 22 S 22 P 4$

Fe19 O-like 2S 2PA ( 2D) $3 P\left(1 F^{*}\right) j=3.0-2 S 22 P 4$

Fe19 0 -like 2S 2P4 ( 2D) 3P (1F*) $j=3.0-2 S 22 \mathrm{P} 4$

Fe19 0 -like 2S2 2P3 (2P*) 3D $\left(3 D^{*}\right) j=2.0-1 S 22 S 22 P 4$

Fe19 0 -1ike 2S2 2P3 ( 2P*) 3D $\left(3 D^{*}\right) j=3.0-1 S 2$ 2S2 2P4

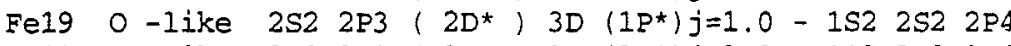

Fe19 0 -like $2 S 22 P^{2}\left(2 D^{*}\right) 3 S\left(3 D^{*}\right) j=3.0-1 S 22 S 22 P^{*}$

Fe19 O-like 2S2 2P3 ( $\left.2 \mathrm{P}^{*}\right)$ 3S $\left(3 \mathrm{P}^{*}\right) j=2.0-2 \mathrm{~S} 22 \mathrm{P} 4$

Fe19 O-like 2S2 2P3 ( $\left.2 D^{*}\right) 3 S\left(3 D^{*}\right) j=2.0-1 S 22 S 22 P^{*}$

Fe19 0 -like 2S2 $2 P^{2}\left(2 P^{\star}\right) 3 S\left(3 P^{\star}\right) j=1.0-2 S 22 P^{4}$ $\left(2 P^{*}\right) j=.5 \quad 15.491$ beryl 100

$\left(2 P^{*}\right) j=1.5 \quad 15.611$ beryl 100

$\left(2 P^{*}\right) j=1.5 \quad 15.623$ bery1 100

$\left(2 P^{\star}\right) j=1.5 \quad 15.764$ beryl 100

$\left(2 P^{*}\right) j=1.5 \quad 15.826$ beryl 100

$\left(2 P^{*}\right) j=.5 \quad 15.869$ beryl 100

(3P) $j=2.0 \quad 1.918 \quad$ quartz 223

(3P) $j=2.0 \quad 1.918 \quad$ Si 220

(3P) $j=2.0 \quad 1.918$ fluorite 220

(3P) $j=2.0 \quad 1.918$ Ge 220

(3P) $j=2.0 \quad 1.918 \quad$ IiF 200

(3P) $j=2.0 \quad 1.918$ Al 200

(3P) $j=2.0 \quad 1.918$ calcite 200

(1S ) $j=.0 \quad 1.931$ quartz 223

(1S) $j=.0 \quad 1.931$ Ge 220

(1S ) $j=.0 \quad 1.931$ LiF 200

(1S ) $j=.0 \quad 1.931$ AI 200

(1s) $j=.0 \quad 1.931$ calcite 200

(3P) $j=2 \quad 0$

9.547 mica 002

(3P $j=2.0 \quad 9.599$ mica 002

(3P) $j=2.0 \quad 9.688$ mica 002

(ID) $j=2.0 \quad 9.696$ mica 002

(ID) $j=2.0$

9.705 mica 002

(3P) $j=2.0$

(3P) $j=2.0$

9.713 mica 002

(1D) $j=2.0$

mica 002

(3P) $j=1.0$

9.752 mica 002

$9.766 \mathrm{mica} 002$

(3P) $j=1.0$

9.799 mica 002

(3P) $j=2.0$

9.842 mica 002

(3P) $j=2.0$

9.842 mica 002

$(3 P) j=.0$

9.911

mica 002

(3P) $j=1.0$

9.926 mica 002

(3P) $j=1.0$

10.564 ADP 101

$(3 P) j=1.0$

10.580

ADP 101

(3P) $j=.0 \quad 10.617 \quad$ ADP 101

(3P) $j=2.0 \quad 10.635$ ADP 101

(3P) $j=2.0 \quad 10.635$ ADP 101

(1D) $j=2.0$

(ID) $j=2.0 \quad 12.990 \quad K A P \quad 100$

(3P) $j=2.0 \quad 13.237 \quad \mathrm{KAP} 100$

(1S) $j=.0 \quad 14.293$ gypsum 020

(3P) $j=2.0 \quad 14.668$

(1D) $j=2.0 \quad 14.706$

(3P) $j=2.0 \quad 14.735$

(1D) $j=2.0 \quad 14.806$
$15.954 \quad 76.163$

$15.954 \quad 78.098$

$15.954 \quad 78.308$

$15.954 \quad 81.149$

$15.954 \quad 82.737$

$15.954 \quad 84.083$

$2.024 \quad 71.375$

$3.840 \quad 87.385$

$3.862 \quad 83.348$

$4.000 \quad 73.536$

$4.027 \quad 72.283$
4.048

$4.048 \quad 71.375$

$6.071 \quad 71.403$

$2.024 \quad 72.564$

$4.000 \quad 74.906$

$4.027 \quad 73.542$

$4.048 \quad 72.564$

$6.071 \quad 72.594$

$19.942 \quad 73.231$

$19.942 \quad 74.300$

$19.942 \quad 76.317$

$19.942 \quad 76.512$

$19.942 \quad 76.736$

$19.942 \quad 76.938$

$19.942 \quad 77.220$

$19.942 \quad 77.969$

$19.942 \quad 78.362$

$19.942 \quad 79.342$

$19.942 \quad 80.774$

$19.942 \quad 80.774$

$19.942 \quad 83.711$

$19.942 \quad 84.554$

$10.640 \quad 83.148$

$10.640 \quad 83.912$

$10.640 \quad 86.232$

$10.640 \quad 88.243$

$10.640 \quad 88.243$

$26.116 \quad 84.150$

$26.634 \quad 77.277$

$26.634 \quad 83.717$

$26.634 \quad 84.887$

$15.185 \quad 70.264$

$15.185 \quad 75.006$

$15.185 \quad 75.571$

$15.185 \quad 76.017$

$15.185 \quad 77.172$

Page 66 
Fe19 $0-1$ ike $2 S 2$ 2P3 (2D*) $3 S\left(3 D^{*}\right) j=1.0-2 S 22 P 4$

Fe19 0 -like 2S2 2P3 ( 2D*) 3S $\left(3 D^{*}\right) j=2.0-2 S 22 P 4$

Fe19 0 -1ike 2S2 $2 P^{2}\left(4 S^{*}\right) 3 S\left(3 S^{*}\right) j=1.0-1 S 22 S 22 P 4$

Fe19 0 -Iike 2S2 2P3 (2D*) 3S (1D*) $j=2.0-2 S 22 P 4$

Fe19 O-like 2S2 2P3 ( $\left.2 D^{*}\right) 3 S\left(1 D^{\star}\right) j=2.0-2 S 22 P 4$

Fe19 0 -like 2S2 2P3 (2D*) $3 S\left(3 D^{*}\right) j=3.0-2 S 22 P 4$

Fe19 O-like 2S2 2P3 (2D*) $3 S\left(3 D^{*}\right) j=3.0-2 S 22 P 4$

Fe19 O-like 2S2 2P3 ( $\left.2 D^{*}\right) 3 S\left(3 D^{*}\right) j=2.0-2 S 22 P 4$

Fe19 0 -like 2S2 2P3 (2D*) 3S $\left(3 D^{*}\right) j=2.0-2 S 22 P 4$

Fe19 O -like 2S2 2P3 (4S*) 3S $\left(3 S^{*}\right) j=1.0-2 S 22 P 4$

Fe19 0 -like 2S2 2P3 ( $\left.4 S^{\star}\right) 3 S\left(3 S^{*}\right) j=1.0-2 S 22 P 4$

Fe19 O-like 2S2 $2 \mathrm{P} 3$ (4S*) $3 S^{*}\left(3 S^{*}\right) j=1.0-2 S 22 P 4$

Fe19 0 -like 2S2 2P3 ( $\left.4 S^{*}\right) 3 S\left(3 S^{*}\right) j=1.0-2 S 22 P 4$

Fe19 0 -like 2S2 2P3 (4S*) 3S $\left(5 S^{*}\right) j=2.0-1 S 22 S 22 P 4$

Fe19 0 -like 2S2 2P3 (4S*) $3 S\left(5 S^{*}\right) j=2.0-2 S 22 \mathrm{P} 4$

Fe2O N-like $1 S$ 2S2 2P4 (2P) $j=1.5-1 S 2 \quad 2 S 2 \quad 2 P 3$

Fe20 N-like 1S 2S2 2P4 (2P) j=1.5 - 1S2 2S2 2P3

Fe20 N -like 15 2S2 284

Fe20 $N$-like $1 \mathrm{~S}$ 2S2 $2 \mathrm{P} 4 \quad$ (2P $) j=1.5-1 \mathrm{~S} 2$ 2S2 $2 \mathrm{P} 3$

Fe20 N-like $1 \mathrm{~S}$ 2S2 $2 \mathrm{P} 4 \quad(2 \mathrm{P}) \mathrm{j}=1.5-1 \mathrm{~S} 2$ 2S2 $2 \mathrm{P} 3$

Fe20 N-1ike 1S 2S2 2P4

Fe20 N -Iike $1 S$ 2S2 $2 \mathrm{P} 4 \quad$ (2P) $j=1.5-1 \mathrm{~S} 2$ 2S2 $2 \mathrm{P} 3$

Fe20 $N$-like $1 S$ 2S2 $2 P 4 \quad(2 P) j=1.5-1 S 2 \quad 2 S 2 \quad 2 P 3$

Fe20 N-like 1S 2S2 2P4 (2P) $j=1.5-1 S 2 \quad 2 S 2 \quad 2 P 3$

Fe20 N-like 1S 2S2 2P4 (2P) $j=1.5-1 S 2 \quad 2 S 2 \quad 2 P 3$

Fe2O N -like 1S 2S2 $2 \mathrm{P} 4 \quad$ (2P) $\mathrm{j}=1.5-1 \mathrm{~S} 2$ 2S2 $2 \mathrm{P} 3$

Fe20 $N$-like $1 \mathrm{~S}$ 2S2 2P4 (2P ) j=1.5 - 1S2 2S2 2P3

Fe20 N-like 1S 2S2 2P4 (2P) j=1.5 - 1S2 2S2 2P3

Fe20 N -like is 2S2 2P4. (2P) $j=1.5-1 S 22 S 2$ 2P3

Fe20 N-like 1S 2S2 2P4 (4P ) $j=1.5-1 S 2 \quad 2 S 2 \quad 2 P 3$

Fe20 N-1ike 1S 2S2 2P4 (4P) j=1.5 - 1S2 2S2 2P3

Fe20 N-like 1S 2S2 2P4 (4P) j=1.5 - 1S2 2S2 2P3

Fe20 N-like 1S 2S2 2P4 (4P) $j=1.5-1 S 22 S 2$ 2P3

Fe20 N-like 1S 2S2 2P4 (4P ) j=1.5 - 1S2 2S2 2P3

Fe20 N -like 1S 2S2 2P4

Fe20 N-like $1 \mathrm{~S}$ 2S2 $2 \mathrm{P} 4$

Fe20 N-like 1S 2S2 2P4

Fe20 N-like 1S 2S2 2P4

Fe20 N-like 1S 2S2 2P4

Fe20 N-like 1S 2S2 2P4

Fe20 N -like 1S 2S2 2PA

Fe20 N-like 1S 2S2 2P4

Fe20 N-like is 2S2 2P4

Fe20 N-Iike 2S2 $2 P 2$ ( $3 P$ ) $5 D(4 F) j=2.5-2 S 22 P 3$

Fe20 N-1ike 2S2 2P2 ( $3 P$ ) 5D (4P) j=2.5 - 2S2 2P3

Fe20 N-like $2 \mathrm{~S} 22 \mathrm{P} 2$ ( $1 \mathrm{D}) 4 \mathrm{D}(2 \mathrm{D}) \mathrm{j}=1.5-1 \mathrm{~S} 22 \mathrm{~S} 22 \mathrm{P} 3$

$(4 P) j=1.5-1 S 22 S 22 P 3$

j=1.5 - 1S2 2S2 $2 P 3$

$(4 P) j=1.5-1 S 22 S 22 P 3$

$(4 \mathrm{P}) j=1.5-1 \mathrm{~S} 22 \mathrm{~S} 22 \mathrm{P} 3$

$(4 \mathrm{P}) j=1.5-1 S 22 \mathrm{~S} 22 \mathrm{P} 3$

(4P) $j=1.5-1 S 2 \quad 252 \quad 2 P 3$
(3P) $j=.0 \quad 14.900$ gypsum 020

(3P) $j=1.0 \quad 14.929$

(3P) $j=2.0 \quad 14.966$

(1D) $j=2.0 \quad 14.995$

(ID ) $j=2.0 \quad 14.995$

(1D) $j=2.0 \quad 15.042$

(ID ) $j=2.0 \quad 15.042$

(ID ) $j=2.0 \quad 15.111$

15.111

(3P) $j=$.

15.138

$(3 P) j=.0$

15.138

(3P) $j=1.0 \quad 15.172$

(3P) $j=1.0 \quad 15.172$

(3P) $j=2.0 \quad 15.193$

(3P) $j=1.0$

$\left(2 D^{*}\right) j=2.5$

$\left(2 D^{\star}\right) j=2$.

$\left(2 D^{*}\right) j=2.5$

$\left(2 D^{*}\right) j=2.5$

$\left(2 D^{*}\right) j=2.5$

$\left(2 D^{\star}\right) j=2.5$

$\left(2 D^{*}\right) j=2.5$

$\left(2 D^{*}\right) j=2.5$

$\left(2 D^{*}\right) j=2.5$

$\left(2 D^{*}\right) j=2.5$

$\left(2 D^{*}\right) j=2.5$

$\left(2 D^{\star}\right) j=2.5$

$\left(2 D^{*}\right) j=2$.

$\left(2 D^{\star}\right) j=2.5$

$\left(4 S^{*}\right) j=1.5$

$\left(4 S^{\star}\right) j=1.5$

$\left(4 S^{\star}\right) j=1.5$

$\left(4 S^{\star}\right) j=1.5$

$\left(4 S^{*}\right) j=1.5$

$\left(4 S^{\star}\right) j=1.5$

$\left(4 S^{\star}\right) j=1.5$

$\left(4 S^{\star}\right) j=1.5$

$\left(4 S^{\star}\right) j=1.5$

$\left(4 S^{\star}\right) j=1.5$

$\left(4 S^{\star}\right) j=1.5$

$\left(4 S^{\star}\right) j=1.5$

$\left(4 S^{\star}\right) j=1.5$

$\left(4 S^{\star}\right) j=1.5$

$\left(2 P^{*}\right) j=1.5$

$\left(2 P^{*}\right) j=1.5$

$\left(4 S^{\star}\right) j=1.5$
15.413

1.905

1.905

1.905

1.905

1.905
1.905

1.905
1.905

1.905

1.905

1.905

1.905

1.905

1.905

1. 907

1.907

1.907

1.907

1.907

1.907

1.907

1.907

1.907

1.907

1.907

1.907

.389 mica 002

9.440 mica 002

9.871 mica 002
1.905
$15.185 \quad 78.882$

$15.185 \quad 79.464$

$15.185 \quad 80.257$

$15.185 \quad 80.927$

15.95470 .033

$15.185 \quad 82.131$

$15.954 \quad 70.533$

$15.185 \quad 84.341$

$15.954 \quad 71.291$

$15.185 \quad 85.491$

$15.954 \quad 71.596$

$15.185 \quad 87.629$

$15.954 \quad 71.987$

$15.954 \quad 72.232$

$15.954 \quad 75.036$

2.02470 .255

$3.840 \quad 82.833$

$3.862 \quad 80.587$

$4.000 \quad 72.270$

$4.027 \quad 71.105$

$4.048 \quad 70.255$

$6.071 \quad 70.281$

2.02470 .255

$3.840 \quad 82.833$

$3.862 \quad 80.587$

$4.000 \quad 72.270$

$4.027 \quad 71.105$

$4.048 \quad 70.255$

$6.071 \quad 70.281$

2.02470 .423

$3.840 \quad 83.329$

$3.862 \quad 80.957$

$4.000 \quad 72.459$

$4.027 \quad 71.282$

$4.048 \quad 70.423$

6.07170 .450

2.02470 .423

$3.840 \quad 83.329$

$3.862 \quad 80.957$

$4.000 \quad 72.459$

$4.027 \quad 71.282$

$4.048 \quad 70.423$

6.07170 .450

$19.942 \quad 70.327$

$19.942 \quad 71.217$

$19.942 \quad 81.879$

Page 67 
Fe20 N -like 2S2 2P2 ( $3 P$ ) $4 D(2 D) j=1.5-1 S 22 S 22 P 3$ Fe20 N-like 2S2 2P2 ( $1 D$ ) $4 D(2 D) j=1.5-2 S 22 P 3$

Fe20 N-like $2 S 2$ 2P2 ( $1 D$ ) $4 D(2 D) j=2.5-1 S 22 S 22 P 3$

Fe20 N-like 2S2 2P2 ( $1 D$ ) $4 D$ (2D) $j=1.5-1 S 22 S 22 P 3$

Fe20 N-like 2S2 2P2 ( $3 P$ ) $4 D(4 F) j=2.5-1 S 22 S 22 P 3$

Fe20 N-like 2S2 2P2 ( $3 P$ ) $4 D(2 D) j=1.5-2 S 22 P 3$

Fe20 N-like 2S2 2P2 ( $3 P$ ) $4 D(2 D) j=2.5-2 S 22 P 3$

Fe20 N-1ike 2S2 2P2 ( $3 P$ ) $4 D(4 P) j=2.5-1 S 22 S 22 P 3$

Fe20 N-like 2S2 2P2 ( $3 P$ ) $4 D(4 P) j=1.5-2 S 22 P 3$

Fe20 N-like 2S2 $2 \mathrm{P} 2(3 \mathrm{P}) 4 \mathrm{D}(2 \mathrm{~F}) \mathrm{j}=3.5-1 S 22 \mathrm{~S} 22 \mathrm{P} 3$

Fe20 N-like 2S2 2P2 ( $3 P$ ) $4 D(2 F) j=2.5-2 S 22 P 3$

Fe20 N-like 2S2 2P2 ( $3 P$ ) $4 D(2 D) j=1.5-2 S 22 P 3$

Fe20 N-1ike 2S2 2P2 ( $3 P$ ) $4 D(4 P) j=1.5-2 S 22 P 3$

Fe20 N-like 2S2 2P2 ( $3 P$ ) $4 D(4 D) j=2.5-2 S 22 P 3$

Fe20 N-like $2 S$ 2P3 $\left(3 D^{*}\right) 3 D\left(4 P^{*}\right) j=1.5-2 S$ 2P4

Fe20 N-like $2 S 2 P 3\left(3 D^{*}\right) 3 D\left(4 P^{*}\right) j=1.5-2 S 2 P 4$

Fe20 N-like 2S 2P3 (3D*) 3D $\left(4 P^{*}\right) j=1.5-2 S$ 2P4

Fe20 N-like 2S 2P3 (3D*) 3D (4P*) $j=1.5-2 S$ 2P4

Fe20 N-like 2S2 2P2 ( ID) 3D (2F) $j=2.5-2 S 22 P 3$

Fe20 N-Iike 2S2 2P2 ( ID) 3D (2F) $j=2.5-2 S 22 P 3$

Fe20 N-like 2S2 2P2 ( ID) 3D (2F) $j=2.5-2 S 22 P 3$

Fe20 N-like $2 S 2 F 3\left(3 D^{*}\right) 3 D\left(2 F^{*}\right) j=3.5-2 S \quad 2 P 4$

Fe20 N-like $2 S 2 P 3\left(3 D^{*}\right) 3 D\left(2 F^{*}\right) j=3.5-2 S 2 P 4$

Fe20 N-like $2 S$ 2P3 $\left(3 D^{*}\right) 3 D\left(2 F^{*}\right) j=3.5-2 S \quad 2 P 4$

Fe20 N-like 2S2 2P2 ( 3P) 3D (4P) $j=1.5-1 S 22 S 22 P 3$

Fe20 N-like 2S2 $2 P 2(3 P) 3 D(4 P) j=1.5-1 S 22 S 22 P 3$

Fe2O N-like 2S2 2P2 ( $3 P$ ) 3D (4P) $j=1.5-1 S 22 S 22 P 3$

Fe20 N -1ike 2S2 2P2 ( 1D ) 3D (2P) j=1.5 - 1S2 2S2 2P3

Fe20 N-1ike 2S2 2P2 ( $1 D$ ) 3D (2P) j=1.5 - 1S2 2S2 2P3

Fe20 N-like 2S2 2P2 ( $1 D$ ) 3D (2P) $j=1.5-1 S 22 S 22 P 3$

Fe20 N-like 2S2 2P2 ( $1 D$ ) 3D (2D) $j=1.5-2 S 22 P 3$

Fe20 N -like 2S2 2P2 (ID) 3D (2D) $j=1.5-2 S 22 P 3$

Fe20 N-1ike 2S2 2P2 ( 1D) 3D (2D) $j=1.5-2 S 22 P 3$

Fe20 N-like 2S2 2P2 ( $1 D$ ) 3D (2F) $j=3.5-1 S 22 S 22 \mathrm{P} 3$

Fe2O N-1ike 2S2 2P2 ( $1 D)$ 3D (2F) j=3.5 - 1S2 2S2 2P3

Fe20 N-like $2 S$ 2P3 ( $\left.3 D^{*}\right) 3 D\left(4 S^{*}\right) j=1.5-2 S$ 2P4

Fe20 N -like $2 S$ 2P3 $\left(3 D^{\star}\right) 3 D\left(4 S^{\star}\right) j=1.5-2 S \quad 2 P 4$

Fe20 N-Iike $2 S 22 \mathrm{P} 2$ ( ID) $3 D(2 D) j=2.5-1 S 22 S 22 \mathrm{P} 3$

Fe20 $N$-like 2S2 2P2 ( $1 D$ ) 3D (2D) $j=2.5-1 S 22 S 22 P 3$

Fe20 N-like 2S2 2P2 ( $1 S$ ) $3 D$ (2D) $j=2.5-2 S 22 P 3$

Fe20 N-like 2S2 2P2 ( $1 S$ ) $3 D(2 D) j=2.5-2 S 22 P 3$

Fe20 N-like $2 S \quad 2 P 3\left(3 D^{*}\right) 3 D\left(4 D^{*}\right) j=3.5-2 S \quad 2 P 4$

Fe20 N-like $2 S 2 P^{2}\left(3 D^{*}\right) 3 D\left(4 D^{*}\right) j=3.5-2 S 2 P 4$

Fe20 N-like 2S2 2P2 ( ID) 3D (2G) $j=3.5-1 S 22 S 22 P 3$

$F \in 20 N$-like $2 S 22 P 2$ ( $1 D$ ) $3 D(2 G) j=3.5-1 S 22 S 22 P 3$

Fe20 N-like 2S2 2P2 ( $3 P$ ) 3D (2D) $j=2.5-2 S 22 P 3$ $\left(4 S^{*}\right) j=1.5 \quad 9.953$ mica 002

$\left(2 D^{*}\right) j=1.5 \quad 10.008$ ADP 101

$\left(2 D^{\star}\right) j=2.5 \quad 10.034 \quad$ ADP 101

$\left(2 D^{*}\right) j=2.5 \quad 10.047 \quad A D P \quad 101$

$\left(4 S^{\star}\right) j=1.5 \quad 10.058 \quad$ ADP 101

$\left(2 D^{*}\right) j=1.5 \quad 10.095 \quad$ ADP 101

(2D*) $j=1.5 \quad 10.116$ ADP 101

$\left(4 S^{*}\right) j=1.5 \quad 10.121 \quad A D P 101$

$\left(2 D^{*}\right) j=1.5 \quad 10.128$ ADP 101

$\left(2 D^{*}\right) j=2.5 \quad 10.159 \quad$ ADP 101

(2D*) $j=1.5 \quad 10.177 \quad$ ADP 101

$\left(2 P^{*}\right) j=.5 \quad 10.222$ ADP 101

$\left(2 P^{\star}\right) j=1.5 \quad 10.322 \quad$ ADP 101

$\left(2 P^{\star}\right) j=1.5 \quad 10.344$ ADP 101

(4P) $j=2 \quad 5 \quad 12.393 \quad$ TAP 100

(4P) $j=2.5 \quad 12.393 \quad$ RAP 100

(4P) $j=1.5 \quad 12.494$ TAP 100

(4P) $j=1.5 \quad 12.494 \quad \operatorname{RAP} 100$

$\left(2 D^{*}\right) j=1.5 \quad 12.763 \quad$ TAP 100

$\left(2 D^{\star}\right) j=1.5 \quad 12.763 \quad \operatorname{RAP} 100$

$\left(2 D^{\star}\right) j=1.5 \quad 12.763 \quad$ KAP 100

(4P) $j=2.5 \quad 12.812 \quad$ TAP 100

(4P) $j=2.5 \quad 12.812$ RAP 100

$(4 P) j=2.5 \quad 12.812 \quad K A P \quad 100$

$\left(4 S^{\star}\right) j=1.5 \quad 12.818 \quad$ TAP 100

$\left(4 S^{\star}\right) j=1.5 \quad 12.818 \quad$ RAP 100

$\left(4 S^{\star}\right) j=1.5 \quad 12.818 \quad \mathrm{KAP} 100$

$\left(2 D^{*}\right) j=2.5 \quad 12.834$ TAP 100

$\left(2 D^{\star}\right) j=2.5 \quad 12.834 \quad$ RAP 100

$\left(2 D^{*}\right) j=2.5 \quad 12.834 \quad \mathrm{KAP} 100$

$\left(2 D^{*}\right) j=1.5 \quad 12.857 \quad$ TAP 100

(2D*) $j=1.5 \quad 12.857 \quad$ RAP 100

$\left(2 D^{*}\right) j=1.5 \quad 12.857 \quad$ KAP 100

$\left(2 D^{*}\right) j=2.5 \quad 12.888 \quad \operatorname{RAP} 100$

$\left(2 D^{*}\right) j=2.5 \quad 12.888 \quad \operatorname{KAP} 100$

(4P) $j=2.5 \quad 12.909 \quad$ RAP 100

$(4 P) j=2.5 \quad 12.909$ KAP 100

$\left(2 D^{*}\right) j=2.5 \quad 12.924 \quad \operatorname{RAP} 100$

(2D*) $j=2.5 \quad 12.924$ KAP 100

$\left(2 P^{\star}\right) j=1.5 \quad 12.946 \quad \operatorname{RAP} 100$

$\left(2 P^{\star}\right) j=1.5 \quad 12.946 \quad K A P \quad 100$

(4P) $j=2.5 \quad 12.958 \quad \operatorname{RAP} 100$

(4P ) $j=2.5 \quad 12.958 \quad \operatorname{KAP} 100$

$\left(2 D^{*}\right) j=2.5 \quad 12.970 \quad$ RAP 100

$\left(2 D^{*}\right) j=2.5 \quad 12.970 \quad \operatorname{KAP} 100$

$\left(2 D^{*}\right) j=1.5 \quad 12.983 \quad \operatorname{RAP} 100$
$19.942 \quad 86.557$

$10.640 \quad 70.153$

$10.640 \quad 70.569$

$10.640 \quad 70.781$

$10.640 \quad 70.962$

$10.640 \quad 71.582$

$10.640 \quad 71.944$

$10.640 \quad 72.031$

$10.640 \quad 72.153$

$10.640 \quad 72.706$

$10.640 \quad 73.035$

$10.640 \quad 73.887$

$10.640 \quad 75.957$

$10.640 \quad 76.454$

$25.763 \quad 74.170$

$26.116 \quad 71.636$

$25.763 \quad 75.911$

$26.116 \quad 73.099$

$25.763 \quad 82.222$

$26.116 \quad 77.798$

$26.634 \quad 73.415$

$25.763 \quad 84.046$

$26.116 \quad 78.861$

26.63474 .171

$25.763 \quad 84.309$

$26.116 \quad 78.998$

$26.634 \quad 74.266$

$25.763 \quad 85.078$

$26.116 \quad 79.372$

$26.634 \quad 74.521$

$25.763 \quad 86.466$

$26.116 \quad 79.934$

$26.634 \quad 74.897$

$26.116 \quad 80.745$

$26.634 \quad 75.417$

$26.116 \quad 81.336$

$26.634 \quad 75.781$

$26.116 \quad 81.785$

$26.634 \quad 76.046$

$26.116 \quad 82.490$

26.63476 .444

$26.116 \quad 82.905$

$26.634 \quad 76.666$

$26.116 \quad 83.344$

$26.634 \quad 76.892$

$26.116 \quad 83.856$ 
Fe20 N-1ike 2S2 2P2 (3P) 3D (2D) j=2.5-2S2 $2 \mathrm{P} 3$

Fe20 N-like $2 S$ 2P3 ( $\left.3 D^{*}\right) 3 D\left(4 D^{*}\right) j=2.5-2 S \quad 2 P 4$

Fe20 N-1ike $2 S$ 2P3 $\left(3 D^{*}\right) 3 D\left(4 D^{*}\right) j=2.5-2 S \quad 2 P 4$

Fe20 N-like 2S2 2P2 ( $1 D$ ) 3D (2S $) j=.5-2 S 22 P 3$

Fe20 N-like 2S2 2P2 ( $3 \mathrm{P})$ 3D (2F) $j=3.5-1 \mathrm{~S} 22 \mathrm{~S} 22 \mathrm{P} 3$

Fe20 N-like $2 S 2 P 3\left(3 P^{*}\right) 3 D\left(2 D^{*}\right) j=2.5-2 S \quad 2 P 4$

Fe20 N-like $2 S$ 2P3 (3P*) 3D $\left(2 D^{*}\right) j=2.5-2 S 2 P 4$

Fe20 N-1ike 2S 2P3 (3P*) 3D (2F*) $j=3.5-2 S$ 2P4

Fe20 N-like 2S2 $2 \mathrm{P} 2$ ( $3 P$ ) $3 D(2 P) j=1.5-2 S 22 \mathrm{P} 3$

Fe20 N-like $2 S$ 2P3 ( $\left.3 D^{*}\right) 3 D\left(4 P^{*}\right) j=1.5-2 S$ 2P4

Fe20 N-like $2 S$ 2P3 ( $\left.1 D^{\star}\right) 3 D\left(2 F^{\star}\right) j=2.5-2 S$ 2P4

Fe20 N-like 2S2 $2 P 2$ ( $3 P$ ) 3D (2P) $j=1.5-1 S 22 S 22 P 3$

Fe20 $N$-like 2S 2P3 ( 5S*) 3D $\left(4 D^{*}\right) j=1.5-2 S$ 2P4

Fe20 N-like $2 S$ 2P3 (1 $\left.D^{*}\right) 3 D\left(2 S^{*}\right) j=.5-2 S$ 2P4

Fe20 N-like 2S2 2P2 ( $3 P$ ) $3 D(2 D) j=2.5-2 S 22 P 3$

Fe20 N-like 2S2 $2 \mathrm{P} 2(3 \mathrm{P}) 3 \mathrm{~S}(2 \mathrm{P}) \mathrm{j}=.5-2 \mathrm{~S} 22 \mathrm{P} 3$

Fe21 $C$-like is 2S2 $2 P 3 \quad\left(1 P^{*}\right) j=1.0-1 S 22 S 22 P 2$

Fe21 C -1ike 1S 2S2 2P3

Fe21 C-like is 252 2P3

Fe21 C -like 1S 2S2 2P3

Fe21 C -like 1s '2S2 $2 P 3$

Fe2I C-like is $252 \quad 2 P 3$

Fe21 C -iike 1S 2S2 $2 P 3$

Fe21 C -like 1S 2S2 2P3

Fe21 C -like 1S 2S2 2P3

Fe21 C -like 1s 2S2 2P3

Fe21 C -like 1s 2S2 2P3

Fe21 C -like 1S2 2S2 2P

Fe21 C -like 1S2 2S2 2P

Fe21 C -like 1S2 2S2 2P

Fe21 C -like 1S2 2S2 2P

Fe21 C -like 1S2 2S2 2P

Fe21 C-like 1S2 2S2 2P

Fe21 C -like 1S2 2S2 2P

Fe21 C -like IS2 2S2 2P

Fe2I C-like 1S2 2S2 2P

Fe21 C-like 1S2 2S2 2P

Fe21 C -like 1S2 2S2 2P

Fe21 C -like 1S2 2S2 2P

Fe21 C-like 1S2 2S2 2P

Fe21 C -like IS2 2S2 2P

Fe21 C-like 152 2S2 $2 P$

Fe21 C-like 1S2 2S2 2P

Fe21 C -like 152 2S2 2P

Fe21 C-like 1S2 2S2 2P

Fe2I $\dot{c}$-like IS2 2S2 2P $\left(1 P^{*}\right) j=1.0-1 S 2$ 2S2 2P2

$\left(1 P^{*}\right) j=1.0-1 S 2 \quad 2 S 2 \quad 2 P 2$

$\left(3 S^{*}\right) j=1.0-1 S 2$ 2S2 2P2

$\left(3 S^{*}\right) j=1.0-1 S 2$ 2S2 2P2

$\left(3 S^{*}\right) j=1.0-1 S 2$ 2S2 2P2

$\left(3^{*}\right) j=1.0-1 S 2$ 2S2 2P2

$\left(3 D^{*}\right) j=3.0-1 \mathrm{~S} 2$ 2S2 2P2

$\left(3 D^{*}\right) j=3.0-1 S 2$ 2S2 2P2

$\left(3 D^{*}\right) j=3.0-1 S 2 \quad 2 S 22 P 2$

$\left(3 D^{*}\right) j=3.0-1 S 2$ 2S2 2P2

$\left(3 P^{*}\right) j=1.0-1 S 2$ 2S2 2P2

$\left(3 P^{\star}\right) j=1.0-1 S 2 \quad 2 S 22 \mathrm{P} 2$

$\left(3 P^{*}\right) j=1.0-1 \mathrm{~S} 2$ 2S2 $2 \mathrm{P} 2$

$\left(3 \mathrm{P}^{*}\right) j=1.0-1 \mathrm{~S} 2$ 2S2 2P2

$\left(3 P^{*}\right) j=1.0-1 S 2$ 2S2 $2 \mathrm{P} 2$

$\left(3 P^{*}\right) j=1.0-1 S 22 S 22 P 2$

$\left(3 P^{*}\right) j=1.0-1 S 2$ 2S2 2P2

$\left(3 \mathrm{P}^{*}\right) j=1.0-1 \mathrm{~S} 2$ 2S2 2P2

$\left(3 P^{*}\right) j=1.0-1 S 2$ 2S2 2P2

$\left(3 \mathrm{P}^{*}\right) j=1.0-1 \mathrm{~S} 2$ 2S2 2P2

$\left(3 \mathrm{P}^{*}\right) j=1.0-1 \mathrm{~S} 2$ 2S2 2P2

$\left(3 D^{*}\right) j=2.0-1 S 2$ 2S2 $2 P 2$

$\left(3 D^{*}\right) j=2.0-1 \mathrm{~S} 2$ 2S2 2P2

$\left(3 D^{*}\right) j=2.0-1 S 22 S 22 P 2$

$\left(3 D^{*}\right) j=2.0-1 S 2$ 2S2 $2 F 2$

$\left(3 D^{*}\right) j=2.0-1 S 2 \quad 2 S 2 \quad 2 P 2$

$\left(3 D^{*}\right) j=2.0-1 S 2 \quad 2 S 2 \quad 2 P 2$

$\left(3 D^{*}\right) j=2.0-1 S 2$ 2S2 2P2 $\left(3 D^{*}\right) j=2.0-1 \mathrm{~S} 2$ 2S2 $2 \mathrm{P} 2$

\begin{tabular}{|c|c|c|}
\hline$\left.D^{*}\right) j=$ & 12.983 & KAP 1 \\
\hline $4 P, j=2.5$ & 12.995 & RAP 100 \\
\hline $4 P \quad j=2.5$ & 12.995 & KAP 100 \\
\hline$\left.D^{*}\right) j=1.5$ & 13.070 & KAP 100 \\
\hline$\left.D^{*}\right) j=2.5$ & 13.082 & KAP 100 \\
\hline D $) j=1.5$ & 13.111 & KAP 100 \\
\hline D $) j=2.5$ & 13.138 & $\operatorname{KAP} 100$ \\
\hline$j j=2.5$ & 13.159 & KAP 100 \\
\hline$\left.D^{*}\right) j=1.5$ & 13.183 & KAP 100 \\
\hline$P / j=1.5$ & 13.194 & KAP 100 \\
\hline$P / j=1.5$ & 13.232 & $\operatorname{KAP} 100$ \\
\hline$\left.D^{*}\right) j=2.5$ & 13.247 & KAP 100 \\
\hline$S / j=.5$ & 13.279 & KAP 100 \\
\hline$P \mid j=.5$ & 13.292 & KAP 100 \\
\hline$p * / j=1.5$ & 13.298 & KAP 100 \\
\hline$\left.P^{*}\right) j=1.5$ & 14.387 & gypsum 020 \\
\hline D $j j=2.0$ & 1.891 & $\mathrm{Si} 220$ \\
\hline D $j j=2.0$ & 1.891 & Eluorite 220 \\
\hline D $i j=2.0$ & 1.891 & Ge 220 \\
\hline P $j j=1.0$ & 1.894 & $\mathrm{Si} 220$ \\
\hline$P \mid j=1.0$ & 1.894 & Eluorite 220 \\
\hline$j=1.0$ & 1.894 & Ge 220 \\
\hline$j j=1.0$ & 1.894 & LiF 200 \\
\hline$j j=2.0$ & 1.896 & $\mathrm{Si} 220$ \\
\hline$P \mid j=2.0$ & 1.896 & Eluorite 220 \\
\hline$P \mid j=2.0$ & 1.896 & $\mathrm{Ge} 220$ \\
\hline$j j=2.0$ & 1.896 & LiF 200 \\
\hline$j j=.0$ & 8.472 & quartz 100 \\
\hline$j j=.0$ & 8.472 & PET 002 \\
\hline$j j=.0$ & 8.472 & EDT 020 \\
\hline$i j=.0$ & 8.472 & TAP 100 \\
\hline$j j=.0$ & 8.472 & RAP 100 \\
\hline$j j=.0$ & 8.472 & KAP 100 \\
\hline$j j=1.0$ & 8.521 & PET 002 \\
\hline$j j=1.0$ & 8.521 & EDT 020 \\
\hline$j j=1.0$ & 8.521 & TAP 100 \\
\hline$i j=1.0$ & 8.521 & RAP 100 \\
\hline$\lambda j=1.0$ & 8.521 & KAP 100 \\
\hline$j j=1.0$ & 8.558 & PET 002 \\
\hline$j j=1.0$ & 8.558 & EDT 020 \\
\hline$j j=1.0$ & 8.558 & TAP 100 \\
\hline$j j=1 . c$ & 8.558 & RAP 100 \\
\hline$j j=1.0$ & 8.558 & KAP 100 \\
\hline$j j=2 . c$ & 8.590 & PET 002 \\
\hline$j=2$. & 8.590 & EDT 020 \\
\hline 1$]-2.0$ & 8.590 & RAP 100 \\
\hline
\end{tabular}

$26.634 \quad 77.141$

$26.115 \quad 84.370$

$26.634 \quad 77.375$

26.63478 .948

$26.634 \quad 79.220$

$26.634 \quad 79.909$

$26.634 \quad 80.595$

$26.634 \quad 81.165$

$26.634 \quad 81.865$

$26.634 \quad 82.207$

$26.634 \quad 83.523$

$26.634 \quad 84.123$

$26.634 \quad 85.671$

$26.634 \quad 86.489$

$26.634 \quad 86.939$

$15.185 \quad 71.343$

$3.840 \quad 80.029$

3.86278 .318

$4.000 \quad 70.997$

$3.840 \quad 80.560$

3.86278 .766

$4.000 \quad 71.262$

$4.027 \quad 70.161$

$3.840 \quad 80.931$

$3.862 \quad 79.075$

$4.000 \quad 71.442$

$4.027 \quad 70.329$

8.51284 .443

$8.742 \quad 75.723$

$8.808 \quad 74.123$

$25.763 \quad 80.586$

$26.116 \quad 76.704$

26.63472 .605

$8.742 \quad 77.089$

$8.808 \quad 75.334$

$25.763 \quad 82.856$

$26.116 \quad 78.188$

$26.634 \quad 73.697$

$8.742 \quad 78.224$

$8.808 \quad 76.316$

$25.763 \quad 85.236$

$26.116 \quad 79.444$

26.63474 .570

$8.742 \quad 79.300$

$8.808 \quad 77.226$

$26.116 \quad 80.663$

Page 69 


\begin{tabular}{|c|c|c|c|c|c|c|c|c|c|c|}
\hline 21 & $\mathrm{C}$ & -like & $1 \mathrm{~S} 2$ & $2 \mathrm{~S} 2$ & $2 \mathrm{P}$ & & $\left(3 D^{\star}\right) j=2.0$ & $-1 s 2$ & $2 s 2$ & $2 \mathrm{P} 2$ \\
\hline $\mathrm{Fe} 21$ & c & -1ike & $1 S 2$ & $2 \mathrm{~S} 2$ & $2 P$ & & $\left(3 P^{*}\right) j=2.0$ & $-1 s 2$ & $2 \mathrm{~S} 2$ & $2 \mathrm{P} 2$ \\
\hline Fe21 & C & -like & $1 S 2$ & $2 s 2$ & $2 \mathrm{P}$ & & $\left(3 p^{*}\right) j=2.0$ & $-1 s 2$ & $2 \mathrm{~S} 2$ & $2 \mathrm{P} 2$ \\
\hline Fe21 & C & -1ike & 152 & $2 S 2$ & $2 \mathrm{P}$ & & $\left(3 P^{*}\right) j=2.0$ & $-1 s 2$ & $2 S 2$ & $2 \mathrm{P} 2$ \\
\hline $\mathrm{Fe} 21$ & C & -like & $1 S 2$ & $2 \mathrm{~S} 2$ & $2 \mathrm{P}$ & & $\left(3 P^{*}\right) j=2.0$ & - IS2 & $2 \mathrm{~S} 2$ & $2 \mathrm{P} 2$ \\
\hline Fe21 & $\mathrm{C}$ & -like & $1 \mathrm{~S} 2$ & $2 S 2$ & $2 \mathrm{P}$ & & $\left(3 F^{*}\right) j=3.0$ & -152 & $2 S 2$ & $2 \mathrm{P} 2$ \\
\hline e21 & C & -1ike & $1 S 2$ & $2 S 2$ & $2 \mathrm{P}$ & & $\left(3 F^{*}\right) j=3.0$ & $-1 s 2$ & $2 \mathrm{~s} 2$ & $2 \mathrm{P} 2$ \\
\hline$e 21$ & C & -Iike & 152 & 252 & $2 \mathrm{P}$ & & $\left(3 F^{*}\right) j=3.0$ & $-1 S 2$ & $2 \mathrm{~s} 2$ & $2 \mathrm{P} 2$ \\
\hline$e 21$ & C & -like & $1 S 2$ & $2 S 2$ & $2 \mathrm{P}$ & & $\left(3 F^{\star}\right) j=3.0$ & $-1 s 2$ & $2 S 2$ & $2 \mathrm{P} 2$ \\
\hline e21 & C & -like & 1S2 & 252 & $2 \mathrm{P}$ & & *) $j=1.0$ & $-1 S 2$ & $2 \mathrm{~s} 2$ & $2 \mathrm{P} 2$ \\
\hline e21 & C & -like & 152 & $2 S 2$ & $2 \mathrm{P}$ & & $\left\langle 3 P^{*}\right\rangle j=1.0$ & $-1 S 2$ & $2 \mathrm{~s} 2$ & $2 \mathrm{P} 2$ \\
\hline e21 & c & -like & 152 & $2 s 2$ & $2 P$ & & $\left(3 P^{*}\right) j=1.0$ & $-1 S 2$ & $2 \mathrm{~S} 2$ & P2 \\
\hline Fe21 & C & -like & $1 S 2$ & $2 s 2$ & $2 \mathrm{P}$ & & $\left(3 P^{\star}\right) j=1.0$ & $-1 s 2$ & $2 \mathrm{~s} 2$ & 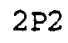 \\
\hline Ee21 & C & -Iike & $1 S 2$ & 252 & $2 P$ & & $\left(1 D^{*}\right) j=2.0$ & $-1 s 2$ & $2 s 2$ & $2 \mathrm{P} 2$ \\
\hline $\mathrm{Fe} 21$ & C & -1ike & 152 & $2 \mathrm{~S} 2$ & $2 \mathrm{P}$ & & $\left(3 D^{\star}\right) j=2.0$ & $-1 S 2$ & $2 \mathrm{~S} 2$ & P2 \\
\hline Fe21 & C & -like & $1 S 2$ & $2 s 2$ & $2 \mathrm{P}$ & & $\left(3 D^{*}\right) j=3.0$ & -152 & 252 & $2 P 2$ \\
\hline Fe21 & C & -like & $1 S 2$ & $2 S 2$ & $2 P$ & & $\left(1 D^{*}\right) j=2.0$ & -152 & 52 & 282 \\
\hline$F \in 21$ & C & -like & 152 & $2 s 2$ & $2 P$ & & $\left(3 P^{*}\right) j=2.0$ & -152 & $2 \mathrm{~s} 2$ & $P 2$ \\
\hline $\mathrm{Fe} 21$ & C & -like & $1 S 2$ & $2 s 2$ & $2 \mathrm{P}$ & & $\left(3 P^{\star}\right) j=2.0$ & $-1 S 2$ & $2 \$ 2$ & $2 \mathrm{P} 2$ \\
\hline $\mathrm{Fe} 21$ & C & -like & $2 S 2$ & $2 \mathrm{~S} 2$ & $2 \mathrm{P}$ & & $\left(3 F^{\star}\right) j=3.0$ & -152 & $2 \$ 2$ & $2 P 2$ \\
\hline Fe21 & C & -like & $1 \mathrm{~S} 2$ & $2 \mathrm{~S} 2$ & $2 P$ & & $\left(3 P^{*}\right) j=1.0$ & $-1 s 2$ & $2 s 2$ & $P 2$ \\
\hline Fe2I & c & -like & $1 S 2$ & $2 S$ & $2 \mathrm{P} 2$ & $2 \mathrm{P}$ & $(3 D) j=3.0$ & $-1 S 2$ & 25 & $2 P 3$ \\
\hline $\mathrm{Fe} 21$ & C & -like & $1 S 2$ & $2 \mathrm{~S} 2$ & $2 \mathrm{P}$ & & $\left(3 P^{\star}\right) j=2.0$ & -152 & $2 s 2$ & $2 \mathrm{P} 2$ \\
\hline Fe21 & C & -like & 1S2 & $2 \mathrm{~S} 2$ & $2 P$ & & $\left(3 P^{\star}\right) j=.0$ & $-1 S 2$ & $2 s 2$ & $2 \mathrm{P} 2$ \\
\hline Fe21 & C & -like & $1 \mathrm{~s} 2$ & $2 \mathrm{~S}$ & $2 \mathrm{P} 2$ & $4 P$ & )$(5 P) j=2.0$ & $-1 S 2$ & $2 S$ & $2 \mathrm{P3}$ \\
\hline $\mathrm{Fe} 21$ & C & -like & $1 S 2$ & $2 S$ & $2 \mathrm{P} 2$ & $(4 P$ & )$(5 P) j=2.0$ & $-1 S 2$ & S & $2 \mathrm{P} 3$ \\
\hline $\mathrm{Fe} 21$ & C & -like & $1 \mathrm{~S} 2$ & $2 S 2$ & $2 P$ & & $\left(3 P^{*}\right) j=1.0$ & $-1 S 2$ & $2 s 2$ & $P 2$ \\
\hline $\mathrm{Fe} 21$ & $c$ & -like & $1 S 2$ & $2 \mathrm{~S} 2$ & $2 \mathrm{P}$ & & $\left(3 p^{*}\right) j=1.0$ & $-1 s 2$ & 52 & 292 \\
\hline Fe21 & C & -like & IS2 & $2 S$ & $2 \mathrm{P} 2$ & $2 \mathrm{D}$ & )$(3 D) j=3.0$ & $-1 S 2$ & $2 S$ & P 3 \\
\hline Fe21 & C & -like & 152 & $2 S$ & 2P2 & $(2 D$ & )$(3 D) j=3.0$ & $-1 S 2$ & $2 S$ & 283 \\
\hline Fe21 & C & -like & $1 \mathrm{~S} 2$ & $2 S$ & $2 \mathrm{P} 2$ & $(2 D$ & )$(3 D) j=2.0$ & $-1 S 2$ & $2 S$ & $2 \mathrm{P} 3$ \\
\hline$F e 21$ & C & -like & $1 S 2$ & $2 S$ & $2 \mathrm{P} 2$ & $(2 D$ & )$(3 D) j=2.0$ & $-1 S 2$ & $2 \mathrm{~S}$ & $2 \mathrm{P} 3$ \\
\hline e21 & c & -like & $1 S 2$ & $2 S$ & 2P2 & $(2 \mathrm{D}$ & )$(3 F) j=3.0$ & $-1 S 2$ & $2 \mathrm{~s}$ & $2 \mathrm{P} 3$ \\
\hline Fe21 & C & -like & 152 & $2 S$ & $2 \mathrm{P} 2$ & $2 \mathrm{D}$ & )$(3 F) j=3.0$ & $-1 S 2$ & $2 S$ & $2 \mathrm{P3}$ \\
\hline Fe21 & C & -like & $1 \mathrm{~S} 2$ & $2 S$ & $2 \mathrm{P} 2$ & $2 \mathrm{D}$ & )$(3 D) j=3.0$ & $-1 s 2$ & $2 S$ & 2 P3 \\
\hline Fe21 & C & -1ike & 152 & $2 S$ & $2 \mathrm{P} 2$ & $2 D$ & )$(3 D) j$ & $-1 S 2$ & $2 S$ & $2 \mathrm{P3}$ \\
\hline $\mathrm{Fe} 21$ & c & -like & 152 & $2 S$ & $2 \mathrm{P} 2$ & $2 D$ & )$(3 F) j=4.0$ & -152 & $2 S$ & $2 \mathrm{P} 3$ \\
\hline Fe21 & C & -like & $1 \mathrm{~s} 2$ & $2 S$ & $2 \mathrm{P} 2$ & $2 \mathrm{D}$ & )$(3 F) j=4.0$ & $-1 \mathrm{~s} 2$ & $2 \mathrm{~S}$ & $2 \mathrm{P} 3$ \\
\hline Fe21 & $c$ & -like & $1 \mathrm{~S} 2$ & $2 S$ & $2 P 2$ & $2 P$ & )$(3 D) j=3.0$ & $-1 s 2$ & $2 \mathrm{~S}$ & $2 \mathrm{P} 3$ \\
\hline $\mathrm{Fe} 21$ & C & -1ike & $1 \mathrm{~S} 2$ & $2 S$ & $2 \mathrm{P} 2$ & $(2 \mathrm{P}$ & )$(3 D) j=3.0$ & $-1 S 2$ & $2 \mathrm{~S}$ & $2 \mathrm{P} 3$ \\
\hline Fe21 & $c$ & -like & 152 & $2 \$ 2$ & $2 \mathrm{P}$ & & $\left(1 P^{*}\right) j=1.0$ & $-1 s 2$ & $2 s 2$ & $2 \mathrm{P} 2$ \\
\hline Fe21 & C & -like & $1 \mathrm{~S} 2$ & $2 S 2$ & $2 \mathrm{P}$ & & $\left\langle 1 P^{*}\right\rangle j=1.0$ & - 1S2 & $2 \$ 2$ & $2 \mathrm{P} 2$ \\
\hline Fe2I & C & -like & 152 & $2 \mathrm{~s} 2$ & $2 P$ & & $\left(1 F^{*}\right) j=3.0$ & $-1 \mathrm{~S} 2$ & $2 \mathrm{~s} 2$ & $2 \mathrm{P} 2$ \\
\hline $\mathrm{e} 21$ & C & -like & $1 S 2$ & $2 \mathrm{~S} 2$ & $2 \mathrm{P}$ & & $\left(1 F^{*}\right) j=3.0$ & $-1 \mathrm{~s} 2$ & $2 \mathrm{~s} 2$ & $2 \mathrm{P} 2$ \\
\hline Fe21 & C & -1ike & $1 \mathrm{~S} 2$ & $2 S$ & $2 \mathrm{P} 2$ & $2 \mathrm{P}$ & $(1 F) j=3.0$ & $-1 S 2$ & $2 S$ & $2 \mathrm{P3}$ \\
\hline $\mathrm{Fe} 21$ & C & -like & $1 \mathrm{~s} 2$ & $2 S$ & $2 \mathrm{P} 2$ & $(2 P$ & )$(1 F) j=3.0$ & $-1 S 2$ & $2 S$ & $2 \mathrm{P} 3$ \\
\hline
\end{tabular}

(3P $j=2.0 \quad 8.590 \quad \mathrm{KAP} 100$ (3P) $j=1.0$ 8.610 PET 002 (3P) $j=1.0$ (3P) $j=1.0$ 8.610 EDT 020 8.610 RAP 100 (3P) $j=1.0 \quad 8.610 \quad \operatorname{KAP} 100$ (3P) $j=2.0 \quad 8.643$ PET 002 (3P) $j=2.0$ (3P) $j=2.0 \quad 8.643$ RAP 100 (3P) $j=2.0 \quad 8.643 \quad \mathrm{KAP} 100$ (1S) $j=.0 \quad 8.741 \quad$ PET 002 (1S $) j=.0 \quad 8.741$ EDT 020 (1S) $j=.0 \quad 8.741$ KAP 100 (3P) $j=1.0 \quad 9.421$ mica 002 (3P) $j=1.0 \quad 9.433$ mica 002 (3P) $j=1.0 \quad 9.451$ mica 002 (3P) $j=2.0 \quad 9.460$ mica 002 (3P) $j=2.0 \quad 9.475$ mica 002 (3P) $j=1.0 \quad 9.518$ mica 002 (3P) $j=2.0 \quad 9.559$ mica 002 (3P) $j=2.0 \quad 9.581$ mica 002 (3P) $j=.0 \quad 12.145 \quad$ TAP 100 $\left(3 D^{\star}\right) j=3.0 \quad 12.201$ TAP 100 (3P) $j=1.0 \quad 12.248 \quad$ TAP 100 (3P) $j=1.0 \quad 12.264$ TAP 100 $\left(5 S^{\star}\right) j=2.0 \quad 12.291$ TAP 100 $\left(5 S^{*}\right) j=2.0 \quad 12.291$ RAP 100 (3P) $j=2.0 \quad 12.322$ TAP 100 (3P) $j=2.0 \quad 12.322$ RAP 100 $\left(3 D^{\star}\right) j=2.0 \quad 12.346 \quad$ TAP 100 $\left(3 D^{*}\right) j=2.0 \quad 12.346 \quad \operatorname{RAP} 100$ $(3 D *) j=2.0 \quad 12.355$ TAP 100 $\left(3 D^{*}\right) j=2.0 \quad 12.355 \quad \operatorname{RAP} 100$ $\left(3 D^{*}\right) j=2.0 \quad 12.371$ TAP 100 $\left(3 D^{*}\right) j=2.0 \quad 12.371$ RAP 100

$\left(3 D^{*}\right) j=3$

$\left(3 D^{*}\right) j=3.0 \quad 12.387 \quad \operatorname{RAP} 100$ $\left(3 D^{*}\right) j=3.0 \quad 12.398 \quad$ TAP 100 $\left(3 D^{*}\right) j=3.0 \quad 12.398$ RAP 100 $(3 P *) j=2.0 \quad 12.411$ TAP 100 $\left(3 P^{\star}\right) j=2.0 \quad 12.411$ RAP 100 (1D) $j=2.0 \quad 12.429 \quad$ TAP 100 (1D ) $j=2.0 \quad 12.429 \quad$ RAP 100 (1D) $j=2.0 \quad 12.436 \quad$ TAP 100 (1D) $j=2.0 \quad 12.436 \quad$ RAP 100 (1D*) $j=2.0 \quad 12.451$ TAP 100 $\left(1 D^{*}\right) j=2.0 \quad 12.451$ RAP 100
$26.634 \quad 75.366$

$8.742 \quad 80.031$

$8.808 \quad 77.828$

$26.116 \quad 81.513$

$26.634 \quad 75.886$

8.74281 .369

$8.808 \quad 78.892$

$26.116 \quad 83.139$

26.63476 .788

$8.742 \quad 89.134$

$8.808 \quad 82.929$

$26.634 \quad 79.921$

$19.942 \quad 70.881$

$19.942 \quad 71.093$

$19.942 \quad 71.414$

$19.942 \quad 71.577$

$19.942 \quad 71.852$

$19.942 \quad 72.663$

$19.942 \quad 73.472$

$19.942 \quad 73.922$

$25.763 \quad 70.532$

$25.763 \quad 71.293$

$25.763 \quad 71.956$

$25.763 \quad 72.188$

$25.763 \quad 72.584$

$26.116 \quad 70.265$

$25.763 \quad 73.051$

$26.116 \quad 70.671$

$25.763 \quad 73.421$

$26.116 \quad 70.992$

$25.763 \quad 73.562$

$26.116 \quad 71.114$

$25.763 \quad 73.816$

$26.116 \quad 71.332$

$25.763 \quad 74.073$

$26.116 \quad 71.552$

$25.763 \quad 74.252$

$26.116 \quad 71.706$

$25.763 \quad 74.467$

$26.116 \quad 71.888$

$25.763 \quad 74.768$

$26.116 \quad 72.144$

$25.763 \quad 74.887$

$26.116 \quad 72.244$

$25.763 \quad 75.146$

$26.116 \quad 72.462$ 
Fe21 C-like 1S2 2S 2P2 ( $2 P)(3 F) j=3.0-1 S 2 \quad 2 S 2 P 3$ Fe21 $C$-like 1S2 2S 2P2 ( $2 P$ ) $(3 F) j=3.0-1 S 2$ 2S $2 P 3$ Fe21 C -like 1S2 2S2 $2 P \quad\left(3 F^{*}\right) j=2.0-1 S 2$ 2S2 $2 P 2$ Fe21 C-like 1S2 2S2 $2 P \quad\left(3 F^{*}\right) j=2.0-1 S 2$ 2S2 2P2 Fe21 C -like 1S2 2S2 2P (3F*) j=2.0 - 1S2 2S2 2P2 Fe21 $C$-like 1S2 2S 2P2 ( 2D ) (1F) $j=3.0$ - IS2 2S 2P3 Fe21 $C$-like $1 S 2$ 2S $2 P 2(2 D)(1 F) j=3.0-1 S 22 S$ 2P3 Fe21 C -like 1S2 2S 2P2 ( $2 D)(1 F) j=3.0-1 S 2$ 2S $2 P 3$ Fe21 $C$-like 1S2 2S 2P2 ( $2 P$ ) (1D) $j=2.0-1 S 22 S$ 2P3 Fe21 C-like 1S2 2S. 2P2 ( $2 P$ )(ID $) j=2.0-1 S 2$ 2S $2 P 3$ Fe21 $C$-like 1S2 $2 \mathrm{~S} \cdot 2 \mathrm{P} 2(2 \mathrm{P})(1 \mathrm{D}) \mathrm{j}=2.0-1 \mathrm{~S} 2$ 2S $2 \mathrm{P} 3$ Fe21 $C$-like $1 S 2$ 2S $2 P 2(4 P)(3 D) j=3.0-1 S 22 S 2 P 3$ Fe21 $C$-like 1S2 2S 2P2 ( $4 P$ ) (3D) $j=3.0-1 S 2$ 2S $2 P 3$ Fe21 C -like IS2 2S 2P2 ( $4 \mathrm{P})(3 \mathrm{D}) \mathrm{j}=3.0-1 \mathrm{~S} 2$ 2S $2 \mathrm{P} 3$ Fe21 $C$-like 1S2 2S 2P2 ( $2 D)(3 D) j=2.0-1 S 2$ 2S 2P3 Fe21 $C$-like 1S2 2S 2P2 (2D) (3D) $j=2.0-1 S 2 \quad 2 S$ 2P3 Fe21 C-like 1S2 2S 2P2 ( 2D ) (3D) $j=2.0-1 S 22 S$ 2P3 Fe21 $C$-like 1S2 2S 2P2 ( 2D ) (3D) $j=3.0-1 S 22 S$ 2P3 Fe21 $C$-like IS2 $2 S$ 2P2 ( $2 D)(3 D) j=3.0-1 S 2$ 2S $2 P 3$ Fe21 $C$-like 1S2 2S $2 P 2(2 D)(3 D) j=3.0-1 S 22 S$ 2P3 Fe21 C-like 1S2 2S 2P2 ( $4 P$ ) (3D) $j=3.0-1 S 2$ 2S 2P3 Fe21 $C$-like $1 S 2$ 2S $2 P 2(4 P)(3 D) j=3.0-1 S 22 S \quad 2 P 3$ Fe21 $C$-like 1S2 2S 2P2 ( $4 \mathrm{P})(3 \mathrm{D}) j=3.0-1 \mathrm{~S} 2$ 2S $2 \mathrm{P} 3$ Fe21 $C$-like 1S2 $2 S$ 2P2 ( $4 P$ ) (3F) $j=4.0-1 S 2$ 2S $2 P 3$ Fe21 $C$-1ike 1S2 2S 2P2 ( $4 P)(3 F) j=4.0-1 S 2$ 2S 2P3 Fe21 $C$-like 1S2 2S 2P2 ( $4 P$ ) (3F) $j=4.0-1 S 22 S$ 2P3 Fe21 $C$-like IS2 2S 2P2 ( $4 P$ ) (3F) j=3.0 - 1S2 2S $2 \mathrm{P} 3$ Fe21 $C$-like 1S2 2S 2P2 ( $4 \mathrm{P})(3 F) j=3.0-1 S 2$ 2S $2 \mathrm{P} 3$ Fe21 $C$-like $1 S 2$ 2S $2 P 2(4 P)(3 F) j=3.0-1 S 22 S$ 2P3 Fe21 C-like 1S2 2S2 $2 P \quad\left(1 D^{*}\right) j=2.0-1 S 2$ 2S2 $2 P 2$ Fe21 C-like 1S2 2S2 $2 P \quad\left(1 D^{*}\right) j=2.0-1 S 2$ 2S2 $2 P 2$ Fe21 $C$-like 1S2 2S2 2P $\quad\left(1 D^{*}\right) j=2.0-1 S 2$ 2S2 2P2 Fe21 C-like 1S2 2S2 2P (3P*)j=1.0 - 1S2 2S2 2P2 Fe21 C-like 1S2 2S2 $2 P \quad\left(3 P^{*}\right) j=1.0$ - 1S2 2S2 $2 P 2$ Fe21 C -like 1S2 2S2 2P (3P*)j=1.0 - 1S2 2S2 2P2 Fe21 $C$-like 1S2 2S 2P2 ( $4 P$ ) (3F) $j=2.0-1 S 2$ 2S $2 P 3$ Fe21 C-like 1S2 2S $2 R 2$ ( $4 P$ ) $(3 F) j=2.0-1 S 2 \quad 2 S$ 2P3 Fe21 C-like 1S2 2S 2P2 ( $4 \mathrm{P})(3 F) j=2.0-1 S 2$ 2S $2 \mathrm{P} 3$ Fe21 $C$-like 1S2 2S 2P2 ( $2 \mathrm{P})(3 \mathrm{~F}) j=3.0-1 S 22 \mathrm{~S} 2 \mathrm{P} 3$ Fe21 $C$-like 1S2 2S 2P2 ( $2 P$ ) (3F ) $j=3.0-1 S 2$ 2S $2 P 3$ Fe21 C-like 1S2 2S 2P2 ( $2 P$ ) (3F) $j=3.0-1 S 22 S$ 2P3 Fe21 $C$-like 1S2 2S 2P2 ( 2D )(1D) $j=2.0-1 S 2 \quad 2 S$ 2P3 Fe21 $C$-like $1 S 2$ 2S 2P2 ( $2 D)(1 D) j=2.0-1 S 22 S$ 2P3 Fe21 $C$-like 1S2 2S 2P2 (2D) (1D) $j=2.0-1 S 22 S$ 2P3 Fe21 $C$-like 1S2 2S 2P2 ( $4 \mathrm{P})(5 \mathrm{P}) \mathrm{j}=2.0-1 S 22 \mathrm{~S} 2 \mathrm{P} 3$ Fe21 C -like 1S2 2S 2P2 ( $4 \mathrm{P})(5 \mathrm{P}) j=2.0-1 S 22 S 2 \mathrm{P} 3$ $\left(3 P^{\star}\right) j=2.0 \quad 12.463 \quad$ TAP 100 $(3 P *) j=2.0$ (3P) $j=1.0 \quad 12.519 \quad$ TAP 100 (3P) $j=1.0 \quad 12.519 \quad \operatorname{RAP} 100$ (3P) $j=1.0 \quad 12.519 \quad \mathrm{KAP} 100$ $\left(3 P^{*}\right) j=2.0 \quad 12.548$ TAP 100 $\left(3 P^{*}\right) j=2.0 \quad 12.548 \quad \operatorname{RAP} 100$ $\left(3 P^{*}\right) j=2.0 \quad 12.548 \quad \mathrm{KAP} 100$ $\left(1 P^{*}\right) j=1.0 \quad 12.575$ TAP 100 $\left(12^{\star}\right) j=1.0 \quad 12.575$ RAP 100 $\left(1 P^{*}\right) j=1.0 \quad 12.575 \quad \mathrm{KAP} 100$ $\left(3 D^{*}\right) j=2.0 \quad 12.581 \quad \operatorname{TAP} 100$ $\left(3 D^{*}\right) j=2.0 \quad 12.581 \quad \operatorname{RAP} 100$ $\left(3 D^{*}\right) j=2.0 \quad 12.581 \quad$ KAP 100 $\left(3 P^{*}\right) j=1.0 \quad 12.586$ TAP 100 $\left(3 P^{\star}\right) j=1.0 \quad 12.586$ RAP 100 $\left(3 P^{*}\right) j=1.0 \quad 12.586 \quad \mathrm{KAP} 100$ $\left(3 P^{*}\right) j=2.0 \quad 12.606$ TAP 100 $\left(3 P^{*}\right) j=2.0 \quad 12.606$ RAP 100 $\left(3 P^{\star}\right) j=2.0 \quad 12.606 \quad$ KAP 100 $\left(3 D^{*}\right) j=3.0 \quad 12.623$ TAP 100 $\left(3 D^{*}\right) j=3.0 \quad 12.623$ RAP 100 (3D*) $j=3.0 \quad 12.623 \quad \mathrm{KAP} 100$ $\left(3 D^{*}\right) j=3.0 \quad 12.681$ TAP 100 $\left(3 D^{*}\right) j=3.0 \quad 12.681 \quad \operatorname{RAP} 100$ $\left(3 D^{*}\right) j=3.0 \quad 12.681$ KAP 100 $\left(3 D^{*}\right) j=2.0 \quad 12.699 \quad$ TAP 100 $\left(3 D^{*}\right) j=2.0 \quad 12.699 \quad \operatorname{RAP} 100$ (3D*) $j=2.0 \quad 12.699$ KAP 100 (1D) $j=2.0 \quad 12.714 \quad$ TAP 100 (ID) $j=2.0 \quad 12.714$ RAP 100 (1D) $j=2.0 \quad 12.714 \quad \operatorname{KAP} 100$ (1S ) $j=.0 \quad 12.726$ TAP 100 (1S) $j=.0 \quad 12.726$ RAP 100 (1S ) $j=.0 \quad 12.726$ KAP 100 $\left(3 D^{*}\right) j=1.0 \quad 12.743$ TAP 100 (3D*) $j=1.0 \quad 12.743 \quad$ RAP 100 $\left(3 D^{*}\right) j=1.0 \quad 12.743 \quad \mathrm{KAP} 100$ $\left(1 D^{*}\right) j=2.0 \quad 12.756 \quad$ TAP 100 $\left(1 D^{*}\right) j=2.0 \quad 12.756 \quad$ RAP 100 $\left\{1 D^{*}\right\} j=2.0 \quad 12.756 \quad \mathrm{KAP} 100$ $\left(1 D^{*}\right) j=2.0 \quad 12.777 \quad$ TAP 100 (1D*) $j=2.0 \quad 12.777 \quad$ RAP 100 (1D*) $j=2.0 \quad 12.777 \quad \mathrm{KAP} 100$ $\left(3 D^{*}\right) j=3.0 \quad 12.789 \quad$ TAP 100 $\left(3 D^{*}\right) j=3.0 \quad 12.789 \quad \operatorname{RAP} 100$
$25.763 \quad 75.355$ $\begin{array}{ll}26.116 & 72.637\end{array}$ $25.763 \quad 76.375$ $26.116 \quad 73.480$ $26.634 \quad 70.064$ $25.763 \quad 76.934$ $26.116 \quad 73.934$ $26.634 \quad 70.434$ $25.763 \quad 77.476$ $26.116 \quad 74.368$ $26.634 \quad 70.784$ $25.763 \quad 77.600$ $26.116 \quad 74.466$ $26.634 \quad 70.862$ $25.763 \quad 77.704$

$26.116 \quad 74.548$ $26.634 \quad 70.928$ 25.76378 .129

$26.116 \quad 74.881$

$26.634 \quad 71.193$ $25.763 \quad 78.502$ $26.116 \quad 75.169$ $26.634 \quad 71.421$ $25.763 \quad 79.878$ $26.116 \quad 76.199$ $26.634 \quad 72.221$ $25.763 \quad 80.344$ $26.116 \quad 76.534$ $26.634 \quad 72.476$ $25.763 \quad 80.750$ $26.116 \quad 76.819$ $26.634 \quad 72.692$ $25.763 \quad 81.088$ $26.116 \quad 77.052$ $26.634 \quad 72.866$ $25.763 \quad 81.591$ $26.116 \quad 77.390$ $26.634 \quad 73.116$ $25.763 \quad 81.996$ $26.116 \quad 77.654$ $26.634 \quad 73.310$ $25.763 \quad 82.697$ $26.116 \quad 78.092$ $26.634 \quad 73.628$ $25.763 \quad 83.130$ $26.116 \quad 78.350$ 
Fe21 C-like 1S2 2S 2P2 ( $4 \mathrm{P})(5 \mathrm{P}) \mathrm{J}=2.0-1 \mathrm{~S} 2$ 2S $2 \mathrm{P} 3$ Fe21 C-like 1S2 2S 2P2

Fe21 C -like 1S2 2S 2P2

Fe22 B -like 1S 2S2 2P2

Fe22 B -like 1S 2S2 2P2

Fe22 B -like 1S 2S2 2P2

$\mathrm{Fe} 22$ B -like 1S 2S2 2P2

Fe22 B -like 1S 2S2 2P2

Fe22 B-Iike 1S 2S2 2P2

Fe22 B -like 1S 2S2 2P2

Fe22 B-like is 2S2 2P2

Fe22 B -like 1S 2S2 2P2

Fe22 B -like 1S 2S2 2F2

Fe22 B -like is 2S2 2P2

$\mathrm{Fe} 22$ B-like 1s 2S2 $2 \mathrm{P} 2$

Fe22 B -like 1S 2S2 2P2

Fe22 B -like 1S 2S2 2P2

Fe22 B-like 1S 2S2 2P2

Fe22 B-like 1S 2S2 $2 \mathrm{P} 2$

Fe22 B -like 1S 2S2 2P2

Fe22 B-like is 2S2 2P2

Fe22 B-like 1S 2S2 $2 \mathrm{P} 2$

Fe22 B-like 1S 2S2 2P2

Fe22 B -like 1S 2S2 2P2

Fe22 B -like 1S 2S2 2P2

Fe22 B -like 1S 2S2 2P2

Fe22 B -like 1S2 2S 2P ( $\left.1 P^{*}\right) 4\left(2 D^{*}\right) j=2.5-1 S 2$ 2S $2 P^{*}$

Fe22 B -like 1S2 2S $2 P\left(1 P^{*}\right) 4\left(2 D^{*}\right) j=2.5-1 S 2$ 2S $2 P_{2}$

Fe22 B -like IS2 $2 S 2 P\left(1 P^{*}\right) 4\left(2 D^{*}\right) j=2.5-1 S 22 S$ 2P2

Fe22 B -like 1S2 2S 2P ( $\left.1 P^{*}\right) 4\left(2 F^{*}\right) j=2.5-1 S 22 S$ 2P2

Fe22 B -like 1S2 2S 2P(1 $\left.P^{*}\right) 4\left(2 F^{*}\right) j=2.5-1 S 2$ 2S 2P2

Fe22 B - like 1S2 $2 S 2 P\left(3 P^{*}\right) 4\left(4 D^{*}\right) j=2.5-1 S 2$ 2S $2 \mathrm{P} 2$

Fe22 B-like 1S2 2S2 4P (2P*)j=1.5 - 1S2 2S 2P2

Fe22 B -like 1S2 2S2 4P $\quad\left(2 P^{*}\right) j=.5-152$ 2S $2 P^{*}$

Fe22 B -like 1S2 2S2 $4 P \quad\left(2 P^{\star}\right) j=.5-1 S 2$ 2S $2 P 2$

Fe22 B -1ike 1S2 2S2 4P (2P*) $j=1.5-1 S 2$ 2S $2 \mathrm{P} 2$

Fe22 B-like 1S2 2S 2P ( $\left.3 P^{*}\right) 3\left(2 P^{*}\right) j=1.5-1 S 2$ 2S 2P2

Fe22 B -like 1S2 2S $2 \mathrm{P}\left(1 \mathrm{P}^{*}\right) 3\left(2 \mathrm{P}^{*}\right) j=.5-1 S 2$ 2S $2 \mathrm{P}^{*}$

Fe22 B-like $1 S 2$ 2S $2 P\left(1 P^{*}\right) 3\left(2 D^{*}\right) j=1.5-1 S 2$ 2S $2 P^{*}$

Fe22 B -like 1S2 $2 S 2 P\left(3 P^{*}\right) 3\left(2 P^{*}\right) j=.5-1 S 22 S$ 2P2

Fe22 B-1ike 1S2 2S $2 P\left(3 P^{*}\right) 3\left(2 P^{*}\right) j=.5-1 S 22 S$ 2P2

Fe22 B -like 1S2 2S 2P $\left(3 P^{*}\right) 3\left(2 P^{*}\right) j=1.5-1 S 2$ 2S 2P2

Fe22 B -like 1S2 2S 2P( $\left.3 P^{\star}\right) 3\left(2 P^{\star}\right) j=1.5-1 S 2$ 2S $2 P^{*}$

Fe22 B-like 1S2 2S 2P ( $\left.3 P^{*}\right) 3\left(4 D^{*}\right) j=1.5-1 S 2$ 2S 2P2

Fe22 B -like 1S2 2S $2 \mathrm{P}\left(3 \mathrm{P}^{*}\right) 3\left(4 \mathrm{D}^{*}\right) \mathrm{j}=1.5-1 \mathrm{~S} 2$ 2S 2P2

Fe22 B-like 1S2 $2 S 2 P\left(3 P^{*}\right) 3\left(4 D^{*}\right) j=1.5-1 S 22 S 2 P 2$ $\left(3 D^{*}\right) j=3.0 \quad 12.789 \quad \operatorname{KAP} 100$

(3P) $j=2.0 \quad 13.070$ KAP 100

(3P) $j=2.0 \quad 13.146 \quad K A P 100$

$\left(2 P^{*}\right) j=.5 \quad 1.875$ Si 220

$\left(2 P^{*}\right) j=.5 \quad 1.875$ fluorite 220

$\left(2 P^{\star}\right) j=5$. 1.875 NaCl 200

$\left(2 P^{\star}\right) j=.5 \quad 1.877$ Si 220

$\left(2 P^{\star}\right) j=.5 \quad 1.877$ fluorite 220

$\left(2 P^{\star}\right) j=.5$

$\left(2 P^{*}\right) j=1.5$

$\left(2 P^{*}\right) j=1.5$

$\left(2 P^{*}\right) j=1.5$

$\left(2 P^{\star}\right) j=1.5$

$\left(2 P^{\star}\right) j=1.5$

$\left(2 P^{*}\right) j=1.5$

$\left(2 P^{*}\right) j=1.5$

$\left(2 P^{\star}\right) j=1.5$

$\left(2 P^{\star}\right) j=1.5$

$\left(2 P^{\star}\right) j=1.5$

$\left(2 P^{\star}\right) j=1.5$

$\left(2 P^{\star}\right) j=1.5$

$\left(2 P^{*}\right) j=1.5$

$\left(2 P^{\star}\right) j=1.5$

$\left(2 P^{*}\right) j=$

$\left(2 P^{\star}\right) j=1.5$

$\left(2 P^{*}\right) j=1.5$

$(4 P) j=1.5$

( $4 P) j=1.5$

$(4 P) j=1.5$

$(4 P) j=2.5$

$(4 P) j=2.5$

(2P) $j=1.5$

(2D) $j=1.5$

(2D) $j=1.5$

(2s) $j=.5$

(2P) $j=.5$

1.879 Si 220

1.879 fluorite 220

1.879 NaCl 200

1.882 si 220

1.882 fluorite 220

1.882 Ge 220

1.885 Si 220

1.885 fluorite 220

1.885 Ge 220

1.886 Si 220

1.886 fluorite 220

1.886 Ge 220

1.936 quartz 223

1.936 Ge 220

1.936 LiF 200

1.936 Al 200

1.936 calcite 200

8.736 PET 002

8.736 EDT 020

8.736 KAP 100

8.786 EDT 020

8.786 KAP 100

9.412 mica 002

9.663 mica 002

9.675 mica 002

9.785 mica 002

9.894 mica 002

$\begin{array}{lll}5 & 12.193 \text { TAP } 100\end{array}$

(2P ) $j=1.5 \quad 12.231$ TAP 100

(2P) $j=.5 \quad 12.259$ TAP 100

(2S ) $j=.5 \quad 12.322$ TAP 100

(2S ) $j=.5 \quad 12.322 \quad$ RAP 100

(2P ) $j=.5 \quad 12.380$ TAP 100

(2P ) $j=.5 \quad 12.380$ RAP 100

(2P $) j=.5 \quad 12.653$ TAP 100

(2P $) j=\begin{array}{lll}5 & 12.653 \\ \text { RAP } 100\end{array}$

(2P) $j=.5 \quad 12.653$ KAP 100
$26.634 \quad 73.812$

26.63478 .948

$26.634 \quad 80.808$

$3.840 \quad 77.571$

3.86276 .168

$5.641 \quad 85.684$

$3.840 \quad 77.851$

3.86276 .418

5.64186 .588

$3.840 \quad 78.138$

$3.862 \quad 76.673$

$5.641 \quad 87.842$

$3.840 \quad 78.582$

$3.862 \quad 77.065$

$4.000 \quad 70.220$

$3.840 \quad 79.043$

$3.862 \quad 77.469$

$4.000 \quad 70.476$

$3.840 \quad 79.201$

$3.862 \quad 77.606$

$4.000 \quad 70.562$

$2.024 \quad 73.043$

$4.000 \quad 75.466$

$4.027 \quad 74.052$

$4.048 \quad 73.043$

$6.071 \quad 73.074$

$8.742 \quad 87.877$

$8.808 \quad 82.669$

$26.634 \quad 79.739$

$8.808 \quad 85.950$

$26.634 \quad 81.744$

$19.942 \quad 70.724$

$19.942 \quad 75.722$

$19.942 \quad 76.004$

$19.942 \quad 78.916$

$19.942 \quad 82.875$

$25.763 \quad 71.183$

$25.763 \quad 71.714$

$25.763 \quad 72.115$

$25.763 \quad 73.051$

$26.116 \quad 70.671$

$25.763 \quad 73.960$

$26.116 \quad 71.456$

$25.763 \quad 79.192$

$26.116 \quad 75.693$

$26.634 \quad 71.831$ 
Fe22 B -1ike 1S2 2S2 3P

Fe22 B -like 1S2 2S2 3S

Fe23 Be-like 1s 252 2P

Fe23 Be-like 1s 2s2 2P

Fe23 Be-like 1S 2S2 2P

Fe23 Be-like is $2522 \mathrm{P}$

Fe23 Be-like 1s2 2P

Fe23 Be-like 1S2 2P

Fe23 Be-like 1S2 2S 5P

Fe23 Be-like 1S2 2S 5P

$\mathrm{Fe} 23$ Be-like 152 2S

Fe23 Be-like 152 2S

Fe23 Be-like 1S2 2P

Fe23 Be-like 1S2 2P

Fe23 Be-like 1S2 2P

Fe23 Be-like 1S2 2P

Fe23 Be-like 1S2 2P

Fe23 Be-like 1S2 2S

Fe23 Be-like 1S2 2S

Fe23 Be-like 1S2 2P

Fe23 Be-like 1S2 2P

$\mathrm{Fe} 23$ Be-like 1S2 2S 5P

Fe23 Be-like 1S2 2S 5P

Fe23 Be-like 1S2 2S 5P

Fe23 Be-like 1S2 2S 5P

Fe23 Be-like 1S2 2S 5P

Fe23 Be-like 1S2 2S 5P

Fe23 Be-like 1S2 2S 5P

Fe23 Be-like IS2 2S 5P

Fe23 Be-like 1S2 2S 5P

Fe23 Be-like 1S2 2S 5P

$\mathrm{Fe} 23$ Be-like 1S2 2P

Fe23 Be-like 1s2 2P

Fe23 Be-like 1S2 2P

$\mathrm{Fe} 23$ Be-like 1S2 2P

Fe23 Be-like IS2 2P

Fe23 Be-like 1S2 2P

Fe23 Be-like IS2 2P

Fe23 Be-like 1S2 2P

Fe23 Be-like 1S2 2P

Fe23 Be-like 1S2 2P

Fe23 Be-likse 1S2 2P

Fe23 Be-like IS2 2S

$\mathrm{Fe} 23$ Be-1ike 1S2 2S

Fe23 Be-like IS2 2S

Fe23 Be-like 152 2S $\left(2 \mathrm{P}^{*}\right) \mathrm{j}=1.5-1 \mathrm{~S} 22 \mathrm{~S} 2 \mathrm{P} 2$

(2S $) j=.5-1 S 22 \mathrm{P} 3$

$\left(1 P^{\star}\right) j=1.0-1 S 2252$

$\left(1 P^{*}\right) j=1.0-1 S 2252$

$\left(1 P^{*}\right) j=1.0-152252$

$\left(1 P^{\star}\right) j=1.0-1 S 2252$

$5 P(3 D) j=3.0-1 S 22 S 2 P$

$5 P(3 D) j=3.0-1 S 2 \quad 2 S \quad 2 P$

(1P*) $j=1.0-1 S 22 S 2$

$\left(1 P^{\star}\right) j=1.0-1 S 2252$

5D (3D) $j=2.0-1 S 22 S 2 P$

$5 D(3 D) j=3.0-1 S 2$ 2S $2 P$

$5 D\left(1 D^{\star}\right) j=2.0-1 S 2 \quad 2 P 2$

$5 D\left(3 D^{*}\right) j=3.0-1 S 2 \quad 2 P 2$

5D $\left(3 D^{*}\right) j=2.0-1 S 2 \quad 2 P 2$

5D $\left(1 F^{*}\right) j=3.0-1 S 2 \quad 2 \mathrm{P} 2$

5D $\left(3 D^{\star}\right) j=2.0-1 S 2 \quad 2 P 2$

$5 D(1 D) j=2.0-1 S 22 S 2 P$

5D (1D) $j=2.0-1 S 2$ 2S 2P

$5 D\left(3 D^{\star}\right) j=2.0-1 S 2 \quad 2 P 2$

$5 D\left(3 D^{\star}\right) j=2.0-1 S 2$

$\left(3 P^{*}\right) j=1.0-1 S 2$

$\left(3 P^{*}\right) j=1.0-1 S 2$

$\left(3 P^{*}\right) j=1.0-1 \mathrm{~S} 2$

$\left(3 P^{*}\right) j=1.0-1 S 2$

$\left(3 P^{*}\right) j=1.0-1 S 2$

$\left(3 P^{*}\right) j=1.0-152$

$\left(1 P^{*}\right) j=1.0-152$

$\left(1 P^{*}\right) j=1.0-1 S 2$

$\left(1 P^{\star}\right) . j=1.0-1 S 2$

$\left(1 P^{\star}\right) j=1.0-1 S 2$

$4 P(3 D) j=3.0-1 S 2$ 2S $2 P$

$4 P(3 D) j=3.0-1 S 22 S \quad 2 P$

$4 P(3 D) j=3.0-1 S 2$ 2S $2 P$

$4 P(3 D) j=3.0-1 S 22 S \quad 2 P$

$4 P(3 D) j=2.0-1 S 22 S \quad 2 P$

$4 P(3 D) j=2.0-1 S 2$ 2S $2 P$

$4 P$ (3D) $j=2.0-1 S 22 S 2 P$

$4 P(3 D) j=2.0-1 S 22 S \quad 2 P$

$4 P(3 D) j=2.0-1 S 22 S \quad 2 P$

$4 P(3 D) j=2.0-1 S 2$ 2S $2 P$

$\left(1 P^{*}\right) j=1.0-1 \mathrm{~S} 2 \quad 2 \mathrm{~S} 2$

$\left(1 P^{*}\right) j=1.0-1 \mathrm{~S} 22 \mathrm{~S} 2$

$\left(1 P^{*}\right) j=1.0-1 \mathrm{~S} 22 \mathrm{~S} 2$

$\left(1 P^{*}\right) j=1.0-1 S 22 S 2$
(2D) $j=1.5 \quad 13.095 \quad \operatorname{KAP} 100$

$\left(2 P^{*}\right) j=1.5 \quad 14.859$ gypsum 020

(1S $) j=.0 \quad 1.870$ Si 220

(1S ) $j=.0 \quad 1.870$ fluorite 220

(1s $) j=.0 \quad 1.870 \quad \mathrm{NaCl} 200$

(1s) $j=.0 \quad 1.870$ Insb 111

$\left(3 P^{*}\right) j=2.0 \quad 7.445$ InSb 111

$\left(3 P^{*}\right) j=2.0 \quad 7.445$ gypsum 020

(1S) $j=.0 \quad 7.472$ InSb 111

(1s) $j=.0 \quad 7.472$ gypsum 020

$\left(3 P^{*}\right) j=1.0$

$\left(3 P^{\star}\right) j=2.0$

(3P) $j=1.0$

(3P) $j=2.0$

(3P) $j=1.0$

(1D) $j=2.0$

(3P) $j=2.0$

$\left(1 P^{\star}\right) j=1.0$

$\left(1 P^{*}\right) j=1.0$

(ID) $j=2.0$

(ID) $j=2.0$

(3P) $j=1.0$

(3P) $j=1.0$

(3P) $j=1.0$

(3P) $j=2.0$

(3P) $j=2.0$

(3P) $j=2.0$

(ID) $j=2.0$

(ID) $j=2.0$

(ID) $j=2.0$

(1D) $j=2$.

(3P*) $j=2.0$

$\left(3 P^{*}\right) j=2.0$

$\left(3 P^{*}\right) j=2.0$

$\left(3 P^{*}\right) j=2.0$

$\left(3 P^{*}\right) j=2.0$

$\left(3 P^{*}\right) j=1.0$

$\left(3 P^{*}\right) j=1.0$

$\left(3 P^{*}\right) j=1.0$

$\left(3 P^{*}\right) j=1.0$

$\left(3 P^{*}\right) j=1.0$

$(3 P \star) j=1.0$

(1s) $j=.0$

(Is) $j=.0$

(1S) $j=.0$

(1s) $j=.0$
7.680 beryl 100

7.733 beryl 100

7.755 beryl 100

7.778 beryl 100

.826 beryl 100

7.849 beryl 100

7.854 beryl 100

7.883 topaz 002

7.883 beryl 100

7.935 topaz 002

7.935 beryl 100

8.159 topaz 002

8.159 quartz 100

8.159 TAP 100

8.180 topaz 002

8.180 guartz 100

8.180 TAP 100

.210 topaz 002

8.210 quartz 100

8.210 TAP 100

8.210 RAP 100

8.273 topaz 002

8.273 guartz 100

8.273 PET 002

8.273 TAP 100

8.273 RAP 100

8.289 topaz 002

8.289 quartz 100

8.289 PET 002

8.289 EDT 020

8.289 TAP 100

289 RAP 100

8.303 topaz 002

8.303 quartz 100

8.303 PET 002

8.303 EDT 020
$26.634 \quad 79.524$

15.18578 .106

$3.840 \quad 76.896$

3.86275 .560

$5.641 \quad 83.990$

$7.481 \quad 89.063$

$7.481 \quad 84.377$

$15.185 \quad 78.688$

$7.481 \quad 87.189$

$15.185 \quad 79.778$

$15.954 \quad 74.316$

$15.954 \quad 75.792$

$15.954 \quad 76.451$

$15.954 \quad 77.175$

$15.954 \quad 78.834$

$15.954 \quad 79.722$

$15.954 \quad 79.925$

$8.374 \quad 70.282$

$15.954 \quad 81.195$

8.37471 .365

$15.954 \quad 84.118$

8.37476 .989

$8.512 \quad 73.441$

$25.763 \quad 71.820$

$8.374 \quad 77.643$

$8.512 \quad 73.945$

$25.763 \quad 72.275$

8.37478 .642

$8.512 \quad 74.692$

$25.763 \quad 72.945$

$26.116 \quad 70.579$

$8.374 \quad 81.092$

8.51276 .390

$8.742 \quad 71.147$

$25.763 \quad 74.442$

$26.116 \quad 71.867$

$8.374 \quad 81.829$

8.51276 .856

$8.742 \quad 71.474$

$8.808 \quad 70.233$

$25.763 \quad 74.845$

$26.116 \quad 72.209$

$8.374 \quad 82.534$

$8.512 \quad 77.277$

8.74271 .765

$8.808-70.504$ 


\begin{tabular}{|c|c|c|c|}
\hline $\mathrm{Fe} 23$ & Be-like & 152 & $2 S$ \\
\hline $\mathrm{Fe} 23$ & Be-like & $1 S 2$ & $2 S^{\circ}$ \\
\hline e23 & Be-like & IS2 & $2 S$ \\
\hline Pe23 & Be-like & 152 & $2 S$ \\
\hline $\mathrm{Fe} 23$ & Be-like & $1 S 2$ & $2 \mathrm{~S}$ \\
\hline Fe23 & Be-like & $1 S 2$ & $2 S$ \\
\hline Fe23 & Be-like & 152 & $2 S$ \\
\hline $\mathrm{Fe} 23$ & Be-like & $1 \mathrm{~S} 2$ & $2 S$ \\
\hline Fe23 & Be-like & 152 & $2 S$ \\
\hline $\mathrm{Fe} 23$ & Be-1ike & 152 & $2 S$ \\
\hline Fe23 & Be-like & $1 S 2$ & $2 s$ \\
\hline$F \in 23$ & Be-like & $1 \mathrm{~S} 2$ & $2 S$ \\
\hline Fe23 & Be-like & 152 & $2 S$ \\
\hline Fe23 & Be-1ike & $1 \mathrm{S2}$ & $2 S$ \\
\hline Fe23 & Be-like & IS2 & $2 S$ \\
\hline $\mathrm{Fe} 23$ & Be-like & 152 & 25 \\
\hline $\mathrm{Fe} 23$ & Be-like & 152 & $2 S$ \\
\hline$F \in 23$ & Be-like & 152 & 25 \\
\hline $\mathrm{Fe} 23$ & Be-like & 152 & $2 \mathrm{P}$ \\
\hline Fe23 & Be-like & $1 \mathrm{~S} 2$ & $2 P$ \\
\hline Fe23 & Be-like & IS2 & $2 P$ \\
\hline Fe23 & Be-like & 152 & $2 \mathrm{P}$ \\
\hline$F e 23$ & Be-like & $1 S 2$ & 29 \\
\hline Fe23 & Be-like & $1 \mathrm{~S} 2$ & $2 S$ \\
\hline Fe23 & Be-like & IS2 & $2 S$ \\
\hline Fe23 & Be-like & $1 \mathrm{~S} 2$ & $2 S$ \\
\hline Fe23 & Be-like & 1S2 & $2 s$ \\
\hline Fe23 & Be-like & $1 S 2$ & $2 S$ \\
\hline $\mathrm{Fe} 23$ & Be-like & IS2 & $2 S$ \\
\hline Fe23 & Be-Iike & $1 \mathrm{~S} 2$ & $2 S$ \\
\hline Fe23 & Be-like & 1s2 & $2 S$ \\
\hline $\mathrm{Fe} 23$ & Be-like & 152 & $2 \mathrm{P}$ \\
\hline Fe23 & Be-like & 152 & $2 P$ \\
\hline Fe23 & Be-like & $1 S 2$ & $2 \mathrm{P}$ \\
\hline Fe23 & Be-like & $1 S 2$ & $2 \mathrm{P}$ \\
\hline Fe23 & Be-Iike & $1 \mathrm{S2}$ & $2 P$ \\
\hline$F e 23$ & Be-like & 152 & $2 P$ \\
\hline Fe23 & Be-like & 152 & $2 P$ \\
\hline $\mathrm{Fe} 23$ & Be-like & 152 & $2 P$ \\
\hline Fe23 & Be-like & IS2 & $2 \mathrm{P}$ \\
\hline $\mathrm{Fe} 23$ & Be-like & $1 \mathrm{~S} 2$ & $2 P$ \\
\hline $\mathrm{Fe} 23$ & Be-like & $1 S 2$ & $2 P$ \\
\hline Fe23 & Be-like & 152 & $2 P$ \\
\hline $\mathrm{Fe} 23$ & Be-like & 152 & $2 P$ \\
\hline Fe23 & Be-like & 152 & $2 P$ \\
\hline $\mathrm{Fe} 23$ & Be-1ike & 1S2 & $2 \mathrm{P}$ \\
\hline
\end{tabular}

$\left(1 P^{*}\right) j=1.0-1 S 2 \quad 2 S 2$ $\left(1 P^{*}\right) j=1.0-1 S 22 S 2$ $\left(3 P^{*}\right) j=1.0-1 \mathrm{~S} 22 \mathrm{~S} 2$ $\left(3 P^{\star}\right) j=1.0-1 S 2 \quad 2 S 2$ $\left(3 P^{*}\right) j=1.0-1 \mathrm{~S} 22 \mathrm{~S} 2$ $\left(3 P^{*}\right) j=1.0-1 S 2$ 2S2 $\left(3 P^{*}\right) j=1.0-1 S 22 S 2$ $\left(3 P^{\star}\right) j=1.0-1 S 2 \quad 2 S 2$ 4D (3D) $j=1.0-1 S 2$ 2S $2 P$ $4 D$ (3D) $j=1.0-1 S 2$ 2S $2 P$ $4 D(3 D) j=1.0-1 S 22 S \quad 2 P$ $4 D$ (3D) $j=1.0-1 S 22 S \quad 2 P$ 4D (3D) $j=1.0-1 S 2$ 2S $2 P$ $4 D$ (3D) $j=2.0-1 S 22 S \quad 2 P$ $4 D(3 D) j=2.0-1 S 22 S \quad 2 P$ $4 D(3 D) j=2.0-1 S 22 S \quad 2 P$ $4 D(3 D) j=2.0-1 S 2$ 2S $2 P$ $4 D(3 D) j=2.0-1 S 22 S \quad 2 E$ $4 D\left(3 P^{\star}\right) j=1.0-1 S 2 \quad 2 P 2$ $4 D\left(3 P^{\star}\right) j=1.0-1 S 2 \quad 2 F 2$ $4 D\left(3 P^{\star}\right) j=1.0-1 S 2 \quad 2 P 2$ $4 D\left(3 P^{*}\right) j=1.0-1 S 2 \quad 2 P 2$ 4D $\left(3 P^{\star}\right) j=1.0-1 S 2 \quad 2 P 2$ 4D (ID) $j=2.0-1 S 2$ 2S $2 P$ $4 D(1 D) j=2.0-1 S 22 S 2 P$ $4 D$ (ID) $j=2.0-1 S 22 S \quad 2 P$ $4 D(1 D) j=2.0-1 S 2$ 2S $2 P$ $4 D(3 D) j=3.0-1 S 22 S 2 P$ $4 D$ (3D) $j=3.0-1 S 2$ 2S $2 P$ 4D (3D) $j=3.0-1 S 2$ 2S $2 P$ 4D (3D) $j=3.0-1 S 2$ 2S $2 P$ 4D $\left(3 P^{*}\right) j=2.0-1 S 2 \quad 2 P 2$ 4D $\left(3 P^{\star} Y j=2.0-1 S 2 \quad 2 P 2\right.$ $4 D(3 P *) j=2.0-1 S 2 \quad 2 P 2$ $4 \mathrm{D}\left(3 \mathrm{P}^{\star}\right) j=2.0-1 \mathrm{~S} 2 \quad 2 \mathrm{P} 2$ $4 D\left(I D^{*}\right) j=2.0-1 \mathrm{~S} 2 \quad 2 \mathrm{P} 2$ $4 D\left(1 D^{\star}\right) j=2.0-1 S 2 \quad 2 P 2$ $4 D\left(1 D^{*}\right) j=2.0-1 S 2 \quad 2 P 2$ $4 D\left(1 D^{*}\right) j=2.0-1 S 2 \quad 2 P^{2}$ $4 D\left(3 P^{*}\right) j=2.0-1 S 2 \quad 2 P 2$ $4 D\left(3 F^{\star}\right) j=2.0-1 S 2 \quad 2 P 2$ $4 \mathrm{D}\left(3 \mathrm{P}^{*}\right) j=2.0-1 \mathrm{~S} 2 \quad 2 \mathrm{P} 2$ $4 D\left(3 P^{\star}\right) j=2.0-1 S 2 \quad 2 P 2$ $4 D\left(3 D^{*}\right) j=3.0-1 S 2 \quad 2 P 2$ $4 D\left(3 D^{*}\right) j=3.0-1 S 2 \quad 2 P 2$ $4 D\left(3 D^{*}\right) j=3.0-1 S 2 \quad 2 P 2$
(1S ) $j=0 \quad 8.303$ TAP 100

(Is) $j=.0$

(1s) $j=.0$

(1s) $j=.0$

(1s) $j=.0$

(1s) $j=.0$

(1S) $j=.0$

(1S) $j=.0$

$\left(3 P^{*}\right) j=.0$

$\left(3 P^{*}\right) j=.0$

$\left(3 P^{\star}\right) j=.0$

$\left(3 P^{*}\right) j=.0$

$\left(3 P^{*}\right) j=.0$

$\left(3 P^{\star}\right) j=1.0$

$\left(3 P^{*}\right) j=1.0$

$\left(3 P^{*}\right) j=1.0$

$\left(3 P^{*}\right) j=1.0$

$\left(3 P^{*}\right) j=1.0$

(3P) $j=.0$

(3P) $j=.0$

$(3 P) j=.0$

$(3 P) j=.0$

(3P) $j=.0$

$\left(3 P^{*}\right) j=2.0$

$\left(3 P^{*}\right) j=2.0$

$\left(3 P^{\star}\right) j=2.0$

$\left(3 P^{*}\right) j=2.0$

$\left(3 P^{\star}\right) j=2.0$

$\left(3 P^{*}\right) j=2.0$

$\left(3 P^{\star}\right) j=2.0$

$\left(3 P^{\star}\right) j=2.0$

(3P) $j=1.0$

(3P) $j=1.0$

(3P) $j=1.0$

(3P) $j=1.0$

(3P) $j=1.0$

(3P) $j=1.0$

(3P) $j=1.0$

(3P) $j=1.0$

(3P) $j=2.0$

(3P) $j=2.0$

(3P) $j=2.0$

(3P) $j=2.0$

(3P) $j=2.0$

(3P) $j=2.0$

(3P) $j=2.0$
8.316 topaz 002

816 guartz 100

8.316 EDT 020

8.316 TAP 100

.316 RAP 100

8.529 PET 002

8.529 EDT 020

529 TAP 100

8.529 RAP 100

8.529 KAP 100

550 PET 002

8.550 EDT 020

8.550 RAP 100

8.550 KAP 100

8.575 PET 002

8.575 EDT 020

.575 TAP 100

8.575 RAP 100

8.575 KAP 100

8.601 PET 002

8.601 EDT 020

8.601 RAP 100

8.601 KAP 100

8.614 PET 002

8.614 EDT 020

8.614 RAP 100

8.614 KAP 100

8.630 PET 002

8.630 EDT 020

8.630 RAP 100

$8.630 \mathrm{KAP} 100$

8.643 PET 002

8.643 EDT 020

8.643 RAP 100

8.643 KAP 100

8.664 PET 002

8.664 EDT 020

8.664 RAP 100

8.664 KAP 100

8.672 PET 002

8.672 EDT 020

8.672 RAP 100
8.303 TAP 100

8.316 PET 002

8.550 TAP 100 $\begin{array}{lll}25.763 & 75.206\end{array}$

$26.116 \quad 72.513$

$8.374 \quad 83.253$

$8.512 \quad 77.681$

$8.742 \quad 72.040$

$8.808 \quad 70.759$

$25.763 \quad 75.550$

$26.116 \quad 72.800$

$8.742 \quad 77.326$

$8.808 \quad 75.540$

$25.763 \quad 83.299$

$26.116 \quad 78.448$

$26.634 \quad 73.881$

$8.742 \quad 77.970$

$8.808 \quad 76.098$

$25.763 \quad 84.632$

$26.116 \quad 79.160$

$26.634 \quad 74.377$

$8.742 \quad 78.783$

$8.808 \quad 76.792$

$25.763 \quad 86.888$

$26.116 \quad 80.073$

26.63474 .988

$8.742 \quad 79.696$

$8.808 \quad 77.554$

$26.116 \quad 81.120$

$26.634 \quad 75.650$

$8.742 \quad 80.183$

$8.808 \quad 77.952$

$26.116 \quad 81.693$

$26.634 \quad 75.992$

$8.742 \quad 80.819$

$8.808 \quad 78.462$

$26.116 \quad 82.457$

26.63476 .426

$8.742 \quad 81.369$

$8.808 \quad 78.892$

$26.116 \quad 83.139$

$26.634 \quad 76.788$

$8.742 \quad 82.340$

$8.808 \quad 79.625$

$26.116 \quad 84.414$

$26.634 \quad 77.394$

$8.742 \quad 82.744$

$8.808 \quad 79.918$

$26.116 \quad 84.984$ 


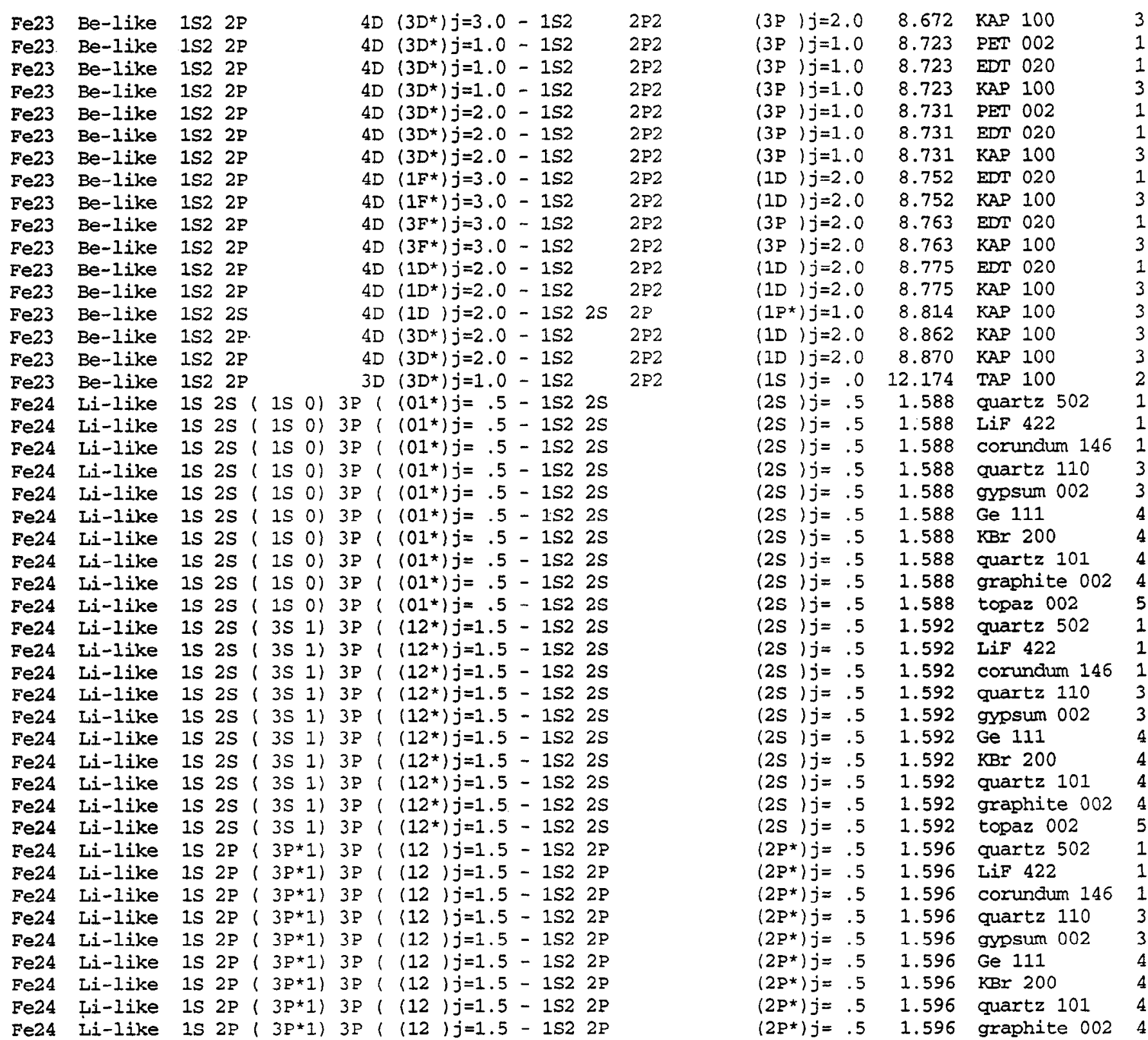

$26.634 \quad 77.633$

$8.742 \quad 86.222$

$8.808 \quad 82.034$

$26.634 \quad 79.278$

$8.742 \quad 87.125$

$8.808 \quad 82.418$

26.63479 .559

$8.808 \quad 83.536$

$26.634 \quad 80.335$

$8.808 \quad 84.206$

$26.634 \quad 80.768$

$8.808 \quad 85.039$

$26.634 \quad 81.264$

$26.634 \quad 83.116$

$26.634 \quad 86.560$

$26.634 \quad 87.567$

$25.763 \quad 70.922$

$1.624 \quad 77.913$

$1.652 \quad 73.999$

$1.660 \quad 73.063$

$4.912 \quad 75.899$

$4.990 \quad 72.690$

$6.532 \quad 76.518$

$6.584 \quad 74.745$

$6.687 \quad 71.787$

$6.696 \quad 71.555$

$8.374 \quad 71.473$

$1.624 \quad 78.607$

$1.652 \quad 74.511$

$1.660 \quad 73.544$

4.91276 .486

$4.990 \quad 73.159$

$6.532 \quad 77.134$

$6.584 \quad 75.283$

$6.687 \quad 72.231$

$6.696 \quad 71.992$

$8.374 \quad 71.908$

$1.624 \quad 79.345$

$1.652 \quad 75.039$

$1.660 \quad 74.038$

$4.912 \quad 77.099$

$4.990 \quad 73.642$

$6.532 \quad 77.780$

$6.584 \quad 75.842$

$6.687 \quad 72.686$

$6.696 \quad 72.441$ 


\begin{tabular}{|c|c|c|c|c|c|c|c|c|}
\hline 24 & Li-like & $1 S$ & $2 P 1$ & $3 p * 1)$ & $3 P$ & $(12) j=1.5$ & $-1 s 2$ & . \\
\hline $\mathrm{Fe} 24$ & Li-like & 1S & $2 \mathrm{P} 2$ & & & $(2 S) j=.5$ & - 152 & $2 P$ \\
\hline e24 & Li-like & 1s & 2P2 & & & $(2 S) j=.5$ & $-1 s 2$ & $2 P$ \\
\hline 224 & Li-like & is & $2 \mathrm{P} 2$ & & & $(2 S) j=.5$ & 152 & $P$ \\
\hline 24 & Li-like & 1s & $2 P 2$ & & & $(2 s) j=.5$ & -152 & $2 P$ \\
\hline$e 24$ & Li-like & $1 \mathrm{~S}$ & $2 \mathrm{P} 2$ & & & $(2 S) j=.5$ & $-1 s 2$ & $2 \mathrm{P}$ \\
\hline$e 24$ & Li-Iike & 1S & $2 \mathrm{P} 2$ & & & $(2 s) j=.5$ & $-1 S 2$ & 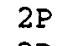 \\
\hline$\in 24$ & Li-1ike & 1s & $2 \mathrm{P} 2$ & & & $(2 s) j=.5$ & $-1 s 2$ & \\
\hline Fe24 & Li-like & 1s & $2 \mathrm{P} 2$ & & & $(2 S) j=.5$ & $-1 s 2$ & $P$ \\
\hline Fe24 & Li-like & 1s & $(2 S$ & )$<2 S$ & $2 P$ & $\left(\left\langle 2 P^{*}\right) j=1.5\right.$ & $-1 s 2$ & $2 s$ \\
\hline Fe24 & Li-like & 1S & $12 S$ & )$(2 S$ & $2 \mathrm{P}$ & $\left(\left(2 P^{*}\right) j=1.5\right.$ & $-1 s 2$ & 25 \\
\hline Fe24 & Li-like & is & $12 S$ & )$(2 S$ & $2 \mathrm{P}$ & $\left(\left(2 P^{\star}\right) j=1.5\right.$ & $-1 s 2$ & S \\
\hline $\mathrm{Fe} 24$ & Li-like & Is & $(2 S$ & )$(2 S$ & $2 \mathrm{P}$ & $\left(\left(2 P^{\star}\right) j=1.5\right.$ & -152 & $S$ \\
\hline Fe24 & Li-like & $1 S$ & $2 \mathrm{P} 2$ & & & $(2 \mathrm{P}) j=1.5$ & $-1 S 2$ & $P$ \\
\hline e24 & Li-like & 15 & $2 \mathrm{P} 2$ & & & $(2 P) j=1.5$ & -152 & \\
\hline Fe24 & Li-like & 1s & $2 \mathrm{P} 2$ & & & $(2 P) j=1.5$ & $-1 s 2$ & \\
\hline Fe24 & Li-like & is & $2 \mathrm{P} 2$ & & & $(2 P) j=1.5$ & $-1 s 2$ & \\
\hline Fe24 & Li-like & IS & $(2 S$ & )$(2 S$ & $2 P$ & $\left(\left(2 P^{*}\right) j=1.5\right.$ & $-1 s 2$ & \\
\hline $\mathrm{Fe} 24$ & Ii-like & 1S & $12 S$ & )$(2 \mathrm{~s}$ & $2 \mathrm{P}$ & $\left(\left(2 P^{*}\right) j=1.5\right.$ & 152 & \\
\hline Fe24 & Li-like & $1 S$ & $12 S$ & )$(25$ & $2 P$ & $\left(\left(2 P^{\star}\right) j=1.5\right.$ & -152 & \\
\hline Fe24 & Li-like & Is & $(2 \mathrm{~S}$ & )$(2 \mathrm{~S}$ & $2 \mathrm{P}$ & $\left(\left(2 q^{*}\right) j=1.5\right.$ & $-1 S 2$ & \\
\hline Fe24 & Li-like & $1 S$ & $(2 S$ & )$(25$ & $2 \mathrm{P}$ & $\left(\left(2 P^{*}\right) j=.5\right.$ & $-1 s 2$ & \\
\hline Fe24 & Li-like & $1 S$ & $12 S$ & )$(2 S$ & $2 \mathrm{P}$ & $\left(\left(2 P^{*}\right) j=.5\right.$ & $-1 s 2$ & \\
\hline Fe24 & Li-like & $1 \mathrm{~S}$ & $12 S$ & )$(2 \mathrm{~s}$ & $2 \mathrm{P}$ & $\left(\left(2 P^{*}\right) j=.5\right.$ & -152 & \\
\hline Fe24 & Li-like & $1 S$ & $(2 S$ & )$(2 S$ & $2 \mathrm{P}$ & $\left(\left(2 P^{*}\right) j=.5\right.$ & $-1 s 2$ & \\
\hline Fe24 & Li-like & $1 S$ & $2 \mathrm{P} 2$ & & & $(2 P) j=1.5$ & $-1 S 2$ & - \\
\hline $\mathrm{Fe} 24$ & Li-like & $1 s$ & $2 \mathrm{P} 2$ & & & $(2 P) j=1.5$ & $-1 s 2$ & \\
\hline Fe24 & Li-like & 1S & $2 \mathrm{P} 2$ & & & $(2 P) j=1.5$ & $-1 S 2$ & ? \\
\hline Fe24 & Li-like & is & $2 P 2$ & & & $(2 P) j=1.5$ & -152 & \\
\hline Fe24 & Li-like & $1 \mathrm{~S}$ & $2 \mathrm{P} 2$ & & & $(2 D) j=1.5$ & $-1 S 2$ & \\
\hline Fe24 & Li-like & 1s & $2 \mathrm{P} 2$ & & & $\langle 2 D\rangle j=1.5$ & $-1 S 2$ & \\
\hline $\mathrm{Fe} 24$ & Li-like & $1 S$ & $2 \mathrm{P} 2$ & & & $(2 D) j=1.5$ & - IS2 & \\
\hline $\mathrm{Fe} 24$ & Li-like & 15 & $2 \mathrm{P} 2$ & & & $(2 D) j=1.5$ & -152 & \\
\hline Fe24 & Li-1ike & $1 \mathrm{~S}$ & $2 \mathrm{P} 2$ & & & (2D) $j=2.5$ & $-1 s 2$ & \\
\hline $\mathrm{Fe} 24$ & Li-like & Is & $2 \mathrm{P} 2$ & & & $(2 D) j=2.5$ & -152 & $p$ \\
\hline Fe24 & Li-like & 15 & $2 \mathrm{P} 2$ & & & $\{2 D\rangle j=2.5$ & $-1 S 2$ & \\
\hline Fe24 & Li-like & $1 \mathrm{~S}$ & $2 \mathrm{P} 2$ & & & $(2 D) j=2.5$ & $-1 S 2$ & \\
\hline Fe24 & Li-like & 15 & $2 \mathrm{P} 2$ & & & $(4 P) j=2.5$ & $-1 s 2$ & \\
\hline Fe24 & Li-like & 1s & $2 \mathrm{P} 2$ & & & $(4 P) j=2.5$ & $-1 s 2$ & \\
\hline Fe24 & Li-1ike & 15 & $2 \mathrm{P} 2$ & & & $(4 P) j=2.5$ & $-1 s 2$ & \\
\hline Fe24 & Li-like & $1 \mathrm{~S}$ & 2P2 & & & $(4 P) j=1.5$ & $-1 s 2$ & \\
\hline Fe24 & Li-like & $1 \mathrm{~s}$ & 2P2 & & & $(4 P) j=1.5$ & $-1 s 2$ & \\
\hline Fe24: & Li-like & $1 \mathrm{~S}$ & $2 \mathrm{P} 2$ & & & $(48) j=1.5$ & $-1 s 2$ & \\
\hline Fe24 & Li-like & 152 & $9 \mathrm{P}$ & & & $\left(2 P^{*}\right) j=1.5$ & $-1 S 2$ & \\
\hline Fe24 & li-1ike & $1 S 2$ & $9 P$ & & & $\left\langle 2 P^{\star}\right\rangle j=1.5$ & $-1 s 2$ & \\
\hline Fe24 & Ei-like & $1 S 2$ & $9 P$ & & & $\left(2 P^{*}\right) j=1.5$ & $-1 S 2$ & \\
\hline
\end{tabular}

\begin{tabular}{|c|c|c|c|}
\hline$\left(2 P^{\star}\right) j=.5$ & 1.596 & topaz 002 & \\
\hline$\left(2 P^{*}\right) j=.5$ & 1.852 & Si 220 & \\
\hline$\left(2 P^{*}\right) j=.5$ & 1.852 & Eluorite & 220 \\
\hline$\left(2 p^{*}\right) j=.5$ & 1.852 & $\mathrm{NaCl} 200$ & \\
\hline$\left(2 \mathrm{P}^{*}\right) j=.5$ & 1.852 & InSb 111 & \\
\hline$\left(2 P^{*}\right) j=1.5$ & 1.856 & Si 220 & \\
\hline$\left(2 P^{*}\right) j=1.5$ & 1.856 & Eluorite & 220 \\
\hline$\left(2 P^{*}\right) j=1.5$ & 1.856 & $\mathrm{NaCl} 200$ & \\
\hline$\left(2 P^{*}\right) j=1.5$ & 1.856 & InSb 111 & \\
\hline es $j j=.5$ & 1.856 & Si 220 & \\
\hline$(2 S) j=.5$ & 1.856 & fluorite & 220 \\
\hline$(2 S) j=.5$ & 1.856 & $\mathrm{NaCl} 200$ & \\
\hline$(2 S) j=.5$ & 1.856 & InSb 111 & \\
\hline$\left(2 P^{\star}\right) j=.5$ & 1.857 & Si 220 & \\
\hline$\left(2 P^{*}\right) j=.5$ & 1.857 & Eluorite & 220 \\
\hline$\left(2 P^{\star}\right) j=.5$ & 1.857 & $\mathrm{NaCl} 200$ & \\
\hline$\left(2 P^{\star}\right) j=.5$ & 1.857 & Insb 111 & \\
\hline$(2 s) j=.5$ & 1.858 & Si 220 & \\
\hline$(2 s) j=.5$ & 1.858 & fluorite & 220 \\
\hline$(2 S) j=.5$ & 1.858 & $\mathrm{NaCl} 200$ & \\
\hline$(2 s) j=.5$ & 1.858 & Insb 111 & \\
\hline$(2 s) j=.5$ & 1.860 & Si 220 & \\
\hline (2s) $j=.5$ & 1.860 & fluorite & 220 \\
\hline (2S) j= .5 & 1.860 & $\mathrm{NaCl} 200$ & \\
\hline$(2 S) j=.5$ & 1.860 & Insb 111 & \\
\hline$\left(2 P^{\star}\right) j=1.5$ & 1.862 & $\mathrm{Si} 220$ & \\
\hline$\left(2 P^{*}\right) j=1.5$ & 1.862 & fluorite & 220 \\
\hline$\left(2 P^{\star}\right) j=1.5$ & 1.862 & $\mathrm{NaCl} 200$ & \\
\hline$(2 \mathrm{P} *) j=1.5$ & 1.862 & InSb 111 & \\
\hline$\left(2 P^{*}\right) j=.5$ & 1.863 & Si 220 & \\
\hline$\left(2 P^{\star}\right) j=.5$ & 1.863 & fluorite & 220 \\
\hline$\left(2 P^{*}\right) j=.5$ & 1.863 & $\mathrm{NaCl} 200$ & \\
\hline$\left(2 P^{*}\right) j=.5$ & 1.863 & Insb 111 & \\
\hline$\left(2 P^{*}\right) j=1.5$ & 1.865 & Si 220 & \\
\hline$\left(2 p^{\star}\right) j=1.5$ & 1.865 & fluorite & 220 \\
\hline$\left(2 P^{*}\right) j=1.5$ & 1.865 & $\mathrm{NaCl} 200$ & \\
\hline$\left(2 P^{*}\right) j=1.5$ & 1.865 & InSb 111 & \\
\hline$\left(2 P^{*}\right) j=1.5$ & 1.872 & Si 220 & \\
\hline$\left(2 P^{*}\right) j=1.5$ & 1.872 & Eluorite & 220 \\
\hline$(2 p *) j=1.5$ & 1.872 & $\mathrm{NaCl} 200$ & \\
\hline$\left(2 P^{*}\right) j=1.5$ & 1.873 & Si 220 & \\
\hline$\left(2 P^{*}\right) j=1.5$ & 1.873 & fluorite & 220 \\
\hline $\left.2 P^{*}\right) j=1.5$ & 1.873 & $\mathrm{NaCl} 200$ & \\
\hline$j=.5$ & 6.365 & Ge 111 & \\
\hline$j j=.5$ & 6.365 & $\mathrm{KBr} 200$ & \\
\hline s $j j=.5$ & 6.365 & quartz 1 & \\
\hline
\end{tabular}

$\begin{array}{ll}8.374 & 72.354 \\ 3.840 & 74.706 \\ 3.862 & 73.554 \\ 5.641 & 80.041 \\ 7.481 & 81.989 \\ 3.840 & 75.165 \\ 3.862 & 73.979 \\ 5.641 & 80.772 \\ 7.481 & 82.923 \\ 3.840 & 75.165 \\ 3.862 & 73.979 \\ 5.641 & 80.772 \\ 7.481 & 82.923 \\ 3.840 & 75.282 \\ 3.862 & 74.087 \\ 5.641 & 80.964 \\ 7.481 & 83.176 \\ 3.840 & 75.400 \\ 3.862 & 74.195 \\ 5.641 & 81.161 \\ 7.481 & 83.439 \\ 3.840 & 75.638 \\ 3.862 & 74.415 \\ 5.641 & 81.566 \\ 7.481 & 83.999 \\ 3.840 & 75.881 \\ 3.862 & 74.637 \\ 5.641 & 81.993 \\ 7.481 & 84.616 \\ 3.840 & 76.004 \\ 3.862 & 74.750 \\ 5.641 & 82.214 \\ 7.481 & 84.953 \\ 3.840 & 76.253 \\ 3.862 & 74.977 \\ 5.641 & 82.678 \\ 7.481 & 85.706 \\ 3.840 & 77.161 \\ 3.862 & 75.800 \\ 5.641 & 84.604 \\ 3.840 & 77.296 \\ 3.862 & 75.922 \\ 5.641 & 84.938 \\ 6.532 & 77.016 \\ 6.584 & 75.181 \\ 6.687 & 72.147\end{array}$




\begin{tabular}{|c|c|c|c|}
\hline 24 & Li-like & $1 \mathrm{~S} 2$ & $9 P$ \\
\hline $\mathrm{Fe} 24$ & Li-like & $1 \mathrm{~s} 2$ & $9 P$ \\
\hline Fe24 & Li-like & $1 \mathrm{~s} 2$ & $9 P$ \\
\hline Fe24 & Li-like & $1 S 2$ & $9 P$ \\
\hline Fe24 & Li-like & 152 & $9 P$ \\
\hline Fe24 & Ii-Iike & $1 S 2$ & $8 P$ \\
\hline Fe24 & Li-like & $1 \mathrm{~S} 2$ & $8 P$ \\
\hline Fe24 & Li-like & 1S2 & $8 P$ \\
\hline Fe24 & Li-like & 152 & $8 P$ \\
\hline Fe24 & Li-1ike & 152 & $8 P$ \\
\hline Fe24 & Li-like & 152 & $8 P$ \\
\hline Fe24 & Li-like & 152 & $8 P$ \\
\hline Fe24 & Li-like & 152 & 90 \\
\hline $\mathrm{Fe} 24$ & Li-like & $1 S 2$ & $9 D$ \\
\hline Fe24 & Li-like & $1 S 2$ & $9 D$ \\
\hline Fe24 & Li-like & 152 & 90 \\
\hline Fe24 & Li-like & $1 S 2$ & 90 \\
\hline Fe24 & Li-like & $1 S 2$ & $9 D$ \\
\hline Fe24 & Li-like & 152 & $9 D$ \\
\hline $\mathrm{Fe} 24$ & Li-like & $1 S 2$ & $7 P$ \\
\hline Fe24 & Li-like & $1 \mathrm{~s} 2$ & $7 P$ \\
\hline Fe24 & Li-like & $1 S 2$ & $7 P$ \\
\hline Fe24 & Li-like & 152 & $7 P$ \\
\hline $\mathrm{Fe} 24$ & Li-like & $1 \mathrm{~S} 2$ & $7 P$ \\
\hline Fe24 & Li-like & 152 & $8 D$ \\
\hline Fe24 & Li-like & 152 & $8 D$ \\
\hline Fe24 & Li-like & $1 S 2$ & $8 D$ \\
\hline Fe24 & Li-like & 152 & $8 D$ \\
\hline Fe24 & Li-1ike & $1 S 2$ & $8 D$ \\
\hline Fe24 & Li-1ike & 152 & $8 D$ \\
\hline Fe24 & Li-1ike & $1 S 2$ & $6 D$ \\
\hline Fe24 & Li-like & 152 & $6 s$ \\
\hline Fe24 & Li-like & 152 & $5 P$ \\
\hline $\mathrm{Fe} 24$ & Li-like & 152 & $5 P$ \\
\hline Fe24 & Li-like & 152 & $5 D$ \\
\hline Fe24 & Li-like & $1 s 2$ & $5 D$ \\
\hline Fe24 & Li-like & 152 & $5 S$ \\
\hline $\mathrm{Fe} 24$ & Li-1ike & $1 S 2$ & 55 \\
\hline Fe24 & Li-1ike & $1 s 2$ & $5 D$ \\
\hline Fe24 & Li-like & 152 & 50 \\
\hline Fe24 & Li-like & $1 S 2$ & $5 S$ \\
\hline Fe24 & Li-like & $1 S 2$ & $5 S$ \\
\hline Fe24. & Li-like & 152 & $4 \mathrm{P}$ \\
\hline Fe24 & Li-1ike & 152 & $4 P$ \\
\hline Fe24 & Ii-like & 152 & $4 \mathrm{D}$ \\
\hline Fe24 & Li-like & 152 & $4 D$ \\
\hline
\end{tabular}

s. $\left(2 P^{\star}\right) j=1.5-1 S 22 S$ $\left(2 P^{*}\right) j=1.5-1522 S$ $\left(2 \mathrm{P}^{*}\right) j=1.5-1 \mathrm{~S} 22 \mathrm{~S}$ $\left(2 P^{\star}\right) j=1.5-1522 S$ $\left(2 P^{*}\right) j=1.5-1 S 22 S$ $\left(2 P^{*}\right) j=1.5-1 S 22 S$ $\left(2 P^{*}\right\rangle j=1.5-1 S 22 S$ $\left(2 P^{*}\right) j=1.5-1 S 22 S$ $\left(2 P^{*}\right) j=1.5-1 S 22 S$ $\left(2 P^{*}\right) j=1.5-1 S 22 S$ $\left(2 P^{*}\right) j=1.5-1 S 22 S$ $\left(2 P^{*}\right) j=1.5-1 S 22 S$ (2D) $j=1.5-1 S 22 \mathrm{P}$ (2D) $j=1.5-1 S 22 P$ (2D) $j=1.5-1 S 22 P$ (2D) $j=1.5-1 S 22 P$ (2D) $j=1.5-1 S 22 P$ (2D) $j=1.5-1 S 22 P$ (2D) $j=1.5-1 S 22 P$ $\left(2 P^{*}\right) j=1.5-1 S 22 S$ $\left(2 P^{\star}\right) j=1.5-1 S 22 S$ $\left(2 P^{*}\right) j=1.5-1 S 22 S$ $\left(2 P^{*}\right) j=1.5-1 S 22 S$ $\left(2 P^{\star}\right) j=1.5-1 S 22 S$ (2D) $j=1.5-1 S 22 P$ (2D ) $j=1.5-1 S 22 P$ (2D) $j=1.5-1 \mathrm{~S} 22 \mathrm{P}$ (2D ) $j=1.5-1 S 22 P$ (2D) $j=2.5-1 S 22 P$ (2D) $j=2.5-1 S 22 P$ (2D ) $j=2.5-1 S 22 P$ (2S) $j=.5-1 S 22 P$ $\left(2 P^{*}\right) j=1.5-1 S 22 S$ $\left(2 P^{\star}\right) j=1.5-1 S 22 S$ (2D) $j=1.5-1 S 22 P$ (2D) $j=1.5-1 S 22 \mathrm{P}$ (2S ) $j=.5-1 S 22 P$ (2S) $j=.5-1 S 22 R$ (2D) $j=2.5-1 S 22 P$ (2D) $j=2.5-1 S 22 P$ (2S $) j=.5-1 S 22 \mathrm{P}$ (2S) $j=.5-1 S 22 P$ $\left(2 P^{\star}\right) j=1.5-1 S 2$ 2S $\left(2 P^{\star}\right) j=.5-1 S 22 S$ (2D ) $j=1.5-1 S 22 P$ (2D) $j=1.5-1 S 22 P$

\begin{tabular}{|c|c|c|}
\hline SS $j j=.5$ & 6.365 & graphite 002 \\
\hline $2 s) j=.5$ & 6.365 & mica 002 \\
\hline $25 j=.5$ & 6.365 & $\operatorname{TAP} 100$ \\
\hline $5 j j=.5$ & 6.365 & $\operatorname{RAP} 100$ \\
\hline$j j=.5$ & 6.365 & KAP 100 \\
\hline$j=.5$ & 6.451 & Ge 111 \\
\hline$j=.5$ & 6.451 & $\mathrm{KBr} 200$ \\
\hline$j=.5$ & .451 & quartz 101 \\
\hline$j=.5$ & 6.451 & graphite 002 \\
\hline$j=.5$ & 6.451 & mica 002 \\
\hline$j=.5$ & 6.451 & $\operatorname{RAP} 100$ \\
\hline$j=.5$ & 6. & KAP 100 \\
\hline$j=.5$ & 6.527 & Ge 111 \\
\hline$j=.5$ & 6.527 & $\mathrm{KBr} 200$ \\
\hline$j=.5$ & 6.527 & quartz 101 \\
\hline$j=.5$ & 6.527 & graphite 002 \\
\hline$j=.5$ & 6.527 & mica 002 \\
\hline$j=.5$ & 6.527 & RAP 100 \\
\hline$j=.5$ & 6.527 & KAP 100 \\
\hline$j=.5$ & 6.583 & $\mathrm{KBr} 200$ \\
\hline$j=.5$ & 6.583 & quartz 101 \\
\hline$j=.5$ & 6.583 & graphite 002 \\
\hline$j=.5$ & 6.583 & mica 002 \\
\hline$j=.5$ & 6.583 & KAP 100 \\
\hline$j=.5$ & 6.617 & quartz 101 \\
\hline$j=.5$ & 6.617 & graphite 002 \\
\hline *) $j=.5$ & 6.617 & mica 002 \\
\hline *) $j=.5$ & 6.617 & KAP 100 \\
\hline$j=1.5$ & 6.672 & quartz 101 \\
\hline$=1.5$ & 6.672 & graphite 002 \\
\hline$=1.5$ & 7.033 & Insb 111 \\
\hline$j=1.5$ & 7.066 & InStb 111 \\
\hline$j=.5$ & 7.169 & InSto 111 \\
\hline$j j=.5$ & 7.169 & gypsum 020 \\
\hline$\left.{ }^{*}\right) j=.5$ & 7.370 & InSb 111 \\
\hline$j j=.5$ & 7.370 & gypsum 020 \\
\hline .5 & 7.391 & Insb 111 \\
\hline .5 & 7.391 & gypsum 020 \\
\hline$j=1.5$ & 7.438 & InSt 111 \\
\hline$j=1.5$ & 7.438 & gypsum 020 \\
\hline *) $j=1.5$ & 7.462 & Insb 111 \\
\hline$\Rightarrow j=1.5$ & 7.462 & gypsum 020 \\
\hline .5 & 7.983 & topaz 002 \\
\hline .5 & 7.993 & topaz 002 \\
\hline .5 & 8.231 & topaz 002 \\
\hline .2 & 8.231 & quartz 100 \\
\hline
\end{tabular}

$6.696 \quad 71.910$ $19.942 \quad 73.241$ $25.763 \quad 81.204$ $26.116 \quad 77.131$ $26.634 \quad 72.925$ $6.532 \quad 80.968$ $6.584 \quad 78.464$ $6.687 \quad 74.733$ $6.696 \quad 74.453$ $19.942 \quad 76.040$ $26.116 \quad 81.135$ 26.63475 .659 $6.532 \quad 87.758$

$6.584 \quad 82.455$

$6.687 \quad 77.441$

$6.696 \quad 77.100$

$19.942 \quad 79.081$

$26.116 \quad 88.582$

26.63478 .594

$6.584 \quad 89.001$

$6.687 \quad 79.882$

$6.696 \quad 79.459$

$19.942 \quad 82.022$

26.63481 .364

$6.687 \quad 81.702$

$6.696 \quad 81.190$

$19.942 \quad 84.524$

$26.634 \quad 83.600$

6.68786 .162

$6.696 \quad 85.147$

$7.481 \quad 70.071$

$7.481 \quad 70.826$

$7.481 \quad 73.394$

$15.185 \quad 70.773$

$7.481 \quad 80.118$

$15.185 \quad 76.095$

$7.481 \quad 81.104$

$15.185 \quad 76.770$

$7.481 \quad 83.854$

$15.185 \quad 78.422$

$\begin{array}{ll}7.481 & 85.916\end{array}$

$15.185 \quad 79.362$

$8.374 \quad 72.422$

$8.374 \quad 72.650$

$8.374 \quad 79.396$

8.51275 .237 


\begin{tabular}{|c|c|c|}
\hline 24 & Li-like & $1 S 2$ \\
\hline Fe24 & Li-1ike & 152 \\
\hline $\mathrm{Fe} 24$ & Li-like & 152 \\
\hline Ee24 & Ii-like & 152 \\
\hline e24 & Li-like & 152 \\
\hline 24 & Ii-1ike & 152 \\
\hline e24 & Li-like & $1 S 2$ \\
\hline e24 & Li-like & 1s2 \\
\hline e24 & Ii-like & $1 \mathrm{~s} 2$ \\
\hline$e 24$ & Li-like & $1 \mathrm{~s} 2$ \\
\hline Pe24 & I-i-1ike & $1 S 2$ \\
\hline e24 & Li-1ike & $1 S 2$ \\
\hline e24 & Li-like & 152 \\
\hline e24 & Li-like & $1 \mathrm{~S} 2$ \\
\hline$e 24$ & Li-like & 152 \\
\hline e24 & Li-like & $1 \mathrm{~S} 2$ \\
\hline e24 & Li-1ike & $1 \mathrm{~S} 2$ \\
\hline $\mathrm{Fe} 24$ & Li-like & 152 \\
\hline Pe24 & Li-like & $1 \mathrm{~S} 2$ \\
\hline e24 & Li-like & $1 \mathrm{~S} 2$ \\
\hline Pe24 & Li-like & $1 S 2$ \\
\hline ge24 & Li-like & $1 \mathrm{~S} 2$ \\
\hline e24 & Ii-like & $1 s 2$ \\
\hline ge25 & He-like & 1s \\
\hline Fe25 & He-like & is \\
\hline Fe25 & He-like & is \\
\hline Fe25 & He-like & 15 \\
\hline Fe25 & He-like & 1S \\
\hline Fe25 & He-like & 15 \\
\hline $\mathrm{Fe} 25$ & He-like & 1S \\
\hline Fe25 & He-like & 15 \\
\hline e25 & Fe-like & IS \\
\hline$e 25$ & ike & is \\
\hline e25 & He-like & 1s \\
\hline Re25 & He-like & Is \\
\hline Pe25 & He-like & is \\
\hline Fe25 & He-like & 15 \\
\hline Fe25 & He-like & 15 \\
\hline Fe25 & Fe-like & 1s \\
\hline Fe25 & He-like & 1s \\
\hline e25 & He-like & 1s \\
\hline Fe25 & He-like & 15 \\
\hline Ge25. & He-like & IS \\
\hline e25 & He-1i & \\
\hline e25 & $\mathrm{He}-1$ & \\
\hline e25 & He-like & 15 \\
\hline
\end{tabular}

(2D) $j=1.5-1 S 22 P$ (2D) $j=1.5-1 S 22 P$ (2D) $j=1.5-1 S 22 P$ (2S ) $j=.5-1 S 22 P$ (2S) $j=.5-1 S 22 \mathrm{P}$ (2S ) $j=.5-1 S 22 P$ (2S) $j=.5-1 S 22 P$ (2S ) $j=.5-1 S 22 \mathrm{P}$ (2S) $j=.5-1 S 22 P$ (2D) $j=2.5-1 S 22 P$ (2D) $j=2.5-1 S 22 P$ (2D) $j=2.5-1 S 22 \mathrm{P}$ (2D) $j=2.5-1 S 22 P$ (2D ) $j=2.5-1 S 22 P$ (2D) $j=2.5-1 S 22 P$ (2S) $j=.5-1 S 22 P$ (2S ) $j=.5-1 S 22 P$ (2S ) $j=.5-1 S 22 P$ (2S) $j=.5-1 S 22 \mathrm{P}$ $(2 S) j=.5-1 S 22 P$ (2S ) $j=.5-1 S 22 P$ (2S ) $j=.5-1 S 22 \mathrm{P}$ $\left(2 P^{*}\right) j=1.5-1 S 22 S$ $\left(1 P^{*}\right) j=1.0-1 S 2$

$\left(1 P^{*}\right) j=1.0-152$

$\left(1 P^{*}\right) j=1.0-1 \mathrm{~S} 2$

$\left(1 P^{*}\right) j=1.0-1 \mathrm{~S} 2$

$\left(1 P^{*}\right) j=1.0-1 \mathrm{~s} 2$

$\left(1 P^{*}\right) j=1.0-1 S 2$

$\left(1 P^{\star}\right) j=1.0-1 \mathrm{~S} 2$

$\left(1 p^{\star}\right) j=1.0-152$

$\left(1 P^{*}\right) j=1.0-1 S 2$

$\left(1 P^{*}\right) j=1.0-152$

$\left(1 P^{\star}\right) j=1.0-1 S 2$

$\left(1 P^{*}\right) j=1.0-1 S 2$

$(1 P *) j=1.0-1 S 2$

$\left(1 P^{*}\right) j=1.0-152$

$\left(1 P^{*}\right) j=1.0-1 S 2$

$\left(1 p^{*}\right) j=1.0-1 \mathrm{~s} 2$

$\left(1 P^{*}\right) j=1.0-1 \mathrm{~S} 2$

$\left(1 P^{*}\right) j=1.0-152$

$\left(1 P^{*}\right) j=1.0-1 \mathrm{~S} 2$

$\left(1 P^{*}\right) j=1.0-1 S 2$

$\left(1 P^{\star}\right) j=1.0-1 S 2$

$\left(1 P^{*}\right) j=1.0-1 S 2$

$\left(1 P^{*}\right) j=1.0-1 \mathrm{~S} 2$ $\left(2 P^{*}\right) j=.5$ $\left(2 P^{*}\right) j=.5$ $\left(2 P^{\star}\right) j=.5$ $\left(2 P^{\star}\right) j=.5$

$(2 P *) j=$

$\left(2 \mathrm{P}^{*}\right) j=.5$

$\left(2 P^{\star}\right) j=$

$\left(2 P^{\star}\right) j=.5$

$\left(2 P^{*}\right) j=.5$

$\left(2 P^{\star}\right) j=1.5$

$\left(2 P^{*}\right) j=1.5$

$\left(2 P^{*}\right) j=1.5$

$\left(2 P^{\star}\right) j=1.5$

$\left(2 P^{\star}\right) j=1.5$

$\left(2 P^{\star}\right) j=1.5$

$\left(2 P^{\star}\right) j=1.5$

$\left(2 P^{*}\right) j=1.5$

$\left(2 P^{\star}\right) j=1.5$

$\left(2 P^{*}\right) j=1.5$

$\left(2 P^{*}\right) j=1.5$

$\left(2 P^{\star}\right) j=1.5$

$\left(2 P^{*}\right) j=1.5$

(2S) $j=$.

(1S) $j=.0$

(Is) $j=.0$

(1s) $j=.0$

(1s) $j=$.

(is) $j=.0$

(1s) $j=.0$

(1s) $j=.0$

(is) $j=.0$

(1S) $j=.0$

(1s) $\mathrm{j}=.0$

(1s) $j=.0$

(1s) $j=.0$

(1s) $j=.0$

(1s) $j=.0$

(1s) $j=.0$

(1s) $j=.0$

(1s) $j=.0$

(1s) $j=.0$

(1s) $j=.0$

(1s) $j=.0$

(1s) $j=.0$

(1s) $j=.0$

(1s) $j=.0$
8.231 PET 002

8.231 TAP 100

8.231 RAP 100

285 topaz 002

285 guartz 100

8.285 PET 002

8.285 EDT 020

285 TAP 100

8.285 RAP 100

8.315 topaz 002

315 cuartz 100

8.315 PET 002

8.315 EDT 020

8.315 TAP 100

315 RAP 100

8.371 topaz 002

8.371 quartz 100

8.371 PET 002

371 EDT 020

8.371 TAP 100

8.371 RAP 100

10.619 ADP 101

calcite 422

1.443 guartz 112

1.443 calcite 200

1.443 Insb 111

1.461 calcite 422

1.461 quartz 211

1.461 tungsten 1103

1.461 quartz 112

1.461 topaz 200

1.461 calcite 200

1.461 InSb 111

1.495 calcite 422

1.495 quartz 211

1.495 quartz 112

1.495 topaz 200

1.495 Al 111

1.495 calcite 200

1.495 Si 111

1.495 sylvite 200

1.495 Eluorite 111

1.495 InSt 111

1.573 quartz 502
$8.742 \quad 70.313$

$25.763 \quad 73.429$

$26.116 \quad 70.999$

$8.374 \quad 81.639$

$8.512 \quad 76.738$

$8.742 \quad 71.392$

$8.808 \quad 70.156$

$25.763 \quad 74.743$

$26.116 \quad 72.123$

$8.374 \quad 83.195$

$8.512 \quad 77.649$

$8.742 \quad 72.018$

$8.808 \quad 70.739$

$25.763 \quad 75.523$

$26.116 \quad 72.777$

$8.374 \quad 88.466$

8.51279 .557

$8.742 \quad 73.248$

$8.808 \quad 71.876$

$25.763 \quad 77.102$

$26.116 \quad 74.069$

$26.634 \quad 70.543$

$10.640 \quad 86.400$

$3.034 \quad 72.030$

$4.476 \quad 75.275$

$4.564 \quad 71.534$

$6.071 \quad 71.943$

$7.481 \quad 74.675$

$3.034 \quad 74.383$

$3.082 \quad 71.457$

$4.476 \quad 78.300$

$4.564 \quad 73.810$

$4.638 \quad 70.912$

$6.071 \quad 74.283$

$7.481 \quad 77.547$

$3.034 \quad 80.230$

3.08275 .965

$4.564 \quad 79.324$

$4.638 \quad 75.242$

$4.676 \quad 73.567$

$6.071 \quad 80.067$

$6.271 \quad 72.477$

$6.292 \quad 71.881$

$6.308 \quad 71.442$

$7.481 \quad 87.705$

$1.624 \quad 75.603$ 
(18*) $j=1.0-152$

$\left(1 P^{*}\right) j=1.0-1 S 2$

$\left(1 P^{*}\right) j=1.0-1 S 2$

$\left(1 P^{*}\right) j=1.0-1 S 2$

$\left(1 P^{*}\right) j=1.0-1 S 2$

$\left(1 P^{*}\right) j=1.0-152$

$\left(1 P^{*}\right) j=1.0-1 S 2$

(1P*) $j=1.0-1 \mathrm{~s} 2$

$\left(3 P^{*}\right) j=1.0-152$

$\left(3 P^{*}\right) j=1.0-1 S 2$

$\left(3 p^{*}\right) j=1.0-1 \mathrm{~s} 2$

$\left(3 P^{*}\right) j=1.0-1 S 2$

$\left(3 P^{*}\right) j=1.0-152$

$\left(3 P^{*}\right) j=1.0-1 \mathrm{~s} 2$

$\left(3 P^{*}\right) j=1.0-1 S 2$

$\left(3 P^{*}\right) j=1.0-152$

$\left(3 \mathrm{P}^{*}\right) j=1.0-1 \mathrm{~S} 2$

$\left(3 P^{*}\right) j=1.0-1 S 2$

$\left(3 p^{*}\right) j=1.0-1 \mathrm{~s} 2$

(1S ) $j=.0-1 S 2 P$

(1S) $j=.0-152 P$

(1S) $j=.0-1 S 2 P$

(1S ) $j=.0-1 S 2 P$

$\left(1 P^{*}\right) j=1.0$ - IS $2 S$

$\left(1 P^{*}\right) j=1.0-15 \quad 25$

$\left(1 P^{*}\right) j=1.0-1 S \quad S$

$\left(1 P^{*}\right) j=1.0-1 S \quad 2 S$

$\left(3 P^{*}\right) j=2.0-1 S \quad 2 S$

$\left(3 P^{*}\right) j=2.0-1 S \quad 2 S$

$\left(3 P^{*}\right) j=2.0-1 S 2 S$

$\left(3 P^{*}\right) j=2.0-1 S \quad 2 S$

(1D) $j=2.0-1 S \quad 2 P$

(1D) $j=2.0-1 S 2 P$

(1D) $j=2.0-1 S 2 P$

(1D) $j=2.0-1 S 2 P$

(3P) $j=2.0-1 S 2 P$

(3P) $j=2.0-1 S \quad 2 P$

(3P) $j=2.0-1 S \quad 2 P$

(3P) $j=2.0-1 S 2 P$

$\left(3 P^{*}\right) j=1.0-1 S 2$

$\left(3 P^{*}\right\rangle j=1.0-1.52$

$\left(3 P^{\star}\right) j=1.0-152$

$\left(3 P^{*}\right) j=1.0-1 \mathrm{~S} 2$

$\left(3 P^{*}\right) j=1.0-1 S 2$

$\left(3 P^{*}\right) j=1.0-1 S 2$

$\left(3 P^{*}\right) j=1.0-1 \mathrm{~S} 2$

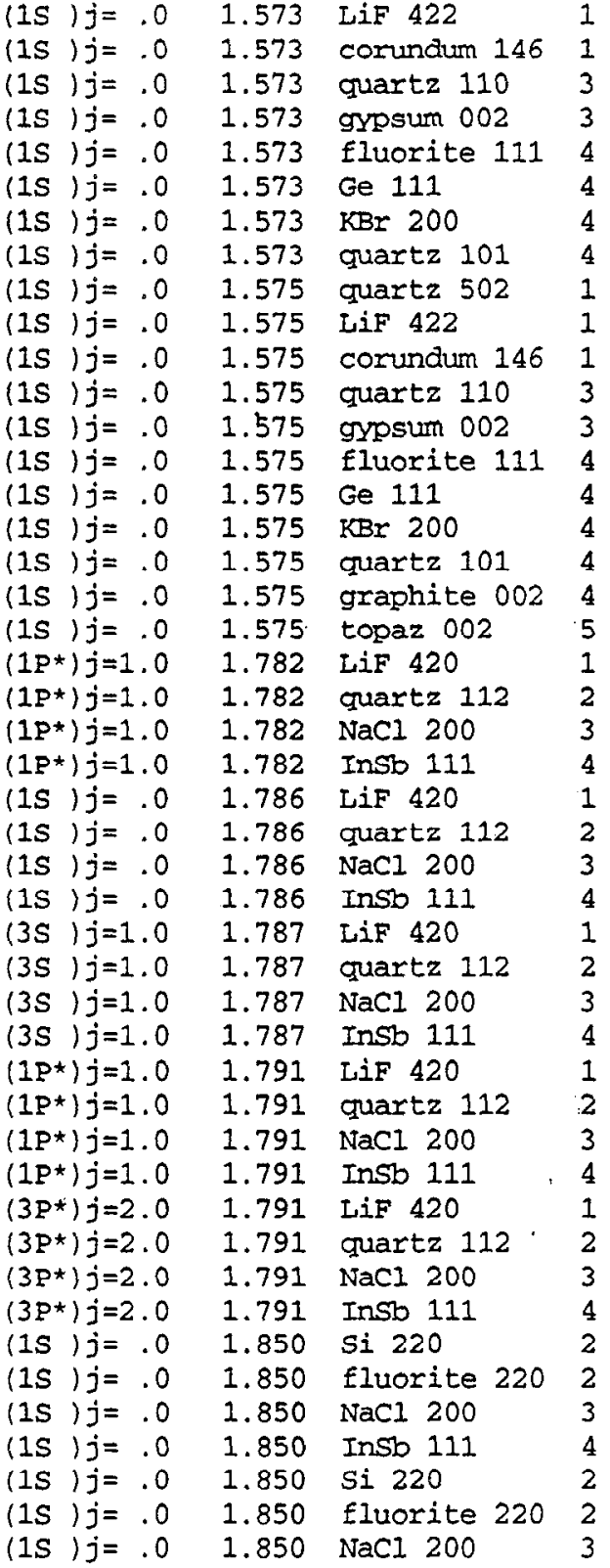

$\begin{array}{ll}1.652 & 72.209\end{array}$

$1.660 \quad 71.368$

$4.912 \quad 73.885$

$4.990 \quad 71.030$

$6.308 \quad 85.918$

6.53274 .420

$6.584 \quad 72.872$

$6.687 \quad 70.208$

$1.624 \quad 75.890$

1.65272 .438

1.66071 .585

$4.912 \quad 74.140$

$4.990 \quad 71.244$

$6.308 \quad 87.114$

$6.532 \quad 74.684$

$6.584 \quad 73.110$

$6.687 \quad 70.412$

$6.696 \quad 70.197$

$8.374 \quad 70.121$

$1.801 \quad 81.670$

$3.636 \quad 78.579$

$5.641 \quad 71.388$

$7.481 \quad 72.329$

1.80182 .600

$3.636 \quad 79.234$

$5.641 \quad 71.774$

$7.481 \quad 72.737$

$1.801 \quad 82.851$

$3.636 \quad 79.404$

$5.641 \quad 71.872$

$7.481 \quad 72.841$

$1.801 \quad 83.959$

$3.636 \quad 80.113$

$5.641 \quad 72.268$

$7.481 \quad 73.261$

$1.801 \quad 83.959$

$3.636 \quad 80.113$

$\begin{array}{ll}5.641 & 72.268\end{array}$

$7.481 \quad 73.261$

$3.840 \quad 74.481$

$3.862 \quad 73.346$

$5.641 \quad 79.695$

$7.481 \quad 81.561$

$3.840 \quad 74.481$

$3.862 \quad 73.346$

5.64179 .695 
$\left(3 P^{*}\right) j=1.0-1 S 2$

$\left(3 P^{*}\right) j=2.0-152$

$\left(3 P^{*}\right) j=2.0-1 S 2$

$\left(3 P^{*}\right) j=2.0-1 s 2$

$\left(3 P^{*}\right) j=2.0-1 S 2$

$\left(3 P^{*}\right) j=1.0-1 S 2$

$\left(3 P^{*}\right) j=1.0-1 S 2$

$\left(3 P^{*}\right) j=1.0-1 S 2$

$\left(3 P^{*}\right) j=1.0-1 S 2$

(3S) $j=1.0-152$

(3s) $j=1.0-1 s 2$

(3S) $j=1.0-1 S 2$

(3s) $j=1.0-1 s 2$

(1S) $j=.0-1 S \quad 2 P$

$\left(3 P^{*}\right) j=2.0-1 S 2 S$

$\left(3 P^{*}\right) j=1.0-1 S$ 2S

$\left(1 P^{*}\right) j=1.0-1 S 2 S$

$\left(2 P^{*}\right) j=1.5-1 S$

$(2 P *) j=1.5-1 S$

$\left(2 P^{\star}\right) j=1.5-15$

$(2 p *) j=1.5-1 S$

$\left(2 \mathrm{P}^{*}\right) j=1.5-1 \mathrm{~S}$

$\left(2 P^{\star}\right) j=1.5-1 S$

$\left(2 P^{\star}\right) j=1.5-1 S$

$\left(2 P^{*}\right) j=1.5-1 S$

$\left(2 P^{\star}\right) j=1.5-1 S$

$\left(2 P^{*}\right) j=1.5-1 S$

$\left(2 P^{*}\right) j=1.5-1 S$

$\left(2 P^{*}\right) j=1.5-1 S$

$(2 P *) j=1.5-1 S$

$\left(2 p^{*}\right) j=1.5-1 \mathrm{~s}$

$\left(2 P^{*}\right) j=1.5-1 S$

$\left(2 P^{*}\right) j=.5-15$

$\left(2 P^{\star}\right) j=.5-1 S$

$\left(2 P^{*}\right) j=.5-1 S$

$\left(2 P^{*}\right) j=.5-15$

$\left(2 \mathrm{P}^{\star}\right) j=.5-1 \mathrm{~s}$

$\left(2 P^{*}\right) j=.5-1 S$

$\left(2 P^{*}\right) j=.5-1 S$

$\left(2 P^{*}\right) j=.5-1 s$

$\left(2 P^{*}\right) j=.5-1 S$

$\left(2 P^{\star}\right) j=1.5-1 S$

$\left(2 P^{\star}\right) j=1.5-1 S$

$\left(2 P^{\star}\right) j=1.5-1 S$

$\left(2 P^{*}\right) j=1.5-15$

$\left(2 p^{\star}\right) j=.5-1 S$

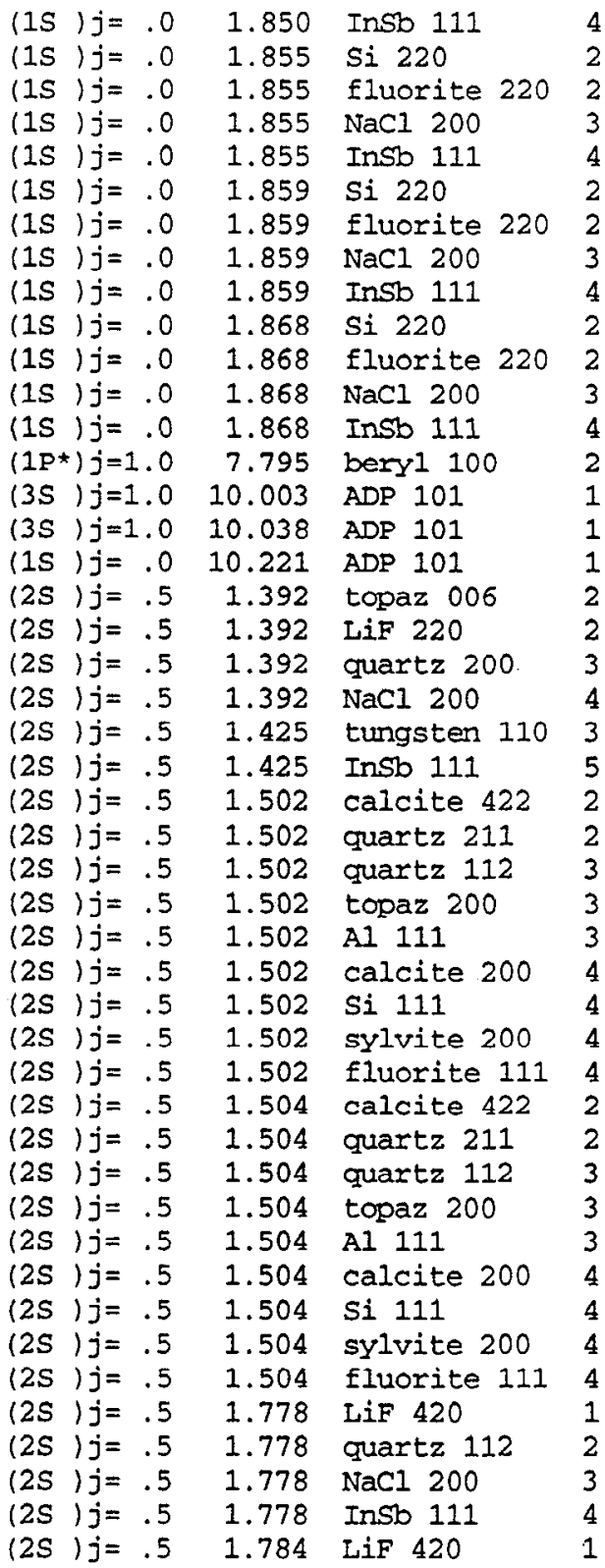

$7.481 \quad 81.561$

$3.840 \quad 75.049$

$3.862 \quad 73.872$

5.64180 .584

$7.481 \quad 82.678$

$3.840 \quad 75.519$

$3.862 \quad 74.305$

$5.641 \quad 81.361$

$7.481 \quad 83.712$

$3.840 \quad 76.635$

$3.862 \quad 75.324$

$5.641 \quad 83.434$

$7.481 \quad 87.189$

$15.954 \quad 77.737$

$10.640 \quad 70.074$

$10.640 \quad 70.634$

$10.640 \quad 73.867$

$2.795 \quad 84.915$

$2.848 \quad 77.830$

$4.246 \quad 79.582$

$\begin{array}{ll}5.641 & 80.772\end{array}$

$4.476 \quad 72.764$

$7.481 \quad 72.253$

$3.034 \quad 81.936$

3.08277 .082

$4.564 \quad 80.856$

$4.638 \quad 76.298$

$4.676 \quad 74.503$

6.07181 .739

$6.271 \quad 73.348$

$6.292 \quad 72.720$

$6.308 \quad 72.259$

$3.034 \quad 82.494$

$3.082 \quad 77.419$

$4.564 \quad 81.343$

$4.638 \quad 76.614$

$4.676 \quad 74.781$

$6.071 \quad 82.282$

6.27173 .605

$6.292 \quad 72.967$

$5.308 \quad 72.499$

1.801 .80 .833

$3.636 \quad 77.959$

5.64171 .010

$7.481 \quad 71.929$

$1.801 \quad 82.121$ 


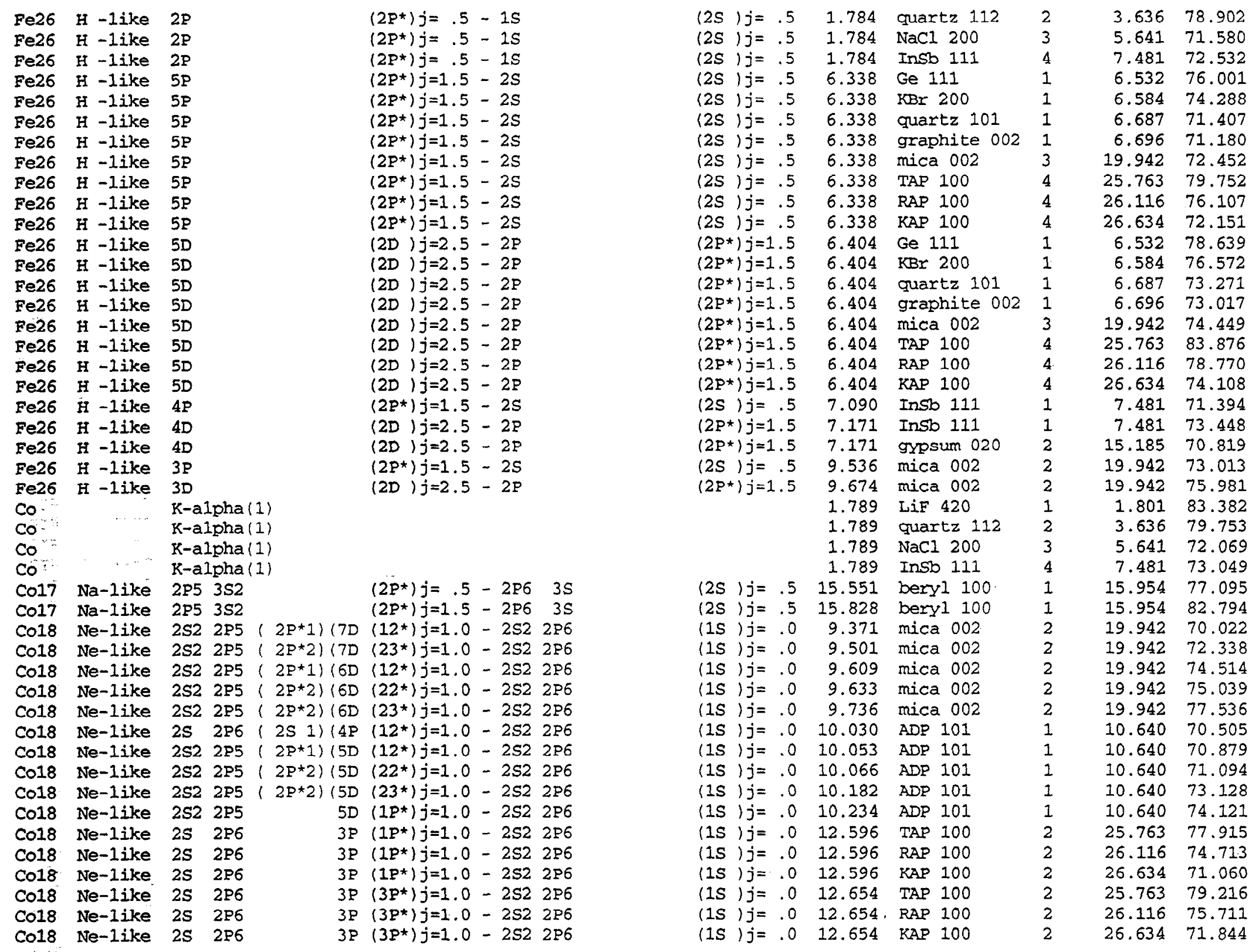


Co18 Ne-1ike 2S2 2P5 ( $2 \mathrm{P} * 1)$ 3S $\left(11^{*}\right) j=1.0-2 \mathrm{~S} 22 \mathrm{P} 6$ Co18 Ne-1ike 2S2 $2 P 5(2 P * 1) 3 S\left(11^{*}\right) j=1.0-2 S 22 P 6$ Co18 Ne-1ike 2S2 2P5 ( $\left.2 P^{*} 2\right)$ 3S $(21 *) j=1.0-2522 \mathrm{P} 6$ Co19 F-1ike 2S2 2P4 ( $1 S$ ) $4 D$ (2D) $j=1.5-2 S 22 \mathrm{P} 5$ Co19 F-like 2S2 2P4 ( ID) $4 D(2 D) j=1.5-2 S 22 P 5$ Co19 F-like 2S2 2P4 ( ID) $4 D(2 D) j=2.5-2 S 22 P 5$ Co19 F-like 2S2 2P4 ( $3 P$ ) $4 D(4 P) j=2.5-2 S 2$ 2P5 Co19 F-like 2S2 2P4 ( $3 P$ ) $4 D(4 F) j=2.5-2 S 22 P 5$ Co19 F-like 2S2 2P4 ( ID) $4 D(2 S) j=.5-2 S 22 P 5$ Co19 F-like 2S2 2P4 ( $3 P$ ) 4D (2F) $j=2.5-2 S 22 P 5$ Co19 F-like 2S2 2P4 (1D) $4 S(2 D) j=2.5-2 S 2$ 2P5 Co19 F-like 2S2 2P4 ( $3 P$ ) $4 S(4 P) j=.5-2 S 22 P 5$ Co19 F-like 2S 2P5 ( $\left.3 P^{*}\right) 3 P(4 P) j=2.5-2 S 22 P 5$ Co19 F-like 2S 2P5 ( $\left.3 P^{*}\right) 3 P(2 P) j=1.5-2 S 22 P 5$ Co19 F-like 2S 2P5 (3P*) 3P (2S $) j=.5-2 S 22 P 5$ Co19 F-like 2S 2P5 ( $\left.3 P^{*}\right) 3 P(2 D) j=2.5-2 S 22 P 5$ Co19 $F$-like $2 S$ 2P5 ( $\left.3 P^{*}\right) 3 P(2 D) j=1.5-2 S 22 P 5$ Co19 F-like 2S 2P5 ( $\left.3 P^{*}\right)$ 3P (4D) $j=1.5-2 S 22 \mathrm{P5}$ Co19 F-like 2S 2P5 ( $\left.3 P^{*}\right) 3 P(4 D) j=2.5-2 S 22 P 5$ Co19 F-like $2 S$ 2P5 ( $\left.3 P^{*}\right) 3 P(4 D) j=2.5-2 S 22 P 5$ Co19 F-like 2S 2P5 (3P*) 3P (4P) j=1.5 - 2S2 2P5 Co19 F-like 2S 2P5 (3P*) 3P (4P) j=1.5 - 2S2 2P5 Co19 $F$-like $2 S 2 P 5(3 P *) 3 P(2 P) j=.5-2 S 22 P 5$ Co19 F-like 2S 2P5 ( $\left.3 P^{\star}\right)$, $P P(2 P) j=.5-2 S 22 P 5$ Co19 F-like 2S2 2P4 ( $1 S$ ) 3D (2D) j=2.5 - 2S2 $2 P 5$ Co19 F-like 2S2 2P4 ( 15 ) 3D (2D) $j=2.5-2 S 22 \mathrm{P} 5$ Co19 F-like 2S2 2P4 ( $1 S$ ) $3 D$ (2D) $j=2.5-2522 P 5$ Co19 F-1ike 2S2 2P4 ( $1 S$ ) 3D (2D) $j=1.5-2 S 22 \mathrm{P} 5$ Co19 F-like 2S2 2P4 ( $1 S$ ) 3D (2D) j=1.5 - 2S2 2P5 Co19 F-like 2S2 $2 P 4$ ( $1 S$ ) $3 D$ (2D) $j=1.5-2 S 22 P 5$ Co19 F-like 2S2 2P4 ( $1 D$ ) 3D (2P) $j=.5-2522 P 5$ Co19 F-like 2S2 2P4 (1D) 3D (2P)j=.5- $2522 P 5$ Co19 F-like 2S2 2P4 ( 1D) $3 D(2 P) j=.5-2 S 22 P 5$ Co19 F-like 2S2 2P4 ( $1 D$ ) 3D (2D) $j=1.5-2 S 22 P 5$ Co19 F-like 2S2 2P4 ( $1 D$ ) 3D (2D) j=1.5 - 2S2 2P5 Co19 F-like 2S2 2P4 ( 1D) 3D (2D) j=2.5 - 2S2 2P5 Co19 F-like 2S2 2P4 ( $1 D$ ) 3D (2D) $j=2.5-2 S 22 P 5$ Co19 F-like 2S2 2P4 ( $1 D$ ) $3 D(2 S) j=.5-2 S 22 P 5$ Co19 F-like 2S2 2P4 (1D) 3D (2S) j=.5-2S2 2P5 Co19 F-like 2S2 2P4 ( $3 P$ ) 3D (2D) $j=2.5-2 S 22 P 5$ Co19 F-like 2S2 2P4 ( $1 D$ ) 3D (2D) $j=1.5-2 S 22 P 5$ Co19 F-like 2S2 2P4 ( $3 P$ ) 3D (4D) $j=1.5-2 S 22 P 5$ Co19. F-like 2S2 2P4 ( ID) $3 D(2 P) j=1.5-2 S 22 P 5$ Co19 F-like 2S2 2P4 ( $3 P$ ) 3D (4P) $j=2.5-2 S 22 P 5$ Co19 F-like 2S2 2P4 ( 1D) 3D (2S) j=.5- 2S2 2P5 Co19 F-like 2S2 2P4 ( $3 \mathrm{P}$ ) $3 \mathrm{D}(2 \mathrm{~F}) j=2.5-2 \mathrm{~S} 22 \mathrm{P} 5$ (1s $) j=.0 \quad 15.170$ gypsum 020 (1s) $j=.0 \quad 15.170$ beryl 100 (1S) $j=.0 \quad 15.439$ beryl 100 $\left(2 P^{*}\right) j=.5 \quad 10.206$ ADP 101

$(2 P *) j=1.5 \quad 10.275$ ADP 101

$\left(2 P^{*}\right) j=1.5 \quad 10.290$ ADP 101

$\left(2 P^{\star}\right) j=1.5 \quad 10.373 \quad$ ADP 101

$(2 P *) j=1.5$ (20.406 ADP 101

$\left(2 P^{*}\right) j=.5 \quad 10.428$ ADP 101

$\left(2 P^{*}\right) j=1.5 \quad 10.477 \quad$ ADP 101

$(2 P *) j=1.5 \quad 10.568$ ADP 101

$(2 \mathrm{P} *) \mathrm{j}=$

$\begin{array}{lll}\left(2 P^{*}\right) j=1.5 & 10.583 & \text { ADP } 101 \\ \left(2 P^{*}\right) j=1.5 & 12.155 & \text { TAP } 100\end{array}$

$\left(2 P^{*}\right) j=1.5 \quad 12.168$ TAP 100

$\left(2 P^{*}\right) j=5.512 .193$ TAP 100

$(2 P *) j=1.5 \quad 12.212$ TAP 100

$\left(2 P^{\star}\right) j=.5 \quad 12.224$ TAP 100

$\left(2 P^{*}\right) j=1.5 \quad 12.238$ TAP 100

(2P*) j=1.5 12.281 TAP 100

$\left(2 P^{*}\right) j=1.5 \quad 12.281 \quad \operatorname{RAP} 100$

$\langle 2 P * j=.5 \quad 12.300$ TAP 100

$\left(2 P^{*}\right) j=\begin{array}{lll}5 & 12.300 \quad \text { RAP } 100\end{array}$

$\left(2 P^{*}\right) j=.5 \quad 12.338$ TAP 100

$\left(2 P^{*}\right) j=.5 \quad 12.338$ RAP 100

$\left(2 P^{*}\right) j=1.5 \quad 12.700$ TAP 100

(2P*) $j=1.5 \quad 12.700$ RAP 100

$\left(2 P^{*}\right) j=1.5 \quad 12.700$ KAP 100

$\left(2 P^{*}\right) j=.5 \quad 12.828$ TAP 100

$(2 P *) j=.512 .828$ RAP 100

$\left(2 P^{*}\right) j=.512 .828$ KAP 100

$\left(2 P^{*}\right) j=1.5 \quad 12.876$ TAP 100

$\left(2 P^{*}\right) j=1.5 \quad 12.876 \quad \operatorname{RAP} 100$

$\left(2 P^{*}\right) j=1.5 \quad 12.876 \quad \mathrm{KAP} 100$

$\left(2 P^{*}\right) j=1.5 \quad 12.890$ RAP 100

$\left(2 P^{\star}\right) j=1.5 \quad 12.890 \quad$ KAP 100

(2P*) $j=1.5 \quad 12.942$ RAP 100

$\left(2 P^{*}\right) j=1.5 \quad 12.942 \quad \operatorname{KAP} 100$

$\left(2 P^{*}\right) j=1.5 \quad 12.985$ RAP 100

$(2 P *) j=1.5 \quad 12.985 \quad K A P 100$

$\left(2 P^{*}\right) j=1.5 \quad 13.084 \quad \mathrm{KAP} 100$

$\left(2 P^{*}\right) j=.5 \quad 13.097$ KAP 100

$\left(2 P^{*}\right) j=1.5 \quad 13.123$ KAP 100

$\left(2 P^{*}\right) j=.5 \quad 13.151$ KAP 100

$\left(2 P^{*}\right) j=1.5 \quad 13.183$ KAP 100

$\left(2 P^{*}\right) j=.5 \quad 13.192$ KAP 100

$(2 p *) j=1.5 \quad 13.240 \quad \mathrm{KAP} 100$
$15.185 \quad 87.453$

$15.954 \quad 71.963$

$15.954 \quad 75.402$

$10.640 \quad 73.579$

$10.640 \quad 74.949$

$10.640 \quad 75.263$

$10.640 \quad 77.137$

$10.640 \quad 77.961$

$10.640 \quad 78.543$

$10.640 \quad 79.958$

$10.640 \quad 83.331$

$10.640 \quad 84.067$

$25.763 \quad 70.665$

$25.763 \quad 70.841$

$25.763 \quad 71.183$

$25.763 \quad 71.446$

$25.763 \quad 71.615$

$25.763 \quad 71.813$

$25.763 \quad 72.436$

$26.116 \quad 70.135$

$25.763 \quad 72.719$

$\begin{array}{ll}26.116 & 70.382\end{array}$

$25.763 \quad 73.297$

$26.116 \quad 70.885$

$25.763 \quad 80.370$

$26.116 \quad 76.553$

$26.634 \quad 72.491$

$25.763 \quad 84.776$

$26.116 \quad 79.230$

$26.634 \quad 74.425$

$25.763 \quad 88.326$

$26.116 \quad 80.423$

$26.634 \quad 75.214$

$26.116 \quad 80.799$

$26.634 \quad 75.452$

$26.116 \quad 82.357$

$26.634 \quad 76.371$

$26.116 \quad 83.939$

$26.634 \quad 77.179$

$26.634 \quad 79.266$

26.63479 .571

$26.634 \quad 80.208$

$26.634 \quad 80.944$

$26.634 \quad 81.865$

$26.634 \quad 82.143$

$26.634 \quad 83.836$ 
Co19 F-1ike 2S2 2P4 (3P) 3D (4P) $j=1.5-2 S 22 \mathrm{P} 5$

Co19 $F$-like 2S2 2P4 (3P) $3 D(4 P) j=.5-2 S 22 P 5$

Co19 $F$-like 2S2 2P4 (3P) $3 D(2 P) j=1.5-2 S 22 P 5$

Co19 F-like 2S2 2P4 ( 3P ) 3S $(2 P) j=.5-2 S 22 P 5$

Co19 F-like 2S2 2P4 (3P) ) 3S (4P) $j=1.5-2 S 2$ 2P5

Co19 F-1ike 2S2 2P4 ( ID) 3S (2D) $j=1.5-2 S 22 P 5$

Co19 F -like 2S2 2P4 (3P) 3S (2P) $j=1.5-2 S 2$ 2P5

Co19 $F$-like $2 S 22 \mathrm{P} 4(3 \mathrm{P})$ ) $3 \mathrm{~S}(2 \mathrm{P}) j=.5-2 \mathrm{~S} 2 \mathrm{PP} 5$

Co19 $F$-like 2S2 2P4 (3P) $3 S(4 P) j=2.5-2 S 22 P 5$

Co19 F - like 2S2 2P4 ( 3P ) 3S (2P) $j=1.5$ - 2S2 $2 \mathrm{PS}$

Co20 o-1ike 2S2 2P3 ( 2P*) $4 \mathrm{D}\left(3 \mathrm{P}^{*}\right) j=1.0-2 \mathrm{~S} 22 \mathrm{P} 4$

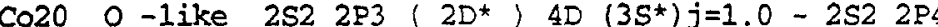

Co20 O-like 2S2 2P3 (2D*) 4D (3D*) $j=3.0-2 S 22 \mathrm{PA}$

Co20 O-like 2S2 $2 P 3\left(2 D^{*}\right) 4 D\left(3 D^{*}\right) j=3.0-2 S 22 P 4$

Co20 O-like 2S2 2P3 (2D*) 4D $\left(3 F^{*}\right) j=3.0-2 S 22 P 4$

Co20 O-like 2S2 2P3 (2D*) $4 \mathrm{D}\left(3 D^{*}\right) j=2.0-2 \mathrm{~S} 22 \mathrm{P}$

Co20 o-like 2S2 $2 P^{2}\left(4 S^{*}\right) 4 D\left(3 D^{*}\right) j=3.0-2 S 22 P 4$

Co20 o-like 2S2 2P3 ( $\left.4 S^{*}\right) 4 D\left(3 D^{*}\right) j=3.0-2 S 22 P 4$

Co20 o-1ike 2S2 2P3 (2D*) $4 \mathrm{D}\left(3 \mathrm{D}^{*}\right) j=2.0-2 \mathrm{~S} 22 \mathrm{P} 4$

Co20 0 -like 2S2 2P3 ( $\left.4 S^{*}\right) 4 D\left(3 D^{*}\right) j=1.0-2 S 2$ 2P4

Co20 O-like 2S2 2P3 ( $\left.4 S^{*}\right) 4 D\left(3 D^{*}\right) j=1.0-2 S 22 \mathrm{PA}$

Co20 O-like 2S2 2P3 (2P*) $3 D\left(3 P^{*}\right) j=1.0-2 S 22 P^{*}$

Co20 O-like 2S2 2P3 ( 2P*) 3D $\left(3 \mathrm{P}^{*}\right) j=2.0-2 \mathrm{~S} 2 \quad 2 \mathrm{P} 4$

Co20 o-like 2S2 2P3 ( 2D*) 3D $\left(1 F^{*}\right) j=3.0-2 S 22 P 4$

Co20 o-like 2S2 2P3 (2P*) 3D $\left(3 P^{*}\right) j=.0-2 S 22 P 4$

Co20 0 -like 2S2 2P3 (2P*) $3 D\left(3 P^{*}\right) j=0-2 S 22 P 4$

Co20 0 -1ike $2 S 22 \mathrm{P} 3\left(2 \mathrm{P}^{*}\right) 3 \mathrm{D}\left(3 \mathrm{P}^{*}\right) j=1.0-2 \mathrm{~S} 2 \quad 2 \mathrm{P} 4$

Co20 o -1ike 2S2 2P3 ( $\left.2 P^{*}\right) 3 D\left(3 P^{*}\right) j=1.0-2 S 22 P 4$

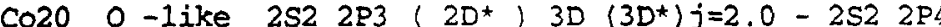

Co20 O-like 2S2 2P3 (2D*) 3D $\left(3 D^{*}\right) j=2.0-2 S 22 P 4$

Co20 0 -1ike 2S2 $2 \mathrm{P} 3\left(2 D^{*}\right) 3 D\left(3 D^{*}\right) j=3.0-2 \mathrm{~S} 22 \mathrm{PP}$

Co20 0 -like 2S2 2P3 ( $\left.2 D^{*}\right) \quad 3 D\left(3 D^{*}\right) j=3.0-2 S 22 P 4$

Co20 o-like 2S2 2P3 (2D*) 3D $\left(3 D^{*}\right) j=3.0-2 S 22 P 4$

Co20 0 -like 2S2 $2 P^{2}\left(2 D^{*}\right) 3 D\left(3 D^{*}\right) j=3.0-2 S 22 P 4$

Co20 o -like 2S2 2P3 (2P*) 3D $\left(3 P^{*}\right) j=2.0-2 S 22 P 4$

Co20 o-1ike 2S2 2P3 (2P*) 3D $\left(3 P^{*}\right) j=2.0-2 S 22 P^{*}$

Co20 O-like 2S2 2P3 ( 2P*) 3D (3P*) j=1.0 - 2S2 2P4

Co20 O-like 2S2 2P3 ( 2P*) 3D (3P*) j=1.0 - 2S2 2P4

Co20 o-like 2S2 2P3 ( 2D*) 3D (3D*) j=2.0 - 2S2 $2 D^{*} 4$

Co20 0 -like 2S2 2P3 ( 2D*) $3 D\left(3 D^{*}\right) j=2.0-2 S 22 P 4$

Co20 0 -like 2S2 2P3 ( $\left.2 P^{*}\right) \quad 3 D\left(3 P^{*}\right) j=2.0-2 S 22 \mathrm{PA}$

Co20 o -1ike 2S2 2P3 (2P*) 3D (3P*) j=2.0 - 2S2 $2 P^{*} 4$

Co20 0 -1ike 2S2 2P3 ( 2P*) 3D (1P*) j=1.0 - 2S2 $2 P^{*} 4$

Co20 0 -1ike 2S2 2P3 ( 2P*) 3D $\left(1 P^{*}\right) j=1.0-2 S 22 P 4$

$\operatorname{co} 20$ o-like 2S2 2P3 (2P*) 3D (1P*)j=1.0 - 2S2 2P4

Co20 0 -like 2S2 $2 P^{2}\left(4 S^{*}\right) \quad 3 D\left(3 D^{*}\right) j=3.0-2 S 22 P 4$ $\left(2 P^{*}\right) j=1.5 \quad 13.289$ KAP 100

$\left(2 P^{*}\right) j=.5 \quad 13.314$ KAP 100

$\left(2 P^{*}\right) j=1.5 \quad 14.303 \quad$ gypsum 020

$\left(2 P^{*}\right) j=1.5 \quad 14.355$

$\left(2 P^{*}\right) j=.5 \quad 14.423$

$\left(2 P^{*}\right) j=1.5 \quad 14.534$

$\left(2 F^{*}\right) j=.5 \quad 14.557$

$\left(2 P^{*}\right) j=1.5 \quad 14.594$

$\left(2 p^{*}\right) j=15$

(3P) $j=1$.

14.794

(3P) $j=2.0$

9.633

(3P) $j=2$.

9.681

(3P) $j=2.0$

.694

9.694

(3P $j=1.0$

9.828

( $3 P) ~ j=2.0$

9.856

9.856

9.900

9.924

(1D) $j=2.0$

10.020

(3P) $j=2.0$

(3P) $j=1.0$

12.144

(3P) $j=2$.

12.258

(3P) $j=1.0$

12.281

(3P) $j=1$.

12.281

(3P) $j=1$.

(3P) $j=1$.

(3P) $j=2.0$

12.300

12.300

(3P) $j=2.0 \quad 12.331$

(3P) $j=2$.

12.348

(3P) $j=2.0$

(3P) $j=2.0$

12.348

(3P) $j=2$.

12.348

(3P) $j=1$.

(3P) $j=1.0$

12.382

(1D) $j=2$

12.382

(1D) $j=2.0$

12.420

. $0 \quad 12.493$

(3P) $j=1.0 \quad 12.493$

(1D) $j=2.0$

12.513

(1D) $j=2.0 \quad 12.513$

(1s) $j=$.

12.551

(1s) $j=$

(1S) $j=.0$

(3P) $j=2.0 \quad 12.606 \quad$ TAP 100
$26.634 \quad 84.605$

$26.634 \quad 86.284$

$26.634 \quad 88.784$

$15.185 \quad 70.376$

$15.185 \quad 70.969$

$15.185 \quad 71.772$

$15.185 \quad 73.162$

$15.185 \quad 73.464$

$15.185 \quad 73.962$

$15.185 \quad 76.970$

$19.942 \quad 75.039$

$19.942 \quad 76.148$

$19.942 \quad 76.463$

$19.942 \quad 76.463$

$19.942 \quad 77.697$

$19.942 \quad 80.285$

$19.942 \quad 81.290$

$19.942 \quad 81.290$

$19.942 \quad 83.158$

$19.942 \quad 84.435$

$10.640 \quad 70.344$

$25.763 \quad 70.518$

$25.763 \quad 71.813$

$25.763 \quad 72.101$

$25.763 \quad 72.436$

$26.116 \quad 70.135$

$25.763 \quad 72.719$

$26.116 \quad 70.382$

$25.763 \quad 73.189$

$26.116 \quad 70.791$

$25.763 \quad 73.453$

$26.116 \quad 71.019$

$25.763 \quad 73.453$

$26.116 \quad 71.019$

$25.763 \quad 73.992$

$26.116 \quad 71.483$

$25.763 \quad 74.617$

$26.116 \quad 72.016$

$25.763 \quad 75.893$

$26.116 \quad 73.084$

$25.763 \quad 76.262$

$26.116 \quad 73.388$

$25.763 \quad 76.993$

$26.116 \quad 73.982$

$26.634 \quad 70.472$

$25.763 \quad 78.129$

Page 83 


\begin{tabular}{|c|c|c|c|c|c|c|c|c|c|}
\hline $\mathrm{CO} 20$ & 0 -like & $2 \$ 2$ & $2 \mathrm{P} 3$ & $\left(4 S^{\star}\right)$ & $3 D$ & $\left(3 D^{*}\right) j=3.0$ & $-2 s 2$ & $2 P 4$ & \\
\hline Co2O & o-like & $2 \$ 2$ & $2 \mathrm{P} 3$ & $\left(4 S^{\star}\right)$ & $3 D$ & $\left(3 D^{*}\right) j=3.0$ & $-2 s 2$ & $2 P 4$ & \\
\hline Co2O & o-like & 252 & $2 \mathrm{P} 3$ & $\left(45^{\star}\right)$ & $3 D$ & $\left(3 D^{*}\right) j=2.0$ & $-2 s 2$ & $2 \mathrm{P} 4$ & \\
\hline $\operatorname{Co} 20$ & 0 -like & $2 S 2$ & $2 \mathrm{P} 3$ & $\left(4 S^{*}\right)$ & $3 D$ & $\left(3 D^{*}\right) j=2.0$ & $-2 s 2$ & $2 P 4$ & \\
\hline $\mathrm{CO} 20$ & 0 -like & $2 \$ 2$ & $2 \mathrm{P} 3$ & $\left(4 S^{*}\right)$ & $3 D$ & $\left(3 D^{*}\right) j=2.0$ & $-2 s 2$ & $2 \mathrm{P} 4$ & \\
\hline $\operatorname{Co} 20$ & 0 -like & $2 \$ 2$ & $2 P 3$ & $(2 \mathrm{P} *)$ & $3 S$ & $\left(3 P^{*}\right) j=1.0$ & $-2 s 2$ & $2 P 4$ & \\
\hline $\mathrm{Co} 20$ & o-like & $2 \$ 2$ & $2 \mathrm{P} 3$ & $\left(2 P^{*}\right)$ & $3 S$ & $\left(3 p^{*}\right) j=2.0$ & $-2 s 2$ & $2 P 4$ & \\
\hline $\mathrm{CO} 20$ & 0 -like & $2 \$ 2$ & $2 \mathrm{P} 3$ & $\left(2 D^{*}\right)$ & 35 & $\left(1 D^{*}\right) j=2.0$ & $-2 s 2$ & $2 P 4$ & \\
\hline $\operatorname{Co} 24$ & Be-like & $1 S 2$ & $2 P$ & & $3 P$ & $(3 S) j=1.0$ & $-1 s 2$ & $2 S$ & $2 P$ \\
\hline Co24 & Be-like & $1 S 2$ & $2 \mathrm{P}$ & & $3 S$ & $(3 D) j=3.0$ & $-1 s 2$ & $2 S$ & $2 \mathrm{P}$ \\
\hline $\operatorname{co2} 24$ & Be-like & 152 & $2 \mathrm{P}$ & & $3 P$ & $(1 \mathrm{P}) j=1.0$ & $-1 s 2$ & $2 s$ & $2 P$ \\
\hline $\mathrm{CO} 24$ & Be-like & $1 S 2$ & $2 s$ & $3 P$ & & $\left(3 P^{*}\right) j=1.0$ & $-1 s 2$ & $2 S 2$ & \\
\hline Co24 & Be-like & 152 & $2 P$ & & $3 s$ & $(3 D) j=1.0$ & -152 & $2 S$ & $2 P$ \\
\hline $\mathrm{CO} 24$ & Be-like & $1 S 2$ & $2 S$ & & 35 & $(1 S) j=.0$ & -152 & $2 S$ & $2 \mathrm{P}$ \\
\hline $\operatorname{Co} 24$ & Be-like & 1,52 & $2 \mathrm{P}$ & & $3 P$ & $(10) j=2.0$ & -152 & $2 S$ & $2 \mathrm{P}$ \\
\hline Co24 & Be-like & $1 S 2$ & $2 S$ & $3 D$ & & $(3 D) j=2.0$ & $-1 s 2$ & $2 s$ & $2 \mathrm{P}$ \\
\hline Co24 & Be-like & 1.52 & $2 P$ & & $3 P$ & $(1 P) j=1.0$ & $-1 s 2$ & $2 S$ & $2 P$ \\
\hline $\operatorname{Co} 24$ & Be-like & $1 S 2$ & $2 \mathrm{P}$ & & $3 D$ & $\left(3 P^{*}\right) j=.0$ & $-1 s 2$ & & $2 \mathrm{P} 2$ \\
\hline $\operatorname{Co} 24$ & Be-like & 152 & $2 S$ & 30 & & $(3 D) j=3.0$ & $-1 s 2$ & $2 S$ & $2 P$ \\
\hline $\mathrm{Co} 24$ & Be-like & $1 \mathrm{~S} 2$ & $2 S$ & $3 D$ & & $(3 D) j=2.0$ & $-1 s 2$ & $2 S$ & $2 \mathrm{P}$ \\
\hline $\operatorname{Co24}$ & Be-like & 152 & $2 \mathrm{P}$ & & $3 D$ & $\left(1 D^{*}\right) j=2.0$ & $-1 s 2$ & & $2 \mathrm{P} 2$ \\
\hline Co24 & Be-like & 152 & $2 \mathrm{P}$ & & $3 D$ & $\left(3 P^{\star}\right) j=2.0$ & $-1 s 2$ & & $2 \mathrm{P} 2$ \\
\hline $\operatorname{co2} 25$ & Li-1ike & $1 \mathrm{S2}$ & $4 P$ & & & $\left(2 P^{\star}\right) j=1.5$ & $-1 s 2$ & $2 S$ & \\
\hline Co25 & Li-1ike & $1 \$ 2$ & $4 \mathrm{P}$ & & & $\left(2 P^{*}\right) j=1.5$ & $-1 s 2$ & $2 S$ & \\
\hline $\operatorname{co} 25$ & Li-1ike & $1 S 2$ & $4 \mathrm{P}$ & & & $\left(2 P^{\star}\right) j=.5$ & $-1 s 2$ & $2 S$ & \\
\hline Co25 & Li-like & $1 S 2$ & $4 \mathrm{P}$ & & & $\left(2 P^{*}\right) j=.5$ & $-1 s 2$ & $2 S$ & \\
\hline Co25 & Li-like & $1 S 2$ & $4 \mathrm{D}$ & & & (2D) $j=1.5$ & $-1 s 2$ & $2 \mathrm{P}$ & \\
\hline $\operatorname{Co} 25$ & Li-like & $1 S 2$ & $4 D$ & & & (2D) $j=1.5$ & $-1 s 2$ & $2 P$ & \\
\hline $\operatorname{Co} 25$ & Li-like & 152 & $4 \mathrm{~S}$ & & & (2s) $j=.5$ & -152 & $2 P$ & \\
\hline Co25 & li-like & $1 \mathrm{~s} 2$ & $4 D$ & & & (2D) $j=2.5$ & $-1 s 2$ & $2 P$ & \\
\hline $\operatorname{co} 25$ & Li-like & $1 S 2$ & $4 S$ & & & (2s) $j=.5$ & $-1 s 2$ & $2 P$ & \\
\hline Co25 & Li-like & 152 & $3 P$ & & & $\left(2 P^{*}\right) j=1.5$ & $-1 s 2$ & 2.5 & \\
\hline Co25 & Li-like & $1 S 2$ & $3 P$ & & & $\left(2 P^{\star}\right) j=.5$ & -152 & $2 S$ & \\
\hline Co25 & Li-1ike & 152 & $3 D$ & & & (2D) $j=1.5$ & $-1 s 2$ & $2 \mathrm{P}$ & \\
\hline $\operatorname{co} 25$ & Li-1ike & $1 S 2$ & $3 D$ & & & $(2 D) j=2.5$ & $-1 s 2$ & $2 P$ & \\
\hline Co25 & Li-like & $1 S 2$ & $3 s$ & & & $(25) j=.5$ & -152 & $2 P$ & \\
\hline $\operatorname{co} 25$ & Li-like & 152 & $3 s$ & & & (2s) $j=.5$ & -152 & $2 \mathrm{P}$ & \\
\hline $\operatorname{Co} 26$ & He-1ike & 15 & $5 P$ & & & $\left(1 P^{\star}\right) j=1.0$ & $-1 s 2$ & & \\
\hline $\mathrm{Co26}$ & He-like & $1 S$ & $5 P$ & & & $\left(1 P^{*}\right) j=1.0$ & $-1 s 2$ & & \\
\hline Co26. & He-like & $1 \mathrm{~S}$ & $5 p$ & & & $\left(1 P^{\star}\right) j=1.0$ & $-1 s 2$ & & \\
\hline $\operatorname{Co2} 6$ & He-like & $1 S$ & $5 P$ & & & $\left(1 P^{*}\right) j=1.0$ & $-1 s 2$ & & \\
\hline $\operatorname{co} 26$ & He-like & 15 & $5 P$ & & & $\left(1 P^{\star}\right) j=1.0$ & $-1 \$ 2$ & & \\
\hline $\operatorname{co} 26$ & He-like & $1 \mathrm{~S}$ & $5 P$ & & & $\left(1 P^{\star}\right) j=1.0$ & $-1 S 2$ & & \\
\hline Co26 & He-like & $1 \mathrm{~S}$ & $5 P$ & & & $\left(1 P^{*}\right) j=1.0$ & $-1 s 2$ & & \\
\hline Co26 & He-like & $1 S$ & $5 P$ & & & $\left(3 P^{*}\right) j=1.0$ & -152 & & \\
\hline $\operatorname{co} 26$ & He-like & 1s & $5 P$ & & & $\left(3 P^{*}\right) j=1.0$ & $-1 S 2$ & & \\
\hline
\end{tabular}

(3P) $j=2.0 \quad 12.606 \quad$ RAP 100 (3P $) j=2.0 \quad 12.606$ KAP 100 (3P) $j=2.0 \quad 12.628$ TAP 100 (3P $) j=2.0 \quad 12.628$ RAP 100 (3P) $j=2.0 \quad 12.628 \quad K A P \quad 100$ (3P) $j=2.0 \quad 13.157 \quad K A P 100$ (3P) $j=1.0 \quad 13.240 \quad \mathrm{KAP} 100$ (3P) $j=2.0 \quad 13.314 \quad \mathrm{KAP} 100$ $\left(3 P^{*}\right) j=2.0 \quad 10.053 \quad$ ADP 101 $\left(3 P^{*}\right) j=2.0 \quad 10.066$ ADP 101 $\left(3 P^{*}\right) j=1.0 \quad 10.080$ ADP 101 (1S) $j=.0 \quad 10.115$ ADP 101 $\left(3 P^{*}\right) j=1.0 \quad 10.156 \quad$ ADP 101 $\left(1 P^{*}\right) j=1.0 \quad 10.182$ ADP 101 $\left(1 P^{*}\right) j=1.0 \quad 10.265$ ADP 101 $\left(3 P^{\star}\right) j=1.0 \quad 10.445$ ADP 101 $\left(1 P^{*}\right) j=1.0 \quad 10.503 \quad$ ADP 101 (3P) $j=1.0 \quad 10.543$ ADP 101 $\left(3 P^{*}\right) j=2.0 \quad 10.552 \quad A D P \quad 101$ $\left(3 P^{*}\right) j=2.0 \quad 10.571$ ADP 101 (3P) $j=1.0 \quad 10.582$ ADP 101 (3P ) $j=2.0 \quad 10.593 \quad$ ADP 101 (2S $) j=.5 \quad 7.364$ InSD 111 (2s) $j=.5 \quad 7.364$ gypsum 020 (2S ) $j=.5 \quad 7.374 \quad$ InSb 111 (2s) $j=.5 \quad 7.374$ gypsum 020 $\left(2 P^{\star}\right) j=.5 \quad 7.583$ gypsum 020 $(2 P *) j=.5 \quad 7.583$ bery 100 $\left(2 P^{*}\right) j=.5 \quad 7.629$ beryl 100 $\left(2 P^{*}\right) j=1.5 \quad 7.667$ beryl 100 $\left(2 P^{\star}\right) j=1.5 \quad 7.718$ beryl 100 (2S) $j=.5 \quad 9.795$ mica 002 (2S ) $j=.5 \quad 9.838$ mica 002 $\left(2 p^{*}\right) j=.5 \quad 10.159$ ADP 101 $\left(2 P^{\star}\right) j=1.5 \quad 10.303 \quad$ ADP 101 $\left(2 P^{*}\right) j=.5 \quad 10.367 \quad$ ADP 101 $\left(2 P^{*}\right) j=1.5 \quad 10.533$ ADP 101

(1S) $j=.0 \quad 1.351$ topaz 303

(1S) $j=.0 \quad 1.351$ corundum 030 (1S ) $j=.0 \quad 1.351$ guartz 203

(1s) $j=.0 \quad 1.351$ topaz 006

(1S $) j=.0 \quad 1.351$ LiF 220

(1s) $j=.0 \quad 1.351$ quartz 200

(1S ) $j=.0 \quad 1.351 \quad \mathrm{NaCl} 200$

(1S) $j=.0 \quad 1.352$ topaz 303

(1s) $j=.0$
$26.116 \quad 74.881$ 26.63471 .193

$25.763 \quad 78.614$

$26.116 \quad 75.255$

$26.634 \quad 71.489$

$26.634 \quad 81.109$

$26.634 \quad 83.836$

$26.634 \quad 88.784$

$10.640 \quad 70.879$

$10.640 \quad 71.094$

$10.640 \quad 71.328$

$10.640 \quad 71.926$

$10.640 \quad 72.652$

$10.640 \quad 73.128$

$10.640 \quad 74.743$

$10.640 \quad 79.014$

$10.640 \quad 80.796$

$10.640 \quad 82.257$

$10.640 \quad 82.626$

$10.640 \quad 83.471$

$10.640 \quad 84.015$

$10.640 \quad 84.613$

$7.481 \quad 79.853$

$15.185 \quad 75.908$

7.481
150.298

$15.185 \quad 76.221$

$15.185 \quad 87.133$

$15.954 \quad 71.917$

$15.954 \quad 73.014$

$15.954 \quad 73.974$

$15.954 \quad 75.360$

$19.942 \quad 79.219$

$19.942 \quad 80.631$

$10.640 \quad 72.706$

$10.640 \quad 75.541$

$10.640 \quad 76.993$

$10.640 \quad 81.868$

$2.712 \quad 85.078$

$2.748 \quad 79.502$

$2.749 \quad 79.390$

$2.795 \quad 75.178$

$2.848 \quad 71.575$

$4.246 \quad 72.659$

$5.641 \quad 73.333$

$2.712 \quad 85.598$

$2.748 \quad 79.733$ 


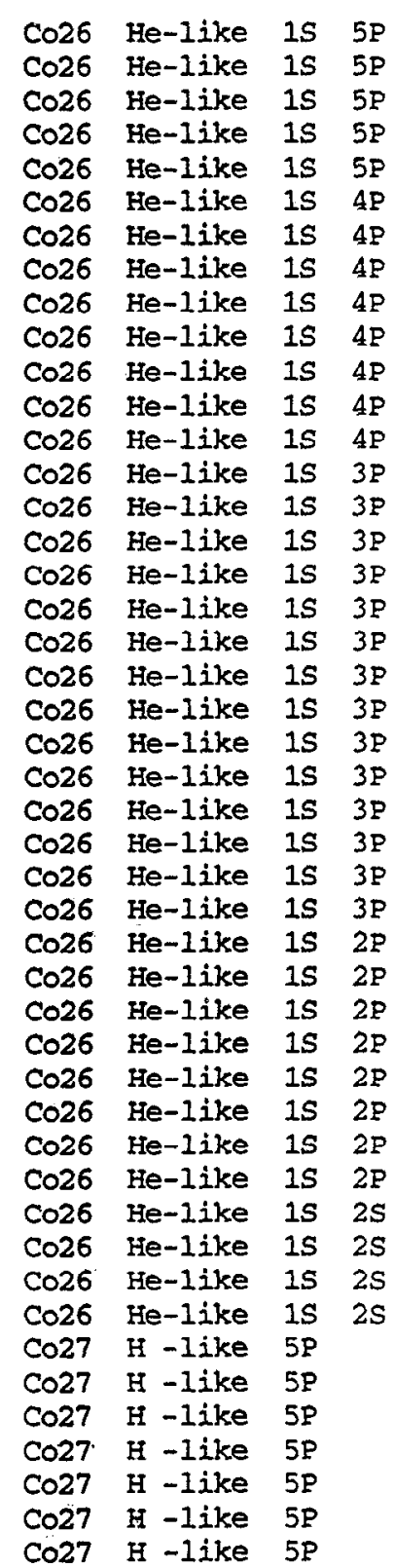

$\left(3 P^{*}\right) j=1.0-152$

$\left(3 P^{\star}\right) j=1.0-152$

$\left(3 P^{*}\right) j=1.0-152$

$(3 P *) j=1.0-1 S 2$

$\left(3 P^{\star}\right) j=1.0-1 S 2$

$\left(1 P^{*}\right) j=1.0-152$

$\left(1 P^{\star}\right) j=1.0-1 S 2$

$\left(1 P^{*}\right) j=1.0-1 S 2$

$\left(1 P^{*}\right) j=1.0-152$

$\left(3 P^{*}\right) j=1.0-152$

$\left(3 P^{\star}\right) j=1.0-1 S 2$

$\left(3 P^{*}\right) j=1.0-152$

$\left(3 P^{\star}\right) j=1.0-152$

$\left(1 P^{*}\right) j=1.0-152$

$\left(1 P^{*}\right) j=1.0-152$

$(1 P *) j=1.0-1 S 2$

$\left(1 P^{\star}\right) j=1.0-1.52$

$\left(1 P^{*}\right) j=1.0-1 S 2$

$\left(1 P^{*}\right) j=1.0-1 S 2$

$\left(1 P^{\star}\right) j=1.0-1 S 2$

$\left(3 P^{\star}\right) j=1.0-1.52$

$\left(3 P^{*}\right) j=1.0-1 S 2$

$\left(3 P^{*}\right) j=1.0-1 S 2$

$\left(3 P^{\star}\right) j=1.0-1 S 2$

$\left(3 P^{*}\right) j=1.0-152$

$\left(3 P^{*}\right) j=1.0-1 \mathrm{~S} 2$

$\left(3 P^{*}\right) j=1.0-1 \mathrm{~S} 2$

$(1 P *) j=1.0-1 S 2$

$\left(1 P^{*}\right) j=1.0-1 \mathrm{~S} 2$

$\left(1 P^{*}\right) j=1.0-1 S 2$

$\left(1 P^{*}\right) j=1.0^{\prime}-1 S 2$

$\left(3 p^{*}\right) j=1.0-1 \mathrm{~s} 2$

$\left(3 P^{*}\right) j=1.0-1 S 2$

$\left(3 P^{*}\right) j=1.0-152$

$\left(3 P^{*}\right) j=1.0-1 S 2$

(3S) $j=1.0-1 S 2$

(3S) $j=1.0-1 s 2$

(3S) $j=1.0-152$

(3s) $j=1.0-1 \mathrm{~s} 2$

$\left(2 P^{*}\right) j=1.5-1 S$

$\left(2 P^{\star}\right) j=1.5-1 S$

$\left(2 P^{*}\right) j=1.5-1 s$

$\left(2 P^{*}\right) j=1.5-1 S$

$\left(2 P^{*}\right) j=1.5-1 S$

$\left(2 P^{*}\right) j=1.5-1 \mathrm{~S}$

$\left(2 P^{\star}\right) j=1.5-1 S$

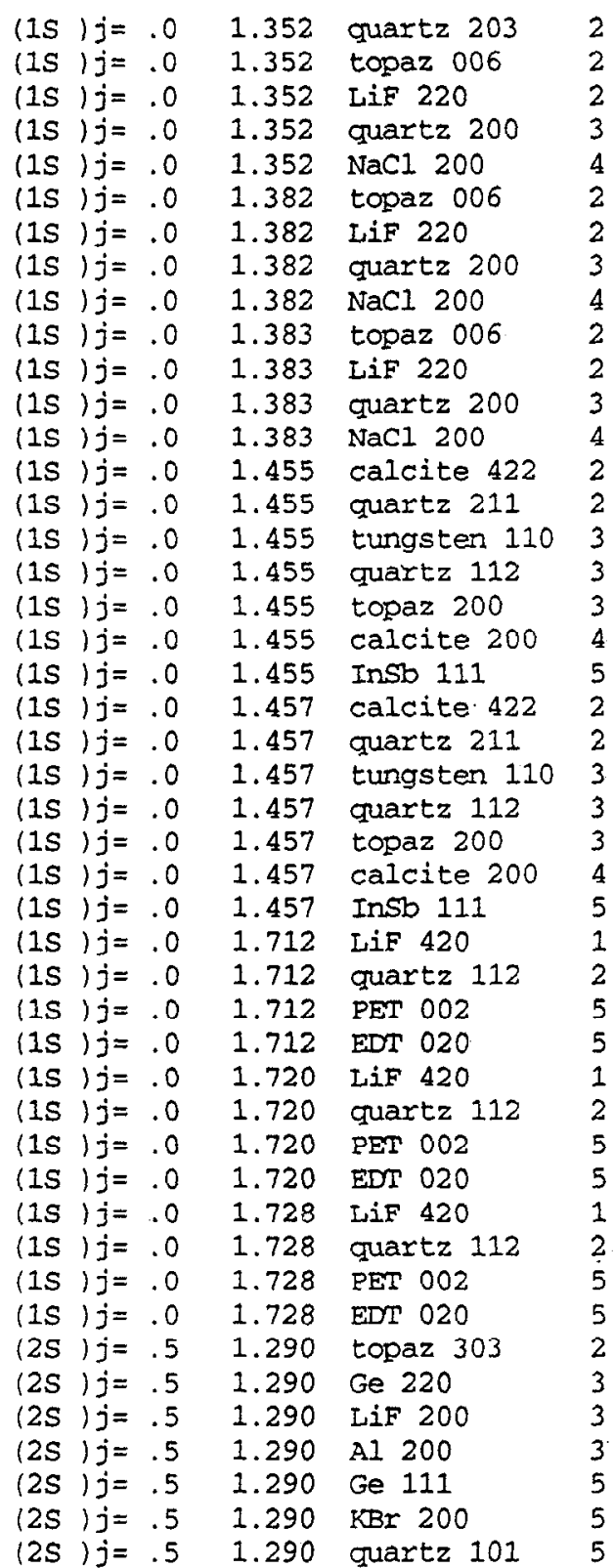

$2.749 \quad 79.619$ $2.795 \quad 75.339$

$2.848 \quad 71.702$

$4.246 \quad 72.795$

$5.641 \quad 73.475$

$2.795 \quad 81.459$

$2.848 \quad 76.050$

$4.246 \quad 77.540$

5.64178 .512

$2.795 \quad 81.739$

$2.848 \quad 76.218$

$4.246 \quad 77.729$

5.64178 .718

3.03473 .563

3.08270 .768

$4.476 \quad 77.213$

$4.564 \quad 73.018$

$4.638 \cdot 70.244$

$6.071 \quad 73.467$

$7.481 \quad 76.523$

$3.034 \quad 73.832$

$3.082 \quad 70.995$

$4.476 \quad 77.565$

4.56473 .278

4.63870 .464

$6.071 \quad 73.734$

7.48176 .856

$1.801 \quad 71.912$

$3.636 \quad 70.338$

$8.742 \quad 78.288$

$8.808 \quad 76.371$

$1.801 \quad 72.751$

3.63671 .102

$8.742 \quad 79.659$

$8.808 \quad 77.524$

$1.801 \quad 73.631$

$3.636 \quad 71.896$

$8.742 \quad 81.239$

8.808 .78 .792

$2.712 \quad 72.050$

$4.000 \quad 75.353$

$4.027 \quad 73.948$

$4.048 \quad 72.946$

$6.532 \quad 80.912$

6.58478 .421

$6.687 \quad 74.700$

Page 85 


\begin{tabular}{|c|c|c|}
\hline Co27 & H -like & $5 P$ \\
\hline Co27 & H-like & $4 \mathrm{P}$ \\
\hline $\mathrm{Co} 27$ & H -like & $4 P$ \\
\hline Co27 & H-like & $4 \mathrm{P}$ \\
\hline Co27 & H-like & $4 \mathrm{P}$ \\
\hline Co27 & H-like & $4 P$ \\
\hline Co27 & H -like & $4 P$ \\
\hline $\operatorname{Co} 27$ & H -like & $4 P$ \\
\hline $\operatorname{Co} 27$ & н-like & $4 P$ \\
\hline Co27 & H -like & $4 P$ \\
\hline Co27 & H -like & $3 P$ \\
\hline $\mathrm{Co} 27$ & H-like & $3 P$ \\
\hline Co27 & H -like & $3 P$ \\
\hline Co27 & H-like & $3 P$ \\
\hline $\operatorname{co} 27$ & H-like & $3 P$ \\
\hline $\operatorname{co} 27$ & H-like & $3 P$ \\
\hline $\mathrm{Co} 27$ & H -like & $3 P$ \\
\hline $\mathrm{Co} 27$ & $\mathrm{H}$-like & $3 P$ \\
\hline $\operatorname{co} 27$ & H-like & $2 \mathrm{P}$ \\
\hline $\mathrm{Co} 27$ & H -like & $2 P$ \\
\hline $\operatorname{co} 27$ & H -like & $2 P$ \\
\hline $\operatorname{Co} 27$ & H -like & $2 P$ \\
\hline $\mathrm{Co} 27$ & $\mathrm{H}$-1ike & $2 P$ \\
\hline Co27 & H -like & $2 P$ \\
\hline $\mathrm{Co27}$ & H-like & $2 P$ \\
\hline $\operatorname{Co} 27$ & H-like & $2 P$ \\
\hline $\operatorname{co2} 7$ & $\mathrm{H}$-like & $2 P$ \\
\hline $\mathrm{Co} 27$ & H-like & $2 P$ \\
\hline Co27 & H -like & $2 P$ \\
\hline Co27 & H-like & $2 P$ \\
\hline $\operatorname{Co} 27$ & H-like & $2 P$ \\
\hline $\operatorname{Co} 27$ & H -like & $2 \mathrm{P}$ \\
\hline $\operatorname{co} 27$ & H-like & $2 P$ \\
\hline Co27 & H -like & 52 \\
\hline $\operatorname{co} 27$ & H-like & $5 D$ \\
\hline Co27 & н-like & $5 D$ \\
\hline Co27 & H -like & $5 I$ \\
\hline $\operatorname{co} 27$ & H -like & $5 D$ \\
\hline Co27 & H -like & $4 P$ \\
\hline Co27 & H -like & $4 P$ \\
\hline Co27 & $\mathrm{H}$-like & $4 P$ \\
\hline Co27 & H -like & $4 P$ \\
\hline $\mathrm{CO} 27$ & H -like & $4 P$ \\
\hline Co27 & H-like & $4 \mathrm{I}$ \\
\hline $\mathrm{Co} 27$ & H -like & \\
\hline $\operatorname{Co} 27$ & H -like & \\
\hline
\end{tabular}

$\left(2 P^{*}\right) j=1.5-1 \mathrm{~S}$ $\left(2 P^{*}\right) j=1.5-1 S$ $\left(2 P^{\star}\right) j=1.5-1 S$ $\left(2 P^{\star}\right) j=1.5-1 S$ $\left(2 P^{*}\right) j=1.5-1 \mathrm{~S}$ $\left(2 P^{*}\right) j=1.5-1 S$ $\left(2 P^{\star}\right) j=1.5-1 S$ $\left(2 P^{*}\right) j=1.5-1 S$ $\left(2 P^{*}\right) j=1.5-1 S$ $\left(2 P^{*}\right) j=1.5-1 S$ $\left(2 P^{*}\right) j=1.5-1 S$ $\left(2 P^{*}\right) j=1.5-1 S$ $\left(2 P^{*}\right) j=1.5-1 \mathrm{~S}$ $\left(2 P^{*}\right) j=1.5-1 S$ $\left(2 P^{*}\right) j=.5-1 S$ $\left(2 P^{*}\right) j=.5-1 S$ $\left(2 P^{*}\right) j=.5-1 S$ $\left(2 P^{*}\right) j=.5-1 S$ $\left(2 P^{*}\right) j=1.5-1 S$ $\left(2 P^{*}\right) j=1.5-1 S$ $\left(2 P^{*}\right) j=1.5-1 S$ $\left(2 P^{*}\right) j=1.5-1 S$ $\left(2 P^{*}\right) j=1.5-1 S$ $\left(2 P^{*}\right) j=1.5-1 S$ $\left(2 P^{*}\right) j=1.5-1 S$ $\left(2 P^{\star}\right) j=1.5-1 S$ $\left(2 P^{*}\right) j=.5-1 S$ $\left(2 P^{*}\right) j=.5-1 S$ $\left(2 P^{*}\right) j=.5-1 S$ $\left(2 P^{*}\right) j=.5-1 S$ $\left(2 P^{*}\right) j=.5-1 S$ $\left(2 P^{\star}\right) j=.5-1 S$ $\left(2 P^{\star}\right) j=.5-1 S$ $\left(2 P^{\star}\right) j=1.5-2 S$ (2D) $j=2.5-2 P$ (2D) $j=2.5-2 P$ (2D) $j=2.5-2 P$ (2D) $j=2.5-2 P$ $\left(2 P^{*}\right) j=1.5-2 S$ $\left(2 P^{*}\right) j=1.5-2 S$ $\left(2 P^{*}\right) j=1.5-2 S$ $\left(2 P^{*}\right) j=1.5-2 S$ $\left(2 P^{*}\right) j=1.5-2 S$ (2D) $j=2.5-2 P$ (2D) $j=2.5-2 P$ (2D) $j=2.5-2 P$

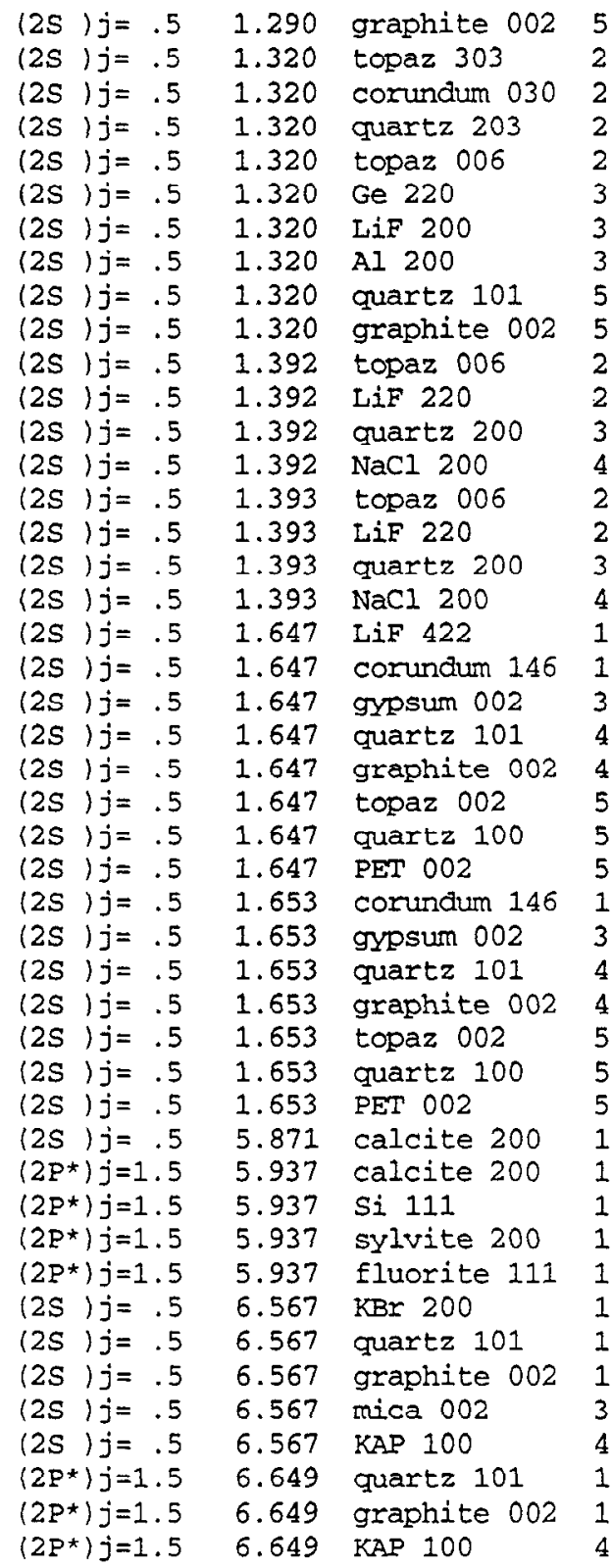

$\begin{array}{ll}6.696 & 74.421\end{array}$

$2.712 \quad 76.768$

$2.748 \quad 73.883$

$2.749 \quad 73.811$

$2.795 \quad 70.829$

$4.000 \quad 81.890$

$4.027 \quad 79.534$

$4.048 \quad 78.031$

$6.687 \quad 80.748$

$6.696 \quad 80.286$

$2.795 \quad 84.915$

$2.848 \quad 77.830$

$4.246 \quad 79.582$

$5.641 \quad 80.772$

$2.795 \quad 85.401$

$2.848 \quad 78.023$

$4.246 \quad 79.808$

$5.641 \quad 81.029$

1.65285 .541

$1.660 \quad 82.825$

$4.990 \quad 81.964$

$6.687 \quad 80.129$

6.69679 .695

$8.374 \quad 79.546$

$8.512 \quad 75.343$

8.74270 .391

$1.660 \quad 84.736$

$4.990 \quad 83.610$

$6.687 \quad 81.411$

$6.696 \quad 80.915$

$8.374 \quad 80.745$

$8.512 \quad 76.164$

$8.742 \quad 70.985$

$6.071 \quad 75.252$

$6.071 \quad 77.940$

$6.271 \quad 71.216$

$6.292 \quad 70.662$

$\begin{array}{lll}6.308 & 70.252\end{array}$

$6.584 \quad 85.882$

$6.687 \quad 79.129$

$6.696 \quad 78.735$

$19.942 \quad 81.083$

$26.634 \quad 80.490$

$6.687 \quad 83.889$

$6.696 \quad 83.207$

$26.634 \quad 86.939$ 


\begin{tabular}{|c|c|c|c|c|c|c|c|c|}
\hline $\operatorname{Co} 27$ & $H$-like & \multicolumn{3}{|l|}{$3 P$} & \multicolumn{4}{|c|}{$\left(2 P^{*}\right) j=1.5-2 S$} \\
\hline $\mathrm{Ni}$ & & $k-a$ & lpha! & & & & & \\
\hline $\mathrm{Ni}$ & & $K-a$ & Iphal & & & & & \\
\hline $\mathrm{Ni}$ & & $K-a$ & Iphal & & & & & \\
\hline $\mathrm{Ni}$ & & $\mathrm{K}-\mathrm{a}$ & lphal & & & & & \\
\hline $\mathrm{Ni}$ & & $\mathrm{k}-\mathrm{a}$ & lphal & & & & & \\
\hline $\mathrm{Ni}$ & & $\mathrm{K}-\mathrm{a}$ & lphal & & & & & \\
\hline $\mathrm{Ni}$ & & $K-a$ & 15 & & & & & \\
\hline $\mathrm{Ni}$ & & $\mathrm{K}-\mathrm{a}$ & Ipha! & & & & & \\
\hline $\mathrm{Ni} 17$ & Mg-Iike & $2 P 5$ & 352 & & $3 D$ & *) $j=1.0$ & $-2 P 6$ & $3 \$ 2$ \\
\hline $\mathrm{Ni17}$ & Mg-iike & $2 \mathrm{P} 5$ & 352 & & $3 D$ & $\left(1 p^{*}\right) j=1.0$ & $-2 P 6$ & 352 \\
\hline $\mathrm{Ni17}$ & Mg-1ike & $2 P 5$ & 352 & & $3 D$ & $\left(1 p^{*}\right) j=1.0$ & $-2 P 6$ & 352 \\
\hline Ni18 & Na-1ike & $2 P 5$ & 352 & & & $\left(2 p^{*}\right) j=1.5$ & $-2 P 6$ & 35 \\
\hline Ni.18 & $\mathrm{Na}-1 i k e$ & $2 \mathrm{P} 6$ & $8 \mathrm{D}$ & & & (2D) $j=1.5$ & $-2 \mathrm{P6}$ & $3 P$ \\
\hline $\mathrm{Ni18}$ & Na-like & $2 \mathrm{P} 6$ & $8 D$ & & & $(2 D)$ & $-2 P 6$ & $3 P$ \\
\hline $\mathrm{Ni18}$ & Na-iike & $2 \mathrm{P} 5$ & $8 D$ & & & $(2 D)$ & $-2 P 6$ & $3 P$ \\
\hline Ni18 & Na-1ike & $2 \mathrm{P} 6$ & $8 D$ & & & $(2 D)=$ & $-2 P 6$ & $3 P$ \\
\hline Ni18 & Na-1ike & $2 P 6$ & $8 D$ & & & $(2 D) j=2.5$ & $-2 P 6$ & $3 P$ \\
\hline Ni18 & Na-like & $2 \mathrm{P} 6$ & $6 \mathrm{P}$ & & & $(2 \mathrm{P} *) j=1.5$ & $-2 P 6$ & $3 S$ \\
\hline Ni18 & $\mathrm{Na}-$ & $2 P 6$ & $6 \mathrm{P}$ & & & $\left(2 P^{*}\right) j=1.5$ & $-2 F 6$ & 35 \\
\hline $\mathrm{Ni} 18$ & $\mathrm{Na}-$ & $2 \mathrm{P} 6$ & $6 P$ & & & $\left(2 p^{*}\right) j=.5$ & $-2 P 6$ & $3 S$ \\
\hline $\mathrm{Ni18}$ & $\mathrm{Na}-$ & $2 P 6$ & $6 P$ & & & $\left(2 P^{*}\right) j=.5$ & $-2 P 6$ & $3 S$ \\
\hline Ni18 & $\mathrm{Na}-1 i k e$ & 2P6 & D7 & & & $(2 D) j=2.5$ & $-2 P 6$ & $3 P$ \\
\hline Ni19 & $\mathrm{Ne}-1$ & 252 & $2 P 5$ & $2 \mathrm{P} \star 2)$ & $8 D$ & $\left(23^{*}\right) j=1.0$ & $-2 s 2$ & $2 P 6$ \\
\hline $\mathrm{Ni19}$ & $\mathrm{Ne}-1$ & $2 \mathrm{~s} 2$ & $2 P 5$ & $2 F$ & $8 D$ & $\left(23^{*}\right) j=1.0$ & $-2 S 2$ & $2 P 6$ \\
\hline Ni19 & $\mathrm{Ne}-$ & $2 \$ 2$ & $2 P 5$ & $2 F$ & $8 D$ & *) $j=1.0$ & $-2 s 2$ & $2 \mathrm{P} 6$ \\
\hline Ni19 & $\mathrm{Ne}-1$ & 252 & $2 \mathrm{P} 5$ & $(2 P * 2)$ & $8 D$ & $=1.0$ & $-2 s 2$ & $2 P 6$ \\
\hline $\mathrm{Ni} 19$ & Ne-1ike & $2 \mathrm{~S} 2$ & $2 P 5$ & $2 P * 2)$ & 80 & $=1.0$ & $-2 s 2$ & $2 P 6$ \\
\hline Ni19 & Ne-like & $2 \mathrm{~s} 2$ & $2 P 5$ & $2 \mathrm{P} * 2)$ & $8 D$ & $=1.0$ & $-2 s 2$ & $2 P 6$ \\
\hline $\mathrm{Ni} 19$ & $\mathrm{Ne}-1$ & $2 \mathrm{~s} 2$ & $2 P 5$ & $(2 P * 1)$ & $7 D$ & $=1.0$ & $-2 s 2$ & $2 \mathrm{P} 6$ \\
\hline Ni19 & Ne-like & $2 \mathrm{~s} 2$ & $2 P 5$ & $(2 P * 1)$ & $7 D$ & $j j=1.0$ & $-2 s 2$ & $2 P 6$ \\
\hline Ni19 & ike & $2 \mathrm{~s} 2$ & $2 P 5$ & $(2 F$ & $7 D$ & $=1.0$ & $-2 s 2$ & $2 P 6$ \\
\hline Ni19 & $\mathrm{Ne}-$ & $2 \mathrm{~s} 2$ & $2 \mathrm{P5}$ & $2 p * 1)$ & $7 D$ & $=1.0$ & $-2 s 2$ & $2 P 6$ \\
\hline Ni19 & Ne- & $2 \$ 2$ & $2 P 5$ & $2 P * 1)$ & $7 D$ & $=1.0$ & $-2 S 2$ & $2 \mathrm{P} 6$ \\
\hline Ni19 & Ne-like & $2 s 2$ & $2 \mathrm{P} 5$ & $(2 P * 2)$ & $7 D$ & $j=1.0$ & $-2 s 2$ & $2 \mathrm{P} 6$ \\
\hline Ni19 & Ne-like & $2 \mathrm{~s} 2$ & $2 P 5$ & $(2 P \star 2)$ & $7 D$ & 1.0 & $-2 s 2$ & $2 \mathrm{P} 6$ \\
\hline Ni19 & $\mathrm{Ne}-$ & $2 \$ 2$ & $2 \mathrm{P} 5$ & $2 P \star 2)$ & $7 D$ & 1.0 & $-2 s 2$ & 296 \\
\hline Ni19 & $\mathrm{Ne}-$ & $2 \mathrm{~S} 2$ & $2 \mathrm{P} 5$ & $2 P * 2)$ & $7 D$ & $=1.0$ & $-2 \$ 2$ & $2 P 6$ \\
\hline Ni19 & $\mathrm{Ne}-$ & $2 \mathrm{~s} 2$ & $2 P 5$ & $2 p * 1)$ & 60 & $(12 *) j=1.0$ & $-2 s 2$ & $2 P 6$ \\
\hline Ni19 & $\mathrm{Ne}-1$ & $2 s 2$ & $2 \mathrm{P} 5$ & $2 p * 1)$ & $6 D$ & c) $j=1.0$ & $-2 s 2$ & $2 P 6$ \\
\hline Nilg & Ne-1 & $2 \mathrm{~s} 2$ & $2 \mathrm{P} 5$ & $(2 p * 1)$ & $6 D$ & $(12 *) j=1.0$ & $-2 s 2$ & $2 P 6$ \\
\hline $\mathrm{Ni} 19$ & Ne-like & $2 \mathrm{~s} 2$ & $2 \mathrm{P} 5$ & $2 P * 2)$ & $6 \mathrm{D}$ & $\left(23^{*}\right) j=1.0$ & $-2 s 2$ & $2 \mathrm{P} 6$ \\
\hline Ni19 & Ne-like & $2 S 2$ & $2 \mathrm{P} 5$ & $2 P \star 1)$ & $4 D$ & $(12 *) j=1.0$ & $-2 s 2$ & $2 P 6$ \\
\hline Ni19 & Ne-like & $2 \$ 2$ & 2P5 & $2 p \star 2)$ & $4 \mathrm{D}$ & $=1.0$ & -252 & $2 \mathrm{P} 6$ \\
\hline Ni19 & $\mathrm{Ne}-$ & $2 \$ 2$ & $2 \mathrm{P} 5$ & $(2 P * 2)$ & 40 & $(22 *) j=1.0$ & $-2 s 2$ & $2 \mathrm{P} 6$ \\
\hline Ni19 & $\mathrm{Ne}-\mathrm{I}$ & $2 s 2$ & $2 \mathrm{P} 5$ & $2 p * 1)$ & $4 S$ & $\left(11^{*}\right) j=1.0$ & $-2 s 2$ & $2 P 6$ \\
\hline
\end{tabular}

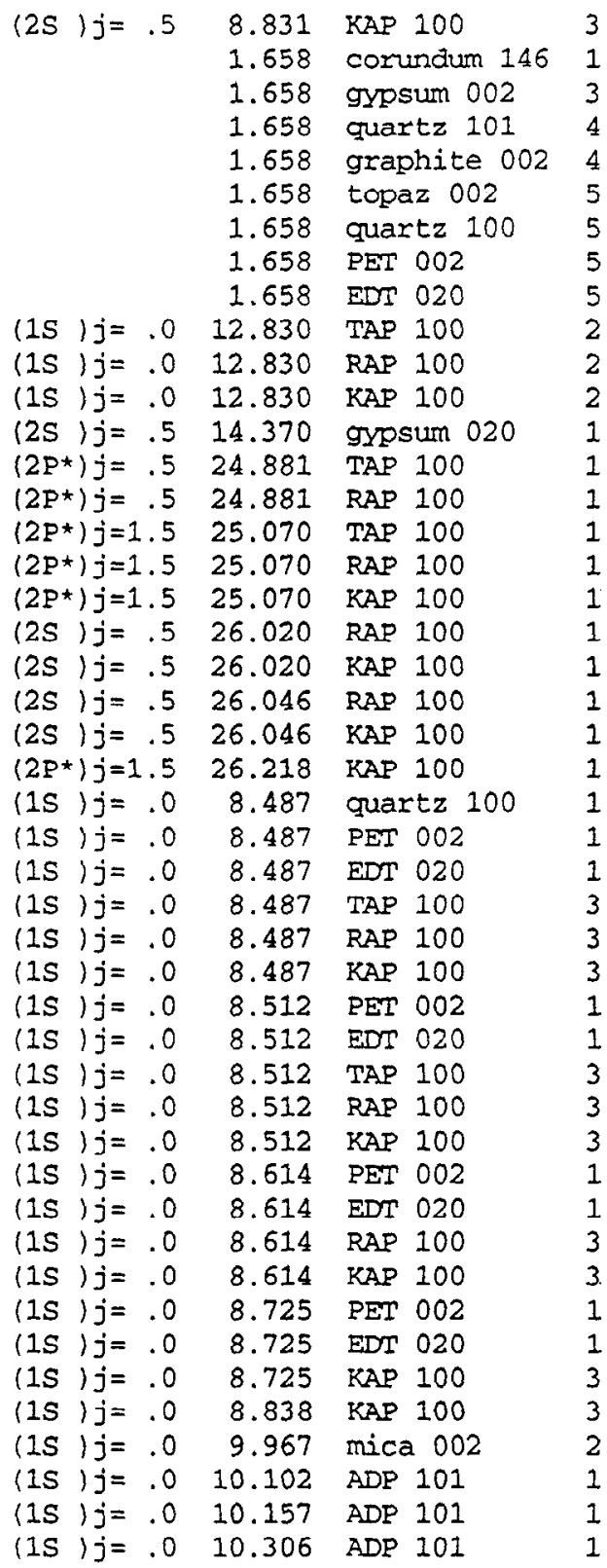

$26.634 \quad 84.102$

$1.660 \quad 87.187$

$4.990 \quad 85.411$

$6.687 \quad 82.646$

$6.696 \quad 82.072$

$8.374 \quad 81.878$

$8.512 \quad 76.886$

$8.742 \quad 71.495$

$8.808 \quad 70.252$

$25.763 \quad 84.875$

$26.116 \quad 79.277$

$26.634 \quad 74.457$

$15.185 \quad 71.143$

$25.763 \quad 74.964$

$26.116 \quad 72.309$

$25.763 \quad 76.681$

$26.116 \quad 73.729$

$26.634 \quad 70.267$

$26.116 \quad 85.086$

$26.634 \quad 77.673$

$26.116 \quad 85.804$

$26.634 \quad 77.938$

$26.634 \quad 79.860$

$8.512 \quad 85.608$

$8.742 \quad 76.127$

$8.808 \quad 74.484$

$25.763 \quad 81.219$

$26.116 \quad 77.141$

$26.634 \quad 72.932$

$8.742 \quad 76.828$

$8.808 \quad 75.104$

$25.763 \quad 82.388$

$26.116 \quad 77.902$

$26.634 \quad 73.491$

$8.742 \quad 80.183$

$8.808 \quad 77.952$

$26.116 \quad 81.693$

$26.634 \quad 75.992$

$8.742 \quad 86.426$

$8.808 \quad 82.128$

$26.634 \quad 79.348$

$26.634 \quad 84.559$

$19.942 \quad 88.377$

$10.640 \quad 71.702$

$10.640 \quad 72.670$

$10.640 \quad 75.606$ 
Ni19 Ne-1ike 2S2 2P5 ( 2P*2) 4S (21*)j=1.0 - 2S2 $2 P 6$ Ni19 Ne-like 2S2 2P5 ( 2P*1) 3P (12*)j=1.0 - 2S2 2P6 Ni19 Ne-like 2S2 2P5 ( $2 P \star 1) 3 P(12 *) j=1.0-2 S 22 P 6$ Ni19 Ne-like 2S2 2P5 $(29 * 2) \quad 3 D(23 *) j=1.0-2 S 22 P 6$ Ni19 Ne-like 2S2 2P5 ( 2P*2) 3D $\left(23^{*}\right) j=1.0-2 S 22 P 6$ Ni19 Ne-like 2S2 $2 P 5(2 P * 2) 3 D(23 *) j=1.0-2 S 22 P 6$ Ni19 Ne-like 2S2 2P5 $(2 P * 2)$ 3D $(22 *) j=1.0-2 S 22 P 6$ Ni19 Ne-like 2S2 2P5 ( $\left.2 P^{*} 2\right)$ 3D $\left(22^{*}\right) j=1.0$ - 2S2 2P6 Ni19 Ne-like 2S2 2P5 ( $2 P * 2)$ 3D (22*) j=1.0 - 2S2 $2 P 6$ Ni19 Ne-like $\quad 8 \quad\left(j j=.0-\frac{8}{8}\right.$ Ni19 Ne-like $8 \quad(\quad) j=.0-$ (

Ni20 F-like 2S2 2P4 ( $1 D$ ) $4 D(2 D) j=2.5-2 S 22 P 5$

Ni20 F-like 2S2 2P4 ( $1 D$ ) $4 D(2 D) j=2.5-2 S 22 P 5$

Ni20 F-like 2S2 2P4 ( $3 P$ ) $4 D(2 P) j=1.5-2 S 22 P 5$

$N i 20 F$-like $2 S 22 P 4(3 P) 4 D(4 P) j=2.5-2 S 22 P 5$

Ni20 F-like 2S2 2P4 ( $1 D$ ) $4 D(2 D) j=1.5-2 S 22 P 5$

Ni20 F-like $2 S 22 P 4(3 P) 4 D(2 D) j=2.5-2 S 22 P 5$

Ni20 F-like 2S2 2P4 ( 3P ) 4D (2P) j=1.5 - 2S2 2P5

Ni20 F-1ike 2S2 2P4 ( $3 P$ ) $4 D(4 F) j=1.5-2 S 22 P 5$

Ni20 F-like $2 S 22 P 4(3 P)$ (SS (4P) $j=1.5-2 S 22 P 5$

Ni20 F-like 2S2 2P4 ( $3 P$ ) $4 S(2 P) j=1.5-2 S 2$ 2P5

Ni20 F-like 2S2 2P4 ( $3 P$ ) 3D (2F) $j=2.5-2 S 22 P 5$

Ni20 $F$-like $2 S 22 P 4(3 P), 3 D(4 P) j=1.5-2 S 22 P 5$

Ni20 F-like 2S2 2P4 ( $3 P$ ) 3D (4P) $j=.5-2 S 22 P 5$

Ni20 F-like 2S2 2P4 ( 3P) 3D (2P) j=1.5 - 2S2 2P5

Ni20 F-like 2S2 2P4 ( ID) 3S (2D) j=2.5 - 2S2 2P5

Ni20 F-like 2S2 2P4 ( $1 D$ ) 3S (2D) $j=2.5-2 S 22 P 5$

Ni20 F-like 2S2 2P4 ( $3 P$ ) 3S (2P) j=.5-2S2 $2 P 5$

Ni20 F-like 2S2 2P4 (3P) 3S $(2 P) j=.5-2 S 22 P 5$

Ni20 F-1ike 2S2 $2 P 4(3 P) 3 S(4 P) j=1.5-2 S 22 P 5$

Ni20 F-like 2S2 2P4 ( $3 P$ ) 3S (4P) $j=.5-2 S 22 P 5$

Ni20 F -like 2S2 2P4 ( ID) 3S (2D) $j=1.5-2 S 22 P 5$

Ni20 F-like 2S2 2P4 ( $3 P$ ) 3S (2P) j=1.5 - 2S2 $2 P 5$

Ni2O $F$-like 2S2 $2 P 4(3 P) 3 S(2 P) j=.5-2 S 22 P 5$

Ni20 F-like 2S2 $2 \mathrm{P} 4(3 \mathrm{P}) 3 \mathrm{~S}(4 \mathrm{P}) \mathrm{j}=2.5-2 \mathrm{~S} 2 \mathrm{PS}$

Ni21 O-Iike 2S2 2P3 ( 2P*) 4D (3D*) j=2.0 - 2S2 2P4

Ni21 0 -like 2S2 2P3 (2P*) $4 D\left(3 D^{*}\right) j=2.0-2 S 22 P 4$

Ni21 O-like 2S2 2P3 ( 2P*) $4 D\left(3 P^{\star}\right) j=1.0-2 S 22 P 4$

Ni21 0 -like 2S2 2P3 ( 2P*) $4 D\left(3 P^{*}\right) j=1.0-2 S 22 P 4$

$\mathrm{Ni} 21$ O-like 2S2 2P3 (2D*) 4D (3S*)j=1.0 - 2S2 2P4

Ni21 0 -like $2 S 22 P^{2}\left(2 D^{*}\right) 4 D\left(3 S^{*}\right) j=1.0-2 S 22 P^{*}$

Ni21 O-like 2S2 2P3 (2P*) 3S (1P*) $j=1.0-2 S 22 P 4$

Ni21. O-like 2S2 2P3 (2D*) 3S (3D*) $j=3.0-2 S 22 D^{*} 4$

Ni21 O-like 2S2 2P3 ( $\left.2 P^{*}\right) 3 S\left(3 P^{*}\right) j=.0-2 S 22 P^{*}$

Ni21 O-like 2S2 2P3 ( 2D*) 3S (3D*) j=2.0 - 2S2 $2 P 4$

$\mathrm{Ni} 21$ O-1ike $2 S 22 \mathrm{P} 3\left(2 D^{*}\right) 3 S\left(3 D^{*}\right) j=2.0-2 S 22 \mathrm{P} 4$
(1S ) $j=.0 \quad 10.417$ ADP 101 (1S) $j=.0 \quad 12.430$ TAP 100 (1S) $j=.0 \quad 12.430$ RAP 100 (1S) $j=.0 \quad 12.654$ TAP 100 (1S) $j=.0 \quad 12.654$ RAP 100 (1S) $j=.0 \quad 12.654$ KRAP 100 (1S) $j=.0 \quad 12.809$ TAP 100 (IS ) $j=.012 .809$ RAP 100 (1S) $j=.0 \quad 12.809 \operatorname{KAP} 100$ $(\quad) j=.0 \quad 9.441$ mica 002 $(\quad j=.0 \quad 9.545$ mica 002 $\left(2 P^{\star}\right) j=1.5 \quad 9.385$ mica 002 $\left(2 P^{*}\right) j=1.5 \quad 9.385$ mica 002 $\left(2 P^{*}\right) j=1.5 \quad 9.446$ mica 002 $\left(2 P^{*}\right) j=1.5 \quad 9.455 \quad \mathrm{mica} 002$ $(2 P *) j=.5 \quad 9.497$ mica 002 $\left(2 P^{*}\right) j=1.5 \quad 9.558$ mica 002 $\left(2 P^{*}\right) j=.5 \quad 9.581$ mica 002 $(2 P *) j=.5 \quad 9.630$ mica 002 $\left(22^{*}\right) j=1.5 \quad 9.693$ mica 002 $\left(2 P^{*}\right) j=1.5 \quad 9.821$ mica 002 $\left(2 P^{*}\right) j=1.5 \quad 12.112$ TAP 100

$\left(2 P^{*}\right) j=1.5 \quad 12.130$ TAP 100 $\left(2 P^{*}\right) j=1.5 \quad 12.157$ TAP 100 $\left(2 P^{*}\right) j=.5 \quad 12.181$ TAP 100 $\left(2 P^{*}\right) j=1.5 \quad 12.927 \quad$ RAP 100 $\left(2 P^{*}\right) j=1.5 \quad 12.927 \quad \operatorname{KAP} 100$ $\left(2 P^{*}\right) j=1.5 \quad 13.032$ RAP 100 (2P*) $j=1.5 \quad 13.032$ KAP 100 $\left(2 P^{*}\right) j=1.5 \quad 13.075$ KAP 100 $\left(2 P^{*}\right) j=1.5 \quad 13.135$ KAP 100 $\left(2 P^{*}\right) j=.5 \quad 13.161$ KAP 100 $\left(2 P^{*}\right) j=1.5 \quad 13.256 \quad K A P \quad 100$ $\left(2 P^{*}\right) j=.5 \quad 13.282 \quad \operatorname{KAP} 100$ $\left(2 P^{\star}\right) j=1.5 \quad 13.309$ KAP 100 (3P) $j=1.0 \quad 8.775 \quad$ EDT 020 (3P) $j=1.0 \quad 8.775$ KAP 100 (3P ) $j=1.0 \quad 8.802$ EDT 020 (3P) $j=1.0 \quad 8.802 \quad \operatorname{KAP} 100$ (3P) $j=2.0 \quad 8.849 \quad \operatorname{KAP} 100$ (3P) $j=2.0 \quad 8.849 \quad \mathrm{KAP} 100$ (1D) $j=2.0 \quad 12.181$ TAP 100 (3P) $j=2.0 \quad 12.208$ TAP 100 (3P) $j=1.0 \quad 12.245$ TAP 100 (3P $) j=2.0 \quad 12.277$ TAP 100 (3P) $j=2.0 \quad 12.277$ RAP 100
$10.640 \quad 78.249$

$25.763 \quad 74.785$ $26.116 \quad 72.158$ $25.763 \quad 79.216$ $26.116 \quad 75.711$ $26.634 \quad 71.84$ $25.763 \quad 83.918$ $26.116 \quad 78.793$

$26.634 \quad 74.123$ $19.942 \quad 71.235$ $19.942 \quad 73.191$ $19.942 \quad 70.259$ $19.942 \quad 70.259$ $19.942 \quad 71.325$ $19.942 \quad 71.487$ $19.942 \quad 72.262$ $19.942 \quad 73.452$ $19.942 \quad 73.922$ $19.942 \quad 74.972$ 19.94276 .439 $19.942 \quad 80.049$ $25.763 \quad 70.096$ $25.763 \quad 70.332$ $25.763 \quad 70.692$ $25.763 \quad 71.018$ $26.116 \quad 81.877$ $26.634 \quad 76.099$ $26.116 \quad 86.384$ 26.63478 .125 $26.634 \quad 79.060$ $26.634 \quad 80.517$ $26.634 \quad 81.221$ 26.63484 .514 $26.634 \quad 85.845$ $26.634 \quad 88.014$ $8.808 \quad 85.039$

$26.634 \quad 81.264$

$8.808 \quad 87.885$

$26.634 \quad 82.498$

$26.634 \quad 85.368$

$26.634 \quad 85.368$ $25.763 \quad 71.018$ $25.763 \quad 71.391$ $25.763 \quad 71.913$ $25.763 \quad 72.378$ $26.116 \quad 70.083$ 
Ni21 o-1ike 2S2 2P3 ( 2D*) $3 S\left(1 D^{*}\right) j=2.0-2 S 22 P 4$

$\mathrm{Ni21} O$-like 2S2 $2 \mathrm{P} 3\left(2 D^{*}\right) 3 S\left(1 D^{*}\right) j=2.0-2 S 22 \mathrm{P4}$

Ni21 o-like 2S2 $2 P^{2}$ ( $\left.4 S^{*}\right)$ ) $3 S\left(3 S^{*}\right) j=1.0-2 S 22 P 4$

Ni21 0 -1ike 2S2 2P3 (4S*) 3S (3S*)j=1.0 - 2S2 2P4

Ni21 O-like $2 S 22 \mathrm{P} 3\left(2 D^{*}\right) 3 S\left(3 D^{*}\right) j=2.0-2 S 22 \mathrm{P4}$

Ni21 o-like 2S2 $2 P^{2}\left(2 D^{*}\right) 3 S\left(3 D^{*}\right) j=2.0-2 S 22 P 4$

Ni21 0 -like 2S2 $2 P^{2}\left(2 D^{*}\right) 3 S\left(1 D^{*}\right) j=2.0-2 S 22 P 4$

Ni21 o-like 2S2 2P3 ( 2D*) 3S (1D*)j=2.0-2S2 2P4

Ni21 0 -like 2S2 2P3 (4S*) 3S (3S*) j=1.0 - 2S2 $2 P 4$

Ni21 o-like 2S2 2P3 ( $\left.4 S^{*}\right) 3 S\left(3 S^{*}\right) j=1.0-2 S 22 P 4$

Ni21 o-like 2S2 2P3 ( $\left.4 S^{*}\right)$ 3S $\left(3 S^{*}\right) j=1.0-2 S 22 P 4$

Ni21 O-like 2S2 $2 \mathrm{P} 3\left(\mathrm{SS}^{*}\right)$ ) $3 S^{\left(3 S^{*}\right) j=1.0-2 S 22 \mathrm{PA}}$

Ni21 O-like 2S2 2P3 ( $\left.4 S^{*}\right) 3 S\left(3 S^{*}\right) j=1.0-2 S 22 P 4$

Ni21 O-like 2S2 2P3 (4S*) 3S (3S*) $j=1.0-2 S 22 \mathrm{P} 4$

$\mathrm{Ni22} \mathrm{N}-1 \mathrm{ike} \quad 10$

Ni22 N -like

Ni23 C-like 2S2 2P 3D

Ni23 C-like 2S2 $2 P \quad 3 D$

$\mathrm{Ni23} C$-like 2S2 $2 \mathrm{P}$ 3D

$\mathrm{Ni23} C$-like 2S2 2P 3D

Ni23 C-like 252 2P $3 D$

Ni24 B-like $2 S 2 P\left(3 P^{*}\right) 3 P(2 P) j=.5-2 S 22 P$

Ni24 B-like $2 S$ 2P ( $\left.P^{*}\right) 3 P(2 P) j=1.5-2 S 22 P$

Ni24 $B$-like $2 S$ 2P (1 $\left.P^{*}\right) 3 D\left(2 D^{*}\right) j=2.5-2 S 2 P 2$

Ni24 B -like $2 S 2 P\left(3 P^{*}\right) 3 P(2 D) j=1.5-2 S 22 P$

$\mathrm{Ni24} B-1 \mathrm{ike} 2 \mathrm{~S} 2 \mathrm{P}\left(3 \mathrm{P}^{*}\right) 3 \mathrm{P}$ (2D) $\mathrm{j}=2.5-2 \mathrm{~S} 2 \mathrm{PP}$

Ni24 B-like $2 S$ 2P $\left(1 P^{*}\right) 3 D\left(2 D^{*}\right) j=2.5-2 S \quad 2 P 2$

Ni24 B-like $2 S$ 2P ( $\left.3 P^{*}\right) 3 D\left(4 D^{*}\right) j=3.5-2 S \quad 2 P 2$

Ni24 B-like 2S 2P (1 $\left.P^{*}\right) 3 D\left(2 F^{*}\right) j=3.5-2 S$ 2P2

Ni24 B-like $2 S 2 P\left(3 P^{*}\right) 3 D\left(4 P^{*}\right) j=2.5-2 S \quad 2 P 2$

Ni24 B-like $2 S \quad 2 P\left(1 P^{\star}\right) 3 D\left(2 D^{\star}\right) j=2.5-2 S$ 2P2

Ni24 B-like $2 S$ 2P (3P*) $3 D\left(2 F^{*}\right) j=2.5-2 S$ 2P2

$\mathrm{Ni24} B$-like $2 S$ 2P (3P*) $3 D\left(2 D^{*}\right) j=2.5-2 S .2 P 2$

Ni25 Be-like $1 S 22 S \quad 3 P\left(3 P^{*}\right) j=1.0-1 S 22 S 2$

Ni25 Be-like 1S2 2S 3S (1S) $j=.0-1 S 2$ 2S $2 P$

Ni25 Be-like 1s2 2P

Ni25. Be-like 152 2S

Ni25 Be-like 1S2 2s

Ni25 Be-like 1s2 2P

Ni25 Be-like 1S2 2P

Ni25 Be-like 1S2 2S

$\mathrm{Ni25}$ Be-like 1s2 2s

Ni25 Be-like 1S2 $2 \mathrm{P}$

Ni25 Be-like 1S2 2P

Ni25 Be-like 1S2 2 P

Ni25. Be-like 1S2 2P
(3P) $j=1.0 \quad 12.370$ TAP 100

(3P) $j=1.0 \quad 12.370$ RAP 100

(3P) $j=2.0 \quad 12.435$ TAP 100

(3P) $j=2.0 \quad 12.435$ RAP 100

(3P) $j=1.0 \quad 12.472$ TAP 100

(3P $) j=1.0 \quad 12.472$ RAP 100

(1D) $j=2.0 \quad 12.502 \quad$ TAP 100

(1D $) j=2.0012 .502$ RAP 100

(3P $) j=.0 \quad 12.592$ TAP 100

(3P $) j=.0 \quad 12.592 \quad \operatorname{RAP} 100$

(3P) $j=0 \quad 12.592$ KAP 100

(3P) $j=1.0 \quad 12.656 \quad$ TAP 100

(3P) $j=1.0 \quad 12.656$ RAP 100

(3P) $j=1.0 \quad 12.656$ KAP 100

$(\quad j=.0 \quad 8.798 \quad$ EDT 020

$(\quad j=.0 \quad 8.798 \quad K A P 100$

(3P) $j=2.0 \quad 10.450 \quad$ ADP 101

(1D) $j=2.0 \quad 10.490 \quad$ ADP 101

(3P) $j=2.0 \quad 12.693 \quad$ TAP 100

(3P) $j=2.0 \quad 12.693 \quad$ RAP 100

(3P) $j=2.0 \quad 12.693 \quad \mathrm{KAP} 100$

$\left\langle 2 P^{*}\right\rangle j=.5 \quad 9.690$ mica 002

$(2 P \star) j=1.5 \quad 9.740$ mica 002

(2D) $j=1.5 \quad 9.883$ mica 002

$\left(2 P^{*}\right) j=.5 \quad 9.900$ mica 002

$\left(2 P^{*}\right) j=1.5 \quad 9.920$ mica 002

(2D) $j=2.5 \quad 9.933$ mica 002

(4P ) $j=2.5 \quad 9.966$ mica 002

(2D) $j=2.5 \quad 10.000 \quad$ ADP 101

$(4 P) j=2.5 \quad 10.100 \quad$ ADP 101

(2P) $j=1.5 \quad 10.195$ ADP 101

(2D) $j=1.5 \quad 10.261$ ADP 101

(2D) $j=2.5 \quad 10.350$ ADP 101

(1S) $j=.0 \quad 9.390$ mica 002

$\left(1 P^{\star}\right) j=1.0 \quad 9.407$ mica 002

$\left(1 P^{*}\right) j=1.0 \quad 9.471$ mica 002

$\left(3 P^{*}\right) j=.0 \quad 9.601$ mica 002

$\left(3 P^{*}\right) j=1.0 \quad 9.633$ mica 002

$\left(1 P^{\star}\right) j=1.0 \quad 9.695$ mica 002

(3P) $j=1.0 \quad 9.707$ mica 002

$\left(3 P^{*}\right) j=2.0 \quad 9.744$ mica 002

$\left(3 P^{*}\right) j=2.0 \quad 9.753$ mica 002

(3P) $j=2.0 \quad 9.759$ mica 002

(3P) $j=2.0 \quad 9.776$ mica 002

(1D) $j=2.0 \quad 9.860$ mica 002

(3P) $j=2.0 \quad 9.873$ mica 002
$25.763 \quad 73.800$

$26.116 \quad 71.318$

$25.763 \quad 74.870$

$26.116 \quad 72.230$

$25.763 \quad 75.514$

$26.116 \quad 72.770$

$25.763 \quad 76.058$

$26.116 \quad 73.220$

$25.763 \quad 77.830$

$26.116 \quad 74.647$

$26.634 \quad 71.007$

$25.763 \quad 79.263$

$26.116 \quad 75.746$

$26.634 \quad 71.872$

$8.808 \quad 87.270$

$26.634 \quad 82.302$

$10.640 \quad 79.156$

$10.640 \quad 80.368$

$25.763 \quad 80.186$

$26.116 \quad 76.421$

$26.634 \quad 72.391$

$19.942 \quad 76.365$

$19.942 \quad 77.643$

$19.942 \quad 82.382$

$19.942 \quad 83.158$

$19.942 \quad 84.203$

$19.942 \quad 84.996$

$19.942 \quad 88.185$

$10.640 \quad 70.026$

$10.640 \quad 71.668$

$10.640 \quad 73.371$

$10.640 \quad 74.661$

$10.640 \quad 76.592$

$19.942 \quad 70.344$

$19.942 \quad 70.637$

$19.942 \quad 71.778$

$19.942 \quad 74.343$

$19.942 \quad 75.039$

$19.942 \quad 76.488$

$19.942 \quad 76.786$

$19.942 \quad 77.751$

$19.942 \quad 77.997$

$19.942 \quad 78.164$

$19.942 \quad 78.650$

$19.942 \quad 81.443$

$19.942 \quad 81.960$ 


\begin{tabular}{|c|c|c|c|}
\hline $\mathrm{Ni25}$ & Be-like & $1 S 2$ & $2 P$ \\
\hline Ni25 & Be-like & $1 S 2$ & $2 S$ \\
\hline Ni25 & Be-like & $1 S 2$ & $2 S$ \\
\hline $\mathrm{Ni25}$ & Be-like & $1 S 2$ & $2 \mathrm{P}$ \\
\hline Ni25 & Be-like & 152 & $2 S$ \\
\hline $\mathrm{Ni25}$ & Be-like & $1 S 2$ & $2 P$ \\
\hline $\mathrm{Ni26}$ & Li-like & 152 & $4 S$ \\
\hline $\mathrm{Ni26}$ & Li-like & $1 S 2$ & $4 \mathrm{D}$ \\
\hline $\mathrm{Ni26}$ & Li-like & 152 & $4 S$ \\
\hline $\mathrm{Ni26}$ & Ii-like & $1 \$ 2$ & $4 S$ \\
\hline $\mathrm{Ni} 26$ & Li-like & $1 S 2$ & $3 D$ \\
\hline Ni26 & Li-like & $1 S 2$ & 3D \\
\hline $\mathrm{Ni26}$ & Li-like & 152 & $3 D$ \\
\hline $\mathrm{Ni26}$ & Li-like & $1 S 2$ & $3 s$ \\
\hline $\mathrm{Ni} 26$ & Li-like & 152 & $3 s$ \\
\hline $\mathrm{Ni27}$ & He-like & 1s & $6 P$ \\
\hline $\mathrm{Ni27}$ & He-1ike & 1S & $6 P$ \\
\hline $\mathrm{Ni} 27$ & He-like & 15 & $6 P$ \\
\hline $\mathrm{Ni27}$ & He-like & $1 s$ & $6 P$ \\
\hline $\mathrm{Ni27}$ & He-like & $1 s$ & $6 P$ \\
\hline $\mathrm{Ni27}$ & He-like & 15 & $6 P$ \\
\hline $\mathrm{Ni} 27$ & He-like & 15 & $6 P$ \\
\hline $\mathrm{Ni27}$ & He-like & $1 S$ & $6 \mathrm{P}$ \\
\hline Ni27 & He-like & $1 s$ & $5 P$ \\
\hline $\mathrm{Ni27}$ & He-like & 15 & $5 P$ \\
\hline Ni27 & He-like & $1 \mathrm{~S}$ & $5 P$ \\
\hline $\mathrm{Ni27}$ & He-like & 1s & $5 P$ \\
\hline Ni27 & He-like & $1 S$ & $5 P$ \\
\hline Ni27 & He-1ike & 15 & $5 P$ \\
\hline Ni27 & He-1ike & $1 \mathrm{~s}$ & $5 P$ \\
\hline Ni27 & He-like & 15 & $5 \mathrm{P}$ \\
\hline Ni27 & He-like & 1S & $4 \mathrm{P}$ \\
\hline $\mathrm{Ni27}$ & He-like & $1 \mathrm{~S}$ & $4 P$ \\
\hline Ni27 & He-1ike & is & $4 P$ \\
\hline $\mathrm{Ni27}$ & He-1ike & 15 & $4 \mathrm{P}$ \\
\hline $\mathrm{Ni} 27$ & He-like & 15 & $4 P$ \\
\hline Ni27 & He-like & 15 & $4 \mathrm{P}$ \\
\hline $\mathrm{Ni27}$ & He-1ike & $1 S$ & $4 P$ \\
\hline $\mathrm{Ni27}$ & He-like & 15 & $4 \mathrm{P}$ \\
\hline $\mathrm{Ni27}$ & He-1ike & 1s & $4 P$ \\
\hline Ni27 & He-like & 1S & $3 P$ \\
\hline $\mathrm{Ni27}$ & He-like & 15 & $3 P$ \\
\hline $\mathrm{Ni27}$ & He-like & is & $3 P$ \\
\hline $\mathrm{Ni27}$ & He-like & $1 S$ & $3 P$ \\
\hline Ni27 & He-like & 1s & $3 P$ \\
\hline $\mathrm{Ni27}$ & He-like & 1s & $3 P$ \\
\hline
\end{tabular}

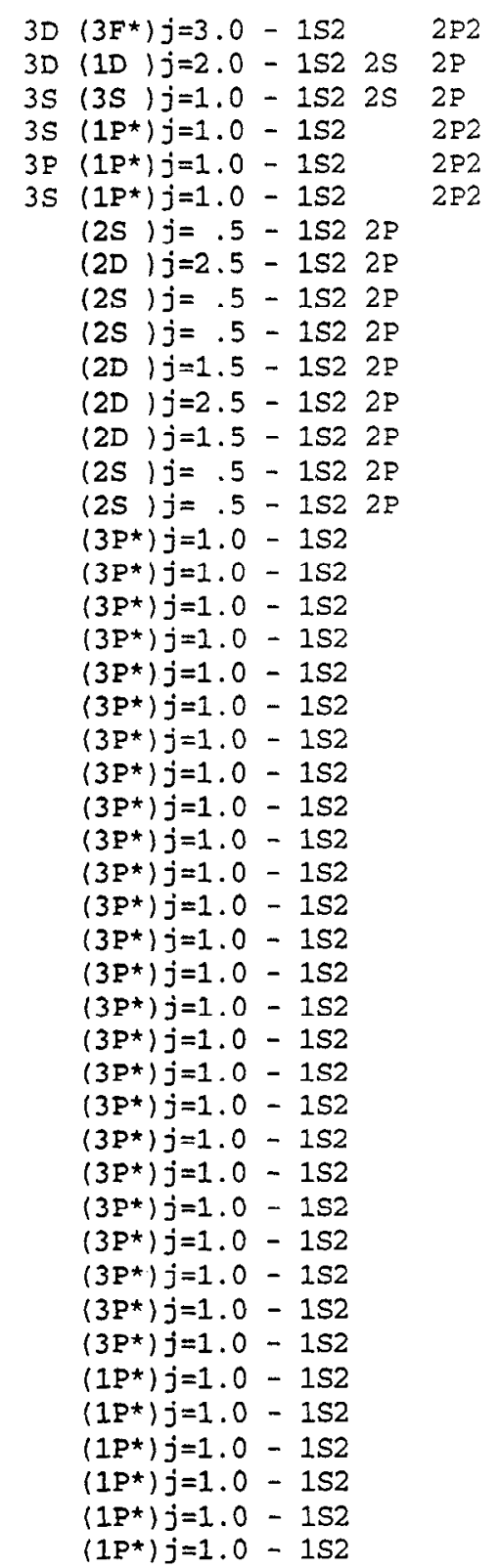

$19.942 \quad 85.063$ $19.942 \quad 88.377$

$10.640 \quad 71.719$

$10.640 \quad 74.970$

$10.640 \quad 76.685$

$10.640 \quad 81.717$

$7.481 \quad 70.411$

$7.481 \quad 71.418$

$7.481 \quad 72.583$

$\begin{array}{ll}15.185 & 70.075\end{array}$

$19.942 \quad 70.344$

$19.942 \quad 72.994$

$19.942 \quad 73.291$

$19.942 \quad 73.573$

$19.942 \quad 77.430$

$3.840 \quad 75.282$

$3.862 \quad 74.087$

$4.990 \quad 82.925$

$6.271 \quad 80.781$

$6.292 \quad 79.669$

$6.308 \quad 78.900$

6.53271 .377

$6.584 \quad 70.078$

$3.840 \quad 78.432$

$3.862 \quad 76.933$

$4.000 \quad 70.136$

$6.271 \quad 88.977$

$\begin{array}{ll}6.292 & 85.207\end{array}$

$6.308 \quad 83.708$

$6.532 \quad 73.717$

$6.584 \quad 72.234$

2.71271 .114

$3.862 \quad 85.298$

$4.000 \quad 74.207$

$4.027 \quad 72.901$

$4.048 \quad 71.960$

$6.532 \quad 79.139$

6.58476 .990

$6.687 \quad 73.602$

$6.696 \quad 73.342$

$2.712 \quad 84.608$

$2.748 \quad 79.275$

$2.749 \quad 79.166$

$2.795 \quad 75.019$

$2.848 \quad 71.448$

$4.246 \quad 72.523$ 


\begin{tabular}{|c|c|c|c|c|}
\hline $\mathrm{Ni27}$ & He-like & is $3 P$ & $\left(1 P^{\star}\right) j=1.0$ & $-1 s 2$ \\
\hline $\mathrm{Ni27}$ & He-like & 15 & $\left(3 p^{*}\right) j=1.0$ & $-1 s 2$ \\
\hline $\mathrm{Ni} 27$ & He-like & IS & $\left(3 R^{\star}\right) j=1.0$ & $-1 s 2$ \\
\hline $\mathrm{Ni27}$ & He-like & $1 S$ & $\left(3 P^{*}\right) j=1.0$ & $-1 s 2$ \\
\hline $\mathrm{Ni27}$ & He-like & 1s & $\left(3 P^{\star}\right) j=1.0$ & -152 \\
\hline $\mathrm{Ni27}$ & He-like & 1S & $\left(3 P^{*}\right) j=1.0$ & -152 \\
\hline $\mathrm{Ni27}$ & He-like & 1s & $\left(3 P^{*}\right) j=1.0$ & -152 \\
\hline Ni27 & He-like & $1 S$ & $\left(3 P^{*}\right) j=1.0$ & -152 \\
\hline $\mathrm{Ni27}$ & He-like & 1S & $\left(1 P^{\star}\right) j=1.0$ & $-1 S 2$ \\
\hline Ni27 & He-like & $1 S \quad 2 P$ & $\left(1 P^{*}\right) j=1.0$ & $-1 s 2$ \\
\hline $\mathrm{Ni27}$ & He-like & 1s & $\left(1 P^{\star}\right) j=1.0$ & $-1 s 2$ \\
\hline $\mathrm{Ni27}$ & He-1ike & $1 S$ & $\left(1 P^{*}\right) j=1.0$ & $-1 S 2$ \\
\hline Ni27 & He-like & $1 S$ & $\left(1 P^{\star}\right) j=1.0$ & $-1 S 2$ \\
\hline $\mathrm{Ni27}$ & He-like & 1s & $\left(1 P^{\star}\right) j=1.0$ & $-1 s 2$ \\
\hline Ni27 & He-like & $1 S$ & $(1 P *) j=1.0$ & $-1 s 2$ \\
\hline $\mathrm{Ni27}$ & He-like & 1s & $\left(1 P^{*}\right) j=1.0$ & $-1 s 2$ \\
\hline Ni27 & He-like & $1 s$ & $\left(1 p^{*}\right) j=1.0$ & -152 \\
\hline Ni27 & He-like & 15 & $\left(1 P^{*}\right) j=1.0$ & $-1 s 2$ \\
\hline Ni27 & He-like & 15 & $\left(3 P^{*}\right) j=1.0$ & $-1 s 2$ \\
\hline Ni27 & He-like & 1S & $\left(3 P^{\star}\right) j=1.0$ & $-1 s 2$ \\
\hline Ni27 & He-1ike & 1s & $\left(3 P^{*}\right) j=1.0$ & -152 \\
\hline $\mathrm{Ni27}$ & He-like & 15 & $\left(3 P^{*}\right) j=1.0$ & $-1 S 2$ \\
\hline $\mathrm{Ni27}$ & He-like & 1s & $\left(3 P^{\star}\right) j=1.0$ & $-1 s 2$ \\
\hline Ni27 & He-like & 1S & $\left(3 P^{*}\right) j=1.0$ & $-1 s 2$ \\
\hline Ni27 & He-like & 15 & $\left(3 P^{*}\right) j=1.0$ & $-1 s 2$ \\
\hline $\mathrm{Ni27}$ & He-like & 15 & $\left(3 P^{*}\right) j=1.0$ & -152 \\
\hline $\mathrm{Ni27}$ & He-like & $1 S$ & $\left(3 P^{\star}\right) j=1.0$ & $-1 S 2$ \\
\hline $\mathrm{Ni27}$ & He-like & 1S & $\left(3 P^{\star}\right) j=1.0$ & -152 \\
\hline $\mathrm{Ni27}$ & He-like & is & $(3 s) j=1.0$ & $-1 s 2$ \\
\hline $\mathrm{Ni27}$ & He-like & 15 & $(3 s) j=1.0$ & $-1 s 2$ \\
\hline $\mathrm{Ni27}$ & He-like & 1s & $(3 s) j=1.0$ & $-1 S 2$ \\
\hline Ni27 & He-1ike & 1s & $(3 s) j=1.0$ & $-1 s 2$ \\
\hline $\mathrm{Ni27}$ & He-like & 15 & (3s) $j=1.0$ & $-1 s 2$ \\
\hline $\mathrm{Ni27}$ & He-like & $1 \mathrm{~S}$ & (3s) $j=1.0$ & $-1 s 2$ \\
\hline $\mathrm{Ni27}$ & He-like & 15 & $(3 s) j=1.0$ & $-1 s 2$ \\
\hline Ni27 & He-like & 15 & (3s) $j=1.0$ & $-1 s 2$ \\
\hline $\mathrm{Ni27}$ & He-1ike & 15 & (3s) $j=1.0$ & $-1 s 2$ \\
\hline $\mathrm{Ni27}$ & He-like & is & $(3 s) j=1.0$ & $-1 s 2$ \\
\hline $\mathrm{Ni27}$ & He-like & $1 s$ & (3s) $j=1.0$ & $-1 s 2$ \\
\hline $\mathrm{Ni28}$ & H -like & $5 P$ & $\left(2 P^{*}\right) j=1.5$ & $-1 s$ \\
\hline $\mathrm{Ni28}$ & H -like & $5 P$ & $\left(2 P^{\star}\right) j=1.5$ & $-1 S$ \\
\hline $\mathrm{Ni28}$ & H -like & $5 P$ & $\left(2 P^{*}\right) j=1.5$ & $-1 s$ \\
\hline $\mathrm{Ni28}$ & H -like & $5 P$ & $\left(2 P^{*}\right) j=1.5$ & $-1 s$ \\
\hline $\mathrm{Ni28}$ & H -like & $5 P$ & $\left(2 P^{*}\right) j=1.5$ & $-1 S$ \\
\hline $\mathrm{Ni28}$ & H -like & $5 P$ & $\left(2 P^{*}\right) j=1.5$ & $-1 s$ \\
\hline $\mathrm{Ni28}$ & $\mathrm{H}$-like & $\mathbf{P}$ & $\left(2 P^{\star}\right) j=1.5$ & \\
\hline
\end{tabular}

\begin{tabular}{|c|c|c|c|}
\hline (1s) $j=$ & .0 & 1.350 & $\mathrm{NaCl} 200$ \\
\hline (1s) $j=$ & .0 & 1.352 & topaz 303 \\
\hline 1s $) j=$ & .0 & 1.352 & corundum 030 \\
\hline 1s $/ j=$ & .0 & 1.352 & guartz 203 \\
\hline$(15) j=$ & .0 & 1.352 & topaz 006 \\
\hline$(15) j=$ & .0 & 1.352 & LiF 220 \\
\hline (s)j= & .0 & 1.352 & quartz 200 \\
\hline$S / j=$ & .0 & 1.352 & $\mathrm{NaCl} 200$ \\
\hline Ls $/ j=$ & .0 & 1.588 & quartz 502 \\
\hline$j=$ & .0 & 1.588 & LiF 422 \\
\hline$j=$ & .0 & 1.588 & corundum 146 \\
\hline$j=$ & .0 & 1.588 & quartz 110 \\
\hline$j=$ & .0 & 1.588 & gypsum 002 \\
\hline$j=$ & .0 & 1.588 & Ge 111 \\
\hline$j=$ & .0 & 1.588 & $\mathrm{KBr} 200$ \\
\hline$j=$ & .0 & 1.588 & quartz 101 \\
\hline$j=$ & .0 & 1.588 & graphite 002 \\
\hline$j=$ & .0 & 1.588 & topaz 002 \\
\hline$j=$ & .0 & 1.596 & guartz 502 \\
\hline$J=$ & .0 & 1.596 & LiF 422 \\
\hline$j=$ & .0 & 1.596 & corundum 146 \\
\hline$j=$ & .0 & 1.596 & quartz 110 \\
\hline$y=$ & .0 & 1.596 & gypsum 002 \\
\hline$j=$ & .0 & 1.596 & Ge 111 \\
\hline$=$ & .0 & 1.596 & $\mathrm{KBr} 200$ \\
\hline$j=$ & .0 & 1.596 & quartz 101 \\
\hline$=$ & .0 & 1.596 & graphite 002 \\
\hline$=$ & .0 & 1.596 & topaz 002 \\
\hline 1. & .0 & 1.603 & guartz 502 \\
\hline$j=$ & .0 & 1.603 & LiF 422 \\
\hline $1 j=$ & .0 & 1.603 & corundum 146 \\
\hline (1s) $j=$ & .0 & 1.603 & quartz 110 \\
\hline$j=$ & .0 & 1.603 & gypsum 002 \\
\hline 1 & .0 & 1.603 & Ge 111 \\
\hline$j=$ & .0 & 1.603 & $\mathrm{KBr} 200$ \\
\hline (1 & .0 & 1.603 & quartz 101 \\
\hline 11S & .0 & 1.603 & graphite 002 \\
\hline 110 & .0 & 1.603 & topaz 002 \\
\hline$i j$ & .0 & 1.603 & quartz 100 \\
\hline 17 & .5 & 1.198 & quartz 220 \\
\hline 12 & .5 & 1.198 & quartz 112 \\
\hline 125 & .5 & 1.198 & quartz 110 \\
\hline & .5 & 1.198 & gypsum 002 \\
\hline & .5 & 1.198 & calcite 200 \\
\hline & .5 & 1.198 & Si 111 \\
\hline & .5 & 1.198 & sylvite 200 \\
\hline
\end{tabular}

$5.641 \quad 73.192$

$2.712 \quad 85.598$

$2.748 \quad 79.733$

$2.749 \quad 79.619$

$2.795 \quad 75.339$

$2.848 \quad 71.702$

$4.246 \quad 72.795$

$5.641 \quad 73.475$

1.62477 .913

$1.652 \quad 73.999$

$1.660 \quad 73.063$

4.91275 .899

$4.990 \quad 72.690$

$6.532 \quad 76.518$

6.58474 .745

$6.687 \quad 71.787$

$6.696 \quad 71.555$

$8.374 \quad 71.473$

1.62479 .345

1.652. 75.039

$1.660 \quad 74.038$

$4.912 \quad 77.099$

$4.990 \quad 73.642$

$6.532 \quad 77.780$

$6.584 \quad 75.842$

$6.687 \quad 72.686$

$6.696 \quad 72.441$

$8.374 \quad 72.354$

$1.624 \quad 80.776$

$1.652 \quad 76.010$

1.66074 .942

$4.912 \quad 78.246$

$4.990 \quad 74.521$

$6.532 \quad 79.001$

6.58476 .875

$6.687 \quad 73.511$

$6.696 \quad 73.253$

8.37473 .162

$8.512 \quad 70.324$

$2.451 \quad 77.839$

$3.636 \quad 81.283$

$4.912 \quad 77.309$

$4.990 \quad 73.806$

6.07180 .630

$6.271 \quad 72.783$

6.29272 .176 
$\left(2 P^{\star}\right) j=1.5-1 S$

$\left(2 P^{*}\right) j=1.5-15$

$\left(2 P^{*}\right) j=1.5-1 S$

$(2 P *) j=1.5-15$

$\left(2 P^{*}\right) j=1.5-1 S$

$\left(2 P^{*}\right) j=1.5-15$

$\left(2 P^{*}\right) j=1.5-15$

$\left(2 P^{*}\right) j=1.5-1 S$

$\left(2 P^{*}\right) j=1.5-1 S$

$\left(2 P^{*}\right) j=1.5-1 S$

$\left(2 P^{*}\right) j=1.5-1 S$

$\left(2 P^{*}\right) j=1.5-1 S$

$\left(2 Q^{*}\right) j=1.5-15$

$\left(2 P^{*}\right) j=1.5-15$

$\left(2 P^{*}\right) j=1.5-1 \mathrm{~s}$

$\left(2 P^{*}\right) j=1.5-1 S$

$(2 p *) j=1.5-15$

$\left(2 P^{*}\right) j=1.5-1 S$

$\left(2 P^{*}\right) j=.5-1 S$

$\left(2 P^{*}\right) j=.5-1 S$

$\left(2 P^{*}\right) j=.5-15$

$\left(2 P^{*}\right) j=.5-1 S$

$\left(2 P^{\star}\right) j=.5-1 S$

$\left(2 P^{\star}\right) j=.5-15$

$\left(2 P^{*}\right) j=.5-1 S$

$\left(2 P^{*}\right) j=.5-1 S$

$\left(2 P^{\star}\right) j=.5-1 S$

$\left(2 P^{*}\right) j=.5-1 S$

$\left(2 P^{*}\right) j=1.5-1 S$

$\left(2 P^{*}\right) j=1.5-1 S$

$\left(2 P^{*}\right) j=1.5-15$

$\left(2 P^{*}\right) j=1.5-1 S$

$\left(2 P^{*}\right) j=1.5-1 S$

$\left(2 P^{*}\right) j=1.5-1 S$

$\left(2 P^{*}\right) j=1.5-1 S$

$\left(2 P^{\star}\right) j=.5-1 S$

$\left(2 P^{*}\right) j=.5-1 S$

$\left(2 P^{*}\right) j=.5-1 S$

$\left(2 P^{\star}\right) j=.5-1 S$

$\left(2 P^{*}\right) j=.5-1 S$

$\left(2 P^{*}\right) j=.5-1 S$

$\left(2 P^{\star}\right\rangle j=.5-1 S$

$\left(2 P^{\star}\right) j=.5-1 S$

$\left(2 P^{*}\right) j=1.5-2 S$

(2D) $j=2.5-2 P$

$\left(2 P^{*}\right) j=1.5-2 S$

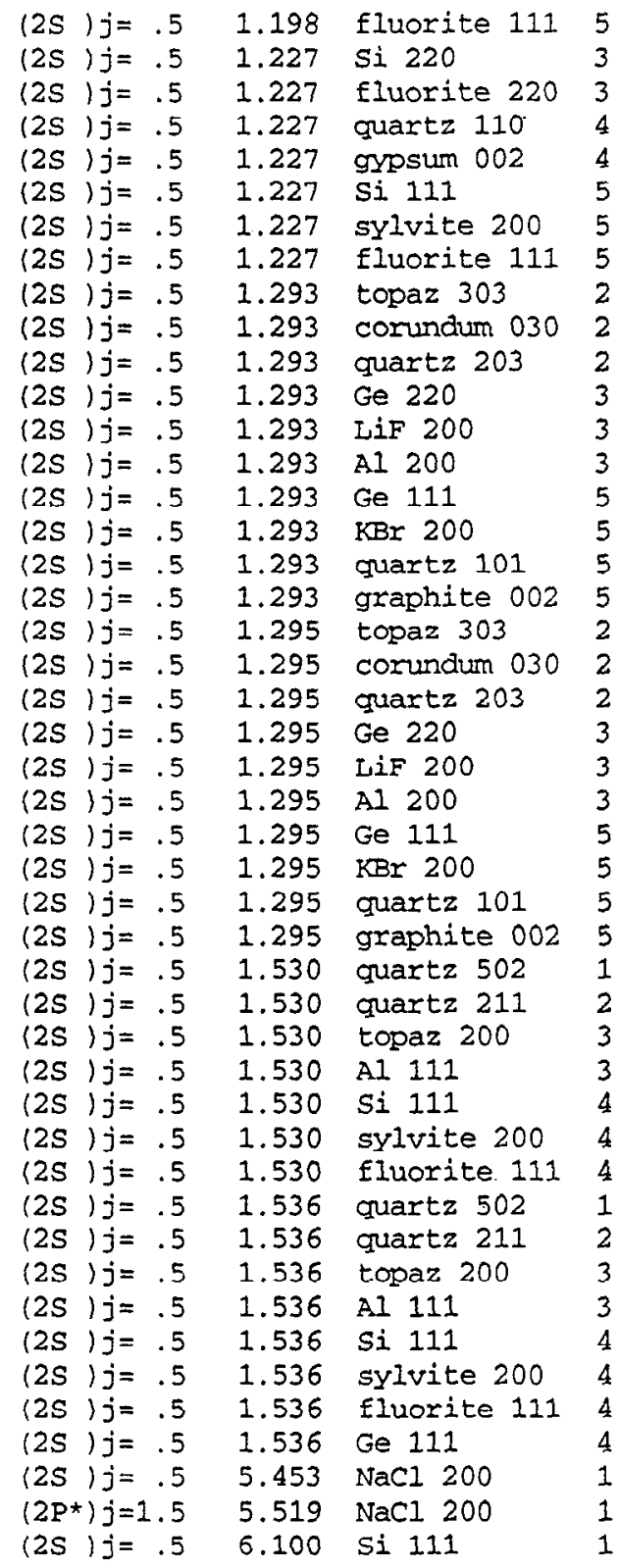

$6.308 \quad 71.730$

$3.840 \quad 73.454$

$3.862 \quad 72.389$

$4.912 \quad 87.688$

$4.990 \quad 79.599$

6.27178 .046

$6.292 \quad 77.174$

6.30876 .550

$2.712 \quad 72.466$

$2.748 \quad 70.228$

$2.749 \quad 70.170$

$4.000 \quad 75.871$

$4.027 \quad 74.418$

$4.048 \quad 73.386$

$6.532 \quad 81.787$

$6.584 \quad 79.090$

$6.687 \quad 75.195$

$6.696 \quad 74.906$

$2.712 \quad 72.749$

$2.748 \quad 70.476$

$2.749 \quad 70.418$

$4.000 \quad 76.228$

$4.027 \quad 74.739$

$4.048 \quad 73.685$

$6.532 \quad 82.425$

6.58479 .560

$6.587 \quad 75.534$

$6.696 \quad 75.239$

1.62470 .410

$3.082 \quad 83.150$

$4.638 \quad 81.750$

$4.676 \quad 78.994$

$6.271 \quad 77.401$

$6.292 \quad 76.572$

$6.308 \quad 75.977$

$1.624 \quad 71.052$

$3.082 \quad 85.383$

$4.638 \quad 83.480$

$4.676 \quad 80.217$

6.27178 .449

$6.292 \quad 77.548$

$6.308 \quad 76.906$

6.53270 .153

$5.641 \quad 75.166$

$5.641 \quad 78.062$

6.27176 .589

Page 92 


\begin{tabular}{|c|c|c|c|c|}
\hline $\mathrm{Ni28}$ & H -like & $4 P$ & & \\
\hline $\mathrm{Ni28}$ & H -like & $4 P$ & & \\
\hline Ni28 & H -like & $4 P$ & & \\
\hline $\mathrm{Ni28}$ & H -like & $4 D$ & & \\
\hline $\mathrm{Ni28}$ & H -like & 40 & & \\
\hline $\mathrm{Ni28}$ & H -like & $4 D$ & & \\
\hline $\mathrm{Ni28}$ & H -like & $4 D$ & & \\
\hline $\mathrm{N} i 28$ & H -like & $4 D$ & & \\
\hline $\mathrm{Ni28}$ & H -like & 40 & & \\
\hline $\mathrm{Ni28}$ & H -like & $3 P$ & & \\
\hline $\mathrm{N} 128$ & H -Iike & $3 P$ & & \\
\hline $\mathrm{Ni28}$ & H -like & $3 P$ & & \\
\hline $\mathrm{Ni28}$ & H-like & $3 P$ & & \\
\hline $\mathrm{Ni28}$ & H -like & $3 D$ & & \\
\hline Ni28 & H -like & $3 D$ & & \\
\hline $\mathrm{Ni28}$ & $\mathrm{H}$-like & 30 & & \\
\hline $\mathrm{Ni28}$ & H -like & $3 D$ & & \\
\hline $\mathrm{Ni} 28$ & H -like & $3 D$ & & \\
\hline $\mathrm{Ni28}$ & H -like & 30 & & \\
\hline Cu. & & $k-a]$ & Ipha (1 & \\
\hline $\mathrm{Cu}$ & & $K-a 1$ & Ipha (1 & \\
\hline Cu & & $\mathrm{k}-\mathrm{al}$ & lpha (1 & \\
\hline $\mathrm{Cu}$ & & $\mathrm{K}-\mathrm{al}$ & 1pha (1 & \\
\hline Cu & $\cdots \quad \cdots$ & $\mathrm{k}-\mathrm{al}$ & Ipha (1 & \\
\hline Cu: & & $k-a l$ & Ipha (1 & \\
\hline $\mathrm{Cu}_{\mathrm{s}}$ & .... & $k-2]$ & Ipha (1 & \\
\hline Cu. & $\ldots$ & $\mathrm{k}-\mathrm{al}$ & Ipha (1 & \\
\hline $\mathrm{Cu}$ & .. & $k-a]$ & Ipha (1 & \\
\hline Cu19 & Na-like & 285 & 352 & \\
\hline Cu19 & Na-like & $2 P 6$ & $8 F$ & \\
\hline Cu19 & $\mathrm{Na}$-Iike & $2 P 6$ & $8 F$ & \\
\hline cu19 & Na-like & $2 \mathrm{P} 6$ & $8 \mathrm{~F}$ & \\
\hline Cu19 & Na-1ike & $2 \mathrm{P} 6$ & $8 F$ & \\
\hline Cu19 & Na-like & $2 P 6$ & $8 F$ & \\
\hline Cu19 & Na-like & $2 \mathrm{P} 6$ & $8 \mathrm{~F}$ & \\
\hline Cu19 & Na-like & $2 P 6$ & $6 D$ & \\
\hline Cu19 & Na-like & $2 \mathrm{PG}$ & $6 \mathrm{D}$ & \\
\hline Cu19 & Na-like & $2 \mathrm{P} 6$ & 60 & \\
\hline Cu19 & Na-like & $2 P 6$ & $6 D$ & \\
\hline Cu19 & Na-like & $2 \mathrm{P} 6$ & $6 D$ & \\
\hline cu19 & Na-like & $2 P 6$ & 60 & \\
\hline Cu19 & Na-like & $2 P 6$ & $7 F$ & \\
\hline $\cos 19$ & Na-like & $2 P 6$ & $7 F$ & \\
\hline $\mathrm{Cu} 20$. & Ne-like & $2 \mathrm{~S} 2$ & $2 \mathrm{P} 5$ & \\
\hline Cu20: & Ne-like & 252 & $2 P 5$ & \\
\hline 0,20 & Ne-like & $2 s 2$ & 275 & \\
\hline
\end{tabular}

$\left(2 P^{*}\right) j=1.5-2 S$ $\left(2 P^{*}\right) j=1.5-2 S$ $\left(2 P^{*}\right) j=1.5-2 S$ (2D) $j=2.5-2 P$ (2D) $j=2.5-2 P$ (2D) $j=2.5-2 P$ (2D) $j=2.5-2 P$ (2D) $j=2.5-2 P$ (2D) $j=2.5-2 P$ $\left(2 P^{\star}\right) j=1.5-2 S$ $\left(2 P^{*}\right) j=1.5-2 S$ $\left(2 P^{*}\right) j=1.5-2 S$ $\left(2 P^{\star}\right) j=1.5-2 S$ (2D) $j=2.5-2 P$ (2D) $j=2.5-2 P$ (2D) $j=2.5-2 P$ (2D) $j=2.5-2 P$ (2D) $j=2.5-2 P$ (2D) $j=2.5-2 P$

$\left(2 P^{*}\right) j=1.5-2 P 6 \quad 3 S$ $\left(2 F^{*}\right) j=2.5-2 P 63 D$ $\left(2 F^{*}\right) j=2.5-2 P 63 D$ $\left(2 F^{*}\right) j=2.5-2 P 63 D$ $\left(2 F^{\star}\right) j=3.5-2 P 63 D$ $\left(2 F^{*}\right) j=3.5-2 P 6 \quad 3 D$ $\left(2 F^{*}\right) j=3.5-2 P 63 D$ (2D) $j=1.5-2 P 6 \quad 3 P$ (2D) $j=1.5-2 P 6 \quad 3 P$ (2D) $j=1.5-2 P 63 P$ (2D) $j=2.5-2 \mathrm{P} 63 \mathrm{P}$ (2D) $j=2.5-2 P 63 P$ (2D) $j=2.5-2 P 6 \quad 3 P$ $\left(2 F^{*}\right) j=2.5-2 P 6 \quad 3 D$ $\left(2 F^{*}\right) j=3.5-2 P 63 D$ $\left(3 P^{*}\right) j=1.0-2 S 2 \quad 2 P 6$ $\left(3 P^{*}\right) j=1.0-2 S 22 P 6$ $\left(3 D^{*}\right) j=1.0-2 S 22 P 6$
(2S) $j=.5$ (2s) $j=.5$ (2S) $j=.5$ $\left(2 P^{\star}\right) j=1.5$ $(2 P *) j=1.5$ $\left(2 P^{*}\right) j=1.5$ $\left(2 P^{\star}\right) j=1.5$ $\left(2 p^{*}\right) j=1.5$ $(2 P *) j=1.5$ (2S) $j=.5$ (2S ) $j=.5$ (2S) $j=.5$ (2s) $j=.5$ $\left(2 P^{*}\right) j=1.5$ $\left(2 P^{*}\right) j=1.5$ $\left(2 P^{*}\right) j=1.5$ $\left(2 P^{\star}\right) j=1.5$ $\left(2 P^{*}\right) j=1.5$ $\left(2 P^{*}\right) j=1.5$

$$
(2 P * j=1.5
$$

6.100 sylvite 200 6.100 fluorite 111 6.100 TAP 100

6.181 Si 111

6.181 sylvite 200

6.181 Eluorite $111 \quad 1$

6.181 Ge 111

6.181 TAP 100

6.181 RAP 100

8.199 topaz 002

8.199 quartz 100

8.199 TAP 100

8.199 RAP 100

8.337 topaz 002

8.337 guartz 100

8.337 PET 002

8.337 EDT 020

8.337 TAP 100

8.337 RAP 100

1.541 quartz 502

1.541 quartz 211

1.541 topaz 200

1.541 AI 111

1.541 quartz 110

1.541 Si 111

1.541 sylvite 200

1.541 fluorite 111

1.541 Ge 111

(2S $j j=.5 \quad 13.110 \quad \operatorname{KAP} 100$

(2D) $j=1.5 \quad 25.142 \quad$ TAP 100

(2D) $j=1.5 \quad 25.142 \quad$ RAP 100

(2D $j j=1.5 \quad 25.142 \quad \mathrm{KAP} 100$

(2D) $j=2.5 \quad 25.175$ TAP 100

(2D) $j=2.5 \quad 25.175 \quad$ RAP 100

(2D $) j=2.5 \quad 25.175$ KAP 100

$\left(2 P^{*}\right) j=.5 \quad 25.297$ TAP 100

$\left(2 P^{*}\right) j=.5 \quad 25.297$ RAP 100

$\left(2 P^{\star}\right) j=.5 \quad 25.297$ KAP 100

$\left(2 P^{*}\right) j=1.5 \quad 25.526$ TAP 100

$\left(2 P^{*}\right) j=1.5 \quad 25.526 \quad \operatorname{RAP} 100$

$\left(2 P^{*}\right) j=1.5 \quad 25.526 \quad \mathrm{KAP} 100$

(2D) $j=1.5 \quad 26.416 \quad \mathrm{KAP} 100$

(2D) $j=2.5 \quad 26.452$ KAP 100

(1s) $j=.0 \quad 7.955$ topaz 002

(Is ) $j=.0 \quad 7.955$ beryl 100

(1S ) $j=.0 \quad 8.070$ topaz 002
$6.292 \quad 75.809$

$6.308 \quad 75.246$

$25.763 \quad 71.279$

6.27180 .281

$6.292 \quad 79.222$

$6.308 \quad 78.483$

6.53271 .132

$25.763 \quad 73.673$

$26.116 \quad 71.209$

$8.374 \quad 78.266$

$8.512 \quad 74.414$

$25.763 \quad 72.696$

$26.116 \quad 70.362$

$8.374 \quad 84.612$

$8.512 \quad 78.362$

$8.742 \quad 72.491$

$8.808 \quad 71.178$

$25.763 \quad 76.123$

$26.116 \quad 73.273$

$1.624 \quad 71.558$

$3.082 \quad 88.694$

$4.638 \quad 85.210$

$4.676 \quad 81.268$

$4.912 \quad 70.207$

$6.271 \quad 79.321$

$6.292 \quad 78.351$

$6.308 \quad 77.666$

$6.532 \quad 70.634$

$26.634 \quad 79.885$

$25.763 \quad 77.394$

$26.116 \quad 74.303$

$26.634 \quad 70.731$

$25.763 \quad 77.735$

$26.116 \quad 74.573$

$26.634 \quad 70.948$

$25.763 \quad 79.086$

$26.116 \quad 75.613$

$26.634 \quad 71.769$

$25.763 \quad 82.222$

$26.116 \quad 77.798$

$26.634 \quad 73.415$

$26.634 \quad 82.664$

$26.634 \quad 83.298$

$8.374 \quad 71.799$

$15.954 \quad 85.744$

$8.374 \quad 74.514$ 


\begin{tabular}{|c|c|c|c|c|c|c|c|c|c|}
\hline 120 & Ne-like & $2 S 2$ & $2 \mathrm{P} 5$ & $6 D$ & & & $\left(3 D^{*}\right) j=1.0$ & $-2 s 2$ & \\
\hline Cu20 & Ne-like & $2 \mathrm{~S} 2$ & 2P5 & $6 D$ & & & $\left(3 D^{\star}\right) j=1.0$ & $-2 s 2$ & \\
\hline $\mathrm{Cu} 20$ & Ne-like & 252 & $2 \mathrm{P} 5$ & $5 D$ & & & $\left(P^{*}\right) j=1.0$ & 2 & \\
\hline Cu2O & Ne-1ike & 252 & $2 \mathrm{P} 5$ & $5 D$ & & & $\left.P^{*}\right) j=1.0$ & $\$ 2$ & \\
\hline Qu20 & Ne-like & $2 \mathrm{~S} 2$ & $2 \mathrm{P} 5$ & 50 & & & $\left.{ }^{*}\right) j=1.0$ & S2 & \\
\hline 20 & Ne-1ike & $2 \mathrm{~s} 2$ & $2 \mathrm{P} 5$ & $5 D$ & & & $j=1.0$ & 2 & \\
\hline 20 & Ne-like & $2 \mathrm{~S} 2$ & 2P5 & $5 D$ & & & *) $j=$ & 32 & \\
\hline 2020 & Ne-like & $2 \$ 2$ & $2 \mathrm{P} 5$ & 5D & & & *) $j=1.0$ & - & \\
\hline 20 & Ne-like & $2 S$ & $2 P 6$ & $4 \mathrm{P}$ & & & $p * j=1.0$ & & \\
\hline $2 u 20$ & Ne-like & $2 \mathrm{~S}$ & $2 \mathrm{P} 6$ & $4 P$ & & & $\left(1 P^{*}\right) j=1.0$ & & \\
\hline 20 & Ne-1ike & $2 S$ & P6 & $4 \mathrm{P}$ & & & $1 P * \mid j=1.0$ & 52 & \\
\hline $\mathrm{Cu} 20$ & Ne-like & $2 S$ & $2 P 6$ & $4 P$ & & & $\left.1 P^{*}\right) j=1.0$ & 82 & \\
\hline Cu20 & Ne-like & $2 s$ & $2 P 6$ & $4 P$ & & & *j $j=1.0$ & s2 & \\
\hline $\mathrm{Cu} 20$ & Ne-like & $2 s$ & $2 P 6$ & $4 P$ & & & $1 P^{\star} j j=1.0$ & $\mathrm{~s} 2$ & \\
\hline $\mathrm{Cu} 20$ & Ne-like & 25 & $2 P 6$ & $4 \mathrm{P}$ & & & $\left.3 P^{*}\right) j=1.0$ & $\$ 2$ & \\
\hline $\mathrm{Cu} 20$ & Ne-like & $2 S$ & $2 \mathrm{P} 6$ & $4 P$ & & & $3 P^{\star} j j=1.0$ & $\$ 2$ & \\
\hline Cu20 & Ne-1ike & $2 \mathrm{~s}$ & $2 P 6$ & $4 \mathrm{P}$ & & & $3 P *) j$ & $\$ 2$ & \\
\hline Cu2O & Ne-like & $2 S$ & $2 \mathrm{P} 6$ & $4 \mathrm{P}$ & & & 1.0 & S2 & \\
\hline Cu2O & Ne-like & $2 S$ & $2 \mathrm{P} 6$ & $4 P$ & & & 1.0 & S2 & \\
\hline Cu20 & Ne-like & $2 S$ & $2 P 6$ & $4 \mathrm{P}$ & & & *) $j=1.0$ & S2 & \\
\hline Cu2O & Ne-like & 252 & $2 \mathrm{PS}$ & 5D & & & $j=1.0$ & $\$ 2$ & \\
\hline Cu2 20 & Ne-like & $2 \mathrm{~s} 2$ & P5 & $5 \mathrm{D}$ & & & k) $j=1.0$ & & \\
\hline $\mathrm{Cu} 20$ & Ne-like & $2 \mathrm{~s} 2$ & 5 & 5D & & & $\left(3 D^{*}\right) j=1.0$ & $\$ 2$ & \\
\hline 20 & Ne-like & 252 & 95 & $5 D$ & & & *) $j=1.0$ & S2 & \\
\hline 220 & Ne-like & $2 \mathrm{~S} 2$ & P5 & $5 D$ & & & $j=1.0$ & 252 & \\
\hline Cu20 & Ne-like & 252 & 25 & $5 D$ & & & $j j=1.0$ & $-2 S 2$ & \\
\hline 2020 & Ne-like & $2 \mathrm{~s} 2$ & P5 & 45 & & & $j=1.0$ & $-2 s 2$ & \\
\hline 2020 & Ne-like & 252 & P5 & $4 S$ & & & $j=1.0$ & $-2 S 2$ & \\
\hline Cu2O & Ne-like & $2 s 2$ & 55 & $4 S$ & & & $j=1.0$ & $-2 s 2$ & \\
\hline Cu20 & Ne-like & $2 s$ & 06 & $3 P$ & & & $j=1.0$ & $-2 s 2$ & \\
\hline Cu20 & Ne-like & $2 s 2$ & P5 & 35 & & & 1.0 & $-2 s 2$ & \\
\hline Cu2O & Ne-like & 252 & P5 & 35 & & & .0 & $-2 s 2$ & \\
\hline $\mathrm{Cu} 20$ & Ne-like & 252 & 205 & 35 & & & $\left(1 P^{*}\right) j$ & $-2 s 2$ & \\
\hline $\mathrm{Cu} 20$ & $\mathrm{Ne}-1$ & $2 \mathrm{~S} 2$ & 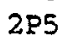 & $3 s$ & & & 1.0 & $-2 s 2$ & \\
\hline $\mathrm{Cu} 20$ & Ne-like & $2 \mathrm{~S} 2$ & $2 \mathrm{P} 5$ & $3 S$ & & & $\left(3 P^{*}\right) j$ & $S 2$ & \\
\hline Cu2O & Ne-like & $2 \mathrm{~S} 2$ & & 35 & & & $\left(3 P^{*}\right) j=1.0$ & 252 & \\
\hline Cu21 & F-like & 252 & & $(1 S$ & ) & $4 D$ & $(2 D) j=2.5$ & $2 s 2$ & \\
\hline Cu21 & F-like & $2 \mathrm{~s} 2$ & 24 & ( 15 & ) & $4 D$ & $(2 D) j=2.5$ & $-2 s 2$ & \\
\hline Cu21 & F-like & $2 s 2$ & P4 & ( $1 S$ & 1 & $4 D$ & (2D) $j=2.5$ & $-2 s 2$ & \\
\hline Cu21 & F-like & 252 & 24 & ( IS & ) & $4 D$ & $(2 D) j=2.5$ & $-2 s 2$ & \\
\hline $\mathrm{Cu} 21$ & F-1ike & $2 \mathrm{~S} 2$ & $P 4$ & ( 15 & ) & $4 D$ & $(2 D) j=2.5$ & $-2 s 2$ & \\
\hline Cu21 & F-like & 252 & $P 4$ & ( 15 & i & $4 D$ & (2D) $j=2.5$ & $-2 s 2$ & \\
\hline Cu21: & F-like & $2 s 2$ & P4 & ( 15 & ) & $4 D$ & $(2 D) j=1.5$ & $-2 s 2$ & \\
\hline Cu21 & F-like & $2 \mathrm{~S} 2$ & 294 & i 15 & ) & $4 D$ & (2D $) j=1.5$ & $-2 s 2$ & \\
\hline Cu21. & F-like & $2 s 2$ & & ( Is & ) & $4 D$ & $(2 D) j=1.5$ & $-2 s 2$ & \\
\hline Cu21 & F-like & 252 & P4 & ( 15 & ; & $4 D$ & $(2 D) j=1.5$ & $-2 S 2$ & \\
\hline
\end{tabular}

\begin{tabular}{|c|c|c|}
\hline$(1 S) j=.0$ & 8.070 & quartz 100 \\
\hline$(1 s) j=.0$ & 8.070 & $\operatorname{TAP} 100$ \\
\hline$(1 s) j=.0$ & 8.330 & topaz 002 \\
\hline$j=.0$ & 8.330 & quartz 100 \\
\hline$j j=.0$ & 8.330 & PET 002 \\
\hline$j j=.0$ & 8.330 & EDT 020 \\
\hline$j j=.0$ & 8.330 & TAP 100 \\
\hline$j j=.0$ & 8.330 & RAP 100 \\
\hline$j j=.0$ & 8.383 & quartz 100 \\
\hline$j=.0$ & 8.383 & PET 002 \\
\hline$(1 s) j=.0$ & 8.383 & EDT 020 \\
\hline$S) j=.0$ & 8.383 & TAP 100 \\
\hline$j=.0$ & 8.383 & RAP 100 \\
\hline$j j=.0$ & 8.383 & $\operatorname{KAP} 100$ \\
\hline$j=.0$ & 8.395 & quartz 100 \\
\hline$j j=.0$ & 8.395 & PET 002 \\
\hline$j=.0$ & 8.395 & EDT 020 \\
\hline$j=.0$ & 8.395 & TAP 100 \\
\hline$j=.0$ & 8.395 & RAP 100 \\
\hline$s) j=.0$ & 8.395 & KAP 100 \\
\hline$S) j=.0$ & 8.444 & quartz 100 \\
\hline$j=.0$ & 8.444 & PET 002 \\
\hline$j=.0$ & 8.444 & EDT 020 \\
\hline$j j=.0$ & 8.444 & TAP 100 \\
\hline$j=.0$ & 8.444 & RAP 100 \\
\hline$j=.0$ & 8.444 & KAP 100 \\
\hline$j=.0$ & 9.371 & mica 002 \\
\hline$j=.0$ & 9.423 & mica 002 \\
\hline$j j=.0$ & 9.522 & mica 002 \\
\hline$j=.0$ & 10.599 & ADP 101 \\
\hline$j j=.0$ & 12.573 & TAP 100 \\
\hline$j=.0$ & 12.573 & RAP 100 \\
\hline$j=.0$ & 12.573 & KAP 100 \\
\hline$j j=.0$ & 12.830 & TAP 100 \\
\hline$j j=.0$ & 12.830 & RAP 100 \\
\hline$j j=.0$ & 12.830 & KAP 100 \\
\hline$j=1.5$ & 8.437 & quartz 100 \\
\hline$j=1.5$ & 8.437 & PET 002 \\
\hline$i j=1.5$ & 8.437 & EDT 020 \\
\hline$j=1.5$ & 8.437 & $\operatorname{TAP} 100$ \\
\hline *) $j=1.5$ & 8.437 & RAP 100 \\
\hline *) $j=1.5$ & 8.437 & KAP 100 \\
\hline$\left.{ }^{*}\right) j=.5$ & 8.554 & PET 002 \\
\hline$+j=.5$ & 8.554 & EDT 020 \\
\hline$j=.5$ & 8.554 & TAP 100 \\
\hline$j=.5$ & 8.554 & $\operatorname{RAP} 100$ \\
\hline
\end{tabular}

$8.512 \quad 71.455$ $25.763 \quad 70.005$ $8.374 \quad 84.124$

$8.512 \quad 78.130$

$8.742 \quad 72.340$

$8.808 \quad 71.037$

$25.763 \quad 75.929$

$26.116 \quad 73.114$

$8.512 \quad 80.012$

$8.742 \quad 73.523$

$8.808 \quad 72.129$

$\begin{array}{lll}25.763 & 77.466\end{array}$

$26.116 \quad 74.360$

$26.634 \quad 70.777$

$8.512 \quad 80.489$

$8.742 \quad 73.803$

$8.808 \quad 72.385$

$25.763 \quad 77.840$

$26.116 \quad 74.655$

$26.634 \quad 71.014$

$8.512 \quad 82.753$

$8.742 \quad 74.997$

$8.808 \quad 73.471$

$25.763 \quad 79.505$

$26.116 \quad 75.925$

$26.634 \quad 72.011$

$\begin{array}{ll}19.942 & 70.022\end{array}$

$19.942 \quad 70.916$

$19.942 \quad 72.740$

$10.640 \quad 84.968$

$25.763 \quad 77.435$

$26.116 \quad 74.335$

$26.634 \quad 70.757$

$25.763 \quad 84.875$

$26.116 \quad 79.277$

$26.634 \quad 74.457$

$8.512 \quad 82.388$

$8.742 \quad 74.821$

$8.808 \quad 73.311$

$\begin{array}{ll}25.763 & 79.252\end{array}$

$26.116 \quad 75.737$

$26.634 \quad 71.865$

$8.742 \quad 78.096$

$8.808 \quad 76.207$

$25.763 \quad 84.925$

$26.116 \quad 79.301$

Page 94 
Cu21 F-like 2S2 2P4 ( 1S ) 4D (2D) $j=1.5-2 S 22 P 5$ Cu21 $F$-like 2S2 2P4 ( $1 D$ ) $4 D(2 D) j=1.5-2 S 22 P 5$ Cu21 F-like 2S2 2P4 (1D) 4D (2D) $j=1.5-2 S 22 P 5$ Cu21 F-like 2S2 2P4 (1D) 4D (2D) $j=1.5-2 S 22 P 5$ Cu21 F-like 2S2 2P4 ( $1 D$ ) $4 D(2 D) j=1.5-2 S 2$ 2P5 Cu21 F-like 2S2 2P4 ( $1 D$ ) $4 D(2 D) j=1.5-2 S 22 P 5$ Cu21 F-like 2S2 2P4 ( 1D) 4D (2D) j=2.5 - 2S2 2P5 Cu21 F-like 2S2 2P4 ( ID) $4 D(2 D) j=2.5-2 S 22 P 5$ Cu21 F-like 2S2 2P4 ( $1 D$ ) $4 D$ (2D) $j=2.5-2 S 22 P 5$ Cu21 F-like 2S2 2P4 ( 1D) 4D (2D) j=2.5 - 2S2 2P5 Cu21 F-like 2S2 2P4 ( ID) 4D (2D) j=2.5 - 2S2 2P5 Cu21 F-like 2S2 2P4 ( ID) 4D (2D) $j=2.5-2 S 22 P 5$ Cu21 F-like 2S2 2P4 ( $1 D$ ) $4 D(2 D) j=2.5-2 S 22 P 5$ Cu21 F-like 2S2 2P4 (1D) 4D (2D) $j=2.5-2 S 22 P 5$ Cu21 F-like 2S2 2P4 ( $3 P$ ) $4 D(4 P) j=2.5-2 S 22 P 5$ Cu21 F-like 2S2 2P4 ( 3P) $4 D(4 P) j=2.5-2 S 22 P 5$ Cu21 F-1ike 2S2 2P4 (3P) $4 D(4 P) j=2.5-2 S 22 P 5$ Cu21 $F$-like 2S2 2P4 ( $3 P$ ) $4 D(4 P) j=2.5-2 S 22 P 5$ Cu21 F-like 2S2 2P4 ( 3P) $4 D(4 F) j=2.5-2 S 22 P 5$ Cu21 F-like 2S2 2P4 (3P) 4D (4F) j=2.5-2S2 2P5 Cu21 F-like 2S2 2P4 ( $3 P$ ) $4 D(4 F) j=2.5-2 S 22 P 5$ Cu21 F-like 2S2 2P4 ( 3P) $4 D(4 F) j=2.5-2 S 22 P 5$ Cu21 $F$-like 2S2 2P4 ( $1 D)$ 4D (2D) $j=1.5-2 S 22 P 5$ Cu21 F-1ike 2S2 2P4 (1D) 4D (2D) j=1.5 - 2S2 2P5 Cu21 F-like 2S2 2P4 ( 1D) 4D (2D) $j=1.5-2 S 2$ 2P5 Cu21 F-like 2S2 2P4 ( 3P) 4D (2D) $j=2.5-2 S 22 P 5$ Cu21 F-like 2S2 2P4 ( 3P) 4D (2D) j=2.5 - 2S2 2P5 Cu21 F-like 2S2 2P4 (3P) 4D (2P) j=1.5 - $2 S 22 P 5$ Cu21 F-like 2S2 2P4 ( 3P) $4 D(2 P) j=1.5-2 S 22 P 5$ Cu21 F-like 2S2 2P4 ( 1D) 4S (2D) $j=2.5$ - 2S2 2P5 Cu21 F-like 2S2 2P4 (1D) 4S (2D) j=2.5 - 2S2 2P5 Cu21 F-like 2S2 2P4 ( 3P) 4S (4P) j=1.5 - 2S2 2P5 Cu21 F-like $2 S$ 2P5 ( $\left.1 P^{*}\right) 3 P(2 D) j=2.5-2 S 22 P 5$ Cu21 F-like 2S 2P5 (1P*) 3P $(2 P) j=1.5-2 S 22 P 5$ Cu21 F-like 2S 2P5 (1P*) 3P (2P) j=.5-2S2 2P5 Cu21 F-like 2S 2P5 (1P*) 3P (2D) $j=1.5-2 S 2$ 2P5 ci21 F-like $2 S$ 2P5 (3P*) 3P (4P) $j=2.5-2 S 22 P 5$ Cu21 $F$-like $2 S$ 2P5 (3P*) 3P (2P) $j=.5-2 S 22 P 5$ Cu21 F-like 2S 2P5 ( $\left.3 P^{*}\right) 3 P(2 P) j=1.5-2 S 22 P 5$ Cu21 $F$-like 2S 2P5 ( $\left.3 P^{*}\right) 3 P(2 S) j=.5-2 S 2$ 2P5 Cu21 F-like $2 S$ 2P5 (3P*) 3P (2D) $j=2.5-2 S 22 P 5$ Cu21 $F$-like 2S 2P5 ( $\left.3 P^{*}\right) 3 P(2 D) j=1.5-2 S 22 P 5$ Cu21 F-like 2S $2 P 5\left(3 P^{*}\right) 3 P(4 D) j=1.5-2 S 22 P 5$ Cu21 F-like 2S 2P5 (3P*) 3P (4D) j=2.5 - 2S2 2P5 Cu21 F-like 2S 2P5 (3P*) 3P $44 P) j=1.5-2 S 22 P 5$ Cu21 F-like 2S2 $2 P 4$ ( $1 S$ ) $3 D$ (2D $j j=2.5-2 S 22 P 5$
$(2 P *) j=.5 \quad 8.554 \quad K A P 100$ $\left\{2 P^{*}\right\} j=1.5 \quad 8.574 \quad$ PET 002 $\left(2 P^{*}\right) j=1.5$ $\left(2 P^{*}\right) j=1.5$ $\left(2 P^{*}\right) j=1.5$ $(2 P *) j=1.5$ $\left(2 P^{*}\right) j=1.5$ $\left(2 P^{*}\right) j=1.5$ $\left(2 P^{\star}\right) j=1.5$ $\left(2 P^{*}\right) j=1.5$ $(2 P *) j=1.5$ $\left(2 P^{*}\right) j=1.5$ $\left(2 P^{*}\right) j=1.5$ $\left(2 P^{*}\right) j=1.5$ $\left(2 P^{*}\right) j=1$. $(2 P *) j=1.5$ $\left(2 P^{*}\right) j=1.5$ $\left(2 P^{*}\right) j=1.5$ $(2 P *) j=1.5$ $\left(2 P^{*}\right) j=1.5$ $(2 P *) j=1.5$ $\left(2 P^{*}\right) j=1.5$ $\left(2 P^{*}\right) j=.5$ $\left(2 P^{\star}\right) j=.5$ $\left(2 P^{*}\right) j=.5$ $\left(2 P^{*}\right) j=1.5$ $\left(2 P^{*}\right) j=1.5$ $\left(2 P^{*}\right) j=.5$ $\left(2 P^{\star}\right) j=.5$ $\left(2 P^{*}\right) j=1.5$ $\left(2 P^{*}\right) j=1.5$ $\left(2 P^{*}\right) j=1.5$ $\left(2 P^{*}\right) j=1.5$ (2P*) $j=$ $\left(2 P^{\star}\right) j=.5$ $\left(2 p^{*}\right) j=$ $\left(2 P^{*}\right) j=1$. $\left(2 P^{*}\right) j=1$.

8.574 EDT 020

8.574 TAP 100

8.574 RAP 100

$8.574 \mathrm{KAP} 100$

8.591 PET 002

8.591 EDT 020

8.591 RAP 100

8.591 KAP 100

8.591 PET 002

8.591 EDT 020

8.591 RAP 100

8.591 KAP 100

8.652 PET 002

8.652 EDT 020

8.652 RAP 100

8.652 KAP 100

8.691 PET 002

8.691 EDT 020

8.691 RAP 100

8.691 KAP 100

8.707 PET 002

8.707 EDT 020

8.707 KAP 100

8.754 EDT 020

8.754 KAP 100

8.777 EDT 020

8.777 KAP 100

8.808 KAP 100

8.811 KAP 100

8.870 KAP 100

9.912 mica 002

10.057 ADP 101

10.074 ADP 101

10.121 ADP 101

10.203 ADP 101

$\left(2 P^{*}\right) j=1.5 \quad 10.260 \quad$ ADP 101

$\left(2 P^{\star}\right) j=.5 \quad 10.282 \quad$ ADP 101

$\left(2 P^{*}\right) j=1.5 \quad 10.291 \quad$ ADP 101

$\left(2 P^{*}\right) j=.5 \quad 10.306 \quad$ ADP 101

$(2 P *) j=1.5 \quad 10.316 \quad$ ADP 101

$\left(2 P^{*}\right) j=1.5 \quad 10.354 \quad$ ADP 101

$\left(2 P^{*}\right) j=.5 \quad 10.392 \quad$ ADP 101

$\left\langle 2 P^{*}\right\rangle j=1.5 \quad 10.625$ ADP 101
$26.634 \quad 74.473$

$8.742 \quad 78.749$

$8.808 \quad 76.764$

$25.763 \quad 86.767$

$26.116 \quad 80.035$

$26.634 \quad 74.963$

$8.742 \quad 79.335$

$8.808 \quad 77.255$

$26.116 \quad 80.704$

26.63475 .392

$8.742 \quad 79.335$

$8.808 \quad 77.255$

$26.116 \quad 80.704$

$26.634 \quad 75.392$

$8.742 \quad 81.771$

$8.808 \quad 79.201$

$26.116 \quad 83.655$

$26.634 \quad 77.044$

$8.742 \quad 83.808$

$8.808 \quad 80.651$

$26.116 \quad 86.712$

$26.634 \quad 78.219$

$8.742 \quad 84.871$

$8.808 \quad 81.315$

$26.534 \quad 78.736$

$8.808 \quad 83.652$

$26.634 \quad 80.413$

$8.808 \quad 85.192$

$26.634 \quad 81.349$

$26.634 \quad 82.800$

$26.634 \quad 82.956$

$26.634 \quad 87.567$

$19.942 \quad 83.764$

$10.640 \quad 70.945$

$10.640 \quad 71.228$

$10.640 \quad 72.031$

$10.640 \quad 73.522$

$10.640 \quad 74.121$

$10.640 \quad 74.641$

$10.640 \quad 75.095$

$10.640 \quad 75.285$

$10.640 \quad 75.606$

$10.640 \quad 75.824$

$10.640 \quad 76.685$

$10.640 \quad 77.605$

$10.640 \quad 86.957$ 
Cu21 F-like 2S2 2P4 ( 3P) 3S (2P) $j=1.5-2 S 22 P 5$ Cu21 F-like 2S2 2P4 ( $3 P$ ) 3S (2P) $j=.5-2 S 22 P 5$ Cu21 F-like 2S2 2P4 ( 3P) 3S (4P) $j=2.5-2 S 2$ 2P5 Cu21 $F$-like 2S2 2P4 ( 3P) 3S (4P) $j=1.5-2 S 22 P 5$ Cu22 O-like 2S2 2P3 ( $\left.2 P^{*}\right) 4 D\left(3 D^{*}\right) j=2.0-2 S 22 P 4$ Cu22 0 -like 2S2 2P3 ( $\left.2 P^{*}\right) 4 D\left(3 D^{*}\right) j=2.0-2 S 22 P^{*}$

Cu22 O-like 2S2 2P3 (2P*) 4D (3D*) j=2.0 - 2S2 2P4

Cu22 O-like 2S2 2P3 (2D*) $4 D\left(3 D^{*}\right) j=3.0-2 S 22 P 4$

Cu22 $O$-like 2S2 2P3 ( 2D*) $4 D\left(3 D^{*}\right) j=3.0-2 S 22 P 4$

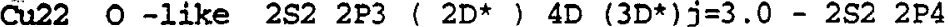

Cu22 0 -like 2S2 $2 P^{2}\left(2 D^{*}\right) 4 D\left(3 D^{*}\right) j=3.0-2 S 22 P 4$

Cu22 0 -like 2S2 2P3 (2D*) $4 D\left(3 D^{*}\right) j=3.0-2 S 22 P 4$

Cu22 o-1ike 2S2 2P3 ( $\left.2 D^{*}\right) 4 D\left(3 D^{*}\right) j=3.0-2 S 22 \mathrm{P} 4$

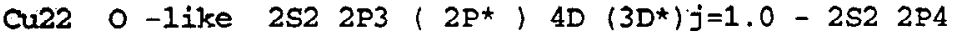

Cu22 0 -like 2S2 $2 P^{2}\left(2 P^{*}\right) 4 D\left(3 D^{*}\right) j=1.0-2 S 22 P 4$

$\mathrm{Cu} 220$-like 2S2 $2 P^{2}\left(2 P^{*}\right) 4 D\left(3 D^{*}\right) j=1.0-2 S 22 P 4$

Ci22 0 -like 2S2 2P3 (2D*) 4D (3F*) j=3.0- 2S2 2P4

Ci22 0 -like 2S2 $2 P^{2}\left(2 D^{*}\right) 4 D\left(3 F^{*}\right) j=3.0-2 S 22 P 4$

Ci22 0 -like 2S2 2P3 (2D*) 4D (3F*) j=3.0 - 2S2 2P4

cu22 O-like 2S2 2P3 (2P*) $4 D\left(3 F^{*}\right) j=3.0-2 S 22 P^{*}$

Cu22 0 -like 2S2 2P3 ( 2P*) 4D (3F*) j=3.0-2S2 2P4

Civ22 0 -like 2S2 2P3 ( 2P*) $4 D\left(3 F^{*}\right) j=3.0-2 S 22 P^{*}$

Cu22 0 -1ike 2S2 2P3 ( 2P*) 4D (3F*) j=3.0 - 2S2 2P4

Cu22 0 -like 2S2 2P3 (2P*) $4 D\left(3 F^{*}\right) j=3.0-2 S 22 P 4$

Cu22 0 -like 2S2 $2 P^{2}\left(4 S^{*}\right) 4 D\left(3 D^{*}\right) j=3.0-2 S 22 P 4$

Cu22 O-like 2S2 2P3 ( 4S*) $4 D\left(3 D^{*}\right) j=3.0-2 S 22 P 4$

Cu22 0 -like 2S2 2P3 ( $\left.4 S^{*}\right) 4 D\left(3 D^{*}\right) j=3.0-2 S 22 P 4$

cu22 O-like 2S2 $2 P^{2} 3$ ( $\left.4 S^{*}\right) 4 D \cdot\left(3 D^{*}\right) j=3.0-2 S 22 P 4$

Cu22 0 -like $2 S 22 \mathrm{P}^{\prime}\left(4 S^{*}\right) 4 \mathrm{D}\left(3 \mathrm{D}^{*}\right) j=3.0-2 \mathrm{S2} 2 \mathrm{P4}$

Cu22 0 -like 2S2 2P3 ( $\left.4 S^{*}\right) 4 D\left(3 D^{*}\right) j=3.0-2 S 22 P 4$

Cu22 O-like 2S2 2P3 ( $\left.4 S^{*}\right) 4 D\left(3 D^{*}\right) j=3.0-2 S 22 P 4$

Cu22 0 -like 2S2 $2 \mathrm{P}^{2}\left(\mathrm{SS}^{*}\right) 4 \mathrm{D}\left(3 \mathrm{D}^{*}\right) j=3.0-2 S 22 \mathrm{P} 4$

$\mathrm{Cu} 22 \mathrm{O}$-like $2 \mathrm{S2} 2 \mathrm{P} 3\left(4 \mathrm{~S}^{*}\right) 4 \mathrm{D}\left(3 \mathrm{D}^{*}\right) \mathrm{j}=3.0-2 \mathrm{S2} 2 \mathrm{P} 4$

Cu22 0 -like 2S2 2P3 (4S*) 4D $\left(3 D^{*}\right) j=3.0-2 S 22 P 4$

Ci22 0 -like 2S2 $2 P^{2}\left(2 D^{*}\right) 4 D\left(1 F^{*}\right) j=3.0-2 S 22 P 4$

Ci22 0 -like 2S2 2P3 ( 2D*) $4 D\left(1 F^{*}\right) j=3.0-2 S 22 P 4$

Cii22 0 -like 2S2 $2 P_{3}\left(2 D^{*}\right) 4 D\left(1 F^{*}\right) j=3.0-2 S 22 P 4$

Cu22 O-like 2S2 2P3 ( 2D*) 4D (1F*) j=3.0-2S2 2P4

Cu22 $O$-like 2S2 2P3 ( 2D*) $4 D\left(1 D^{*}\right) j=3.0-2 S 22 P 4$

Ci22 $O$-like 2S2 2P3 ( 2D*) $4 D\left(1 F^{*}\right) j=3.0-2 S 22 P 4$

Ci22 0 -like 2S2 2P3 (4S*) $4 D\left(3 D^{\star}\right) j=1.0-2 S 22 P 4$

Cu22 0 -like 2S2 2P3 ( 4S*) 4D (3D*)j=1.0 - 2S2 2P4

$\mathrm{Cu} 220$-like 2S2 2P3 (4S*) 4D (3D*)j=1.0-2S2 2P4

Cu22 0 -like 2S2 2P3 (4S*) $4 D\left(3 D^{*}\right) j=1.0-2 S 22 P 4$

Cu22 .0 -like 2S2 2P3 ( $\left.4 S^{*}\right) 4 D\left(3 D^{*}\right) j=1.0-2 S 22 P 4$

Cu22 0 -like $2 S 22 \mathrm{P} 3\left(4 S^{*}\right) 4 \mathrm{D}\left(3 D^{*}\right) j=1.0-2 S 22 \mathrm{P} 4$ $\left(2 P^{*}\right) j=1.5 \quad 12.140 \quad$ TAP 100

$\left(2 \mathrm{P}^{*}\right) j=.5 \quad 12.165$ TAP 100

$\left(2 P^{*}\right) j=1.5 \quad 12.186$ TAP 100

$\left(2 P^{*}\right) j=.5 \quad 12.203$ TAP 100

(3P) $j=1.0 \quad 8.086$ topaz 002

(3P) $j=1.0 \quad 8.086$ quartz 100

(3P ) $j=1.0 \quad 8.086$ TAP 100

(3P) $j=2.0 \quad 8.125$ topaz 002

(3P) $j=2.0 \quad 8.125$ quartz 100

(3P) $j=2.0$

(3P) $j=2.0$

(3P) $j=2.0$

(3P) $j=2.0$

(3P) $j=1.0$

(3P) $j=1.0$

(3P) $j=1.0$

(3P) $j=2.0$

( $3 P) j=2.0$

(3P) $j=2.0$

(ID) $j=2.0$

(1D) $j=2.0$

(ID) $j=2.0$

(ID) $j=2.0$

(1D) $j=2.0$

( $3 P$ ) $j=2.0$

(3P) $j=2.0$

(3P) $j=2.0$

( $3 P$ ) $j=2.0$

(3P) $j=2.0$

(3P) $j=2.0$

8.125

8.125

8.125

8.125

8.158

8.158

8.158

8.171

8.171

8.171

8.222
8.222

AP 100

topaz 002

quartz 100

TAP 100

topaz 002

quartz 100

topaz 002

quartz 100

TAP 100

8.222

topaz 002

8.222

8.275

PET 002

8.275

quartz 100

275

PET 002

(3P) $j=2.0$

(3P) $j=2.0$

(3P) $j=2.0$

(3P) $j=2.0$

(1D) $j=2.0$

(ID) $j=2.0$

(ID) $j=2.0$

(ID) $\mathrm{j}=2.0$

(ID) $j=2.0$

(1D) $j=2.0$

(3P ) $j=.0$

(3P) $j=.0$

(3P) $j=.0$

(3P) $j=.0$

$(3 P) j=.0$

(3P) $j=.0$
8.275 topaz 002

275 quartz 100

8.275 PET 002

8.275 TAP 100

8.275 RAP 100

8.281 topaz 002

8.281 quartz 100

8.281 PET 002

8.281 EDT 020

8.281 TAP 100

8.281 RAP 100

8.333 topaz 002

8.333 quartz 100

8.333 PET 002

8.333 EDT 020

8.333 TAP 100
8.333 RAP 100
$25.763 \quad 70.465$

$25.763 \quad 70.800$

$25.763 \quad 71.086$

$25.763 \quad 71.321$

$8.374 \quad 74.930$

$8.512 \quad 71.797$

$\begin{array}{ll}25.763 & 70.319\end{array}$

$8.374 \quad 75.993$

$8.512 \quad 72.657$

$25.763 \quad 71.107$

$8.374 \quad 75.993$

$8.512 \quad 72.657$

$25.763 \quad 71.107$

$8.374 \quad 76.958$

$8.512 \quad 73.418$

$25.763 \quad 71.799$

$8.374 \quad 77.358$

$8.512 \quad 73.727$

$25.763 \quad 72.079$

$8.374 \quad 79.067$

$8.512 \quad 75.001$

8.74270 .139

$\begin{array}{ll}25.763 & 73.220\end{array}$

$26.116 \quad 70.818$

$8.374 \quad 81.181$

$8.512 \quad 76.448$

$8.742 \quad 71.188$

$25.763 \cdot 74.492$

$26.116 \quad 71.909$

$8.374 \quad 81.181$

$8.512 \quad 76.448$

$8.742 \quad 71.188$

$25.763 \quad 74.492$

$26.116 \quad 71.909$

$8.374 \quad 81.453$

8.51276 .621

$8.742 \quad 71.310$

$8.808 \quad 70.080$

$25.763 \quad 74.642$

$26.116 \quad 72.037$

$8.374 \quad 84.328$

$8.512 \quad 78.229$

$8.742 \quad 72.405$

$8.808 \quad 71.098$

$25.763 \quad 76.012$

$26.116 \quad 73.182$ 
$\mathrm{Cu} 22$ - -like 2S2 $2 \mathrm{P} 3\left(4 S^{*}\right) 4 \mathrm{D}\left(3 \mathrm{D}^{*}\right) j=2.0-2 S 22 \mathrm{P} 4$

Cu22 0 -like 2S2 $2 \mathrm{P}^{2}\left(\mathrm{SS}^{\star}\right) 4 \mathrm{D}\left(3 \mathrm{D}^{\star}\right) j=2.0-2 S 22 \mathrm{P} 4$

Cu22 O-like 2S2 2P3 (4S*) 4D (3D*)j=2.0-2S2 2P4

Cu22 0 -like 2S2 $2 P^{2}\left(4 S^{*}\right) 4 D\left(3 D^{*}\right) j=2.0-2 S 22 P 4$

Cu22 0 -like 2S2 2P3 ( 4S*) $4 D\left(3 D^{*}\right) j=2.0-2 S 22 P 4$

Cu22 O-like 2S2 2P3 (4S*) $4 D\left(3 D^{*}\right) j=2.0-2 S 22 P 4$

Cu22 0 -like 2S2 2P3 ( $\left.2 P^{*}\right) 3 D\left(1 D^{*}\right) j=2.0-2 S 22 P^{*}$

Cu22 0 -like 2S2 2P3 ( $\left.2 P^{*}\right) 3 D\left(3 D^{*}\right) j=2.0-2 S 22 P 4$

Cu22 0 -like 2S2 2P3 (2P*) 3D (3D*) j=1.0 - 2S2 $2 \mathrm{P} 4$

Cu22 0 -like 2S2 2P3 ( 2P*) 3D (3F*) $j=3.0-2 S 22 P^{*} 4$

Cu22 0 -1ike 2S2 2P3 $\left(2 P^{\star}\right) 3 D\left(3 P^{*}\right) j=.0-2 S 22 P 4$

Cu22 0 -1ike 2S2 2P3 ( 2D*) 3D (3S*) j=1.0 - 2S2 2P4

Cu22 0 -like 2S2 $2 P_{3}\left(2 D^{*}\right) 3 D\left(3 S^{*}\right) j=1.0-2 S 22 P 4$

Cu22 0 -like $2 S 22 P^{2}\left(2 D^{*}\right) 3 D\left(3 P^{*}\right) j=2.0-2 S 22 P 4$

Cu22 0 -like 2S2 2P3 ( 2D*) 3D $\left(3 D^{*}\right) j=3.0-2 S 22 P 4$

Cu22 0 -like 2S2 2P3 ( 2D*) 3D (3D*) j=3.0-2S2 2P4

Cu22 0 -like 2S2 2P3 ( 2P*) 3D (3D*) $j=1.0-2 S 22 P 4$

Cu22 0 -like $2 S 22 P^{2}\left(2 D^{*}\right) 3 D\left(1 P^{*}\right) j=1.0-2 S 22 P 4$

Cu22 0 -like 2S2 2P3 ( $\left.2 D^{*}\right) 3 D\left(3 G^{*}\right) j=3.0-2522 P 4$

Cu22 0 -like 2S2 2P3 (2D*) 3D $\left(3 F^{*}\right) j=3.0-2 S 22 P 4$

Cu22 0 -like 2S2 $2 P^{2} 3\left(2 D^{*}\right) 3 D\left(1 D^{*}\right) j=2.0-2 S 22 P 4$

Cu22 O-like 2S2 2P3 (2P*) 3D $\left(3 F^{*}\right) j=2.0-2 S 22 P 4$

Cu22 0 -like 2S2 2P3 (2. $\left.D^{*}\right) 3 D\left(3 P^{*}\right) j=1.0-2 S 22 P 4$

Cu22 0 -like 2S2 2P3 ( 2D*) 3D $\left(3 P^{*}\right) j=.0-2 S 22 P 4$

Cu22 0 -like 2S2 2P3 (2D*) 3D (1F*) j=3.0-2S2 2P4

Cu22 0 -like 2S2 $2 \mathrm{P} 3\left(4 S^{*}\right) 3 D\left(3 D^{*}\right) j=3.0-2 S 22 \mathrm{P} 4$

Cu22 0 -like 2S2 $2 \mathrm{P} 3$ ( $\left.4 S^{*}\right)$ 3D (3D*) $j=3.0-2 S 22 \mathrm{P} 4$

Cu23 N-like 2S2 2P2 3D (2P) $j=1.5-2 S 22 P 3$

Cu24 C-like 2S2 2P $3 S$

Cu24 C-like 2S2 2P $3 S$

Cu24 C-like 2S2 2P $3 S$

Cu24 C-like 2S2 2P $3 S$

Cu26 Be-like 152 2P

Cu26. Be-like 1s2 2P

Cu26 Be-like 1S2 2P

Cu26 Be-like 1S2 2P

Cu26 Be-like 1S2 2P

Cu26 Be-like 1S2 2F

Cu26 Be-like 1S2 2P

Cu26 Be-like 1S2 2P

Cu26 Be-like 1S2 29

Cu26 Be-like 1S2 2P

Cu26- Be-like 1522 P

Cu26. Be-like 1S2 2P

Cu26 Be-like 1S2 2P

Cu26 Be-like 1S2 2P

$\left(3 P^{*}\right) j=2.0-2 S 22 P 2$

$\left(1 P^{*}\right) j=1.0-2 S 22 P 2$

(1P*) $j=1.0-2 S 22 P 2$

$3 P(3 P) j=1.0-1 S 22 S$ $3 P(3 P) j=1.0-1 S 22 S$ $3 P(3 P) j=1.0-1 S 22 S$ $3 P(3 P) j=1.0-1 S 22 S$ $3 P(3 P) j=1.0-1 S 22 S$ $3 P(I D) j=2.0-1 S 22 S$ $3 P(I D) j=2.0-1 S 22 S$ $3 P(1 D) j=2.0-1 S 22 S$ $3 P$ (ID) $j=2.0-1 S 22 S$ $3 P$ (ID) $j=2.0-1 S 22 S$ $3 P$ (3S) $j=1.0-1522 S$ 3P (3S) $j=1.0-1 S 22 S$ $3 P(3 S) j=1.0-1522 s$ $3 P \quad$ (3S $) j=1.0-1 S 22 S$
(3P) $j=1.0$

(3P) $j=1.0$

(3P) $j=1.0$

(3P) $j=1.0$

(3P) $j=1.0$

(3P) $j=1.0$

(3P) $j=1.0$

(3P) $j=1.0$

(3P ) $j=1.0$

(3P) $j=2.0$

(3P) $j=1.0$

(3P) $j=2.0$

(3P) $j=2.0$

(3P) $j=2.0$

(3P) $j=2.0$

(1D) $j=2.0$

(3P) $j=2.0$

(3P) $j=2.0$

(3P) $j=2.0$

(3P) $j=2.0$

(1D) $j=2.0$

(3P ) $j=1.0 \quad 10.562 \quad$ ADP 101

(1D) $j=2.0 \quad 10.597 \quad$ ADP 101

(3P ) $j=2.0 \quad 10.611 \quad$ ADP 101

(3P) $j=2.0 \quad 10.611$ ADP 101

$\left(2 P^{*}\right) j=1.5 \quad 9.772$ mica 002

(3P) $j=.0 \quad 10.103$ ADP 101

(1D ) $j=2.0 \quad 10.170$ ADP 101

(3P) $j=1.0 \quad 10.229 \quad$ ADP 101

(1D) $j=2.0 \quad 10.400$ ADP 101
$2 \mathrm{P}\left(3 \mathrm{P}^{*}\right) j=1.0 \quad 8.530$ PET 002

$2 P\left(3 P^{*}\right) j=1.0 \quad 8.530$ EDT 020

$2 P(3 P *) j=1.0 \quad 8.530$ TAP 100

$2 P\left(3 P^{*}\right) j=1.0 \quad 8.530 \quad \operatorname{RAP} 100$

$2 P(3 P *) j=1.0 \quad 8.530$ KAP 100

$2 P\left(3 P^{*}\right) j=2.0 \quad 8.551$ PET 002

$2 P\left(3 P^{*}\right) j=2.0 \quad 8.551$ EDT 020

$2 P(3 P *) j=2.0 \quad 8.551$ TAP 100

$2 P(3 P *) j=2.0 \quad 8.551 \quad R A P 100$

$2 P(3 P *) j=2.0 \quad 8.551$ KAP 100

$2 P\left(3 P^{*}\right) j=2.0 \quad 8.616$ PET 002

$2 P\left(3 P^{*}\right) j=2.0 \quad 8.616$ EDT 020

$2 P(3 P *) j=2.0 \quad 8.616 \quad \operatorname{RAP} 100$

$2 P\left(3 P^{*}\right) j=2.0 \quad 8.616 \quad K A P \quad 100$
$8.512 \quad 80.090$

$8.742 \quad 73.569$

$8.808 \quad 72.171$

$25.763 \quad 77.528$

$26.116 \quad 74.409$

$26.634 \quad 70.816$

$10.640 \quad 74.991$

$10.640 \quad 75.824$

$10.640 \quad 76.091$

$10.640 \quad 76.408$

$10.640 \quad 76.732$

$10.640 \quad 77.089$

$10.640 \quad 77.089$

$10.640 \quad 77.605$

$10.640 \quad 77.961$

$10.640 \quad 77.961$

$10.640 \quad 78.382$

10.64078 .818

$10.640 \quad 80.729$

$10.640 \quad 80.931$

$10.640 \quad 81.495$

$10.640 \quad 82.419$

$10.640 \quad 82.584$

$10.640 \quad 83.058$

$10.640 \quad 84.847$

$10.640 \quad 85.769$

$10.640 \quad 85.769$

$19.942 \quad 78.534$

$10.640 \quad 71.719$

$10.640 \quad 72.907$

$10.640 \quad 74.023$

$10.640 \quad 77.808$

$8.742 \quad 77.356$

$8.808 \quad 75.567$

$25.763 \quad 83.356$

$26.116 \quad 78.481$

$26.634 \quad 73.905$

$8.742 \quad 78.001$

$8.808 \quad 76.125$

$25.763 \quad 84.703$

$26.116 \quad 79.195$

26.63474 .401

$8.742 \quad 80.260$

$8.808 \quad 78.015$

$26.116 \quad 81.785$

$26.634 \quad 76.046$ 


\begin{tabular}{|c|c|c|c|c|c|c|c|c|c|}
\hline Cu26 & Be-like & $1 S 2$ & $2 \mathrm{P}$ & & & $3 P$ & $(3 D) j=3.0$ & $-1 S 2$ & $2 S$ \\
\hline Cu26 & Be-like & $1 S 2$ & $2 P$ & & & $3 P$ & $(3 D) j=3.0$ & $-1 s 2$ & 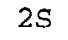 \\
\hline Cu26 & Be-like & $1 S 2$ & $2 P$ & & & $3 P$ & $(3 D) j=3.0$ & $-1 s 2$ & $2 S$ \\
\hline 2126 & Be-like & $1 \mathrm{~S} 2$ & $2 \mathrm{P}$ & & & $3 P$ & (3D & $-1 s 2$ & 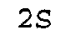 \\
\hline 26 & Be-like & 152 & $2 P$ & & & $3 P$ & $13 P$ & $-1 s 2$ & 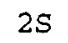 \\
\hline 126 & Be-like & $1 \mathrm{~S} 2$ & $2 \mathrm{P}$ & & & $3 P$ & $(3 P)$ & - & \\
\hline$u 26$ & Be-like & $1 S 2$ & $2 \mathrm{P}$ & & & $3 P$ & 2.0 & $\$ 2$ & \\
\hline 126 & Be-like & LS2 & $2 \mathrm{P}$ & & & $3 P$ & 2.0 & $S 2$ & \\
\hline 426 & Be-like & 152 & $2 S$ & & & $3 F$ & 1.0 & $\$ 2$ & \\
\hline Cu26 & Be-like & 152 & $2 S$ & & & $3 P$ & 1.0 & S2 & \\
\hline Cu26 & Be-like & $1 S 2$ & $2 S$ & & & $3 P$ & $\left(1 \mathrm{P}^{*}\right)$ & $-2 s 2$ & \\
\hline 26 & Be-like & 152 & $2 S$ & & & $3 P$ & $\left(1 P^{\star}\right) j=1.0$ & $-2 s 2$ & \\
\hline $\mathrm{Cu} 26$ & Be-Iike & $1 \$ 2$ & $2 P$ & & & $3 P$ & $(3 D) j=1.0$ & $-1 S 2$ & 2 \\
\hline Cu26 & Be-like & $1 \mathrm{~S} 2$ & $2 P$ & & & $3 P$ & $(3 D$ & $-1 s 2$ & $2 \mathrm{~S}$ \\
\hline $\mathrm{Cu} 26$ & Be-like & $1 \mathrm{~S} 2$ & $2 P$ & & & $3 P$ & (3D & $-1 s 2$ & $2 S$ \\
\hline $\mathrm{Cu} 26$ & Be-like & 152 & $2 \mathrm{P}$ & & & $3 P$ & $(3 D)$ & & $2 \mathrm{~s}$ \\
\hline Cu26 & Be-like & $1 S 2$ & $2 S$ & & & $3 P$ & 1.0 & $-2 s 2$ & \\
\hline 26 & Be-like & $1 \mathrm{~S} 2$ & $2 \mathrm{~S}$ & & & $3 P$ & 1.0 & S & \\
\hline$u 26$ & Be-like & $1 \mathrm{~S} 2$ & $2 S$ & & & $3 P$ & 1.0 & $-2 s 2$ & \\
\hline 26 & Be-like & LS2 & $2 S$ & & & $3 P$ & *) $j=1.0$ & $-2 s 2$ & \\
\hline u26 & Be-like & 152 & $2 S$ & & & $3 \mathrm{~s}$ & $(1 s) j=.0$ & $-1 s 2$ & 25 \\
\hline$u 26$ & Be-1ike & $1 S 2$ & $2 S$ & & & $3 S$ & $(1 s) j=.0$ & 152 & $s$ \\
\hline 26 & Be-like & 152 & $2 S$ & & & 35 & $=.0$ & IS2 & 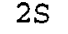 \\
\hline 426 & Be-like & 152 & $2 P$ & & & $3 \mathrm{P}$ & $(1 D) j=2.0$ & 152 & 25 \\
\hline Cu26 & Be-like & $1 s 2$ & $2 \mathrm{P}$ & & & $3 P$ & $(1 D) j=2.0$ & 152 & 28 \\
\hline Cu26 & Be-like & $1 s 2$ & $2 \mathrm{P}$ & & & $3 D$ & $\left(3 P^{\star}\right) j=2.0$ & S2 & $2 \mathrm{P} 2$ \\
\hline Cu26 & Be-like & $1 S 2$ & $2 \mathrm{P}$ & & & $3 s$ & $\left(1 P^{\star}\right) j=1.0$ & $S 2$ & $2 \mathrm{P} 2$ \\
\hline Cu26 & Be-like & 152 & $2 \mathrm{P}$ & & & 35 & $\left(1 P^{\star}\right) j=1.0$ & 152 & $2 \mathrm{P} 2$ \\
\hline Cu27 & Li-Iike & 15 & 125 & $2 P$ & $1 P^{\star}$ & 11 & $\left(2 P^{*}\right) j=.5$ & -152 & $2 S$ \\
\hline Cu27 & Li-like & is & (2S & $2 \mathrm{P}$ & $\left(1 p^{\star}\right.$ & 11 & $\left(2 P^{*}\right) j=.5$ & $-1 s 2$ & $2 S$ \\
\hline 2027 & Li-1ike & 15 & 125 & $2 \mathrm{P}$ & $\left(1 P^{*}\right.$ & 11 & $\left(2 \mathrm{P}^{\star}\right) j=.5$ & -152 & $2 S$ \\
\hline 27 & Li-Iike & 15 & 125 & $2 \mathrm{P}$ & $\left(1 \mathrm{P}^{\star}\right.$ & 11 & $\left(2 P^{\star}\right) j=.5$ & -152 & $2 s$ \\
\hline ג27 & like & 15 & 125 & $2 \mathrm{P}$ & $\left(1 P^{*}\right.$ & 11 & $* j=.5$ & .52 & $2 s$ \\
\hline 227 & like & $1 S$ & $12 \mathrm{~S}$ & $2 \mathrm{P}$ & $\left(1 p^{*}\right.$ & 11 & $\left(2 p^{*}\right) j=.5$ & S2 & $2 S$ \\
\hline Lu27 & ike & $1 S$ & <2s & $2 P$ & $\left(1 P^{*}\right.$ & 11 & $\left(2 \mathrm{P}^{*}\right)$ & $-1 s 2$ & $2 S$ \\
\hline Qi27 & like & $1 S$ & $12 S$ & $2 P$ & $\left(1 P^{*}\right.$ & 1) & $\left(2 P^{\star}\right)$ & -152 & $2 S$ \\
\hline $\mathrm{Cu27}$ & ike & 15 & $12 S$ & $2 \mathrm{P}$ & $\left(1 P^{\star}\right.$ & 11 & $\left(2 \mathrm{P}^{\star}\right) j=.5$ & LS2 & $2 \mathrm{~s}$ \\
\hline Cu27 & Li-like & 1s & (2S & $2 \mathrm{P}$ & $\left(1 P^{\star}\right.$ & 11 & $\left(2 P^{*}\right) j=.5$ & $-1 s 2$ & $2 S$ \\
\hline Cu27 & Like & is & (2S & $2 \mathrm{P}$ & $\left(1 P^{*}\right.$ & 11 & $\left(2 \mathrm{P}^{*}\right) j=.5$ & -152 & $2 S$ \\
\hline $\mathrm{Cu} 27$ & Li-like & 1s & $12 S$ & $2 \mathrm{P}$ & $\left(3 P^{\star}\right.$ & 11 & $\left(2 P^{*}\right) j=.5$ & $-1 s 2$ & $2 \mathrm{~S}$ \\
\hline Cu27 & Li-like & $1 S$ & $12 S$ & $2 \mathrm{P}$ & $\left(3 P^{*}\right.$ & 11 & $\left(2 p^{*}\right) j=.5$ & $-1 s 2$ & $2 S$ \\
\hline 27 & Li-like & $1 S$ & $12 S$ & $2 \mathrm{P}$ & $\left(3 P^{*}\right.$ & 11 & $\left(2 p^{*}\right) j=.5$ & $-1 s 2$ & $2 \mathrm{~s}$ \\
\hline 427 & ike & 15 & $(25$ & $2 P$ & $\left(3 P^{\star}\right.$ & 11 & $\left(2 p^{*}\right) j=.5$ & $-1 s 2$ & $2 \mathrm{~s}$ \\
\hline 127 & Li-like & is & (2S & $2 \mathrm{P}$ & $\left(3 P^{*}\right.$ & 11 & $\left(2 \mathrm{P}^{*}\right) j=.5$ & $-1 s 2$ & $2 S$ \\
\hline 227 & Li-like & IS & $12 S$ & $2 \mathrm{P}$ & $\left(3 P^{\star}\right.$ & 1) & $\left(2 P^{\star}\right) j=.5$ & $-1 s 2$ & $2 S$ \\
\hline$u 2^{\circ}$ & Li-like & $1 S$ & $(2 S$ & $2 \mathrm{P}$ & $3 P^{*}$ & 1 & $j=$ & 152 & $2 . s$ \\
\hline
\end{tabular}

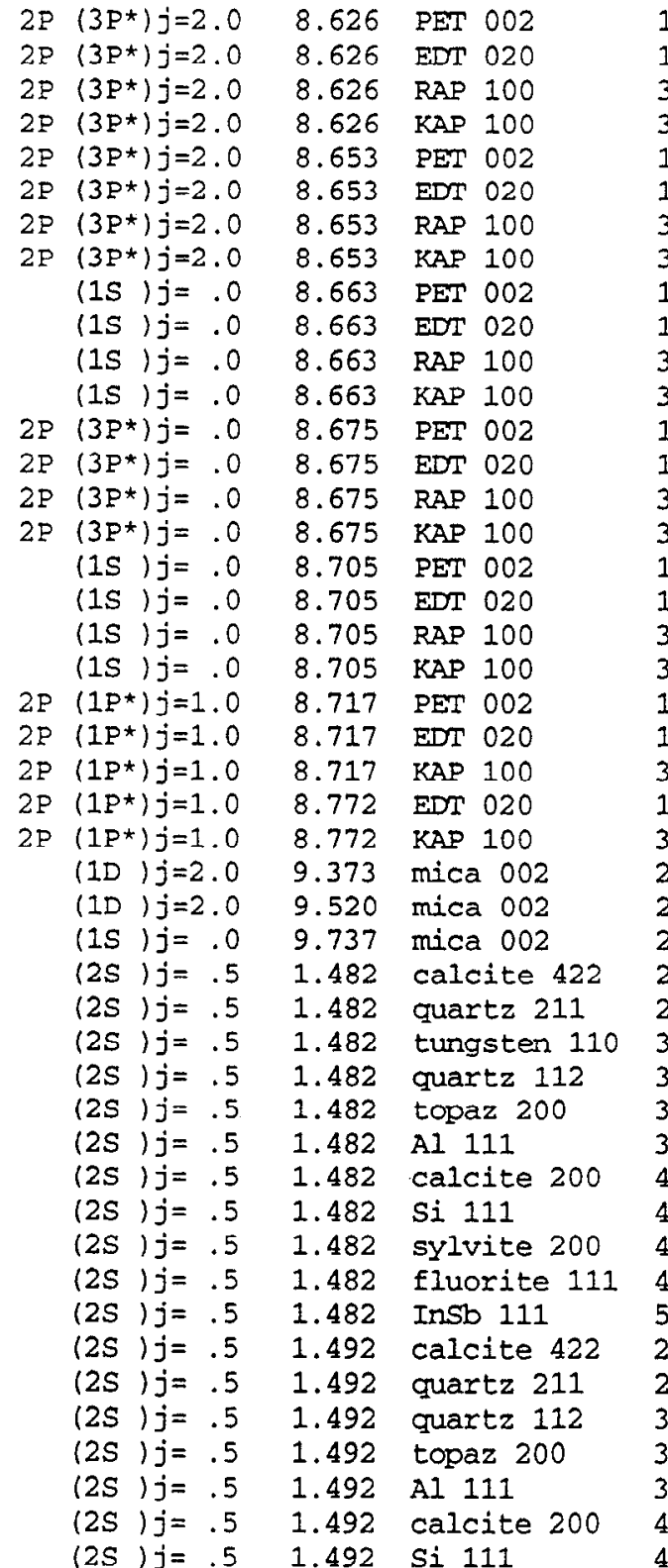

$\begin{array}{rl}8.742 & 80.656 \\ 8.808 & 78.332 \\ 26.116 & 82.259 \\ 26.634 & 76.316 \\ 8.742 & 81.817 \\ 8.808 & 79.235 \\ 26.116 & 83.714 \\ 26.634 & 77.073 \\ 8.742 & 82.291 \\ 8.808 & 79.589 \\ 26.116 & 84.347 \\ 26.634 & 77.365 \\ 8.742 & 82.902 \\ 8.808 & 80.031 \\ 26.116 & 85.216 \\ 26.634 & 77.724 \\ 8.742 & 84.727 \\ 8.808 & 81.229 \\ 26.116 & 89.499 \\ 26.634 & 78.670 \\ 8.742 & 85.666 \\ 8.808 & 81.757 \\ 26.634 & 79.072 \\ 8.808 & 84.818 \\ 26.634 & 81.137 \\ 19.942 & 70.056 \\ 19.942 & 72.702 \\ 19.942 & 77.563 \\ 3.034 & 77.668 \\ 3.082 & 74.094 \\ 4.476 & 83.363 \\ 4.564 & 76.943 \\ 4.638 & 73.456 \\ 4.676 & 71.955 \\ 6.071 & 77.540 \\ 6.271 & 70.962 \\ 6.292 & 70.416 \\ 6.308 & 70.011 \\ 7.481 & 82.100 \\ 3.034 & 79.584 \\ 3.082 & 75.513 \\ 4.564 & 78.730 \\ 4.638 & 74.812 \\ 4.676 & 73.182 \\ 6.071 & 79.431 \\ 6.271 & 72.116\end{array}$ 
Cu27 Li-like is $\left(2 S 2 P\left(3 P^{*}\right)\right)\left(2 P^{*}\right) j=.5-1 S 22 S$

Cu27 Li-like $1 \mathrm{~S}$ (2S $2 \mathrm{P}\left(3 \mathrm{P}^{*}\right)(1)\left(2 \mathrm{P}^{*}\right) j=.5-1 \mathrm{~S} 2 \mathrm{j}$

Cu27 Li-like is (2S $\left.2 \mathrm{P}\left(3 \mathrm{P}^{*}\right)\right)\left(2 \mathrm{P}^{*}\right) \mathrm{j}=.5-1522 \mathrm{~S}$

Cu27 Li-like 1S2 $3 \mathrm{P}$

Cu27 Li-like 1S2 3P

Cu27 Li-like 1S2 $3 \mathrm{P}$

Cu27 Li-like 1S2 3P

Cu27 Li-like 1S2 39

Cu27 Li-like 1S2 $3 F$

Cu27 Li-like 1S2 $3 P$

Cu27 Li-like 1S2 $3 P$

Cu27 Li-like 1S2 $3 \mathrm{P}$

Cu27 Li-like 1S2 $3 \mathrm{P}$

Cu27 Li-like 1S2 $3 P$

Cu27 Li-like 1S2 $3 \mathrm{P}$

Cu28 He-like is $5 P$

Cu28 He-like 1s 5P

Cu28 He-like is $5 \mathrm{P}$

Cu28 He-like is $5 P$

Cu28 He-like 15 $5 P$

Cu28 He-like 1S $5 P$

Cu28 He-like is $4 P$

Cu28 He-like is $4 P$

cu28 He-like 15 $4 \mathrm{P}$

Cu28 He-like 15 4P

Cu28 He-like 15 4F

Cu28 He-like 15 $4 P$

Cu28 He-like is $4 F$

Cu28 He-like is $4 P$

Cu28 He-like is $3 P$

Cu28 He-like 15 $3 F$

cu28 He-like is $3 \mathrm{~F}$

Cu28 He-like 1s $3 P$

Cu28 He-like is $3 P$

Cu28 He-like is $3 F$

Cu28 He-like 1S 3P

Cu28 He-like 15 $3 F$

Cu28 He-like is $2 \mathrm{P}$

Cu28 He-like is $2 \mathrm{P}$

Cu28 He-like is $2 \mathrm{P}$

Cu28 He-like is $2 P$

cu28 He-like 1S $2 F$

Cu28 He-like is $2 \mathrm{P}$

Cu28 He-like 1S 2P

cu28 He-like is $2 \mathrm{P}$

Cu28 He-like 1S 2 P

\begin{tabular}{|c|c|c|c|}
\hline$j=$ & .5 & 1.492 & sylvite 200 \\
\hline$j=$ & .5 & 1.492 & fluorite 111 \\
\hline$j=$ & .5 & 1.492 & Insto 111 \\
\hline$j=$ & .5 & 8.406 & quartz 100 \\
\hline$j=$ & .5 & 8.406 & PET 002 \\
\hline$j=$ & .5 & 8.406 & EDT 020 \\
\hline$j=$ & .5 & 8.406 & TAP 100 \\
\hline$j=$ & .5 & 8.406 & RAP 100 \\
\hline$j=$ & .5 & 8.406 & KAP 100 \\
\hline$j=$ & .5 & 8.448 & quartz 100 \\
\hline$j=$ & .5 & 8.448 & PET 002 \\
\hline$j=$ & .5 & 8.448 & EDT 020 \\
\hline$j=$ & .5 & 8.448 & TAP 100 \\
\hline$j=$ & .5 & 8.448 & RAP 100 \\
\hline$j=$ & .5 & 8.448 & KAP 100 \\
\hline$j=$ & .0 & 1.166 & quartz 310 \\
\hline$j=$ & .0 & 1.166 & quartz 220 \\
\hline$j=$ & .0 & 1.166 & quartz 112 \\
\hline$j=$ & .0 & 1.166 & Al 111 \\
\hline$j=$ & .0 & 1.166 & quartz 110 \\
\hline$j=$ & .0 & 1.166 & calcite 200 \\
\hline$j=$ & .0 & 1.193 & quartz 220 \\
\hline$j=$ & .0 & 1.193 & quartz 112 \\
\hline$j=$ & .0 & 1.193 & quartz 110 \\
\hline$j=$ & .0 & 1.193 & gypsum 002 \\
\hline$j=$ & .0 & 1.193 & calcite 200 \\
\hline$j=$ & .0 & 1.193 & Si 111 \\
\hline$j=$ & .0 & 1.193 & sylvite 200 \\
\hline$j=$ & .0 & 1.193 & Eluorite 111 \\
\hline$j=$ & .0 & 1.257 & Si 220 \\
\hline$j=$ & .0 & 1.257 & Eluorite 220 \\
\hline$j=$ & .0 & 1.257 & Ge 220 \\
\hline$j j=$ & .0 & 1.257 & sylvite 200 \\
\hline 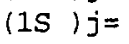 & .0 & 1.257 & Eluorite 111 \\
\hline$i=$ & .0 & 1.257 & Ge 111 \\
\hline$j j=$ & .0 & 1.257 & $\mathrm{KBr} 200$ \\
\hline$j=$ & .0 & 1.257 & quartz .101 \\
\hline$j=$ & .0 & 1.478 & calcite 422 \\
\hline$i=$ & .0 & 1.478 & quartz 211 \\
\hline 1 & .0 & 1.478 & tungsten 110 \\
\hline & .0 & 1.478 & quartz 112 \\
\hline & .0 & 1.478 & topaz 200 \\
\hline & .0 & 1.478 & AI 111 \\
\hline & .0 & 1.478 & calcite 200 \\
\hline & .0 & 1.478 & Si 111 \\
\hline & .0 & 1.478 & InSb 111 \\
\hline
\end{tabular}

$6.292 \quad 71.533$

$6.308 \quad 71.103$

$7.481 \quad 85.706$

$8.512 \quad 80.948$

$8.742 \quad 74.063$

$8.808 \quad 72.623$

$25.763 \quad 78.194$

$26.116 \quad 74.931$

$26.634 \quad 71.233$

8.51282 .970

$8.742 \quad 75.098$

$8.808 \quad 73.562$

$25.763 \quad 79.652$

$26.116 \quad 76.034$

$26.634 \quad 72.095$

$2.360 \quad 81.165$

$2.451 \quad 72.073$

$3.636 \quad 74.164$

$4.676 \quad 85.894$

$4.912 \quad 71.716$

6.07173 .802

$2.451 \quad 76.775$

$3.636 \quad 79.841$

$4.912 \quad 76.288$

$4.990 \quad 73.002$

$6.071 \quad 79.278$

$6.271 \quad 72.027$

$6.292 \quad 71.447$

$6.308 \quad 71.019$

$3.840 \quad 79.122$

$3.862 \quad 77.537$

$4.000 \quad 70.519$

$6.292 \quad 87.297$

$6.308 \quad 85.106$

$6.532 \quad 74.193$

$6.584 \quad 72.667$

$6.687 \quad 70.032$

3.03476 .980

$3.082 \quad 73.560$

$4.476 \quad 82.145$

$4.564 \quad 76.292$

$4.638 \quad 72.943$

$4.676 \quad 71.486$

$6.071 \quad 76.858$

6.27170 .519

$7.481 \quad 81.054$ 


\begin{tabular}{|c|c|c|}
\hline Cu28 & He-like & $1 \mathrm{~s}$ \\
\hline Cu28 & He-like & is \\
\hline Cu28 & He-like & 1S \\
\hline $\mathrm{Cu} 28$ & He-1ike & $1 s$ \\
\hline Cu28 & Fe-like & 1s \\
\hline Cu28 & He-like & $1 \mathrm{~S}$ \\
\hline Cu28 & He-like & $1 \mathrm{~s}$ \\
\hline Cu28 & He-like & $1 s$ \\
\hline $\mathrm{Cu28}$ & He-like & Is \\
\hline $\operatorname{cu28}$ & He-Iike & $1 S$ \\
\hline Cu28 & He-like & $1 S$ \\
\hline Cu28 & He-like & 1s \\
\hline Cu28 & He-like & $1 s$ \\
\hline Cu28 & He-1ike & 15 \\
\hline Cu28 & He-1ike & is \\
\hline Cu28 & He-like & is \\
\hline Cu28 & He-like & $1 S$ \\
\hline 0,28 & He-like & 15 \\
\hline Cu28 & He-1ike & 1s \\
\hline $\mathrm{Cu} 28$ & He-1ike & 15 \\
\hline Cu28 & He-1ike & 15 \\
\hline cu29 & H-like & $5 P$ \\
\hline Cu29 & H -1ike & $5 P$ \\
\hline $\cos 29$ & H -1ike & $5 P$ \\
\hline Cu29 & H -like & $5 P$ \\
\hline Cu29 & $\mathrm{H}$-1ike & $5 P$ \\
\hline Cu29 & H -like & $5 P$ \\
\hline Cu29 & H -1ike & $5 P$ \\
\hline cu29 & H -1ike & $4 \mathrm{P}$ \\
\hline Cu29 & H-1ike & $4 \mathrm{P}$ \\
\hline Cu29 & E-1ike & $4 \mathrm{P}$ \\
\hline Cu29 & H -like & $4 \mathrm{P}$ \\
\hline Cu29 & H -1ike & $4 P$ \\
\hline Cu29 & $\mathrm{H}-1$ ike & $4 \mathrm{P}$ \\
\hline 0029 & H -like & $3 P$ \\
\hline $\mathrm{Cu} 29$ & $\mathrm{H}$-1ike & $3 P$ \\
\hline Cu29 & H -like & $3 P$ \\
\hline Cu29 & H -1ike & $3 P$ \\
\hline Cu29 & H -1ike & $3 P$ \\
\hline $\mathrm{Cu} 29$ & H-like & $3 P$ \\
\hline Cu29 & $H-1 i k e$ & $3 P$ \\
\hline 0229 & H-1ike & $3 \mathrm{P}$ \\
\hline Cu29 & $\mathrm{H}$-1ike & $3 P$ \\
\hline Cu29 & H -like & $2 \mathrm{P}$ \\
\hline Cu29. & $\mathrm{H}$-like & $2 \mathrm{P}$ \\
\hline Cu29 & H-like & $2 P$ \\
\hline
\end{tabular}

(3P*) $j=1.0-152$ $\left(3 P^{*}\right) j=1.0-1 S 2$ $\left(3 P^{*}\right) j=1.0-1 S 2$ $\left(3 P^{*}\right) j=1.0-1 S 2$ $\left(3 P^{*}\right) j=1.0-1 S 2$ $\left(3 P^{*}\right) j=1.0-1 S 2$ $\left(3 P^{\star}\right) j=1.0-1 S 2$ $\left(3 P^{\star}\right) j=1.0-1 \mathrm{~S} 2$ $\left(3 P^{*}\right) j=1.0-1 S 2$ (3P*) $j=1.0-1 S 2$ $\left(3 P^{*}\right) j=1.0-1 s 2$ (3S) $j=1.0-1 S 2$ (3s) $j=1.0-1 s 2$ (3s) $j=1.0-1 s 2$ (3S) $j=1.0-1 S 2$ (3s) $j=1.0-1 s 2$ (3s) $j=1.0-152$ (3S) $j=1.0-1 S 2$ (3s) $j=1.0-1 s 2$ (3s) $j=1.0-1 s 2$ (3S) $j=1.0-1 S 2$ $\left(2 P^{*}\right) j=1.5-1 S$ $\left(2 P^{*}\right) j=1.5-1 \mathrm{~s}$ $\left(2 \mathrm{P}^{*}\right) j=1.5-1 \mathrm{~S}$

$\left(2 P^{*}\right) j=1.5-1 \mathrm{~s}$

$\left(2 P^{*}\right) j=1.5-1 S$

$\left(2 P^{*}\right) j=1.5-1 S$

$\left(2 \mathrm{P}^{*}\right) j=1.5-1 \mathrm{~S}$

$\left(2 P^{\star}\right) j=1.5-1 S$

$\left(2 P^{\star}\right) j=1.5-1 S$

(2P*) j=1.5 - 1S

$\left(2 P^{*}\right) j=1.5-1 S$

$\left(2 P^{*}\right) j=1.5-1 S$

$\left(2 P^{*}\right) j=1.5-1 S$

$\left(2 P^{*}\right) j=1.5-1 S$

$\left(2 P^{*}\right) j=1.5-1 S$

$\left(2 P^{*}\right) j=1.5-1 \mathrm{~S}$

$\left(2 \mathrm{P}^{*}\right) j=1.5-1 \mathrm{~S}$

$\left(2 P^{*}\right) j=1.5-1 S$

$\left(2 P^{*}\right) j=1.5-1 S$

$\left(2 P^{\star}\right) j=1.5-1 S$

$\left(2 P^{\star}\right) j=1.5-1 S$

$\left(2 P^{\star}\right) j=1.5-1 S$

$\left(2 P^{*}\right) j=1.5-1 \mathrm{~S}$

$\left(2 P^{*}\right) j=1.5-1 S$

$\left(2 P^{*}\right) j=.5-1 S$

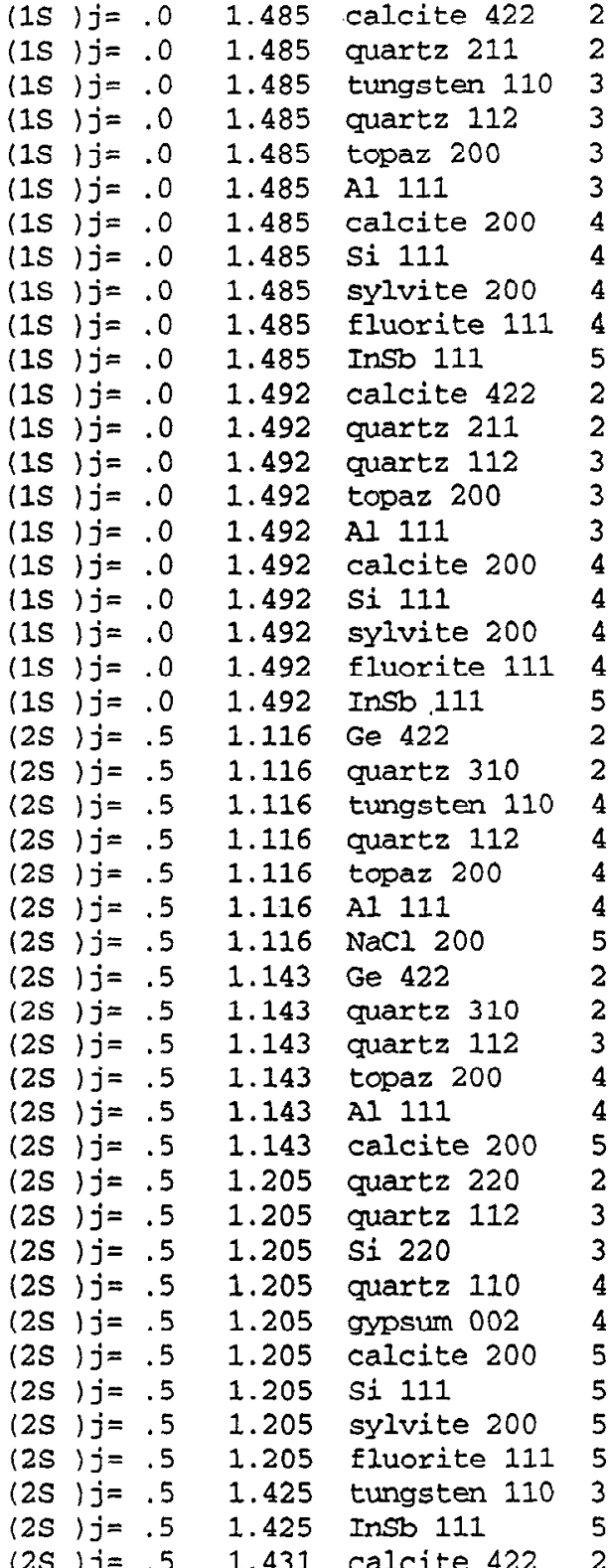

$3.034 \quad 78.211$

$3.082 \quad 74.506$

$4.476 \quad 84.448$

$4.564 \quad 77.453$

$4.638 \quad 73.851$

$4.676 \quad 72.314$

6.07178 .076

6.27171 .301

6.29270 .744

$6.308 \quad 70.332$

$7.481 \quad 82.985$

$3.034 \quad 79.584$

$3.082 \quad 75.513$

4.56478 .730

$4.638 \quad 74.812$

$4.676 \quad 73.182$

6.07179 .431

$6.271 \quad 72.116$

$6.292 \quad 71.533$

$6.308 \quad 71.103$

$7.481 \quad 85.706$

$2.310 \quad 75.068$

$2.360 \quad 71.043$

$4.476 \quad 85.804$

$4.564 \quad 77.984$

$4.638 \quad 74.256$

$4.676 \quad 72.681$

5.64181 .566

$2.310 \quad 81.734$

$2.360 \quad 75.614$

$3.636 \quad 70.574$

$4.638 \quad 80.323$

$4.676 \quad 77.893$

6.07170 .281

$2.451 \quad 79.505$

$3.636 \quad 83.839$

$\begin{array}{ll}3.840 & 70.289\end{array}$

4.91278 .893

$4.990 \quad 75.001$

6.07182 .942

$6.271 \quad 73.898$

6.29273 .249

$6.308 \quad 72.773$

$4.476 \quad 72.764$

$7.481 \quad 72.253$

$3.034 \quad 70.615$ 


\begin{tabular}{|c|c|c|c|c|c|c|c|c|}
\hline Cu29 & H -like & $2 P$ & & & & $\left(2 P^{*}\right) j=.5$ & $-1 s$ & \\
\hline $\operatorname{cus} 29$ & H -like & $2 P$ & & & & $\left(2 P^{\star}\right) j=.5$ & -15 & \\
\hline $\mathrm{Cu} 29$ & H-1ike & $2 P$ & & & & $\left(2 P^{*}\right) j=.5$ & $-1 s$ & \\
\hline $\mathrm{Cu} 29$ & H -like & $2 \mathrm{P}$ & & & & $\left(2 P^{*}\right) j=.5$ & $-1 S$ & \\
\hline $\operatorname{cus} 29$ & H -like & $5 P$ & & & & $(2 P *) j=1.5$ & $-2 s$ & \\
\hline 0,29 & H-like & $5 P$ & & & & $\left(2 P^{\star}\right) j=1.5$ & $-2 S$ & \\
\hline $\mathrm{Cu29}$ & H -like & $5 P$ & & & & $\left(2 P^{*}\right) j=1.5$ & $-2 S$ & \\
\hline cu29 & H -like & $5 P$ & & & & $(2 P *) j=1.5$ & $-2 S$ & \\
\hline Cu29 & H -like & $5 P$ & & & & $(2 P *) j=1.5$ & $-2 s$ & \\
\hline Cu29 & H -like & $5 D$ & & & & $(2 D) j=2.5$ & $-2 P$ & \\
\hline $\mathrm{Cu} 29$ & H-like & 50 & & & & $(2 D) j=2.5$ & $-2 P$ & \\
\hline $\mathrm{Cu} 29$ & H -like & $5 D$ & & & & $\langle 2 D)_{j=2.5}$ & $-2 P$ & \\
\hline $\mathrm{Cu29}$ & H-like & $5 D$ & & & & $(2 D) j=2.5$ & $-2 P$ & \\
\hline Cu29 & H -1ike & $5 D$ & & & & $(2 D) j=2.5$ & $-2 p$ & \\
\hline Cu29 & H-like & $4 D$ & & & & $(2 D) j=2.5$ & $-2 P$ & \\
\hline $\mathrm{Cu} 29$ & H -like & $3 P$ & & & & $\left(2 P^{*}\right) j=1.5$ & -25 & \\
\hline Cu29 & H-like & $3 D$ & & & & $(2 D) j j=2.5$ & $-2 F$ & \\
\hline $\mathrm{Zn}$ & . & $\mathrm{K}-\mathrm{a}$ & lpha! & & & & & \\
\hline $\ln \cdots$ & & $\mathrm{K}-\mathrm{a}$ & Ipha! & & & & & \\
\hline $\mathrm{Zn}$ & ?. & $\mathrm{K}-\mathrm{a}$ & Ipha! & 1) & & & & \\
\hline $\operatorname{zn} \ldots$ & $\therefore$ & $\mathrm{K}-\mathrm{a}$ & lphal & 1) & & & & \\
\hline $\mathrm{Zn}$ & “. & $\mathrm{K}-\mathrm{a}$ & Iphal & & & & & \\
\hline $\mathrm{Zn} 20$ & Na-like & $2 P 6$ & $5 P$ & & & $\left(2 p^{*}\right) j=1.5$ & $-2 P 6$ & $3 S$ \\
\hline $\mathrm{zn} 20$ & Na-like & $2 \mathrm{P} 6$ & $5 P$ & & & $(2 P \star) j=1.5$ & $-2 P 6$ & $3 S$ \\
\hline $\ln 20$ & Na-like & $2 P 6$ & $5 P$ & & & $\left(2 P^{*}\right) j=.5$ & $-2 P 6$ & $3 S$ \\
\hline $\sin 20$ & $\mathrm{Na-like}$ & $2 P 6$ & $5 P$ & & & $\left(2 P^{\star}\right) j=.5$ & $-2 P 6$ & $3 s$ \\
\hline $\operatorname{Zn} 20$ & $\mathrm{Na}-1 \mathrm{ike}$ & $2 P 6$ & $6 F$ & & & $\left(2 F^{\star}\right) j=2.5$ & $-2 P 6$ & 30 \\
\hline $\mathrm{Zn} 20$ & Na-like & $2 \mathrm{P} 6$ & $6 F$ & & & $\left(2 F^{*}\right) j=2.5$ & $-2 P 6$ & $3 D$ \\
\hline $\operatorname{zn} 20$ & $\mathrm{Na-like}$ & $2 \mathrm{P} 6$ & $6 F$ & & & $\left(2 F^{\star}\right) j=3.5$ & $-2 P 6$ & $3 D$ \\
\hline $\mathrm{zn} 20$ & Na-like & $2 \mathrm{P} 6$ & $6 \mathrm{~F}$ & & & $\left(2 F^{\star}\right) j=3.5$ & $-2 P 6$ & $3 D$ \\
\hline $\ln 20$ & Na-like & $2 P 6$ & $5 D$ & & & $(2 D) j=1.5$ & $-2 P 6$ & $3 P$ \\
\hline $\ln 20$ & Na-Iike & $2 P 6$ & $5 D$ & & & $(2 D) j=2.5$ & $-2 P 6$ & $3 P$ \\
\hline $\operatorname{zn} 21$ & Ne-like & 252 & $2 P 5$ & $(2 P \star 2)$ & $8 D$ & $\left(23^{*}\right) j=1.0$ & -252 & $2 P 6$ \\
\hline $\mathrm{Zn} 21$ & Ne-like & 252 & $2 \mathrm{P} 5$ & $(2 P * 1)$ & $7 D$ & $\left(12^{*}\right) j=1.0$ & $-2 s 2$ & $2 P 6$ \\
\hline $\operatorname{zn} 21$ & Ne-like & 252 & $2 \mathrm{P5}$ & $(2 P * 2)$ & $7 D$ & $\left(23^{*}\right) j=1.0$ & $-2 s 2$ & $2 \mathrm{P} 6$ \\
\hline $\operatorname{Zn} 21$ & Ne-like & 252 & $2 \mathrm{P} 5$ & $(2 P * 2)$ & $7 D$ & $\left(23^{*}\right) j=1.0$ & $-2 s 2$ & $2 \mathrm{R} 6$ \\
\hline $\mathrm{Zn} 21$ & Ne-like & 252 & $2 \mathrm{P} 5$ & $(2 P * 1)$ & $6 \mathrm{D}$ & $(12 *) j=1.0$ & $-2 s 2$ & 2P6 \\
\hline $\operatorname{Zn} 21$ & Ne-like & 252 & 2P5 & $(2 P \star 1)$ & $6 \mathrm{D}$ & $\left(12^{\star}\right) j=1.0$ & -252 & $2 \mathrm{P} 6$ \\
\hline $\mathrm{Zn} 21$ & Ne-like & 252 & $2 \mathrm{P5}$ & $(2 \mathrm{P} * 2)$ & $6 D$ & $\left(23^{*}\right) j=1.0$ & $-2 s 2$ & $2 \mathrm{P} 6$ \\
\hline $\operatorname{Zn} 21$ & Ne-like & 252 & 2P5 & $(2 P * 2)$ & $6 \mathrm{D}$ & $\left(23^{*}\right) j=1.0$ & $-2 S 2$ & $2 \mathrm{P} 6$ \\
\hline $\operatorname{Zn} 21$ & Ne-like & 252 & $2 \mathrm{P5}$ & $\left(2 P^{\star} 1\right)$ & 50 & $\left\langle 12^{*}\right\rangle j=1.0$ & $-2 \mathrm{~S} 2$ & $2 \mathrm{P} 6$ \\
\hline $\operatorname{Zn} 21$ & Ne-like & $2 s$ & $2 \mathrm{P} 6$ & & $4 \mathrm{P}$ & $\left(1 P^{*}\right) j=1.0$ & $-2 S 2$ & 2P6 \\
\hline $\operatorname{Zn} 21$ & Ne-Iike & 252 & $2 \mathrm{P} 5$ & $(2 \mathrm{P} * 2)$ & $5 D$ & $\left(23^{*}\right) j=1.0$ & $-2 s 2$ & $2 \mathrm{P} 6$ \\
\hline $\operatorname{Zn} 21$ & Ne-like & $2 S 2$ & 2P5 & $(2 P \star 1)$ & $4 D$ & $(12 *) j=1.0$ & $-2 S 2$ & $2 \mathrm{P} 6$ \\
\hline $\operatorname{Zn} 21$ & Ne-like & 252 & $2 P 5$ & $\left(2 P^{*} 1\right)$ & $4 \mathrm{D}$ & $\left(12^{*}\right) j=1.0$ & $-2 s 2$ & $2 \mathrm{P} 6$ \\
\hline $\ln 21^{\circ}$ & Ne-like & 252 & $2 \mathrm{P} 5$ & $(2 \mathrm{P} * 1)$ & $4 \mathrm{D}$ & $\left(12^{*}\right) j=1.0$ & $-2 S 2$ & $2 \mathrm{P} 6$ \\
\hline
\end{tabular}

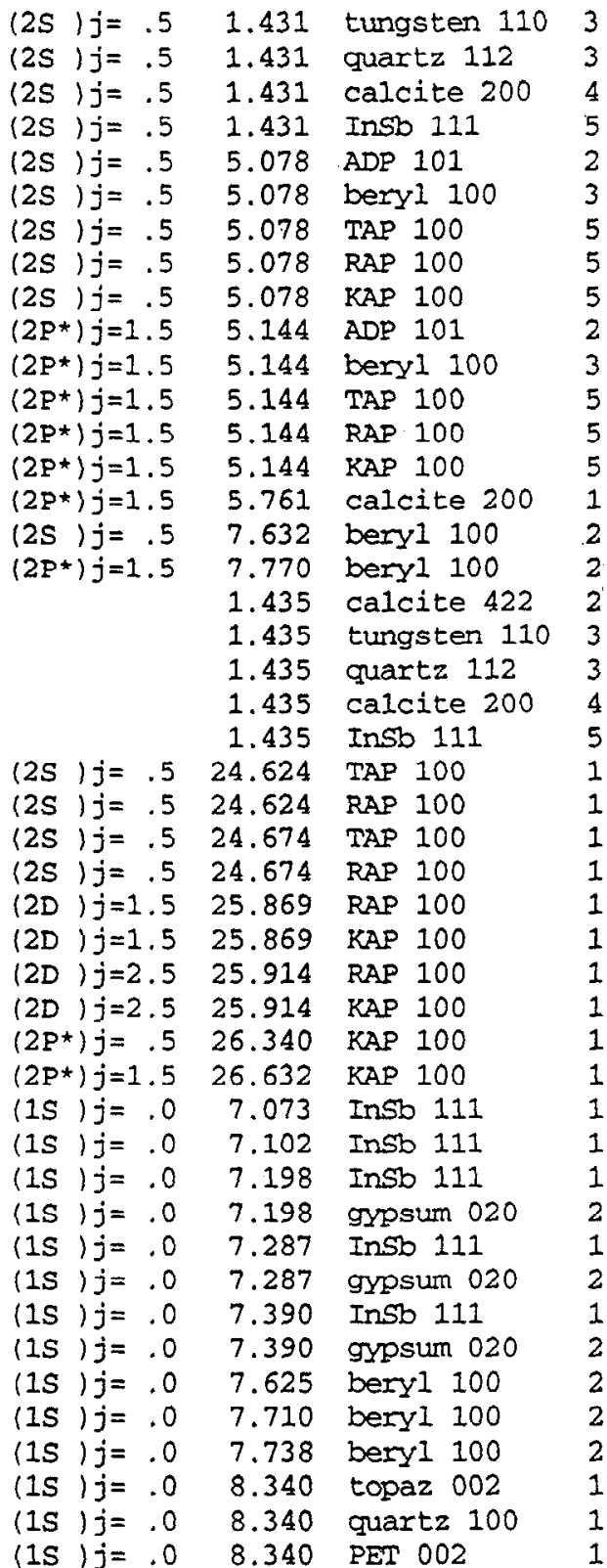

$4.476 \quad 73.560$

$4.564 \quad 70.156$

6.07170 .535

$\begin{array}{lll}7.481 & 73.023\end{array}$

$10.640 \quad 72.652$

$15.954 \quad 72.721$

$25.763 \quad 80.238$

$26.116 \quad 76.459$

$26.634 \quad 72.419$

$10.640 \quad 75.221$

$15.954 \quad 75.303$

$25.763 \quad 86.689$

$26.116 \quad 80.010$

$26.634 \quad 74.946$

6.07171 .611

$15.954 \quad 73.088$

$15.954 \quad 76.919$

3.03471 .099

$4.476 \quad 74.140$

4.56470 .628

6.07171 .016

$7.481 \quad 73.583$

$25.763 \quad 72.899$

$26.116 \quad 70.539$

$25.763 \quad 73.282$

$26.116 \quad 70.871$

$26.116 \quad 82.114$

$26.634 \quad 76.234$

$26.116 \quad 82.869$

$26.634 \quad 76.647$

$26.634 \quad 81.479$

$26.634 \quad 89.298$

$7.481 \quad 70.990$

$7.481 \quad 71.684$

$7.481 \quad 74.190$

$15.185 \quad 71.449$

$7.481 \quad 76.923$

$15.185 \quad 73.691$

$7.481 \quad 81.054$

$15.185 \quad 76.737$

$15.954 \quad 72.916$

$.15 .954 \quad 75.134$

$15.954 \quad 75.939$

$8.374 \quad 84.835$

$8.512 \quad 78.462$

$8.742 \quad 72.557$ 
Zn21 Ne-1ike 2S2 2P5 ( 2P*1) 4D (12*) $j=1.0-2 S 22 P 6$

Zn21 Ne-like 2S2 2P5 ( $\left.2 P^{*} 1\right) 4 D(12 *) j=1.0-2 S 22 P 6$

Zn21 Ne-like 2S2 2P5 (2P*1) 4D (12*) j=1.0 - 2S2 $2 P 6$

$\mathrm{Zn21}$ Ne-1ike 2S2 $2 \mathrm{P} 5(2 \mathrm{P} * 2)$ 4D $\left(23^{*}\right) j=1.0-2 \mathrm{~S} 22 \mathrm{P} 6$

$\mathrm{Zn21}$ Ne-like 2S2 $2 \mathrm{P} 5(2 \mathrm{P} * 2) 4 \mathrm{D}\left(23^{*}\right) \mathrm{j}=1.0-2 \mathrm{~S} 22 \mathrm{P} 6$

Zn21 Ne-like 2S2 $2 P 5(2 P * 2) 4 D(23 *) j=1.0-2 S 22 P 6$

Zn21 Ne-like 2S2 2P5 ( 2P*2) 4D $(23 *) j=1.0-2 S 22 \mathrm{P} 6$

Zn21 Ne-like 2S2 2P5 ( 2P*2) 4D $\left(23^{*}\right) j=1.0-2 S 22 \mathrm{P} 6$

$\mathrm{Zn} 21$ Ne-like 2S2 $2 \mathrm{P} 5(2 \mathrm{P} * 2) 4 \mathrm{D}(23 *) j=1.0-2 \mathrm{~S} 22 \mathrm{P} 6$

$\mathrm{Zn21}$ Ne-like 2S2 2P5 ( $2 \mathrm{P} * 1) \quad 4 S\left(11^{*}\right) j=1.0-2 S 2 \quad 2 P 6$

Zn21 Ne-1ike 2S2 2P5 ( $\left.2 P^{*} 1\right)$ 4S $\left(11^{*}\right) j=1.0-2 S 22 \mathrm{P} 6$

$\operatorname{Zn} 21$ Ne-like 2S2 2P5 ( $\left.2 P^{* 1}\right)$ 4S $\left(11^{*}\right) j=1.0-2 \mathrm{~S} 2 \quad 2 \mathrm{P} 6$

Zn21 Ne-like 2S2 2P5 ( $\left.2 P^{*} 1\right)$ 4S (11*) $j=1.0-2 S 22 P 6$

Zn21 Ne-like 2S2 2P5 ( 2P*2) 4S $\left(22^{\star}\right) \mathrm{j}=1.0-2 \mathrm{~S} 2$ 2P6

Zn21 Ne-like 2S2 2P5 ( 2P*2) 4S $(22 *) j=1.0-2 S 22 P 6$

Zn21 Ne-like 2S2 2P5 ( $\left.2 P^{*} 2\right)$ 4S $\left(22^{*}\right) j=1.0-2 S 22 P 6$

Zn21 Ne-like $2 S$ 2P6 $3 P\left(1 P^{*}\right) j=1.0-252 \quad 2 P 6$

Zn21 Ne-like 2S $2 \mathrm{P} 6 \quad 3 \mathrm{P}\left(3 P^{*}\right) j=1.0-2 \mathrm{~S} 22 \mathrm{P} 6$

Zn21 Ne-like 2S2 $2 \mathrm{PS}(2 \mathrm{P} * 1)$ 3D $\left(12^{*}\right) j=1.0-2 \mathrm{~S} 22 \mathrm{P} 6$

$\mathrm{Zn} 22$ F-like 2S2 $2 \mathrm{P} 4$ ( $1 S$ ) $4 \mathrm{D}(2 \mathrm{D}) \mathrm{j}=2.5-2 \mathrm{~S} 2 \mathrm{2P5}$

Zn22 F-like 2S2 2P4 (1S) $4 D(2 D) j=1.5-2 S 22 P 5$

Zn22 F-1ike 2S2 2P4 ( $1 D$ ) $4 D$ (2D) $j=1.5-2 S 2$ 2P5

$\mathrm{Zn} 22 \mathrm{~F}$-like 2S2 2P4 ( $1 D$ ) $4 D(2 D) j=1.5-2 S 22 \mathrm{P5}$

$\mathrm{Zn22} F$-like 2S2 2P4 ( $1 D$ ) $4 D(2 D) j=2.5-2 S 22 P 5$

Zn22 F-1ike 2S2 2P4 (1D) 4D (2D) j=2.5 - 2S2 2P5

Zn22 $F$-like $2 S 22 \mathrm{P} 4$ ( $1 \mathrm{~S}$ ) $4 \mathrm{~S}(2 \mathrm{~S}) j=.5-2 \mathrm{~S} 22 \mathrm{P} 5$

Zn22 F-like 2S2 2P4 ( $1 S$, $4 S$ (2S) $j=.5-2 S 22 \mathrm{P5}$

2n22 F-like 2S2 2P4 (3P) 4D (4F) j=2.5-2S2 2P5

Zn22 $F$-1ike 2S2 2P4 ( ID) $4 D$ (2D) $j=1.5-2 S 22 P 5$

Zn22 F-like 2S2 2P4 ( ID) $4 D(2 D) j=1.5-2 S 22 P 5$

$\mathrm{Zn22} F$-like 2S2 2P4 ( 3P) $4 \mathrm{D}(2 \mathrm{D}) \mathrm{j}=2.5-2 \mathrm{~S} 2$ 2P5

Zn22 F-like 2S2 2P4 (3P) $4 D(2 D) j=2.5-2 S 2$ 2P5

Zn22 F-1ike 2S2 2P4 ( $3 P$ ) $4 D(2 P) j=1.5-2 S 22 \mathrm{P} 5$

$\mathrm{Zn} 22 \mathrm{~F}$-like 2S2 2P4( $\mathrm{PP}$ ) $4 \mathrm{D}(2 \mathrm{P}) \mathrm{j}=1.5-2 \mathrm{~S} 22 \mathrm{PS}$

$\mathrm{Zn} 22 \mathrm{~F}$-like 2S2 2P4 ( 1D) 4S (2D) j=2.5 - 2S2 2P5

$\mathrm{Zn22}$-1ike 2S2 2P4 ( ID) $4 S(2 D) j=2.5-2 S 22 P 5$

$\mathrm{Zn22} F$-like 2S2 2P4 ( 1D) $4 S$ (2D) $j=2.5-2 S 22 \mathrm{P} 5$

$\mathrm{Zn22} F$-like 2S2 2P4 ( $3 P$ ) $4 S(4 P) j=1.5-2 S 22 P 5$

Zn22 $F-1$ - ike 2S2 2P4 ( $3 P$ ) $4 S(4 P) j=1.5-2 S 22 P 5$

Zn22 F-like 2S2 2P4 ( $3 P$ ) $4 S(4 P) j=1.5-2 S 22 P 5$

$\mathrm{Zn} 22 \mathrm{~F}$-like 2S2 2P4 ( ID) $4 S(2 D) j=1.5-2 S 22 P 5$

Zn22 F-like 2S2 2P4 ( 1D) 4S (2D) $j=1.5-2 S 2$ 2P5

$\mathrm{Zn22} F$-like 2S2 2P4 ( 1D) $4 S(2 D) j=1.5-2 S 22 \mathrm{P} 5$

$\mathrm{Zn} 22$ F-like $2 S 22 \mathrm{P} 4(\mathrm{ID}) 4 \mathrm{~S}(2 \mathrm{D}) \mathrm{j}=1.5-2 \mathrm{~S} 2 \mathrm{2P5}$

$\mathrm{Zn} 22 \mathrm{~F}$-like 2S2 2P4 (3P) $4 S(2 P) j=1.5-2 S 22 P 5$

$\mathrm{Zn} 22 \mathrm{~F}$-like $2 \mathrm{S2} 2 \mathrm{P} 4(3 \mathrm{P}) 4 \mathrm{~S}(2 \mathrm{P}) \mathrm{j}=1.5-2 \mathrm{~S} 2 \mathrm{2P5}$
(1S) $j=.0 \quad 8.340 \quad$ EDT 020

(1S) $j=.0 \quad 8.340$ TAP 100

(1S) $j=.0 \quad 8.340$ RAP 100

(1s) $j=.0 \quad 8.467$ cuartz 100

(1S) $j=.0 \quad 8.467$ PET 002

(1S) $j=.0 \quad 8.467$ EDT 020

(1S) $j=.0 \quad 8.467$ TAP 100

(1S) $j=0 \quad 8.467$ RAP 100

(1S) $j=.0 \quad 8.467 \quad \operatorname{KAP} 100$

(1S) $j=.0 \quad 8.658$ PET 002

(1S) $j=.0 \quad 8.658$ EDT 020

(1S) $j=.0 \quad 8.658$ RAP 100

(1s) $j=.0 \quad 8.658 \quad \mathrm{KAP} 100$

(1S) $j=.0 \quad 8.722 \quad$ PET 002

(1S) $j=.0 \quad 8.722$ EDT 020

(1S) $j=.0 \quad 8.722$ KAP 100

(IS ) $j=.0 \quad 9.762$ mica 002

(1S) $j=.0 \quad 9.815 \mathrm{mica} 002$

(1S) $j=.0 \quad 10.462 \quad$ ADP 101

$\left(2 P^{*}\right) j=1.5 \quad 7.747$ beryl 100

$\left(2 P^{*}\right) j=.5 \quad 7.847$ bery 100

$\left(2 P^{\star}\right) j=1.5 \quad 7.879$ topaz 002

$\left(2 P^{*}\right) j=1.5 \quad 7.879$ bery 100

$\left(2 P^{*}\right) j=1.5 \quad 7.890$ topaz 002

$\left(2 P^{*}\right) j=1.5 \quad 7.890$ beryl 100

$\left(2 P^{*}\right) j=1.5 \quad 7.941$ topaz 002

$\left(2 P^{*}\right) j=1.5 \quad 7.941$ beryl 100

$\left(2 P^{*}\right) j=1.5 \quad 7.993$ topaz 002

$\left(2 P^{*}\right) j=.5 \quad 8.005$ topaz 002

$\left(2 P^{*}\right) j=.5 \quad 8.005$ quartz 100

$\left(2 P^{*}\right) j=1.5 \quad 8.047$ topaz 002

$\left(2 P^{*}\right) j=1.5 \quad 8.047$ quartz 100

$\left(2 P^{*}\right) j=.5 \quad 8.069$ topaz 002

$\left(2 P^{*}\right) j=.5 \quad 8.069$ quartz 100

$\left(2 \mathrm{P}^{\star}\right) j=1.5 \quad 8.086$ topaz 002

(2P*) $j=1.5 \quad 8.086$ quartz 100

$\left(2 P^{*}\right) j=1.5 \quad 8.086 \quad$ TAP 100

$\left(2 P^{\star}\right) j=1.5 \quad 8.154$ topaz 002

$\left(2 P^{*}\right) j=1.5$

$\left(2 P^{\star}\right) j=1.5$

$\left(2 P^{\star}\right) j=.5$

$\left(2 P^{*}\right) j=.5$

$\left(2 P^{*}\right) j=.5$

$\left(2 P^{*}\right) j=.5$

$\left(2 P^{*}\right) j=1.5$

8.154 quartz 100

8.154 TAP 100

8.198 topaz 002

8.198 quartz 100

8.198 TAP 100

8.198 PAP 100

$\left(2 P^{*}\right) j=1.5$
$8.808 \quad 71.239$

$25.763 \quad 76.206$

$26.116 \quad 73.342$

$8.512 \quad 84.106$

$8.742 \quad 75.591$

$8.808 \quad 74.005$

$25.763 \quad 80.384$

$26.116 \quad 76.562$

$26.634 \quad 72.498$

$8.742 \quad 82.051$

$8.808 \quad 79.411$

$26.116 \quad 84.022$

$26.634 \quad 77.218$

$8.742 \quad 86.124$

$8.808 \quad 81.987$

$26.634 \quad 79.243$

$19.942 \quad 78.248$

$19.942 \quad 79.852$

$10.640 \quad 79.505$

15.95476 .208

$15.954 \quad 79.642$

8.374. 70.201

$15.954 \quad 81.010$

8.37470 .425

$15.954 \quad 81.530$

$8.374 \quad 71.494$

$15.954 \quad 84.555$

$8.374 \quad 72.650$

8.37472 .928

$8.512 \quad 70.125$

$8.374 \quad 73.935$

8.51270 .974

$8.374 \quad 74.489$

$8.512 \quad 71.434$

$8.374 \quad 74.930$

$8.512 \quad 71.797$

$25.763 \quad 70.319$

$8.374 \quad 76.838$

$8.512 \quad 73.324$

$25.763 \quad 71.714$

$8.374 \quad 78.232$

$8.512 \quad 74.389$

$25.763 \quad 72.674$

$26.116 \quad 70.343$

$8.374 \quad 80.411$

$8.512 \quad 75.940$

Page 102 
Zn22 F-like 2S2 2P4 ( $3 P$ ) $4 S(2 P) j=1.5-2 S 22 P 5$ Zn22 F-1ike 2S2 2P4 ( $3 P$ ) $4 S(2 P) j=1.5-2 S 22 P 5$

Zn22 F-like 2S2 2P4 ( $3 P$ ) $4 S(2 P) j=1.5-2 S 22 P 5$

$\mathrm{Zn} 22 \mathrm{~F}$-like $2 \mathrm{~S} 2 \mathrm{P5}\left(3 \mathrm{P}^{\star}\right) 3 \mathrm{P}(4 \mathrm{D}) \mathrm{j}=.5-2 S 22 \mathrm{P5}$

Zn22 $F$-like 2S 2P5 (3P*) $3 P(2 P) j=.5-2 S 22 P 5$

Zn22 F-like $2 S 2 P 5(3 P *) 3 P(2 P) j=1.5-2 S 22 P 5$

$\mathrm{Zn} 22 \mathrm{~F}$-like $2 S 2 P 5\left(3 P^{*}\right) 3 P,(2 D) j=1.5-2 S 22 P 5$

Zn22 F-like 2S 2P5 (3P*) 3P (4D) $j=1.5-2 S 2$ 2P5

$2 n 22 F-1 i k e$ 2S 2P5 (3P*) 3P (4D)j=2.5 - $2 S 22 P 5$

Zn22 $F$-like 2S 2P5 ( $3 P *) 3 P(4 P) j=1.5-2 S 22 P 5$

$\mathrm{Zn} 22 \mathrm{~F}$-1ike $2 \mathrm{S2} 2 \mathrm{P} 4$ ( $1 \mathrm{~S}$ ) $3 \mathrm{D}$ (2D) $\mathrm{j=1.5}-2 \mathrm{S2} 2 \mathrm{P5}$

Zn22 F-like 2S2 2P4 ( $1 S$ ) 3D (2D) j=2.5 - 2S2 $2 P 5$

Zn22 F-1ike 2S2 2P4 ( $1 S$ ) 3D (2D) $j=1.5-2 S 22 P 5$

Zn22 F-1ike 2S2 2P4 ( ID) 3D (2D) $j=1.5-2 S 22 P 5$

$\mathrm{Zn22} F$-like 2S2 2P4 ( 1D) 3D (2P) j=1.5 - 2S2 2P5

Zn22 F -like 2S2 2P4 ( 1D) 3D (2S) j=.5-2S2 2P5

Zn22 F-like 2S2 2P4 ( $3 P$ ) 3D (2D) $j=2.5-2 S 22 P 5$

Zn22 F-like 2S2 $2 P 4(3 P)$ 3D (2P) $j=1.5-2 S 22 P 5$

$\mathrm{Zn22} F$-like 2S2 $2 \mathrm{P} 4$ ( $1 \mathrm{D}) 3 \mathrm{D}(2 \mathrm{P}) \mathrm{j}=.5-2 \mathrm{~S} 2 \mathrm{2P5}$

$\mathrm{Zn22} F$-like 2S2 2P4 ( 3P) 3D (4D) $j=.5-2 S 22 \mathrm{P5}$

Zn22 F-like 2S2 2P4 ( 3P) 3D (4P) $j=2.5-2 S 22 P 5$

$\mathrm{Zn22} F$-like 2S2 2P4 (1D) 3D (2P) $j=1.5-2 S 2$ 2P5

$\mathrm{Zn22} F$-like 2S2 2P4 ( $1 D$ ) $3 \mathrm{D}(2 \mathrm{~S}) j=.5-2 S 22 \mathrm{P} 5$

Zn22 F-like 2S2 $2 \mathrm{P} 4$ ( 3P) 3D (2F) j=2.5 - 2S2 $2 \mathrm{P} 5$

$\mathrm{Zn22} F$-like 2S2 2P4 ( 3P) 3D (4P) $j=1.5-2 S 22 P 5$

Zn22 F-like 2S2 2P4 ( 3P ) 3D (4P) $j=.5-2 S 22 P 5$

Zn22 $F$-like $2 S 22 P 4$ ( $3 P$ ) 3D (2P) $j=1.5-2 S 22 P 5$

Zn22 $F$-like 2S2 2P4 ( 3P) 3D (4D) $j=1.5-2 S 22 P 5$

$\operatorname{Zn} 22 \mathrm{~F}$-like 2S2 2P4 ( $3 P$ ) 3D (4F) $j=1.5-2 S 22 P 5$

Zn22 $F-1$-ike 2S2 2P4 ( $3 P$ ) 3D (4P) $j=1.5-2 S 22 P 5$

$\mathrm{Zn} 22 \mathrm{~F}$-like $2 S 22 \mathrm{P} 4(3 \mathrm{P}), 3 D(4 P) j=.5-2 S 22 \mathrm{PS}$

$\mathrm{Zn} 22 \mathrm{~F}$-like 2S2 2P4 ( $1 S$ ) $3 S(2 S) j=.5-2 S 22 \mathrm{P} 5$

Zn23 0 -like 2S2 2P3 (2P*) $4 D\left(3 D^{*}\right) j=1.0-2 S 22 P^{*}$

$\operatorname{Zn} 230$-like $2 S 22 P^{2}\left(2 P^{*}\right) 4 D\left(3 D^{*}\right) j=1.0-2 S 22 P 4$

$\mathrm{Zn} 230$-like $2 S 22 \mathrm{P} 3\left(2 D^{*}\right) 4 \mathrm{D}\left(3 D^{*}\right) j=3.0-2 S 22 \mathrm{P4}$

Zn23 0 -like 2S2 2P3 (2D*) 4D (3F*)j=3.0- 2S2 2P4

Zn23 0 -like 252 2P3 ( 2D*) $4 D\left(3 F^{*}\right) j=3.0-2 S 22 P 4$

$\mathrm{Zn} 230$-like 2S2 $2 \mathrm{P} 3\left(2 \mathrm{P}^{*}\right) 4 \mathrm{D}\left(3 \mathrm{~F}^{*}\right) j=3.0-2 \mathrm{~S} 22 \mathrm{P} 4$

$\mathrm{Zn23} 0$-like 2S2 2P3 ( 2P*) 4D $\left(3 F^{*}\right) j=3.0-2 \mathrm{~S} 22 \mathrm{P} 4$

Zn23 0 -like 2S2 2P3 (4S*) 4D (3D*) j=3.0-2S2 2P4

$\operatorname{Zn23} 0$-like 2S2 $2 \mathrm{P} 3\left(4 S^{*}\right) 4 \mathrm{D}\left(3 D^{*}\right) j=3.0-2 S 22 \mathrm{P4}$

Zn23 0 -like 2S2 2P3 (2D*) $4 D\left(1 F^{*}\right) j=3.0-2 S 22 P 4$

$\operatorname{Zn23} 0$-like 2S2 2P3 (4S*) 4D (3D*)j=1.0 - 2S2 2P4

$\mathrm{Zn} 23 \mathrm{O}-\mathrm{-like} 2 \mathrm{~S} 2 \mathrm{2P3}\left(4 \mathrm{~S}^{*}\right) 4 \mathrm{D}\left(3 \mathrm{D}^{*}\right) \mathrm{j}=2.0-2 \mathrm{~S} 22 \mathrm{P4}$

Zn23 o-like 2S2 2P3 ( 2P*) 3D $\left(3 P^{*}\right) j=2.0-2 S 22 P 4$

Zn23 0 -like 2S2 $2 P^{2}\left(2 P^{*}\right) 3 D\left(1 D^{*}\right) j=2.0-2 S 22 P 4$

\begin{tabular}{|c|c|c|}
\hline$\left(2 P^{*}\right) j=1.5$ & 8.257 & PET 002 \\
\hline$\left(2 p^{\star}\right) j=1.5$ & 8.257 & TAP 100 \\
\hline$\left(2 P^{*}\right) j=1.5$ & 8.257 & $\operatorname{RAP} 100$ \\
\hline$\left(2 \mathrm{P}^{\star}\right) j=1.5$ & 9.425 & mica 002 \\
\hline$\left(2 \mathrm{P}^{*}\right) j=1.5$ & 9.440 & mica 002 \\
\hline$\left(2 P^{*}\right) j=1.5$ & 9.468 & mica 002 \\
\hline$\left(2 P^{\star}\right) j=.5$ & 9.507 & mica 002 \\
\hline$\left(2 P^{*}\right) j=1.5$ & 9.518 & mica 002 \\
\hline$\left(2 P^{*}\right) j=1.5$ & 9.562 & mica 002 \\
\hline$\left(2 q^{*}\right) j=1.5$ & 9.601 & mica 002 \\
\hline$\left(2 P^{*}\right) j=1.5$ & 9.751 & mica 002 \\
\hline$\left(2 P^{*}\right) j=1.5$ & 9.795 & mica 002 \\
\hline$\left(2 \mathrm{P}^{\star}\right) j=.5$ & 9.947 & mica 002 \\
\hline$\left(2 P^{*}\right) j=1.5$ & 9.962 & mica 002 \\
\hline$\left(2 p^{*}\right) j=1.5$ & 10.007 & ADP 101 \\
\hline$\left(2 p^{\star}\right) j=1.5$ & 10.022 & ADP 101 \\
\hline$\left(2 P^{*}\right) j=1.5$ & 10.099 & ADP 101 \\
\hline$\left(2 P^{*}\right) j=1.5$ & 10.118 & ADP 101 \\
\hline$\left(2 P^{\star}\right) j=.5$ & 10.144 & ADP 101 \\
\hline$\left(2 P^{*}\right) j=1.5$ & 10.158 & ADP 101 \\
\hline$\left(2 P^{\star}\right) j=1.5$ & 10.201 & ADP 101 \\
\hline$\left(2 P^{*}\right) j=.5$ & 10.206 & $A D P 101$ \\
\hline$\left(2 P^{*}\right) j=.5$ & 10.229 & ADP 101 \\
\hline$\left(2 P^{*}\right) j=1.5$ & 10.245 & $A D P 101$ \\
\hline$\left(2 P^{*}\right) j=1.5$ & 10.262 & ADP 101 \\
\hline$\left(2 P^{*}\right) j=1.5$ & 10.285 & ADP 101 \\
\hline$\left\langle 2 P^{*}\right\rangle j=.5$ & 10.307 & ADP 101 \\
\hline$\left(2 P^{*}\right) j=.5$ & 10.353 & ADP 101 \\
\hline$\left(2 P^{*}\right) j=.5$ & 10.408 & ADP 101 \\
\hline$\left(2 P^{*}\right) j=.5$ & 10.472 & ADP 101 \\
\hline$\left(2 P^{\star}\right) j=.5$ & 10.496 & ADP 101 \\
\hline$\left(2 P^{*}\right) j=1.5$ & 10.561 & ADP 101 \\
\hline$(3 P) j=1.0$ & 7.445 & Insb 111 \\
\hline$\langle 3 P\rangle j=1.0$ & 7.445 & gypsum 020 \\
\hline$(3 P) j=2.0$ & 7.486 & gypsum 020 \\
\hline$(3 P) j=2.0$ & 7.525 & gypsum 020 \\
\hline$(3 P) j=2.0$ & 7.525 & beryl 100 \\
\hline (1D) $j=2.0$ & 7.583 & gypsum 020 \\
\hline (ID ) $j=2.0$ & 7.583 & beryl 100 \\
\hline$(3 P) j=2.0$ & 7.623 & beryl 100 \\
\hline$(3 P) j=2.0$ & 7.623 & beryl 100 \\
\hline (1D) $j=2.0$ & 7.631 & beryl 100 \\
\hline$(3 P) j=.0$ & 7.688 & beryl 100 \\
\hline$(3 P) j=1.0$ & 7.741 & beryl 100 \\
\hline$(3 P) j=2.0$ & 9.499 & mica 002 \\
\hline & 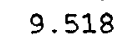 & -0 \\
\hline
\end{tabular}
$25.763 \quad 74.049$

$26.116 \quad 71.532$

$19.942 \quad 70.951$

$19.942 \quad 71.217$

$19.942 \quad 71.723$

$19.942 \quad 72.452$

$19.942 \quad 72.663$

$19.942 \quad 73.533$

$19.942 \quad 74.343$

$19.942 \quad 77.942$

$19.942 \quad 79.219$

$19.942 \quad 86.024$

$19.942 \quad 87.565$

$10.640 \quad 70.137$

$10.640 \quad 70.376$

$10.640 \quad 71.651$

$10.640 \quad 71.978$

$10.640 \quad 72.437$

$10.640 \quad 72.688$

$10.640 \quad 73.484$

$10.640 \quad 73.579$

$10.640 \quad 74.023$

$10.640 \quad 74.339$

$10.640 \quad 74.682$

$10: 640 \quad 75.158$

$10.640 \quad 75.628$

$10.640 \quad 76.662$

$10.640 \quad 78.013$

$10.640 \quad 79.805$

$10.640 \quad 80.563$

$10.640 \quad 83.014$

$\begin{array}{ll}7.481 & 84.377\end{array}$

$15.185 \quad 78.688$

$\begin{array}{ll}15.185 & 80.392\end{array}$

$15.185 \quad 82.354$

$\begin{array}{ll}15.954 & 70.620\end{array}$

$15.185 \quad 87.133$

$15.954 \quad 71.917$

$15.954 \quad 72.867$

$15.954 \quad 72.867$

$15.954 \quad 73.063$

$15.954 \quad 74.530$

$15.954 \quad 76.028$

$19.942 \quad 72.300$

$19.942 \quad 72.663$ 


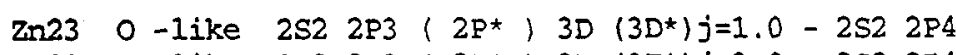

$\mathrm{Zn} 230$-1ike 2S2 $2 P_{3}\left(2 P^{*}\right) 3 D\left(3 F^{*}\right) j=3.0-2 S 22 P^{*}$

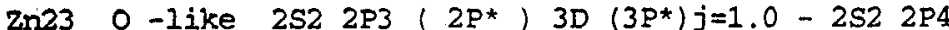

Zn23 O-like $2 S 22 P 3\left(2 D^{*}\right) 3 D\left(3 S^{*}\right) j=1.0-2 S 22 P 4$

Zn23 O-like 2S2 2P3 ( 2D*) 3D $\left(3 P^{*}\right) j=2.0-2 S 22 P 4$

$\mathrm{Zn} 230$-1ike 2S2 $2 \mathrm{P} 3\left(2 \mathrm{P}^{*}\right)$ 3D $\left(1 F^{*}\right) j=3.0-2 \mathrm{~S} 2 \mathrm{2P4}$

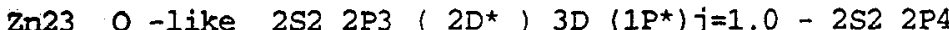

Zn23 0 -like 2S2 $2 P 3\left(2 P^{*}\right) 3 D\left(3 D^{*}\right) j=2.0-2 S 22 P 4$

$\mathrm{Zn} 230$-like $2 S 22 \mathrm{P} 3\left(2 D^{*}\right) 3 D\left(3 G^{*}\right) j=3.0-2 S 22 P 4$

Zn23 0 -like $2 S 22 P^{2}\left(2 D^{*}\right) 3 D\left(3 F^{*}\right) j=3.0-2 S 22 P 4$

Zn23 O-like 2S2 $2 P^{2}$ ( $\left.2 P^{*}\right) 3 D\left(3 D^{*}\right) j=2.0-2 S 22 P 4$

$\operatorname{Zn} 230$-like $2 S 22 \mathrm{P} 3\left(2 \mathrm{P}^{*}\right) 3 \mathrm{D}\left(3 \mathrm{D}^{*}\right) j=3.0-2 \mathrm{~S} 2 \mathrm{PP4}$

Zn23 o-like 2S2 2P3 (2P*) 3D $\left(3 F^{*}\right) j=2.0-2 S 22 P 4$

Zn23 O-like 2S2 $2 P^{2} 3\left(2 D^{*}\right) 3 D\left(3 P^{*}\right) j=2.0-2 S 22 P 4$

$\mathrm{Zn} 230$-like $2 S 22 \mathrm{P}^{2}\left(2 \mathrm{D}^{*}\right) 3 \mathrm{D}\left(3 \mathrm{P}^{*}\right) \mathrm{j}=.0-2 S 22 \mathrm{P}^{*}$

$\mathrm{Zn} 230$-1ike 2S2 $2 \mathrm{P} 3\left(2 \mathrm{P}^{*}\right)$ ) 3D (1P*)j=1.0 - 2S2 2P4

$\ln 230$-1ike $2 S 22 P^{2}\left(2 D^{*}\right) 3 D\left(1 D^{*}\right) j=2.0-2 S 22 P 4$

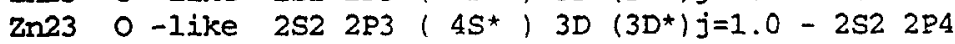

$\mathrm{z} 230$-1ike $2 S 22 \mathrm{P} 3\left(2 \mathrm{P}^{*}\right) 3 \mathrm{~S}\left(3 \mathrm{P}^{*}\right) \mathrm{j}=2.0-2 \mathrm{~S} 2 \mathrm{2P4}$

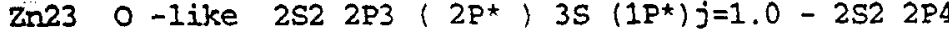

Zn23 O-1ike $2 S 22 P 3\left(2 D^{*}\right) 3 S\left(3 D^{*}\right) j=3.0-2 S 22 P 4$

$\mathrm{Zn} 23$. 0 -1ike $2 S 22 \mathrm{P}^{2}\left(2 \mathrm{P}^{*}\right) 3 S\left(3 \mathrm{P}^{*}\right) \mathrm{j}=.0-2 S 22 \mathrm{P} 4$

$\mathrm{zn} 230-1 \mathrm{ike} 2 S 22 \mathrm{P} 3\left(2 D^{*}\right) 3 S\left(1 D^{*}\right) j=2.0-2 S 22 \mathrm{P} 4$

Zn23 O-like 2S2 2P3 (4S*) 3S $\left(3 S^{*}\right) j=1.0-2 S 22 P 4$

zn23 0 -like $2 S 22 P^{2}\left(2 D^{*}\right) 3 S\left(3 D^{*}\right) j=1.0-2 S 22 P 4$

$\mathrm{Zn} 230$-like $2 S 22 \mathrm{P} 3\left(2 D^{*}\right) 3 S\left(1 D^{*}\right) j=2.0-2 S 22 \mathrm{P} 4$

zi23 O-like 2S2 2P3 ( 2D*) 3S $\left(3 D^{*}\right) j=2.0-2 S 22 D^{*}$

7 27 Be-like $1522 P \quad 3 P(3 P) j=2.0-15225$

$\mathrm{Zn27}$ Be-like 1S2 $2 \mathrm{P} \quad 3 \mathrm{P}(3 \mathrm{P}) j=2.0-1522 \mathrm{~S}$

Zn27 Be-1ike 1S2 2P

207 Be-1ike 1S2 2P

Zn27 Be-1ike 1S2 2P

$\begin{array}{lll}\text { Zn27 Be-like } 1 S 2 & 2 P \\ \text { Zn27 } & \text { Be-like 1S2 2P }\end{array}$

2727 Be-1ike 152 2P

$\mathrm{Zin} 27$ Be-like 1S2 2P

Zn27 Be-1ike 1s2 2s

in27 Be-like 1S2 2S

2027 Be-like 152 2S

Zn27 Be-like 1s2 25

$2 n 27$ Be-like 1S2 2P

in27 Be-1ike 1S2 2P

$\operatorname{Zn27}$ Be-like 1S2 2P

zn27 Be-like 1S2 $2 \mathrm{~S}$

2027 Be-like 152 2S

2n27 Be-like 1S2 2S

Zn27 Be-like 1S2 2P
(3P) $j=1.0 \quad 9.534$ mica 002

(3P) $j=2.0$

(3P) $j=1.0$

(3P) $j=2.0$

(3P) $j=2.0$

(1D) $j=2.0$

(3P) $j=2.0$

(3P) $j=1.0$

(3P) $j=2.0$

(3P) $j=2.0$

(1D) $j=2.0$

(1D) $j=2.0$

(1D) $j=2.0$

(3P) $j=1.0$

(3P) $j=1.0$

(1s) $j=.0$

(1D) $j=2.0$

(3P) $j=.0$

(3P) $j=1$.

(1D) $j=2.0$

(3P) $j=2.0$

(3P) $j=1$.

(3P) $j=1.0$

(3P) $j=2.0$

(3P) $j=1.0$

(1D) $j=2.0$

(3P) $j=1.0$

$2 P\left(3 P^{*}\right) j=1.0$

$2 P(3 P *) j=1.0$

$2 P\left(3 P^{*}\right) j=.0$

$2 P\left(3 P^{*}\right) j=.0$

$2 P\left(3 P^{*}\right) j=2.0$

$2 P\left(3 P^{*}\right) j=2.0$

$2 P\left(3 P^{*}\right) j=1.0$

$2 P\left(3 P^{*}\right) j=1.0$

(1s) $j=.0$

(1s) $j=.0$

(1s) $j=.0$

(1S) $j=.0$

$2 P\left(3 P^{*}\right) j=2.0$

$2 P\left(3 P^{*}\right) j=2.0$

$2 P\left(3 P^{*}\right) j=2.0$

2P $\left(1 P^{*}\right) j=1.0$

$2 P\left(1 P^{*}\right) j=1.0$

$2 P\left(1 P^{*}\right) j=1.0$

$2 P\left(1 P^{\star}\right) j=1.0$
9.541 mica 002

9.557 mica 002

.568 mica 002

9.586 mica 002

9.601 mica 002

.628 mica 002

.635 mica 002

9.686 mica 002

9.695 mica 002

9.713 mica 002

9.723 mica 002

9.744 mica 002

9.753 mica 002

9.761 mica 002

9.794 mica 002

823 mica 002

9.893 mica 002

10.229 ADP 101

10.285 ADP 101

10.307 ADP 101

10.380 ADP 101

10.539 ADP 101

10.557 ADP 101

10.569 ADP 101

10.574 ADP 101

939 topaz 002

7.939 beryl 100

7.999 topaz 002

7.999 quartz 100

8.009 topaz 002

8.009 quartz 100

8.034 topaz 002

8.034 quartz 100

8.056 topaz 002

8.056 quartz 100

8.056 topaz 002

8.056 quartz 100

8.078 topaz 002

8.078 quartz 100

8.078 TAP 100

8.092 topaz 002

8.092 quartz 100

8.092 TAP 100

8.147 topaz 002
$19.942 \quad 72.974$

$19.942 \quad 73.112$

$19.942 \quad 73.432$

$19.942 \quad 73.655$

$19.942 \quad 74.026$

$19.942 \quad 74.343$

$19.942 \quad 74.928$

$19.942 \quad 75.084$

$19.942 \quad 76.268$

$19.942 \quad 76.488$

$19.942 \quad 76.938$

$19.942 \quad 77.194$

$19.942 \quad 77.751$

$19.942 \quad 77.997$

$19.942 \quad 78.220$

$19.942 \quad 79.188$

$19.942 \quad 80.116$

$19.942 \quad 82.829$

$10.640 \quad 74.023$

$10.640 \quad 75.158$

$10.640 \quad 75.628$

$10.640 \quad 77.308$

$10.640 \quad 79.866$

$10.640 \quad 82.099$

$10.640 \quad 82.839$

$10.640 \quad 83.377$

$10.640 \quad 83.615$

$8.374 \quad 71.451$

$15.954 \quad 84.405$

$8.374 \quad 72.788$

$8.512 \quad 70.007$

$8.374 \quad 73.021$

$8.512 \quad 70.204$

$8.374 \quad 73.617$

$8.512 \quad 70.707$

$8.374 \quad 74.160$

8.51271 .161

$8.374 \quad 74.160$

8.51271 .161

$8.374 \quad 74.721$

$8.512 \quad 71.625$

$25.763 \quad 70.161$

$8.374 \quad 75.088$

8.51271 .926

$25.763 \quad 70.438$

$8.374 \quad 76.629$ 
Zn27 Be-1ike 1s2 2P

Zn27 Be-like 1s2 $2 \mathrm{P}$

$\mathrm{z} n 27$ Be-like 1s2 2S

Zn27 Be-like 152 2S

$\operatorname{Zn} 27$ Be-like 1s2 2S

Zn27 Be-like 1s2 2S

zn27 Be-like 152 2S

$\mathrm{Zn} 27$ Be-like 152 2S

Zn27 Be-like 1s2 2S

zn27 Be-like 1s2 2S

Zn27 Be-like 1s2 2S

Zn27 Be-like 1s2 25

zn27 Be-like 152 2S

Zn27 Be-like 1S2 2P

Zn27 Be-like 1S2 2P

Z 27 Be-like 1s2 2P

Zn27 Be-like 1s2 2P

Zi227 Be-1ike 1S2 2P

Zn27 Be-like 1S2 2P

zi27 Be-like 1S2 2P

Zn27 Be-like 1S2 2P

Zn27 Be-like 1S2 2P

Zn27 Be-like 1S2 2P

Zn27 Be-like 1S2 2P

Zin27 Be-like 152 2P

Zn27 Be-like 1S2 2P

Zn27: Be-like 1S2 2P

Zn27 Be-like 1S2 2P

Zn27 Be-like 1S2 2P

Zn27 Be-1ike 152 2P

Zn27 Be-like 1S2 2P

Zn27 Be-1ike 1s2 2s

Zn27 Be-like 1s2 2S

Zn27 Be-like 152 2S

Zn27 Be-like 1S2 2S

Zn27 Be-like 152 2S

Zn27 Be-like 1s2 2S

Zn27 Be-like 1S2 2P

Zn27 Be-like 1S2 2P

zin27. Be-like 1S2 2P

Zn27 Be-like 152 2P

Zn27. Be-1ike 1S2 2P

Zn27 Be-1ike 1S2 2P

Zn27 Be-like 1s2 2P

Zn27 Be-like 1S2 2P

Zn27: Be-like 1S2 2P
3P (1D) $j=2.0-1 s 22 s$ $3 P(1 D) j=2.0-1 S 22 S$ 3D (3D) $j=1.0-1 S 22 S$ 3D (3D) $j=1.0-1 S 22 S$ 3D (3D) $j=1.0-1 S 22 S$ 3D (3D) $j=1.0-1 S 22 S$ 3D (3D) $j=1.0-1 S 22 S$ 3D (3D) $j=1.0-1 S 22 S$ 3D (3D) $j=1.0-1 S 22 S$ 3D (3D) $j=1.0-1 S 22 S$ 3D (3D) $j=1.0-1 S 22 S$ 3D (3D) $j=1.0-1 S 22 S$ 3D (3D) $j=1.0-1 S 22 S$ 3P (1P) $j=1.0-1 S 2$ 2S $3 P(1 P) j=1.0-1522 S$ $3 P(1 P) j=1.0-1 S 22 S$ $3 P(1 P) j=1.0-1 S 22 S$ $3 P(1 P) j=1.0-1 S 22 S$ $3 P(1 P) j=1.0-1 S 22 S$ 3D $\left(3 D^{*}\right) j=2.0-1 S 22 P 2$ 3D $\left(3 D^{*}\right) j=2.0-1 S 22 P 2$ 3D $\left(3 D^{*}\right) j=2.0-1 S 22 P 2$ 3D $\left(3 D^{*}\right) j=2.0-152 \quad 2 P 2$ $3 D\left(3 D^{*}\right) j=2.0-1 S 22 P 2$ 3D $\left(3 D^{*}\right) j=2.0-1 S 22 \mathrm{P} 2$ $3 P(3 D) j=1.0-1 S 22 S$ 3P (3D) $j=1.0-1 S 22 S$ 3P (3D) $j=1.0-1 S 22 S$ $3 P(3 D) j=1.0-1 S 22 S$ $3 P$ (3D) $j=1.0-1 \mathrm{~S} 2 \mathrm{SS}$ 3P (3D) $j=1.0-1 S 22 S$ 3D (3D) $j=1.0-1 S 22 S$ 3D (3D) $j=1.0-1 S 22 S$ 3D (3D) $j=1.0-1 S 22 S$ 3D (3D) $j=1.0-1 S 22 S$ 3D (3D) $j=1.0-1 S 22 S$ 3D (3D) $j=1.0-1 S 22 S$ 3D $\left(1 F^{\star}\right) j=3.0-1 S 22 P 2$ 3D (1F*) $j=3.0-1 S 22 \mathrm{P} 2$ 3D (1F*) $j=3.0-1 S 22 \mathrm{F2}$ 3D $\left(1 F^{\star}\right) j=3.0-1522 P 2$ 3D $\left(1 F^{*}\right) j=3.0-1 S 22 P 2$ 3D $\left(1 F^{*}\right) j=3.0-152 \quad 2 \mathrm{P} 2$ 3D $\left(3 D^{*}\right) j=1.0-1 S 22 P 2$ 3D $\left(3 D^{*}\right) j=1.0-1.522 . P 2$ 3D $\left(3 D^{*}\right) j=1.0-1522 \mathrm{P} 2$

\begin{tabular}{|c|c|c|c|}
\hline $2 P$ & $\left(1 P^{\star}\right) j=1.0$ & 8.147 & quartz 100 \\
\hline $2 P$ & $\left(1 P^{\star}\right) j=1.0$ & 8.147 & TAP 100 \\
\hline $2 \mathrm{P}$ & $\left(3 p^{\star}\right) j=.0$ & 8.249 & topaz 002 \\
\hline $2 P$ & $\left(3 P^{\star}\right) j=.0$ & 8.249 & quartz 100 \\
\hline $2 \mathrm{P}$ & $\left(3 p^{*}\right) j=.0$ & 8.249 & PET 002 \\
\hline $2 P$ & $\left(3 P^{\star}\right) j=.0$ & 8.249 & TAP 100 \\
\hline$P$ & $\left(3 P^{*}\right) j=.0$ & 8.249 & RAP 100 \\
\hline P & $\left(3 P^{*}\right) j=1.0$ & 8.286 & topaz 002 \\
\hline & $\left(3 P^{*}\right) j=1.0$ & 8.286 & quartz 100 \\
\hline P & $\left(3 P^{\star}\right) j=1.0$ & 8.286 & PET 002 \\
\hline$P$ & $\left(3 P^{*}\right) j=1.0$ & 8.286 & EDS 020 \\
\hline$P$ & $\left(3 P^{\star}\right) j=1.0$ & 8.286 & TAP 100 \\
\hline$P$ & $\left(3 P^{*}\right) j=1.0$ & 8.286 & RAP 100 \\
\hline$P$ & $\left(1 P^{\star}\right) j=1.0$ & 8.340 & topaz 002 \\
\hline$P$ & $\left(1 P^{*}\right) j=1.0$ & 8.340 & quartz 100 \\
\hline$P$ & $\left(1 P^{*}\right) j=1.0$ & 8.340 & PET 002 \\
\hline $2 P$ & $\left(1 P^{\star}\right) j=1.0$ & 8.340 & $\mathrm{EDT} 020$ \\
\hline$P$ & $(1 p *) j=1.0$ & 8.340 & TAP 100 \\
\hline$P$ & $\left(1 P^{*}\right) j=1.0$ & 8.340 & RAP 100 \\
\hline & $(3 P) j=2.0$ & 8.389 & quartz 100 \\
\hline & $(3 P) j=2.0$ & 8.389 & PET 002 \\
\hline & $(3 P) j=2.0$ & 8.389 & EDT 020 \\
\hline & $(3 P) j=2.0$ & 8.389 & TAP 100 \\
\hline & $(3 P) j=2.0$ & 8.389 & RAP 100 \\
\hline & $(3 P) j=2.0$ & 8.389 & KAP 100 \\
\hline$P$ & $\left(1 p^{*}\right) j=1.0$ & 8.402 & quartz 100 \\
\hline$P$ & $\left(1 P^{\star}\right) j=1.0$ & 8.402 & PET 002 \\
\hline$P$ & $\left(1 p^{*}\right) j=1.0$ & 8.402 & EDT 020 \\
\hline $2 P$ & $\left(1 P^{*}\right) j=1.0$ & 8.402 & TAP 100 \\
\hline$P$ & $(1 P *) j=1.0$ & 8.402 & RAP 100 \\
\hline $2 P$ & $\left(1 P^{*}\right) j=1.0$ & 8.402 & KAP 100 \\
\hline 29 & $\left\langle 3 p^{*}\right\rangle j=2.0$ & 8.414 & quartz 100 \\
\hline$P$ & $\left(3 P^{*}\right) j=2.0$ & 8.414 & PET 002 \\
\hline$Z P$ & $\left(3 P^{*}\right) j=2.0$ & 8.414 & EDT 020 \\
\hline $2 P$ & $\left(3 P^{*}\right) j=2.0$ & 8.414 & TAP 100 \\
\hline$P$ & $\left(3 P^{*}\right) j=2.0$ & 8.414 & RAP 100 \\
\hline & $\left(3 P^{*}\right) j=2.0$ & 8.414 & $\operatorname{KAP} 100$ \\
\hline & (ID ) j=2.0 & 8.483 & quartz 100 \\
\hline & $(1 D) j=2.0$ & 8.483 & PET 002 \\
\hline & $(1 D) j=2.0$ & 8.483 & EDT 020 \\
\hline & (ID) $j=2.0$ & 8.483 & TAP 100 \\
\hline & (1D) $j=2.0$ & 8.483 & RAP 100 \\
\hline & (1D) $j=2.0$ & 8.483 & KAP 100 \\
\hline & $\langle 3 P\rangle j=1.0$ & 8.500 & quartz 100 \\
\hline & $(3 P) j=1.0$ & 8.500 & PET 002 \\
\hline & $13 P$ & 8.500 & EDT 020 \\
\hline
\end{tabular}

$8.512 \quad 73.160$ $25.763 \quad 71.566$ $8.374 \quad 80.088$ $8.512 \quad 75.720$

$8.742 \quad 70.666$ $25.763 \quad 73.856$ $26.116 \quad 71.366$ $8.374 \quad 81.686$

$8.512 \quad 76.768$

$8.742 \quad 71.413$

$8.808 \quad 70.175$

$25.763 \quad 74.768$

$26.116 \quad 72.144$

$8.374 \quad 84.835$

8.51278 .462

$8.742 \quad 72.557$

$8.808 \quad 71.239$

$25.763 \quad 76.206$

$26.116 \quad 73.342$

$8.512 \quad 80.248$

$8.742 \cdot 73.662$

$8.808 \quad 72.256$

$25.763 \quad 77.652$

$26.116 \quad 74.507$

$26.634 \quad 70.895$

$8.512 \quad 80.779$

$8.742 \quad .73 .968$

$8.808 \quad 72.536$

$25.763 \quad 78.064$

$26.116 \quad 74.830$

$26.634 \quad 71.153$

$8.512 \quad 81.297$

$8.742 \quad 74.255$

$8.808 \quad 72.798$

$25.763 \quad 78.458$

$26.116 \quad 75.135$

$26.634 \quad 71.394$

$8.512 \quad 85.269$

$8.742 \quad 76.018$

$8.808 \quad 74.387$

$25.763 \quad 81.045$

$26.116 \quad 77.023$

$26.634 \quad 72.845$

$8.512 \quad 86.957$

$8.742 \quad 76.487$

$8.808 \quad 74.803$ 
Zn27 Be-1ike 1S2 2P

Zn27 Be-like 1S2 2P

Zn27 Be-1ike 1S2 2P

Zn27 Be-like 1S2 2P

Zn27 Be-like 1S2 2P

Zn27 Be-1ike 1S2 2P

Zn27 Be-like 1s2 2P

Zn27 Be-like 1S2 2P

Zn27 Be-like 1S2 2S

Zn27 Be-like 1S2 2S

Zn27 Be-1ike 1s2 $2 \mathrm{~s}$

Zn27 Be-like 1s2 2S

Zn27 Be-like 1S2 2S

Zn27 Be-like 1s2 2S

Zn27 Be-like 1S2 2S

Zn27 Be-like 1s2 2S

Zn27 Be-like 1S2 2S

Zn27 Be-like 1s2 2S

Zn27 Be-like 1S2 2P

Zn27 Be-like 1S2 2P

Zn27 Be-like 1S2 2P

Zn27 Be-like 1S2 2P

Zn27 Be-like 1s2 25

Zn27 Be-like 1S2 2S

in27 Be-like 1S2 2S

Zn27 Be-like 1S2 2S

Zn27 Be-like 1s2 2P

Zn27 Be-like 1S2 2P

Zn27 Be-like 1S2 2P

Zn27 Be-1ike 1S2 2P

$\ln 28$ Li-like $15\left(2 S 2 P\left(1 P^{*}\right)\right)\left(2 P^{*}\right) j=.5-1522 S$

Zn28 Li-like 1S (2S $\left.2 P^{2}\left(1 P^{*}\right)\right)\left(2 P^{*}\right) j=.5-1 S 22 S$

Zn28 Li-like is (2S $\left.2 P^{2}\left(1 P^{*}\right)\right)\left(2 P^{*}\right) j=.5-1 S 22 S$

$\mathrm{Zn} 28$ Li-1ike $1 \mathrm{~S}\left(2 \mathrm{~S} 2 \mathrm{P}\left(1 \mathrm{P}^{*}\right)\right)\left(2 \mathrm{P}^{*}\right) j=.5-1 S 2 \mathrm{~S}$

Zn28 Li-1ike 1S (2S 2P (3P*)) $\left(2 P^{*}\right) j=.5-1 S 2$ 2S

Zn28 Li-like 1S (2S $\left.2 P^{2}\left(3 P^{*}\right)\right)\left(2 P^{*}\right) j=5-1 S 22 S$

Zn28 Li-like is (2S $\left.2 P^{2}\left(3 P^{*}\right)\right)\left(2 P^{*}\right) j=.5-1 S 22 S$

Zn28 Li-like $1 S\left(2 S 2 P\left(3 P^{*}\right)\right)\left(2 P^{*}\right) j=.5-1 S 2 S$

Zn29 He-like 1s 5p

Zn29 He-like 15 SP

$\mathrm{Zn} 29$ He-like $1 \mathrm{~S} 5 \mathrm{P}$

Zn29 He-like 1s 5P

Zn29 He-like 15 $5 P$

Zn29 He-like is $4 P$

Zn29 He-like 1S $4 P$

Zn29 He-like 15 4P $\left(3 P^{*}\right) j=1,0-1 S 2$

$(3 P *) j=1.0-1 s 2$

$\left(3 P^{\star}\right) j=1.0-1 S 2$

$\left(3 P^{*}\right) j=1.0-1 S 2$

$\left(3 P^{*}\right) j=1.0-1 S 2$

$\left(3 P^{*}\right) j=1.0-1 S 2$

$\left(3 P^{*}\right) j=1.0-152$

$\left(3 P^{\star}\right) j=1.0-1 S 2$
(3P) $j=1.0$

(3P) $j=1.0$

(3P) $j=1.0$

(3P) $j=2.0$

( $3 P$ ) $j=2.0$

(3P) $j=2.0$

(3P) $j=2.0$

(3P) $j=2.0$

2P $\left(3 P^{*}\right) j=1.0$

$2 P\left(3 P^{*}\right) j=1.0$

$2 P\left(3 P^{*}\right) j=1.0$

$2 P \quad\left(3 P^{\star}\right) j=1.0$

$2 P\left(3 P^{*}\right) j=1.0$

$2 P\left\langle 1 P^{*}\right) j=1.0$

$2 P\left(1 P^{*}\right) j=1.0$

$2 P\left(1 P^{*}\right) j=1.0$

$2 P\left(1 P^{*}\right) j=1.0$

$2 P\left(1 P^{*}\right) j=1.0$

(1s) $j=.0$

(1s) $j=.0$

(1s) $j=.0$

(1s) $j=.0$

$2 P\left(3 P^{*}\right) j=2.0$

$2 P\left(3 P^{\star}\right) j=2.0$

$2 P\left(3 P^{*}\right) j=2.0$

$2 P(3 P *) j=2.0$

(1D) $j=2.0$

(ID) $j=2.0$

(1D) $j=2.0$

(ID) $j=2.0$

(2S) $j=.5$

(2s) $j=.5$

(2s) $j=.5$

(2S) $j=.5$

(2S) $j=.5$

(2s) $j=.5$

(2S) $j=.5$

(2s) $j=.5$

(1s) $j=.0$

(1s) $j=.0$

(1s) $j=.0$

(1s) $j=.0$

(1s) $j=.0$

(1s) $j=.0$

(1s) $j=.0$

(1s) $j=.0$
8.500 TAP 100

8.500 RAP 100

8.500 KAP 100

550 PET 002

8.550 EDT 020

8.550 TAP 100

8.550 RAP 100

$8.550 \mathrm{KAP} 100$

8.566 PET 002

8.566 EDT 020

8.566 TAP 100

8.566 RAP 100

8.566 KAP 100

8.576 PET 002

8.576 EDT 020

8.576 TAP 100

8.576 RAP 100

8.576 KAP 100

8.658 PET 002

8.658 EDT 020

8.658 RAP 100

8.658 KAP 100

8.702 PET 002

8.702 EDT 020

8.702 RAP 100

8.702 KAP 100

8.720 PET 002

8.720 EDT 020

8.720 KAP 100

8.856 KAP 100

1.382 topaz 006

1.382 LiF 220

1.382 quartz 200

$1.382 \mathrm{NaCI} 200$

1.387 topaz 006

1.387 LIF 220

1.387 quartz 200

$1.387 \mathrm{NaCl} 200$

1.087 Ge 422

1.087 tungsten 110

1.087 quartz 112

$1.087 \mathrm{NaCl} 200$

1.113 Ge 422

1.113 quartz 310

1.113
$25.763 \quad 81.806$

$26.116 \quad 77.531$

$26.634 \quad 73.220$

$8.742 \quad-77.970$

$8.808 \quad 76.098$

$25.763 \quad 84.632$

$26.116 \quad 79.160$

$26.634 \quad 74.377$

$8.742 \quad 78.484$

$8.808 \quad 76.538$

$25.763 \quad 85.929$

$26.116 \quad 79.735$

$26.634 \quad 74.765$

$8.742 \quad 78.817$

$8.808 \quad 76.820$

$25.763 \quad 87.013$

$26.116 \quad 80.111$

$26.634 \quad 75.013$

$8.742 \quad 82.051$

$8.808 \quad 79.411$

$26.116 \quad 84.022$

$26.634 \quad 77.218$

$8.742 \quad 84.517$

$8.808 \quad 81.102$

$26.116 \quad 88.414$

$26.634 \quad 78.572$

$8.742 \quad 85.934$

$8.808 \quad 81.894$

$26.634 \quad 79.174$

$26.634 \quad 85.966$

$2.795 \quad 81.459$

$2.848 \quad 76.050$

$4.246 \quad 77.540$

$5.641 \quad 78.512$

$2.795 \quad 82.972$

$2.848 \quad 76.910$

$4.246 \quad 78.516$

$5.641 \quad 79.582$

$2.217 \quad 78.697$

$2.310 \quad 70.241$

$4.476 \quad 76.265$

$4.564 \quad 72.302$

$5.641 \quad 74.468$

$2.310 \quad 74.501$

2.36070 .600

$4.476 \quad 84.064$ 


\begin{tabular}{|c|c|c|c|c|c|c|}
\hline $\ln 29$ & He-like & 1S & $4 P$ & $\left(3 P^{\star}\right) j=1.0$ & $-1 S 2$ & \\
\hline $\ln 29$ & He-like & 1S & $4 P$ & $\left(3 P^{*}\right) j=1.0$ & -152 & \\
\hline $\ln 29$ & He-like & 1s & $4 \mathrm{P}$ & $\left(3 P^{*}\right) j=1.0$ & $-1 \$ 2$ & \\
\hline 29 & He-1ike & 1s & $4 P$ & $\left(3 P^{\star}\right) j=1.0$ & -152 & \\
\hline $\ln 29$ & He-like & 1s & $3 p$ & $\left(3 P^{\star}\right) j=1.0$ & -152 & \\
\hline $\ln 29$ & He-like & $1 \mathrm{~s}$ & $3 P$ & $\left(3 P^{\star}\right) j=1.0$ & $-1 s 2$ & \\
\hline $\ln 29$ & He-like & 1s & $3 p$ & $\left(3 P^{\star}\right) j=1.0$ & -152 & \\
\hline $\ln 29$ & He-like & 1S & $3 P$ & $\left(3 P^{*}\right) j=1.0$ & $-1 s 2$ & \\
\hline $2 \pi 29$ & He-like & 1S & $3 P$ & $\left(3 P^{*}\right) j=1.0$ & $-1 s 2$ & \\
\hline $\ln 29$ & He-like & is & $2 \mathrm{P}$ & $\left(1 P^{\star}\right) j=1.0$ & $-1 s 2$ & \\
\hline $\ln 29$ & He-1ike & 15 & $2 P$ & $\left(1 P^{\star}\right) j=1.0$ & $-1 s 2$ & \\
\hline $\ln 29$ & He-like & is & $2 P$ & $\left(1 P^{*}\right) j=1.0$ & $-1 s 2$ & \\
\hline $\ln 29$ & He-like & 15 & $2 P$ & $\left(1 P^{*}\right) j=1.0$ & $-1 s 2$ & \\
\hline $\ln 29$ & $\mathrm{He}-1 \mathrm{ike}$ & 1S & $2 P$ & $\left(3 P^{*}\right) j=1.0$ & $-1 S 2$ & \\
\hline $\ln 29$ & He-like & is & $2 \mathrm{P}$ & $\left(3 P^{*}\right) j=1.0$ & $-1 S 2$ & \\
\hline $\ln 29$ & He-like & 15 & $2 P$ & $\left(3 P^{*}\right) j=1.0$ & -152 & \\
\hline $\mathrm{Zn} 29$ & He-like & 1s & $2 \mathrm{P}$ & $\left(3 P^{*}\right) j=1.0$ & -152 & \\
\hline $\mathrm{Zn} 29$ & He-like & 1S & 25 & $\langle 3 s\rangle j=.0$ & $-1 s 2$ & \\
\hline $\mathrm{Z} 229$ & He-like & 1S & $2 S$ & $(3 s) j=.0$ & $-1 s 2$ & \\
\hline $2 \pi 29$ & He-like & 15 & $2 S$ & $(3 s) j=.0$ & -152 & \\
\hline $\ln 29$ & He-like & 1S & $2 S$ & $(3 s) j=.0$ & - $1 S 2$ & \\
\hline $\mathrm{Ga}$ & & $\mathrm{K}-\mathrm{a}$ & lpha (1) & & & \\
\hline $\mathrm{Ga}$ & & $\mathrm{K}-\mathrm{a}]$ & lpha (1) & & & \\
\hline $\mathrm{Ga}$ & & K-a & Ipha (I) & & & \\
\hline $\mathrm{Ga}$ & & $\mathrm{k}-\mathrm{a}$ & Ipha (1) & & & \\
\hline $\mathrm{Ga}$ & & $\mathrm{K}-\mathrm{a}$ & lpha (1) & & & \\
\hline $\mathrm{Ga}$ & & $k-a$ & lpha (1) & & & \\
\hline $\mathrm{Ga}$ & & $\mathrm{K}-\mathrm{a}$ & lpha (1) & & & \\
\hline $\mathrm{Ga}$ & & $\mathrm{K}-\mathrm{a}$ & lpha (1) & & & \\
\hline $\mathrm{Ga}$ & & $K-a$ & 1pha (1) & & & \\
\hline Ga21 & Na-like & 225 & $3 P<3 D / 2 D$ & 3)) $(03) j=2.5$ & $-2 P 6$ & $3 P$ \\
\hline Ga21 & Na-like & $2 \mathrm{P} 5$ & $3 S 13 D<2 D$ & 2)) $\left(12^{*}\right) j=1.5$ & $-2 P 6$ & $3 S$ \\
\hline Ga21 & Na-like & $2 \mathrm{P} 5$ & $(2 \mathrm{P} * 1)(3 \mathrm{D} 2$ & $\left(I\left(14^{*}\right) j=3.5\right.$ & $-2 P G$ & $3 D$ \\
\hline $\mathrm{Ga} 21$ & Na-like & 2P5 & $(2 \mathrm{P} * 1)(3 \mathrm{D} 2$ & $\left(3\left(13^{*}\right) j=2.5\right.$ & $-2 P 6$ & 3D \\
\hline Ga21 & $\mathrm{Na}-$ like & $2 \mathrm{P5}$ & $3 S<3 D<2 D$ & 3)) $(13 *) j=1.5$ & $-2 P 6$ & $3 S$ \\
\hline $\mathrm{Ga} 21$ & Na-like & 2P5 & $3 P<3 D<2 D$ & 2)) (12) j=1.5 & $-2 P 6$ & $3 P$ \\
\hline Ga21 & $\mathrm{Na-like}$ & $2 \mathrm{P} 5$ & $3 P<3 D<2 D$ & 3) $(23, j=2.5$ & $-2 P 6$ & $3 P$ \\
\hline Ga21 & Na-like & $2 \mathrm{P} 5$ & $(2 \mathrm{P} * 1)(3 \mathrm{D} 2$ & $(3(12 *) j=2.5$ & $-2 P 6$ & $3 D$ \\
\hline Ga21 & Na-like & 2P5 & $(2 P \star 2)(3 D 2$ & $\left(1\left(20^{*}\right) j=1.5\right.$ & $-2 P 6$ & 3D \\
\hline Ga21 & $\mathrm{Na-like}$ & $2 P 5$ & $(2 \mathrm{~F} * 2)(3 \mathrm{D} 2$ & $\left(3\left(24^{*}\right) j=2.5\right.$ & $-2 P 6$ & $3 \mathrm{D}$ \\
\hline $\mathrm{Ga} 21$ & Na-like & $2 \mathrm{P} 5$ & $(2 \mathrm{~F} * 2)(3 \mathrm{D} 2$ & $\left(3\left(24^{*}\right) j=2.5\right.$ & $-2 P 6$ & $3 D$ \\
\hline Ga21 & Na-like & 2P5 & $(2 P \star 2)(3 D 2$ & $\left(1\left(24^{*}\right) j=3.5\right.$ & $-2 P 6$ & $3 D$ \\
\hline Ga21 & $\mathrm{Na-like}$ & $2 P 6$ & $5 D$ & $(2 D) j=2.5$ & $-2 P 6$ & $3 P$ \\
\hline $\mathrm{Ga} 22$ & Ne-like & $2 \mathrm{~S} 2$ & $2 P 5(2 P * 1)$ & $6 D(12 *) j=1.0$ & $-2 s 2$ & $2 P 6$ \\
\hline $\mathrm{Ga22}$ & Ne-like & $2 \mathrm{~S} 2$ & $2 \mathrm{P} 5 \quad(2 \mathrm{P} \star 2)$ & 5D $\left(23^{*}\right) j=1.0$ & $-2 s 2$ & $2 \mathrm{PG}$ \\
\hline $\mathrm{Ga} 22$ & Ne-like & $2 \mathrm{~s} 2$ & $2 P 5(2 P * 2)$ & $5 D\left(22^{\star}\right) j=1.0$ & $-2 s 2$ & $2 P 6$ \\
\hline
\end{tabular}

\begin{tabular}{|c|c|c|}
\hline$(1 s) j=.0$ & 1.113 & quartz 112 \\
\hline (1s) $j=.0$ & 1.113 & topaz 200 \\
\hline (1s) $j=.0$ & 1.113 & Al 111 \\
\hline$j=.0$ & 1.113 & $\mathrm{NaCl} 200$ \\
\hline$j j=.0$ & 1.172 & quartz 310 \\
\hline$j j=.0$ & 1.172 & quartz 220 \\
\hline$S / j=.0$ & 1.172 & quartz 112 \\
\hline $1 S j j=.0$ & 1.172 & quartz 110 \\
\hline (S $j j=.0$ & 1.172 & calcite 200 \\
\hline (s) $j=.0$ & 1.378 & topaz 006 \\
\hline$S i j=.0$ & 1.378 & LiF 220 \\
\hline$s i j=.0$ & 1.378 & quartz 200 \\
\hline$j=.0$ & 1.378 & $\mathrm{NaC1} 200$ \\
\hline$j=.0$ & 1.385 & topaz 006 \\
\hline$j=.0$ & 1.385 & LiF 220 \\
\hline s $j=.0$ & 1.385 & quartz 200 \\
\hline Ls $j j=.0$ & 1.385 & $\mathrm{NaCl} 200$ \\
\hline$j=.0$ & 1.391 & topaz 006 \\
\hline$j=.0$ & 1.391 & LIF 220 \\
\hline s $j j=.0$ & 1.391 & quartz 200 \\
\hline$S i j=.0$ & 1.391 & $\mathrm{NaCl} 200$ \\
\hline & 1.340 & topaz 303 \\
\hline & 1.340 & corundum 030 \\
\hline & 1.340 & quartz 203 \\
\hline & 1.340 & topaz 006 \\
\hline & 1.340 & LiF 220 \\
\hline & 1.340 & LiF 200 \\
\hline & 1.340 & Al 200 \\
\hline & 1.340 & quartz 200 \\
\hline & 1.340 & $\mathrm{NaCl} 200$ \\
\hline$j=1.5$ & 9.685 & mica 002 \\
\hline$j=.5$ & 9.720 & mica 002 \\
\hline$j=2.5$ & 9.739 & mica 002 \\
\hline$j j=2.5$ & 9.752 & mica 002 \\
\hline$j=.5$ & 9.772 & mica 002 \\
\hline *) $j=.5$ & 9.780 & mica 002 \\
\hline$j=1.5$ & 9.791 & mica 002 \\
\hline$j=1.5$ & 9.812 & mica 002 \\
\hline$j=2.5$ & 9.905 & mica 002 \\
\hline$j=1.5$ & 9.920 & mica 002 \\
\hline$j j=2.5$ & 9.939 & mica 002 \\
\hline$j=2.5$ & 9.961 & mica 002 \\
\hline$=1.5$ & 24.326 & TAP 100 \\
\hline .0 & 6.695 & graphite 002 \\
\hline .0 & 7.119 & InSb 111 \\
\hline$=0$ & 7.130 & Insb 111 \\
\hline
\end{tabular}

$\begin{array}{rl}4.564 & 77.281 \\ 4.638 & 73.719 \\ 4.676 & 72.194 \\ 5.641 & 80.584 \\ 2.360 & 83.324 \\ 2.451 & 73.008 \\ 3.636 & 75.239 \\ 4.912 & 72.630 \\ 6.071 & 74.850 \\ 2.795 & 80.417 \\ 2.848 & 75.397 \\ 4.246 & 76.811 \\ 5.641 & 77.723 \\ 2.795 & 82.331 \\ 2.848 & 76.560 \\ 4.246 & 78.116 \\ 5.641 & 79.142 \\ 2.795 & 84.472 \\ 2.848 & 77.641 \\ 4.246 & 79.360 \\ 5.641 & 80.522 \\ 2.712 & 81.217 \\ 2.748 & 77.246 \\ 2.749 & 77.154 \\ 2.795 & 73.522 \\ 2.848 & 70.234 \\ 4.027 & 86.694 \\ 4.048 & 83.293 \\ 4.246 & 71.235 \\ 5.641 & 71.852 \\ 19.942 & 76.244 \\ 19.942 & 77.117 \\ 19.942 & 77.616 \\ 19.942 & 77.969 \\ 19.942 & 78.534 \\ 19.942 & 78.767 \\ 19.942 & 79.097 \\ 19.942 & 79.754 \\ 19.942 & 83.404 \\ 19.942 & 84.203 \\ 19.942 & 85.408 \\ 19.942 & 87.434 \\ 25.763 & 70.773 \\ 6.696 & 89.010 \\ 7.481 & 72.103 \\ 7.481 & 72.379\end{array}$


Ga22 Ne-1ike 2S2 2P5 ( 2P*1) 4D (12*)j=1.0 - 2S2 $2 \mathrm{P} 6$ Ga22 Ne-like 2S2 2P5 ( $2 P * 2)$ 4D $\left(23^{*}\right) j=1.0-2 S 22 P 6$ Ga22 Ne-like 2S2 2P5 $(2 P * 2) 4 D\left(22^{*}\right) j=1.0-2 S 22 P 6$ Ga22 Ne-like $2 S 22 P 5(2 P * 1) 4 S\left(11^{*}\right) j=1.0-2 S 22 P 6$ Ga22 Ne-like 2S2 2P5 ( 2P*1) 4S (11*)j=1.0 - 2S2 2P6 Ga22 Ne-like 2S2 $2 P 5\left(2 P^{\star} 2\right) 4 S\left(22^{\star}\right) j=1.0-2 S 22 P 6$ Ga22 Ne-like 2S2 2P5 ( 2P*2) 4S $\left(22^{\star}\right) j=1.0-2 S 2$ 2P6 Ga22 Ne-1ike 2S2 2P5 (2P*1) 3D (12*)j=1.0 - 2S2 2P6 Ga22 Ne-like 2S2 2P5 $(2 P * 2)$ 3D $\left(23^{*}\right) j=1.0-2 S 22 P 6$ Ga22 Ne-like 2S2 $2 P 5(2 P * 2) 3 D\left(22^{*}\right) j=1.0-2 S 22 P 6$ Ga22 Ne-1ike 2S2 2P5 ( 2P*1) 3S $\left(11^{*}\right) j=1.0-2 \mathrm{~S} 2$ 2P6 Ga23 F-like 2S2 2P2 ( IS ) $4 D(2 D) j=1.5-2 S 22 P 5$ Ga23 $F$-like 2S2 2P2 ( $1 S$ ) $4 D(2 D) j=1.5-2 S 22 P 5$ Ga23 F-like 2S2 2P2 (ID) $4 D(2 D) j=1.5-2 S 22 P 5$ Ga23 F-like 2S2 2P2 (ID) $4 D(2 D) j=1.5-2 S 22 P 5$ Ga23 $F$-like 2S2 2P2 ( $1 D$ ) $4 D(2 D) j=2.5-2 S 22 P 5$ Ga23 $F$-like 2S2 2P2 ( 1D) 4D (2D) $j=2.5-2 S 22 P 5$ Ga23 F-like $2 S 22 \mathrm{P} 2(1 \mathrm{D}) 4 \mathrm{D}(2 \mathrm{D}) j=2.5-2 \mathrm{~S} 2$ 2P5 Ga23 F-1ike 2S2 2P2 ( 1D) $4 D(2 D) j=2.5-2 S 2$ 2P5 Ga23 F-like 2S2 $2 P 2$ ( $3 P$ ) $4 D(4 P) j=2.5-2 S 22 P 5$ Ga23 F-1ike 2S2 2P2 ( $3 P$ ) $4 D(4 P) j=2.5-2 S 2$ 2P5 Ga23 F-like 2S2 2P4 ( $3 P$ ) $4 D(4 D) j=2.5-2 S 22 P 5$ Ga23 F-like 2S2 2P4 ( $3 P$ ) $4 D(4 D) j=2.5-2 S 22 P 5$ Ga23 F-like 2S2 2P2 ( $3 P$ ) $4 D(4 F) j=2.5-2 S 22 P 5$ Ga23 F-like 2S2 2P2 ( $3 P$ ) $4 D(4 F) j=2.5-2 S 22 P 5$ Ga23 F-like 2S2 2P2 ( $1 D) 4 D(2 S) j=.5-2 S 22 P 5$ Ga23 $F$-like 2S2 2P2 ( ID) $4 D(2 S) j=.5-2 S 22 P 5$ Ga23 F-like 2S2 2P2 ( 3P) 4D (2D) j=2.5 - 2S2 2P5 Ga23 F-like 2S2 2P2 ( $3 P$ ) $4 D(2 D) j=2.5-2 S 22 P 5$ Ga23 $F$-like $2 S$ 2P5 ( $1 P$ ) $3 P(2 D) j=2.5-2 S 22 P 5$ Ga23 F-like 2S 2P5 ( $1 P$ ) $3 P(2 D) j=2.5-2 S 22 P 5$ Ga23 F-like 2S 2P5 ( $1 P$ ) $3 P(2 D) j=2.5-2 S 2$ 2P5 Ga23 F-like 2S 2P5 ( $1 P$ ) 3P (2D) $j=2.5-2 S 22 P 5$ Ga23 F-like 2S 2P5 ( 1P ) 3P (2D) j=2.5 - 2S2 2P5 Ga23 $F$-like 2S 2P5 ( IP) 3P (2D) j=2.5 - 2S2 2P5 Ga23 $F$-like 2S 2P5 ( $1 P$ ) 3P (2P) $j=1.5-2 S 22 P 5$ Ga23 $F$-like 2S 2P5 ( $1 P$ ) $3 P(2 P) j=1.5-2 S 22 P 5$ Ga23 $F$-like 2S 2P5 ( $1 P$ ) 3P (2P) $j=1.5-2 S 22 P 5$ Ga23 F -like $2 S$ 2P5 ( $1 P$ ) 3P (2P) $j=1.5-2 S 22 P 5$ Ga23 F-like 2S 2P5 ( $1 P$ ) 3P (2P) $j=.5-2 S 22 P 5$ Ga23 F-like 2S 2P5 ( IP ) 3P (2P) $j=.5-2 S 2$ 2P5 Ga23. F-like 2S 2P5 ( 1P ) 3P (2P )j=.5-2S2 2P5 Ga23 F-like 2S 2P5 ( 1P) 3P (2P) j=.5-2S2 2P5 Ga23 F-like 2S 2P5 ( $1 P$ ) 3P (2D $) j=1.5-2 S 22 P 5$ Ga23 F-like 2S 2P5 ( $1 P$ ) 3P (2D) $j=1.5-2 S 22 P 5$ Ga23. ' $F$ "-like 2S 2P5 ( $1 P$ ) 3P (2D $j j=1.5-2 S 2$ 2P5
(1S $) j=.0$

(1s) $j=.0$

(1s) $j=.0$

(1s) $j=.0$

(1s) $j=.0$

(1s) $j=.0$

(1s) $j=.0$

(1s) $j=0$

(1s) $j=.0$

(1s) $j=.0$

(1s) $j=.0$

$\left(2 P^{*}\right) j=.5$

$\left(2 P^{*}\right) j=.5$

$\left(2 P^{*}\right) j=1.5$

$\left(2 P^{*}\right) j=1.5$

$\left(2 P^{\star}\right) j=1.5$

$\left(2 P^{*}\right) j=1.5$

$\left(2 P^{*}\right) j=1.5$

$\left(2 P^{*}\right) j=1.5$

$\left(2 P^{*}\right) j=1.5$

$\left(2 P^{*}\right) j=1.5$

$\left(2 p^{*}\right) j=1.5$

$(2 P *) j=1.5$

$\left(2 P^{\star}\right) j=1.5$

$\left(2 P^{*}\right) j=1.5$

$\left(2 P^{*}\right) j=.5$

$\left(2 P^{*}\right) j=.5$

$\left(2 P^{*}\right) j=1.5$

$\left(2 P^{*}\right) j=1.5$

$\left(2 P^{*}\right) j=1.5$

$\left(2 P^{*}\right) j=1.5$

$\left(2 P^{*}\right) j=1.5$

$\left(2 P^{\star}\right) j=1.5$

$\left(2 P^{*}\right) j=1.5$

$\left(2 P^{*}\right) j=1.5$

$\left(2 P^{*}\right) j=.5$

$\left(2 P^{*}\right) j=.5$

$\left(2 P^{*}\right) j=.5$

$\left(2 P^{*}\right) j=.5$

$\left(2 P^{*}\right) j=.5$

$\left(2 P^{*}\right) j=.5$

$\left(2 P^{*}\right) j=.5$

$\left(2 P^{*}\right) j=.5$

$\left(2 P^{*}\right) j=.5$

$\left(2 P^{*}\right) j=.5$

$\left(2 P^{*}\right) j=.5$
7.673 beryl 100

7.794 beryl 100

7.827 beryl 100

7.887 topaz 002

7.887 bery 100

8.028 topaz 002

8.028 quartz 100

9.643 mica 002

9.842 mica 002

9.961 mica 002

10.583 ADP 101

7.234 Insb 111

7.234 gypsum 020

7.264 InSb 111

7.264 gypsum 020

7.275 InSb 111

7.275 gypsum 020

7.275 InSb 111

7.275 gypsum 020

7.319 InSb 111

7.319 gypsum 020

7.371 Insb 111

7.371 gypsum 020

7.386 InSb 111

7.386 gypsum 020

7.404 Insb 111

7.404 gypsum 020

7.424 InSb 111

7.424 gypsum 020

8.466 quartz 100

8.466 PET 002

8.466 EDT 020

8.466 TAP 100

8.466 RAP 100

8.466 KAP 100

8.617 PET 002

8.617 EDT 020

8.617 RAP 100

8.617 KAP 100

8.633 PET 002

8.633 EDT 020

8.633 RAP 100

8.633 KAP 100

8.672 PET 002

8.672 EDT 020

8.672 RAP 100
$15.954 \quad 74.131$

$15.954 \quad 77.704$

$15.954 \quad 78.871$

8.37470 .364

$15.954 \quad 81.385$

$8.374 \quad 73.472$

$8.512 \quad 70.586$

$19.942 \quad 75.263$

$19.942 \quad 80.774$

$19.942 \quad 87.434$

$10.640 \quad 84.067$

$7.481 \quad 75.236$

$15.185 \quad 72.323$

$7.481 \quad 76.166$

$15.185 \quad 73.084$

$7.481 \quad 76.523$

$15.185 \quad 73.372$

$7.481 \quad 76.523$

$15.185 \quad 73.372$

$7.481 \quad 78.055$

$15.185 \quad 74.575$

7.48180 .162

$15.185 \quad 76.126$

$7.481 \quad 80.859$

$15.185 \quad 76.606$

$7.481 \quad 81.772$

$15.185 \quad 77.206$

$\begin{array}{ll}7.481 & 82.923\end{array}$

$\begin{array}{lll}15.185 & 77.907\end{array}$

$8.512 \quad 84.041$

$8.742 \quad 75.564$

$8.808 \quad 73.981$

$25.763 \quad 80.344$

$26.116 \quad 76.534$

$26.634 \quad 72.476$

$8.742 \quad 80.299$

$8.808 \quad 78.046$

$26.116 \quad 81.831$

26.63476 .073

$8.742 \quad 80.943$

$8.808 \quad 78.560$

$26.116 \quad 82.609$

$26.634 \quad 76.508$

$8.742 \quad 82.744$

$8.808 \quad 79.918$

$26.116 \quad 84.984$ 
Ga23 F-like $2 S$ 2P5 ( $1 P$ ) 3P (2D) $j=1.5-2 S 22 P 5$ Ga23 F-like 2S 2P5 ( $3 P$ ) 3P (4P) $j=2.5-2 S 22 P 5$ Ga23 F-1ike 2S 2P5 ( $3 P$ ) 3P (4P) $j=2.5-2 S 2$ 2P5 Ga23 F-like 2S 2P5 ( 3P) 3P (4P) $j=2.5-2 S 22 P 5$ Ga23 F-like 2S 2P5 ( $3 P)$ 3P (2P) $j=.5-2 S 22 P 5$ Ga23 $F$-like 2S 2P5 ( 3P) 3P (2P ) $j=.5-2 S 2$ 2P5 Ga23 F-like 2S 2P5 ( 3P) 3P $(2 P) j=.5-2 S 22 P 5$ Ga23 $F$-like 2S 2P5 ( $3 P$ ) $3 P(2 P) j=1.5-2 S 22 P 5$ Ga23 F-like 2S 2P5 ( 3P) 3P (2P) j=1.5 - 2S2 2P5 Ga23 F-like 2S 2P5 ( 3P) 3P (2D) $j=2.5-2 S 22 P 5$ Ga23 $F$-like 2S 2P5 ( $3 P$ ) $3 P(2 D) j=2.5-2 S 22 P 5$ Ga23 $F$-like $2 S$ 2P5 ( $3 P$ ) $3 P(2 S) j=.5-2 S 22 P 5$ Ga23 $F$-like 2S 2P5 ( 3P ) 3P (2S) $j=.5-2 S 22 P 5$ Ga23 F-like 2S 2P5 ( $3 P$ ) $3 P(4 D) j=2.5-2 S 22 P 5$ Ga23 F-like 2S2 2P2（1D) 3D (2P) $j=.5-2 S 22 P 5$ Ga23 : F-like 2S2 2P2 ( $1 D$ ) 3D (2D) $j=1.5-2 S 2$ 2P5 Ga23 F-like 2S2 2P4 (3P) 3D (4P) $j=2.5-2 S 22 P 5$ Ga23 F-like 2S2 2P4 ( 3P) 3D (4F) $j=1.5-2 S 22 P 5$ Ga23 F-like 2S2 2P2 ( ID) 3D (2P) j=1.5 - 2S2 2P5 Ga23 F-like 2S2 2P2 ( $1 D$ ) 3D (2S) $j=.5-2 S 22 P 5$ Ga23 F-like 2S2 2P2 ( 3P) 3D (4P) $j=1.5-2 S 2$ 2P5 Ga23 $F$-like 2S2 2P2 ( $3 P$ ) $3 D(4 P) j=.5-2 S 22 P 5$ Ga23 $F$-like 2S2 $2 P 2(3 P) 3 D(2 P) j=1.5-2 S 22 P 5$ Ga23 F-like 2S2 2P4 ( $3 P$ ) 3D (4D) $j=2.5$ - 2S2 2P5 Ga23 F-like 2S2 2P2 ( $1 S$ ) $3 S$ (2S ) $j=.5-2 S 22 P 5$ Ga23 $F$-like 2S2 2P2 ( 1D ) 3S (2D) $j=2.5-2 S 2$ 2P5 Ga23 F-like $2 S 22 P 2(3 P)$ 3S $(2 P) j=.5-2 S 22 P 5$ Ga23 $F$-like 2S2 $2 P 2(3 P)$, $3 S(4 P) j=1.5-2 S 22 P 5$ Ga23 $F$-like 2S2 2P2 ( $3 P$ ) $3 S(4 P) j=.5-2 S 22 P 5$ Ga23 F-like 2S2 2P2 (1D) 3S (2D) $j=1.5-2 S 22 P 5$ Ga23 F-like 2S2 2P2 ( 3P ) 3S (2P) $j=1.5-2 S 22 P 5$ Ga23 F-like 2S2 2P2 ( $3 P$ ) 3S (2P) $j=.5-2 S 22 P 5$ Ga23 F-like 2S2 2P2 ( $3 P$ ) $3 S(4 P) j=2.5-2 S 22 P 5$ Ga23 F-like 2S2 2P2 ( $3 P$ ) 3S (4P) $j=1.5-2 S 22 P 5$ Ga24 O-like 2S2 2P3 (2P*) 3D (3D*) j=2.0 - 2S2 2P4 Ga24 O-like 2S2 2P3 ( 2P*) 3D $\left(3 D^{*}\right) j=2.0-2 S 22 P 4$ Ga24 0 -1ike 2S2 2P3 ( 2D*) 3D $\left(1 F^{*}\right) j=3.0-2 S 22 P 4$ Ga24 0 -like 2S2 2P3 (2P*) 3D (3P*) j=1.0 - 2S2 2P4 Ga24 O-like 2S2 2P3 (2D*) 3D (3P*) j=2.0 - 2S2 2P4 Ga24 0 -like 2S2 $2 P^{2}\left(2 P^{*}\right) 3 S\left(3 P^{*}\right) j=2.0-2 S 22 P 4$ Ga24 O-like 2S2 2P3 (2D*) $3 S\left(3 D^{*}\right) j=3.0-2 S 22 P 4$ Ga24 0 -like 2S2 2P3 ( 2D*) 3S $\left(3 D^{*}\right) j=3.0-2 S 22 P 4$ Ga24 0 -like 2S2 2P3 ( $\left.2 D^{*}\right) 3 S\left(3 D^{*}\right) j=2.0-2 S 22 P 4$ Ga24 o-like 2S2 $2 P^{2}\left(2 P^{*}\right) 3 S\left(3 P^{*}\right) j=.0-2 S 22 P 4$ Ga24 0 -like 2S2 $2 P 3\left(2 D^{*}\right) 3 S\left(3 D^{*}\right) j=1.0-2 S 22 P 4$ Ga24. o-like $2 S 22 \mathrm{P} 3\left(2 D^{*}\right) 3 S\left(1 D^{*}\right) j^{\prime}=2.0-2 S 22 \mathrm{P} 4$ $\left(2 P^{*}\right) j=5 \quad 8.672$ KAP 100

$\left(2 P^{*}\right) j=1.5$

$\left(2 P^{*}\right) j=1.5$

$\left(2 P^{*}\right) j=1.5$

8.712 PET 002

8.712 EDT 020

$\left(2 P^{*}\right) j=1.5$

8.712 KAP 100

$\left.2 P^{\star}\right) j=1.5$

$\left(2 P^{*}\right) j=1.5$

8.730 PET 002

8.730 EDT 020

8.730 KAP 100

8.761 EDT 020

8.761 KAP 100

8.786 EDT 020

$\left(2 P^{\star}\right) j=1.5$

$\left(2 P^{*}\right) j=1.5$

$\left(2 P^{*}\right) j=1.5$

$\left(2 P^{*}\right) j=.5$

8.786 KAP 100

8.807 EDT 020

$\left(2 P^{*}\right) j=1.5$

$(2 P *) j=.5$

$\left(2 P^{\star}\right) j=.5$

$\left(2 P^{*}\right) j=1.5$

$\left(2 P^{*}\right) j=1.5$

$\left(2 P^{*}\right) j=.5$

$\left(2 p^{*}\right) j=.5$

$\left(2 P^{\star}\right) j=1.5$

$\left(2 P^{*}\right) j=1.5$

$\left(2 P^{*}\right) j=$

$\left(2 P^{\star}\right) j=1.5$

$\left(2 P^{*}\right) j=.5$

$\left(2 P^{*}\right) j=1$.

$\left(2 P^{*}\right) j=1.5$

8.807 KAP 100

8.846 KAP 100

9.381 mica 002

9.395 mica 002

9.421 mica 002

9.431 mica 002

9.442 mica 002

9.470 mica 002

9.486 mica 002

9.507 mica 002

9.529 mica 002

9.548 mica 002

9.961 mica 002

10.013 ADP 101

$\left(2 P^{*}\right) j=1.5 \quad 10.109 \quad$ ADP 101

$\left(2 P^{\star}\right) j=1.5 \quad 10.198 \quad$ ADP 101

$\left\langle 2 P^{*}\right\rangle j=.5 \quad 10.239$ ADP 101

(2P*) $j=1.5 \quad 10.298 \quad$ ADP 101

$\left(2 P^{\star}\right) j=.5 \quad 10.319 \quad$ ADP 101

$\left(2 P^{*}\right) j=1.5 \quad 10.333 \quad$ ADP 101

$\left(2 P^{*}\right) j=.5 \quad 10.347 \quad$ ADP 101

(3P $j j=1.0 \quad 8.807$ EDT 020

(3P) $j=1.0 \quad 8.807 \quad K A P ~ 100$

(3P) $j=2.0 \quad 8.827 \quad \mathrm{KAP} 100$

(3P) $j=1.0 \quad 8.846 \quad \operatorname{KAP} 100$

(3P) $j=2.0 \quad 8.864 \quad \operatorname{KAP} 100$

(3P) $j=1.0 \quad 9.442$ mica 002

(3P) $j=2.0 \quad 9.508$ mica 002

(3P) $j=2.0 \quad 9.508$ mica 002

(3P ) $j=2.0 \quad 9.575$ mica 002

(3P ) $j=1.0 \quad 9.609$ mica 002

(3P) $j=.0 \quad 9.668$ mica 002

(3P) $j=1.0$

9.685 mica 002
$26.634 \quad 77.633$

$8.742 \quad 85.252$

$8.808 \quad 81.533$

$26.634 \quad 78.903$

$8.742 \quad 86.998$

$8.808 \quad 82.369$

$26.634 \quad 79.523$

$8.808 \quad 84.078$

$26.634 \quad 80.688$

$8.808 \quad 85.950$

$26.634 \quad 81.744$

$8.808 \quad 89.137$

$26.634 \quad 82.749$

$26.634 \quad 85.134$

19.94270 .191

$19.942 \quad 70.430$

$19.942 \quad 70.881$

$19.942 \quad 71.057$

$19.942 \quad 71.253$

$19.942 \quad 71.760$

$19.942 \quad 72.056$

$19.942 \quad 72.452$

$19.942 \quad 72.876$

$19.942 \quad 73.251$

$19.942 \quad 87.434$

$10.640 \quad 70.232$

$10.640 \quad 71.379$

$10.640 \quad 71.822$

$10.640 \quad 73.427$

$10.640 \quad 74.220$

$10.640 \quad 75.434$

$10.640 \quad 75.890$

$10.640 \quad 76.203$

$10.640 \quad 76.523$

$8.808 \quad 89.137$

$26.634 \quad 82.749$

$26.634 \quad 83.856$

$26.634 \quad 85.134$

$26.634 \quad 86.782$

$19.942 \quad 71.253$

$19.942 \quad 72.471$

$19.942 \quad 72.471$

$19.942 \quad 73.798$

$19.942 \quad 74.514$

$19.942 \quad 75.839$

$19.942 \quad 76.244$ 
Ga24 0 -1ike 2S2 2P3 ( 4S*) 3S (3S*) j=1.0 - 2S2 2P4

Ga24 O-1ike 2S2 2P3 ( 2D*) 3S $\left(3 D^{*}\right) j=1.0-2 S 22 P 4$

Ga24 0 -like 2S2 $2 P^{2}\left(2 D^{*}\right) 3 S\left(3 D^{*}\right) j=2.0-2 S 22 P 4$

Ga24 0 -like 2S2 2P3 ( $\left.4 S^{*}\right) 3 S\left(3 S^{*}\right) j=1.0-2 S 2$ 2P4

Ga24 0 -1ike 2S2 $2 P_{3}\left(4 S^{*}\right) 3 S\left(3 S^{*}\right) j=1.0-2 S 22 P 4$

Ga28. Be-like $1 S 2$ 2P 3D (3D*) $j=2.0-1 S 22 P 2$

Ga28 Be-like 1S2 2P 3D

Ga28 Be-1ike 1S2 2P 3D

Ga28 Be-like 1S2 2P 3D

Ga28 Be-like 1S2 2P 3D

Ga28 Be-like 1S2 2P 3D

Ga28 Be-like 1S2 2P 3D

Ga28 Be-like 1S2 2P 3D

Ga30 He-like 1S 3P

Ga30 He-Iike 1S 3P

Ga30 He-like is $3 P$

Ga30 He-1ike is $3 P$

Ga30 He-like 1s 3P

Ga30 He-like 1S $3 P$

Ga30 He-1ike is $2 \mathrm{P}$

Ga30 He-like 1S 2P

Ga30 He-like IS 2P

Ga30 He-like is $2 P$

Ga30 He-like 1S $2 P$

Ga30 He-like IS 2P

Ga30 He-1ike 1S 2P

Ga30 He-like 1s 2P

Ga30 He-like 1S 2P

Ga30 He-like 1S 2P

Ga30 He-like 1s 2P

Ga30 He-like 1s 2P

Ga30 He-like 1S 2P

Ga30 He-Iike IS 2P

Ga30 He-like is $2 P$

Ga30 He-like 1S 2P

Ga30 He-like 1S 2P

Ga30 He-like 1S 2P

Ga30 He-like is $2 S$

Ga30 He-1ike 1s 2S

Ga30 He-like 1s 2s

Ga30 He-like 15 2S

Ga30 He-like 1s 2S

Ga3.0 He-1ike 1S 2S

Ga3O He-like 1s $2 S$

Ga30 He-like 1s 2S

Ga30 :He-like 1s 2S $\left(3 P^{\star}\right) j=2.0-1 S 2 \quad 2 P 2$

$\left(3 P^{*}\right) j=2.0-1 S 22 P 2$

$\left(3 F^{*}\right) j=2.0-1 S 22 P 2$

$\left(3 P^{*}\right) j=2.0-1 S 22 \mathrm{P} 2$

$(3 P *) j=2.0-1 S 22 P 2$

$\left(3 P^{\star}\right) j=2.0-1 S 22 P 2$

(3P*) $j=1.0-1 S 2$

$\left(3 P^{*}\right) j=1.0-1 S 2$

$\left(3 P^{\star}\right) j=1.0-1 \mathrm{~s} 2$

$\left(3 P^{\star}\right) j=1.0-152$

$\left(3 p^{*}\right) j=1.0-152$

$\left(3 P^{*}\right) j=1.0-1 S 2$

$\left(1 P^{*}\right) j=1.0-1 \mathrm{~S} 2$

$\left(1 P^{*}\right) j=1.0-1 \mathrm{~s} 2$

$\left(1 p^{*}\right) j=1.0-1 \mathrm{~S} 2$

$\left(1 P^{*}\right) j=1.0-1 \mathrm{~S} 2$

$\left(1 P^{*}\right) j=1.0-152$

$\left(1 P^{\star}\right) j=1.0-1 S 2$

$\left(1 P^{*}\right) j=1.0-152$

$\left(3 P^{*}\right) j=1.0-1 S 2$

(3P*) $j=1.0-152$

$\left(3 P^{*}\right) j=1.0-1 S 2$

$\left(3 P^{\star}\right) j=1.0-152$

$\left(3 P^{*}\right) j=1.0-1 S 2$

$\left(3 p^{*}\right) j=1.0-1 s 2$

$\left(3 P^{*}\right) j=1.0-1 S 2$

$\left(3 P^{*}\right) j=1.0-1 S 2$

$\left(3 p^{*}\right) j=1.0-152$

$\left(3 P^{*}\right) j=1.0-1 S 2$

(3s) $j=1.0-1 s 2$

(3s) $j=1.0-1 s 2$

(3s) $j=1.0-1 s 2$

(3s) $j=1.0-1 s 2$

(3S ) $j=1.0-1 S 2$

(3S) $j=1.0-1 S 2$

(3s) $j=1.0-1 s 2$

(3s) $j=1.0-1 s 2$

(3S) $j=1.0-152$ $\left(3 F^{*}\right) j=2.0-1 S 22 P 2$

$\left(1 P^{*}\right) j=1.0-152$
(3P) $j=2.0$

(3P) $j=1.0$

(3P) $j=1.0$

(3P) $j=.0$

(3P) $j=1.0$

(3P) $j=2.0$

(3P) $j=2.0$

(3P) $j=2.0$

(3P) $j=2.0$

(3P) $j=2.0$

(1D) $j=2.0$

(1D) $j=2.0$

(ID) $j=2.0$

(1s) $j=.0$

(1s) $j=.0$

(1s) $j=.0$

(1s) $j=.0$

(1s) $j=.0$

(1s) $j=.0$

(1s) $j=.0$

(1s) $j=.0$

(1s) $j=.0$

(IS) $j=.0$

(1s) $j=.0$

(1s) $j=.0$

(IS ) $j=.0$

(1s) $j=.0$

(1s) $j=.0$

(1s) $j=.0$

(1s) $j=.0$

(1s) $j=.0$

(1s) $j=.0$

(1s) $j=.0$

(1S) $j=.0$

(IS) $j=.0$

(Is) $j=.0$

(1s) $j=.0$

(1s) $j=.0$

(1s) $j=.0$

(1s) $j=.0$

(Is) $j=.0$

(1s) $j=.0$

(1s) $j=.0$

(1s) $j=.0$

(1s) $j=.0$

(1s) $j=.0$
9.739 mica 002

9.761 mica 002

9.772 mica 002

9.859 mica 002

946 mica 002

7.790 beryl 100

7.950 topaz 002

7.950 beryl 100

8.000 topaz 002

8.000 quartz 100

8.110 topaz 002

8.110 ouartz 100

8.110 TAP 100

1.096 Si 422

1.096 Ge 422

1.096 tungsten 110

1.096 quartz 112

1.096 topaz 200

$1.096 \mathrm{NaCl} 200$

1.288 topaz 303

1.288 Ge 220

1.288 LiF 200

1.288 A. 200

1.288 Ge 111

$1.288 \mathrm{KBr} 200$

1.288 quartz 101

1.288 graphite 002

1.295 topaz 303

1.295 corundum 030

1.295 quartz 203

1.295 Ge 220

1.295 LiF 200

1.295 A1 200

1.295 Ge 111

$1.295 \mathrm{KBr} 200$

1.295 guartz 101

1.295 graphite 002

1.300 topaz 303

1.300 corundum 030

1.300 quartz 203

1.300 Ge 220

1.300 LiF 200

1.300 Al 200

1.300 Ge 111

$1.300 \mathrm{KBr} 200$

1.300 guartz 101
$19.942 \quad 77.616$

$19.942 \quad 78.220$

$19.942 \quad 78.534$

$19.942 \quad 81.404$

$19.942 \quad 85.942$

$15.954 \quad 77.569$

$8.374 \quad 71.689$

$15.954 \quad 85.285$

$8.374 \quad 72.812$

$8.512 \quad 70.026$

$8.374 \quad 75.575$

$8.512 \quad 72.321$

$25.763 \quad 70.800$

$2.217 \quad 81.387$

$2.310 \quad 71.608$

$4.476 \quad 78.363$

$4.564 \quad 73.855$

$4.638 \quad 70.950$

$5.641 \quad 76.278$

$2.712 \quad 71.778$

$4.000 \quad 75.016$

$4.027 \quad 73.643$

$4.048 \quad 72.659$

6.53280 .372

6.58477 .995

$6.687 \quad 74.379$

$6.696 \quad 74.106$

$2.712 \quad 72.749$

$2.748 \quad 70.476$

$2.749 \quad 70.418$

$4.000 \quad 76.228$

$4.027 \quad 74.739$

$4.048 \quad 73.685$

6.53282 .425

$6.584 \quad 79.560$

$6.687 \quad 75.534$

$6.696 \quad 75.239$

$2.712 \quad 73.476$

$2.748 \quad 71.110$

$2.749 \quad 71.049$

$4.000 \quad 77.161$

$4.027 \quad 75.572$

$4.048 \quad 74.459$

$6.532 \quad 84.326$

$6.584 \quad 80.838$

$6.687 \quad 76.418$ 


\begin{tabular}{|c|c|c|c|c|c|c|c|c|}
\hline Ga30 & He-like & is & \multicolumn{3}{|l|}{$2 S$} & $(3 S) j=1.0$ & \multicolumn{2}{|l|}{$-1 s 2$} \\
\hline Ga31 & H -like & $3 P$ & & & & $\left(2 p^{\star}\right) j=1.5$ & $-1 S$ & \\
\hline Ga31 & H -Iike & $3 P$ & & & & $\left(2 P^{*}\right) j=1.5$ & $-1 s$ & \\
\hline Ga31 & H -like & $3 P$ & & & & $\left(2 P^{\star}\right) j=1.5$ & $-1 S$ & \\
\hline Ga31 & $\mathrm{H}-\mathrm{like}$ & $2 \mathrm{P}$ & & & & $\left(2 P^{\star}\right) j=1.5$ & $-1 s$ & \\
\hline Ga31. & H -like. & $2 P$ & & & & $\left(2 P^{*}\right) j=1.5$ & $-1 S$ & \\
\hline Ga31 & H -like & $2 P$ & & & & $\left(2 P^{\star}\right) j=1.5$ & $-1 S$ & \\
\hline Ga31 & $\mathrm{H}$-like & $2 P$ & & & & $\left(2 P^{\star}\right) j=1.5$ & $-1 S$ & \\
\hline Ga31 & H -like & $2 P$ & & & & $\left(2 P^{\star}\right) j=1.5$ & $-1 s$ & \\
\hline Ga31 & H -like & $2 P$ & & & & $\left(2 P^{\star}\right) j=1.5$ & $-1 S$ & \\
\hline Ga31 & H -like & $2 P$ & & & & $\left(2 P^{\star}\right) j=1.5$ & $-1 S$ & \\
\hline Ga31 & H -like & $2 P$ & & & & $\left(2 p^{*}\right) j=1.5$ & $-1 S$ & \\
\hline Ga31 & H -like & $2 P$ & . & & & $\left(2 P^{\star}\right) j=.5$ & $-1 S$ & \\
\hline Ga31 & $\mathrm{H}$-like & $2 P$ & & & & $\left(2 P^{\star}\right) j=.5$ & $-1 s$ & \\
\hline Ga31 & H -like & $2 P$ & & & & $\left(2 P^{*}\right) j=.5$ & $-1 s$ & \\
\hline Ga31 & H -like & $2 P$ & & & & $\left(2 P^{*}\right) j=.5$ & -15 & \\
\hline Ga31 & H -like & $2 P$ & & & & $\left(2 P^{\star}\right) j=.5$ & -15 & \\
\hline Ga31 & H -like & $2 P$ & & & & $(2 \mathrm{P} * q j=.5$ & $-1 s$ & \\
\hline Ga31 & H-like & $2 P$ & & & & $\left(2 \mathrm{P}^{\star}\right) j=.5$ & $-1 S$ & \\
\hline $\mathrm{Ge}$ & & $\mathrm{K}-\mathrm{a}]$ & lpha( & & & & & \\
\hline $\mathrm{Ge}$ & & $\mathrm{K}-\mathrm{a}]$ & Iphal & & & & & \\
\hline $\mathrm{Ge}$ & & $\mathrm{K}-\mathrm{a}$ & Iphal & & & & & \\
\hline $\mathrm{Ge}$ & & $\mathrm{K}-\mathrm{a}]$ & Iphar & & & & & \\
\hline $\mathrm{Ge}$ & & $\mathrm{K}-\mathrm{a}]$ & Iphal & (1) & & & & \\
\hline $\mathrm{Ge}$ & & $\mathrm{K}-\mathrm{a}]$ & Ipha ( & (1) & & & & \\
\hline Ge & & $\mathrm{K}-\mathrm{a}]$ & Iphal & (1) & & & & \\
\hline Ge & & $\mathrm{K}-\mathrm{a}]$ & Ipha( & & & & & \\
\hline Ge21 & Mg-like & $3 s$ & $5 F$ & & & $\left(3 F^{\star}\right) j=4.0$ & -35 & $3 D$ \\
\hline Ge22 & Na-like & $2 \mathrm{P} 6$ & $6 \mathrm{D}$ & & & $(2 D) j=2.5$ & $-2 P \sigma$ & $3 P$ \\
\hline Ge22 & Na-like & $2 \mathrm{P} 6$ & $7 F$ & & & $\left(2 F^{*}\right) j=3.5$ & $-2 P 6$ & $3 D$ \\
\hline Ge22 & Na-like & $2 \mathrm{P} 6$ & $5 F$ & & & $\left(2 F^{*}\right) j=2.5$ & $-2 P 6$ & $3 D$ \\
\hline Ge22 & Na-like & $2 \mathrm{P} 6$ & $5 F$ & & & $\left(2 F^{\star}\right) j=2.5$ & $-2 P 6$ & $3 D$ \\
\hline $\mathrm{Ge} 22$ & $\mathrm{Na-like}$ & $2 \mathrm{P} 6$ & $5 F$ & & & $\left(2 F^{\star}\right) j=3.5$ & $-2 P 6$ & 3D \\
\hline Ge22 & Na-like & $2 \mathrm{P} 6$ & $5 F$ & & & $\left(2 F^{*}\right) j=3.5$ & $-2 \mathrm{P} 6$ & $3 D$ \\
\hline Ge23 & Ne-like & $2 s 2$ & $2 \mathrm{P} 5$ & $(2 \mathrm{P} * 1)$ & 8D & $(12 *) j=1.0$ & $-2 s 2$ & $2 \mathrm{P} 6$ \\
\hline $\mathrm{Ge} 23$ & Ne-like & $2 S 2$ & $2 \mathrm{P} 5$ & $(2 \mathrm{P} * 1)$ & $8 D$ & $\left(12^{*}\right) j=1.0$ & $-2 s 2$ & $2 \mathrm{P} 6$ \\
\hline Ge23 & Ne-like & 252 & 285 & $(2 \mathrm{P} * 1)$ & $8 D$ & $\left(12^{\star}\right) j=1.0$ & $-2 s 2$ & $2 \mathrm{P} 6$ \\
\hline Ge23 & Ne-like & 252 & $2 \mathrm{P} 5$ & $(2 \mathrm{P} * 2)$ & $9 D$ & $\left(23^{*}\right) j=1.0$ & $-2 s 2$ & $2 \mathrm{P} 6$ \\
\hline Ge23, & Ne-like & 252 & $2 \mathrm{P5}$ & $(2 \mathrm{P} \star 2)$ & 90 & $\left(23^{*}\right) j=1.0$ & $-2 s 2$ & $2 \mathrm{P} 6$ \\
\hline $\mathrm{Ge} 23$ & Ne-like & $2 s 2$ & $2 \mathrm{P} 5$ & $(2 \mathrm{P} * 2)$ & $9 D$ & $\left(23^{*}\right) j=1.0$ & $-2 s 2$ & $2 \mathrm{P} 6$ \\
\hline Ge23 & Ne-like & 252 & $2 \mathrm{P} 5$ & $(2 \mathrm{P} \star 2)$ & $9 D$ & $\left(23^{*}\right) j=1.0$ & $-2 s 2$ & $2 \mathrm{P} 6$ \\
\hline Ge23 & Ne-like & $2 \mathrm{~S} 2$ & $2 \mathrm{P} 5$ & $(2 \mathrm{P} * 2)$ & $8 D$ & $\left(23^{*}\right) j=1.0$ & $-2 s 2$ & $2 \mathrm{P} 6$ \\
\hline Ge23 & Ne-like & $2 \$ 2$ & $2 P 5$ & $(2 q * 2)$ & $8 D$ & $\left(23^{*}\right) j=1.0$ & $-2 s 2$ & $2 \mathrm{P} 6$ \\
\hline $\mathrm{Ge} 23$ & Ne-like & $2 s 2$ & $2 \mathrm{P} 5$ & $(2 P * 2)$ & $8 D$ & $\left(23^{*}\right) j=1.0$ & $-2 s 2$ & $2 \mathrm{P} 6$ \\
\hline Ge23 & Ne-like & 252 & $2 \mathrm{P} 5$ & $(2 P \star 2)$ & $8 D$ & $\left(23^{*}\right) j=1.0$ & $-2 \$ 2$ & $2 \mathrm{P} 6$ \\
\hline Ge23 & Ne-like & $2 S 2$ & 285 & $(2 P \star I)$ & $7 D$ & $\left(12^{*}\right) j=1.0$ & $-2 s 2$ & $2 \mathrm{P} 6$ \\
\hline
\end{tabular}

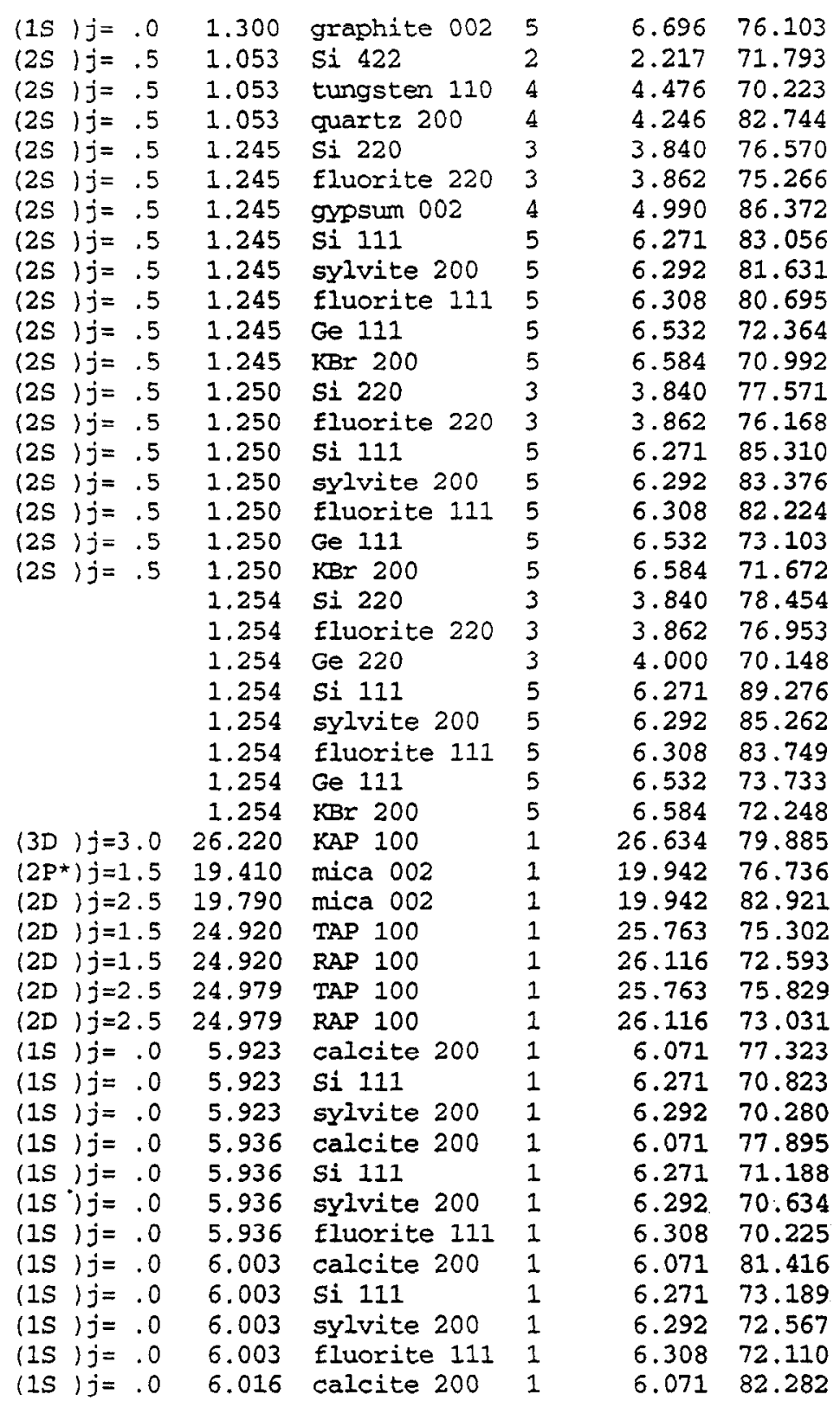


Ge23 Ne-like 2S2 2P5 ( 2P*1) 7D (12*) $j=1.0-2 \mathrm{~S} 22 \mathrm{P} 6$ Ge23 Ne-like 2S2 2P5 $(2 P \star 1) 7 D(12 *) j=1.0-2 S 22 P 6$ Ge23 Ne-like 2S2 2P5 ( 2P*1) 7D (12*) j=1.0 - 2S2 2P6 Ge23 Ne-like 2S2 2P5 ( 2P*2) 7D $(23 *) j=1.0-2 S 22 P 6$ Ge23 Ne-1ike 2S2 2P5 ( 2P*2) 7D $\left(23^{*}\right) j=1.0-2 S 2$ 2P6 Ge23 Ne-like 2S2 2P5 ( $\left.2 P^{*} 2\right) 7 D(23 *) j=1.0-2 S 22 P 6$ Ge23 Ne-like 2S2 2P5 ( 2P*2) 7D $(23 *) j=1.0-2 S 2$ 2P6 Ge23 Ne-like 2S2 2P5 ( $\left.2 P^{\star} 1\right)$ 6D $(12 \star) j=1.0$ - 2S2 2P6 Ge23 Ne-1ike 2S2 2P5 ( 2P*1) 6D (12*)j=1.0 - 2S2 2P6 Ge23 Ne-Iike 2S2 2P5 ( $\left.2 P^{*} 1\right) 6 D(12 *) j=1.0-2 S 22 P 6$ Ge23 Ne-1ike 2S2 2P5 ( 2P*1) 6D (12*) j=1.0 - 2S2 2P6 Ge23 Ne-1ike 2S2 2P5 (2P*1) 6D $\left(12^{\star}\right) j=1.0-2 S 22 P 6$ Ge23 Ne-1ike 2S2 2P5 ( 2P*1) 6D $(12 *) j=1.0-2 S 22 P 6$ Ge23 Ne-like 2S2 2P5 ( 2P*2) 6D $(23 *) j=1.0-2 S 22 P 6$ Ge23 Ne-like 2S2 2P5 ( $\left.2 P^{*} 2\right) 6 D(23 *) j=1.0-2 S 22 P 6$ Ge23 Ne-like 2S2 2P5 ( $\left.2 P^{\star} 2\right)$ 6D $\left(23^{*}\right) j=1.0-2 \mathrm{~S} 2$ 2P6 Ge23 Ne-1ike 2S2 2P5 ( $2 P \star 2) 6 D(23 *) j=1.0-2 S 22 P 6$ Ge23 Ne-like 2S2 2P5 ( $2 P * 2) 6 D(23 *) j=1.0-2 S 22 P 6$ Ge23 Ne-1ike 2S2 2P5 $(2 P \star 2) 6 D(23 *) j=1.0-2 S 22 P 6$ Ge23 Ne-like 2S2 2P5 ( $\left.2 P^{\star} \star 2\right) 6 D\left(23^{\star}\right) j=1.0-2 S 22 P 6$ Ge23 Ne-1ike 2S2 2P5 ( $2 P * 2) 6 D(23 *) j=1.0-2 S 22 P 6$ $\mathrm{Ge} 23$ Ne-like 2S2 $2 \mathrm{P} 5$ ( $2 P * 1)$ 5D $(12 *) j=1.0-2 S 22 P 6$ Ge23 Ne-like 2S2 2P5 ( $2 P * 1)$ 5D $\left(12^{*}\right) j=1.0-2 S 22 P 6$ Ge23 Ne-1ike 2S2 2P5 ( $2 P * 1)$ 5D $\left(12^{\star}\right) j=1.0-2 S 22 P 6$ Ge23 Ne-like 2S2 2P5 (2P*1) 5D $(12 *) j=1.0-2 S 22 P 6$ Ge23 Ne-like 2S2 2P5 ( $\left.2 P^{*} 1\right)$ 5D $\left(12^{*}\right) j=1.0-2 S 2$ 2P6 Ge23 Ne-like 2S2 2P5 ( $2 P * 1)$ 5D $(12 *) j=1.0-2 S 22 P 6$ Ge23 Ne-like 2S2 2P5 ( $2 P \star 1)$ 5D (12*) $j=1.0$ - 2S2 2P6 Ge23 Ne-1ike $2 S 22 P 5(2 P * 2) 5 D(23 *) j=1.0-2 S 22 P 6$ Ge23 Ne-1ike 2S2 2P5 ( $2 P * 2)$ 5D $\left(23^{*}\right) j=1.0-2 S 22 P 6$ Ge23 Ne-1ike 2S2 2P5 ( $2 P * 2)$ 5D $(23 *) j=1.0-2 S 22 P 6$ Ge23 Ne-like 2S2 2P5 ( $2 P * 2) 5 D(23 *) j=1.0-2 S 22 P 6$ Ge23 Ne-1ike 2S2 2P5 ( $2 P \star 2) 5 D(23 *) j=1.0-2 S 22 P 6$ Ge23 Ne-like 2S2 2P5 ( $2 P * 2)$ 5D $(22 *) j=1.0-2 S 22 P 6$ Ge23 Ne-like 2S2 2P5 ( $\left.2 P^{*} 2\right)$ 5D $\left(22^{*}\right) j=1.0-2 \mathrm{~S} 22 \mathrm{P} 6$ Ge23 Ne-1ike 2S2 2P5 (2P*2) 5D $\left(22^{\star}\right) j=1.0-2 S 22 P 6$ Ge23 Ne-1ike 2S2 2P5 ( $2 P \star 2)$ 5D $\left(22^{\star}\right) j=1.0-2 S 22 P 6$ Ge23 Ne-like 2S2 $2 P 5$ ( $\left.2 P^{*} 1\right) 4 D\left(12^{\star}\right) j=1.0-2 S 22 P 6$ Ge23 Ne-like 2S2 2P5 ( $\left.2 P^{\star} 2\right)$ 4D $\left(23^{*}\right) j=1.0-2 S 2$ 2P6 Ge23 Ne-like 2S2 2P5 (2P*2) 4D $(23 *) j=1.0-2 S 22 P 6$ Ge23 Ne-like 2S2 $2 P 5$ (2P*1) $4 S(11 *) j=1.0-2522 P 6$ Ge23 Ne-like 2S2 2P5 ( $\left.2 P^{\star} 1\right)$ 4S $\left(11^{\star}\right) j=1.0-2 S 22 P 6$ Ge23. Ne-1ike 2S2 2P5 ( $\left.2 P^{*} 2\right)$ 4S $\left(22^{*}\right) j=1.0-2 S 22 P 6$ Ge23 Ne-like 2S2 2P5 ( 2P*2) 4S $\left(22^{\star}\right) j=1.0$ - 2S2 2P6 Ge23 Ne-like 2S 2P6 3P (1P*) $j=1.0-2 S 22 P 6$ Ge23 Ne-like 2S $2 \mathrm{PG} \quad 3 \mathrm{P}\left(1 \mathrm{P}^{*}\right) \mathrm{j}=1.0-2 \mathrm{~S} 2 \mathrm{PP6}$ (1s) $j=.0$

(1S) $j=.0$

(1s) $j=.0$

(1s) $j=.0$

(1S) $j=.0$

(1S $) j=.0$

(Is ) $j=.0$

(1s) $\mathrm{j}=.0$

(1s) $j=.0$

(1s) $j=.0$

(1S) $j=.0$

(1S) $j=.0$

(1s) $j=.0$

(1s) $j=.0$

(1S) $j=.0$

(1s) $j=.0$

(1s) $j=.0$

(1s) $j=.0$

(1s) $j=.0$

(1s) $j=.0$

(1s) $j=.0$

(1s) $j=.0$

(1s) $j=.0$

(1s) $j=.0$

(Is) $j=.0$

(1s) $j=.0$

(1s) $j=.0$

(1s) $j=.0$

(1s) $j=.0$

(1s) $\mathrm{j}=$.

(1s) $j=.0$

(1s) $j=.0$

(1s) $j=.0$

(1s) $j=.0$

(1S) $j=.0$

(1S) $j=.0$

(1S) $j=.0$

(1s) $j=.0$

(1s ) $j=.0$

(1s) $j=.0$

(1S) $j=.0$

(1s) $j=.0$

(1s) $j=.0$

(1s) $j=.0$

(1s) $j=.0$

(1s) $j=.0$
6.016 Si 111

016 sylvite 200

6.016 fluorite 111

6.112 Si 111

6.112 sylvite 200

6.112 fluorite 111

6.112 TAP 100

6.177 Si 111

6.177 sylvite 200

6.177 fluorite 111

6.177 Ge 111

6.177 TAP 100

6.177 RAP 100

6.275 sylvite 200

6.275 fluorite 111

6.275 Ge 111

$6.275 \mathrm{KBr} 200$

6.275 mica 002

6.275 TAP 100

6.275 RAP 100

6.275 KAP 100

6.464 Ge 111

6.464 KBr 200

6.464 guartz 101

6.464 graphite 002

6.464 mica 002

6.464 RAP 100

6.464 KAP 100

6.574 quartz 101

6.574 graphite 002

6.574 mica 002

6.574 KAP 100

6.584 quartz 101

6.584 graphite 002

6.584 mica 002

6.584 KAP 100

7.081 InSb 111

7.205 InSb 111

7.205 gypsum 020

7.359

7.359

InSb 111

7.411 gypsum 020

8.369 topaz 002

8.369 quartz 100
$6.271 \quad 73.605$

$6.292 \quad 72.967$

$6.308 \quad 72.499$

$6.271 \quad 77.070$

$6.292 \quad 76.262$

$6.308 \quad 75.680$

$25.763 \quad 71.615$

$\begin{array}{ll}6.271 & 80.067\end{array}$

$6.292 \quad 79.029$

$6.308 \quad 78.303$

6.53271 .024

$25.763 \quad 73.546$

$26.116 \quad 71.100$

$6.292 \quad 85.787$

$6.308 \quad 84.137$

$6.532 \quad 73.874$

$6.584 \quad 72.377$

$19.942 \quad 70.732$

$25.763 \quad 76.973$

$26.116 \quad 73.966$

$26.634 \quad 70.459$

6.53281 .725

$6.584 \quad 79.044$

$6.687 \quad 75.162$

$6.696 \quad 74.874$

$19.942 \quad 76.512$

$26.116 \quad 81.908$

$26.634 \quad 76.117$

$6.584 \quad 86.842$

$6.687 \quad 79.452$

6.69679 .046

$19.942 \quad 81.481$

$26.634 \quad 80.862$

$6.687 \quad 79.931$

$6.696 \quad 79.506$

$19.942 \quad 82.085$

$26.634 \quad 81.421$

$7.481 \quad 71.179$

$7.481 \quad 74.388$

$15.185 \quad 71.616$

$\begin{array}{lll}7.481 & 79.638\end{array}$

15.185
7.45 .754

$7.481 \quad 82.156$

$\begin{array}{lll}15.185 & 77.447\end{array}$

$8.374 \quad 88.020$

8.51279 .483 


\begin{tabular}{|c|c|c|c|c|c|c|c|c|c|}
\hline$e 23$ & Je-like & 25 & $2 P 6$ & & & 3 & $\left(1 p^{\star}\right) j=1.0$ & $-2 \$ 2$ & 6 \\
\hline e23 & Ne-like & $S$ & $2 \mathrm{P} 6$ & & & & $\left(1 P^{*}\right) j=1.0$ & $-2 s 2$ & 25 \\
\hline 23 & Je-like & S & $2 P 6$ & & & & *) $j=1.0$ & & \\
\hline 23 & le-like & & $2 p 6$ & & & & 1.0 & & \\
\hline 23 & e-like & & 6 & & & & .0 & & \\
\hline 23 & $\mathrm{Ne}-$ & & & & & & .0 & & \\
\hline 23 & ike & & & & & p & 1.0 & & \\
\hline$e 23$ & e- & & 96 & & & 5 & 1.0 & & \\
\hline$e 23$ & $e-$ & & $2 P 6$ & & & $P$ & .0 & s2 & \\
\hline e23 & e- & & & & & $\mathrm{P}$ & & & \\
\hline$e 23$ & e- & & $2 P 6$ & & & $P$ & 1.0 & S2 & \\
\hline$e 23$ & e- & $\$ 2$ & $2 \mathrm{P5}$ & $2 F$ & & $S$ & 1.0 & & \\
\hline$e 23$ & $\mathrm{Ne}-$ & & $2 P 5$ & & & S & *) $j=1.0$ & & \\
\hline$e 23$ & $\mathrm{Ne}-3$ & & & & & & $j=.0$ & 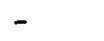 & \\
\hline 23 & $\mathrm{Ne}-1$ & & & & & & & 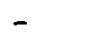 & \\
\hline e24 & F-like & S2 & 254 & 15 & 1 & $4 D$ & $(2 D) j=2.5$ & & \\
\hline 24 & $F-1$ & $\$ 2$ & $2 P 4$ & is & 1 & D: & $(2 D) j=$ & & \\
\hline 24 & $E-1 i]$ & $S 2$ & $2 P 4$ & ( $1 \mathrm{~s}$ & ) & $4 D$ & $(2 D) j$ & & \\
\hline & $F-i i]$ & s2 & $2 P 4$ & ( $1 \mathrm{~s}$ & ) & 10 & (2D) & & \\
\hline Ge24 & $F-1 i$ & s2 & $2 \mathrm{P} 4$ & $(3 P$ & 1 & $4 \mathrm{~S}$ & $\{2 P\} j$ & & \\
\hline Ge24 & $F-1 i$ & 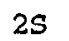 & $2 P 5$ & 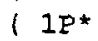 & ) & $P$ & $(2 D)$ & & \\
\hline \pm & $F-1 i$ & $S$ & $2 P 5$ & $(1$ & 1 & $3 P$ & $(2 P)=$ & & \\
\hline 24 & $F-1$ & $s$ & $2 P 5$ & 11 & ) & $\mathbf{P}$ & $(2 P) j=1.5$ & 52 & \\
\hline 24 & $F-1$ & & 85 & 11 & $j$ & $3 P$ & $(2 P) j=.5$ & - & \\
\hline 24 & $F-1$ & $S$ & 5 & $i 1$ & 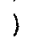 & $\mathrm{p}$ & $(2 P)]$ & - & \\
\hline 24 & $F-1$ & S & 5 & 11 & ) & $P$ & $(2 D)$ & - & \\
\hline 24 & $F-$ & & 5 & $i 1$ & $j$ & $P$ & $(2 D)$ & 52 & \\
\hline & $F-1$ & & $P 5$ & $<3$ & $j$ & $P$ & $(4 P) j$ & - & \\
\hline$e 24$ & $F-1$ & & 5 & 13 & ) & $3 P$ & $(4 \mathrm{P})$ & - & \\
\hline Ge24 & $F$ & & 2P5 & & j & $P$ & $(4 P)$ & & \\
\hline e24 & $F$ & & & & $j$ & $P$ & $(2 E$ & - & \\
\hline Ge24 & $F$ & c & 5 & & 1 & $3 \mathrm{P}$ & $j=.5$ & & \\
\hline Ge24 & $F-1$ & $\sigma$ & $2 \mathrm{P} 5$ & & 1 & $3 P$ & $(2 P) j=.5$ & & \\
\hline Ge24 & $F-1$ & & $2 \mathrm{P} 5$ & & ) & $3 P$ & $(2 P)$ & & \\
\hline Ge24 & $F-1$ & $s$ & $2 \mathrm{P} 5$ & & i & $3 P$ & $(2 P)$ & & \\
\hline Ge24 & F-like & $s$ & $2 \mathrm{P} 5$ & 13 & 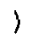 & $3 P$ & $(2 P) j$ & & \\
\hline Ge24 & $F-1 i]$ & & $2 \mathrm{P} 5$ & & I & $3 P$ & $(2 D)$ & & \\
\hline Ge24 & $F-1$ & $s$ & $2 P 5$ & & j & $3 P$ & $(2 D) j$ & - & \\
\hline G & $F-1$ & $2 S$ & $2 \mathrm{P} 5$ & & $i$ & $3 P$ & (2D) $j=1.5$ & - & \\
\hline & $F-1$ & 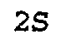 & $2 \mathrm{P} 5$ & 3. & $\therefore$ & $3 P$ & $(2 s) j=.5$ & - & \\
\hline 024 & $F-1$ & $2 s$ & $2 \mathrm{P} 5$ & 13 & 1 & $3 P$ & $(25) j$ & s2 & \\
\hline $\mathrm{Ge} 24$ & $F-1$ & s & & & i) & $3 P$ & $(2 s) j=.5$ & 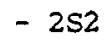 & \\
\hline & $F-1$ & $2 S$ & $2 \mathrm{P}$ & & & $3 p$ & $(2 S)$ & . & \\
\hline & $E-$ & & & & & $3 P$ & 2.5 & t & \\
\hline & $F-1$ & & 21 & & & $3 P$ & (4D) $j=2.5$ & & \\
\hline & & & 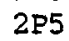 & $3 P$ & & $3 P$ & $(4 D) j=2.5$ & . & \\
\hline
\end{tabular}

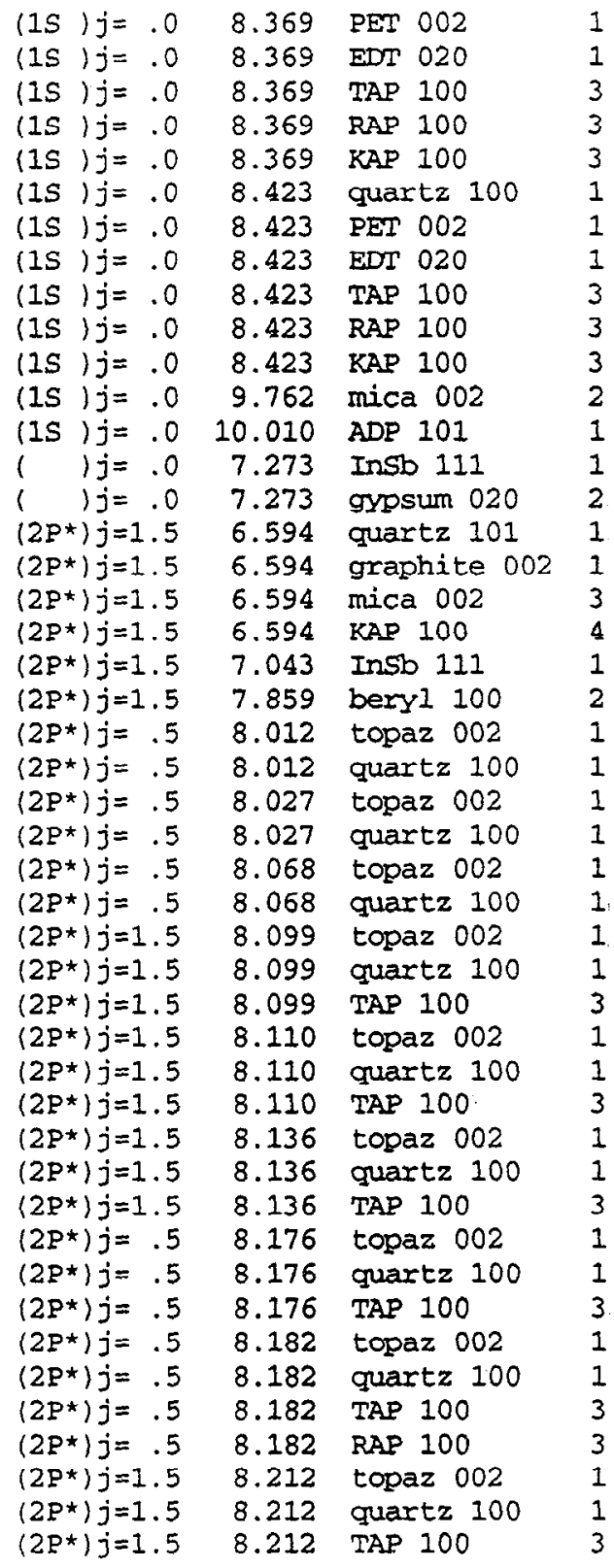

$\begin{array}{rr}8.742 & 73.203 \\ 8.808 & 71.834 \\ 25.763 & 77.043 \\ 26.116 & 74.021 \\ 26.634 & 70.504 \\ 8.512 & 81.707 \\ 8.742 & 74.474 \\ 8.808 & 72.997 \\ 25.763 & 78.762 \\ 26.116 & 75.368 \\ 26.634 & 71.577 \\ 19.942 & 78.248 \\ 10.640 & 70.185 \\ 7.481 & 76.457 \\ 15.185 & 73.319 \\ 6.687 & 80.433 \\ 6.696 & 79.987 \\ 19.942 & 82.737 \\ 26.634 & 82.019 \\ 7.481 & 70.297 \\ 15.954 & 80.133 \\ 8.374 & 73.092 \\ 8.512 & 70.264 \\ 8.374 & 73.448 \\ 8.512 & 70.565 \\ 8.374 & 74.463 \\ 8.512 & 71.413 \\ 8.374 & 75.276 \\ 8.512 & 72.079 \\ 25.763 & 70.578 \\ 8.374 & 75.575 \\ 8.512 & 72.321 \\ 25.763 & 70.800 \\ 8.374 & 76.307 \\ 8.512 & 72.907 \\ 25.763 & 71.335 \\ 8.374 & 77.516 \\ 8.512 & 73.848 \\ 25.763 & 72.188 \\ 8.374 & 77.707 \\ 8.512 & 73.994 \\ 25.763 & 72.319 \\ 26.116 & 70.032 \\ 8.374 & 78.712 \\ 8.512 & 74.743 \\ 25.763 & 72.990\end{array}$


Ge24 F-like 2S 2P5 ( $\left.3 P^{*}\right) 3 P(4 D) j=2.5-2 S 22 P 5$

Ge24 F-like 2S2 2P4 ( 1D) 3D (2P) $j=.5-2 S 22 P 5$

Ge24 F-like 2S2 2P4 ( $1 D$ ) 3D (2P) $j=.5-2 S 22 P 5$

Ge24 F-like 2S2 2P4 ( 1D) 3D (2P) $j=.5-2 S 22 P 5$

Ge24 F-like 2S2 2P4 (1D) 3D (2P) $j=.5-2 S 22 P 5$

Ge24 F-like 2S2 2P4 ( $1 D$ ) 3D (2P) $j=.5-2 S 22 P 5$

Ge24 F-like 2S2 2P4 ( $1 S$ ) 3D (2D) $j=1.5-2 S 22 P 5$

Ge24 F-like 2S2 2P4 ( $1 S$ ) 3D (2D $) j=1.5-2 S 22 P 5$

Ge24 F-like 2S2 2P4 ( $1 S$ ) 3D (2D) $j=1.5-2 S 22 P 5$

Ge24 $F$-like 2S2 2P4 ( $1 S$ ) 3D (2D) $j=1.5-2 S 22 P 5$

Ge24 F-like 2S2 2P4 ( IS) 3D (2D) $j=1.5-2 S 2$ 2P5

Ge24 F-like 2S2 2P4 ( 1D) 3D (2D) $j=2.5$ - 2S2 2P5

Ge24 F-like 2S2 2P4 (1D) 3D (2D) $j=2.5-2 S 22 P 5$

Ge24 F-like 2S2 2P4 ( ID) 3D (2D) $j=2.5-2 S 22 P 5$

Ge24 F-like 2S2 2P4 ( $1 D$ ) 3D (2D) $j=2.5-2 S 22 P 5$

Ge24 F-like 2S2 2P4 ( $1 D$ ) 3D (2D) $j=2.5-2 S 22 P 5$

Ge24 F-like 2S2 2P4 ( $1 D$ ) 3D (2P) $j=1.5-2 S 22 P 5$

Ge24 F-like 2S2 2P4 ( 1D) 3D (2P) $j=1.5-2 S 22 P 5$

Ge24 F-like 2S2 2P4 ( $D$ ) 3D (2P) $j=1.5-2 S 22 P 5$

Ge24 $F$-like 2S2 2P4 ( ID) 3D (2P) $j=1.5-2 S 2$ 2P5

Ge24 F-Iike 2S2 2P4 ( 1D) 3D (2P) $j=1.5$ - 2S2 2P5

Ge24 F-like 2S2 2P4 (ID) 3D (2S) $j=.5-2 S 22 P 5$

Ge24 F-like 2S2 2P4 ( ID) 3D (2S $) j=.5-2 S 22 P 5$

Ge24 F-like 2S2 2P4 ( $1 D$ ) $3 D(2 S) j=.5-2 S 22 P 5$

Ge24 F-like 2S2 2P4 ( $1 D$ ) 3D (2S) $j=.5-2 S 22 P 5$

Ge24 F-like 2S2 2P4 ( $3 P$ ) 3D (2D) $j=2.5-2 S 22 P 5$

Ge24 F-like 2S2 2P4 ( $3 P$ ) 3D (2D) $j=2.5-2 S 22 P 5$

Ge24 F-like 2S2 2P4 (3P) 3D (2D) j=2.5 - 2S2 2P5

Ge24 $F$-like 2S2 2P4 (3P) 3D (2D) $j=2.5-2 S 22 P 5$

Ge24 F-like 2S2 2P4 ( $3 P$ ) 3D (4D) $j=1.5-2 S 22 P 5$

Ge24 F-like 2S2 2P4 ( $3 P$ ) 3D (4D) $j=1.5-2 S 22 P 5$

Ge24 F-like 2S2 2P4 ( $3 P$ ) 3D (4D) $j=1.5-2 S 2$ 2P5

Ge24 $F$-like 2S2 2P4 ( $3 P$ ) 3D (4D) $j=1.5-2 S 22 P 5$

Ge24 F-like 2S2 2P4 ( $3 P$ ) 3D (4D) $j=.5-2 S 22 P 5$

Ge24 F-like 2S2 2P4 (3P) 3D (4D) j=.5-2S2 $2 P 5$

Ge24 F-like 2S2 2P4 (3P) 3D (4D) $j=.5-2 S 22 P 5$

Ge24 F-like 2S2 2P4 ( $3 P)$ 3D (4D) $j=.5-2 S 22 P 5$

Ge24 F-like 2S2 $2 P 4$ ( ID ) 3D $(2 P) j=.5-2 S 22 P 5$

Ge24 F-like 2S2 2P4 (1D) 3D (2P) j=.5 - 2S2 2P5

Ge24 F-like 2S2 2P4 ( $1 D$ ) 3D (2P) $j=.5-2 S 22 P 5$

Ge24 F-like 2S2 2P4 ( $1 D$ ) 3D (2D) $j=1.5-2 S 22 P 5$

Ge24 F-like 2S2 2P4 ( 1D) 3D (2D) $j=1.5-2 S 2$ 2P5

Ge24 F-like 2S2 2P4 (ID) 3D (2D) j=1.5 - 2S2 2P5

Ge24 F-1ike 2S2 2P4 (3P) 3D (4P) j=2.5 - 2S2 2P5

Ge24 F-like 2S2 $2 P 4$ ( $3 P$ ) $3 D(4 P) j=2.5-2 S 22 P 5$

Ge24 :F-like 2S2 $2 \mathrm{P} 4$ ( 3P ) 3D (4P $) j=2.5-2 S 22 \mathrm{PS}$ $\left(2 P^{*}\right) j=1.5$

$(2 P *) j=1.5$

$\left(2 P^{*}\right) j=1.5$

$\left(2 P^{*}\right) j=1.5$

$\left(2 P^{\star}\right) j=1.5$

$\left(2 P^{*}\right) j=1.5$

$\left(2 P^{*}\right) j=.5$

$\left(2 P^{*}\right) j=.5$

$\left(2 P^{\star}\right) j=.5$

$\left(2 P^{*}\right) j=.5$

$(2 P *) j=.5$

$\left(2 P^{*}\right) j=1.5$

$(2 P *) j=1.5$

$\left(2 P^{*}\right) j=1.5$

$\left(2 P^{*}\right) j=1.5$

$\left(2 P^{\star}\right) j=1.5$

$\left(2 P^{\star}\right) j=1.5$

$\left(2 P^{*}\right) j=1.5$

$\left(2 P^{*}\right) j=1.5$

$\left(2 P^{*}\right) j=1.5$

$\left(2 P^{*}\right) j=1.5$

$\left(2 P^{\star}\right) j=1.5$

$\left(2 P^{*}\right) j=1.5$

$\left(2 P^{*}\right) j=1.5$

$\left(2 P^{*}\right) j=1.5$

$\left(2 P^{*}\right) j=1.5$

$\left(2 P^{*}\right) j=1.5$

$\left(2 P^{\star}\right) j=1.5$

$\left(2 P^{*}\right) j=1.5$

$\left(2 P^{*}\right) j=1.5$

$\left(2 P^{*}\right) j=1.5$

$\left(2 \mathrm{P}^{*}\right) j=1.5$

$\left(2 P^{*}\right) j=1.5$

$\left(2 p^{\star}\right) j=1.5$

$\left(2 P^{*}\right) j=1.5$

$\left(2 P^{*}\right) j=1.5$

$\left(2 P^{*}\right) j=1.5$

$\left(2 \mathrm{P}^{\star}\right) j=.5$

$\left(2 P^{*}\right) j=.5$

$\left(2 \mathrm{P}^{*}\right) \mathrm{j}=.5$

$\left(2 P^{*}\right) j=.5$

$\left(2 P^{*}\right) j=.5$

$\left(2 P^{*}\right) j=.5$

$\left(2 P^{*}\right) j=1.5$

$\left(2 P^{*}\right) j=1.5$

$\left(2 P^{*}\right) j=1.5$
8.212 RAP 100

8.512 PET 002

8.512 EDT 020

8.512 TAP 100

8.512 RAP 100

8.512 KAP 100

8.527 PET 002

8.527 EDT 020

8.527 TAP 100

8.527 RAP 100

8.527 KAP 100

8.560 PET 002

8.560 EDT 020

8.560 TAP 100

8.560 RAP 100

8.560 KAP 100

8.567 PET 002

8.567 EDT 020

8.567 TAP 100

8.567 RAP 100

8.567 KAP 100

8.588 PET 002

8.588 EDT 020

8.588 RAP 100

8.588 KAP 100

8.643 PET 002

8.643 EDT 020

8.643 RAP 100

8.643 KAP 100

8.653 PET 002

8.653 EDT 020

8.653 RAP 100

8.653 KAP 100

8.676 PET 002

8.676 EDT 020

8.676 RAP 100

8.676 KAP 100

8.710 PET 002

8.710 EDT 020

$8.710 \mathrm{KAP} 100$

8.724 PET 002

8.724 EDT 020

8.724 KAP 100

8.734 PET 002

8.734 EDT 020

8.734 KAP 100
$26.116 \quad 70.618$

$8.742 \quad 76.828$

$8.808 \quad 75.104$

$25.763 \quad 82.388$

$26.116 \quad 77.902$

$26.634 \quad 73.491$

$8.742 \quad 77.267$

$8.808 \quad 75.488$

$25.763 \quad 83.186$

$26.116 \quad 78.383$

$26.634 \quad 73.835$

$8.742 \quad 78.288$

$8.808 \quad 76.371$

$25.763 \quad 85.400$

$26.116 \quad 79.516$

$26.634 \quad 74.619$

8.74278 .516

$8.808 \quad 76.566$

$25.763 \quad 86.024$

$26.116 \quad 79.772$

$26.634 \quad 74.790$

$8.742 \quad 79.230$

$8.808 \quad 77.167$

$26.116 \quad 80.582$

$26.634 \quad 75.315$

$8.742 \quad 81.369$

$8.808 \quad 78.892$

$\begin{array}{lll}26.116 & 83.139\end{array}$

$26.634 \quad 76.788$

$8.742 \quad 81.817$

$8.808 \quad 79.235$

$26.116 \quad 83.714$

$26.634 \quad 77.073$

$8.742 \quad 82.955$

$8.808 \quad 80.068$

$26.116 \quad 85.295$

$26.634 \quad 77.754$

$8.742 \quad 85.096$

$8.808 \quad 81.445$

$26.634 \quad 78.836$

$8.742 \quad 86.323$

$8.808 \quad 82.081$

$26.634 \quad 79.313$

$8.742 \quad 87.549$

$8.808 \quad 82.568$

$26.634 \quad 79.666$ 
Ge24 F-like 2S2 2P4 ( $1 D$ ) 3D (2P) $j=1.5-2 S 22 P 5$

Ge24 F-like 2S2 2P4 ( $1 D$ ) 3D (2P) $j=1.5$ - 2S2 2P5

Ge24 $F$-like 2S2 2P4 ( $3 P$ ) 3D (2F) $j=2.5-2 S 22 P 5$

Ge24 F-like 2S2 2P4 ( $3 P$ ) 3D (2F) $j=2.5-2 S 22 P 5$

Ge24 $F$-like 2S2 2P4 ( $3 P$ ) 3D (4P) j=1.5 - 2S2 $2 P 5$

Ge24 F-like 2S2 2P4 ( 3P) 3D (4P) $j=1.5-2 S 22 P 5$

Ge24 F-like 2S2 2P4 ( $3 P$ ) 3D (4P) $j=.5-2 S 22 P 5$

Ge24 $F$-like $2 S 22 P 4(3 P)$ 3D (4P) $j=.5-2 S 22 P 5$

Ge24 F-like 2S2 2P4 ( 3P ) 3D (2P) $j=1.5-2 S 22 P 5$

Ge24 F-like 2S2 2P4 ( $3 P$ ) 3S (4P) $j=.5-2 S 22 P 5$

Ge24 F-like 2S2 2P4 ( $1 D$ ) 3S (2D) $j=1.5-2 S 22 P 5$

Ge24 $F$-like 2S2 2P4 ( 3P) 3S (4P) $j=1.5-2 S 22 P 5$

Ge24 F-like 2S2 2P4 ( $3 P$ ) $3 S(4 P) j=2.5-2 S 22 P 5$

Ge24 F-like 2S2 2P4 ( $3 P$ ) 3S (4P) $j=1.5-2 S 22 P 5$

Ge25 0 -like 2S2 2P3 ( 2P*) 3D $\left(3 D^{*}\right) j=2.0-2 S 22 P 4$

Ge25 O-like 2S2 2P3 ( 2P*) 3D (3D*) $j=2.0-2 S 22 P 4$

Ge25 O-like 2S2 $2 P^{2}$ ( 2P*) $3 D\left(3 D^{*}\right) j=2.0-2 S 22 P 4$

Ge25 O-like 2S2 2P3 ( $\left.2 D^{*}\right)$ 3D $\left(1 F^{*}\right) j=3.0-2 S 22 P^{*}$

Ge25 O-like 2S2 2P3 ( $\left.2 D^{*}\right) 3 D\left(1 F^{*}\right) j=3.0-2 S 22 P 4$

Ge25 O-like 2S2 2P3 ( $\left.2 D^{*}\right) 3 D\left(1 F^{*}\right) j=3.0-2 S 22 P 4$

Ge25 O-like 2S2 2P3 ( $\left.2 D^{*}\right)$ ) 3D (1F*) $j=3.0-2 S 2$ 2P4

Ge25 O-like 2S2 2P3 ( $\left.2 P^{*}\right) 3 D\left(3 P^{*}\right) j=1.0-2 S 22 P 4$

Ge25 0 -like 2S2 2P3 ( 2P*) 3D $\left(3 P^{*}\right) j=1.0-2 S 22 P 4$

Ge25 O -like 2S2 2P3 ( 2P*) 3D $\left(3 P^{\star}\right) j=1.0-2 S 22 P 4$

Ge25 0 -like 2S2 2P3 (2P*) 3D (3P*) $j=1.0-2 S 22 P^{*}$

Ge25 $O$-like 2S2 2P3 ( $\left.2 D^{*}\right) 3 D\left(3 P^{*}\right) j=2.0-2 S 22 P 4$

Ge25 0 -like $2 S 22 P 3\left(2 D^{*}\right) 3 D\left(3 P^{*}\right) j=2.0-2 S 22 P 4$

Ge25 O-like 2S2 2P3 ( 2D*) 3D $\left(3 P^{*}\right) j=2.0-2 S 22 P 4$

Ge25 O-like 2S2 2P3 (2D*) 3D (3P*) $j=2.0-2 S 22 \mathrm{P4}$

Ge25 O-like 2S2 $2 P^{2}\left(2 D^{*}\right) 3 D\left(3 P^{*}\right) j=2.0-2 S 22 P 4$

Ge25 O-like 2S2 2P3 ( 2D*) 3D $\left(3 D^{*}\right) j=3.0-2 S 22 P 4$

Ge25 0 -like 2S2 2P3 (2D*) 3D (3D*) j=3.0 - 2S2 2P4

Ge25 O-like 2S2 2P3 ( 2D*) 3D (3D*) $j=3.0-2 S 22 P 4$

Ge25 O-like $2 S 22 P 3\left(2 D^{\star}\right) 3 D\left(3 D^{*}\right) j=3.0-2 S 22 P 4$

Ge25 O-like 2S2 2P3 ( 2D*) 3D $\left(3 D^{*}\right) j=3.0-2 S 22 P 4$

Ge25 O-like 2S2 2P3 ( $\left.2 D^{*}\right) 3 D\left(1 P^{*}\right) j=1.0-2 S 22 P 4$

Ge25 O-like 2S2 2P3 ( 2D*) $3 D\left(1 P^{*}\right) j=1.0-2 S 22 P 4$

Ge25 $0-1 i k e$ 2S2 2P3 ( $\left.2 D^{*}\right) 3 D\left(1 P^{*}\right) j=1.0-2 S 22 P 4$

Ge25 0 -like 2S2 2P3 ( $\left.2 D^{*}\right)$ 3D ( $\left.1 P^{*}\right) j=1.0-2 S 22 P 4$

Ge25 O-like $2 S 22 P 3$ ( $\left.2 D^{*}\right) 3 D\left(1 P^{*}\right) j=1.0-2 S 22 P 4$

Ge25 O-like 2S2 2P3 ( 2D*) 3D (3S*) $j=1.0-2 S 22 \mathrm{P4}$

Ge25 O-like 2S2 2P3 ( $\left.2 D^{*}\right) 3 D\left(3 S^{*}\right) j=1.0-2 S 22 P 4$

Ge25 O-like 2S2 2P3 (2D*) $3 D\left(3 S^{*}\right) j=1.0-2 S 22 P 4$

Ge25 O-like 2S2 2P3 (2D*) 3D $\left(3 S^{*}\right) j=1.0-2 S 22 P 4$

Ge25 o-like 2S2 2P3 (2D*) 3D (3S*) $j=1.0-2 S 2$ 2P4

Ge25 $O$-like 2S2 2P3 ( $\left.2 D^{\star}\right) 3 D\left(3 S^{\star}\right) j=1.0-2 S 22 P 4$ $\left(2 P^{\star}\right) j=$

$\left(2 P^{*}\right) j=.5$

$\left(2 P^{*}\right) j=1.5$

$(2 P *) j=1.5$

$\left(2 P^{*}\right) j=1.5$

$\left(2 P^{\star}\right) j=1.5$

$\left(2 P^{\star}\right) j=1.5$

$\left(2 P^{*}\right) j=1.5$

$\left(2 P^{*}\right) j=.5$

$\left(2 P^{*}\right) j=1.5$

$\left(2 P^{*}\right) j=.5$

$\left(2 P^{*}\right) j=1.5$

$\left(2 P^{*}\right) j=1.5$

$\left(2 P^{*}\right) j=.5$

(3P) $j=1.0$

(3P) $j=1.0$

(3P) $j=1.0$

(3P) $j=2.0$

(3P) $j=2.0$

(3P) $j=2.0$

(3P) $j=2.0$

(3P) $j=1.0$

(3P) $j=1.0$

(3P) $j=1.0$

(3P) $j=1.0$

(3P) $j=2.0$

(3P) $j=2.0$

(3P) $j=2.0$

(3P) $j=2.0$

(3P) $j=2.0$

(3P) $j=2.0$

(3P) $j=2.0$

(3P) $j=2.0$

(3P) $j=2.0$

(3P) $j=2.0$

(3P) $j=2.0$

(3P) $j=2.0$

(3P) $j=2.0$

(3P) $j=2.0$

(3P) $j=2.0$

$\langle 3 P\rangle j=.0$

(30 $) j=0$

(3P) $j=.0$

(3P) $j=.0$

(3P) $j=.0$

(3P) $j=.0$
8.754 EDT 020

8.754 KAP 100

8.767 EDT 020

8.767 KAP 100

.788 EDT 020

8.788 KAP 100

8.805 EDT 020

8.805 KAP 100

$8.826 \mathrm{KAP} 100$

9.439 mica 002

9.481 mica 002

9.533 mica 002

9.553 mica 002

9.762 mica 002

8.176 topaz 002

8.176 quartz 100

8.176 TAP 100

8.200 topaz 002

8,200 quartz 100

8.200 TAP 100

8.200 RAP 100

212 topaz 002

8.212 quartz 100

8.212 TAP 100

8.212 RAP 100

8.233 topaz 002

8.233 quartz 100

8.233 PET 002

8.233 TAP 100

8.233 RAP 100

8.246 topaz 002

8.246 quartz 100

8.246 PET 002

8.246 TAP 100

8.246 RAP 100

8.274 topaz 002

8.274 quartz 100

8.274 PET 002

8.274 TAP 100

8.274 RAP 100

8.302 topaz 002

8.302 quartz 100

8.302 PET 002

8.302 EDT 020

8.302 TAP 100

8.302 RAP 100
$8.808 \quad 83.652$

$26.634 \quad 80.413$

$8.808 \quad 84.470$

$26.634 \quad 80.930$

$8.808 \quad 86.138$

$26.634 \quad 81.835$

$8.808 \quad 88.505$

$26.634 \quad 82.647$

$26.634 \quad 83.796$

$19.942 \quad 71.199$

$19.942 \quad 71.963$

$19.942 \quad 72.955$

$19.942 \quad 73.351$

$19.942 \quad 78.248$

$8.374 \quad 77.516$

$8.512 \quad 73.848$

$25.763 \quad 72.188$

$8.374 \quad 78.300$

$8.512 \quad 74.439$

$25.763 \quad 72.719$

$\begin{array}{ll}26.116 & 70.382\end{array}$

$8.374 \quad 78.712$

$8.512 \quad 74.743$

$25.763 \quad 72.990$

$26.116 \quad 70.618$

$8.374 \quad 79.471$

8.51275 .290

$8.742 \quad 70.352$

$25.763 \quad 73.476$

$26.116 \quad 71.039$

$8.374 \quad 79.969$

8.51275 .638

$8.742 \quad 70.607$

$25.763 \quad 73.784$

$26.116 \quad 71.305$

$8.374 \quad 81.137$

8.51276 .419

$8.742 \quad 71.167$

$25.763 \quad 74.467$

$26.116 \quad 71.888$

$8.374 \quad 82.481$

$8.512 \quad 77.247$

$8.742 \quad 71.744$

$8.808 \quad 70.485$

$25.763 \quad 75.180$

$26.116 \quad 72.491$

Page 115 
Ge25 O-like 2S2 2P3 ( 2D*) 3D (3G*)j=3.0-2S2 2P4 Ge25 0 -like 2S2 2P3 ( 2D*) 3D $\left(3 G^{*}\right) j=3.0-2 S 2$ 2P4 Ge25 0 -like 2S2 2P3 ( 2D*) 3D $\left(3 G^{*}\right) j=3.0-2 S 22 P 4$ Ge25 0 -1ike 2S2 2P3 ( 2D*) 3D (3G*) $j=3.0-2 S 22 \mathrm{PA}$ Ge25 0 -like 2S2 2P3 ( 2D*) 3D $\left(3 G^{*}\right) j=3.0-2 S 22 P 4$ Ge25 0 -like $2522 P 3\left(2 D^{*}\right) 3 D\left(3 G^{*}\right) j=3.0-2 S 22 P 4$ Ge25 O-like 2S2 2P3 ( 2D*) 3D $\left(3 F^{*}\right) j=3.0-2 S 2$ 2P4 Ge25 o-like 2S2 2P3 (2D*) 3D $\left(3 F^{*}\right) j=3.0-2 S 22 P 4$ Ge25 0 -like 2S2 2P3 ( 2D*) 3D (3F*) j=3.0-2S2 2P4 Ge25 O-like 2S2 2P3 ( 2D*) 3D (3F*) j=3.0- 2S2 2P4 Ge25 O-like 2S2 2P3 ( 2D*) 3D $\left(3 F^{*}\right) j=3.0-2 S 22 P 4$ Ge25 O-like 2S2 $2 P 3\left(2 D^{*}\right) 3 D\left(3 F^{\star}\right) j=3.0-2 S 22 P 4$

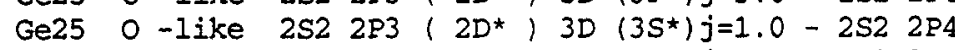
Ge25 O-like 2S2 2P3 (2D*) 3D (3S*) j=1.0 - 2S2 2P4 Ge25 0 -1ike 2S2 2P3 (2D*) 3D $\left(3 S^{*}\right) j=1.0-2 S 22 P 4$ Ge25 O-like 2S2 2P3 ( 2D*) 3D (3S*) j=1.0 - 2S2 2P4 Ge25 O-like 2S2 2P3 ( 2D*) 3D (3S*) j=1.0 - 2S2 2P4 Ge25 O -like 2S2 2P3 ( 2D*) 3D $\left\{3 S^{*}\right\rangle j=1.0-2 S 22 P 4$ Ge25 O-like 2S2 2P3 ( $\left.2 P^{*}\right)$ 3D $\left(3 P^{*}\right) j=2.0-2 S 22 P 4$ Ge25 0 -like 2S2 $2 P^{2}\left(2 P^{*}\right) 3 D\left(3 P^{*}\right) j=2.0-2 S 22 P^{*}$ Ge25 0 -1ike 2S2 2P3 ( $\left.2 P^{*}\right) 3 D\left(3 P^{*}\right) j=2.0-2 S 22 P 4$ Ge25 O-like 2S2 2P3 ( 2P*) 3D (3P*) j=2.0 - 2S2 2P4 Ge25 0 -like $2 S 22 P^{2}$ ( $\left.2 P^{*}\right) 3 D\left(3 P^{*}\right) j=2.0-2 S 22 P^{*}$ Ge25 O-like 2S2 2P3 ( $\left.2 P^{*}\right) 3 D\left(3 P^{*}\right) j=2.0-2 S 22 P 4$ Ge25 0 -like 2S2 2P3 ( 2D*) 3D $\left(3 D^{*}\right) j=.0-2 S 22 P 4$ Ge25 0 -like 2S2 $2 P^{2}$ ( $\left.2 D^{*}\right) 3 D\left(3 P^{*}\right) j=.0-2 S 22 P 4$ Ge25 O-1ike 2S2 2P3 ( 2D*) 3D $\left(3 P^{*}\right) j=.0-2 S 22 P 4$ Ge25 0 -1ike 2S2 2P3 ( 2D*) 3D $\left(3 P^{*}\right) j=.0-2 S 22 P 4$ Ge25 O-like 2S2 2P3 ( 2D*) 3D $\left(3 P^{*}\right) j=.0-2 S 22 P 4$ Ge25 0 -like 2S2 2P3 ( 2D*) 3D $\left(3 P^{*}\right) j=.0-2 S 22 P 4$ Ge25 O-like 2S2 $2 \mathrm{P} 3\left(\mathrm{SS}^{*}\right)$ 3D $\left(3 \mathrm{D}^{*}\right) j=3.0-2 S 22 \mathrm{P} 4$ Ge25 O-like 2S2 2P3 ( $\left.4 S^{*}\right)$ 3D $\left(3 D^{*}\right) j=3.0-2 S 2$ 2P4 Ge25 0 -1ike 2S2 2P3 ( $\left.4 S^{*}\right) 3 D\left(3 D^{*}\right) j=3.0-2 S 22 P 4$ Ge25 O-like 2S2 $2 P 3\left(4 S^{*}\right) 3 D\left(3 D^{*}\right) j=3.0-2 S 22 P 4$ Ge25 0 -like 2S2 2P3 ( $\left.4 S^{*}\right) 3 D\left(3 D^{*}\right) j=3.0-2 S 22 P 4$ Ge25 O-like 2S2 2P3 (4S*) 3D (3D*) $j=3.0-2 S 22 P 4$ Ge25 O-like 2S2 2P3 ( 2P*) 3D $\left(3 F^{*}\right) j=3.0-2 S 22 P 4$ Ge25 0 -like 2S2 2P3 (2P*) $3 D\left(3 F^{*}\right) j=3.0-2 S 22 P 4$ Ge25 O-like 2S2 2P3 ( 2P*) 3D $\left(3 F^{*}\right) j=3.0-2 S 22 P 4$ Ge25 0.-like 2S2 2P3 (2P*) 3D $\left(3 F^{\star}\right) j=3.0-2 S 22 P 4$ Ge25 O-like 2S2 2P3 ( $\left.2 P^{*}\right) 3 D\left(3 F^{*}\right) j=3.0-2 S 22 P 4$ Ge25 o -like 2S2 2P3 ( 2P*) 3D $\left(3 F^{*}\right) j=3.0-2 S 22 P 4$ Ge25 0 -like 2S2 $2 P^{2}$ ( $\left.2 P^{*}\right) 3 D\left(3 F^{*}\right) j=3.0-2 S 22 P 4$ Ge25 O-like 2S2 2P3 ( $\left.2 P^{*}\right) 3 D\left(3 F^{*}\right) j=3.0-2 S 22 P 4$ Ge25 0 -like 2S2 2P3 (2P*) 3D $\left(3 F^{\star}\right) j=3.0-2 S 22 P 4$ Ge25 0 -like 2S2 $2 P^{2}\left(2 P^{*}\right) 3 D\left(3 F^{*}\right) j=3.0-2 S 22 P 4$
(3P) $j=2.0$ (3P) $j=2.0$ (3P) $j=2.0$ (3P) $j=2.0$

(3P) $j=2.0$ (3P) $j=2.0$ (3P) $j=2.0$

(3P) $j=2.0$ (3P) $j=2.0$ (3P) $j=2.0$ (3P) $j=2.0$ (3P) $j=2.0$ (3P) $j=1.0$ (3P) $j=1.0$ (3P) $j=1.0$ (3P) $j=1.0$ (3P) $j=1.0$ (3P) $j=1.0$

(1D) $j=2.0$ (1D) $j=2.0$ (ID) $j=2.0$ (ID) $j=2.0$

(1D) $j=2.0$ (1D) $j=2.0$ (3P) $j=1.0$ (3P) $j=1.0$ (3P) $j=1.0$ (3P) $j=1.0$ (3P) $j=1.0$ (3P) $j=1.0$ (3P) $j=2.0$ (3P) $j=2.0$ (3P) $j=2.0$ (3P) $j=2.0$ ( $3 P$ ) $j=2.0$ (3P) $j=2.0$ (1D) $j=2.0$ (1D) $j=2.0$ (ID) $j=2.0$ (1D) $j=2.0$ (1D) $j=2.0$ (ID) $j=2.0$ (1D) $j=2.0$ (ID) $j=2.0$ (1D) $j=2.0$ (1D) $j=2$.0
8.315 topaz 002

8.315 quartz 100

8.315 PET 002

8.315 EDT 020

8.315 TAP 100

8.315 RAP 100

8.324 topaz 002

8.324 guartz 100

8.324 PET 002

8.324 EDT 020

8.324 TAP 100

8.324 RAP 100

8.379 quartz 100

8.379 PET 002

8.379 EDT 020

8.379 TAP 100

8.379 RAP 100

8.379 KAP 100

8.394 guartz 100

8.394 PET 002

8.394 EDT 020

8.394 TAP 100

8.394 RAP 100

8.394 KAP 100

8.410 quartz 100

8.410 PET 002

8.410 EDT 020

8.410 TAP 100

8.410 RAP 100

8.410 KAP 100

8.427 quartz 100

8.427 PET 002

8.427 EDT 020

8.427 TAP 100

8.427 RAP 100

8.427 KAP 100

8.439 quartz 100

8.439 PET 002

8.439 EDT 020

8.439 TAP 100

8.439 RAP 100

8.439 KAP 100

8.439 cuartz 100

8.439 PET 002

8.439 EDT 020

8.439 TAP 100
$8.374 \quad 83.195$

$8.512 \quad 77.649$

$8.742 \quad 72.018$

$8.808 \quad 70.739$

$25.763 \quad 75.523$

$26.116 \quad 72.777$

$8.374 \quad 83.736$

$8.512 \quad 77.936$

$8.742 \quad 72.210$

$8.808 \quad 70.918$

$25.763 \quad 75.765$

$26.116 \quad 72.979$

$8.512 \quad 79.858$

$8.742 \quad 73.431$

$8.808 \quad 72.044$

$25.763 \quad 77.344$

$26.116 \quad 74.262$

$26.634 \quad 70.699$

$8.512 \quad 80.449$

$8.742 \quad 73.779$

$8.808 \quad 72.363$

$25.763 \quad 77.809$

$26.116 \quad 74.630$

26.63470 .994

8.51281 .121

$8.742 \quad 74.159$

$8.808 \quad 72.710$

$25.763 \quad 78.325$

$26.116 \quad 75.033$

$26.634 \quad 71.313$

$8.512 \quad 81.896$

$8.742 \quad 74.572$

$8.808 \quad 73.086$

$25.763 \quad 78.900$

$26.116 \quad 75.472$

26.63471 .659

$8.512 \quad 82.491$

$8.742 \quad 74.871$

$8.808 \quad 73.357$

$\begin{array}{ll}25.763 & 79.323\end{array}$

$26.116 \quad 75.791$

$26.634 \quad 71.907$

8.512. 82.491

$8.742 \quad 74.871$

$8.808 \quad 73.357$

$25.763 \quad 79.323$ 
Ge25 O-like 2S2 $2 \mathrm{P} 3\left(2 \mathrm{P}^{*}\right) 3 \mathrm{D}\left(3 \mathrm{~F}^{*}\right) \mathrm{j}=3.0-2 \mathrm{~S} 22 \mathrm{P} 4$ Ge25 0 -Iike $2 S 22 \mathrm{P} 3\left(2 P^{*}\right) 3 D\left(3 F^{*}\right) j=3.0-2 S 22 P 4$ Ge25 O-like 2S2 2P3 (2D*) 3D (1D*) j=2.0 - 2S2 2P4 Ge25 O-Iike 2S2 2P3 ( 2D*) 3D (1D*) j=2.0- $2 S 22 \mathrm{P} 4$ Ge25 0 -like 2S2 2P3 (2D*) $3 D\left(1 D^{*}\right) j=2.0-2 S 22 P 4$ Ge25 0 -Iike 2S2 2P3 (2D*) 3D $\left(1 D^{*}\right) j=2.0-2 S 22 P 4$ Ge25 0 -like $2522 \mathrm{P3}\left(2 D^{*}\right) 3 D\left(1 D^{*}\right) j=2.0-2 S 22 \mathrm{P4}$ Ge25 0 -like 2S2 2P3 ( 2D*) 3D (1D*) $j=2.0-2 S 22 P 4$ Ge25 0 -like $2 S 22 \mathrm{P} 3$ ( $\left.4 S^{*}\right) 3 D\left(3 D^{*}\right) j=1.0-2 S 22 P 4$ Ge25 0 -like 2S2 2P3 (4S*) 3D $\left(3 D^{*}\right) j=1.0-2 S 22 P 4$ Ge25 0 -like $2 S 22 P^{2}\left(4 S^{*}\right) 3 D\left(3 D^{*}\right) j=1.0-2 S 22 P 4$ Ge25 0 -like 2S2 2P3 ( $\left.4 S^{*}\right) 3 D\left(3 D^{*}\right) j=1.0-2 S 22 P 4$ Ge25 0 -like 2S2 2P3 ( $\left.4 S^{*}\right)$ 3D $\left(3 D^{*}\right) j=1.0-2 S 22 P 4$ Ge25 0 -like $2 S 22 P 3\left(2 P^{*}\right) 3 S\left(3 P^{*}\right) j=2.0-2 S 22 P 4$ Ge25 O-like $2 S 22 P^{2}$ (2P*) $3 S\left(3 P^{*}\right) j=2.0-2 S 22 P^{*}$ Ge25 0 -Iike $2 S 22 P^{2}\left(2 P^{*}\right) 3 S\left(1 P^{*}\right) j=1.0-2 S 22 P 4$ Ge25 0 -like $2 S 22 P^{2}\left(2 P^{*}\right) 3 S\left(1 P^{*}\right) j=1.0-2 S 22 P 4$

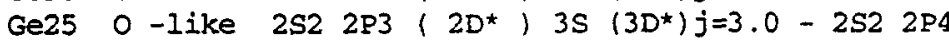
Ge25 0 -like $2 S 22 \mathrm{P3}\left(2 D^{*}\right) 3 S\left(3 D^{*}\right) j=3.0-2 S 22 \mathrm{P} 4$ Ge29 Be-like $1 S 2$ 2S 3P (3P*) j=1.0 - 1S2 $2 S 2$ Ge29 Be-like 1s2 2S Ge29 Be-like 1S2 2S Ge29 Be-like 152 2S

Ge29 Be-like 1S2 2P Ge29 Be-like 152 2P Ge29 Be-like 1S2 2P

Ge29 Be-like 1S2 2P Ge29 Be-like 1S2 2S Ge29 Be-like 1S2 2S Ge29 Be-like 1S2 2S Ge29 Be-like 1S2 2S Ge29 Be-like 1S2 2P Ge29 Be-like 1S2 2P Ge29 Be-like 1S2 2S Ge29 Be-like 1s2 2S Ge29 Be-like 1S2 2S

Ge29 Be-like 1S2 2S Ge29 Be-like 1S2 2P Ge29 Be-like 1S2 2P Ge29 Be-like IS2 2S Ge29 Be-like 1S2 2S Ge29 Be-like IS2 2P Ge29 Be-like 1S2 2P Ge31 He-like $1 \mathrm{~S} 3 \mathrm{P}$ Ge31 He-like IS $3 P$ Ge31 He-like is 2P

$\left(3 P^{*}\right) j=1.0-152$

$\left(3 P^{*}\right) j=1.0-1 S 2$

$\left(1 P^{*}\right) j=1.0-1 S 2$
(1D) $j=2.0$ (1D) $j=2.0$ (1D) $j=2.0$ (1D) $j=2.0$ (ID) $j j=2.0$ (1D) $j=2.0$ (1D) $j=2.0$ (ID) $j=2.0$ (3P) $j=.0$ (3P) $j=.0$ $(3 P) j=.0$ (3P) $j=.0$ (3P) $j=.0$ (3P) $j=1.0$ (3P) $j=1.0$ (ID) $j=2.0$ (1D) $j=2.0$

(3P) $j=2.0$ (3P) $j=2.0$ (1s) $j=.0$ $2 P\left(1 P^{\star}\right) j=1.0$ $2 P\left(3 P^{*}\right) j=1.0$

$2 P\left(3 P^{\star}\right) j=1.0$

(3P) $j=1.0$

(3P) $j=1.0$

(3P) $j=2.0$

(3P) $j=2.0$ $2 P(3 P *) j=2.0$ 2P $\left(3 P^{*}\right) j=2.0$ $2 P\left(3 P^{\star}\right) j=2.0$ $2 P(3 P *) j=2.0$

(ID) $j=2.0$ (ID) $j=2.0$ $2 P(3 P *) j=1$ $2 P\left(3 P^{*}\right) j=1.0$ $2 P(1 P *) j=1.0$

$2 P\left(1 P^{\star}\right) j=1.0$

(IS) $j=.0$ (1s) $j=.0$ $2 P\left(3 P^{*}\right) j=2.0$ $2 P\left(3 P^{*}\right) j=2.0$

(1D) $j=2.0$ (1s) $j=.0$

(Is ) $j=.0$

(1s) $j=.0$

(1S) $j=.0$
8.439 RAP 100 8.439 KAP 100 8.448 quartz 100

8.448 PET 002

8.448 EDT 020

8.448 TAP 100

8.448 RAP 100

8.448 KAP 100

8.512 PET 002

8.512 EDT 020

8.512 TAP 100

8.512 RAP 100

$8.512 \mathrm{KAP} 100$

8.754 EDT 020

8.754 KAP 100

8.805 EDT 020

8.805 KAP 100

8.825

8.825 KAP 100

7.041 InSb 111

7.081 Insto 111

7.205 InSto 111

7.205 gypsum 020

7.230

7.274

Insb 111

74 gypsum 020

7.281 InSt 111

7.281 gypsum 020

7.333 InSb 111

7.333 gypsum 020

7.377 InSb 111

7.377 gypsum 020

7.428 InSb 111

7.428 gypsum 020

7.442 InSb 111

7.442 gypsum 020

7.520 gypsum 020

7.520 beryl 100

7.562 gypsum 020

7.562 beryl 100

7.696 beryl 100

7.852 beryl 100

1.026 quartz 211
1.026

1.206 quartz 200 quartz 220
$26.116 \quad 75.791$

$26.634 \quad 71.907$

$8.512 \quad 82.970$

$8.742 \quad 75.098$

$8.808 \quad 73.562$

$25.763 \quad 79.652$

$26.116 \quad 76.034$

$26.634 \quad 72.095$

$8.742 \quad 76.828$

$8.808 \quad 75.104$

$\begin{array}{ll}25.763 & 82.388\end{array}$

$26.116 \quad 77.902$

$26.634 \quad 73.491$

$8.808 \quad 83.652$

$26.634 \quad 80.413$

$8.808 \quad 88.505$

$26.634 \quad 82.647$

$26.634 \quad 83.736$

$26.634 \quad 83.736$

$\begin{array}{ll}7.481 & 70.251\end{array}$

$7.481 \quad 71.179$

$7.481 \quad 74.388$

$15.185 \quad 71.616$

$7.481 \quad 75.116$

$15.185 \quad 72.224$

$7.481 \quad 76.490$

$15.185 \quad 73.346$

$7.481 \quad 76.722$

$15.185 \quad 73.531$

$7.481 \quad 78.584$

$15.185 \quad 74.977$

$7.481 \quad 80.435$

$15.185 \quad 76.316$

$7.481 \quad 83.176$

$15.185 \quad 78.051$

$\begin{array}{ll}7.481 & 84.147\end{array}$

$15.185 \quad 78.573$

$15.185 \quad 82.076$

$15.954 \quad 70.512$

$15.185 \quad 84.863$

$\begin{array}{lll}15.954 & 71.437\end{array}$

$15.954 \quad 74.747$

$15.954 \quad 79.844$

$3.082 \quad 87.081$

$4.246 \quad 75.140$

2.45179 .765 


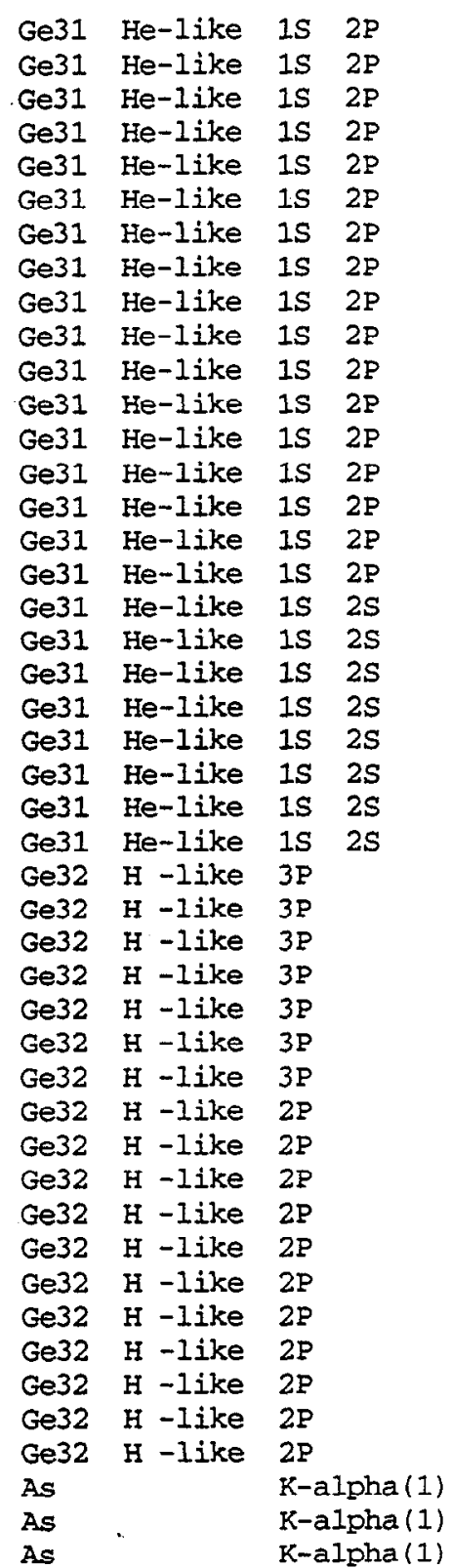

$\left(1 P^{\star}\right) j=1.0-1 S 2$

$\left(1 P^{*}\right) j=1.0-1 S 2$

$\left(1 P^{*}\right) j=1.0-1 \mathrm{~S} 2$

$\left(1 P^{*}\right) j=1.0-1 S 2$

$\left(1 P^{*}\right) j=1.0-1 \mathrm{~s} 2$

$\left(1 P^{*}\right) j=1.0-1 S 2$

$\left(1 P^{*}\right) j=1.0-1 S 2$

$\left(1 P^{*}\right) j=1.0-1 S 2$

$\left(3 P^{*}\right) j=1.0-1 S 2$

$\left(3 P^{*}\right) j=1.0-1 \mathrm{~S} 2$

$\left(3 P^{\star}\right) j=1.0-1 S 2$

$\left\langle 3 P^{\star}\right\rangle j=1.0-152$

$\left(3 P^{*}\right) j=1.0-1 S 2$

$\left(3 P^{\star}\right) j=1.0-1 S 2$

$\left(3 P^{*}\right) j=1.0-1 s 2$

$(3 P *) j=1.0-1 S 2$

$\left(3 P^{\star}\right) j=1.0-1 S 2$

(3S ) $j=1.0-1 S 2$

(3S ) $j=1.0$ - 1S2

(3s) $j=1.0$ - 1s2

(3s) $j=1.0-1 s 2$

(3s) $j=1.0$ - 1s2

(3S) $j=1.0-1 S 2$

(3s) $j=1.0-1 s 2$

(3S) $j=1.0-1 S 2$

$\left(2 P^{*}\right) j=1.5-1 S$

$\left(2 P^{*}\right) j=1.5-1 S$

$\left(2 P^{*}\right) j=1.5-1 S$

$\left(2 P^{*}\right) j=1.5-1 S$

$\left(2 P^{\star}\right) j=1.5-1 S$

$\left(2 P^{*}\right) j=1.5-1 S$

$\left(2 P^{*}\right) j=1.5-1 S$

$\left(2 P^{*}\right) j=1.5-1 S$

$\left(2 P^{*}\right) j=1.5-1 S$

$\left(2 P^{\star}\right) j=1.5-1 S$

$\left(2 P^{\star}\right) j=1.5-1 S$

$\left(2 P^{*}\right) j=1.5-1 S$

$\left(2 P^{\star}\right) j=1.5-1 S$

$\left(2 P^{*}\right) j=.5-1 s$

$\left(2 P^{\star}\right) j=.5-1 S$

$\left(2 P^{\star}\right) j=.5-1 S$

$\left(2 P^{\star}\right) j=.5-1 S$

$\left(2 P^{*}\right) j=.5-1 S$

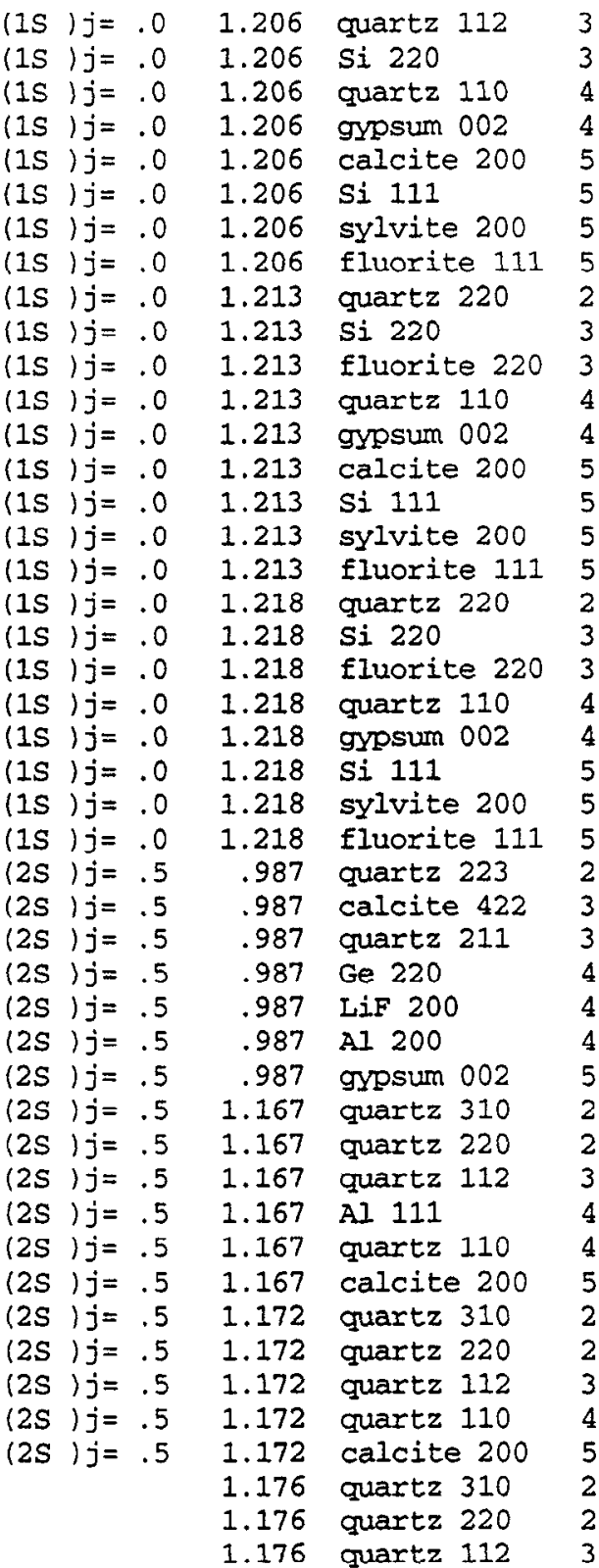

$\begin{array}{ll}3.636 & 84.296 \\ 3.840 & 70.422 \\ 4.912 & 79.138 \\ 4.990 & 75.180 \\ 6.071 & 83.337 \\ 6.271 & 74.064 \\ 6.292 & 73.407 \\ 6.308 & 72.927 \\ 2.451 & 81.810 \\ 3.840 & 71.380 \\ 3.862 & 70.434 \\ 4.912 & 81.035 \\ 4.990 & 76.494 \\ 6.071 & 87.453 \\ 6.271 & 75.274 \\ 6.292 & 74.563 \\ 6.308 & 74.045 \\ 2.451 & 83.658 \\ 3.840 & 72.094 \\ 3.862 & 71.110 \\ 4.912 & 82.683 \\ 4.990 & 77.515 \\ 6.271 & 76.201 \\ 6.292 & 75.442 \\ 6.308 & 74.893 \\ 2.024 & 77.238 \\ 3.034 & 77.406 \\ 3.082 & 73.892 \\ 4.000 & 80.751 \\ 4.027 & 78.632 \\ 4.048 & 77.238 \\ 4.990 & 81.485 \\ 2.360 & 81.487 \\ 2.451 & 72.225 \\ 3.636 & 74.338 \\ 4.676 & 86.648 \\ 4.912 & 71.865 \\ 6.071 & 73.972 \\ 2.360 & 83.324 \\ 2.451 & 73.008 \\ 3.636 & 75.239 \\ 4.912 & 72.630 \\ 6.071 & 74.850 \\ 2.360 & 85.222 \\ 2.451 & 73.643 \\ 3.636 & 75.981 \\ & \end{array}$ 
1.176 quartz 110

1.176 gypsum 002

1.176 calcite 200

8.312 topaz 002

$\left(2 p^{*}\right) j=1.5$

$\left(2 P^{*}\right) j=1.5$

$\left\langle 2 P^{*}\right\rangle j=1.5$

$(2 P *) j=1.5$

$\left(2 P^{\star}\right) j=1.5$

$\left(2 P^{*}\right) j=1.5$

(2S) $j=.5$

(2s) $j=.5$

(2s) $j=.5$

(2s) $j=.5$

(2S) $j=.5$

(2S) $j=.5$

(2D) $j=1.5$

(2D) $j=1.5$

(2D) $j=1.5$

(2D) $j=1.5$

(2D) $j=1.5$

(2D) $j=1.5$

(2D) $j=2.5$

(2D) $j=2.5$

(2D) $j=2.5$

(2D) $j=2.5$

(2D) $j=2.5$

(2D) $j=2.5$

(2D) $j=2.5$

(2D) $j=2.5$

(2D) $j=2.5$

(2D) $j=2.5$

(2D) $j=2.5$

(2D) $j=2.5$

(2D) $j=2.5$

$\left(2 P^{*}\right) j=.5$

$\left(2 P^{*}\right) j=.5$

$\left(2 P^{*}\right) j=.5$

$\left(2 P^{\star}\right) j=.5$

$\left(2 P^{*}\right) j=.5$

$\left(2 P^{*}\right) j=.5$

$\left(2 P^{\star}\right) j=1.5$

$(2 P *) j=1.5$

$\left(2 P^{*}\right) j=1.5$

$\left(2 P^{\star}\right) j=1.5$

$\left(2 P^{*}\right) j=1.5$

$(2 P *) j=1.5$
8.312 quartz 100

8.312 PET 002

8.312 EDT 020

8.312 TAP 100

8.312 RAP 100

8.332 topaz 002

8.332 quartz 100

8.332 PET 002

8.332 EDT 020

8.332 TAP 100

8.332 RAP 100

8.341 topaz 002

8.341 quartz 100

8.341 PET 002

8.341 EDT 020

8.341 TAP 100

8.341 RAP 100

8.356 topaz 002

8.356 quartz 100

8.356 PET 002

8.356 EDT 020

8.356 TAP 100

8.356 RAP 100

8.356 KAP 100

8.374 guartz 100

8.374 PET 002

8.374 TAP 100

8.374 RAP 100

8.374 KAP 100

392 guartz 100

8.392 PET 002

8.392 TAP 100

8.392 RAP 100

8.392 KAP 100

8.406 quartz 100

8.406 PET 002

8.406 EDT 020

8.406 TAP 100

8.406 RAP 100

8.406 KAP 100
8.374 EDT 020

8.392 EDT 020
$4.912 \quad 73.250$

$4.990 \quad 70.494$

$\begin{array}{ll}6.071 & 75.571\end{array}$

$8.374 \quad 83.024$

$8.512 \quad 77.555$

$8.742 \quad 71.955$

$8.808 \quad 70.680$

$25.763 \quad 75.443$

$26.116 \quad 72.711$

$8.374 \quad 84.259$

8.51278 .196

$8.742 \quad 72.383$

$8.808 \quad 71.078$

$25.763 \quad 75.984$

$26.116 \quad 73.159$

$8.374 \quad 84.912$

$8.512 \quad 78.496$

$8.742 \quad 72.579$

$8.808 \quad 71.259$

$25.763 \quad 76.234$

$26.116 \quad 73.365$

$8.374 \quad 86.243$

8.51279 .014

8.74272 .910

$8.808 \quad 71.565$

$25.763 \quad 76.661$

$26.116 \quad 73.714$

$26.634 \quad 70.255$

$8.512 \quad 79.669$

$8.742 \quad 73.316$

$8.808 \quad 71.939$

$25.763 \quad 77.192$

$26.116 \quad 74.141$

26.63470 .601

8.51280 .368

$8.742 \quad 73.732$

$8.808 \quad 72.321$

$25.763 \quad 77.746$

$26.116 \quad 74.581$

$26.634 \quad 70.954$

$8.512 \quad 80.948$

$8.742 \quad 74.063$

$8.808 \quad 72.623$

$25.763 \quad 78.194$

$26.116 \quad 74.931$

$26.634 \quad 71.233$ 
As23 Na-like 2P5 $(2 P * 1)(3 D 2(12 *) j=2.5-2 P 63 D$ As23 Na-1ike 2P5 (2P*1) (3D2 ( $\left(12^{\star}\right) j=2.5-2 P 6$ 3D As23 Na-like 2P5 ( 2P*1) (3D2 ( $(12 *) j=2.5-2 P 6$ 3D As23 Na-1ike 2P5 (2P*1) (3D2) ( $\left.12^{\star}\right) j=2.5-2 P 6$ 3D As23 Na-1ike 2P5 $(2 P * 1)$ (3D2 ( $\left(12^{*}\right) j=2.5-2 P 6$ 3D As23 Na-1ike 2P5 ( 2P*1) (3D2) (12*)j=2.5 - 2P6 3D As23 Na-like 2P5 $\left(2 P^{*} 2\right)$ (3D2 ( $\left(20^{*}\right) j=1.5-2 P 6$ 3D As23 Na-like 2P5 $\left(2 P^{\star} 2\right)$ (3D2) ( $\left(20^{\star}\right) j=1.5-2 P 6$ 3D As23 Na-like 2P5 (2P*2) (3D2 ( $(20 *) j=1.5-2 P 6$ 3D As23 Na-like 2P5 $(2 P * 2) \quad\left(3 D 2\left(\left(20^{*}\right) j=1.5-2 P 6\right.\right.$ 3D As23 Na-like 2P5 ( 2P*2) (3D2 ( $(20 *) j=1.5-2 P 6$ 3D As23 Na-like 2P5 (2P*2) (3D2 ( $\left(20^{*}\right) j=1.5-2 P 6$ 3D As23 Na-like 2P5 $(2 P * 2) \quad(3 D 2)(20 *) j=1.5-2 P 6$ 3D As23 Na-like $2 P 5(2 P * 2)(3 D 2)(20 *) j=1.5-2 P 6$ 3D As23 Na-like 2P5 ( 2P*2) (3D2 ( $(20 *) j=1.5-2 P 6$ 3D As23 Na-like 2P5 (2P*2) (3D2 ( $\left(20^{*}\right) j=1.5-2 P 6$ 3D As23 Na-like 2P5 ( $\left.2 P^{*} 2\right)$ (3D2 ( $(20 *) j=1.5-2 P 6$ 3D As23 Na-like 2P5 $(2 P * 2)$ (3D2 ( $(24 *) j=2.5-2 P 6$ 3D As23 Na-1ike 2P5 ( 2P*2) (3D2) (24*) j=2.5 - 2P6 3D As23 Na-1ike 2P5 $(2 P \star 2) \quad$ (3D2 ( $\left(24^{\star}\right) j=2.5-2 P 6$ 3D As23 Na-like 2P5 $(2 P * 2)$ (3D2 ( $(24 *) j=2.5-2 P 6$ 3D As23 Na-like 2P5 $(2 P * 2)$ (3D2 ( $\left(24^{\star}\right) j=2.5-2 P 6$ 3D As23 Na-1ike 2P5 $(2 P * 2) \quad(3 D 2)(24 *) j=2.5-2 P 63 D$ As23 Na-like 2P5 (2P*2) (3D2) (24*) $j=2.5-2 P 6$ 3D As23 Na-1ike 2P5 ( $2 P * 2)$ (3D2 ( $\left(24^{*}\right) j=2.5-2 P 6$ 3D As23 Na-like 2P5 $(2 P * 2)$ (3D2 $(24 *) j=2.5-2 P 6$ 3D As23 Na-1ike 2P5 $\left(2 P^{*} 2\right) \quad$ (3D2 $\left(24^{*}\right) j=2.5-2 P 6 \quad 3 D$ As23 Na-like 2P5 $(2 P * 2) \quad(3 D 2)\left(24^{*}\right) j=3.5-2 P 6 \quad 3 D$ As23 Na-1ike 2P5 ( $2 P \star 2)$ (3D2 ( $\left(24^{\star}\right) j=3.5-2 P 6$ 3D As23 Na-like 2P5 $(2 P * 2)$ (3D2 ( $\left(24^{*}\right) j=3.5-2 P 6$ 3D As23 Na-1ike 2P5 $\left(2 P^{*} 2\right) \quad(3 D 2)\left(24^{*}\right) j=3.5-2 P 6 \quad 3 D$ As23 Na-like 2P5 $(2 P * 2)$ (3D2 ( $(24 *) j=3.5-2 P 6$ 3D As23 Na-like $2 P 6 \quad 5 P \quad\left(2 P^{\star}\right) j=.5-2 P 6 \quad 3 S$ As23 Na-like 2P6 $6 F \quad\left(2 F^{*}\right) j=3.5-2 P 6$ 3D As23 Na-like 2P6 4P (2P*) $j=1.5-2 P 6 \quad 3 s$ As24 Ne-like 2S2 2P5 ( 2P*2) 6D (23*) $j=1.0-2 S 22 P 6$ As24 Ne-like 2S2 2P5 ( $\left.2 P^{\star} * 2\right) 6 D\left(22^{\star}\right) j=1.0-2 S 22 P 6$ As24 Ne-like 2S2 2P5 ( 2P*1) 5D $(12 *) j=1.0-2 S 22 P 6$ As24 Ne-like 2S2 2P5 ( 2P*1) 5D (12*) j=1.0 - 2S2 2P6 As24 Ne-like 2S2 2P5 (2P*1) 5D $\left(12^{\star}\right) j=1.0-2 S 22 P 6$ AS24 Ne-like 2S2 2P5 ( $\left.2 P^{*} 1\right)$ 5D $(12 *) j=1.0-2 S 2$ 2P6 As24 Ne-like 2S2 2P5 ( $2 P * 2)$ 5D $\left(23^{*}\right) j=1.0-2 S 22 P 6$ As24 Ne-like 2S2 2P5 ( 2P*2) 5D (23*) j=1.0 - 2S2 2P6 As24 Ne-like 2S2 2P5 ( 2P*2) 5D $\left(23^{*}\right) j=1.0-2 S 2$ 2P6 As24 Ne-like 2S2 2P5 ( 2P*2) 5D $\left(23^{*}\right) j=1.0-2 S 22 P 6$ As24 Ne-like 2S 2P6 4P (1P*) $j=1.0-2 S 2 \quad 2 P 6$
(2D) $j=1.5$ (2D) $j=1.5$

(2D) $j=1.5$ (2D) $j=1.5$ (2D) $j=1.5$ (2D) $j=1.5$ (2D) $j=1.5$ (2D) $j=1.5$ (2D) $j=1.5$ (2D) $j=1.5$ (2D) $j=1.5$ (2D) $j=1.5$

(2D) $j=2.5$ (2D) $j=2.5$ (2D) $j=2.5$ (2D) $j=2.5$ (2D) $j=2.5$ (2D) $j=1.5$ (2D) $j=1.5$

(2D) $j=1.5$

(2D) $j=1.5$

(2D) $j=1.5$

(2D) $j=2.5$

(2D) $j=2.5$

(2D) $j=2.5$

(2D) $j=2.5$

(2D) $j=2.5$

(2D) $j=2.5$

(2D) $j=2.5$

(2D) $j=2.5$

(2D) $j=2.5$

(2D) $j=2.5$

(2S) $j=.5$

(2D) $j=2.5$

(2S) $j=.5$

(1s) $j=.0$

(IS) $j=.0$

(1s) $j=.0$

(1s) $j=.0$

(1s) $j=.0$

(1s ) $j=.0$

(1s) $j=.0$

(1s) $j=.0$

(1s) $j=.0$

(1s) $j=.0$

(1s) $j=.0$
8.422 quartz 100

8.422 PET 002

8.422 EDT 020

8.422 TAP 100

8.422 RAP 100

8.422 KAP 100

8.507 quartz 100

8.507 PET 002

8.507 EDT 020

8.507 TAP 100

8.507 RAP 100

8.507 KAP 100

8.517 PET 002

8.517 EDT 020

8.517 TAP 100

8.517 RAP 100

8.517 KAP 100

8.534 PET 002

8.534 EDT 020

8.534 TAP 100

8.534 RAP 100

8.534 KAP 100

8.543 PET 002

8.543 EDT 020

8.543 TAP 100

8.543 RAP 100

8.543 KAP 100

8.567 PET 002

8.567 EDT 020

8.567 TAP 100

8.567 RAP 100

8.567 KAP 100

19.090 mica 002

19.649 mica 002

$26.495 \mathrm{KAP} 100$

5.801 calcite 200

5.811 calcite 200

5.973 calcite 200

5.973 Si 111

5.973 sylvite 200

5.973 fluorite 111

6.081 si 111

6.081 sylvite 200

6.081 fluorite 111

6.081 TAP 100

6.106 Si 111
$8.512 \quad 81.661$

$8.742 \quad 74.450$

$8.808 \quad 72.975$

$25.763 \quad 78.728$

$26.116 \quad 75.342$

26.63471 .557

$8.512 \quad 88.036$

8.74276 .685

$8.808 \quad 74.978$

$25.763 \quad 82.141$

$26.116 \quad 77.746$

$26.634 \quad 73.378$

$8.742 \quad 76.973$

$8.808 \quad 75.231$

$25.763 \quad 82.645$

$26.116 \quad 78.060$

$26.634 \quad 73.605$

$8.742 \quad 77.476$

$8.808 \quad 75.671$

$\begin{array}{ll}25.763 & 83.591\end{array}$

$26.116 \quad 78.614$

$26.634 \quad 73.998$

$8.742 \quad 77.751$

$8.808 \quad 75.910$

$25.763 \quad 84.154$

$26.116 \quad 78.918$

$26.634 \quad 74.210$

$8.742 \quad 78.516$

$8.808 \quad 76.566$

$25.763 \quad 86.024$

$26.116 \quad 79.772$

$26.634 \quad 74.790$

$19.942 \quad 73.191$

19.94280 .166

$26.634 \quad 84.144$

$6.071 \quad 72.848$

6.07173 .171

$6.071 \quad 79.691$

$6.271 \quad 72.266$

$6.292 \quad 71.677$

6.30871 .243

$6.271 \quad 75.860$

$6.292 \quad 75.120$

$6.308 \quad 74.582$

$25.763 \quad 70.760$

6.27176 .828 


\begin{tabular}{|c|c|c|c|c|c|c|c|c|}
\hline & e-like & is & $2 \mathrm{P} 6$ & & $4 \mathrm{P}$ & $\left(1 P^{\star}\right) j=1.0$ & $-2 s 2$ & . \\
\hline As24 & e-like & $S$ & $2 P 6$ & & $4 P$ & $\left(1 P^{*}\right) j=1.0$ & $-2 s 2$ & \\
\hline 524 & le-like & $\mathbf{S}$ & $2 P 6$ & & $4 P$ & $\left(1 P^{*}\right) j=1.0$ & & \\
\hline 24 & Ne-like & $2 S$ & $2 P 6$ & & $4 P$ & *) $j=1.0$ & 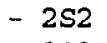 & \\
\hline 24 & le-like & $\mathbf{S}$ & $2 \mathrm{P} 6$ & & $4 P$ & $\left(3 P^{*}\right) j=1.0$ & & \\
\hline 524 & e-like & S & 2P6 & & $4 P$ & $\left(3 P^{*}\right) j=1.0$ & 32 & \\
\hline 24 & e-like & $S$ & 2P6 & & $4 P$ & $\left(3 P^{\star}\right) j=1.0$ & $\$ 2$ & \\
\hline 24 & & S2 & 2P5 & $\left(2 P^{\star} 1\right)$ & $4 \mathrm{D}$ & $\star j j=1.0$ & 2 & \\
\hline 24 & & & 2P5 & $(2 p \star 1)$ & 4D & *) $j=1.0$ & & \\
\hline 24 & $e$ & & & & & 1.0 & & \\
\hline 24 & $\mathrm{Ne}-\mathrm{I}$ & $\mathbf{S 2}$ & 2P5 & $(2 \mathrm{P} \star 1)$ & $4 D$ & 1.0 & 2 & \\
\hline 24 & e- & $\$ 2$ & $2 \mathrm{P} 5$ & $(2 \mathrm{p} * 1)$ & $4 D$ & 1.0 & & \\
\hline 24 & e- & $\$ 2$ & $2 \mathrm{P} 5$ & $(2 P \star 2)$ & $4 D$ & *) $j=1.0$ & & \\
\hline 24 & ke & $\$ 2$ & $2 \mathrm{P} 5$ & $(2 \mathrm{p} * 2)$ & $4 \mathrm{D}$ & *) $j=1.0$ & & \\
\hline 4 & ke & $\mathbf{S}$ & 2P6 & & $3 P$ & $\left(1 P^{\star}\right) j=1.0$ & & \\
\hline 24 & e & & $2 \mathrm{P} 6$ & & & $\left(3 P^{*}\right) j$ & & \\
\hline 24 & e- & 2 & 21 & $(2 \mathrm{p} * 1)$ & & 1.0 & & \\
\hline 24 & $\mathrm{Ne}-$ & 2 & 2P5 & $(2 p \star 1)$ & $D$ & 1.0 & & \\
\hline 24 & $\mathrm{Ne}-$ & 2 & 5 & $(2 p \star 1)$ & & 1.0 & 2 & \\
\hline$\$ 24$ & $\mathrm{Ne}-$ & $\mathrm{S} 2$ & $2 \mathrm{P} 5$ & $\left(2 p^{\prime} 1\right)$ & & $j=1.0$ & 2 & \\
\hline 524 & $\mathrm{Ne}-$ & 252 & 2P5 & $(2 p * 1)$ & o & 1.0 & & \\
\hline$\$ 24$ & $\mathrm{Ne}-$ & $2 S 2$ & 2P5 & $(2 \mathrm{P} * 2)$ & D & $j=1.0$ & & \\
\hline$s 24$ & $\mathrm{Ne}-$ & $\$ 2$ & $2 \mathrm{P} 5$ & $\left(2 p^{\star} 2\right)$ & $D$ & $j=1.0$ & & \\
\hline s24 & $\mathrm{Ne}-$ & 252 & 2P5 & $(28 * 2)$ & $D$ & *) $j=1.0$ & & \\
\hline 24 & $\mathrm{Ne}-$ & $2 S 2$ & 2P5 & $(2 \mathrm{P} \star 2)$ & $3 D$ & $=1.0$ & & \\
\hline 524 & $\mathrm{Ne}-$ & 252 & 21 & $(2 \mathrm{P} * 2)$ & $3 D$ & $j=1.0$ & & \\
\hline$s 24$ & $\mathrm{Ne}-$ & 252 & 2P5 & $(2 P * 2)$ & $3 D$ & $\left(23^{*}\right) j=1.0$ & & \\
\hline 824 & $\mathrm{Ne}$ & 52 & 2P5 & $(2 P * 2)$ & $3 D$ & $\left(22^{*}\right) j=1.0$ & & \\
\hline As24 & $\mathrm{Ne}$ & $\mathbf{S 2}$ & 2 & $(2 P \star 2)$ & 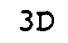 & 1.0 & - & \\
\hline As24 & $\mathrm{Ne}-$ & 252 & $2 P 5$ & $2 p * 2)$ & $3 D$ & $j=1.0$ & 2 & \\
\hline As24 & $\mathrm{Ne}-$ & S2 & & $2 P \star 2)$ & $3 D$ & $j=1.0$ & 4 & \\
\hline As24 & $\mathrm{Ne}-1$ & $2 S 2$ & $2 \mathrm{P} 5$ & $(2 \mathrm{P} * 2)$ & 3D & $\left(22^{\star}\right) j=1.0$ & & \\
\hline As25 & F-like & 252 & & $(15$ & 4D & $(2 D$ & & \\
\hline As25 & F-like & $2 s 2$ & $2 P 4$ & ( 15 & 4D & $(2 D) j=1.5$ & & \\
\hline As25 & F-like & $2 S 2$ & $2 \mathrm{P} 4$ & $(15)$ & 4D & $(2 D) j=1.5$ & & \\
\hline 25 & F-like & 252 & $2 P 4$ & ( 1s & D & (2D) $j=1.5$ & & \\
\hline As25 & $F-1$ & $2 \$ 2$ & $2 \mathrm{P} 4$ & 15 & $4 D$ & $(2 D)$ & S2 & \\
\hline As25 & $F-1 j$ & $2 s 2$ & $2 \mathrm{P} 4$ & 1s & $4 D$ & $(2 D) j=1.5$ & $\mathbf{S 2}$ & \\
\hline As25 & $F-1 i$ & $2 \mathrm{~S} 2$ & $2 \mathrm{P} 4$ & is & $4 D$ & (2D) $j=1.5$ & -2 & \\
\hline As 25 & $F-1 i$ & 252 & 2P4 & $1 D$ & 4D & $(2 D) j=2.5$ & 52 & \\
\hline As25 & $F-I i$ & $2 S 2$ & $2 \mathrm{P} 4$ & $1 D$ & $4 D$ & (2D) $) j=2.5$ & - & \\
\hline As 25 & F - like & 252 & $2 \mathrm{P} 4$ & $1 D$ & $4 D$ & $(2 D) j=2.5$ & - & \\
\hline As25 & F-like & $2 S 2$ & $2 \mathrm{P} 4$ & $1 D$ & 4D & $(2 D) j=2.5$ & & \\
\hline & $\mathbf{F}-1$ & $2 \mathrm{~S} 2$ & 2P4 & $1 D$ & $4 \mathrm{D}$ & $(2 D) j=2.5$ & & \\
\hline & - & $2 \mathrm{~S} 2$ & & $1 D$ & 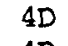 & $(2 D) j=2.5$ & 52 & \\
\hline & - & 0 & & ID & 4D & $(2 D) j=2.5$ & 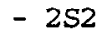 & \\
\hline
\end{tabular}

(1s) $j=.0 \quad 6.106$ sylvite $200 \quad 1$ (1s) $j=.0 \quad 6.106$ fluorite $111 \quad 1$ (1s) $j=.0$ (1s) $j=.0$ (1s) $j=.0$ (1s) $j=.0$ (1s) $j=.0$ (1S) $j=.0$ (1s) $j=.0$ (1s) $j=.0$ (1S) $j=.0$ (1s) $j=.0$ (1s) $j=.0$ (1s) $j=.0$ (1s) $j=.0$ (1s) $j=.0$ (1s) $j=.0$ (1s) $j=.0$ (1s) $j=.0$ (1s) $j=.0$ (1s) $j=.0$ (1S) $j=.0$ (1s) $j=.0$ (1s) $j=.0$ (1S) $j=.0$ (1s) $j=.0$ (1s) $j=.0$ (1s) $j=.0$ (1s) $j=.0$ (1s) $j=.0$ (1s) $j=.0$ (IS) $j=.0$ $\left(2 P^{*}\right) j=.5$ $\left(2 P^{*}\right) j=.5$ $\left(2 P^{*}\right) j=.5$ $\left(2 P^{*}\right) j=.5$ $\left(2 P^{\star}\right) j=.5$ $\left(2 P^{\star}\right) j=.5$ $\left(2 P^{\star}\right) j=.5$ $\langle 2 P *\rangle j=1.5$ $\left(2 P^{*}\right) j=1.5$ $\left(2 P^{*}\right) j=1.5$ $\left(2 P^{*}\right) j=1.5$ $\left(2 P^{\star}\right) j=1.5$ $\left(2 P^{\star}\right) j=1.5$ $\left(22^{*}\right) j=1.5$
6.106 fluorite 1111

TAP 100

6.117 sylvite 200

6.117 fluorite 111

6.117 TAP 100

6.551 KBr 200

6.551 quartz 101

6.551 graphite 002

6.551 mica 002

6.551 KAP 100

6.676 quartz 101

6.676 graphite 002

7.777 beryl 100

7.829 beryl 100

8.271 topaz 002

8.271

271

100

8.464 quartz 100

8.464 PET 002

8.464 EDT 020

8.464 TAP 100

8.464 RAP 100

8.464 KAP 100

8.567 PET 002

8.567 EDT 020

8.567 TAP 100

8.567

8.567

RAP 100

6.217

6.217

6.217

6.217

6.217

6.217

6.217

6.236

6.236

6.236

6.236

6.236

6.236

6.236
$6.292 \quad 76.034$

$6.308 \quad 75.461$

$25.763 \quad 71.446$

$6.271 \quad 77.276$

$6.292 \quad 76.455$

$6.308 \quad 75.865$

$25.763 \quad 71.756$

$6.584 \quad 84.261$

$6.687 \quad 78.425$

6.69678 .055

$19.942 \quad 80.234$

26.63479 .690

$6.687 \quad 86.713$

$6.696 \quad 85.571$

$15.954 \quad 77.143$

$15.954 \quad 78.946$

$8.374 \quad 81.004$

8.51276 .333

$8.742 \quad 71.107$

$25.763 \quad 74.392$

$26.116 \quad 71.825$

$8.512 \quad 83.912$

$8.742 \quad 75.512$

$8.808 \quad 73.934$

$\begin{array}{lll}25.763 & 80.265\end{array}$

$26.116 \quad 76.477$

$26.634 \quad 72.434$

8.74278 .516

$8.808 \quad 76.566$

$25.763 \quad 86.024$

$26.116 \quad 79.772$

$26.634 \quad 74.790$

$6.271 \quad 82.476$

6.29281 .145

$6.308 \quad 80.256$

6.53272 .134

6.58470 .780

$25.763 \quad 74.853$

$26.116 \quad 72.216$

$6.271 \quad 83.944$

$6.292 \quad 82.350$

$6.308 \quad 81.335$

6.53272 .685

$6.584 \quad 71.288$

$25.763 \quad 75.514$

$26.116 \quad 72.770$ 
As25 F-1ike 2S2 2P4 ( $3 P$ ) $4 D(4 P) j=2.5-2 S 22 P 5$ As25 $F$-like 2S2 2P4 (3P) 4D (4P) j=2.5 - 2S2 2P5 As25 F-iike 2S2 2P4 ( $3 P$ ) 4D (4P) $j=2.5-2 S 22 P 5$ As25 F-like 2S2 $2 P 4$ ( $3 P$ ) $4 D(4 P) j=2.5-2 S 22 P 5$ As25 F -like 2S2 2P4 ( $3 P$ ) $4 D(4 P) j=2.5-2 S 22 P 5$ $A s 25 \quad F-l i k e$ 2S2 2P4 (3P) $4 D(4 P) j=2.5-2 S 22 P 5$ As25 F-like 2S2 2P4 ( $3 P) 4 D(4 P) j=2.5-2 S 22 P 5$ As25 $F$-1ike 2S2 2P4 ( $3 P$ ) 4D (4P) $j=2.5-2 S 22 P 5$ As25 F-like 2S2 2P4 (3P) $4 D(4 F) j=2.5-2 S 2$ 2P5 As25 $F$-like 2S2 2P4 ( $3 P$ ) $4 D(4 F) j=2.5-2 S 22 P 5$ As25 F-like 2S2 2P4 ( $3 P$ ) $4 D(4 F) j=2.5-2 S 22 P 5$ As25 $F$-like 2S2 2P4 ( $3 P$ ) $4 D(4 F) j=2.5-2 S 22 P 5$ As25 F-like 2S2 2P4 (3P) 4D (4F) $j=2.5-2 S 22 P 5$ As25 F-like 2S2 2P4 ( 3P) $4 D(4 F) j=2.5-2 S 22 P 5$ As25 $F$-like 2S2 2P4 (3P) $4 D(4 F) j=2.5-2 S 22 P 5$ As25 F-like 2S2 2P4 ( $3 P$ ) $4 D(4 F) j=2.5-2 S 22 P 5$ As25 F-like 2S2 2P4 ( $1 D$ ) 4D (2D) j=1.5 - 2S2 2P5 As25 $F$-like 2S2 2P4 ( 1D) 4D (2D) $j=1.5$ - 2S2 2P5 As25 F-like 2S2 2P4 (1D) 4D (2D) j=1.5 - 2S2 2P5 As25 F-like 2S2 2P4 ( 1D) $4 D(2 D) j=1.5$ - 2S2 2P5 As25 F-like 2S2 2P4 ( 1D) 4D (2D) j=1.5 - 2S2 2P5 As25 F-like 2S2 2P4 ( 1D) $4 D(2 D) j=1.5-2 S 22 P 5$ As25 F-like 2S2 2P4 ( $1 D$ ) $4 D(2 D) j=1.5$ - 2S2 2P5 As25 F-like 2S2 2P4 ( $1 D$ ) 4D (2D) $j=1.5-2 S 22 P 5$ $A s 25 \quad F$-like 2S2 2P4 ( $1 D$ ) $4 S(2 D) j=2.5-2 S 22 P 5$ As25 F-like 2S2 2P4 (1D) 4S (2D) $j=2.5-2 S 22 P 5$ As25 F-like 2S2 2P4 (1D) $4 S(2 D) j=2.5-2 S 2$ 2P5 As25 F-like 2S2 2P4 ( $1 D$ ) $4 S$ (2D) $j=2.5-2 S 22 P 5$ As25 F-like 2S2 2P4 ( $1 D$ ) $4 S(2 D) j=2.5-2 S 22 P 5$ As25 F-like 2S2 2P4 ( $1 D$ ) $4 S$ (2D) $j=2.5-2 S 2$ 2P5 As25 F-like 2S2 2P4 ( $1 D$ ) $4 S(2 D) j=2.5-2 S 22 P 5$ As25 F-like 2S2 2P4 ( $1 D$ ) $4 S$ (2D) $j=2.5-2 S 22 P 5$ As25 F-Iike $2 S 22 P 4$ ( $3 P$ ) $4 D(2 P) j=1.5-2 S 22 P 5$ As25 $F-1 i k e$ 2S2 2P4 ( $3 P$ ) $4 D$ (2P) $j=1.5-2 S 22 P 5$ As25 F-like 2S2 2P4 ( 3P) 4D (2P) $j=1.5-2 S 22 P 5$ As25 F-like 2S2 2P4 ( $3 P$ ) $4 D(2 P) j=1.5-2 S 22 P 5$ As25 F-like 2S2 2P4 ( $3 P$ ) 4D (2P) j=1.5 - 2S2 2P5 As25 F-like 2S2 2P4 ( 3P) 4D (2P) $j=1.5-2 S 22 P 5$ As25 F-like 2S2 2P4 ( $3 P$ ) 4D (2P) $j=1.5-2 S 22 P 5$ As25 $F$-like 2S2 2P4 ( $3 P$ ) $4 D(2 P) j=1.5-2 S 22 P 5$ As25 $F$-like 2S2 2P4 ( $3 \mathrm{P}) 4 \mathrm{~S}(4 \mathrm{P}) \mathrm{j}=1.5-2 \mathrm{~S} 22 \mathrm{P} 5$ As25. F-1ike 2S2 2P4 ( 3P ) 4S (4P) j=1.5 - 2S2 2P5 As25 F-like 2S2 2P4 ( $3 P$ ) 4S (4P)j=1.5 - 2S2 2P5 As25 F-like 2S2 2P4 ( $3 P$ ) 4S (4P) $j=1.5-2 S 2$ 2P5 As25 F-like 2S2 2P4 ( $3 P$ ) $4 S(4 P) j=1.5-2 S 22 P 5$ As25 $F$-1ike 2S2 $2 \mathrm{P} 4$ ( $3 \mathrm{P}) 4 \mathrm{~S}(4 \mathrm{P}) \mathrm{j}=1.5-2 \mathrm{~S} 22 \mathrm{P} 5$ $\left(2 P^{*}\right) j=1.5$ $\left(2 P^{\star}\right) j=1.5$ $\left(2 P^{\star}\right) j=1.5$ $\left(2 P^{*}\right) j=1.5$ $\left(2 P^{*}\right) j=1.5$ $\left(2 P^{\star}\right) j=1.5$ $\left(2 P^{\star}\right) j=1.5$ $\left(2 P^{*}\right) j=1.5$ $\left(2 P^{\star}\right) j=1.5$ $\left(2 P^{\star}\right) j=1.5$ $\left(2 P^{\star}\right) j=1.5$ $\left(2 P^{\star}\right) j=1.5$ $\left(2 p^{*}\right) j=1.5$ $\left(2 P^{\star}\right) j=1.5$ $\left(2 P^{\star}\right) j=1.5$ $\left(2 P^{*}\right) j=1.5$ $\left(2 P^{*}\right) j=.5$ $\left(2 P^{*}\right) j=.5$ $\left(2 P^{\star}\right) j=.5$ $\left(2 P^{*}\right) j=.5$ $\left(2 P^{*}\right) j=.5$ $\left(2 P^{\star}\right) j=.5$ $\left(2 P^{*}\right) j=.5$ $\left(2 P^{*}\right) j=.5$ $\left(2 P^{\star}\right) j=1.5$ $\left(2 P^{*}\right) j=1.5$ $\left(2 P^{*}\right) j=1.5$ $\left(2 P^{*}\right) j=1.5$ $\left(2 P^{*}\right) j=1.5$ $\left(2 p^{*}\right) j=1.5$ (2P*) $j=1.5$ $\left(2 P^{*}\right) j=1.5$ $\left(2 P^{*}\right) j=.5$ $\left(2 P^{\star}\right) j=.5$ $\left(2 P^{\star}\right) j=.5$ $\left(2 P^{*}\right) j=.5$ (2P*) j= $\left(2 P^{*}\right) j=.5$ $\left(2 P^{*}\right) j=.5$ $\left(2 P^{*}\right) j=.5$ $\left(2 P^{\star}\right) j=1.5$ $\left(2 P^{*}\right) j=1.5$ $\left(2 P^{\star}\right) j=1.5$ $\left(2 P^{*}\right) j=1.5$ $\left(2 P^{*}\right) j=1.5$ $\left(2 P^{\star}\right) j=1.5$
6.274 sylvite $200 \quad 1$ 6.274 fluorite 1111

6.274 Ge 111

$6.274 \mathrm{KBr} 200$

6.274 mica 002

6.274 TAP 100

6.274 RAP 100

6.274 KAP 100

6.331 Ge 111

6.331 KBr 200

6.331 quartz 101

6.331 graphite 002

6.331 mica 002

6.331 TAP 100

6.331 RAP 100

6.331 KAP 100

6.344 Ge 111

$6.344 \mathrm{KBr} 200$

6.344 quartz 101

6.344 graphite 002

6.344 mica 002

6.344 TAP 100

6.344 RAP 100

6.344 KAP 100

6.376 Ge 111

$6.376 \mathrm{KBr} 200$

6.376 guartz 101

6.376 graphite 002

6.376 mica 002

6.376 TAP 100

6.376 RAP 100

6.376 KAP 100

6.390 Ge 111

$6.390 \mathrm{KBr} 200$

6.390 guartz 101

6.390 graphite 002

6.390 mica 002

6.390 TAP 100

6.390 RAP 100

6.390 KAP 100

6.413 Ge 111

$6.413 \mathrm{KBr} 200$

6.413 quartz 101

6.413 graphite 002

6.413 mica 002

6.413 TAP 100
$6.292 \quad 85.665$

$6.308 \quad 84.049$

6.53273 .843

6.58472 .348

$19.942 \quad 70.706$

$25.763 \quad 76.934$

$26.116 \quad 73.934$

26.63470 .434

$6.532 \quad 75.749$

6.58474 .065

$6.687 \quad 71.220$

6.69670 .995

$19.942 \quad 72.253$

$25.763 \quad 79.408$

$26.116 \quad 75.853$

$26.634 \quad 71.955$

6.53276 .220

$6.584 \quad 74.482$

$6.687 \quad 71.569$

$6.696 \quad 71.340$

$19.942 \quad 72.624$ $25.763 \quad 80.056$

$26.116 \quad 76.328$

$26.634 \quad 72.320$

6.53277 .453

6.58475 .560

$6.687 \quad 72.457$

$6.696 \quad 72.215$ $19.942 \quad 73.573$ $25.763 \quad 81.869$

$26.116 \quad 77.572$

$26.634 \quad 73.250$

6.53278 .031

$6.584 \quad 76.057$

$6.687 \quad 72.860$

$6.696 \quad 72.612$

$19.942 \quad 74.005$

$25.763 \quad 82.803$

$26.116 \quad 78.156$

$26.634 \quad 73.674$

$6.532 \quad 79.047$

$6.584 \quad 76.913$

$6.687 \quad 73.541$

$6.696 \quad 73.283$

$19.942 \quad 74.741$

$25.763 \quad 84.679$ 
As25 F-1ike 2S2 2P4 ( $3 P$ ) $4 S(4 P) j=1.5-2 S 22 P 5$ As25 $F$-like 2S2 2P4 ( $3 P$ ) $4 S(4 P) j=1.5-2 S 2$ 2P5 As25 $F$-like 2S2 2P4 ( ID) 4S (2D) $j=1.5-2 S 2$ 2P5 As25 F-like 2S2 2P4 ( 1D) $4 S(2 D) j=1.5-2 S 22 P 5$ As25 F-like 2S2 2P4 ( $1 D$ ) $4 S$ (2D) $j=1.5-2 S 2$ 2P5 As25 F-like 2S2 2P4 ( $1 D$ ) $4 S$ (2D) $j=1.5-2 S 22 P 5$ As25 F-like 2S2 2P4 ( 1D) 4S (2D) $j=1.5-2 S 2$ 2P5 As25 F-like 2S2 2P4 ( $1 D$ ) $4 S$ (2D) $j=1.5$ - 2S2 2P5 As25 F-like 2S2 2PA ( $1 D$ ) 4S (2D) $j=1.5-2 S 22 P 5$ As25 F-like 2S2 2P4 ( 3P ) 4S (2P) $j=1.5$ - 2S2 2P5 As25 F-like 2S2 2P4 (3P) 4S (2P) $j=1.5-2 S 22 P 5$ As25 F-like 2S2 2P4( $3 P$ ) $4 S(2 P) j=1.5-2 S 22 P 5$ As25 F-like 2S2 2P4 ( $3 P$ ) $4 S(2 P) j=1.5-2 S 22 P 5$ As25 F-1ike 2S2 2P4. ( $3 P$ ) $4 S(2 P) j=1.5-2 S 22 P 5$ As25 F-like 2S 2P5 ('IP*) 3P (2D) $j=2.5-2 S 2$ 2P5 As25 F -like $2 S{ }^{\circ} 2 P 5\left(1 P^{*}\right) 3 P(2 D) j=2.5-2 S 22 P 5$ As25 F-like 2S. $2 P 5\left(3 P^{*}\right) 3 P(2 S) j=.5-2 S 2$ 2P5 As25 $F$-like $2 S$ 2P5 (3P*) $3 P(2 S) j=.5-2 S 22 P 5$ As25 F-like 2S 2P5 ( $\left.1 P^{*}\right) 3 P(2 P) j=1.5-2 S 2$ 2P5 As25 F-like 2S 2P5 ( $\left.1 P^{*}\right)$ 3P (2P) j=1.5 - 2S2 2P5 As25 F-like $2 S$ 2P5 (1P*) 3P (2P) j=.5- $2 S 22 P 5$ As25 $F$-like $2 S$ 2P5 ( $\left.1 P^{*}\right) 3 P(2 P) j=.5-2 S 22 P 5$ As25 F-like $2 S$ 2P5 ( $\left.1 P^{*}\right) 3 P(2 D) j=1.5-2 S 22 P 5$ As25 F-like 2S 2P5 (1P*) 3P (2D) $j=1.5-2 S 2$ 2P5 As25 $F$-like $2 S$ 2P5 (3P*) $3 P(4 P) j=2.5-2 S 22 P 5$ As25 F-like 2S 2P5 (3P*) 3P (4P) j=2.5 - 2S2 2P5 As25 F-like 2S 2P5 ( $\left.3 P^{*}\right) 3 P(2 P) j=1.5-2 S 2$ 2P5 As25 $F$-like $2 S$ 2P5 (3P*) $3 P(2 P) j=1.5$ - 2S2 $2 P 5$ As25 $F$-like 2S 2P5 (3P*) $3 P(2 D) j=2.5-2 S 22 P 5$ As25 F-like 2S 2P5 ( $\left.3 P^{*}\right) 3 P(2 D) j=2.5-2 S 2$ 2P5 As25 F-like 2S 2P5 ( $\left.3 P^{*}\right) 3 P(2 D) j=1.5-2 S 22 P 5$ As25 $F$-like $2 S$ 2P5 (3P*) $3 P(2 S) j=.5-2 S 2$ 2P5 As25 F-like $2 S$ 2P5 ( $\left.3 P^{*}\right) 3 P(4 D) j=2.5-2 S 2$ 2P5 As25 F-like 2S 2P5 ( $\left.3 P^{*}\right)$ 3P (4P) $j=1.5-2 S 2$ 2P5 As25 F-like 2S2 2P4 ( $1 D)$ ) 3D (2P) $j=.5-2 S 22 P 5$ As25 F-like 2S2 2P4 (1D) 3D (2P) $j=.5-2 S 22 P 5$ As25 F-like 2S2 $2 P 4$ ( $1 S$ ) 3D (2D) $j=1.5-2 S 22 P 5$ As25 F-like 2S2 2P4 ( $1 S$ ) 3D (2D) $j=1.5-2 S 2$ 2P5 As25 F-like 2S2 2P4 ( 1D) 3D (2D) $j=2.5-2 S 2$ 2P5 As25 F-like 2S2 2P4 ( $1 D)$ 3D (2D) $j=2.5-2 S 22 P 5$ As25 $F$-like 2S2 2P4 (1D) 3D (2S) $j=.5-2 S 22 P 5$ As25 F-like 2S2 2P4 (1D) 3D (2S) j=.5 - 2S2 2P5 As25 F-like 2S2 2P4 ( $3 P)$ 3D (2D) $j=2.5-2 S 22 P 5$ As25 F-like 2S2 2P4 ( 3P) 3D (2D) $j=2.5-2 S 22 P 5$ As25 F-like 2S2 2P4 (3P) 3D (4D) $j=1.5-2 S 2$ 2P5 As25 F-like 2S2 $2 P 4(3 P) 3 D(4 D) j=1.5-2 S 22 P 5$ $\left(2 P^{\star}\right) j=1.5$ $\left(2 P^{\star}\right) j=1.5$ $\left(2 P^{\star}\right) j=.5$ $\left(2 P^{\star}\right) j=.5$ $\left(2 P^{*}\right) j=.5$ $\left(2 P^{*}\right) j=.5$ $\left(2 P^{*}\right) j=.5$ $\left(2 P^{*}\right) j=.5$ $\left(2 P^{\star}\right) j=.5$ $\left(2 P^{\star}\right) j=1.5$ $\left(2 P^{\star}\right) j=1.5$ $\left(2 P^{*}\right) j=1.5$ $\left(2 P^{*}\right) j=1.5$ $\left(2 P^{*}\right) j=1.5$ $\left(2 P^{*}\right) j=1.5$ $\left(2 P^{*}\right) j=1.5$ $\left(2 P^{*}\right) j=1.5$ $\left(2 P^{*}\right) j=1.5$ $\left(2 P^{*}\right) j=.5$ $\left(2 P^{*}\right) j=.5$ $\left(2 P^{*}\right) j=.5$ $\left(2 P^{*}\right) j=.5$ $\left(2 P^{*}\right) j=.5$ $\left(2 P^{*}\right) j=.5$ $\left(2 P^{\star}\right) j=1.5$ (2P*) $j=1.5$ $\left(2 P^{*}\right) j=1.5$ $\left(2 P^{*}\right) j=1.5$ $\left(2 P^{*}\right) j=1.5$ $\left(2 P^{*}\right) j=1.5$ $\left(2 P^{*}\right) j=.5$ $\left(2 P^{*}\right) j=.5$ $\left(2 P^{*}\right) j=1.5$ $\left(2 P^{*}\right) j=.5$ $\left(2 P^{*}\right) j=1.5$ $\left(2 P^{*}\right) j=1.5$ $\left(2 P^{*}\right) j=.5$ $\left(2 P^{*}\right) j=.5$ $\left(2 P^{*}\right) j=1.5$ $\left(2 P^{*}\right) j=1.5$ $\left(2 P^{*}\right) j=1.5$ $\left(2 P^{\star}\right) j=1.5$ $\left(2 P^{*}\right) j=1.5$ $\left(2 P^{*}\right) j=1.5$ $\left(2 P^{*}\right) j=1.5$ $\left(2 P^{\star}\right) j=1.5$
6.413 RAP 100

6.413 KAP 100

6.492 Ge 111

$6.492 \mathrm{KBr} 200$

6.492 quartz 101

6.492 graphite 002

6.492 mica 002

6.492 RAP 100

6.492 KAP 100

$6.551 \mathrm{KBr} 200$

6.551 quartz 101

6.551 graphite 002

6.551 mica 002

6.551 KAP 100

7.306 InSt 111

7.306 gypsum 020

7.438 InSb 111

7.438 gypsum 020

7.459 InSo 111

7.459 gypsum 020

7.473 InSb 111

7.473 gypsum 020

7.515 gypsum 020

7.515 beryl 100

7.529 gypsum 020

7.529 beryl 100

7.569 gypsum 020

7.569 beryl 100

7.581 gypsum 020

7.581 beryl 100

7.602 beryl 100

7.613 beryl 100

7.655 beryl 100

7.701 beryl 100

7.902 topaz 002

7.902 beryl 100

7.923 topaz 002

7.923 beryl 100

7.951 topaz 002

7.951 beryl 100

7.969 topaz 002

7.969 bery 1100

8.020 topaz 002

8.020 quartz 100

8.047 topaz 002

8.047 quartz 100
$26.116 \quad 79.183$

$26.634 \quad 74.393$

$6.532 \quad 83.656$

$6.584 \quad 80.411$

$6.687 \quad 76.129$

$6.696 \quad 75.821$

$19.942 \quad 77.589$

$26.116 \quad 83.897$

$26.634 \quad 77.160$

$6.584 \quad 84.261$

$6.687 \quad 78.425$

$6.696 \quad 78.055$

$19.942 \quad 80.234$

$26.634 \quad 79.690$

$7.481 \quad 77.583$

$15.185 \quad 74.210$

$7.481 \quad 83.854$

$15.185 \quad 78.422$

$\begin{array}{ll}7.481 & 85.605\end{array}$

$15.185 \quad 79.240$

$\begin{array}{ll}7.481 & 87.350\end{array}$

$15.185 \quad 79.821$

$15.185 \quad 81.807$

$15.954 \quad 70.404$

$15.185 \quad 82.585$

$\begin{array}{ll}15.954 & 70.707\end{array}$

$15.185 \quad 85.491$

15.95471 .596

$15.185 \quad 86.846$

$15.954 \quad 71.871$

$15.954 \quad 72.362$

$15.954 \quad 72.625$

$15.954 \quad 73.665$

$15.954 \quad 74.884$

8.37470 .671

$15.954 \quad 82.137$

8.37471 .110

$15.954 \quad 83.329$

8.37471 .711

$15.954 \quad 85.373$

$8.374 \quad 72.108$

$15.954 \quad 87.434$

$8.374 \quad 73.281$

8.51270 .424

8.37473 .935

$8.512 \quad 70.974$ 


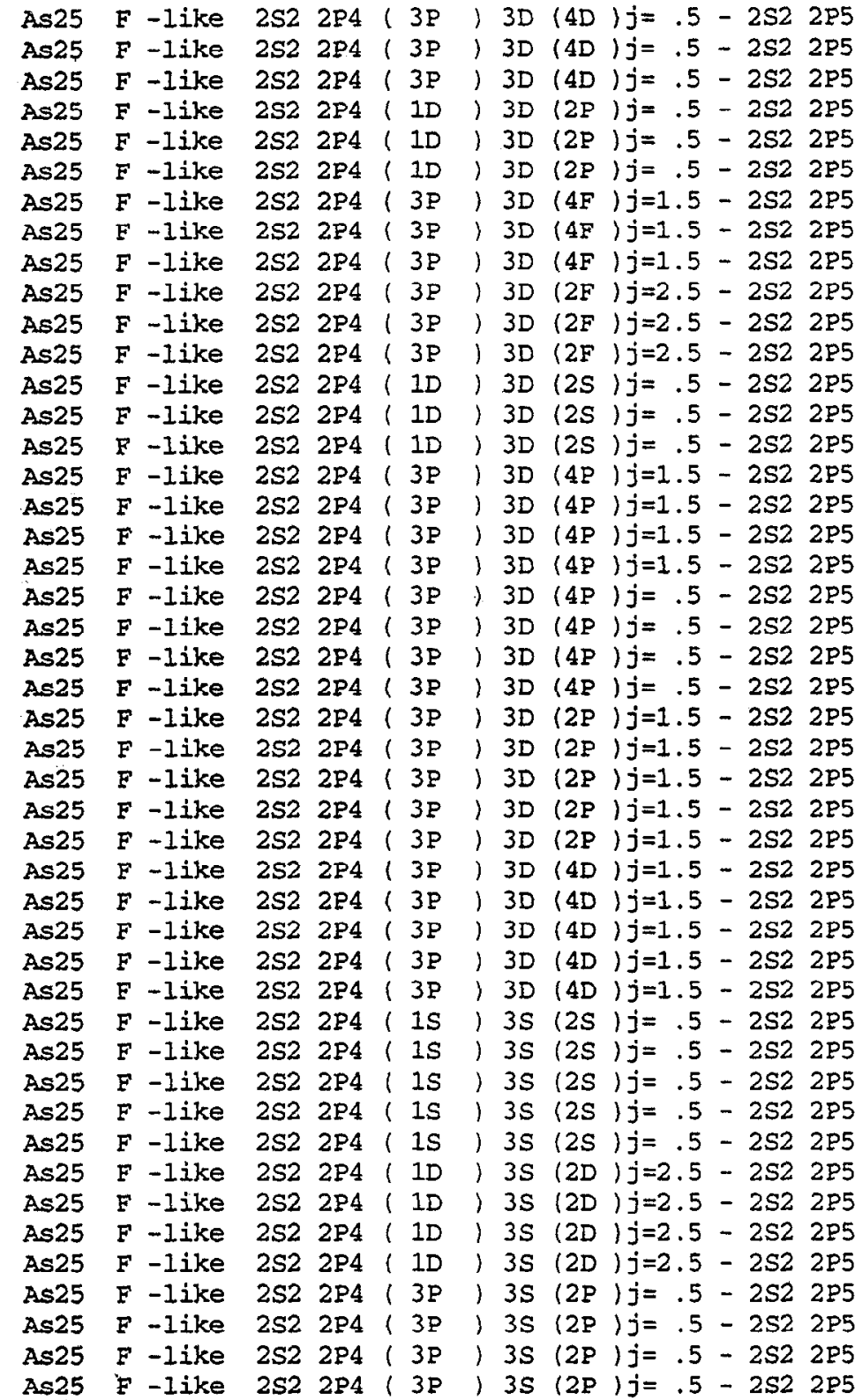

$\left(2 P^{\star}\right) j=1.5$ $\left(2 P^{\star}\right) j=1.5$ $\left(2 P^{*}\right) j=1.5$ $\left(2 P^{*}\right) j=.5$ $\left(2 P^{\star}\right) j=.5$ $\left(2 P^{*}\right) j=.5$ $\left(2 P^{*}\right) j=1.5$ $\left(2 P^{\star}\right) j=1.5$ $\left(2 P^{*}\right) j=1.5$ $\left(2 P^{\star}\right) j=1.5$ $\left(2 p^{*}\right) j=1.5$ $\left(2 P^{\star}\right) j=1.5$ $\left(2 \mathrm{P}^{\star}\right) j=.5$ $\left(2 P^{*}\right) j=.5$ $\left(2 P^{*}\right) j=.5$ $\left(2 P^{*}\right) j=1.5$ $\left(2 \mathrm{P}^{\star}\right) j=1.5$ $\left(2 P^{\star}\right) j=1.5$ $\left(2 P^{*}\right) j=1.5$ $\left(2 P^{\star}\right) j=1.5$ $\left(2 P^{\star}\right) j=1.5$ $\left(2 P^{*}\right) j=1.5$ $\left(2 P^{\star}\right) j=1.5$ $\left(2 P^{\star}\right) j=.5$ $\left(2 P^{\star}\right) j=.5$ $\left(2 P^{\star}\right) j=.5$ $\left(2 P^{*}\right) j=.5$ $\left(2 P^{*}\right) j=.5$ $\left(2 P^{\star}\right) j=.5$ $\left(2 P^{\star}\right) j=.5$ $\left(2 \mathrm{P}^{\star}\right) j=.5$ $\left(2 P^{\star}\right) j=.5$ $\left(2 P^{\star}\right) j=.5$ $\left(2 P^{\star}\right) j=.5$ $\left(2 P^{\star}\right) j=.5$ $\left(2 P^{\star}\right) j=.5$ $\left(2 P^{*}\right) j=.5$ $\left(2 P^{*}\right) j=.5$ $\left(2 P^{\star}\right) j=1.5$ $\left(2 P^{*}\right) j=1.5$ $\left(2 P^{*}\right) j=1.5$ $\left(2 P^{\star}\right) j=1.5$ $\left(2 P^{\star}\right) j=1.5$ $\left(2 P^{*}\right) j=1.5$ $\left(2 P^{\star}\right) j=1.5$ $\left(2 P^{\star}\right) j=1.5$
8.070 topaz 002

8.070 quartz 100

8.070 TAP 100

8.097 topaz 002

8.097 quartz 100

8.097 TAP 100

8.137 topaz 002

8.137 cuartz 100

8.137 TAP 100

8.150 topaz 002

8.150 quartz 100

8.150 TAP 100

8.166 topaz 002

8.166 quartz 100

8.166 TAP 100

8.181 topaz 002

8.181 quartz 100

8.181 TAP 100

8.181 RAP 100

8.200 topaz 002

8.200 guartz 100

8.200 TAP 100

8.200 RAP 100

8.217 topaz 002

8.217 quartz 100

8.217 PET 002

8.217 TAP 100

8.217 RAP 100

8.248 topaz 002

8.248 quartz 100

8.248 PET 002

8.248 TAP 100

8.248 RAP 100

8.534 PET 002

8.534 EDT 020

8.534 TAP 100

8.534 RAP 100

8.534 KAP 100

8.588 PET 002

8.588 EDT 020

8.588 RAP 100

8.588 KAP 100

8.641 PET 002

8.641 EDT 020

8.641 RAP 100

8.641 KAP 100
$8.374 \quad 74.514$

$8.512 \quad 71.455$

$\begin{array}{lll}25.763 & 70.005\end{array}$

$8.374 \quad 75.222$

$8.512 \quad 72.035$

$25.763 \quad 70.538$

$8.374 \quad 76.336$

$8.512 \quad 72.930$

$25.763 \quad 71.356$

$8.374 \quad 76.718$

$8.512 \quad 73.230$

$25.763 \quad 71.629$

$8.374 \quad 77.203$

$8.512 \quad 73.608$

$\begin{array}{lll}25.763 & 71.971\end{array}$

$8.374 \quad 77.675$

$8.512 \quad 73.969$

$\begin{array}{lll}25.763 & 72.297\end{array}$

$26.116 \quad 70.013$

$8.374 \quad 78.300$

$8.512 \quad 74.439$

\begin{tabular}{ll}
$25.763 \quad 72.719$ \\
\hline
\end{tabular}

$26.116 \quad 70.382$

$8.374 \quad 78.888$

$8.512 \quad 74.872$

$8.742 \quad 70.042$

$25.763 \quad 73.105$

$26.116 \quad 70.718$

$8.374 \quad 80.048$

$8.512 \quad 75.693$

$8.742 \quad 70.646$

$25.763 \quad 73.832$

$26.116 \quad 71.346$

$8.742 \quad 77.476$

$8.808 \quad 75.671$

$25.763 \quad 83.591$

$26.116 \quad 78.614$

$26.634 \quad 73.998$

$\begin{array}{lll}8.742 & 79.230\end{array}$

$\begin{array}{ll}8.808 & 77.167\end{array}$

$26.116 \quad 80.582$

$26.634 \quad 75.315$

$8.742 \quad 81.282$

$8.808 \quad 78.825$

$26.116 \quad 83.030$

$26.634 \quad 76.731$ 
As25 $F$-like 2S2 2P4 ( 3P ) 3S (4P) j=1.5 - 2S2 2P5 As25 F-like 2S2 2P4 ( $3 P$ ) 3S (4P) $j=1.5$ - 2S2 2P5 As25 $F$-like 2S2 2P4 ( $3 P$ ) 3S (4P) $j=1.5$ - 2S2 2P5 As25 F-like 2S2 2P4 (3P) 3S (4P) $j=1.5$ - 2S2 2P5 As25 F-like 2S2 2P4 ( 3P ) 3S $(4 P) j=.5-2 S 22 P 5$ As25 $F$-like 2S2 2P4 ( 3P) 3S (4P) $j=.5-2 S 22 P 5$ As25 $F$-1ike 2S2 2P4 ( ID) 3S (2D) $j=1.5-2 S 22 P 5$ As25 $F$-1ike 2S2 2P4 ( $3 P$ ) 3S (2P) $j=1.5-2 S 22 P 5$ As25 F-Iike 2S2 2P4 ( $3 P$ ) $3 S(4 P) j=2.5-2 S 22 P 5$ As26 0 -like 2S2 2P3 ( 2P*) $4 D\left(3 D^{\star}\right) j=2.0-2 S 22 P 4$ As26 O-1ike 2S2 2P3 (2P*) $4 D\left(3 D^{*}\right) j=2.0-2 S 22 P^{*}$ As26 0 -like 2S2 2P3 ( 2P*) $4 D\left(3 D^{*}\right) j=2.0-2 S 2$ 2P4 As26 0 -like 2S2 2P3 ( 2D*) $4 D\left(3 D^{*}\right) j=3.0-2 S 22 P 4$ As26 0 -like 2S2 $2 P^{2}\left(2^{*} D^{*}\right) 4 D\left(3 D^{*}\right) j=3.0-2 S 22 P 4$ As26 0 -1ike 2S2 2P3 (2D*) $4 D\left(3 D^{*}\right) j=3.0-2 S 22 P 4$ As26 0 -iike $2 S 22 P 3\left(2 D^{*}\right) 4 D\left(3 D^{*}\right) j=3.0-2 S 2$ 2PA As26 0 -like 2S2 2P3 (2P*) $4 D\left(3 F^{*}\right) j=3.0-2 S 22 P 4$ As26 0 -like 2S2 2P3 ( 2P*) $4 D\left(3 F^{*}\right) j=3.0-2 S 22 P 4$ As26 0 -like 2S2 $2 P^{2}\left(2 P^{*}\right) 4 D\left(3 F^{*}\right) j=3.0-2 S 22 P 4$ As26 0 -like 2S2 2P3 ( 2P*) $4 D\left(3 F^{*}\right) j=3.0-2 S 22 P 4$ As26 O-like 2S2 $2 P^{2}$ ( $\left.4 S^{*}\right) 4 D\left(3 D^{*}\right) j=3.0-2 S 22 P 4$ As26 0 -like 2S2 $2 P^{2} 3\left(4 S^{*}\right) 4 D\left(3 D^{*}\right) j=3.0-2 S 22 P 4$ $A \in 260$-like 2S2 $2 P 3\left(4 S^{*}\right) 4 D\left(3 D^{*}\right) j=3.0-2 S 22 P 4$ As26 0 -like 2S2 2P3 ( 4S*) 4D $\left(3 D^{*}\right) j=3.0-2 S 22 P 4$ As26 0 -1ike 2S2 $2 P^{2}$ ( $\left.4 S^{*}\right) 4 D\left(3 D^{*}\right) j=1.0-2 S 2$ 2P4 As26 O-like 2S2 $2 P 3\left(4 S^{*}\right) 4 D\left(3 D^{*}\right) j=1.0-2 S 22 P 4$ As26 0 -1ike 2S2 2P3 ( 4S*) 4D $\left(3 D^{*}\right) j=1.0-2 S 22 P 4$ As26 0 -like 2S2.2P3 (4S*) 4D $\left(3 D^{*}\right) j=1.0-2 S 2$ 2P4 As26 O-like 2S2 $2 P^{2} 3\left(4 S^{*}\right) 4 D\left(3 D^{*}\right) j=2.0-2 S 22 P 4$ As26 O-like 2S2 2P3 ( $\left.4 S^{*}\right)$ 4D $\left(3 D^{*}\right) j=2.0-2 S 22 P 4$ As26 0 -like 2S2 2P3 (4S*) 4D (3D*) j=2.0 - 2S2 2P4 As26 O-like 2S2 2P3 ( $\left.4 S^{*}\right) 4 D\left(3 D^{*}\right) j=2.0-2 S 22 P 4$ As26 0 -like 2S2 2P3 ( 4S*) 4D (3D*) j=2.0 - 2S2 2P4 As26 0 -like 2S2 2P3 ( 4S*) 4D (3D*)j=2.0-2S2 2P4 As26 0 -like 2S2 2P3 ( 2P*) 3D (3D*) j=2.0 - 2S2 2P4 As26 0 -1ike 2S2 2P3 ( 2P*) 3D $\left(3 F^{*}\right) j=3.0-2 S 22 P^{*}$ As26 O-like 2S2 2P3 ( $\left.2 D^{*}\right) 3 D\left(3 P^{*}\right) j=1.0-2 S 22 P 4$ As26 0 -like 2S2 2P3 (2P*) 3D $\left(1 F^{*}\right) j=3.0-2 S 22 P 4$ As26 0 -1ike 2S2 2P3 (2P*) 3D $\left(3 P^{*}\right) j=2.0-2 S 22 P 4$ As26 O-like 2S2 2P3 ( $\left.4 S^{*}\right) 3 D\left(3 D^{*}\right) j=3.0-2 S 2$ 2P4 As26 0 -like 2S2 2P3 ( 2D*) 3D $\left(1 F^{*}\right) j=3.0-2 S 22 P 4$ As26 O-like 2S2 2P3 ( 2P*) 3S (3P*)j=2.0 - 2S2 2P4 As26 0 -1ike 2S2 2P3 ( $\left.2 P^{*}\right) 3 S\left(3 P^{*}\right) j=2.0-2 S 22 P 4$ As26 0 -like 2S2 2P3 ( $\left.2 P^{*}\right)$ ) 3S $\left(3 P^{*}\right) j=2.0-2 S 22 P 4$ As26 O-like 2S2 2P3 ( 2D*) 3S (3D*) j=3.0 - 2S2 2P4 $A s 260$-like 2S2 $2 P_{3}\left(2 D^{*}\right) 3 S\left(3 D^{*}\right) j=3.0-2 S 22 P 4$ $\left(2 P^{*}\right) j=1.5$ $\left(2 P^{*}\right) j=1.5$ $\left(2 P^{*}\right) j=1.5$

$\left(2 P^{*}\right) j=1.5$

$\left(2 P^{*}\right) j=1.5$ $\left(2 P^{*}\right) j=1.5$

$\left(2 P^{*}\right) j=.5$

$\left(2 P^{\star}\right) j=1.5$

$\left(2 P^{*}\right) j=1.5$

(3P) $j=1.0$

(3P) $j=1.0$

(3P) $j=1.0$

(3P) $j=2.0$

(3P) $j=2.0$

(3P) $j=2.0$

(3P) $j=2.0$

(ID) $j=2.0$

(1D) $j=2$.

(ID) $j=2$.

(1D) $j=2.0$

(3P) $j=2.0$

(3P) $j=2.0$

(3P) $j=2.0$

(3P) $j=2.0$

(3P) $j=.0$

(3P) $j=.0$

(3P) $j=.0$

(3P) $j=.0$

(3P) $j=1.0$

(3P) $j=1.0$

(3P) $j=1.0$

(3P) $j=1.0$

(3P) $j=1$.

(3P) $j=1.0$

(3P) $j=1.0$

(3P) $j=2.0$

(3P) $j=1$.

(1D) $j=2.0$

(1D) $j=2.0$

(3P) $j=2.0$

(1D) $j=2.0$

(3P) $j=1$.0

(3P) $j=1.0$

(3P) $j=1.0$

(3P) $j=2.0$

(3P) $j=2.0$
8.661 PET 002

8.661 EDT 020

8.661 RAP 100

8.661 KAP 100

8.753 EDT 020

8.753 KAP 100

$8.810 \mathrm{KAP} 100$

$8.855 \mathrm{KAP} 100$

8.873 KAP 100

5.916 calcite 200

5.916 Si 111

5.916 sylvite 200

5.973 calcite 200

5.973 Si 111

5.973 sylvite 200

5.973 fluorite 111

6.047 calcite 200

6.047 Si 111

6.047 sylvite 200

6.047 fluorite 111

6.081 Si 111

6.081 sylvite 200

6.081 fluorite 111

6.081 TAP 100

6.117 Si 111

6.117 sylvite 200

6.117 fluorite 2.11

6.117 TAP 100

6.175 si 111

6.175 sylvite 200

6.175 fluorite 111

6.175 Ge 111

6.175 TAP 100

6.175 RAP 100

7.602 bery 100

7.632 beryl 100

7.655 beryl 100

7.665 beryl 100

7.829 bery 100

7.847 beryl 100

7.861 beryl 100

8.133 topaz 002

8.133 quartz 100

8.133 TAP 100

8.182 topaz 002

8.182 quartz 100
$8.742 \quad 82.194$

$8.808 \quad 79.518$

$26.116 \quad 84.215$

$26.634 \quad 77.306$

$8.808 \quad 83.594$

$26.634 \quad 80.374$

$26.634 \quad 82.904$

$26.634 \quad 85.875$

$26.634 \quad 88.077$

6.07177 .025

$\begin{array}{ll}6.271 & 70.629\end{array}$

6.29270 .092

6.07179 .691

$6.271 \quad 72.266$

$6.292 \quad 71.677$

$6.308 \quad 71.243$

$6.071 \quad 84.904$

$6.271 \quad 74.640$

$6.292 \quad 73.958$

$6.308 \quad 73.461$

6.27175 .860

6.29275 .120

6.308 .74 .582

$25.763 \quad 70.760$

$6.271 \quad 77.276$

$6.292 \quad 76.455$

$6.308 \quad 75.865$

$25.763 \quad 71.756$

$6.271 \quad 79.962$

6.29278 .933

$6.308 \quad 78.214$

$\begin{array}{lll}6.532 & 70.970\end{array}$

$25.763 \quad 73.484$

$26.116 \quad 71.046$

$15.954 \quad 72.362$

$15.954 \quad 73.088$

$15.954 \quad 73.665$

$15.954 \quad 73.922$

$15.954 \quad 78.946$

$15.954 \quad 79.642$

$15.954 \quad 80.217$

$8.374 \quad 76.221$

$8.512 \quad 72.838$

$25.763 \quad 71.272$

$8.374 \quad 77.707$

$8.512 \quad 73.994$

Page 125 
As26 0 -like 2S2 2P3 (2D*) 3S $\left(3 D^{*}\right) j=3.0-2 S 22 P 4$

As26 0 -like 2S2 $2 P^{2}\left(2 D^{*}\right) 3 S\left(3 D^{*}\right) j=3.0-2 S 22 P 4$

As26 $O$-like 2S2 2P3 ( 2P*) $3 S\left(3 P^{*}\right) j=.0-2 S 22 P 4$

As26 0 -like 2S2 2P3 ( $\left.2 P^{*}\right) 3 S\left(3 P^{*}\right) j=.0-2 S 22 P 4$

As26 0 -like 2S2 $2 P^{2}\left(2 P^{*}\right) 3 S\left(3 P^{*}\right) j=.0-2 S 22 P 4$

As26 0 -like 2S2 2P3 (2P*) 3S $\left(3 P^{*}\right) j=.0-2 S 22 P 4$

As26 0 -like 2S2 $2 P^{2}\left(2 P^{*}\right) 3 S\left(3 P^{*}\right) j=.0-2 S 22 P^{*}$

As26 O-like 2S2 $2 P^{2} 3\left(2 P^{*}\right) 3 S\left(3 P^{*}\right) j=.0-2 S 22 P 4$

As26 O-like 2S2 2P3 ( $\left.4 S^{*}\right) 3 S\left(3 S^{*}\right) j=1.0-2 S 22 P 4$

As26 0 -like 2S2 2P3 (4S*) 3S (3S*) $j=1.0-2 S 22 P 4$

As26 O-like 2S2 2P3 ( $\left.S^{*}\right)$ 3S (3S*) $j=1.0$ - 2S2 2P4

As26 0 -like 2S2 2P3 (4S*) 3S (3S*) j=1.0 - 2S2 2P4

As26 O-like 2S2 2P3 ( 4S*) $3 S\left(3 S^{*}\right) j=1.0-2 S 22 P 4$

As26 O-like 2S2 2P3 (4S*) 3S (3S*) j=1.0 - 2S2 2P4

As26 0 -1ike 2S2 2P3 ( 2D*) 3S $\left(1 D^{*}\right) j=2.0-2 S 22 P 4$

As26 0 -like $2 S 22 P 3\left(2 D^{*}\right) 3 S\left(1 D^{*}\right) j=2.0-2 S 22 P 4$

As26 0 -like 2S2 2P3 ( 2D*) 3S (1D*) j=2.0 - 2S2 2P4

As26 0 -like 2S2 2P3 (2D*) 3S (1D*)j=2.0 - 2S2 2P4

As26 O-like 2S2 2P3 ( 2D*) 3S (1D*) $j=2.0-2 S 22 \mathrm{P} 4$

As26 O-like 2S2 2P3 ( $\left.2 D^{*}\right) 3 S\left(1 D^{*}\right) j=2.0-2 S 22 P 4$

As26 0 -like 2S2 2P3 ( 2D*) 3S $\left(3 D^{*}\right) j=2.0-2 S 2$ 2P4

As26 O-like 2S2 2P3 (2D*) 3S (3D*) j=2.0 - 2S2 2P4

As26 0 -like 2S2 2P3 (2D*) 3S (3D*) $j=2.0-2 S 22 \mathrm{P} 4$

As26 0 -like 2S2 2P3 ( $\left.2 D^{*}\right) 3 S\left(3 D^{*}\right) j=2.0-2 S 22 P 4$

As26 0-like 2S2 2P3 (2D*) 3S $\left(3 D^{*}\right) j=2.0-2 S 22 P 4$

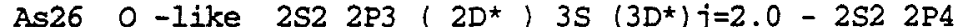

As26 0 -like 2S2 2P3 ( $\left.4 S^{*}\right) 3 S\left(3 S^{*}\right) j=1.0-2 S 2$ 2P4

As26 0 -like 2S2 $2 \mathrm{P3}\left(4 S^{*}\right) 3 S\left(3 S^{\star}\right) j=1.0-2 S 22 \mathrm{P4}$

As26 O-like 2S2 2P3 (4S*) 3S (3S*)j=1.0 - 2S2 2P4

As26 0 -like 2S2 2P3 (4S*) 3S (3S*) $j=1.0-2 S 22 \mathrm{P} 4$

As26 0 -like 2S2 2P3 ( $\left.4 S^{\star}\right) 3 S\left(3 S^{\star}\right) j=1.0-2 S 2$ 2P4

As26 O-like 2S2 2P3 (4S*) 3S (3S*) j=1.0 - 2S2 2P4

As26 0 -like 2S2 2P3 (4S*) 3S (3S*) j=1.0 - 2S2 2P4

As26 0 -like 2S2 2P3 (4S*) 3S (3S*) j=1.0 - 2S2 2P4

As26 0 -like 2S2 2P3 (4S*) 3S (3S*) j=1.0 - 2S2 2P4

As26 0 -like 2S2 $2 \mathrm{P} 3\left(4 S^{*}\right) 3 S\left(3 S^{*}\right) j=1.0-2 S 22 \mathrm{P} 4$

As32 He-like 1S 3P $\left(3 P^{*}\right) j=1.0-152$

As32 He-like is $3 P$

As32 He-like is 3P

As32 He-like is $3 P$

As32 He-like 1s $3 \mathrm{P}$

As32 He-like is $3 P$

As32 He-like 1S 3P

As32 He-like 1S 3P

As32 He-like 1S 2P

As32 He-like IS 2P

$\left(3 P^{*}\right) j=1.0-1 \mathrm{~s} 2$

$\left(1 P^{*}\right) j=1.0-1 \mathrm{~s} 2$

$\left(1 P^{\star}\right) j=1.0-1 \mathrm{~s} 2$
(3P) $j=2.0$

(3P) $j=2.0$

(3P) $j=1.0$

(3P) $j=1.0$

(3P) $j=1.0$

(3P) $j=1.0$

(3P) $j=1.0$

(3P) $j=1.0$

(3P) $j=2.0$

(3P) $j=2.0$

(3P) $j=2.0$

(3P) $j=2.0$

(3P) $j=2.0$

(3P) $j=2.0$

(1D) $j=2.0$

(1D) $j=2.0$

(ID) $j=2.0$

(ID) $j=2.0$

(ID) $j=2.0$

(ID) $j=2.0$

(3P) $j=1.0$

(3P) $j=1.0$

(3P) $j=1.0$

(3P) $j=1.0$

(3P) $j=1.0$

(3P) $j=1.0$

(3P) $j=.0$

(3P) $j=.0$

$(3 P) j=.0$

(3P) $j=.0$

(3P) $j=.0$

(3P) $j=.0$

(3P) $j=1.0$

(3P) $j=1.0$

(3P) $j=1.0$

(3P) $j=1.0$

(1S) $j=.0$

(1s) $j=.0$

(1s) $j=.0$

(1s) $j=.0$

(1s) $j=.0$

(1s) $j=.0$

(1s) $j=.0$

(1s) $j=.0$

(Is) $j=.0$

(1s) $j=.0$
8.182 TAP 100

.182 RAP 100

8.292 topaz 002

quartz 100

292 PET 002

8.292 EDT 020

8.292 TAP 100

8.392 quartz 100

8.392 PET 002

8.392 EDT 020

392 TAP 100

8.392 RAP 100

8.392 KAP 100

422 quartz 100

8.422 PET 002

8.422 EDT 020

8.422 TAP 100

8.422 RAP 100

$8.422 \mathrm{KAP} 100$

8.437 quartz 100

8.437 PET 002

.437 EDT 020

8.437 TAP 100

8.437 RAP 100

8.437

8.491 quartz 100

8.491 PET 002

8.491 EDT 020

491 TAP 100

8.491 RAP 100

8.491 KAP 100

.600 PET 002

8.600 EDT 020

8.600 RAP 100

8.600 KAP 100

963 quartz 223

963 calcite 422

.963 Eluorite 220

.963 Ge 220

.963 LiF 200

.963 Al 200

.963 quartz 110

963
1.132

1.132 gypsum 002

Ge 422

quartz 310
$25.763 \quad 72.319$

$26.116 \quad 70.032$

$8.374 \quad 81.975$

8.51276 .945

$8.742 \quad 71.536$

$8.808 \quad 70.291$

$25.763 \quad 74.922$

$26.116 \quad 72.273$

$8.512 \quad 80.368$

$8.742 \quad 73.732$

$8.808 \quad 72.321$

$25.763 \quad 77.746$

$26.116 \quad 74.581$

$26.634 \quad 70.954$

$8.512 \quad 81.661$

$8.742 \quad 74.450$

$8.808 \quad 72.975$

$25.763 \quad 78.728$

$26.116 \quad 75.342$

$26.634 \quad 71.557$

8.51282 .388

$8.742 \quad 74.821$

$8.808 \quad 73.311$

$25.763 \quad 79.252$

$26.116 \quad 75.737$

$26.634 \quad 71.865$

$8.512 \quad 85.975$

$8.742 \quad 76.237$

$8.808 \quad 74.582$

$25.763 \quad 81.395$

$26.116 \quad 77.260$

$26.634 \quad 73.020$

$8.742 \quad 79.659$

$8.808 \quad 77.524$

$26.116 \quad 81.078$

$26.634 \quad 75.624$

$2.024 \quad 72.098$

$3.034 \quad 72.215$

$3.862 \quad 85.876$

$4.000 \quad 74.365$

$4.027 \quad 73.047$

$4.048 \quad 72.098$

$4.912 \quad 78.595$

$4.990 \quad 74.781$

$2.310 \quad 78.547$

$2.360 \quad 73.602$

Page 126 


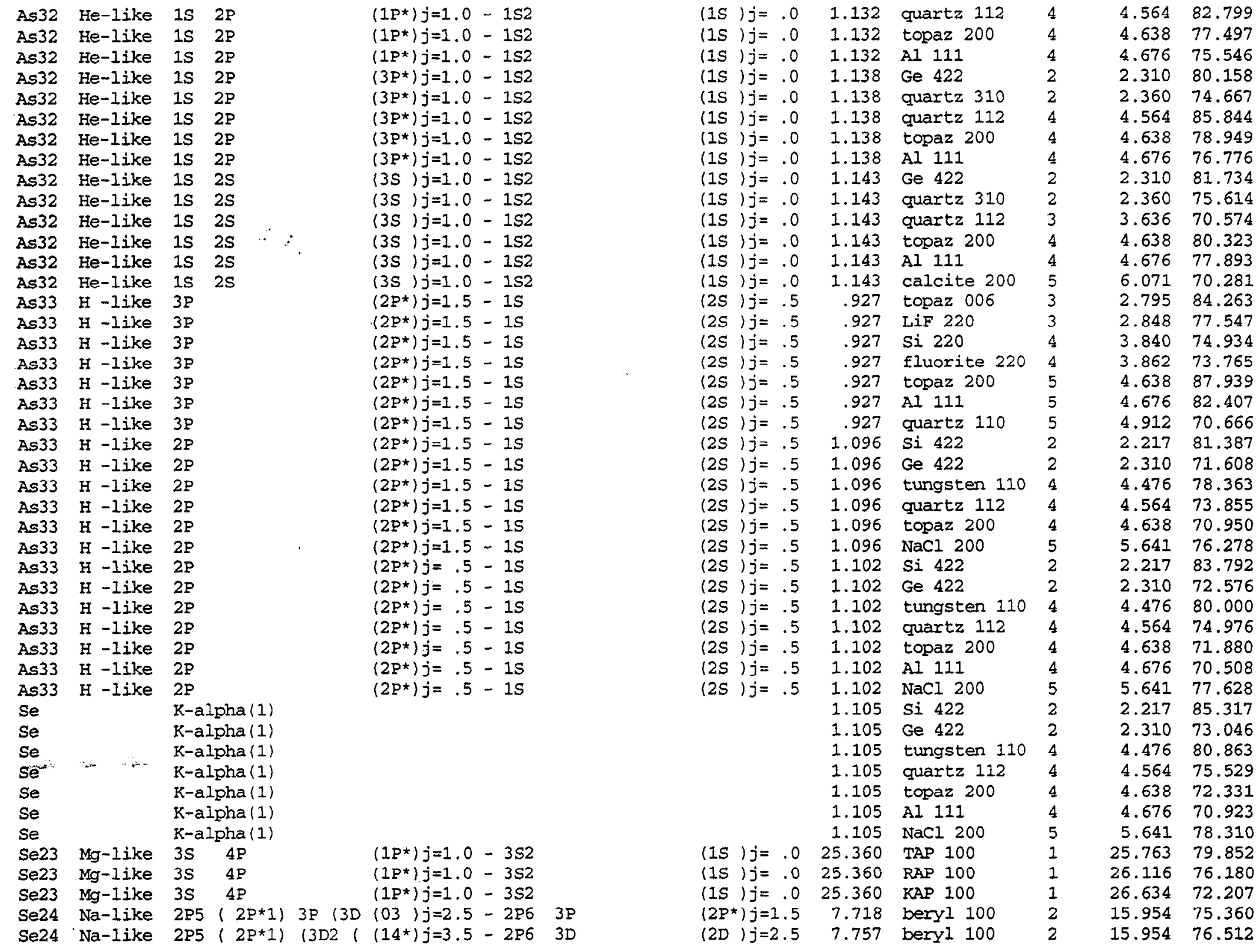


Se24 Na-1ike $2 P 5(2 P * 1)\left(3 D 2\left(\left(13^{*}\right) j=2.5-2 P 6\right.\right.$ 3D Se24 Na-1ike 2P5 ( 2P*1) 3P (3D (12) j=1.5 - 2P6 3P Se24 Na-like 2P5 $(2 P \star 2) \quad 3 P(3 D(23) j=2.5-2 P 6 \quad 3 P$ Se24 Na-1ike 2P5 ( $\left.2 P^{*} 1\right)\left(3 D 2\right.$ ( $\left(12^{*}\right) j=2.5-2 P 6$ 3D Se24 Na-1ike 2P5 $(2 P * 2)(3 D 2)(24 *) j=2.5-2 P 6$ 3D Se24 Na-like 2P5 ( 2P*2) (3D2 ( $\left(24^{*}\right) j=2.5-2 P 6$ 3D Se24 Na-like 2P5 ( $\left.2 P^{*} 2\right)$ (3D2 ( $\left(24^{*}\right) j=3.5-2 P 6$ 3D Se24 Na-1ike 2P5 ( 2P*2) (3D2 ( $\left(24^{*}\right) j=3.5-2 P 6$ 3D Se24 Na-like 2P6 5D (2D) $j=2.5-2 P 6$ 3P Se21 Na-like $2 P 6 \quad 4 P \quad\left(2 P^{*}\right) j=1.5-2 P 6 \quad 35$ Se24 Na-1ike 2P6 $4 \mathrm{P} \quad\left(2 \mathrm{P}^{*}\right) \mathrm{j}=.5-2 \mathrm{P} 6 \quad 3 \mathrm{~S}$ Se24 Na-1ike $2 P 6 \quad 4 P \quad\left(2 P^{*}\right) j=.5-2 P 6 \quad 3 S$ Se24 Na-like 2P6 4D (2D) $j=1.5-2 P 6 \quad 3 P$ Se24 Na-1ike 2P6 4D (2D ) $j=1.5-2 P 6 \quad 3 P$ Se24 Na-like 2P6 4D (2D) $j=2.5-2 P 6 \quad 3 P$ Se25 Ne-1ike 2S2 2P5 ( 2P*2) 7D (23*)j=1.0 - 2S2 2P6 Se25 Ne-1ike 2S2 $2 P 5(2 P \star 2) \quad 7 D\left(23^{\star}\right) j=1.0-2 S 22 P 6$ Se25 Ne-like 2S2 2P5 ( $\left.2 P^{*} 2\right) 7 D\left(23^{*}\right) j=1.0-2 S 22 P 6$ Se25 Ne-1ike 2S2 2P5 (2P*1) 6D $(12 *) j=1.0-2 S 22 P 6$ $\mathrm{Se} 25 \mathrm{Ne}-1 \mathrm{ike} 2 \mathrm{~S} 2 \mathrm{2P5}(2 \mathrm{P} * 1) 6 \mathrm{D}\left(12^{*}\right) \mathrm{j}=1.0-2 \mathrm{~S} 2$ 2P6 Se25 Ne-1ike 2S2 2P5 ( 2P*1) 6D $\left(12^{\star}\right) j=1.0-2 S 22 P 6$ Se25 Ne-like 2S2 2P5 ( $2 P \star 2) 6 D(23 *) j=1.0-2 S 22 P 6$ Se25 Ne-1ike 2S2 2P5 ( 2P*1) 5D $\left(12^{*}\right) \mathrm{j}=1.0-2 \mathrm{~S} 22 \mathrm{P} 6$ Se25 Ne-like 2S2 2P5 ( 2P*1) 4D (12*) j=1.0 - 2S2 2P6 Se25 Ne-1ike 2S2 2P5 ( $\left.2 P^{\star} * 1\right) 4 D\left(12^{*}\right) j=1.0-2 S 22 P 6$ Se25 Ne-like 2S2 2P5 $(2 P * 1)$ 4D $\left(12^{\star}\right) j=1.0-2 S 22 P 6$ Se25 Ne-1ike 2S2 2P5 ( $2 P * 1)$ 4D (12*)j=1.0 - 2S2 2P6 Se25 Ne-like 2S2 $2 P 5(2 P * 2) 4 D(23 *) j=1.0-2 S 22 P 6$ Se25 Ne-1ike 2S2 2P5 ( $\left.2 P^{*} 2\right)$ 4D $\left(23^{*}\right) j=1.0-2 S 22 P 6$ Se25 Ne-like 2S2 2P5 $(2 \mathrm{P} * 2)$ 4D $\left(23^{*}\right) j=1.0-2 \mathrm{~S} 2$ 2P6 Se25 Ne-1ike 2S2 2P5 ( $\left.2 P^{*} 2\right)$ 4D $\left(23^{*}\right) j=1.0-2 S 22 P 6$ Se25 Ne-like 2S2 2PS $(2 P * 2) 4 D(23 *) j=1.0-2 S 22 P 6$ Se25 Ne-1ike 2S2 2P5 ( $2 P * 2) 4 D(23 *) j=1.0-2 S 22 P 6$ Se25 Ne-like 2S2 $2 P 5(2 P * 2) 4 D(23 *) j=1.0-2 S 22 P 6$ Se25 Ne-1ike 2S2 2P5 ( 2P*1) 4S $(11 *) j=1.0-2 S 22 P 6$ Se25 Ne-1ike 2S2 2P5 (2P*1) 4S (11*)j=1.0 - 2S2 2P6 Se25 Ne-like 2S2 2P5 ( $\left.2 P^{*} 1\right)$ 4S $\left(11^{*}\right) j=1.0-2 S 22 \mathrm{P} 6$ Se25 Ne-like 2S2 2P5 ( $\left.2 P^{\star} 1\right)$ 4S $\left(11^{*}\right) j=1.0-2 S 22 P 6$ Se25 Ne-1ike 2S2 2P5 ( $\left.2 P^{*} 1\right) 4 S\left(11^{*}\right) j=1.0-2 S 22 P 6$ Se25 Ne-like 2S2 2P5 ( 2P*1) 4S (11*)j=1.0 - 2S2 2P6 Se25 Ne-1ike 2S2 2P5 ( 2P*1) 4S (11*) j=1.0 - 2S2 2P6 Se25 Ne-like 2S2 $2 \mathrm{P} 5$ ( $\left.2 \mathrm{P}^{*} 1\right)$ 4S $\left(11^{*}\right) j=1.0-2 \mathrm{~S} 2$ 2P6 Se25 Ne-1ike 2S2 2P5 ( 2P*2) 4S $\left(22^{*}\right) j=1.0-2 \mathrm{~S} 2$ 2P6 Se25 Ne-like 2S2 2P5 ( 2P*2) 4S $\left(22^{\star}\right) j=1.0-2 S 22 P 6$ Se25 Ne-1ike 2S2 2P5 ( 2P*2) 4S $(22 *) j=1.0-2 \mathrm{~S} 2$ 2P6 Se25 Ne-like 2S2 $2 \mathrm{P} 5(2 \mathrm{P} * 2) 4 \mathrm{~S}(22 *) j=1.0-2 \mathrm{~S} 22 \mathrm{P} 6$ $\left(2 P^{*}\right) j=.5$

$\left(2 P^{*}\right) j=1$.

(2D) $j=1$.

(2D) $j=2.5$

(2D) $j=2.5$

(2D) $j=2.5$

(2D) $j=2.5$

$\left(2 P^{\star}\right) j=1.5$

(2S) $j=$.

(2S) $j=.5$

(2S) $j=.5$

$\left(2 P^{\star}\right) j=.5$

$\left(2 P^{\star}\right) j=.5$

$\left(2 P^{*}\right) j=1.5$

(1s) $j=$.

(1S) $j=.0$

(1s) $j=0$

(1s) $j=.0$

(1s) $j=.0$

(Is) $j=.0$

(1s) $j=.0$

(1S) $j=.0$

(1s) $j=.0$

(1s) $j=.0$

(1s) $j=.0$

(1S) $j=.0$

(1s) $j=.0$

(1s) $j=.0$

(1S) $j=.0$

(1s) $j=.0$

(Is) $j=.0$

(1s) $j=.0$

(1s) $j=.0$

(1s) $j=.0$

(1s) $j=.0$

(1s) $j=.0$

(1s) $j=.0$

(1s) $j=.0$

(Is) $j=.0$

(1s) $j=.0$

(1s) $j=.0$

(1s) $j=.0$

(1s) $j=.0$

(1s) $j=.0$

(1S ) $j=.0$
7.790 beryl 100

7.805 bery 100

7.821 beryl 100

7.945 topaz 002

7.945 beryl 100

7.969 topaz 002

7.969 beryl 100

18.980 mica 002

24.530 TAP 100

24.730

24.730

25.830

25.830

26.350

5.252

5.252

5.252

5.300

5.300

5.300

5.395

5.54

6.078

6.078

6.078

6.078

6.201

6.201

6.201

6.201

6.201

6.201

6.201

6.322

6.322

6.322

6.322

6.322

6.322

TAP 100

RAP 100

RAP 100

KAP 100

KAP 100

ADP 101

beryl 100

KAP 100

ADP 101

beryl 100

AP 100

$\mathrm{NaCl} 200$

aCl 200

sylvite 200

fluorite 111

TAP 100

Si 111

ylvite 200

luorite 111

Ge 111

KBr 200

AaP 100

RAP 100

e 111

quartz 101

graphite 002

mica 002

6.368

$6.368 \mathrm{KBr} 200$

6.368

6.368
$15.954 \quad 77.305$

$15.954 \quad 77.569$

$15.954 \quad 78.080$

$15.954 \quad 78.650$

$8.374 \quad 71.581$

$15.954 \quad 84.866$

$8.374 \quad 72.108$

$15.954 \quad 87.434$

$19.942 \quad 72.131$

$25.763 \quad 72.202$

$25.763 \quad 73.720$

$26.116 \quad 71.250$

$26.116 \quad 81.513$

$26.634 \quad 75.886$

$26.634 \quad 81.625$

$10.640 \quad 80.829$

$15.954 \quad 80.964$

$26.634 \quad 80.387$

$10.640 \quad 85.030$

$15.954 \quad 85.285$

$26.634 \quad 84.250$

$5.641 \quad 73.017$

$5.641 \quad 79.526$

6.27175 .748

$6.292 \quad 75.014$

$6.308 \quad 74.480$

$25.763 \quad 70.679$

$6.271 \quad 81.431$

$6.292 \quad 80.244$

$6.308 \quad 79.432$

$6.532 \quad 71.682$

$6.584 \quad 70.361$

$25.763 \quad 74.318$

$26.116 \quad 71.762$

$6.532 \quad 75.432$

$6.584 \quad 73.782$

$6.687 \quad 70.982$

6.69670 .760

$19.942 \quad 72.000$

$25.763 \quad 78.981$

$26.116 \quad 75.534$

$26.634 \quad 71.707$

$6.532 \quad 77.134$

$6.584 \quad 75.283$

$6.687 \quad 72.231$

$6.696 \quad 71.992$ 
Se25 Ne-like 2S2 2P5 ( 2P*2) 4S $(22 *) j=1.0-2 S 22 P 6$ Se25 Ne-like 2S2 2P5 ( $2 P * 2) 4 S(22 *) j=1.0-2 S 22 P 6$ Se25 Ne-like 2S2 2P5 ( 2P*2) 4S $\left(22^{\star}\right) j=1.0-2 S 22 P 6$ Se25 Ne-like 2S2 2P5 ( $2 P * 2) 4 S(22 *) j=1.0-2 S 22 P 6$ Se25 Ne-1ike $2 S$ 2P6 3P $(1 P *) j=1.0-2 S 2$ 2P6 Se25 Ne-like 2S 2P6 3P (1P*) $j=1.0-2 S 22 P 6$ Se25 Ne-like 2S 2P6 3P $\left(3 P^{*}\right) j=1.0-2 S 22 P 6$ Se25 Ne-like 2S 2P6 3P (3P*) j=1.0 - 2S2 2P6 Se25 Ne-like 2S2 2P5 ( 2P*1) 3D (12*)j=1.0 - 2S2 2P6 Se25 Ne-Iike 2S2 2P5 ( 2P*2) 3D $(23 *) j=1.0-2 S 2$ 2P6 Se25 Ne-like 2S2 2P5 (2P*2) 3D (23*)j=1.0 - 2S2 2P6 Se25 Ne-like 2S2 2P5 ( 2P*2) 3D (22*) j=1.0 - 2S2 2P6 Se25 Ne-like 2S2 2F5 ( 2P*2) 3D $(22 *) j=1.0-2 S 22 P 6$ Se25 Ne-like 2S2 2P5 ( 2P*1) 3S (11*)j=1.0 - 2S2 2P5 Se25 Ne-like 2S2 2P5 $(2 P * 1)$ 3S $(11 *) j=1.0-2 S 22 P 6$ Se25 Ne-like 2S2 2P5. ( 2P*1) 3S (11*) j=1.0 - 2S2 2P6 Se25 Ne-like 2S2 2P5 ( $\left.2 P^{\star} 1\right)$ 3S (11*)j=1.0 - 2S2 2P6 Se25 Ne-like 2S2 2P5 ( 2P*1) 3S (11*) $j=1.0-2 S 2$ 2P6 Se25 Ne-1ike 2S2 $2 P 5(2 P * 1)$ 3S (11*) $j=1.0-2 S 22 P 6$ Se25 Ne-like $2 S 22 P 5(2 P * 2) 3 S(22 *) j=1.0-2 S 22 P 6$ Se25 Ne-1ike $2 S 22 P 5(2 P * 2) 3 S(22 *) j=1.0-2 S 22 P 6$ Se25 Ne-like $2 S 2$ 2P5 $(2 P \star 2) \quad 3 S(22 *) j=1.0-2 S 22 P 6$ Se25 Ne-1ike 2S2 $2 \mathrm{P} 5\left(2 \mathrm{P}^{*} 2\right)$ 3S $(22 *) j=1.0-2 \mathrm{~S} 22 \mathrm{P} 6$ Se26 F-like 2S2 2P4 ( $1 S$ ) $4 D(2 D) j=2.5-2 S 22 P 5$ Se26 F-like $2 S 22 P 4(1 D) 4 D(2 D) j=2.5-2 S 2$ 2P5 Se26 F-like 2S2 2P4 ( $3 P$ ) 4D (4P) j=2.5 - 2S2 2P5 Se26 F -like 2S2 2P4 ( $3 P$ ) $4 D(4 F) j=2.5-2 S 22 P 5$ Se26 F-like 2S2 2P4 ( $1 D$ ) $4 D$ (2D) j=1.5 - 2S2 2P5 Se26 F-like 2S2 2P4 (1D) 4D (2D) j=1.5 - 2S2 2P5 Se26 F-like 2S2 2P4 ( $3 P) 4 D(2 D) j=2.5-2 S 22 P 5$ Se26 F-like 2S2 2P4 ( $3 P$ ) $4 D(2 D) j=2.5-2 S 22 P 5$ Se26 F-1ike 2S2 2P4 ( $3 P$ ) 4D (2D) j=2.5 - 2S2 2P5 Se26 $F$-like 2S2 2P4 ( $3 P$ ) $4 D(2 D) j=2.5-2 S 22 P 5$ Se26 F-like 2S2 2P4 ( $1 D$ ) $4 S$ (2D) $j=1.5-2 S 22 P 5$ Se26 F-like 2S2 2P4 (1D) 4S (2D) j=1.5 - 2S2 $2 P 5$ Se26 F-like 2S2 2P4 ( 1D) 4S (2D) j=1.5 - 2S2 2P5 Se26 F-like 2S2 2P4 ( $1 D$ ) $4 S(2 D) j=1.5-2 S 22 P 5$ Se26 F-like 2S2 2P4 ( $3 P$ ) $4 S$ (2P) j=1.5 - 2S2 2P5 Se26 F-1ike 2S2 2P4 ( $3 P$ ) $4 S(2 P) j=1.5-2 S 22 P 5$ Se26 F-like 2S2 2P4 ( 3P) 4S (2P) j=1.5 - 2S2 2P5 Se26 F-like 2S2 2P4 ( $3 P$ ) $4 S(2 P) j=1.5-2 S 22 P 5$ Se26 F-like 25 2P5 ( $\left.3 P^{*}\right) 3 P(2 P) j=1.5-2 S 22 P 5$ Se26 F-like 2S 2P5 ( 3P*) 3P (2D) $j=2.5-2 S 2$ 2P5 Se26 $F$-like $2 S$ 2P5 ( $\left.3 P^{\star}\right) 3 P(4 D) j=1.5-2522 P 5$ Se26 F-like 2S 2P5 ( $\left.3 P^{\star}\right) 3 P(2 S) j=.5-2 S 22 P 5$ Se26 $F$-like 2S 2P5 ( $\left.3 P^{*}\right) 3 P(4 D) j=2.5-2 S 22 P 5$
(1S) $j=.0$ (1s) $j=.0$ (1S) $j=.0$ (IS) $j=.0$ (1s) $j=.0$ (1s) $j=.0$ (1s) $j=.0$ (1s) $j=.0$ (1s) $j=.0$ (1s) $j=.0$ (1s) $j=.0$ (1s) $j=.0$ (1s) $j=.0$ (1s) $j=.0$ (1s) $j=.0$ (1s) $j=.0$ (1s) $j=.0$ (1s) $j=.0$ (1s) $j=.0$ (1S) $j=.0$ (1s) $j=.0$ (1s) $j=.0$ (1s) $j=0$ $\left(2 P^{\star}\right) j=1.5$ $\left(2 P^{*}\right) j=1.5$ $\left(2 P^{\star}\right) j=1.5$

$\left(2 P^{*}\right) j=1.5$ $\left(2 P^{\star}\right) j=.5$ $\left(2 P^{*}\right) j=.5$ $\left(2 P^{*}\right) j=1.5$ $\left(2 P^{\star}\right) j=1.5$ $\left(2 P^{*}\right) j=1.5$ $\left(2 P^{*}\right) j=1.5$ $\left(2 P^{*}\right) j=1.5$ $\left(2 p^{*}\right) j=1.5$

$\left(2 P^{*}\right) j=1.5$ $(2 P *) j=1.5$ $(2 p *) j=1.5$ $\left(2 P^{*}\right) j=1.5$ $(2 P *) j=1.5$

$\left(2 P^{\star}\right) j=1.5$

$\left(2 P^{*}\right) j=1.5$ $\left(2 P^{\star}\right) j=1.5$

$\left(2 P^{\star}\right) j=1.5$

$\left(2 P^{*}\right) j=.5$

$(2 P *) j=1.5$
6.368 mica 002

6.368 TAP 100

6.368 RAP 100

6.368 KAP 100

7.243 InSb 111

7.243 gypsum 020

7.294 InSb 111

7.294 gypsum 020

7.685

7.874

7.874

7.967

beryl 100

topaz 002

7.967

topaz 002

eryl 100

8.374

8.374

uartz 100

8.374

PET 002

8.374 KAP 100

8.615 PET 002

EDT 020

8.615

8.615

5.781

RAP 100

KAP 100

5.796

calcite 200

5.829

calcite 200

calcite 200

5.889 calcite 200

5.906 calcite 200

5.906 Si 111

5.930 calcite 200

5.930 Si 111

5.930 sylvite 200

5.930 fluorite 111

6.045 calcite 200

6.045 Si 111

6.045 sylvite 200

6.045 fluorite 111

6.075 Si 111

6.075 sylvite 200

6.075 Eluorite 111

6.075 TAP 100

7.057 Insto 111

7.085 InSb 111

7.097 InSb 111

7.116 InSb 111

7.138 InSb 111
$19.942 \quad 73.331$

$25.763 \quad 81.380$

$26.116 \quad 77.250$

$26.634 \quad 73.013$

7.48175 .509

$15.185 \quad 72.548$

$\begin{array}{ll}7.481 & 77.162\end{array}$

$15.185 \quad 73.881$

$15.954 \quad 74.450$

$8.374 \quad 70.101$

$15.954 \quad 80.783$

$8.374 \quad 72.063$

$15.954 \quad 87.131$

$8.512 \quad 79.669$

$8.742 \quad 73.316$

$8.808 \quad 71.939$

$25.763 \quad 77.192$

$26.116 \quad 74.141$

$26.634 \quad 70.601$

$8.742 \quad 80.222$

$8.808 \quad 77.984$

$26.116 \quad 81.739$

$26.634 \quad 76.019$

$6.071 \quad 72.219$

$6.071 \quad 72.689$

$6.071 \quad 73.768$

$6.071 \quad 75.935$

6.07176 .611

6.27170 .355

$6.071 \quad 77.627$

5.27171 .018

$6.292 \quad 70.470$

$6.308 \quad 70.064$

6.07184 .695

$6.271 \quad 74.571$

$6.292 \quad 73.893$

$6.308 \quad 73.397$

$6.271 \quad 75.637$

$6.292 \quad 74.909$

$6.308 \quad 74.379$

$25.763 \quad 70.598$

$\begin{array}{ll}7.481 & 70.617\end{array}$

$7.481 \quad 71.274$

$.7 .481 \quad 71.563$

$\begin{array}{ll}7.481 & 72.028\end{array}$

$7.481 \quad 72.583$ 
Se26 F-like 2S 2P5 ( $\left.3 P^{*}\right) 3 P$ (4D) $j=2.5-2 S 22 P 5$ Se26 F-like 2S 2P5 (3P*) 3P (4P) $j=1.5-2 S 22 P 5$ Se26 F-1ike $2 S$ 2P5 (3P*) 3P (4P) $j=1.5-2 S 22 P 5$ Se26 F-like 2S2 2P4 ( $1 D)$ 3D (2P) $j=.5-2 S 22 P 5$ Se26 F-like 2S2 2P4 (1D) 3D (2P) $j=.5-2 S 2$ 2P5 Se26 F-like 2S2 2P4 ( $1 D$ ) 3D (2D) j=1.5 - 2S2 2P5 Se26 F-like 2S2 2P4 ( 1D) 3D (2D) $j=1.5-2 S 22 P 5$ Se26 F-1ike 2S2 2P4 ( $1 S$ ) 3D (2D) j=1.5 - 2S2 2P5 Se26 F-Iike 2S2 2P4 ( $1 S$ ) 3D (2D) j=1.5 - 2S2 2P5 Se26 F-like 2S2 2P4 ( 1D) 3D (2D) j=2.5 - 2S2 $2 P 5$ Se26 F-like 2S2 2P4 (1D) 3D (2D) j=2.5 - 2S2 2P5 Se26 F-like 2S2 2P4 ( $1 D$ ) 3D (2S) j=.5-2S2 2P5 Se26 F-like 2S2 2P4 ( $1 D)$ 3D (2S) $j=.5-2 S 22 P 5$ Se26 F-like 2S2 2P4 (3P) 3D (2D) j=2.5 - 2S2 2P5 Se26 F-like $2 S 22 P 4(3 P$ ) 3D (2D) $j=2.5-2 S 22 P 5$ Se26 F-like 2S2 2P4 ( $3 P$ ) 3D (4D) $j=1.5-2 S 22 P 5$ Se26 F-like 2S2 2P4 ( 1D) 3D (2P) J $=.5-2 S 22 P 5$ Se26 F-like 2S2 2P4 ( $1 D$ ) 3D (2P) j=.5 - 2S2 2P5 Se26 F-like 2S2 2P4 ( ID) 3D (2D) j=1.5 - 2S2 2P5 Se26 F-1ike 2S2 2P4 ( 1D) 3D (2D) j=1.5 - 2S2 2P5 Se26 F-like 2S2 2P4 ( $1 D$ ) 3D (2P) j=1.5 - 2S2 2P5 Se26 F-like 2S2 2P4 ( $3 P$ ) 3D (2F) $j=2.5-2 S 22 P 5$ Se26 F-like 2S2 2P4 (3P) 3D (4P) $j=1.5-2 S 22 P 5$ Se26 F-like 2S2 2P4 ( $3 P$ ) $3 D(4 P) j=.5-2 S 22 P 5$ Se26 F-like 2S2 2P4 ( 3P ) 3D (2P) j=1.5 - 2S2 2P5 Se26 F -1ike 2S2 2P4 (3P) 3D (4D) j=1.5 - 2S2 2P5 Se26 F-like 2S2 2P4 ( $1 S$ ) $3 S(2 S) j=.5-2 S 22 P 5$ Se26 F-like 2S2 2P4 ( $1 S$ ) $3 S(2 S) j=.5-2 S 22 P 5$ Se26 F-like 2S2 2P4 ( $1 D)$ 3S (2D) $j=2.5-2 S 22 P 5$ Se26 F-like 2S2 2P4(3P) 3S (2P)j=.5-2S2 2P5 Se26 F-like 2S2 2P4 ( $3 P$ ) $3 S(2 P) j=.5-2 S 22 P 5$ Se26 F-like 2S2 2P4 ( $3 P$ ) 3S (2P) $j=1.5-2 S 22 P 5$ Se26 F-like 2S2 2P4 (3P) 3S (2P) $j=1.5-2 S 22 P 5$ Se26 F-like 2S2 2P4 ( 3P) 3S (4P) $j=.5-2 S 22 P 5$ Se26 $F$-like 2S2 2P4 ( $3 P$ ) 3S $(4 P) j=.5-2 S 22 P 5$ Se26 F-like 2S2 2P4 ( 3P) 3S (4P) $j=.5-2 S 22 P 5$ Se26 F-like 2S2 2P4 ( 1D) 3S (2D) j=1.5 - 2S2 2P5 Se26 F-like 2S2 2P4 ( $1 D$ ) 3S (2D) $j=1.5-2 \mathrm{~S} 22 \mathrm{P} 5$ Se26 F-like 2S2 2PA ( $1 D) 3 S(2 D) j=1.5-2 S 22 P 5$ Se26 F-like 2S2 2P4 ( 1D) 3S (2D) $j=1.5-2 S 22 P 5$ Se26 F-like 2S2 2P4 ( $3 P$ ) $3 S(4 P) j=1.5-2 S 22 P 5$ Se26 F-like 2S2 2P4 ( $3 P$ ) 3S (4P) j=1.5 - 2S2 2P5 Se26 $F$-like 2S2 2P4 ( $3 P$ ) 3S $(4 P) j=1.5-2 S 22 P 5$ Se26 F-like 2S2 2P4 ( $3 P$ ) 3S (4P) $j=1.5-2 S 22 P 5$ Se26 F-like 2S2 $2 P 4$ ( $3 P$ ) 3S (4P) $j=1.5-2 S 22 P 5$ Se26 $F$-like 2S2 $2 \mathrm{P} 4(3 \mathrm{P}) 3 \mathrm{~S}(4 \mathrm{P}) \mathrm{j}=2.5-2 \mathrm{~S} 22 \mathrm{P} 5$ $\left(2 P^{*}\right) j=1.5$ $\left(2 P^{\star}\right) j=.5$

$\left(2 P^{*}\right) j=.5$ $\left(2 P^{\star}\right) j=1.5$ $\left(2 P^{\star}\right) j=1.5$ $\left(2 P^{\star}\right) j=1.5$ $\left(2 P^{*}\right) j=1.5$ $\left(2 P^{*}\right) j=.5$ $\left(2 \mathrm{P}^{*}\right) j=.5$ $\left(2 P^{*}\right) j=1.5$ $\left\langle 2 P^{\star}\right\rangle j-1.5$ $\left(2 P^{*}\right) j=1.5$ $\left(2 P^{*}\right) j=1.5$ $\left(2 P^{\star}\right) j=1.5$ $\left(2 P^{*}\right) j=1.5$ $\left(2 P^{*}\right) j=1.5$ $\left(2 P^{*}\right\rangle j=.5$ $\left(2 P^{*}\right) j=.5$ $\left(2 P^{*}\right) j=.5$ $\left(2 P^{\star}\right) j=.5$

$\left(2 p^{\star}\right) j=.5$ $\left(2 P^{\star}\right) j=1.5$ $\left(2 P^{*}\right) j=1.5$

$\left(2 P^{*}\right) j=1.5$

$\left(2 P^{*}\right) j=.5$

$\left(2 P^{*}\right) j=.5$

$\left(2 P^{*}\right) j=.5$

$\left(2 P^{*}\right) j=.5$

$\left(2 P^{\star}\right) j=1.5$

$(2 P *) j=1.5$

$\left(2 P^{*}\right) j=1.5$

$\left(2 P^{*}\right) j=1.5$

$\left(2 P^{*}\right) j=1.5$

$\left(2 P^{*}\right) j=1.5$

$\left(2 P^{*}\right) j=1.5$

$\left(2 P^{*}\right) j=1.5$

$\left(2 P^{*}\right) j=.5$

$\left(2 P^{*}\right) j=.5$

$\left(2 P^{*}\right) j=.5$

$\left(2 P^{\star}\right) j=.5$

$\left(2 P^{*}\right) j=1.5$

$\left(2 P^{*}\right) j=1.5$

$\left(2 P^{*}\right) j=1.5$

$\left(2 P^{\star}\right) j=1.5$

(2P*) $j=1.5$

$\left(2 P^{\star}\right) j=1.5$
7.138 gypsum 020

7.190 Insto 111

7.190 gypsum 020

7.353 InSb 111

7.353 gypsum 020

7.368 InSb 111

7.368 gypsum 020

7.379 InSb 111

7.379 gypsum 020

7.400 InSb 111

7.400 gypsum 020

7.417 InSb 111

7.417 gypsum 020

7.465 InSb 111

7.465 gyosum 020

7.487 gypsum 020

7.548 gypsum 020

7.548 beryl 100

7.565 gypsum 020

7.565 beryl 100

7.596 bery 100

7.610 beryl 100

7.623 beryl 100

6.637 beryl 100

7.658 beryl 100

7.685 beryl 100

7.945 topaz 002

7.945 beryl 100

7.978 topaz 002

8.026 topaz 002

8.026 quartz 100

8.042 topaz 002

8.042 quartz 100

8.156 topaz 002

8.156 quartz 100

8.156 TAP 100

8.197 topaz 002

8.197 quartz 100

8.197 TAP 100

8.197 RAP 100

8.237 topaz 002

8.237 quartz 100

8.237 PET 002

8.237 TAP 100

8.237 RAP 100

8.259 topaz 002
$15.185 \quad 70.075$

$\begin{array}{ll}7.481 & 73.967\end{array}$

$15.185 \quad 71.260$

$7.481 \quad 79.386$

$15.185 \quad 75.571$

$\begin{array}{ll}7.481 & 80.029\end{array}$

$15.185 \quad 76.032$

$\begin{array}{ll}7.481 & 80.528\end{array}$

$15.185 \quad 76.380$

$7.481 \quad 81.561$

$15.185 \quad 77.070$

$\begin{array}{ll}7.481 & 82.500\end{array}$

$15.185 \quad 77.657$

$7.481 \quad 86.252$

$15.185 \quad 79.485$

$15.185 \quad 80.437$

$15.185 \quad 83.794$

$15.954 \quad 71.124$

$15.185 \quad 85.122$

$15.954 \quad 71.505$

$\begin{array}{lll}15.954 & 72.220\end{array}$

$15.954 \quad 72.553$

$15.954 \quad 72.867$

$15.954 \quad 73.211$

$15.954 \quad 73.742$

$15.954 \quad 74.450$

8.37471 .581

$15.954 \quad 84.866$

$8.374 \quad 72.309$

$8.374 \quad 73.424$

$8.512 \quad 70.545$

$8.374 \quad 73.812$

8.51270 .871

$8.374 \quad 76.898$

$8.512 \quad 73.371$

$25.763 \quad 71.756$

$8.374 \quad 78.199$

$8.512 \quad 74.364$

$25.763 \quad 72.651$

$26.116 \quad 70.323$

$8.374 \quad 79.622$

8.51275 .396

$8.742 \quad 70.430$

$25.763 \quad 73.570$

$26.116 \quad 71.121$

8.37480 .494 
Se26 F-like 2S2 2P4 ( 3P) 3S (4P) $j=2.5-2 S 22 P 5$ Se26 F-like 2S2 2P4 ( $3 P$ ) 3S (4P) $j=2.5-2 S 22 P 5$ Se26 F-like 2S2. 2P4 ( 3P) 3S (4P) j=2.5 - 2S2 2P5 Se26 F-like 2S2 2P4 ( $3 P$ ) 3S (4P) $j=2.5-2 S 2$ 2P5 Se26 F-like 2S2 2P4 (3P) 3S (4P) j=1.5 - 2S2 2P5 Se26 F-like 2S2 2P4 ( 3P) 3S (4P) j=1.5 - 2S2 2P5 Se26 $F$-like 2S2 2P4 ( 3P) 3S (4P) $j=1.5-2 S 22 P 5$ Se26 $F$-like 2S2 2P4 ( 3P) 3S (4P) j=1.5 - 2S2 2P5 Se26 $F$-like 2S2 2P4 ( 3P) 3S (4P) $j=1.5-2 S 2$ 2P5 Se26 F-like 2S2 2P4 (3P) ) 3S (4P) $j=1.5-2 S 2$ 2P5 Se27 O-like 2S2 $2 P^{*}$ ( $\left.2 D^{*}\right) 4 D\left(3 D^{*}\right) j=3.0-2 S 22 P 4$ Se27 0 -Iike 2S2 2P3 ( $\left.4 S^{*}\right) 4 D\left(3 D^{*}\right) j=1.0-2 S 22 P 4$ Se27 O-like 2S2 $2 P 3\left(.4 S^{*}\right) 4 D\left(3 D^{*}\right) j=2.0-2 S 22 P 4$ Se27 0 -like 2S2 2P3 (2P*) 3D (3D*) j=2.0-2S2 2P4 Se27 0 -iike 2S2 $2 P_{3}\left(2 D^{*}\right) 3 D\left(3 S^{*}\right) j=1.0-2 S 22 P 4$ Se27 O-like 2S2 $2 P^{2}\left(2 P^{*}\right) 3 D\left(1 F^{*}\right) j=3.0-2 S 22 P 4$ Se27 O-like 2S2 2P3 (2P*) 3D (1F*) j=3.0-2S2 2P4 Se27 0 -like 2S2 $2 P^{2}\left(2 D^{*}\right) 3 D\left(3 F^{*}\right) j=2.0-2 S 22 P 4$ Se27 O-like 2S2 $2 P_{3}\left(2 D^{*}\right) 3 D\left(3 F^{*}\right) j=2.0-2 S 22 P 4$ Se27 O-1ike 2S2 2P3 (2P*) 3D (3P*)j=1.0-2S2 2P4 Se27 O-like 2S2 2P3(2P*) 3D(3P*)j=1.0-2S2 2P4 Se27 O-like 2S2 $2 P^{2}\left(2 D^{*}\right) 3 D\left(1 P^{*}\right) j=1.0-2 S 22 P 4$ Se27 O-like 2S2 $2 P^{2}\left(2 D^{\star}\right) 3 D\left(1 P^{*}\right) j=1.0-2 S 22 P 4$ Se27 0 -1ike 2S2 2P3 (2D*) $3 D\left(3 G^{*}\right) j=3.0-2 S 22 P 4$ Se27 O-like 2S2 2P3 (2D*) 3D (3G*)j=3.0-2S2 2P4 Se27 O-like 2S2 2P3 ( 2D*) 3D (3F*) j=3.0 - 2S2 2P4 Se27 $0-1$ ike $2 S 22 P^{2}\left(2 D^{*}\right) 3 D\left(3 F^{*}\right) j=3.0-2 S 22 P 4$ Se27 O-like 2S2 2P3 (2D*) 3D $\left(1 D^{*}\right) j=2.0-2 S 22 P 4$ Se27 O-like 2S2 2P3 $\left(2 D^{*}\right)$ 3D $\left(1 D^{*}\right) j=2.0-2 S 2$ 2P4 $S e 270-1 i k e$ 2S2 $2 P_{3}\left(2 P^{*}\right) 3 D\left(3 P^{*}\right) j=2.0-2 S 22 P 4$ Se27 0 -like 2S2 2P3 ( 2P*) 3D (3P*)j=2.0-2S2 2P4 Se27 O-1ike 2S2 2P3 (2P*) 3D $\left(3 F^{*}\right) j=2.0-2 S 22 P 4$ Se27 O-like 2S2 2P3 ( 2P*) 3D $\left(3 F^{*}\right) j=2.0-2 S 22 P 4$ Se27 0 -like 2S2 2P3 (2D*) 3D $\left(3 P^{*}\right) j=.0-2 S 22 P 4$ Se27 O-1ike 2S2 2P3 (2D*) 3D $\left(3 P^{*}\right) j=.0-2 S 22 P^{*}$ Se27 O-like 2S2 $2 P 3\left(4 S^{*}\right) 3 D\left(3 D^{*}\right) j=3.0-2 S 22 P 4$ Se27 0 -1ike 2S2 2P3 (4S*) 3D $\left(3 D^{*}\right) j=3.0-2 S 22 P 4$ Se27 0 -like 2S2 2P3 (2P*) 3D (1P*) $j=1.0-2 S 22 P 4$ Se27 O-like 2S2 2P3 (2P*) 3D (1 $\left.P^{*}\right) j=1.0-2 S 22 P^{*}$ Se27 O-like 2S2 2P3 (4S*) 3D $\left(3 D^{*}\right) j=2.0-2 S 22 P 4$ Se27 O-1ike 2S2 2P3 (4S*) 3D $\left(3 D^{*}\right) j=2.0-2 S 22 P 4$ Se27 O-1ike 2S2 2P3 ( $\left.4 S^{*}\right)$ 3D $\left(3 D^{*}\right) j=1.0-2 S 22 P 4$ Se27 0 -like 2S2 2P3 (4S*) 3D (3D*) $j=1.0-2 S 22 P 4$ Se27 O-like 2S2 2P3 (2P*) 3S (3P*) j=2.0 - 2S2 2P4 Se27 0 -like 2S2 2P3 (2P*) 3S (3P*)j=2.0-2S2 2P4 Se27 o -like $2 S 22 \mathrm{P} 3\left(2 P^{*}\right) 3 S\left(1 P^{*}\right) j=1.0-2 S 22 P 4$ $\left(2 P^{*}\right) j=1.5$

$\left(2 P^{*}\right) j=1.5$

$\left(2 p^{*}\right) j=1.5$

$(2 P *) j=1.5$

$\left(2 P^{\star}\right) j=.5$

$\left(2 P^{*}\right) j=.5$

$\left(2 P^{\star}\right) j=.5$

$\left(2 P^{*}\right) j=.5$

$\left(2 P^{*}\right) j=.5$

$\left(2 P^{\star}\right) j=.5$

(3p) $j=2.0$

(3P) $j=.0$

(3P) $j=1.0$

(3P) $j=1.0$

(3P) $j=2.0$

(1D) $j=2.0$

(1D) $j=2.0$

(3P ) $j=2.0$

(3P) $j=2.0$

(1D) $j=2.0$

(1D) $j=2.0$

(3P) $j=2.0$

(3P) $j=2.0$

(3p) $j=2.0$

(3P) $j=2.0$

(3P) $j=2.0$

(3P) $j=2.0$

(3P) $j=1.0$

(3P) $j=1.0$

(1D) $j=2.0$

(1D) $j=2.0$

(1D) $j=2.0$

(ID) $j=2.0$

(3P) $j=1.0$

(3P) $j=1.0$

(3P) $j=2.0$

(3P) $j=2.0$

(1s) $j=.0$

(1s) $j=.0$

(3P) $j=2.0$

(3P) $j=2.0$

(3P) $j=.0$

(3p) $j=.0$

(3P) $j=1.0$

(3P) $j=1.0$

(1D) $j=2.0$
8.259 quartz 100

8.259 PET 002

8.259 TAP 100

8.259 RAP 100

8.483 guartz 100

8.483 PET 002

8.483 EDT 020

8.483 TAP 100

8.483 RAP 100

8.483 KAP 100

$5.537 \mathrm{NaCl} 200$

5.713 calcite 200

5.781 calcite 200

7.091 Inst 111

1.124 InSb 111

7.138 InSb 111

7.138 gypsum 020

7.150

7.150

7.170

7.170

7.177

7.177

7.207

7.207

7.214

7.214

7.290

7.290

7.294

7.294

7.301

7.301

7.316

7.316

7.329

7.329

7.340

7.340

7.353

7.353

7.400

7.400

7.565

7.565

7.610 Insb 111 gypsum 020 InSb 111

gypsum 020 InSb 111 gypsum 020 InSb 111 gypsum 020 InSt 111

gypsum 020 Insb 111 gypsum 020 InSb 111 gypsum 020 InSb 111 gypsum 020 InSb 111 gypsum 020 Insb 111 gypsum 020 Insb 111 gypsum 020 InSb 111 gypsum 020 InSb 111

gypsum 020 gypsum 020

bery 100 beryl 100
$8.512 \quad 75.996$ $8.742 \quad 70.865$ $25.763 \quad 74.097$ $26.116 \quad 71.573$

$8.512 \quad 85.269$

8.74276 .018

$8.808 \quad 74.387$

$25.763 \quad 81.045$

$26.116 \quad 77.023$

$26.634 \quad 72.845$

5.64178 .981

$6.071 \quad 70.225$

6.07172 .219

7.48171 .418

$\begin{array}{ll}7.481 & 72.228\end{array}$

$7.481 \quad 72.583$

$15.185 \quad 70.075$

$\begin{array}{ll}7.481 & 72.893\end{array}$

$15.185 \quad 70.342$

$\begin{array}{ll}7.481 & 73.421\end{array}$

$15.185 \quad 70.796$

$\begin{array}{ll}7.481 & 73.610\end{array}$

$\begin{array}{ll}15.185 & 70.957\end{array}$

7.48174 .445

$15.185 \quad 71.664$

$7.481 \quad 74.646$

$15.185 \quad 71.832$

$\begin{array}{ll}7.481 & 77.025\end{array}$

$15.185 \quad 73.772$

$7.481 \quad 77.162$

$15.185 \quad 73.881$

$7.481 \quad 77.406$

$15.185 \quad 74.072$

$7.481 \quad 77.944$

$15.185 \quad 74.490$

$\begin{array}{ll}7.481 & 78.430\end{array}$

$15.185 \quad 74.861$

$\begin{array}{ll}7.481 & 78.858\end{array}$

$15.185 \quad 75.182$

$7.481 \quad 79.386$

$15.185 \quad 75.571$

$7.481 \quad 81.561$

$15.185 \quad 77.070$

$15.185 \quad 85.122$

$15.954 \quad 71.505$

$15.954 \quad 72.553$ 
Se27 O-like 2S2 $2 \mathrm{P} 3\left(2 D^{*}\right) 3 S\left(3 D^{*}\right) j=3.0-2 S 22 P 4$ Se27 O-like 2S2 2P3 ( 4S*) 3D (3D*) $j=1.0-2 S 2$ 2PA Se27 O-like 2S2 $2 P 3\left(2 D^{*}\right) 3 S\left(1 D^{*}\right) j=2.0-2 S 22 P 4$ Se27 O-like 2S2 2P3 ( $\left.4 S^{*}\right) 3 S\left(3 S^{*}\right) j=1.0-2 S 22 P 4$ Se27 O-like 2S2 2P3 ( 2P*) 3S (1P*) j=1.0 - 2S2 2P4 Se27 O-like 2S2 2P3 ( 2D*) 3S $\left(3 D^{*}\right) j=2.0-2 S 22 P 4$

Se27 0 -like $2 S 22 P 3\left(2 D^{*}\right) 3 S\left(3 D^{*}\right) j=2.0-2 S 22 P 4$ Se31 Be-like 1S2 2P Se31 Be-like 1S2 2P Se31 Be-like 1S2 2P Se31 Be-like 1S2 2P Se31 Be-like 1S2 2P Se31 Be-like 1S2 2P Se31 Be-like 1S2 2P Se31 Be-like 1S2 2P Se31 Be-like 1S2 2P Se31 Be-like 1S2 2P Se31 Be-like 1S2 $2 \mathrm{P}$ Se31 Be-like 1S2 2P Se31 Be-like 1S2 2P Se31 Be-like 1S2 2P Se31 Be-like 1S2 2P Se31 Be-like 1S2 2P Se31 Be-like 1S2 2P Se31 Be-like 1S2 2P Se31 Be-like 1S2 2P Se31 Be-like 1S2 2P

Se31 Be-like 1S2 2P Se31 Be-like 1S2 2P Se31 Be-like 1S2 2P Se31 Be-like 1S2 2P Se31 Be-like 1S2 2P Se31 Be-like 1S2 2P Se31 Be-like 1S2 2P Se31 Be-like 1S2 2P Se31 Be-like 1S2 2P Se31 Be-like 1S2 2P Se31 Be-like 1S2 2P Se31 Be-like 1S2 2P Se31 Be-like 1S2 2S Se31 Be-like 1s2 2S Se31 Be-like 1s2 2S Se31 Be-like 1S2 2S Se31 Be-like 1S2 25 Se31 Be-like 1S2 2S Së31 Be-like 1s2 2S
(3P) $j=2.0$

(1s) $j=.0$

(3P) $j=1.0$

(3P) $j=2.0$

(1S) $j=.0$

(3P) $j=1.0$

(3P) $j=1.0$

$\left(3 P^{\star}\right) j=1.0$

$\left(3 P^{*}\right) j=1.0$

$\left(3 P^{*}\right) j=1.0$

$\left(3 P^{*}\right) j=1.0$

$2 P\left(3 P^{*}\right) j=2.0$

$2 P\left(3 P^{*}\right) j=2.0$

$2 P\left(3 P^{*}\right) j=2.0$

$2 P\left(3 P^{\star}\right) j=2.0$

$\left(3 P^{\star}\right) j=1.0$

$\left(3 P^{\star}\right) j=1.0$

$\left(3 P^{\star}\right) j=1.0$

$\left(3 P^{*}\right) j=1.0$

$2 P\left(3 P^{*}\right) j=2.0$

2P $\left(3 P^{\star}\right) j=2.0$

2P $\left(3 P^{*}\right) j=2.0$

$2 P\left(3 P^{*}\right) j=2.0$

$2 P\left(3 P^{*}\right) j=2.0$

$2 P\left(3 P^{\star}\right) j=2.0$

2P $\left(1 P^{*}\right) j=1.0$

$2 P\left(1 P^{*}\right) j=1.0$

$2 P\left(1 P^{*}\right) j=1.0$

2P $\left(1 P^{*}\right) j=1.0$

$2 P\left(1 P^{*}\right) j=1.0$

2P $\left(1 P^{*}\right) j=1.0$

2P $(1 P *) j=1.0$

$2 P\left(3 P^{*}\right) j=2.0$

$2 P\left(3 P^{*}\right) j=2.0$

$2 P\left(3 P^{*}\right) j=2.0$

$2 P\left(3 P^{*}\right) j=2.0$

$2 P\left(3 P^{\star}\right) j=2.0$

2P $\left(3 P^{*}\right) j=2.0$

$2 P\left(3 P^{*}\right) j=2.0$

$\left(3 P^{\star}\right) j=1.0$

$\left(3 P^{*}\right) j=1.0$

$\left(3 P^{\star}\right) j=1.0$

$\left(3 P^{*}\right) j=1.0$

$\left(3 P^{\star}\right) j=1.0$

$\left(3 P^{*}\right) j=1.0$

$\left(32^{*}\right) j=1.0$
7.623 beryl 100

7.782 beryl 100

7.801 beryl 100

7.821 beryl 100

7.846 beryl 100

7.874 topaz 002

7.874 beryl 100

6.011 calcite 200

6.011 Si 111

6.011 sylvite 200

6.011 Eluorite 1111

6.048 calcite 200

6.048 Si 111

6.048 sylvite 2001

6.048 fluorite 1111

6.129 Si 111

6.129 sylvite $200 \quad 1$

6.129 Eluorite $111 \quad 1$

6.129 TAP 100

$6.177 \mathrm{Si} 111$

6.177 sylvite 200

6.177 fluorite 1111

6.177 Ge 111

6.177 TAP 100

6.177 RAP 100

6.234 Si 111

6.234 sylvite 200

6.234 fluorite 111

6.234 Ge 111

$6.234 \mathrm{KBr} 200$

6.234 TAP 100

6.234 RAP 100

6.238 Si 111

6.238 sylvite 200

6.238 fluorite 111

6.238 Ge 111

$6.238 \mathrm{KBr} 200$

6.238 TAP 100

6.238 RAP 100

6.308 Ge 111

$6.308 \mathrm{KBr} 200$

6.308 quartz 101

6.308 graphite 002

6.308 mica 002

6.308 TAP 100

6.308 RAP 100
$15.954 \quad 72.867$

$15.954 \quad 77.305$

$15.954 \quad 77.942$

$15.954 \quad 78.650$

$15.954 \quad 79.602$

$8.374 \quad 70.101$

$15.954 \quad 80.783$

$6.071 \quad 81.938$

$6.271 \quad 73.444$

$6.292 \quad 72.812$

$6.308 \quad 72.348$

$6.071 \quad 85.011$

$6.271 \quad 74.674$

$6.292 \quad 73.991$

$6.308 \quad 73.492$

$6.271 \quad 77.784$

$6.292 \quad 76.930$

$6.308 \quad 76.318$

$25.763 \quad 72.101$

$6.271 \quad 80.067$

$6.292 \quad 79.029$

$6.308 \quad 78.303$

$6.532 \quad 71.024$

$25.763 \quad 73.546$

$26.116 \quad 71.100$

$6.271 \quad 83.773$

$6.292 \quad 82.214$

$6.308 \quad 81.215$

$6.532 \quad 72.526$

6.58471 .234

$25.763 \quad 75.443$

$26.116 \quad 72.711$

$6.271 \quad 84.119$

$6.292 \quad 82.488$

$6.308 \quad 81.456$

$6.532 \quad 72.744$

$6.584 \quad 71.343$

$25.763 \quad 75.586$

$26.116 \quad 72.829$

6.53274 .952

6.58473 .351

$6.687 \quad 70.617$

$6.696 \quad 70.400$

$19.942 \quad 71.614$

$25.763 \quad 78.347$

$26.116 \quad 75.050$ 


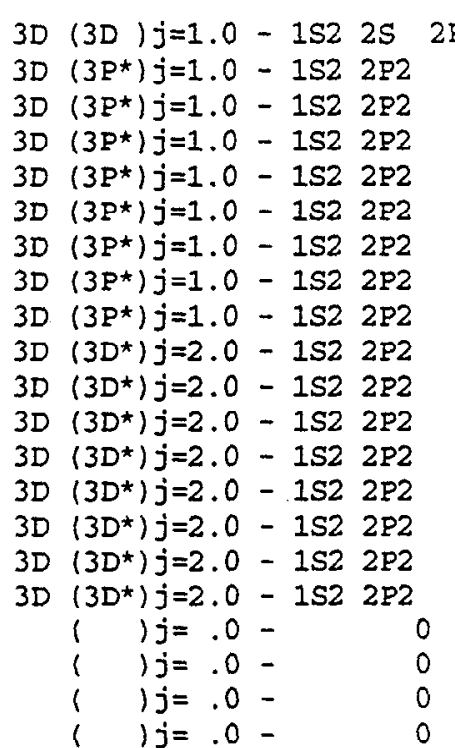

$\left(3 P^{*}\right) j=1.0-1 \mathrm{~S} 2$

$\left(3 P^{*}\right) j=1.0-1 S 2$

$\left(3 P^{*}\right) j=1.0-152$

$\left(3 P^{*}\right) j=1.0-1 S 2$

$\left(3 P^{*}\right) j=1.0-1 S 2$

$\left(3 P^{\star}\right) j=1.0-152$

$\left(3 P^{*}\right) j=1.0-152$

$\left(3 P^{*}\right) j=1.0-152$

$\left(3 P^{*}\right) j=1.0-1 S 2$

$\left(1 P^{*}\right) j=1.0-1 S 2$

$\left(1 P^{*}\right) j=1.0-1 S 2$

$\left(1 P^{*}\right) j=1.0-1 \mathrm{~S} 2$

$\left(3 P^{*}\right) j=1.0-1 S 2$

$\left(3 P^{*}\right) j=1.0-152$

$\left(3 P^{*}\right) j=1.0-152$

(3s) $j=1.0-1 s 2$

(3s) $j=1.0-1 S 2$

(3s) $j=1.0-152$

(3S) $j=1.0-1 \mathrm{~s} 2$

$\left(2 P^{\star}\right) j=1.5-1 S$

$\left(2 P^{*}\right) j=1.5-1 S$

$\left(2 P^{*}\right) j=1.5-1 S$

$\left(2 P^{\star}\right) j=1.5-1 S$

$\left(2 P^{\star}\right) j=1.5-1 S$

$\left(2 P^{*}\right) j=1.5-1 S$ $\left(3 P^{*}\right) j=1.0$

(3P) $j=2.0$

(3P) $j=2.0$

(3P) $j=2.0$

(3P) $j=2.0$

(3P) $j=2.0$

(3P) $j=2.0$

(3P) $j=2.0$

(3P) $j=2.0$

(3P) $j=2.0$

(3P) $j=2.0$

(3P) $j=2.0$

(3P) $j=2.0$

(3P) $j=2.0$

(3P) $j=2.0$

(3P) $j=2$. 0

(3P) $j=2.0$

l $j=.0$

$j j=.0$

$j j=.0$

$j j=.0$

(1s) $j=.0$

(1s) $j=.0$

(1s) $j=.0$

(1s) $j=.0$

(1s) $j=.0$

(1s) $j=.0$

(1s) $j=.0$

(1s) $j=.0$

(IS) $j=.0$

(1s) $j=.0$

(1s) $j=.0$

(1s) $j=.0$

(1s) $j=.0$

(1s) $j=.0$

(1s) $j=.0$

(1s) $j=.0$

(1s) $j=.0$

(1S) $j=.0$

(1s) $j=.0$

(2s) $j=.5$

(2S) $j=.5$

(2s) $j=.5$

(2s) $j=.5$

(2s) $j=.5$

(2s) $j=.5$
6.308 KAP 100

322 Ge 111

$6.322 \mathrm{KBr} 200$

6.322 guartz 101

6.322 graphite 002

6.322 mica 002

6.322 TAP 100

6.322 RAP 100

6.322 KAP 100

6.368 Ge 11

$6.368 \mathrm{KBr} 200$

6.368 quartz 101

6.368 graphite 002

6.368 mica 002

6.368 TAP 100

6.368 RAP 100

6.368 KAP 100

5.952 calcite 200

5.952 Si 111

5.952 sylvite 200

5.952 fluorite 111

905 corundum 030

.905 quartz 203

.905 topaz 006

.905

.905

.905

.905

.905

1.064

1.064 tungsten

$1.064 \mathrm{NaCl} 200$

1.071 Si 422

1.071 tungsten 110

$1.071 \mathrm{NaCl} 200$

1.075 Si 422

1.075 tungsten 110

1.075 quartz 112

$1.075 \mathrm{NaCl} 200$

.872 LiF 420

.872 topaz 303

.872 corundum 030

872

872

.872 quartz 112

tungsten 110
$26.634 \quad 71.327$

$6.532 \quad 75.432$

$6.584 \quad 73.782$

$6.687 \quad 70.982$

6.69670 .760

$19.942 \quad 72.000$

$25.763 \quad 78.981$

$26.116 \quad 75.534$

$26.634 \quad 71.707$

$6.532 \quad 77.134$

6.58475 .283

$6.687 \quad 72.231$

$6.696 \quad 71.992$

$19.942 \quad 73.331$

$25.763 \quad 81.380$

$26.116 \quad 77.250$

$26.634 \quad 73.013$

6.07178 .637

$6.271 \quad 71.646$

6.29271 .078

$6.308 \quad 70.659$

$2.748 \quad 81.112$

$2.749 \quad 80.979$

$2.795 \quad 76.259$

$2.848 \quad 72.421$

$3.636 \quad 84.623$

$3.840 \quad 70.511$

$4.564 \quad 82.504$

$4.638 \quad 77.326$

$4.676 \quad 75.400$

$2.217 \quad 73.710$

$4.476 \quad 71.962$

$5.641 \quad 70.578$

$2.217 \quad 75.054$

$4.476 \quad 73.157$

$5.641 \quad 71.677$

2. $217 \quad 75.878$

$4.476 \quad 73.879$

$4.564 \quad 70.417$

$5.641 \quad 72.335$

$1.801 \quad 75.547$

$2.712 \quad 74.710$

$2.748 \quad 72.169$

$2.749 \quad 72.105$

$3.636 \quad 73.596$

$4.476 \quad 76.927$ 


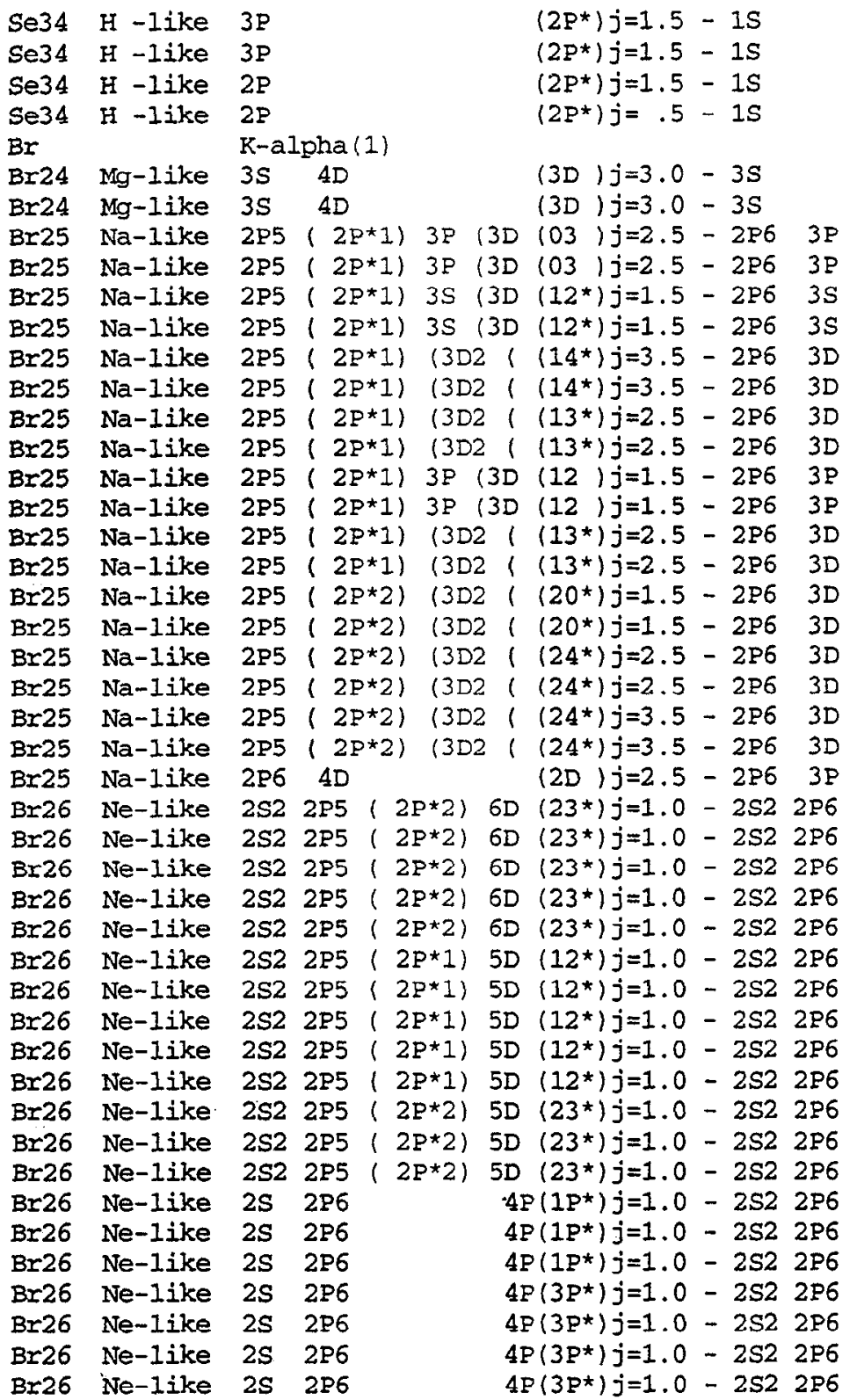

\begin{tabular}{|c|c|c|c|c|c|}
\hline$(2 s) j=.5$ & .872 & quartz 112 & 5 & 4.564 & 72.805 \\
\hline$(2 s) j=.5$ & .872 & topaz 200 & 5 & 4.638 & 70.062 \\
\hline$(2 s) j=.5$ & 1.031 & quartz 200 & 4 & 4.246 & 76.232 \\
\hline$(2 S) j=.5$ & 1.037 & quartz 200 & 4 & 4.246 & 77.666 \\
\hline & 1.040 & quartz 200 & 4 & 4.246 & 78.395 \\
\hline$\left.P^{\star}\right) j=2.0$ & 24.816 & TAP 100 & 1 & 25.763 & 74.417 \\
\hline$\left.P^{*}\right) j=2.0$ & 24.816 & RAP 100 & 1 & 26.116 & 71.846 \\
\hline$\left.P^{*}\right) j=1.5$ & 7.188 & InSb 111 & 1 & 7.481 & 73.911 \\
\hline$\left(2 P^{\star}\right) j=1.5$ & 7.188 & gypsum 020 & 2 & 15.185 & 71.213 \\
\hline (s) $j=.5$ & 7.200 & InSb 111 & 1 & 7.481 & 74.246 \\
\hline$(2 S) j=.5$ & 7.200 & gypsum 020 & 2 & 15.185 & 71.496 \\
\hline$(2 D) j=2.5$ & 7.232 & InSb 111 & 1 & 7.481 & 75.176 \\
\hline (2D) $j=2.5$ & 7.232 & gypsum 020 & 2 & 15.185 & 72.273 \\
\hline$(2 D) j=2.5$ & 7.246 & Insb 111 & 1 & 7.481 & 75.601 \\
\hline$(2 D) j=2.5$ & 7.246 & gypsum 020 & 2 & 15.185 & 72.623 \\
\hline$\left\langle 2 P^{\star}\right\} j=.5$ & 7.254 & InSto 111 & 1 & 7.481 & 75.849 \\
\hline$\left(2 P^{*}\right) j=.5$ & 7.254 & gypsum 020 & 2 & 15.185 & 72.827 \\
\hline 2D $/ j=1.5$ & 7.285 & InSto 111 & 1 & 7.481 & 76.856 \\
\hline (2D) $j=1.5$ & 7.285 & gypsum 020 & 2 & 15.185 & 73.638 \\
\hline (2D) $j=2.5$ & 7.391 & Insb 111 & 1 & 7.481 & 81.104 \\
\hline (2D) $j=2.5$ & 7.391 & gypsum 020 & 2 & 15.185 & 76.770 \\
\hline (2D $) j=2.5$ & 7.413 & InSb 111 & 1 & 7.481 & 82.269 \\
\hline $2 D) j=2.5$ & 7.413 & gypsum 020 & 2 & 15.185 & 77.516 \\
\hline $2 D / j=2.5$ & 7.436 & InSb 111 & 1 & 7.481 & 83.712 \\
\hline$(2 D) j=2.5$ & 7.436 & gypsum 020 & 2 & 15.185 & 78.347 \\
\hline $\left.2 P^{*}\right) j=1.5$ & 24.465 & TAP 100 & 1 & 25.763 & 71.735 \\
\hline is $j j=.0$ & 5.005 & ADP 101 & 2 & 10.640 & 70.185 \\
\hline is $j j=.0$ & 5.005 & gypsum 020 & 3 & 15.185 & 81.419 \\
\hline$j=.0$ & 5.005 & beryl 100 & 3 & 15.954 & 70.244 \\
\hline$j=.0$ & 5.005 & TAP 100 & 5 & 25.763 & 76.253 \\
\hline$j=.0$ & 5.005 & RAP 100 & 5 & 26.116 & 73.380 \\
\hline$j=.0$ & 5.148 & ADP 101 & 2 & 10.640 & 75.391 \\
\hline$j j=.0$ & 5.148 & beryl 100 & 3 & 15.954 & 75.474 \\
\hline$j=.0$ & 5.148 & TAP 100 & 5 & 25.763 & 87.579 \\
\hline$j=.0$ & 5.148 & RAP 100 & 5 & 26.116 & 80.266 \\
\hline$j=.0$ & 5.148 & KAP 100 & 5 & 26.634 & 75.113 \\
\hline (s) $j=.0$ & 5.251 & ADP 101 & 2 & 10.640 & 80.762 \\
\hline LS $j j=.0$ & 5.251 & beryl 100 & 3 & 15.954 & 80.895 \\
\hline$j=.0$ & 5.251 & KAP 100 & 5 & 26.634 & 80.323 \\
\hline$j=.0$ & 5.292 & ADP 101 & 2 & 10.640 & 84.119 \\
\hline$j=.0$ & 5.292 & beryl 100 & 3 & 15.954 & 84.332 \\
\hline$j=.0$ & 5.292 & KAP 100 & 5 & 26.634 & 83.447 \\
\hline$j=.0$ & 5.305 & $\mathrm{NaCl} 200$ & 1 & 5.641 & 70.125 \\
\hline$j=.0$ & 5.305 & ADP 101 & 2 & 10.640 & 85.696 \\
\hline$j=.0$ & 5.305 & beryl 10 & 3 & 15.954 & 85.993 \\
\hline$j=.0$ & 5.305 & KAP 100 & 5 & 26.634 & 84.815 \\
\hline
\end{tabular}


Br26 Ne-like 2S2 2P5 $\left(2 P^{*} 2\right) \quad 4 D\left(23^{*}\right) j=1.0-2 S 2 \quad 2 P 6$ Br26 Ne-like 2S2 2P5 ( $\left.2 P^{*} 1\right)$ 4S $(11 *) j=1.0-2 S 22 P 6$ Br26 Ne-like 2S2 2P5 ( 2P*2) 4S $(22 *) j=1.0-2 S 22 P 6$ Br26 Ne-1ike 2S2 2P5 $\left(2 P^{\star} 2\right)$ 4S $\left(22^{*}\right) j=1.0-2 S 22 P 6$ Br26 Ne-1ike 2S2 2P5 ( 2P*2) 4S (22*) j=1.0 - 2S2 2P6 Br26 Ne-like 2S2 2P5 ( 2P*2) 4S $\left(22^{\star}\right) j=1.0-2 S 2$ 2P6 Br26 Ne-like 2S2 2P5 ( 2P*1) 3D (12*) j=1.0 - 2S2 2P6 Br26 Ne-like 2S2 2P5 ( 2P*1) 3D (12*) j=1.0 - 2S2 2P6 Br26 Ne-like 2S2 2P5 ( 2P*2) 3D $\left(23^{*}\right) j=1.0-2 S 22 P 6$ Br26 Ne-like 2S2 2P5 ( 2P*2) 3D $(23 *) j=1.0-2 S 2$ 2P6 Br26 Ne-like 2S2 2P5 ( 2P*2) 3D $\left(22^{*}\right) j=1.0-2 S 22 P 6$ Br26 Ne-1ike 2S2 2P5 ( 2P*2) 3D (22*) j=1.0 - 2S2 2P6 Br26 Ne-1ike 2S2 2P5: ( $\left.2 P^{*} * 1\right)$ 3S $(11 *) j=1.0-2 S 2$ 2P6 Br26 Ne-like 2S2 2P5 $(2 P \star 2) 3 S(22 *) j=1.0-2 S 2$ 2P6 Br26 Ne-1ike 2S2 2P5 $(2 P * 2) 3 S\left(22^{*}\right) j=1.0-2 S 22 P 6$ Br27 F-like 2S2 2P4 ( $1 S$ ) $4 D$ (2D) $j=2.5-2 S 22 P 5$ Br27 F-like 2S2 2P4 ( $1 D$ ) $4 D(2 F) j=2.5-2 S 22 P 5$ Br27 F-like 2S2 2P4 ( 3P) $4 D(4 P) j=2.5-2 S 22 P 5$ Br27 F-like 2S2 2P4 ( $3 P$ ) $4 D(4 F) j=2.5-2 S 22 P 5$ $B r 27$ - F -like $2 \$ 22 P 4(1 D) 4 S(2 D) j=2.5-2 S 22 P 5$ Br27 F-1ike 2S2 2P4 ( 3P) 4D (2D) $j=2.5-2 S 22 P 5$ Br27 F -like 2S 2P5 ( $\left.1 P^{*}\right) 3 P(2 D) j=2.5-2 S 22 P 5$ Br27 F-like 2S 2P5 ( $1 P^{*}$ ) 3P (2D) $j=2.5-2 S 22 P 5$ Br27 F-like $2 S$ 2P5 ( $\left.1 P^{*}\right) 3 P(2 D) j=2.5-2 S 22 P 5$ Br27 F-like 2S 2P5 ( $\left.1 P^{*}\right) 3 P(2 D) j=2.5-2 S 22 P 5$ Br27 F-like 2S 2P5 ( $\left.1 P^{*}\right) 3 P(2 D) j=2.5-2 S 22 P 5$ Br27 F-like 2S 2P5 ( $\left.1 P^{*}\right) 3 P(2 D) j=2.5-2 S 22 P 5$ Br27 F -like $2 S$ 2P5 ( $\left.1 P^{*}\right) 3 P(2 D) j=2.5-2 S 2$ 2P5 Br27 F-like 2S 2P5 ( $\left.1 P^{*}\right)$, 3P (2D) $j=2.5-2 S 2$ 2P5 Br27 F-like $2 S$ 2P5 ( $\left.P^{\star}\right)$ ) 3P (2P) $j=1.5-2 S 22 P 5$ Br27 F-like 2S 2P5 (1P*) 3P (2P) $j=1.5-2 S 2$ 2P5 Br27 F-like 2S 2P5 ( $\left.1 P^{*}\right)$ ) 3P (2P) $j=1.5-2 S 22 P 5$ Br27 F-like $2 S$ 2P5 ( $\left.1 P^{\star}\right) 3 P(2 P) j=1.5-2 S 22 P 5$ Br27 F -like $2 S$ 2P5 ( $\left.1 P^{*}\right) 3 P(2 P) j=1.5-2 S 2$ 2P5 Br27 F-like 2S 2P5 ( $\left.1 P^{*}\right) 3 P(2 P) j=1.5-2 S 22 P 5$ Br27 F-1ike 2S 2P5 ( $\left.1 P^{*}\right)$ 3P (2P) $j=1.5-2 S 22 P 5$ Br27 F -like $2 S$ 2P5 (1P*) $3 P(2 P) j=.5-2 S 22 P 5$ Br27 F-like 2S 2P5 ( $\left.1 P^{*}\right) 3 P(2 P) j=.5-2 S 22 P 5$ Br27 F-like $2 S$ 2P5 ( $\left.1 P^{*}\right) 3 P(2 P) j=.5-2 S 22 P 5$ Br27 F -like 2S 2P5 (1 $\left.P^{*}\right) 3 P(2 P) j=.5-2 S 22 P 5$ Br27 F-like $2 S 2 P 5\left(1 P^{*}\right) 3 P(2 P) j=.5-2 S 22 P 5$ BI27 F-like 2S 2P5 ( $\left.1 P^{*}\right) 3 P(2 P) j=.5-2522 P 5$ Br27 F-like 2S 2P5 ( $\left.3 P^{*}\right) 3 P(4 P) j=2.5-2 S 22 P 5$ Br27 F-like $2 S$ 2P5 ( $\left.3 P^{*}\right)$ ) $3 P(4 P) j=2.5-2 S 2$ 2P5 Br27 $F$-like $2 S 2 P 5$ ( $\left.3 P^{*}\right) 3 P(4 P) j=2.5-2 S 22 P 5$ Br27 F-like $2 S$ 2P5 ( $\left.3 P^{*}\right) 3 P(4 P) j=2.5-2 S 22 P 5$
(1S) $j=.0$ (1s) $j=.0$ (1S) $j=.0$ (1s) $j=.0$ (1s) $j=.0$ (1s) $j=.0$ (1s) $j=.0$ (1s) $j=.0$ (1s) $j=.0$ (1s) $j=.0$ (1s) $j=.0$ (1s) $j=.0$ (1s) $j=.0$ (IS) $j=.0$ (1S) $j=.0$ $\left(2 P^{*}\right) j=1.5$ $\left(2 P^{*}\right) j=1.5$ $\left(2 P^{\star}\right) j=1.5$ $\left(2 P^{*}\right) j=1.5$ $\left(2 P^{*}\right) j=1.5$ $\left(2 P^{*}\right) j=1.5$ $\left(2 P^{*}\right) j=1.5$ $\left(2 P^{*}\right) j=1.5$ $\left(2 P^{*}\right) j=1.5$ $\left(2 P^{\star}\right) j=1.5$ $\left(2 P^{*}\right) j=1.5$ $\left(2 P^{\star}\right) j=1.5$ $\left(2 P^{*}\right) j=1.5$ $\left(2 P^{\star}\right) j=1.5$ $\left(2 P^{*}\right) j=.5$ $\left(2 P^{*}\right) j=.5$ $\left(2 P^{*}\right) j=.5$ $\left(2 P^{*}\right) j=.5$ $\left(2 P^{*}\right) j=.5$ $\left(2 P^{*}\right) j=.5$ $\left(2 P^{*}\right) j=.5$ $\left(2 P^{*}\right) j=.5$ $\left(2 P^{\star}\right) j=.5$ $\left(2 p^{*}\right) j=.5$ $\left(2 P^{\star}\right) j=.5$ $\left(2 P^{\star}\right) j=.5$ $\left(2 p^{\star}\right) j=.5$

$\left(2 P^{\star}\right) j=1.5$ $\left(2 P^{\star}\right) j=1.5$ $\left(2 P^{*}\right) j=1.5$

$\left(2 P^{\star}\right) j=1.5$
5.771 calcite $200 \quad 1$ 5.793 calcite 200 5.928 calcite 200 5.928 si 111

5.928 sylvite 200 5.928 fluorite 111 7.169 InSb 111

7.169 gypsum 020

7.351 InSb 111

7.351 gypsum 020

7.436 InSb 111

7.436 gypsum 020

7.790

8.030

8.030

beryl 100

5.400 topaz 002

$5.412 \mathrm{NaCl} 200$

$5.429 \mathrm{NaCl} 200$

$5.493 \mathrm{NaCl} 200$

$5.507 \mathrm{NaCl} 200$

$5.530 \mathrm{NaCl} 200$

6.359 Ge 111

$6.359 \mathrm{KBr} 200$

6.359 quartz 101

6.359 graphite 0021

6.359 mica 002

6.359 TAP 100

6.359 RAP 100

6.359 KAP 100

6.512 Ge 111

$6.512 \mathrm{KBr} 200$

6.512 quartz 10

6.512 graphite 002

6.512 mica 002

6.512 RAP 100

6.512 KAP 100

$6.531 \mathrm{Ge} 111$

$6.531 \mathrm{KBr} 200$

6.531 quartz 101

6.531 graphite 002

6.531 mica 002

6.531 KAP 100

$6.556 \mathrm{KBr} 200$

6.556 quartz 101

6.556 graphite 0021

6.556 mica 002
$6.071 \quad 71.913$

$6.071 \quad 72.594$

6.07177 .540

$6.271 \quad 70.962$

$6.292 \quad 70.416$

$6.308 \quad 70.011$

$7.481 \quad 73.394$

$15.185 \quad 70.773$

$7.481 \quad 79.303$

$15.185 \quad 75.510$

$7.481 \quad 83.712$

$15.185 \quad 78.347$

$15.954 \quad 77.569$

$8.374 \quad 73.520$

$8.512 \quad 70.626$

$5.641 \quad 73.192$

$5.641 \quad 73.618$

$5.641 \quad 74.242$

5.64176 .846

$5.641 \quad 77.487$

5.64178 .615

6.53276 .784

$6.584 \quad 74.978$

$6.687 \quad 71.980$

$6.696 \quad 71.745$

$19.942 \quad 73.063$

$25.763 \quad 80.862$

$26.116 \quad 76.897$

$26.634 \quad 72.750$

$6.532 \quad 85.515$

$6.584 \quad 81.519$

$6.687 \quad 76.863$

$6.696 \quad 76.537$

$19.942 \quad 78.419$

$26.116 \quad 85.864$

$26.634 \quad 77.959$

$6.532 \quad 88.997$

$6.584 \quad 82.725$

$6.687 \quad 77.600$

$6.696 \quad 77.254$

$19.942 \quad 79.265$

$26.634 \quad 78.769$

$6.584 \quad 84.714$

$6.687 \quad 78.640$

6.69678 .263

$19.942 \quad 80.491$ 


\begin{tabular}{|c|c|c|c|c|c|c|c|c|c|c|}
\hline $\mathrm{B} \times 27$ & & -like & $2 \mathrm{~S}$ & $2 \mathrm{P5}$ & $3 p^{*}$ & f & $3 P$ & $(4 P) j=2.5$ & -2 & 2 \\
\hline Br27 & & -1ike & $2 S$ & $2 \mathrm{P} 5$ & $1 \mathrm{P}^{*}$ & 1 & $3 P$ & $(2 D) j=1.5$ & -2 & $2 \mathrm{P5}$ \\
\hline 27 & & -like & $S$ & 2P5 & $1 P^{\star}$ & & $3 P$ & $(2 D) j=1.5$ & . & P5 \\
\hline$r 27$ & $F^{\prime}$ & -1ike & $S$ & 25 & $1 P^{*}$ & & $3 P$ & $(2 D) j=1.5$ & 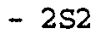 & \\
\hline r27 & $\mathbf{F}$ & -like & is & P5 & $1 P^{*}$ & & $3 P$ & $(2 D$ & 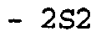 & \\
\hline$r 27$ & $F$ & -like & S & 25 & $1 P^{\star}$ & ) & $3 P$ & $(2 D) j=1.5$ & 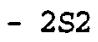 & \\
\hline$=27$ & $F$ & -1ike & $S$ & $2 P 5$ & $3 P^{*}$ & & $3 P$ & $(2 P) j=1.5$ & $-2 s 2$ & \\
\hline 227 & $F$ & -like & $2 S$ & $2 P 5$ & $3 P^{*}$ & & $3 P$ & $(2 P) j=1.5$ & - & \\
\hline 2 & & -like & es & P5 & $3 P^{*}$ & & $3 P$ & $(2 P$ & & \\
\hline-27 & $F$ & -like & $2 S$ & 5 & $3 P^{*}$ & ) & $3 P$ & $(2 \mathrm{P}$ & 52 & \\
\hline 27 & $\mathbf{F}$ & -like & $2 S$ & $2 P 5$ & $3 P^{\star}$ & ) & $3 P$ & $(2 D) j=1.5$ & - & 25 \\
\hline$=27$ & $\mathbf{F}$ & -1ike & $2 S$ & 2P5 & $3 P^{\star}$ & ) & $3 P$ & $(2 D) j=1.5$ & . & \\
\hline r27 & F & -like & es & $2 \mathrm{PS}$ & $3 P^{*}$ & ) & $3 P$ & $(2 D) j=1.5$ & . & \\
\hline 27 & F & -1ike & $2 S$ & P5 & $3 P^{*}$ & 1 & $3 p$ & (2D) $j=1.5$ & . & \\
\hline 27 & $F$ & -1ike & S & P5 & $3 P^{\star}$ & ) & $3 P$ & $(4 D) j=1.5$ & -2 & \\
\hline 227 & $\boldsymbol{F}$ & -like & $2 \mathrm{~s}$ & $P 5$ & $3 P^{*}$ & ) & $3 P$ & $(4 D) j=1.5$ & $2 \mathrm{~S} 2$ & \\
\hline$r 27$ & $\mathbf{F}$ & -like & $2 S$ & $2 P 5$ & $3 P^{*}$ & ) & $3 P$ & $(4 D) j=1.5$ & . & P5 \\
\hline$r 27$ & F & -like & $2 S$ & P5 & $3 p^{*}$ & ) & $3 P$ & $(4 D)$ & 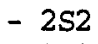 & $\mathrm{Pz}$ \\
\hline 27 & $\mathbf{F}$ & -like & $2 S$ & $\mathrm{P}$ & $3 P^{*}$ & ) & $3 P$ & (4D) & S2 & \\
\hline 227 & $\mathbf{F}$ & -like & $2 S$ & $2 P 5$ & $3 P^{\star}$ & ) & $3 P$ & (4D) $j=2.5$ & 252 & \\
\hline Br27 & $\mathbf{F}$ & -like & $2 \$ 2$ & $2 P 4$ & 10 & 1 & $3 D$ & $(2 P$ & & \\
\hline Br27 & $\mathbf{F}$ & $-1 i k e$ & $2 s 2$ & $2 P 4$ & $1 D$ & ) & $3 D$ & $(2 D)$ & $2 S 2$ & \\
\hline $\mathrm{B} \times 27$ & $\mathbf{F}$ & -like & $2 \mathrm{~S} 2$ & $2 P 4$ & $3 P$ & f & $3 D$ & $(4 F)$ & 252 & PS \\
\hline $3 \times 27$ & $F$ & -like & $2 s 2$ & 2P4 & $3 P$ & ) & $3 D$ & $(2 F)$ & 252 & \\
\hline$\therefore 27$ & $\mathbf{F}$ & -like & $2 s 2$ & $2 \mathrm{P} 4$ & $3 p$ & ) & $3 D$ & $(4 F) j$ & $\$ 2$ & \\
\hline Br27 & $\mathbf{F}$ & -like & $2 s 2$ & $2 \mathrm{P} 4$ & $3 \mathrm{P}$ & ) & $3 \mathrm{D}$ & $(4 P) j=.5$ & s2 & 25 \\
\hline$r 27$ & $F$ & -1ike & $2 \mathrm{~s} 2$ & $2 P 4$ & $3 P$ & ) & $3 D$ & $j=.5$ & 2 & 5 \\
\hline $8 \simeq 27$ & $\mathbf{F}$ & -like & $2 s 2$ & $2 P 4$ & $3 P$ & ) & 30 & $(2 P) j=1.5$ & 32 & P5 \\
\hline $3 r 27$ & F & -1ike & $2 S 2$ & $2 P 4$ & $3 P$ & \} & $3 D$ & $(2 P) j=1.5$ & $-2 s 2$ & P5 \\
\hline 27 & $\mathbf{F}$ & -1ike & 252 & $2 P 4$ & $3 P$ & ) & $3 D$ & $(4 \mathrm{P})$ & $-2 s 2$ & 25 \\
\hline$\Upsilon 27$ & $F$ & -Iike & $2 \$ 2$ & $2 P 4$ & $3 P$ & ) & $3 D$ & $(4 P) j=1.5$ & $2 \mathrm{~S} 2$ & P5 \\
\hline 227 & $F$ & -like & $2 S 2$ & $2 \mathrm{P} 4$ & $3 P$ & ) & $3 \mathrm{D}$ & $(4 P) j=.5$ & 32 & P5 \\
\hline 227 & $F$ & -like & 252 & $2 P 4$ & $3 P$ & ) & $3 D$ & $(4 P) j=.5$ & S2 2 & P5 \\
\hline 527 & $F$ & -like & 252 & 2P4 & 15 & ) & $3 S$ & $(2 s) j=.5$ & $2 S 2$ & P5 \\
\hline 527 & $F$ & -like & $2 \$ 2$ & $2 P 4$ & $1 S$ & ) & $3 S$ & $(2 S) j=.5$ & $-2 s 2$ & 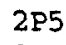 \\
\hline 27 & $F$ & -1ike & 252 & $2 P 4$ & $1 D$ & 7 & $3 S$ & $(2 D) j=2.5$ & $2 S 2$ & P5 \\
\hline 227 & $F$ & -like & $2 s 2$ & 2P4 & 1D & 1 & $3 S$ & $(2 D) j=2.5$ & $-2 s 2$ & P5 \\
\hline 527 & $F$ & -like & $2 s 2$ & $2 P 4$ & $3 P$ & ; & $3 s$ & $(2 P) j=.5$ & 252 & P5 \\
\hline 627 & $\mathbf{F}$ & -like & 252 & $2 P 4$ & $3 P$ & ) & $3 S$ & $(2 P) j=.5$ & $-2 s 2$ & 5 \\
\hline $3 r 27$ & $F$ & -like & $2 S 2$ & $2 P 4$ & $3 P$ & ) & $3 s$ & $(4 P) j=1.5$ & $-2 s 2$ & $2 \mathrm{P} 5$ \\
\hline r27 & $F$ & -like & $2 \$ 2$ & $2 P 4$ & $3 P$ & ) & $3 s$ & $(4 P) j=.5$ & $2 \mathrm{~S} 2$ & P5 \\
\hline 227 & $\mathbf{F}$ & -like & $2 \mathrm{~S} 2$ & $2 P 4$ & ID & ) & 35 & $(2 D) j=1.5$ & 252 & P5 \\
\hline 27 & $F$ & -like & 252 & $2 P 4$ & $3 P$ & 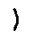 & 35 & $(2 \mathrm{P})$ & is2 & P5 \\
\hline & $F$ & -like & $2 \mathrm{S2}$ & $2 \mathrm{P} 4$ & $3 P$ & & $3 s$ & $(4 \mathrm{P})$ & $2 S 2$ & 20 \\
\hline$r 27$ & $F$ & -like & $2 \mathrm{S2}$ & $2 P 4$ & $3 P$ & & $3 S$ & $=1.5$ & - & $\mathrm{P}$ \\
\hline$r 28$ & 0 & -like & $2 S 2$ & $2 \mathrm{P} 3$ & $2 \mathrm{P}^{\star}$ & ) & $4 D$ & $\left(3 P^{*}\right) j=2.0$ & $S 2$ & P4 \\
\hline
\end{tabular}

\begin{tabular}{|c|c|c|}
\hline$\left(2 P^{*}\right) j=1.5$ & 6.556 & $\operatorname{KAP} 100$ \\
\hline$\left(2 P^{*}\right) j=.5$ & 6.567 & $\mathrm{KBr} 200$ \\
\hline$\left(2 P^{\star}\right) j=.5$ & 6.567 & quartz 101 \\
\hline $\left.2 P^{*}\right) j=.5$ & 6.567 & graphite 002 \\
\hline$\left(2 P^{\star}\right) j=.5$ & 6.567 & mica 002 \\
\hline$\left(2 P^{\star}\right) j=.5$ & 6.567 & KAP 100 \\
\hline$\left(2 P^{\star}\right) j=1.5$ & 6.598 & quartz 101 \\
\hline$\left(2 P^{\star}\right) j=1.5$ & 6.598 & graphite 002 \\
\hline$\left(2 P^{\star}\right) j=1.5$ & 6.598 & mica 002 \\
\hline $\left.2 P^{\star}\right) j=1.5$ & 6.598 & KAP 100 \\
\hline$\left(2 P^{\star}\right) j=.5$ & 6.624 & quartz 101 \\
\hline $\left.2 P^{\star}\right) j=.5$ & 6.624 & graphite 002 \\
\hline$\left(2 \mathrm{P}^{*}\right) j=.5$ & 6.624 & mica 002 \\
\hline$\left(2 P^{\star}\right) j=.5$ & 6.624 & $\operatorname{KAP} 100$ \\
\hline$\left(2 q^{*}\right) j=1.5$ & 6.633 & quartz 101 \\
\hline$\left(2 P^{\star}\right) j=1.5$ & 6.633 & graphite 002 \\
\hline$\left(2 P^{*}\right) j=1.5$ & 6.633 & mica 002 \\
\hline$\left(2 P^{*}\right) j=1.5$ & 6.633 & $\operatorname{KAP} 100$ \\
\hline$\left(2 P^{*}\right) j=1.5$ & 6.674 & quartz 101 \\
\hline$\left(2 P^{*}\right) j=1.5$ & 6.674 & graphite 002 \\
\hline$\left(2 P^{*}\right) j=.5$ & 7.057 & Insb 111 \\
\hline$\left(2 \mathrm{P}^{\star}\right) j=.5$ & 7.071 & InSb 111 \\
\hline$\left(2 P^{*}\right) j=1.5$ & 7.092 & InSb 111 \\
\hline$\left(2 P^{\star}\right) j=1.5$ & 7.111 & InSb 111 \\
\hline$\left(2 P^{*}\right) j=1.5$ & 7.118 & InSb 111 \\
\hline$\left(2 P^{*}\right) j=1.5$ & 7.140 & InSb 111 \\
\hline$\left(2 P^{*}\right) j=1.5$ & 7.140 & gypsum 020 \\
\hline$\left(2 P^{*}\right) j=.5$ & 7.169 & Insb 111 \\
\hline$\left(2 P^{\star}\right) j=.5$ & 7.169 & gypsum 020 \\
\hline$\left(2 P^{*}\right) j=.5$ & 7.330 & InSb 111 \\
\hline$\left(2 p^{\star}\right) j=.5$ & 7.330 & gypsum 020 \\
\hline$\left(2 P^{\star}\right) j=.5$ & 7.348 & InSb 111 \\
\hline$\left(2 P^{\star}\right) j=.5$ & 7.348 & gypsum 020 \\
\hline$\left(2 P^{*}\right) j=.5$ & 7.403 & Insb 111 \\
\hline$\left(2 P^{*}\right) j=.5$ & 7.403 & gypsum 020 \\
\hline$\left(2 P^{*}\right) j=1.5$ & 7.436 & InSb 111 \\
\hline$\left(2 P^{\star}\right) j=1.5$ & 7.436 & gypsum 020 \\
\hline$\left(2 P^{*}\right) j=1.5$ & 7.473 & Insto 111 \\
\hline$\left(2 P^{*}\right) j=1.5$ & 7.473 & gypsum 020 \\
\hline$\left(2 P^{\star}\right) j=1.5$ & 7.488 & gypsum 020 \\
\hline$\left(2 P^{\star}\right) j=1.5$ & 7.608 & beryl 100 \\
\hline$\left(2 P^{*}\right) j=.5$ & 7.651 & beryl 100 \\
\hline$\left(2 Q^{*}\right) j=1.5$ & 7.685 & beryl 100 \\
\hline$\left(2 P^{*}\right) j=1.5$ & 7.704 & beryl 100 \\
\hline$j=.5$ & 7.720 & beryl 100 \\
\hline$=2.0$ & 5.148 & \\
\hline
\end{tabular}

$26.634 \quad 79.934$

$6.584 \quad 85.882$

$6.687 \quad 79.129$

$6.696 \quad 78.735$

$19.942 \quad 81.083$

$26.634 \quad 80.490$

$6.687 \quad 80.642$

$6.696 \quad 80.185$

$19.942 \quad 83.015$

$26.634 \quad 82.270$

$6.687 \quad 82.129$

$6.696 \quad 81.590$

$19.942 \quad 85.198$

$26.634 \quad 84.165$

$6.687 \quad 82.714$

$6.696 \quad 82.134$

$19.942 \quad 86.237$

$26.634 \quad 84.984$

$6.687 \quad 86.427$

$6.696 \quad 85.354$

$7.481 \quad 70.617$

$7.481 \quad 70.943$

$7.481 \quad 71.442$

$7.481 \quad 71.905$

$7.481 \quad 72.078$

$7.481 \quad 72.634$

15.18570 .119

$7.481 \quad 73.394$

$15.185 \quad 70.773$

$\begin{array}{ll}7.481 & 78.469\end{array}$

$15.185 \quad 74.890$

$7.481 \quad 79.180$

$15.185 \quad 75.420$

$7.481 \quad 81.719$

$15.185 \quad 77.172$

$7.481 \quad 83.712$

$\begin{array}{ll}15.185 & 78.347\end{array}$

$\begin{array}{ll}7.481 & 87.350\end{array}$

$15.185 \quad 79.821$

$15.185 \quad 80.483$

$15.954 \quad 72.505$

$15.954 \quad 73.563$

15.95474 .450

$15.954 \quad 74.967$

$15.954 \quad 75.417$

$10.640 \quad 75.391$ 
Br28 O-like 2S2 $2 P^{2}\left(2 P^{*}\right) 4 D\left(3 P^{*}\right) j=2.0-2 S 22 P 4$ Br28 O-like 2S2 2P3 ( 2P*) $4 D\left(3 P^{*}\right) j=2.0-2 S 22 P 4$ Br28 0 -like 2S2 2P3 ( 2P*) $4 D\left(3 P^{*}\right) j=2.0-2 S 2$ 2P4 $\mathrm{B} \times 280$-like $2 S 22 \mathrm{P}^{2}\left(2 \mathrm{P}^{*}\right) 4 D\left(3 \mathrm{P}^{*}\right) j=2.0-2 S 22 \mathrm{P} 4$ Br28 O-like 2S2 2P3 ( 2P*) $4 D\left(3 P^{*}\right) j=2.0-2 S 22 P^{*}$ Br28 O-like 2S2 2P3 (2P*) $4 D\left(3 P^{*}\right) j=2.0-2 S 22 P 4$ Br28 O-like 2S2 $2 P^{2}\left(2 P^{*}\right) 4 D\left(3 P^{*}\right) j=2.0-2 S 22 P 4$ Br28 0 -like 2S2 $2 P 3\left(4 S^{\star}\right) 4 D\left(3 D^{\star}\right) j=3.0-2 S 22 P 4$ Br28 O-like 2S2 $2 P^{2}$ ( $\left.4 S^{*}\right) 4 D\left(3 D^{*}\right) j=3.0-2 S 22 P 4$ Br28 O -like $2522 P 3\left(4 S^{*}\right) 4 D\left(3 D^{\star}\right) j=3.0-2 S 22 P 4$ Br28 O-like 2S2 2P3 ( 2D*) $4 D\left(3 D^{*}\right) j=2.0-2 S 22 P 4$ Br28 0 -like 2S2 2P3 (2D*) $4 D\left(3 D^{*}\right) j=2.0-2 S 22 P 4$ Br28 0 -like 2S2 $2 P_{3}\left(2 D^{\star}\right) \cdot 4 D\left(3 D^{\star}\right) j=2.0-2 S 22 P 4$ Br28 O -like 2S2 2P3 ( $\left.2 D^{*}\right) 4 D\left(3 D^{*}\right) j=2.0-2 S 22 P 4$ Br28 O-like 2S2 $2 P^{3}\left(2 D^{*}\right) 4 D\left(3 D^{*}\right) j=2.0-2 S 22 P 4$ Br28 0 -like 2S2 $2 \mathrm{P} 3\left(2 D^{*}\right) 4 D\left(3 D^{*}\right) j=2.0-2 S 22 P 4$ Br28 O-like 2S2 $2 P^{2}\left(2 D^{*}\right) 4 D\left(3 D^{*}\right) j=2.0-2 S 22 P 4$ Br28. O -1ike 2S2 $2 P^{2}\left(2 D^{\star}\right) 4 D\left(3 D^{*}\right) j=2.0-2 S 22 P 4$ Br28 O-1ike 2S2 2P3 ( $\left.4 S^{*}\right) 4 D\left(3 D^{*}\right) j=1.0-2 S 22 P 4$ Br28 0 -like 2S2 2P3 (4S*) $4 D\left(3 D^{*}\right) j=1.0-2 S 22 P 4$ Br28 O-like $2 S 22 P^{2}\left(4 S^{*}\right) 4 D\left(3 D^{*}\right) j=2.0-2 S 22 P 4$ Br28 O-like 2S2 2P3 ( 2P*) 3D $\left(3 D^{*}\right) j=2.0-2 S 22 P 4$ $B \times 280-1 i k e$ 2S2 2P3 ( $\left.2 P^{*}\right) 3 D\left(3 D^{*}\right) j=2.0-2 S 22 P 4$ Br28 O-1ike 2S2 2P3 ( $\left.2 P^{*}\right) 3 D\left(3 D^{*}\right) j=2.0-2 S 22 P 4$ Br28 O-like 2S2 2P3 (2P*) 3D $\left(3 D^{*}\right) j=2.0-2 S 22 P 4$ $B r 280-1$ ike 2S2 $2 P^{2}\left(2 D^{*}\right) 3 D\left(3 P^{*}\right) j=2.0-2 S 22 P 4$ BI28 O-like 2S2 2P3 ( 2D*) 3D (3P*)j=2.0-2S2 $2 P^{*} 4$ $B r 280-l i k e$ 2S2 $2 P 3\left(2 D^{*}\right) 3 D\left(3 P^{*}\right) j=2.0-2 S 22 P 4$ Br28 O-like 2S2 2P3 (2D*) 3D (3P*)j=2.0-2S2 2P4 Br28 0 -like 2S2 2P3 ( $\left.2 P^{*}\right) 3 S\left(3 P^{*}\right) j=2.0-2 S 22 P 4$ Br28 O-like 2S2 2P3 ( $\left.2 P^{*}\right) 3 S\left(1 P^{*}\right) j=1.0-2 S 22 P 4$ Br28 O-like 2S2 2P3 ( 2P*) 3S $\left(3 P^{*}\right) j=2.0-2 S 22 P 4$ Br28 O-like 2S2 2P3 ( 2P*) 3S $\left(3 P^{*}\right) j=1.0-2 S 22 P^{*}$ Br28 O-like 2S2 2P3 ( 2D*) 3S $\left(3 D^{*}\right) j=2.0-2 S 22 P 4$ Br28 O-like 2S2 2P3 ( 2D*) 3S $\left(3 D^{*}\right) j=2.0-2 S 22 \mathrm{P} 4$ Br28 0 -like 2S2 2P3 (2P*) 3S $\left(3 P^{*}\right) j=.0-2 S 22 P 4$ Br28 0 -like 2S2 $2 P^{2}\left(2 P^{*}\right) 3 S\left(3 P^{*}\right) j=.0-2 S 22 P 4$ Br28 0 -like 2S2 2P3 ( 2P*) $3 S\left(3 P^{*}\right) j=1.0-2 S 22 P 4$ Br28 O -like 2S2 2P3 (2P*) 3S $\left(3 P^{*}\right) j=1.0-2 S 22 P 4$ Br28 O-like 2S2 $2 P 3$ ( $\left.4 S^{*}\right) 3 S\left(3 S^{*}\right) j=1.0-2 S 22 P 4$ Br28 O -1ike 2S2 2P3 (4S*) 3S $\left(3 S^{*}\right) j=1.0-2 S 22 \mathrm{P} 4$ Br28 O-like 2S2 2P3 (2P*) 3S (1P*) $j=1.0-2 S 22 P 4$ Br28 0 -like 2S2 2P3 (2P*) 3S (1P*) j=1.0 - 2S2 2P4 Br28 O -like $2 S 22 \mathrm{P} 3$ (2D*) $3 S\left(3 D^{*}\right) j=2.0-2522 \mathrm{P}$ Br28 O -like 2S2 $2 P^{2}\left(2 D^{*}\right) 3 S\left(3 D^{*}\right) j=2.0-2 S 22 P 4$ BI28 :0 -like 2S2 $2 \mathrm{P} 3$ ( $\left.4 S^{\star}\right) 3 \mathrm{~S}\left(3 \mathrm{~S}^{\star}\right) j=1.0-2 S 22 \mathrm{P} 4$
(3P) $j=2.0$

( $3 P) j=2.0$

(3P) $j=2.0$

(3P) $j=2.0$

( $3 P) j=1.0$

(3P) $j=1.0$

(3P) $j=1.0$

(3P) $j=2.0$

(3P) $j=2.0$

(3P) $j=2.0$

(3P) $j=1.0$

(3P) $j=1.0$

(3P) $j=1.0$

(3P) $j=1.0$

(3P) $j=1.0$

(3P) $j=1.0$

(3P) $j=1.0$

(3P) $j=1.0$

(3P) $j=.0$

$(3 P) j=.0$

(3P) $j=1.0$

(3P) $j=1.0$

(3P) $j=1.0$

(3P) $j=1.0$

(3P) $j=1.0$

(3P) $j=2.0$

(3P) $j=2.0$

(3P) $j=2.0$

(3P) $j=2.0$

(3P) $j=1.0$

(1D) $j=2.0$

(1D) $j=2.0$

(3P) $j=.0$

(3P) $j=2.0$

(3P) $j=2.0$

(3P) $j=1.0$

(3P) $j=1.0$

(1D) $j=2.0$

(1D) $j=2.0$

(3P) $j=2.0$

(3P) $j=2.0$

(1s) $j=.0$

(1s) $j=.0$

(3P) $j=1.0$

(3P) $j=1.0$

$(3 P) j=.0$
5.148 beryl 100

5.148 TAP 100

148 RAP 100

5.148 KAP 100

5.251 ADP 101

5.251 beryl 100

5.251 KAP 100

5.292 ADP 101

5.292 beryl 100

$5.292 \mathrm{KAP} 100$

$5.305 \mathrm{NaCl} 200$

5.305 ADP 101

5.305 beryl 100

$5.305 \mathrm{KAP} 100$

5.305 NaCl 200

5.305 ADP 101

5.305 beryl 100

5.305 KAP 100

$5.325 \mathrm{NaCl} 200$

5.325 KAP 100

$5.400 \mathrm{NaCl} 200$

6.624 quartz 101

6.624 graphite 002

6.624 mica 002

$6.624 \operatorname{RAP} 100$

6.675 quartz 101

6.675 graphite 002

6.675 quartz 101

6.675 graphite 002

7.071 InSb 111

7.110 Insb 111

7.123 InSb 111

7.132 InSt 111

7.169 InSb 111

7.169 gypsum 020

7.246 InSb 111

7.246 gypsum 020

7.271 InSb 111

7.271 gypsum 020

7.285 InSb 111

7.285 gypsum 020

7.351 InSb 111

7.351 gypsum 020

7.370 Insb 111

7.370 gypsum 020

7.403 InSb 111
$15.954 \quad 75.474$

$25.763 \quad 87.579$

$26.116 \quad 80.266$

$26.634 \quad 75.113$

$10.640 \quad 80.762$

$15.954 \quad 80.895$

$26.634 \quad 80.323$

$10.640 \quad 84.119$

$15.954 \quad 84.332$

$26.634 \quad 83.447$

$5.641 \quad 70.125$

$10.640 \quad 85.696$

$15.954 \quad 85.993$

$26.634 \quad 84.815$

$5.641 \quad 70.125$

$10.640 \quad 85.696$

$15.954 \quad 85.993$

$26.634 \quad 84.815$

$5.641 \quad 70.731$

$26.634 \quad 88.510$

$5.641 \quad 73.192$

$6.687 \quad 82.129$

$6.696 \quad 81.590$

$19.942 \quad 85.198$

$26.634 \quad 84.165$

$6.687 \quad 86.567$

$6.696 \quad 85.461$

$6.687 \quad 86.567$

$6.696 \quad 85.461$

$\begin{array}{ll}7.481 & 70.943\end{array}$

$7.481 \quad 71.880$

$7.481 \quad 72.203$

$7.481 \cdot 72.430$

$7.481 \quad 73.394$

$15.185 \quad 70.773$

7.48175 .601

$15.185 \quad 72.623$

$7.481 \quad 76.392$

$15.185 \quad 73.267$

$7.481 \quad 76.856$

$15.185 \quad 73.638$

$\begin{array}{ll}7.481 & 79.303\end{array}$

$15.185 \quad 75.510$

\begin{tabular}{ll}
$7.481 \quad 80.118$ \\
\hline
\end{tabular}

$15.185 \quad 76.095$

$7.481 \quad 81.719$ 
Br28 O -like 2S2 $2 \mathrm{P} 3\left(4 S^{*}\right) 3 S\left(3 S^{*}\right) j=1.0-2 S 22 \mathrm{P} 4$

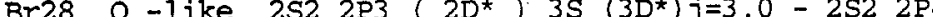

Br28 o-like 2S2 $2 P^{2} 3\left(2 D^{\star}\right) 3 S\left(3 D^{*}\right) j=3.0-2 S 22 P 4$

Br34 He-like is $3 P$

Br34 He-like 15 3P

Br34 He-1ike 1s $3 P$

Br34 He-like 15 2P

Br34 He-like is $2 P$

Br34 He-like 1S $2 \mathrm{P}$

Br34 He-1ike 1s 2P

Br34 He-like 15 2P

Br34 He-like 1S 2P

Br34 He-like 15 2P

Br34 He-like 1S 2P

Br34 He-like 15 2P

Br34 He-like is 2P

Br34 He-like is $2 \mathrm{P}$

Br34 He-like 1s 2S

Br34 He-like 1s 25

Br35 H -like 3P

Br35 H-like 3P

Br35 H-like 3P

Br35 H -like 2P

Br35 H -like 2P

Br35 H-like 2P

Br35 H -like 2P

Br35 $\mathrm{H}$-like 2P

Br35 H-like 2P

Br35 H-like 2P

Br35 H-like 2P

Br35 H-like 2P

Br35 H-like 2P

Br35 H-like 2P

Br35 H-like 2P

Br35 H -like 2P

Br35 H -like 2P

Br35 H -like 2P

Br35 H -like 2P

$\mathrm{Kr}$

$\mathrm{Kr}$

$\mathrm{Kr}$

$\mathrm{Kr}$

$\mathrm{Kr}$

$\mathrm{Kr}$

$\mathrm{Kr}$ $\left(3 P^{*}\right) j=1.0-1 \mathrm{~S} 2$

$\left(3 P^{\star}\right) j=1.0-1 S 2$

$\left(3 P^{*}\right) j=1.0-1 S 2$

$\left(1 p^{*}\right) j=1.0-1 \mathrm{~s} 2$

$\left(1 P^{*}\right) j=1.0-1 S 2$

$\left(1 P^{*}\right) j=1.0-152$

$\left(1 P^{*}\right) j=1.0-1 \mathrm{~s} 2$

$\left(1 P^{\star}\right) j=1.0-1 S 2$

$\left(1 P^{*}\right) j=1.0-1 S 2$

$\left(3 P^{*}\right) j=1.0-1 s 2$

$\left(3 P^{\star}\right) j=1.0-1 S 2$

$\left(3 P^{*}\right) j=1.0-1 S 2$

$\left(3 P^{*}\right) j=1.0-1 S 2$

$\left(3 P^{*}\right) j=1.0-1 S 2$

(3S) $j=1.0-152$

(3S) $j=1.0-1 S 2$

$\left(2 P^{\star}\right) j=1.5-1 S$

$\left(2 P^{*}\right) j=1.5-1 S$

$\left(2 P^{\star}\right) j=1.5-1 S$

$\left(2 P^{\star}\right) j=1.5-1 S$

$\left(2 P^{*}\right) j=1.5-1 S$

$\left(2 P^{\star}\right) j=1.5-1 S$

$\left(2 P^{\star}\right) j=1.5-1 S$

$\left(2 P^{\star}\right) j=1.5-1 S$

$\left(2 P^{\star}\right) j=1.5-1 S$

$\left(2 P^{\star}\right) j=1.5-1 S$

$\left(2 P^{*}\right) j=1.5-1 S$

$\left(2 P^{*}\right) j=.5-1 S$

$\left(2 P^{\star}\right) j=.5-15$

$\left(2 P^{\star}\right) j=.5-1 S$

$\left(2 P^{*}\right) j=.5-1 S$

$\left(2 P^{*}\right) j=.5-1 S$

$\left(2 P^{\star}\right) j=.5-1 S$

$\left(2 P^{*}\right) j=.5-1 S$

$\left(2 P^{*}\right) j=.5-1 S$
K-alpha (1)

K-alpha (1)

K-alpha(1)

K-alpha(1)

K-alpha(1)

$\mathrm{K}$-alpha(1)

K-alpha(1)

K-alpha(1)

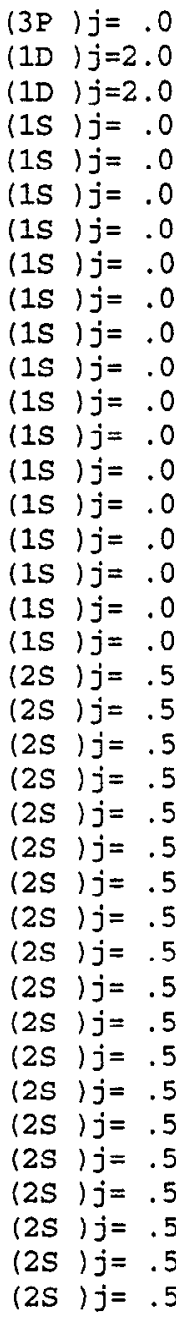

7.403

Inst 111

7.412

gypsum 020

.853 LiF 420

.853 topaz 303

tungster 110

1.002 quartz 223

1.002

calcite 422

1.002

1.002

quartz 211

1.002 Al 200

1.002 quartz 200

1.009

quartz 223

1.009 calcite 422

1.009

quartz 211

1.009 AI 200

1.009 quartz 200

1.012 quartz 211

1.012 quartz 200

.822 LiF 422

822 corundum 146

822

.972

972

quartz 200

quartz 223

972

.972

calcite 422

972 Al 200

.972 quartz 110

.972

978

.978

.978

gypsum 002

quartz 223

978

cuartz 211

978 A1 200

978 quartz 110

978 gypsum 002

.980 quartz 223

980 calcite 422

980 quartz 211

980 Ge 220

980 LiF 200

980 Al 200

980 quartz 110

980 gypsum 002
$15.185 \quad 77.172$

7.48182 .212

$15.185 \quad 77.482$

$1.801 \quad 71.307$

$2.712 \quad 70.662$

$4.476 \quad 72.337$

2.02481 .939

$3.034 \quad 82.210$

$3.082 \quad 77.250$

$4.027 \quad 84.432$

$4.048 \quad 81.939$

$4.246 \quad 70.725$

$2.024 \quad 85.587$

$3.034 \quad 86.107$

$3.082 \quad 79.159$

$4.048 \cdot 85.587$

$4.246 \quad 71.905$

$3.082 \quad 80.088$

4. $246 \quad 72.434$

$1.652 \quad 84.359$

$1.660 \quad 82.039$

$4.246 \quad 75.459$

$2.024 \quad 73.837$

$3.034 \quad 73.968$

$3.082 \quad 71.109$

$4.000 \quad 76.410$

$4.027 \quad 74.902$

$4.048 \quad 73.837$

$4.912 \quad 81.656$

$4.990 \quad 76.893$

$2.024 \quad 75.106$

$3.034 \quad 75.249$

$3.082 \quad 72.172$

$4.000 \quad 77.959$

$4.027 \quad 76.274$

$4.048 \quad 75.106$

$4.912 \quad 84.575$

$4.990 \quad 78.510$

$2.024 \quad 75.599$

$3.034 \quad 75.746$

$3.082 \quad 72.577$

$4.000 \quad 78.579$

$4.027 \quad 76.812$

$4.048 \quad 75.599$

$4.912 \quad 86.165$

$4.990 \quad 79.162$

Page 138 


\begin{tabular}{|c|c|c|c|c|c|c|c|c|}
\hline $\mathrm{Kr} 26$ & $\mathrm{Na}-1 i k e$ & $2 P 5$ & $3 \$ 2$ & & & $\left(2 P^{\star}\right) j=.5$ & $-2 P 6$ & $3 S$ \\
\hline $\mathrm{Kr} 26$ & Na-like & $2 P 5$ & $3 \mathrm{~S} 2$ & & & $\left(2 P^{*}\right) j=.5$ & $-2 P 6$ & $3 S$ \\
\hline $\mathrm{K} r 26$ & $\mathrm{Na-like}$ & $2 P 5$ & $3 S$ & & $3 D$ & $\left(31^{\star}\right) j=2.5$ & $-2 P 6$ & $3 D$ \\
\hline $\mathrm{K} \times 26$ & Na-like & $2 P 5$ & $3 s$ & & $3 D$ & $(31 *) j=2.5$ & $-2 P 6$ & $3 D$ \\
\hline $\mathrm{K} \times 26$ & Na-like & $2 P 5$ & $3 S$ & & $3 D$ & $(29 *) j=3.5$ & $-2 P 6$ & $3 D$ \\
\hline $\mathrm{K} \times 26$ & Na-like & $2 P 5$ & 35 & & $3 D$ & $\left(29^{*}\right) j=3.5$ & $-2 P 6$ & $3 D$ \\
\hline 126 & Na-like & $2 \mathrm{P} 5$ & $3 S$ & & $3 D$ & $\left(28^{*}\right) j=2.5$ & $-2 P 6$ & $3 D$ \\
\hline 26 & Na-like & 2P5 & 35 & & $3 D$ & $\left(28^{*}\right) j=2.5$ & $-2 P 6$ & $3 D$ \\
\hline 626 & Na-like & $2 \mathrm{P} 5$ & $3 \mathrm{~s}$ & & $3 P$ & $j j=2.5$ & $-2 P 6$ & $3 p$ \\
\hline 26 & Na-like & $2 \mathrm{P5}$ & 35 & & $3 P$ & $j=2.5$ & $-2 P 6$ & $3 P$ \\
\hline 26 & Na-like & $2 P 5$ & $3 s$ & & $3 P$ & $j=2.5$ & $-2 P 6$ & $3 P$ \\
\hline$c 26$ & Na-like & $2 P 5$ & $3 s$ & & $3 F$ & $j=2.5$ & $-2 P 6$ & $3 P$ \\
\hline 26 & Na-like & $2 \mathrm{P} 5$ & $3 \mathrm{~S} 2$ & & & $\left(2 P^{*}\right) j=1.5$ & $-2 P 6$ & 35 \\
\hline 26 & Na-like & $2 P 5$ & 352 & & & $\left(2 P^{\star}\right) j=1.5$ & $-2 P 6$ & 35 \\
\hline $\mathrm{Kr26}$ & $\mathrm{Na-like}$ & $2 P 5$ & $3 S$ & & $3 D$ & $\left(25^{\star}\right) j=3.5$ & $-2 P 6$ & $3 D$ \\
\hline $\mathrm{Kr} 26$ & Na-like & $2 \mathrm{P} 5$ & 35 & & $3 D$ & $(24 *) j=3.5$ & $-2 P 6$ & $3 D$ \\
\hline $\mathrm{K} r 26$ & Na-like & $2 P 5$ & 35 & & $3 D$ & $(20 *) j=2.5$ & $-2 P 6$ & $3 D$ \\
\hline $\mathrm{Kr} 26$ & Na-like & $2 \mathrm{P} 5$ & 35 & & $3 P$ & (5) $) j=2.5$ & $-2 P 6$ & $3 P$ \\
\hline $\mathrm{K} \times 26$ & Na-like & 2P5 & $3 s$ & & $3 P$ & $j=1.5$ & $-2 \mathrm{P} 6$ & $3 P$ \\
\hline $\mathrm{Kr} 26$ & Na-like & $2 P 6$ & $4 F$ & & & $\left(2 F^{\star}\right) j=2.5$ & $-2 P 6$ & $3 D$ \\
\hline $\mathrm{Kr} 26$ & $\mathrm{Na}-l i k e$ & $2 P 6$ & $4 F$ & & & $\left(2 F^{*}\right) j=2.5$ & $-2 P 6$ & $3 D$ \\
\hline $\mathrm{Kr} 26$ & Na-like & $2 \mathrm{PG}$ & $4 F$ & & & $\left(2 F^{\star}\right) j=2.5$ & $-2 P 6$ & $3 D$ \\
\hline $\mathrm{Kr} 26$ & Na-Iike & $2 \mathrm{P} 6$ & $4 \mathrm{~F}$ & & & $\left(2 F^{\star}\right) j=3.5$ & $-2 P 6$ & $3 D$ \\
\hline $\mathrm{K}=26$ & Na-like & $2 P 6$ & $4 F$ & & & $\left(2 F^{\star}\right) j=3.5$ & $-2 \mathrm{P} 6$ & $3 D$ \\
\hline $\mathrm{K} r 26$ & Na-like & $2 \mathrm{PG}$ & $4 F$ & & & $\left(2 F^{\star}\right) j=3.5$ & $-2 P 6$ & $3 D$ \\
\hline $\operatorname{Kr} 26$ & Na-like & & 0 & & & $j j=.0$ & - & 0 \\
\hline $\operatorname{Kr26}$ & Na-like & & 0 & & & $j=.0$ & - & 0 \\
\hline$r 27$ & Ne-like & $2 \mathrm{~S} 2$ & $2 \mathrm{P} 5$ & $(2 \mathrm{P} * 1)$ & $6 \mathrm{D}$ & $(12 *) j=1.0$ & $-2 s 2$ & $2 \mathrm{P} 6$ \\
\hline $\mathrm{K} r 27$ & Ne-like & 252 & $2 F 5$ & $(2 P * 1)$ & $6 D$ & $(12 \star) j=1.0$ & $-2 s 2$ & $2 P 6$ \\
\hline$r 27$ & Ne-like & $2 \mathrm{~S} 2$ & $2 \mathrm{P} 5$ & $(2 p * 2\rangle$ & $6 D$ & $(23 *) j=1.0$ & $-2 s 2$ & $2 \mathrm{P} 6$ \\
\hline 27 & Ne-like & 252 & $2 F 5$ & $(2 P * 2)$ & $6 D$ & $\left(23^{*}\right) j=1.0$ & $-2 s 2$ & $2 \mathrm{P} 6$ \\
\hline $\mathrm{K} 227$ & Ne-like & 252 & $2 P 5$ & $(2 P \star 2)$ & $6 D$ & $(23 *) j=1.0$ & $-2 s 2$ & $2 \mathrm{P} 6$ \\
\hline $\mathrm{K} \times 27$ & Ne-like & $2 \mathrm{~s} 2$ & 2P5 & $\left(2 P^{\star 1}\right)$ & $5 D$ & $\left(12^{*}\right) j=1.0$ & 252 & $2 P 6$ \\
\hline $\mathrm{Kr} 27$ & Ne-like & 252 & $2 P 5$ & $(2 P * 1)$ & $5 D$ & $\left(12^{*}\right) j=1.0$ & - & $2 \mathrm{P} 6$ \\
\hline Kr27 & Ne-like & $2 \mathbf{S} 2$ & $2 P 5$ & $\left(2 P^{\star} 1\right)$ & $5 D$ & $\left(12^{*}\right) j=1.0$ & - & $2 \mathrm{P} 6$ \\
\hline $\operatorname{kr27}$ & Ne-1i) & $2 \mathrm{~s} 2$ & 2P5 & $(2 P * 1)$ & $5 D$ & $\left(12^{*}\right) j=1.0$ & $-2 s 2$ & $2 \mathrm{P} 6$ \\
\hline $\mathrm{Kr} 27$ & $\mathrm{Ne}-1$ & $2 \mathrm{~S} 2$ & 2P5 & $(2 p \star 2)$ & $5 D$ & $\left(23^{*}\right) j=1.0$ & $-2 s 2$ & 2P6 \\
\hline $\operatorname{kr} 27$ & Ne-like & $2 \mathrm{~s} 2$ & $2 \mathrm{P} 5$ & $(2 \mathrm{P} * 2)$ & $5 D$ & $\left(23^{*}\right) j=1.0$ & $-2 s 2$ & $2 P 6$ \\
\hline $\operatorname{Kr27}$ & Ne-like & $2 s 2$ & $2 P 5$ & $(2 \mathrm{p} * 2)$ & $5 D$ & $\left(23^{*}\right) j=1.0$ & $-2 s 2$ & $2 \mathrm{PG}$ \\
\hline$K r 27$ & Ne-like & $2 s 2$ & $2 \mathrm{P} 5$ & $(2 \mathrm{p} * 2)$ & $5 D$ & $\left(23^{*}\right) j=1.0$ & $-2 s 2$ & $2 P 6$ \\
\hline Kr27 & Ne-like & $2 s 2$ & $2 P 5$ & $(2 \mathrm{P} \star 2)$ & $5 D$ & $(23 *) j=1.0$ & $-2 s 2$ & $2 \mathrm{P} 6$ \\
\hline $\operatorname{kr} 27$ & Ne-like & $2 \$ 2$ & $2 P 5$ & $(2 \mathrm{P} * 2)$ & $5 D$ & $\left(23^{*}\right) j=1.0$ & $-2 \mathrm{~S} 2$ & $2 \mathrm{P} 6$ \\
\hline $\mathrm{Kr} 27$ & Ne-like & $2 \mathrm{~S} 2$ & $2 P 5$ & $(2 \mathrm{P} \star 1)$ & $4 D$ & $\left(12^{*}\right) j=1.0$ & $-2 S 2$ & $2 \mathrm{P} 6$ \\
\hline r27 & Ne-like & $2 \mathrm{~s} 2$ & $2 \mathrm{P} 5$ & $\left(2 P^{\star 1}\right)$ & $4 \mathrm{D}$ & $(12 *) j=1.0$ & $-2 S 2$ & $2 \mathrm{P} 6$ \\
\hline & $\mathrm{Ne}-1$ i\} & $2 \mathrm{~S} 2$ & $2 \mathrm{P} 5$ & $(2 \mathrm{P} * 1)$ & $4 \mathrm{D}$ & $\left(12^{*}\right) j=1.0$ & $-2 s 2$ & $2 P 6$ \\
\hline & Ne-1ik & $2 s^{2}$ & $2 P 5$ & $(2 \mathrm{P} * 2)$ & $4 D$ & $\left(23^{*}\right) j=1.0$ & $-2 s 2$ & $2 \mathrm{PE}$ \\
\hline
\end{tabular}

\begin{tabular}{|c|c|c|c|c|c|}
\hline$(2 S) j=.5$ & 7.322 & InSb 111 & 1 & 7.481 & 78.166 \\
\hline$\langle 2 s\rangle j=.5$ & 7.322 & gypsum 020 & 2 & 15.185 & 74.660 \\
\hline $2 D / j=2.5$ & 7.350 & InSb 111 & 1 & 7.481 & 79.262 \\
\hline $2 D j j=2.5$ & 7.350 & gypsum 020 & 2 & 15.185 & 75.480 \\
\hline$(2 D) j=2.5$ & 7.376 & Insb 111 & 1 & 7.481 & 80.389 \\
\hline$(2 D) j=2.5$ & 7.376 & gypsum 020 & $\dot{2}$ & 15.185 & 76.284 \\
\hline $2 D / j=2.5$ & 7.384 & InSt 111 & 1 & 7.481 & 80.763 \\
\hline$(2 D) j=2.5$ & 7.384 & gypsum 020 & 2 & 15.185 & 76.541 \\
\hline$\left(2 P^{\star}\right) j=1.5$ & 7.393 & Inso 111 & 1 & 7.481 & 81.203 \\
\hline$\left(2 P^{*}\right) j=1.5$ & 7.393 & gypsum 020 & 2 & 15.185 & 76.836 \\
\hline$\left(2 P^{\star}\right) j=1.5$ & 7.554 & gypsum 020 & 2 & 15.185 & 84.228 \\
\hline $\left.2 P^{*}\right) j=1.5$ & 7.554 & beryl 100 & 2 & 15.954 & 71.258 \\
\hline $2 S / j=.5$ & 7.570 & gypsum 020 & 2 & 15.185 & 85.588 \\
\hline$\langle 2 s\rangle j=.5$ & 7.570 & beryl 100 & 2 & 15.954 & 71.619 \\
\hline$(2 D) j=2.5$ & 7.594 & beryl 100 & 2 & 15.954 & 72.173 \\
\hline$(2 D) j=2.5$ & 7.604 & beryl 100 & 2 & 15.954 & 72.409 \\
\hline$(2 D) j=1.5$ & 7.614 & beryl 100 & 2 & 15.954 & 72.649 \\
\hline$\left(2 P^{*}\right) j=1.5$ & 7.623 & beryl 100 & 2 & 15.954 & 72.867 \\
\hline$\left(2 P^{\star}\right) j=1.5$ & 7.634 & beryl 100 & 2 & 15.954 & 73.137 \\
\hline$(2 D) j=1.5$ & 25.605 & TAP 100 & 1 & 25.763 & 83.651 \\
\hline (2D $) j=1.5$ & 25.605 & $\operatorname{RAP} 100$ & 1 & 26.116 & 78.647 \\
\hline (2D) $j=1.5$ & 25.605 & KAP 100 & 1 & 26.634 & 74.022 \\
\hline$(2 D) j=2.5$ & 25.715 & TAP 100 & 1 & 25.763 & 86.502 \\
\hline (2D $) j=2.5$ & 25.715 & RAP 100 & 1 & 26.116 & 79.947 \\
\hline$(2 D) j=2.5$ & 25.715 & KAP 100 & 1 & 26.634 & 74.905 \\
\hline$j=.0$ & 7.538 & gypsum 020 & 2 & 15.185 & 83.131 \\
\hline$j=.0$ & 7.538 & beryl 100 & 2 & 15.954 & 70.903 \\
\hline (1s) $j=.0$ & 4.598 & topaz 200 & 1 & 4.638 & 82.470 \\
\hline$(1 s) j=.0$ & 4.598 & Al 111 & 1 & 4.676 & 79.520 \\
\hline$(1 s) j=.0$ & 4.690 & guartz 110 & 1 & 4.912 & 72.708 \\
\hline$(1 s) j=.0$ & 4.690 & gypsum 002 & 1 & 4.990 & 70.031 \\
\hline$(1 S) j=.0$ & 4.690 & mica 002 & 4 & 19.942 & 70.174 \\
\hline (1s) $j=.0$ & 4.812 & quartz 110 & 1 & 4.912 & 78.419 \\
\hline$j j=.0$ & 4.812 & gypsum 002 & 1 & 4.990 & 74.650 \\
\hline (1s) $j=.0$ & 4.812 & gypsum 020 & 3 & 15.185 & 71.929 \\
\hline$(1 s) j=.0$ & 4.812 & mica 002 & 4 & 19.942 & 74.840 \\
\hline$\langle 1 S) j=.0$ & 4.911 & quartz 110 & $I$ & 4.912 & 88.844 \\
\hline$j j=.0$ & 4.911 & gypsum 002 & 1 & 4.990 & 79.791 \\
\hline$j j=.0$ & 4.911 & gypsum 020 & 3 & 15.185 & 75.985 \\
\hline (1S) $j=.0$ & 4.911 & mica 002 & 4 & 19.942 & 80.082 \\
\hline$j=.0$ & 4.911 & $\operatorname{TAP} 100$ & 5 & 25.763 & 72.385 \\
\hline$j=.0$ & 4.911 & RAP 100 & 5 & 26.116 & 70.090 \\
\hline$j j=.0$ & 5.277 & ADP 101 & 2 & 10.640 & 82.710 \\
\hline$j=.0$ & 5.277 & beryl 100 & 3 & 15.954 & 82.881 \\
\hline$j=.0$ & 5.277 & $\operatorname{KAP} 100$ & 5 & 26.634 & 82.159 \\
\hline $1 S) j=.0$ & 5.397 & $\mathrm{NaCl} 200$ & 1 & 5.641 & 73.087 \\
\hline
\end{tabular}


Kr27 Ne-1ike 2S2 2P5 ( 2P*1) 4S (11*) j=1.0 - 2S2 2P6 Kr27 Ne-1ike 2S2 2P5 ( 2P*2) 4S (22*) j=1.0 - 2S2 2P6 Kr27 Ne-like 2S 2P6 $\cdots \quad 3 P\left(1 P^{*}\right) j=1.0-2 S 22 P 6$ $\mathrm{K} 227$ Ne-like 2S 2P6 3P (1P*) $j=1.0-2522 \mathrm{P} 6$ Kr27 Ne-like 2S 2P6 3P $\left(1 P^{\star}\right) j=1.0-2 S 2$ 2P6 Kr27 Ne-like 2S 2P6 3P (1P*) j=1.0 - 2S2 2P6 Kr27 Ne-like 2S 2P6

Kr27 Ne-like 2S 2P6

Kr27 Ne-like 2S $2 P 6$

Kr27 Ne-like 2S 2P6

Kr27 Ne-like 25 2P6

$\mathrm{Kr} 27$ Ne-like 2S 2P6

Kr27 Ne-like 2S $2 \mathrm{P} 6$

Kr27 Ne-like 2S 2P6

Kr27 Ne-like 2S 2P6

Kr27 Ne-like 2S $2 P 6$

$\mathrm{Kr27}$ Ne-like 2S 2P6

Kr27 Ne-like 2S $2 \mathrm{PG}$

Kr27 Ne-like 2S2 2P5 ( 2P*1) 3S $(11 *) j=1.0-2 S 2$ 2P6

Kr27 Ne-like 2S2 2P5 ( 2P*1) 3S $(11 *) j=1.0-2 S 2$ 2P6

Kr27 Ne-like 2S2 2P5 $(2 P * 2)$ 3S $\left(22^{*}\right) j=1.0-2 S 22 P 6$

Kr27 Ne-like 2S2 2P5 ( $2 P * 2) \quad 3 S(22 *) j=1.0-2 S 22 P 6$

$\mathrm{K} 2 \mathrm{28} F$-like $2 S$ 2P5 (3P*) 3P (2P) $j=1.5-2 S 2$ 2P5

$K r 28 F-l i k e$ 2S 2P5 (3P*) 3P (2P) j=1.5 - 2S2 2P5

$K r 28 \quad F-1 i k e$ 2S 2P5 ( 3P*) 3P (2P) $j=1.5-2 S 2$ 2P5

Kr28 F-like 2S 2P5 (3P*) 3P (2P) j=1.5 - 2S2 2P5

Kr28 F-like $2 S$ 2P5 (3P*) $3 P(2 S) j=.5-2 S 22 P 5$

$\mathrm{K}+28 \mathrm{~F}$-like $2 \mathrm{~S} 2 \mathrm{P5}\left(3 P^{*}\right) 3 P(2 S) j=.5-2 S 22 \mathrm{PS}$

Kr28 $F$-like 2S 2P5 ( 3P*) $3 P(2 S) j=.5-2 S 22 P 5$

Kr28 F-like 2S 2P5 (3P*) 3P (2S) $j=.5-2 S 2$ 2P5

Kr28 F-like 2S 2P5 ( $3 P *) 3 P(2 S) j=.5-2 S 22 P 5$

Kr28 F-like 2S 2P5 (3P*) 3P (2S) $j=.5-2 S 2$ 2P5

Kr28 $F$-like $2 S$ 2P5 (3P*) $3 P(2 S) j=.5-2 S 22 P 5$

$K \times 28 F$-like $2 S 2 P 5(3 P *) 3 P(2 S) j=.5-2 S 22 P 5$

Kr28 F-like 2S 2P5 (3P*) 3P (2S ) $j=.5-2 S 22 P 5$

Kr28 F-like 2S 2P5 (3P*) 3P (2S ) j=.5 - 2S2 2P5

Kr28 F-like 2S $2 P 5(3 P *) 3 P(2 S) j=.5-2 S 22 P 5$

Kr28 $F$-like 2S 2P5 ( $\left.3 P^{*}\right)$ 3P (2S) $j=.5$ - 2S2 2P5

$K r 28$ F-like 2S 2P5 (1P*) 3P (2D) j=2.5 - 2S2 2P5

Kr28 F-like 2S 2P5 ( $\left.1 P^{*}\right) 3 P(2 D) j=2.5-2 S 22 P 5$

$K r 28$ F-like 2S 2P5 ( $1 P *) ~ 3 P(2 D) j=2.5-2 S 22 P 5$

$K r 28 F-1 i k e$ 2S 2P5 (1P*) $3 P(2 D) j=2.5-2 S 22 P 5$

Kr28 F-like 2S 2P5 (1P*) 3P (2D) $j=2.5-2 S 22 P 5$

$\mathrm{K} r 28 \mathrm{~F}$-like 2S 2P5 ( $\left.1 P^{*}\right) 3 P(2 D) j=2.5-2 S 22 P 5$

$K \times 28$ F -like 2S 2P5 (3P*) 3P (4P) j=1.5 - 2S2 2P5

Kr28 $F$-like 2S $2 P 5\left(3 P^{*}\right) 3 P(4 P) j=1.5-2 S 22 P 5$ (1s) $j=.0$

(1S) $j=.0$

(1S) $j=.0$

(1S) $j=.0$

(1s) $j=.0$

(1s) $j=.0$

(1s) $j=.0$

(1s) $j=.0$

(1s) $j=.0$

(1s) $j=.0$

(1S ) $j=.0$

(1S) $j=.0$

(1s) $j=.0$

(1S) $j=.0$

(IS $) j=.0$

(1s) $j=.0$

(1s) $j=.0$

(1s) $j=.0$

(1S) $j=.0$

(1s) $j=.0$

(1s) $j=.0$

(1s) $j=.0$

$\left(2 P^{*}\right) j=1.5$

$\left(2 P^{\star}\right) j=1.5$

$\left(2 P^{\star}\right) j=1.5$

$\left(2 P^{\star}\right) j=1.5$

$\left(2 P^{*}\right) j=1.5$

$\left(2 P^{*}\right) j=1.5$

$\left(2 P^{\star}\right) j=1.5$

$\left(2 P^{\star}\right) j=1.5$

$\left(2 P^{\star}\right) j=1.5$

$\left(2 P^{*}\right) j=1.5$

$\left(2 P^{\star}\right) j=1.5$

$\left(2 P^{*}\right) j=1.5$

$\left(2 P^{\star}\right) j=1.5$

(2P*) $j=1.5$

$\left(2 P^{*}\right) j=1.5$

$\left(2 P^{\star}\right) j=1.5$

$\left(2 P^{*}\right) j=1.5$

$\left(2 P^{*}\right) j=1.5$

$\left(2 P^{*}\right) j=1.5$

$\left(2 P^{*}\right) j=1.5$

( $\left.2 P^{\star}\right) j=1.5$

$\left(2 P^{\star}\right) j=1.5$

$\left(2 P^{*}\right) j=.5$

$\left(2 P^{*}\right) j=.5$
$5.494 \mathrm{NaCl} 200$

$5.522 \mathrm{NaCl} 200$

$6.336 \mathrm{Ge} 111$

$6.336 \mathrm{KBr} 200$

6.336 quartz 101

6.336 graphite 002

6.336 mica 002

6.336 TAP 100

6.336 RAP 100

6.336 KAP 100

6.385 Ge 111

$6.385 \mathrm{KBr} 200$

6.385 quartz 101

6.385 graphite 002

6.385 mica 002

6.385 TAP 100

6.385 RAP 100

7.269 InSb 111

7.269 grpsum 020

7.507

gypsum 020

7.507 bery1 100

6.129 sylvite 200

6.129 fluorite 111

6.129 TAP 100

6.145 si 111

6.145 sylvite 200

6.145 fluorite 111

6.145 Ge 111

6.145 TAP 100

6.145 RAP 100

6.145 Si 111

6.145 sylvite 200

6.145 fluorite 111

6.145 Ge 111

6.145 TAP 100

6.145 RAP 100

6.166 Si 111

6.166 sylvite 200

6.166 fluorite 111

6.166 Ge 111

6.166 TAP 100

6.166 RAP 100

6.171 Si 111

6.171 sylvite 200
$5.641 \quad 76.891$

5.64178 .210

$6.532 \quad 75.929$

6.58474 .224

$6.687 \quad 71.354$

$6.696 \quad 71.127$

$19.942 \quad 72.395$

$25.763 \quad 79.652$

$26.116 \quad 76.034$

$26.634 \quad 72.095$

$6.532 \quad 77.822$

$6.584 \quad 75.877$

$6.687 \quad 72.715$

$6.696 \quad 72.469$

$19.942 \quad 73.850$

$25.763 \quad 82.456$

$26.116 \quad 77.944$

$26.634 \quad 73.521$

$7.481 \quad 76.327$

$15.185 \quad 73.214$

$15.185 \quad 81.393$

$15.954 \quad 70.234$

6.27177 .784

6.29276 .930

$6.308 \quad 76.318$

$25.763 \quad 72.101$

$6.271 \quad 78.495$

$6.292 \quad 77.591$

$6.308 \quad 76.947$

$6.532 \quad 70.178$

$25.763 \quad 72.570$

$26.116 \quad 70.252$

$6.271 \quad 78.495$

$6.292 \quad 77.591$

$6.308 \quad 76.947$

$6.532 \quad 70.178$

$25.763 \quad 72.570$

$26.116 \quad 70.252$

$6.271 \quad 79.500$

$6.292 \quad 78.514$

$6.308 \quad 77.820$

$\begin{array}{ll}6.532 & 70.729\end{array}$

$25 . .763 \quad 73.204$

$26.116 \quad 70.804$

$6.271 \quad 79.754$

6.29278 .745 
Kr28 F-like 2S 2P5 ( $\left.3 P^{*}\right) 3 P(4 P) j=1.5-2 S 22 P 5$ Kr28 F-like 2S 2P5 ( $\left.3 P^{*}\right) 3 P(4 P) j=1.5-2 S 22 P 5$ $K r 28 \quad F-1 i k e$ 2S 2P5 ( $\left.3 P^{*}\right)$ ) $3 P(4 P) j=1.5-2 S 22 P 5$ $\mathrm{K} 2 \mathrm{28} F$-like $2 \mathrm{~S} 2 \mathrm{P5}$ ( $\left.3 \mathrm{P}^{*}\right)$ ) $3 \mathrm{P}(4 \mathrm{P}) \mathrm{j}=1.5-2 \mathrm{~S} 2 \mathrm{PP}$ Kr28 F-like 2S 2P5 (3P*) 3P (4D) $j=1.5-2 S 2$ 2P5 Kr28 F-like $2 S$ 2P5 ( $\left.3 P^{*}\right)$ ) $3 P$ (4D) $j=1.5-2 S 2$ 2P5 Kr28 F-like 2S 2P5 (3P*) 3P (4D) $j=1.5-2 S 22 P 5$ Kr28 F-like 2S 2P5 (3P*) 3P (4D) $j=1.5-2 S 22 P 5$ Kr28 F-like 2S 2P5 (3P*) 3P (4D) j=1.5 - 2S2 2P5 Kr28 F-like 2S 2P5 (3P*) 3P (4D) $j=1.5-2 S 2$ 2P5 Kr28 F-like 2S 2P5 ( $\left.3 P^{*}\right)$ 3P (4D) $j=1.5-2 S 2$ 2P5 Kr28 F-like 2S 2P5 ( $\left.1 P^{*}\right) 3 P(2 S) j=.5-2 S 22 P 5$ Kr28 $F$-like 2S 2P5 ( $\left.1 P^{*}\right) 3 P(2 S) j=.5-2 S 22 P 5$ $\mathrm{K} \times 28 \mathrm{~F}-\mathrm{lijke} 2 \mathrm{~S} 2 \mathrm{P} 5\left(1 P^{*}\right) 3 P(2 S) j=.5-2 S 2$ 2P5 Kr28 F-like $2 S$ 2P5 ( $\left.1 P^{*}\right) 3 P(2 S) j=.5-2 S 22 P 5$ Kr28 F-like 2S 2P5 ( $\left.1 P^{*}\right) 3 P(2 S) j=.5-2 S 22 P 5$ Kr28 F-like 2S 2P5 ( 1P*) 3P (2S ) $j=.5-2 S 2$ 2P5 Kr28 $F$-like 2S 2P5 ( $\left.1 P^{*}\right) 3 P(2 S) j=.5-2 S 22 P 5$ $\mathrm{Kr} 28 \mathrm{~F}$-like 2S 2P5 (1P*) $3 P(2 S) j=.5-2 S 2$ 2P5 Kr28 F-like 2S $2 P 5\left(1 P^{*}\right) 3 P(2 S) j=.5-2 S 22 P 5$ Kr28 F-like 2S2 2P4 ( $1 D)$ 3D (2P ) $j=.5-2 S 2$ 2P5 Kr28 F-like 2S2 2P4 ( $1 D$ ) 3D (2P) $j=.5-2 S 2$ 2P5 $\mathrm{K} \times 28 \mathrm{~F}$-like 2S2 2P4 ( 1D ) 3D (2P) $j=.5-2 S 22 P 5$ Kr28 F-like 2S2 2P4 ( $1 D)$ 3D (2P) $j=.5-2 S 2$ 2P5 Kr28 F-like 2S2 2P4 ( 1D) 3D (2P) j=.5 - 2S2 2P5 Kr28 F-1ike 2S2 2P4 ( $1 D)$ 3D (2P) $j=.5-2 S 22 P 5$ Kr28 F-like 2S2 2P4 ( $1 D)$ 3D (2P ) $j=.5-2 S 22 P 5$ $K=28 \quad F-1 i k e$ 2S2 2P4 ( $1 D)$ 3D (2P) $j=.5-2 S 22 P 5$ Kr28 F-like 2S2 2P4 ( $1 D)$ 3D (2D) $j=1.5-2 S 2$ 2P5 Kr28 F-like 2S2 2P4（1D） 3D (2D) j=1.5 - 2S2 2P5 $\mathrm{K} \times 28 \mathrm{~F}$-like 2S2 2P4 ( $1 D$ ) 3D (2D) $j=1.5-2 \mathrm{~S} 2$ 2P5 Kr28 F-like 2S2 2P4 ( $1 D$ ) 3D (2D) $j=1.5-2 S 2$ 2P5 Kr28 F-like 2S2 2P4 ( 1D) 3D (2D) j=1.5 - 2S2 2P5 Kr28 F-like 2S2 2P4 ( 1D ) 3D (2D) j=1.5 - 2S2 2P5 Kr28 F-like 2S2 2P4 ( $1 D)$ 3D (2D) $j=1.5-2 S 22 P 5$ Kr28 F-like 2S2 2P4 ( 1D) 3D (2D) j=1.5 - 2S2 2P5 Kr28 F-like 2S2 2P4 ( $1 S$ ) 3D (2D) $j=1.5-2 S 2$ 2P5 $\mathrm{K} r 28 \quad \mathrm{~F}$-1ike 2S2 2P4 ( $1 S$ ) 3D (2D) $j=1.5-2 S 22 \mathrm{P} 5$ $\mathrm{K} \times 28$ F-like 2S2 $2 P 4$ ( $1 S$ ) 3D (2D) $j=1.5-2 S 22 P 5$ $\mathrm{K} \times 28 \mathrm{~F}$-like 2S2 2P4 ( $1 S$ ) 3D (2D) $j=1.5-2 S 2$ 2P5 Kr28 F-iike 2S2 2P4 ( $1 S$ ) 3D (2D) $j=1.5-2 S 22 P 5$ Kr28 F-like 2S2 2P4 ( $1 S$ ) 3D (2D) $j=1.5-2 S 22 P 5$ Kr28 F-like 2S2 2P4 ( 15 ) 3D (2D) $j=1.5-2 S 22 P 5$ Kr28 F-like 2S2 2P4 ( 1D ) 3D (2F) j=2.5 - 2S2 2P5 $K r 28 \quad F-l i k e$ 2S2 2P4 ( $1 D)$ 3D (2F) j=2.5 - 2S2 $2 P 5$ $\mathrm{K} \times 28$ F-like 2S2 2P4 ( $1 D$ ) 3D ( $F$ F $j=2.5-2 S 2$ 2P5 $\left(2 P^{*}\right) j=.5$

$\left(2 P^{\star}\right) j=.5$

$\left(2 P^{\star}\right) j=.5$

$\left(2 P^{\star}\right) j=.5$

$\left(2 P^{*}\right) j=.5$

$(2 \mathrm{p*}) j=.5$

$\left(2 P^{*}\right) j=.5$

$\left(2 P^{\star}\right) j=.5$

$\left(2 P^{\star}\right) j=.5$

$\left(2 P^{\star}\right) j=.5$

$\left(2 P^{*}\right) j=1.5$

$\left(2 P^{\star}\right) j=1.5$

$\left(2 P^{*}\right) j=1$.

$\left(2 P^{\star}\right) j=1.5$

$(2 P *) j=1.5$

$\left(2 P^{\star}\right) j=1.5$

$\left(2 P^{\star}\right) j=1.5$

$\left(2 P^{*}\right) j=1.5$

$\left(2 P^{*}\right) j=1.5$

$\left(2 P^{\star}\right) j=1.5$

$\left(2 P^{*}\right) j=1.5$

$\left(2 P^{*}\right) j=1.5$

$\left(2 P^{\star}\right) j=1.5$

$\left(2 P^{*}\right) j=1.5$

$\left(2 P^{*}\right) j=1.5$

$\left(2 P^{*}\right) j=1.5$

$\left(2 P^{*}\right) j=1.5$

$\left(2 P^{*}\right) j=1.5$

$\left(2 P^{\star}\right) j=1.5$

$\left(2 P^{*}\right) j=1.5$

$\left(2 P^{*}\right) j=1.5$

$\left(2 P^{*}\right) j=1.5$

$\left(2 P^{\star}\right) j=1.5$

$(2 P *) j=1.5$

$\left(2 P^{\star}\right) j=1.5$

$\left(2 p^{\star}\right) j=.5$

$\left(2 P^{\star}\right) j=.5$

$\left(2 P^{\star}\right) j=.5$

$\left(2 P^{\star}\right) j=.5$

$\left(2 P^{\star}\right) j=.5$

$\left(2 P^{\star}\right) j=.5$

$\left(2 P^{*}\right) j=.5$

$\left(2 P^{\star}\right) j=1.5$

$\left(2 P^{\star}\right) j=1.5$

$(2 P \star) j=1.5$
6.171 fluorite $111 \quad 1$

6.171 Ge 111

6.171 TAP 100

6.171 RAP 100

6.214 Si 111

6.214 sylvite 200

6.214 Eluorite 111

6.214 Ge 111

$6.214 \mathrm{KBr} 200$

6.214 TAP 100

6.214 RAP 100

6.259 Si 111

6.259 sylvite 200

6.259 fluorite 111

$6.259 \mathrm{Ge} 111$

$6.259 \mathrm{KBr} 200$

6.259 mica 002

6.259 TAP 100

6.259

6.259 KAP 100

6.418 Ge 111

6.418 KBr 200

6.418 quartz 101

6.418 graphite 002

6.418 mica 002

6.418 TAP 100

6.418 RAP 100

6.418 KAP 100

6.428 Ge 111

$6.428 \mathrm{KBr} 200$

6.428 quartz 101

6.428 graphite 002

6.428 mica 002

6.428 TAP 100

6.428 RAP 100

6.428 KAP 100

6.449 Ge 111

6.449 KBr 200

6.449 quartz 101

6.449 graphite 002

6.449 mica 002

6.449 RAP 100

$6.449 \mathrm{KAP} 100$

6.466 Ge 111

$6.466 \mathrm{KBr} 200$

6.466 quartz 101
$6.308 \quad 78.037$

6.53270 .862

$25.763 \quad 73.359$

$26.116 \quad 70.938$

6.27182 .269

$6.292 \quad 80.969$

$6.308 \quad 80.096$

6.53272 .048

6.58470 .700

$25.763 \quad 74.752$

$26.116 \quad 72.130$

$6.271 \quad 86.455$

$6.292 \quad 84.129$

$6.308 \quad 82.854$

$6.532 \quad 73.377$

$6.584 \quad 71.923$

$1.9 .942 \quad 70.319$

$25.763 \quad 76.356$

$26.116 \quad 73.465$

$26.634 \quad 70.052$

6.53279 .280

$6.584 \quad 77.107$

$6.687 \quad 73.693$

$6.696 \quad 73.432$

$19.942 \quad 74.906$

$25.763 \quad 85.183$

$26.116 \quad 79.420$

26.63474 .554

$6.532 \quad 79.762$

$6.584 \quad 77.503$

$6.687 \quad 74.001$

$6.696 \quad 73.735$

$19.942 \quad 75.241$

$25.763 \quad 86.394$

$26.116 \quad 79.909$

$26.634 \quad 74.880$

6.53280 .856

$\begin{array}{lll}6.584 & 78.377\end{array}$

$6.687 \quad 74.668$

6.69674 .389

$19.942 \quad 75.969$

$26.116 \quad 81.022$

26.63475 .589

$6.532 \quad 81.848$

$6.584 \quad 79.136$

6.68775 .229

Page 141 
Kr28 F-like 2S2 2P4 ( $1 D$ ) 3D (2F) j=2.5 - 2S2 2P5

Kr28 F-like 2S2 2P4 ( $1 D$ ) $3 D(2 F) j=2.5-2 S 22 P 5$

$\mathrm{K} \times 28$ F -like 2S2 $2 P 4$ ( $1 D$ ) 3D (2F) j=2.5 - 2S2 2P5

Kr28 F-like 2S2 2P4 ( $1 D$ ) 3D (2F) $j=2.5$ - 2S2 2P5

$K r 28$ F-like 2S2 $2 P 4(1 D), 3 D(2 S) j=.5-2 S 22 P 5$

Kr28 F-like 2S2 2P4 ( 1D) 3D (2S) $j=.5-2 S 22 P 5$

Kr28 F-like 2S2 2P4 ( 1D ) 3D (2S) j=.5-2S2 2P5

$k=28$-like $2 S 22 P 4(1 D) 3 D(2 S) j=.5-2 S 22 P 5$

Kr28 F-like 2S2 2P4 ( $1 D$ ) $3 D(2 S) j=.5-2 S 22 P 5$

Kr28 F-like 2S2 2P4 ( 1D) 3D (2S) $j=.5-2 S 2$ 2P5

Kr28 F-like 2S2 2P4 ( $1 D)$ 3D (2S) $j=.5-2 S 22 P 5$

$\mathrm{K} 2 \mathrm{28} F$-1ike 2S2 2P4 ( $3 \mathrm{P}) 3 \mathrm{D}(2 \mathrm{~F}) \mathrm{j}=2.5-2 S 22 \mathrm{P5}$

$\mathrm{K} \times 28$ F-like 2S2 2P4 ( 3P ) 3D (2F) $j=2.5-2 S 22 P 5$

Kr28 F-like 2S2 2P4 ( $3 \mathrm{P}$ ) 3D $\langle 2 F) j=2.5-2 \mathrm{~S} 22 \mathrm{P} 5$

$K r 28$ F-like $2 S 22 P 4(3 P) 3 D(2 F) j=2.5-2 S 22 P 5$

Kr28 F-like 2S2 $2 P 4$ ( $3 P$ ) 3D (2F) $j=2.5-2 S 22 P 5$

$\mathrm{K} \times 28 \mathrm{~F}$-1ike $2 \mathrm{~S} 2 \mathrm{2P} 4$ ( $3 \mathrm{P}$ ) $3 \mathrm{D}(2 \mathrm{~F}) j=2.5-2 S 22 \mathrm{P} 5$

Kr28 F-1ike 2S2 2P4 ( 3P) 3D (2F) $j=2.5-2 S 22 P 5$

$\mathrm{K} 2 \mathrm{28} F-1 \mathrm{kke} 2 \mathrm{~S} 22 \mathrm{P} 4(3 \mathrm{P}) 3 \mathrm{D}(4 \mathrm{~F}) \mathrm{j}=2.5-2 \mathrm{~S} 22 \mathrm{P} 5$

$K=28, F-1 i k e$ 2S2 2P4 ( $3 P$ ) $3 D(4 F) j=2.5-2 S 22 P 5$

$\mathrm{K} \times 28$ F-like $2 \mathrm{~S} 22 \mathrm{P} 4$ ( $3 \mathrm{P}$ ) $3 \mathrm{D}(4 \mathrm{~F}) \mathrm{j}=2.5-2 \mathrm{~S} 22 \mathrm{P} 5$

$\mathrm{K} \times 28 \mathrm{~F}$-1ike 2S2 2P4 ( $3 P$ ) 3D ( $4 \mathrm{~F}) j=2.5-2 \mathrm{~S} 22 \mathrm{P} 5$

Kr28 F-like 2S2 2P4 ( $3 P$ ) 3D ( $F F) j=2.5-2 S 22 P 5$

$K i 28 \quad F-l i k e$ 2S2 2P4 ( $3 P$ ) $3 D(4 F) j=2.5-2 S 22 P 5$

$\mathrm{K} \times 28$ F-1ike 2S2 2P4 ( $3 \mathrm{P}) 3 \mathrm{D}(4 \mathrm{~F}) j=2.5-2 S 22 \mathrm{P} 5$

$\mathrm{K} 2 \mathrm{28} \quad \mathrm{F}$-like 2S2 2P4 ( 1D ) 3D (2P) $j=.5-2 S 22 \mathrm{P} 5$

$K \times 28 \quad F-1 i k e$ 2S2 2P4 ( $1 D)$ 3D (2P) $j=.5-2 S 22 P 5$

$K r 28 \quad F-1 i k e$ 2S2 2P4 ( $1 D$ ) 3D (2P $j j=.5-2 S 22 P 5$

$K \times 28$-like 2S2 2P4 ( $1 D$ ) 3D (2P $/ j=.5-2 S 22 P 5$

$\mathrm{K} \times 28 \mathrm{~F}$-1ike $2 \mathrm{~S} 22 \mathrm{P} 4$ ( $1 S$ ) $3 \mathrm{D}$ (2D) $\mathrm{j}=2.5-2 \mathrm{~S} 22 \mathrm{P} 5$

Kr28 F-like 2S2 2P4 (1S) 3D (2D) j=2.5 - 2S2 2P5

$\mathrm{K} \times 28 \quad F-1$-ike 2S2 2P4 ( $1 S$ ) $3 D$ (2D) $j=2.5-2 S 22 P 5$

$\mathrm{K} x 28$ F-like 2S2 2P4 ( $1 S$ ) 3D (2D) $j=2.5-2 S 22 \mathrm{P5}$

Kr28 F-like 2S2 2P4 ( 3P) ) 3D (4F) j=1.5 - 2S2 2P5

Kr28 F-like 2S2 2P4 (3P) $3 D(4 F) j=1.5-2 S 22 P 5$

$\mathrm{K} 2 \mathrm{28} F$-like 2S2 2P4 ( $3 \mathrm{P}) 3 \mathrm{D}(4 F) j=1.5-2 S 22 \mathrm{P} 5$

Kr28 F-like 2S2 2P4 ( 3P) 3D (4F) $j=1.5-2 S 22 P 5$

Kr28 F-like 2S2 2P4 (3P) 3D (2D) $j=2.5-2 S 2$ 2P5

Kr28 $F-1 i k e$ 2S2 2P4 (3P) 3D (2D) $j=2.5-2 S 22 P 5$

$\mathrm{Kr} 28 \mathrm{~F}$-like $2 S 22 \mathrm{P} 4(3 \mathrm{P})$ 3D (4P) $j=1.5-2 S 22 \mathrm{P} 5$

$\mathrm{K} 228$ F-like 2S2 2P4 ( $3 P$ ) 3D (4P) $j=1.5-2 S 22 \mathrm{PS}$

$\mathrm{K}+28$ F-1ike 2S2 2P4 (1S) $3 S(2 \mathrm{~S}) j=.5-2 S 2$ 2P5

$\mathrm{K} 2 \mathrm{28}$ F -1ike 2S2 2P4 (1D) 3S (2D) $j=1.5$ - 2S2 $2 P 5$

$\mathrm{K} \times 28$ F-like 2S2 2P4 ( $1 \mathrm{D}) 3 \mathrm{~S}$ (2D) $\mathrm{j}=1.5-2 \mathrm{~S} 22 \mathrm{P} 5$

$\mathrm{K} \times 28$ F-like 2S2 2P4 (3P) 3S (2P) $j=1.5-2 S 22 \mathrm{P} 5$

$\mathrm{K} \times 28 \cdot \mathrm{F}-1 \mathrm{kke} 2 \mathrm{~S} 2 \mathrm{PP} 4(3 \mathrm{P}) 3 \mathrm{~S}(2 \mathrm{P}) j=1.5-2 \mathrm{~S} 22 \mathrm{P} 5$ $\left(2 P^{\star}\right) j=1.5$ $\left(2 P^{*}\right) j=1.5$ $\left\langle 2 P^{*}\right\rangle j=1.5$

(2P*) $j=1.5$

(2P*) $j=1.5$

(2q*) $j=1.5$

$\left(2 P^{*}\right) j=1.5$

$\left(2 D^{*}\right) j=1.5$

(2P*) $j=1.5$

(2P*) $j=1.5$

$\left(2 P^{*}\right) j=1.5$

$\left(2 P^{*}\right) j=1.5$

$\left(2 P^{\star}\right) j=1.5$

$(2 p *) j=1.5$

$\left(2 P^{*}\right) j=1.5$

$\left(2 P^{*}\right) j=1.5$

$(2 p *) j=1.5$

$\left(2 P^{\star}\right) j=1.5$

(2P*) $j=1.5$

(2P*) $j=1.5$

$\left(2 P^{*}\right) j=1.5$

$\left(2 P^{*}\right) j=1.5$

$\left(2 P^{*}\right) j=1.5$

(2P*) $j=1.5$

$\left(2 P^{*}\right) j=1.5$

$\left(2 P^{*}\right) j=.5$

$\left(2 P^{*}\right) j=.5$

$(2 P *) j=.5$

$\left(2 P^{*}\right) j=.5$

$\left(2 P^{*}\right\rangle j=1.5$

$\left(2 P^{*}\right) j=1.5$

$\left(2 P^{*}\right) j=1.5$

$(2 P *) j=1.5$

$\left(2 P^{*}\right) j=1.5$

$\left(2 P^{*}\right) j=1.5$

$(2 p *) j=1.5$

$\left(2 p^{*}\right) j=1.5$

$\left\langle 2 p^{*}\right\rangle j=1.5$

(2P*) j=1. 5

$\left(2 P^{*}\right) j=1.5$

$\left(2 P^{*}\right) j=1.5$

$\left(2 P^{*}\right) j=1.5$

$\left(2 P^{*}\right) j=5$

$\left(2 P^{\star}\right) j=.5$

$\left(2 P^{*}\right) j=1.5$

$\left(2 P^{*}\right\rangle j=1.5$
6.466 graphite 0021

6.466 mica 002

$6.466 \operatorname{RAP} 100$

6.466 KAP 100

6.479 Ge 111

$6.479 \mathrm{KBr} 200$

6.479 quartz 101

479 graphite 002

6.479 mica 002

6.479 RAP 100

6.479 KAP 100

6.502 Ge 111

$6.502 \mathrm{KBr} 200$

6.502 quartz 101

graphite 0021

6.502 mica 002

6.502 RAP 100

6.502 KAP 100

6.519 Ge 111

$6.519 \mathrm{KBr} 200$

6.519 quartz 101

6.519 graphite 002

6.519 mica 002

6.519 RAP 100

6.519 KAP 100

6.614 quartz 101

614 graphite 0021

6.614 mica 002

6.614 KAP 100

6.626 quartz 101

.626 graphite 002

.626 mica 002

$6.626 \operatorname{KAP} 100$

6.639 quartz 101

6.639 graphite 002

6.639 mica 002

$6.639 \mathrm{KAP} 100$

6.663 quartz 101

6.663 graphite 002

6.678 quartz 101

6.678 graphite 002

.123 InSb 111

.162 InSb 111

7.162 gypsum 020

7.193 InSb 111

7.193 gypsum 020
$6.696 \quad 74.939$

$19.942 \quad 76.586$

$26.116 \quad 82.034$

$26.634 \quad 76.189$

$6.532 \quad 82.696$

$6.584 \quad 79.754$

$6.687 \quad 75.672$

$6.696 \quad 75.374$

$19.942 \quad 77.078$

$26.116 \quad 82.905$

26.63476 .666

$6.532 \quad 84.507$

$6.584 \quad 80.948$

$6.687 \quad 76.491$

$6.696 \quad 76.174$

$19.942 \quad 77.997$

$26.116 \quad 84.788$

$26.634 \quad 77.553$

6.53286 .385

$6.584 \quad 81.942$

$6.687 \quad 77.130$

$6.696 \quad 76.797$

19.94278 .723

$26.116 \quad 86.829$

$26.634 \quad 78.251$

$6.687 \quad 81.526$

$6.596 \quad 81.024$

$19.942 \quad 84.260$

$26.634 \quad 83.372$

$6.687 \quad 82.255$

$6.696 \quad 81.708$

$19.942 \quad 85.408$

$26.634 \quad 84.337$

$6.687 \quad 83.131$

$6.696 \quad 82.519$

$19.942 \quad 87.131$

$26.634 \quad 85.614$

$6.687 \quad 85.144$

$6.696 \quad 84.309$

$6.687 \quad 87.027$

$6.696 \quad 85.798$

$\begin{array}{ll}7.481 & 72.203\end{array}$

$\begin{array}{ll}7.481 & 73.208\end{array}$

$15.185 \quad 70.613$

$7.481 \quad 74.050$

$15.185 \quad 71.331$

Page 142 


\begin{tabular}{|c|c|c|c|c|c|c|}
\hline $\mathrm{Kr} 28$ & $F$-like & $2 s 2$ & $2 P 4) 3 P$ & ) 35 & $(4 P) j=2.5$ & $-2 s 2$ \\
\hline $\mathrm{K} 228$ & F-like & 252 & $2 P 4) 3 P$ & $3 S$ & $(4 P) j=2.5$ & $-2 s 2$ \\
\hline $\mathrm{k} \times 35$ & He-like & 15 & $3 P$ & & $\left(3 P^{*}\right) j=1.0$ & $-1 \$ 2$ \\
\hline $\mathrm{Kr} 35$ & He-like & 1s & $3 P$ & & $\left(3 P^{\star}\right) j=1.0$ & $-1 s 2$ \\
\hline $\mathrm{kr} 35$ & He-like & is & $3 P$ & & $\left(3 P^{*}\right) j=1.0$ & $-1 s 2$ \\
\hline $\mathrm{kr} 35$ & He-like & 15 & $3 P$ & & $\left(3 P^{\star}\right) j=1.0$ & $-1 s 2$ \\
\hline $\mathrm{Kr} 35$ & He-like & 1s & $3 P$ & & $\left(3 R^{\star}\right) j=1.0$ & $-1 S 2$ \\
\hline $\mathrm{K} r 35$ & He-like & 15 & $3 P$ & & $\left(3 P^{*}\right) j=1.0$ & $-1 s 2$ \\
\hline $\mathrm{K} \times 35$ & He-like & 15 & $3 P$ & & $\left(3 P^{*}\right) j=1.0$ & $-1 s 2$ \\
\hline $\mathrm{K} \times 35$ & He-like & $1 S$ & $2 P$ & & $\left(1 p^{\star}\right) j=1.0$ & $-1 s 2$ \\
\hline $\mathrm{Kr} 35$ & He-like & $1 \mathrm{~S}$ & $2 P$ & & $\left(1 P^{\star}\right) j=1.0$ & -1.52 \\
\hline $\mathrm{K} \times 35$ & He-like & 1s & $2 P$ & & $\left(1 P^{*}\right) j=1.0$ & $-1 s 2$ \\
\hline $\mathrm{K} \times 35$ & He-like & $1 s$ & $2 P$ & & $\left(1 \mathrm{P}^{*}\right) j=1.0$ & $-1 s 2$ \\
\hline $\mathrm{K} r 35$ & He-like & IS & $2 P$ & & $\left(1 P^{\star}\right) j=1.0$ & $-1 s 2$ \\
\hline $\mathrm{K} \times 35$ & He-like & 15 & $2 P$ & & $\left(1 P^{*}\right) j=1.0$ & -152 \\
\hline $\mathrm{K} \times 35$ & He-like & 1S & $2 P$ & & $\left(3 P^{*}\right) j=1.0$ & $-1 s 2$ \\
\hline $\mathrm{k} \times 35$ & Fe-like & $1 \mathrm{~s}$ & $2 P$ & & $\left(3 P^{*}\right) j=1.0$ & -152 \\
\hline$K \times 35$ & $\mathrm{He}$-1ike & 1s & $2 \mathrm{P}$ & & $\left(3 P^{\star}\right\rangle j=1.0$ & $-1 s 2$ \\
\hline $\mathrm{K} \times 35$ & He-like & 1s & $2 P$ & & $\left(3 P^{\star}\right) j=1.0$ & $-1 s 2$ \\
\hline $\mathrm{K} \times 35$ & He-like & 1S & $2 P$ & & $\left(3 P^{*}\right) j=1.0$ & $-1 S 2$ \\
\hline $\mathrm{K} \simeq 35$ & He-like & 1s & $2 P$ & & $\left(3 P^{\star}\right) j=1.0$ & -152 \\
\hline$K \times 35$ & He-like & 15 & $2 P$ & & $\left(3 P^{\star}\right) j=1.0$ & $-1 s 2$ \\
\hline $\mathrm{K} \times 35$ & He-like & 1s & $2 P$ & & $\left(3 P^{\star}\right) j=1.0$ & $-1 s 2$ \\
\hline $\mathrm{K} \times 35$ & He-like & 1s & $2 \mathrm{P}$ & & $\left(3 P^{\star}\right) j=1.0$ & -152 \\
\hline $\mathrm{K} \times 35$ & He-like & is & $2 S$ & & (3s) $j=1.0$ & $-1 s 2$ \\
\hline $\mathrm{Kr} 35$ & He-like & is & $2 \mathrm{~s}$ & & (3s) $j=1.0$ & $-1 s 2$ \\
\hline $\mathrm{K} \times 35$ & He-like & is & $2 s$ & & $(3 s) j=1.0$ & -152 \\
\hline $\mathrm{K} \times 35$ & He-like & 15 & $2 S$ & & $(3 s) j=1.0$ & $-1 S 2$ \\
\hline $\mathrm{Kr} 35$ & He-like & $1 \mathrm{~S}$ & $2 S$ & & $(3 S) j=1.0$ & $-1 s 2$ \\
\hline $\mathrm{K} r 35$ & He-like & 1s & $2 s$ & & $(3 s) j=1.0$ & $-1 s 2$ \\
\hline$k \times 35$ & He-like & 15 & 25 & & $(3 s) j=1.0$ & -152 \\
\hline $\mathrm{K} \times 35$ & He-like & $1 \mathrm{~s}$ & 25 & & $(3 s) j=1.0$ & $-1 s 2$ \\
\hline $\mathrm{Kr} 35$ & He-iike & 1s & $2 s$ & & $(3 S) j=1.0$ & $-1 s 2$ \\
\hline$k r 36$ & H -like & $3 P$ & & & $\left(2 P^{*}\right) j=1.5$ & -15 \\
\hline $\mathrm{R} \times 36$ & H -like & $3 P$ & & & $\left(2 P^{\star}\right) j=1.5$ & $-1 S$ \\
\hline $\mathrm{k} \times 36$ & H -like & $3 P$ & & & $\left(2 \mathrm{P}^{\star}\right) j=1.5$ & $-1 s$ \\
\hline $\mathrm{K} \times 36$ & H -like & $3 P$ & & & $\left(2 P^{\star}\right) j=1.5$ & $-1 S$ \\
\hline $\mathrm{k} \times 36$ & $\mathrm{H}$-like & $3 P$ & & & $\left(2 P^{*}\right) j=1.5$ & -15 \\
\hline $\mathrm{K} \times 36$ & H-like & $3 P$ & & & $\left(2 p^{*}\right) j=1.5$ & $-1 S$ \\
\hline $\mathrm{Kr} 36$ & H -like & $3 P$ & & & $\left(2 P^{*}\right) j=1.5$ & $-1 s$ \\
\hline $\mathrm{K} r 36$ & H -like & $2 P$ & & & $\left(2 P^{*}\right) j=1.5$ & $-1 s$ \\
\hline $\mathrm{Kr} 36$ & H -like & $2 P$ & & & $\left(2 P^{*}\right) j=1.5$ & $-1 S$ \\
\hline $\mathrm{K} \times 36$ & H -like & $2 P$ & & & $\left(2 \mathrm{P}^{*}\right) j=1.5$ & $-1 s$ \\
\hline $\mathrm{Kr} 36$ & H -like & $2 P$ & & & $\left(2 P^{\star}\right) j=1.5$ & -15 \\
\hline $\mathrm{k} \times 36$ & H -like & $2 P$ & & & $\left(2 p^{*}\right) j=1.5$ & -15 \\
\hline $\mathrm{Kr} 36$ & H -like & $2 \mathrm{P}$ & & & $\left(2 P^{\star}\right) j=1.5$ & $-1 s$ \\
\hline
\end{tabular}

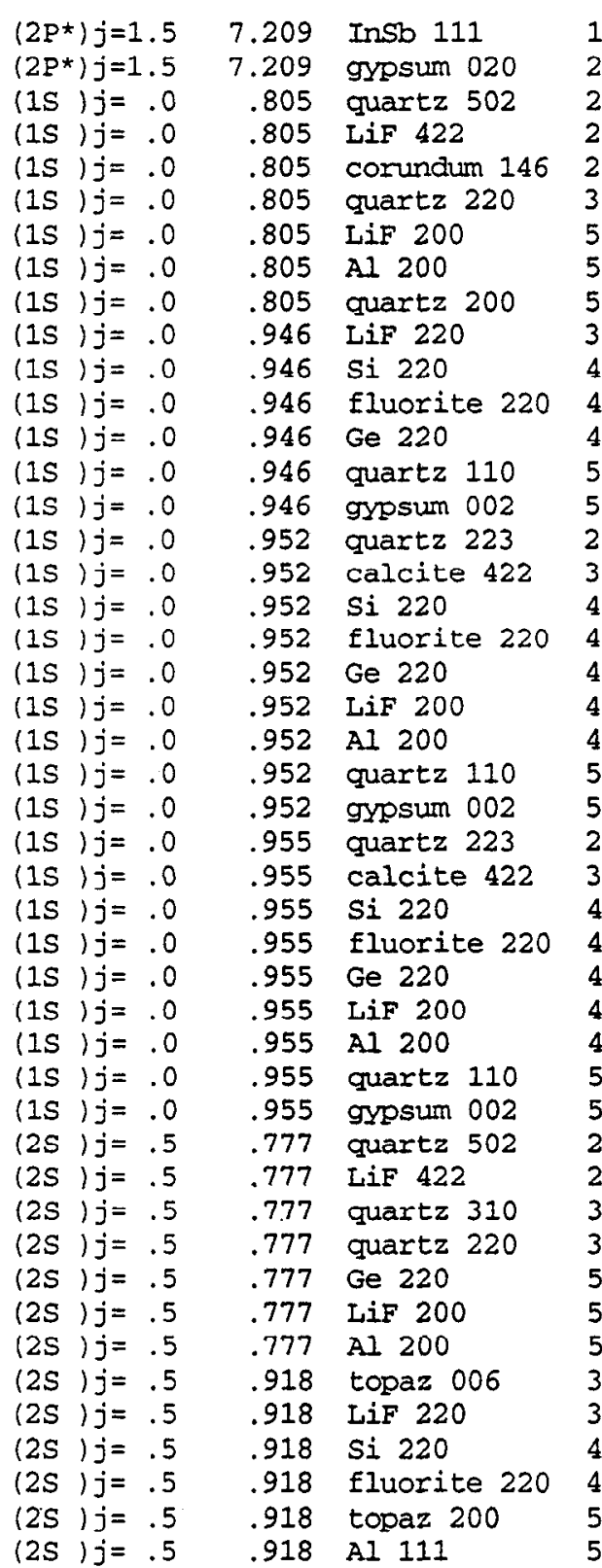

$\begin{array}{rl}7.481 & 74.502 \\ 15.185 & 71.712 \\ 1.624 & 82.471 \\ 1.652 & 77.053 \\ 1.660 & 75.902 \\ 2.451 & 80.168 \\ 4.027 & 88.194 \\ 4.048 & 83.889 \\ 4.246 & 71.433 \\ 2.848 & 85.197 \\ 3.840 & 80.203 \\ 3.862 & 78.465 \\ 4.000 & 71.085 \\ 4.912 & 74.354 \\ 4.990 & 71.423 \\ 2.024 & 70.171 \\ 3.034 & 70.276 \\ 3.840 & 82.598 \\ 3.862 & 80.407 \\ 4.000 & 72.176 \\ 4.027 & 71.017 \\ 4.048 & 70.171 \\ 4.912 & 75.709 \\ 4.990 & 72.536 \\ 2.024 & 70.678 \\ 3.034 & 70.786 \\ 3.840 & 84.150 \\ 3.862 & 81.542 \\ 4.000 & 72.746 \\ 4.027 & 71.549 \\ 4.048 & 70.678 \\ 4.912 & 76.436 \\ 4.990 & 73.120 \\ 1.624 & 73.116 \\ 1.652 & 70.166 \\ 2.360 & 81.009 \\ 2.451 & 71.997 \\ 4.000 & 76.228 \\ 4.027 & 74.739 \\ 4.048 & 73.685 \\ 2.795 & 80.174 \\ 2.848 & 75.238 \\ 3.840 & 72.989 \\ 3.862 & 71.953 \\ 4.638 & 81.750 \\ 4.676 & 78.994\end{array}$




\begin{tabular}{|c|c|c|}
\hline $\mathrm{K} \times 36$ & $\mathrm{H}$-like & 22 \\
\hline $\mathrm{K} \times 36$ & H -like & $2 P$ \\
\hline $\mathrm{K} \times 36$ & $\mathrm{H}$-like & $2 \mathrm{P}$ \\
\hline $\mathrm{K} \times 36$ & H -like & $2 P$ \\
\hline $\mathrm{Kr} 36$ & H -like & $2 P$ \\
\hline $\mathrm{Kr} 36$ & H -like & $2 P$ \\
\hline $\mathrm{Rb}$ & & K-alpha (1) \\
\hline $\mathrm{RB}$ & & $\mathrm{K}$-alpha (1) \\
\hline $\mathrm{Rb}$ & & K-alpha (1) \\
\hline $\mathrm{Rb}$ & & $\mathrm{K}$-alpha (1) \\
\hline $\mathrm{Rb}$ & & K-alpha (I) \\
\hline $\mathrm{Rb}$ & & K-alpha (1) \\
\hline $\mathrm{Rb}$ & & K-alpha (1) \\
\hline $\mathrm{Sr}$ & & $\mathrm{k}$-alpha (1) \\
\hline Sr & & K-alpha (1) \\
\hline Sr & & k-alpha (1) \\
\hline $\mathbf{Y}$ & & K-alpha (1) \\
\hline$Y$ & & K-alpha (1) \\
\hline $\mathrm{ZI}$ & & k-alpha (1) \\
\hline $\mathrm{Zr}$ & & K-alpha $\{1\}$ \\
\hline$\overline{\mathbf{z}} \mathbf{x}$ & & K-alpha(1) \\
\hline $\mathrm{Zr}$ & & K-alpha (1) \\
\hline $\mathrm{zr}$ & & K-alpha (1) \\
\hline $\mathrm{zr}$ & & K-alpha (1) \\
\hline $\mathrm{Zr}$ & & K-alpha (1) \\
\hline$\ddot{z} x$ & & k-alpha (1) \\
\hline $\mathrm{NB}$ & & k-alpha (1) \\
\hline $\mathrm{NB}$ & & $\mathrm{K}$-alpha (1) \\
\hline No & & K-alpha (1) \\
\hline $\mathrm{Nb}$ & & K-alpha (1) \\
\hline $\mathrm{Nb}$ & & $\mathrm{k}$-alpha (1) \\
\hline $\mathrm{Nb}$ & & K-alpha (1) \\
\hline Mo & & K-alpha (I) \\
\hline Mo & & K-alpha (1) \\
\hline Mo & & k-alpha (1 \\
\hline TC & & K-alpha (1 \\
\hline TC & & K-alpha (1 \\
\hline TC & & K-alpha ( 1 \\
\hline TC & & K-alpha (1) \\
\hline TC & & K-alpha ( 1 \\
\hline Ru & & K-alpha (1 \\
\hline Ru & & K-alpha(1 \\
\hline $\mathrm{Rh}$ & & K-alpha ( 1 \\
\hline Pd & & K-alpha (1 \\
\hline$P d$ & . & k-alpha (1 \\
\hline Pa. & - & k-alpha (1 \\
\hline
\end{tabular}

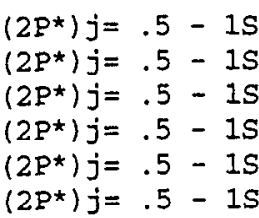

$\left(2 P^{\star}\right) j=.5-15$

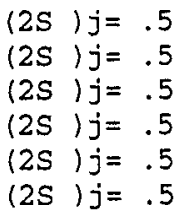

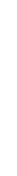

$\begin{array}{ll}2.795 & 82.179 \\ 2.848 & 76.473 \\ 3.840 & 74.041 \\ 3.862 & 72.937 \\ 4.638 & 84.292 \\ 4.676 & 80.735 \\ 2.795 & 83.458 \\ 2.848 & 77.161 \\ 3.840 & 74.615 \\ 3.862 & 73.471 \\ 4.638 & 86.237 \\ 4.676 & 81.783 \\ 4.912 & 70.421 \\ 1.801 & 70.020 \\ 4.476 & 70.975 \\ 4.246 & 85.264 \\ 1.660 & 87.050 \\ 4.246 & 77.447 \\ 1.624 & 75.462 \\ 1.652 & 72.096 \\ 1.660 & 71.260 \\ 2.360 & 87.641 \\ 2.451 & 74.166 \\ 4.000 & 79.265 \\ 4.027 & 77.399 \\ 4.048 & 76.132 \\ 2.310 & 75.717 \\ 2.360 & 71.543 \\ 3.034 & 79.668 \\ 3.082 & 75.572 \\ 3.840 & 76.316 \\ 3.862 & 75.034 \\ 2.217 & 73.701 \\ 2.848 & 85.009 \\ 3.636 & 77.261 \\ 2.712 & 84.608 \\ 2.748 & 79.275 \\ 2.749 & 79.166 \\ 2.795 & 75.019 \\ 2.848 & 71.448 \\ 2.024 & 72.404 \\ 2.712 & 71.536 \\ 3.082 & 84.251 \\ 1.801 & 77.237 \\ 2.360 & 82.919 \\ 2.451 & 72.849\end{array}$




$\begin{array}{ll}\text { Pd } & \text { K-alpha(1) } \\ \text { Pd } & \text { K-alpha(1) } \\ \text { Ag } & \text { K-alpha(1) } \\ \text { Ag } & \text { K-alpha(1) } \\ \text { Ag } & \text { K-alpha(1) } \\ \text { Cd } & \text { K-alpha(1) } \\ \text { Cd } & \text { K-alpha(1) } \\ \text { Cd } & \text { K-alpha(1) } \\ \text { Cd } & \text { K-alpha(1) } \\ \text { Cd } & \text { K-alpha(1) } \\ \text { Cd } & \text { K-alpha(1) } \\ \text { Cd } & \text { K-alpha(1) } \\ \text { Cd } & \text { K-alpha(1) } \\ \text { In } & \text { K-alpha(1) } \\ \text { In } & \text { K-alpha(1) } \\ \text { Sn } & \text { K-alpha(1) }\end{array}$

$\begin{array}{lllll}.586 & \text { calcite 422 } & 5 & 3.034 & 74.774 \\ .586 & \text { quartz 211 } & 5 & 3.082 & 71.781 \\ .559 & \text { Ge 422 } & 4 & 2.310 & 75.617 \\ .559 & \text { quartz 310 } & 4 & 2.360 & 71.466 \\ .559 & \text { LiF 220 } & 5 & 2.848 & 79.141 \\ .535 & \text { quartz 502 } & 3 & 1.624 & 81.227 \\ .535 & \text { LiF 422 } & 3 & 1.652 & 76.300 \\ .535 & \text { corundum 146 } & 3 & 1.660 & 75.210 \\ .535 & \text { Si 422 } & 4 & 2.217 & 74.855 \\ .535 \text { topaz 303 } & 5 & 2.712 & 80.525 \\ .535 \text { corundum 030 } & 5 & 2.748 & 76.764 \\ .535 \text { quartz 203 } & 5 & 2.749 & 76.676 \\ .535 \text { topaz 006 } & 5 & 2.795 & 73.150 \\ .512 \text { quartz 502 } & 3 & 1.624 & 71.084 \\ .512 & \text { topaz 303 } & 5 & 2.712 & 70.758 \\ .491 & \text { quartz 223 } & 4 & 2.024 & 75.828\end{array}$




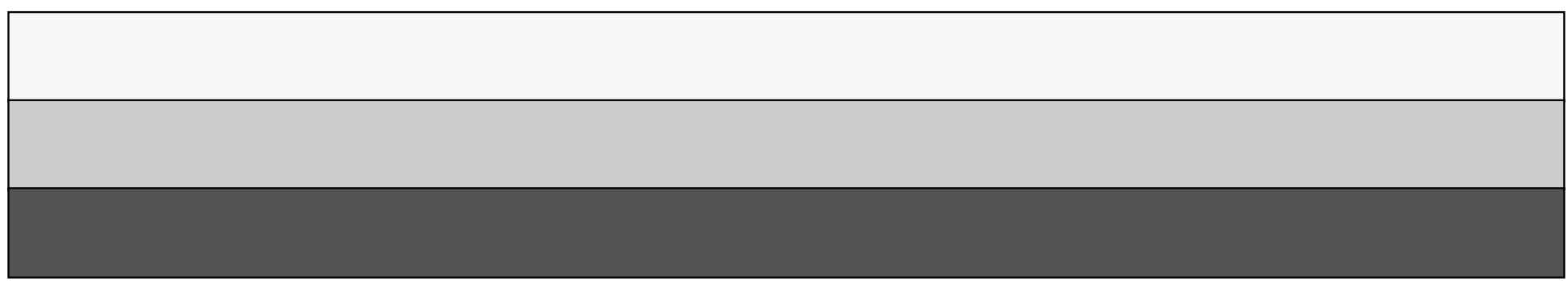

